\title{
Palladium-Catalyzed Regio- and Enantioselective Hydrosulfonylation of 1,3-Dienes with Sulfinic Acids: Scope, Mechanism, and Origin of Selectivity
}

\author{
Qinglong Zhang, Dongfang Dong and Weiwei Zi* \\ State Key Laboratory and Institute of Elemento-Organic Chemistry, College of Chemistry, Nankai \\ University, Tianjin 300071, China
}

Table of Contents

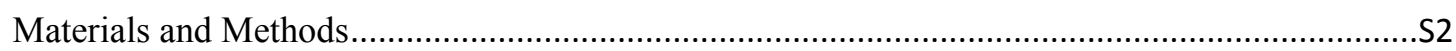

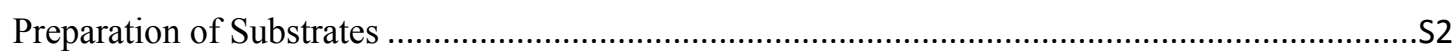

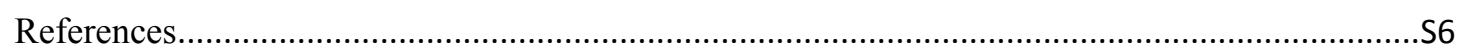

General Procedure for the Hydrosulfonylation Reaction. ......................................................S7

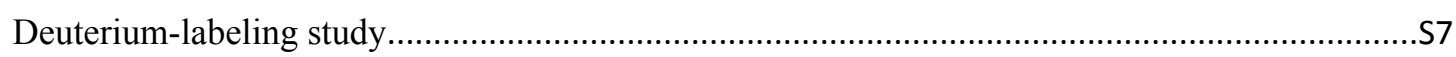

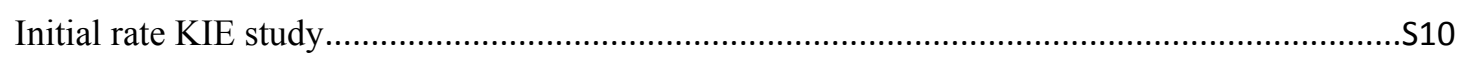

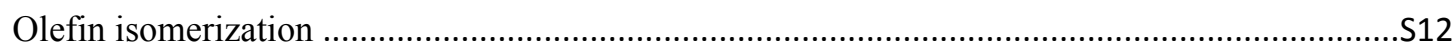

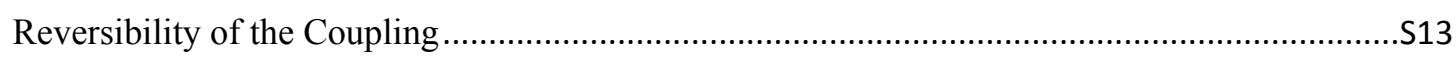

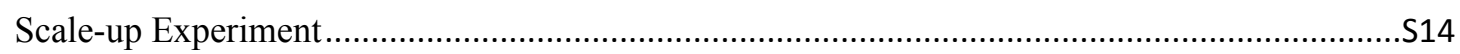

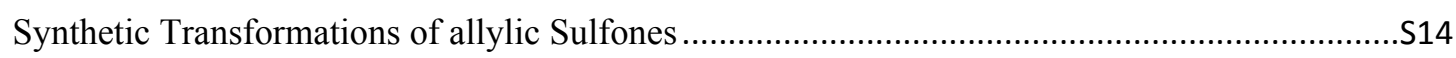

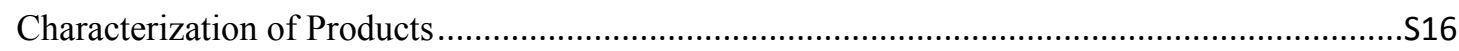

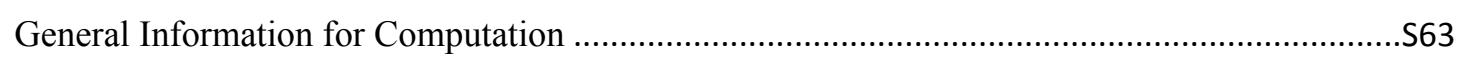

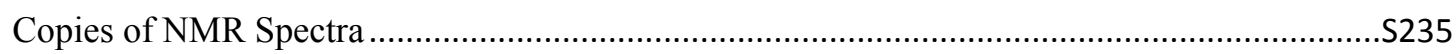




\section{Materials and Methods}

Unless otherwise noted, all reactions were assembled on a Schlenk vacuum line or in a glovebox using oven-dried glassware and were stirred with Teflon-coated magnetic stirring bars. All the ligands were purchased from Strem Chemicals or Sinocompound Catalysts Co., Ltd. and were used as received. Unless otherwise noted, reagents were obtained from commercial sources and used without further purification. THF, $\mathrm{Et}_{2} \mathrm{O}$, toluene were distilled with $\mathrm{Na}$ before using. Dichloromethane was distilled with $\mathrm{CaH}_{2}$ before using. All other dried solvents (DMF, $\mathrm{CH}_{3} \mathrm{CN}, \mathrm{PhH}$, 1,4-dioxane, $\mathrm{Et}_{3} \mathrm{~N}$, DIPEA) were purchased from MERYER (Shanghai) Chemical Technology Co., Ltd. TLC analysis of reaction mixtures was performed on huanghai silica gel 60 F254 TLC plates and visualized by $\mathrm{UV}, \mathrm{I}_{2} /$ silica, and/or ceric ammonium molybdate stain. All work-up and purification procedures were carried out with reagent grade solvents in air. Reaction temperatures above $23{ }^{\circ} \mathrm{C}$ refer to temperatures of an aluminum heating block or a silicon oil bath, which were controlled by an electronic temperature modulator from IKA. Flash chromatography was carried out withsilica gel 200-300 or 300-400 mesh. ${ }^{1} \mathrm{H}$ and ${ }^{13} \mathrm{C}$ NMR spectra were recorded with Bruker AV-400 spectrometers and were referenced to residual ${ }^{1} \mathrm{H}$ and ${ }^{13} \mathrm{C}$ signals of the deuterated

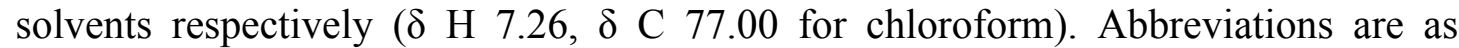
follows: s (singlet), d (doublet), t (triplet), q (quartet), m (multiplet), br (broad). The ee value was determined on Shimadzu LC-20A HPLC workstation (Daicel chiral columns Chiralpak IA, IC, ID, IE, IF (4.6 x $250 \mathrm{~mm})$ ). HRMS was performed on a Varian QFT-ESI instrumental. GS-Mass was performed on ThermoScientific TRACE1300 (ISQ). Optical rotation were determined on a IP-digi300/2 apparatus. Melting points were determined on anX-4B melting point apparatus.

\section{Preparation of Substrates}

The sulfinic acids used in this work are all known compounds and were prepared 
according to literature methods ${ }^{1}$. All 1,3-dienes used in this work were prepared according to literature procedure ${ }^{2}$ and were obtained in $E Z / E E$ mixture form. The ratios of $E Z / E E$ are listed as Figure S1. Pure $(E, Z)-\mathbf{1 a}$ and $(E, E)-\mathbf{1 a}$ used in the mechanistic studies were obtained according to literature methods ${ }^{2 b, 2 c}$.

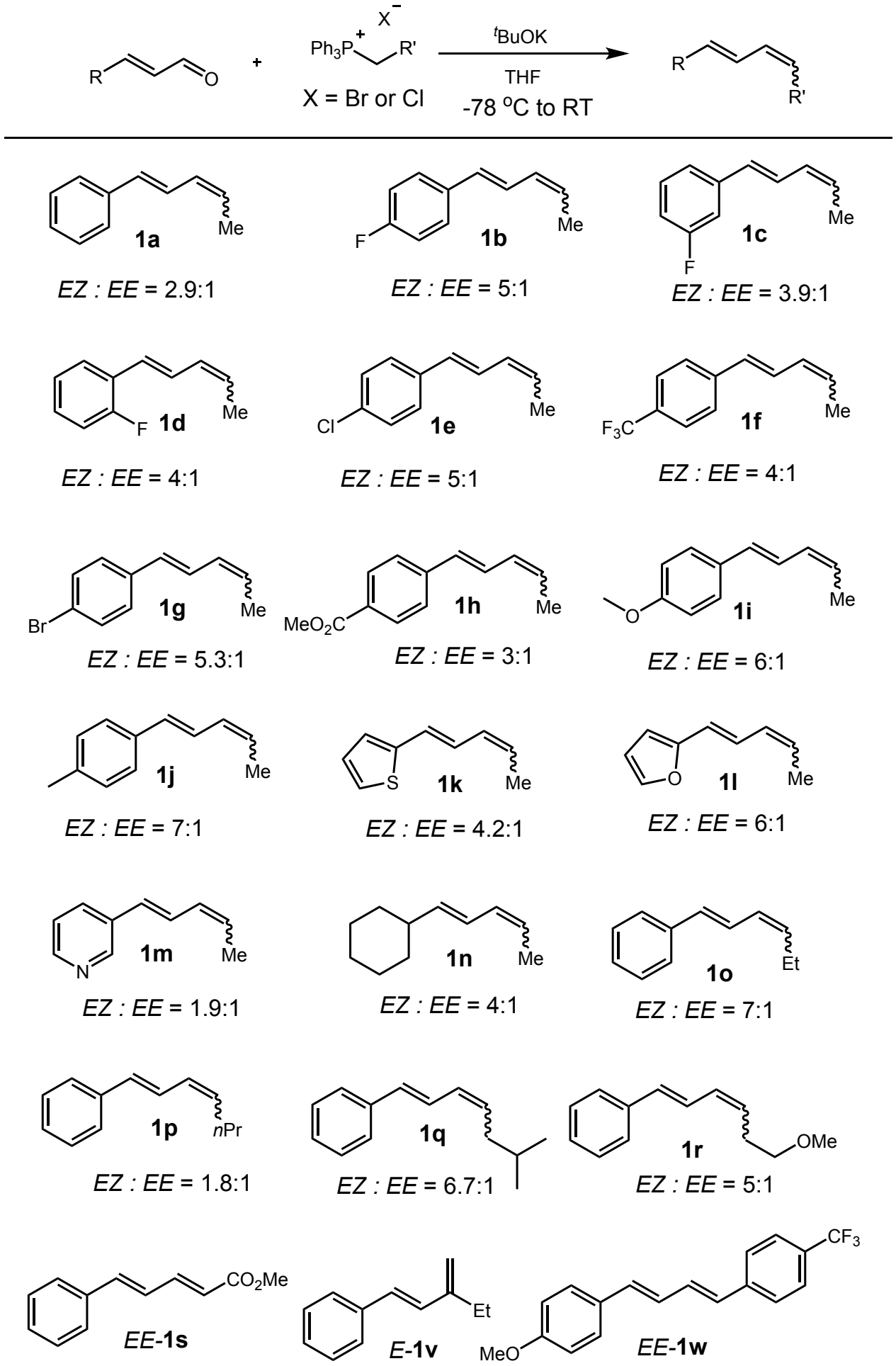

Figure S1 


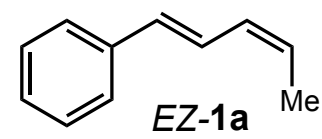

${ }^{1} \mathrm{H}$ NMR (400 MHz, $\left.\mathrm{CDCl}_{3}\right) \delta 7.41(\mathrm{~d}, J=7.5 \mathrm{~Hz}, 2 \mathrm{H}), 7.31(\mathrm{t}, J=7.5 \mathrm{~Hz}, 2 \mathrm{H}), 7.21(\mathrm{dd}, J=13.6,6.2$ $\mathrm{Hz}, 1 \mathrm{H}), 7.09$ (dd, $J=15.5,11.1 \mathrm{~Hz}, 1 \mathrm{H}), 6.52$ (d, $J=15.6 \mathrm{~Hz}, 1 \mathrm{H}), 6.18$ (t, $J=10.9 \mathrm{~Hz}, 1 \mathrm{H}), 5.60$ (dq, $J=14.5,7.2 \mathrm{~Hz}, 1 \mathrm{H}), 1.86(\mathrm{~d}, J=7.2 \mathrm{~Hz}, 3 \mathrm{H})$.

${ }^{13} \mathrm{C}$ NMR $\left(101 \mathrm{MHz}, \mathrm{CDCl}_{3}\right) \delta 137.7,131.9,129.6,128.6,127.3,127.2,126.3,124.2,13.6$.
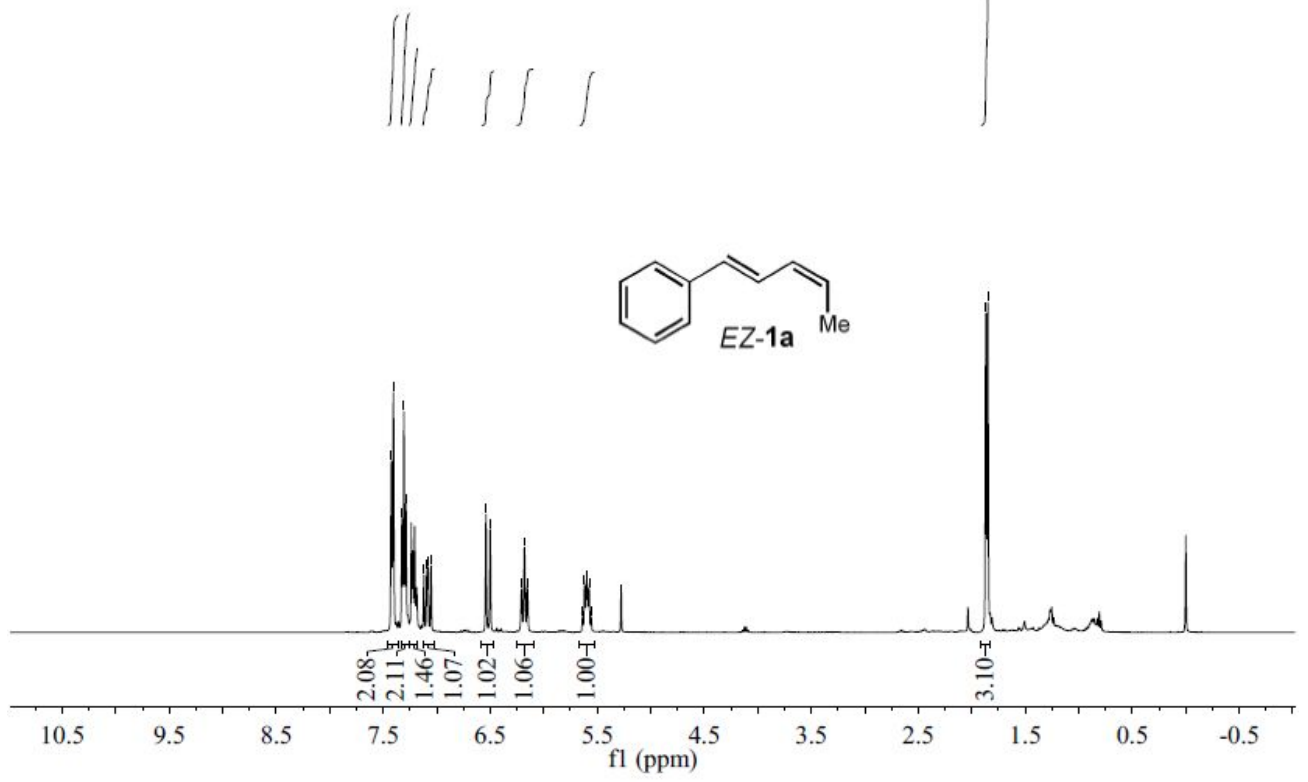

m.

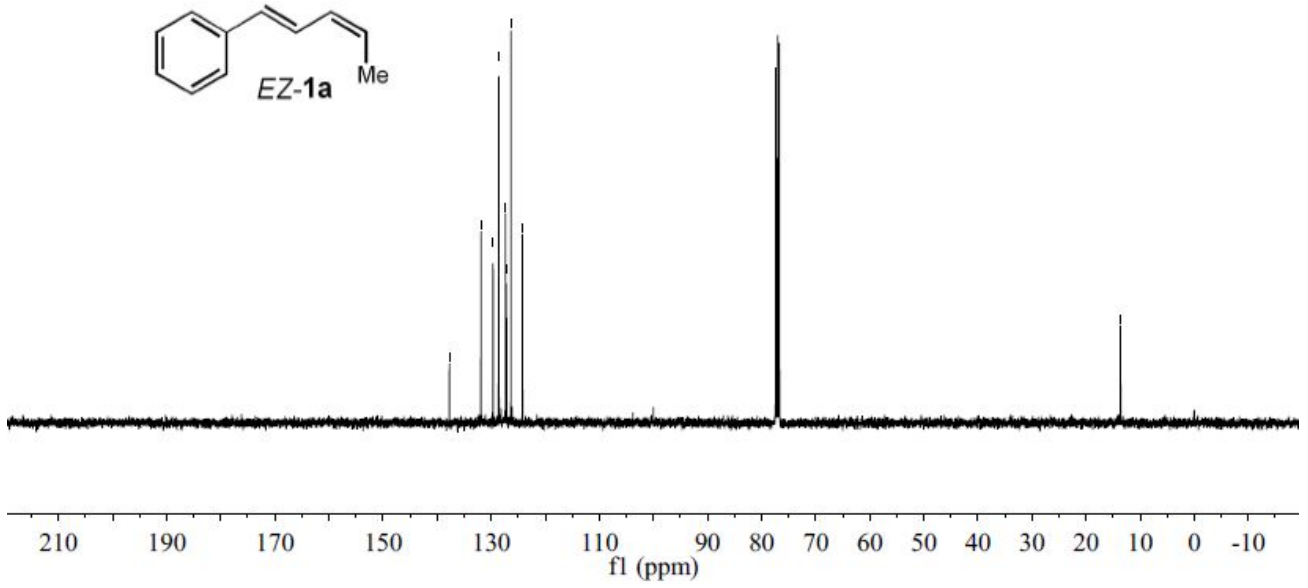




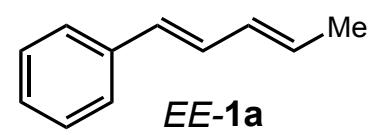

${ }^{1} \mathrm{H}$ NMR (400 MHz, $\left.\mathrm{CDCl}_{3}\right) \delta 7.37(\mathrm{~d}, J=7.4 \mathrm{~Hz}, 2 \mathrm{H}), 7.29(\mathrm{t}, J=7.5 \mathrm{~Hz}, 2 \mathrm{H}), 7.18(\mathrm{t}, J=7.2 \mathrm{~Hz}$, $1 \mathrm{H}), 6.74(\mathrm{dd}, J=15.6,10.5 \mathrm{~Hz}, 1 \mathrm{H}), 6.42(\mathrm{~d}, J=15.7 \mathrm{~Hz}, 1 \mathrm{H}), 6.42$ (d, $J=15.7 \mathrm{~Hz}, 1 \mathrm{H}), 6.27-6.15$ (m, 1H), $5.89-5.74(\mathrm{~m}, 1 \mathrm{H}), 1.82(\mathrm{~d}, J=6.7 \mathrm{~Hz}, 3 \mathrm{H})$.

${ }^{13} \mathrm{C}$ NMR (101 MHz, $\left.\mathrm{CDCl}_{3}\right) \delta 137.7,131.9,130.3,129.8,129.4,128.6,127.1,126.1,18.4$.
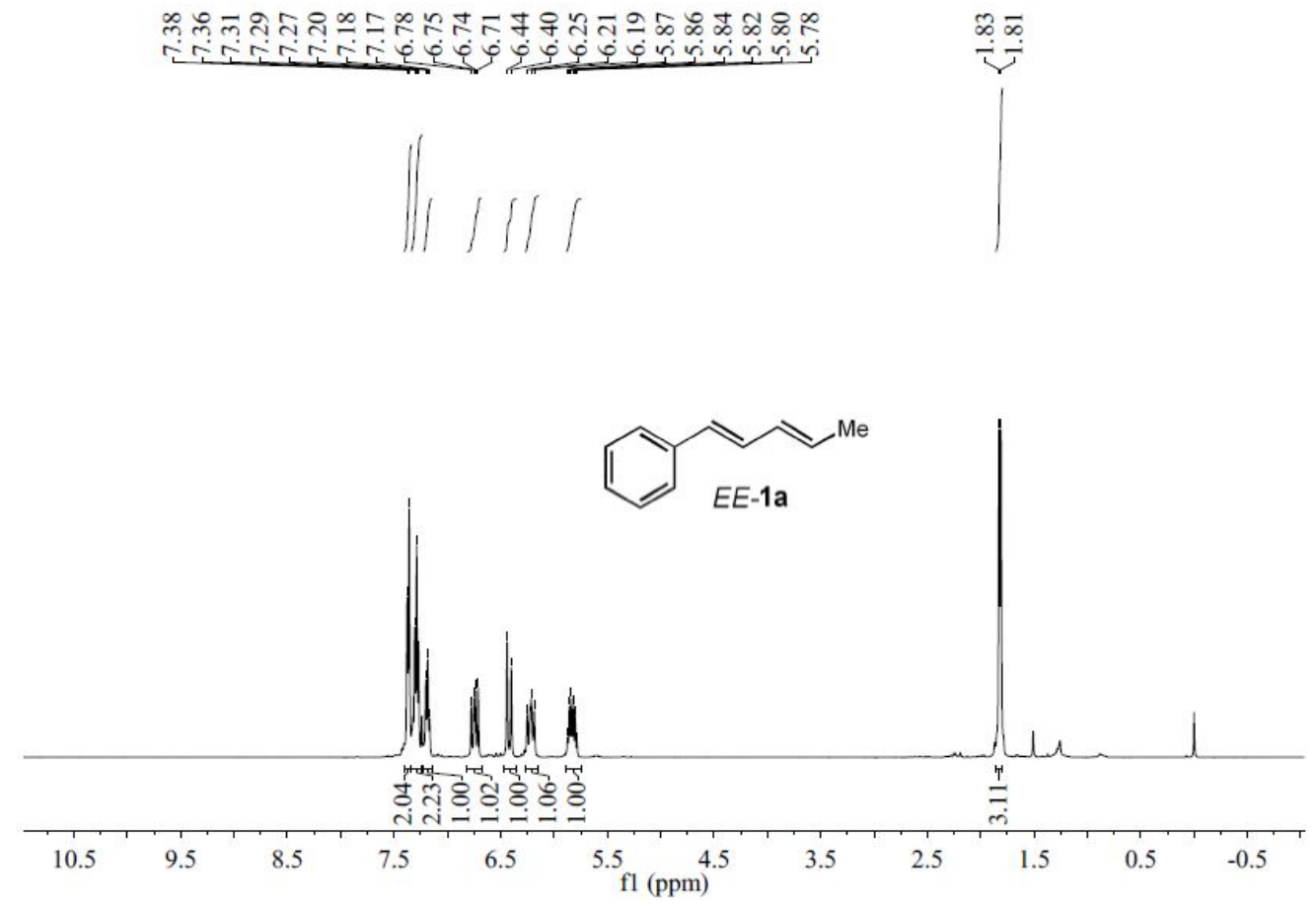

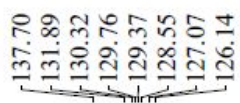

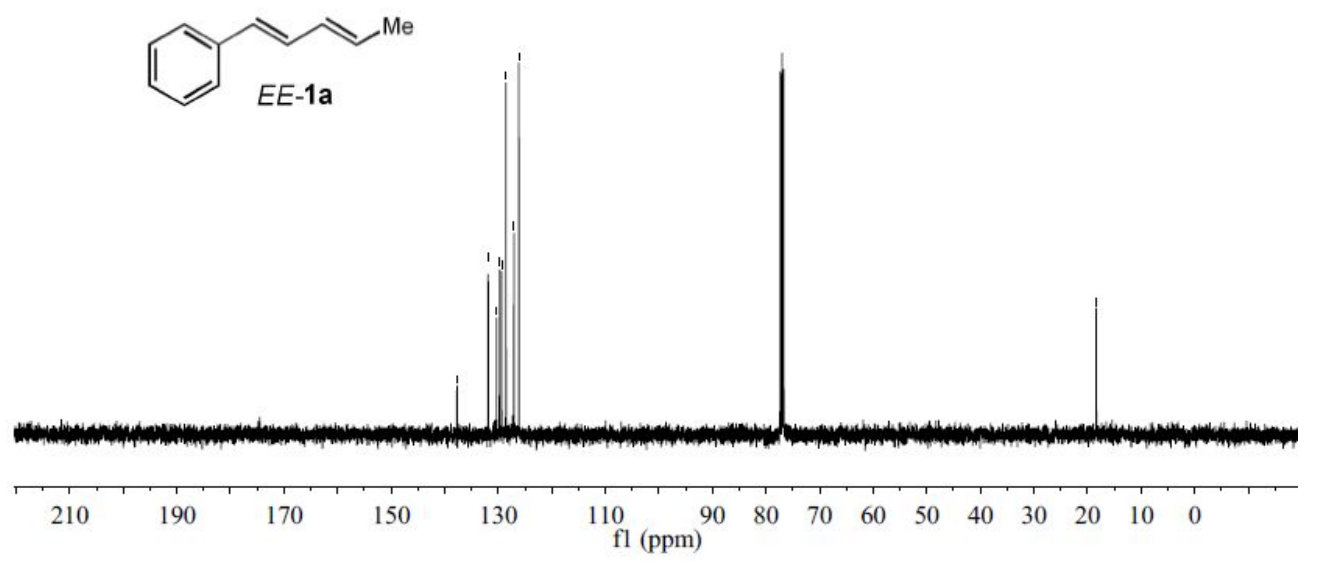




\section{References}

1. a) Qian, P.; Deng, Y.; Mei, H. B.; Han, J. L.; Zhou, J.; Pan, Y. Org. Lett. 2017, 19, 4798-4801.

b) Liu, Y.; Xie, P.; Sun, Z.; Wo, X.; Gao, C.; Fu, W.; Loh, T.-P. Org. Lett. 2018, 20, 5353-5356.

2. a) Ely, R. J.; Morken, J. P. J. Am. Chem. Soc. 2010, 132, 2534-2535. b)Watkins, A. L.; Landis,

C. R. Org. Lett. 2011, 13, 164-167. c) Park, S.; Malcolmson, S. J. ACS Catal. 2018, 8, 8468-8476.

d) Xiong, Q.; Lin, L. L.; Zhao, X. H.; Lang, J. W.; Liu, X. H.; Feng, X. M. J. Org. Chem. 2018, 83, 12527-12534. e) Vijayaraghavan, R. K.; Meskers, S. C. J.; Rahim, M. A.; Das, S. Chem. Commun. 2014, 50, 6530-6533. f) Charette, A. B.; Molinaro, C.; Brochu, C. J. Am. Chem. Soc. 2001, 123, 12168-12175. 


\section{General Procedure for the Hydrosulfonylation Reaction.}

In glovebox, $\mathrm{Pd}_{2}(\mathrm{dba})_{3} \cdot \mathrm{CHCl}_{3}(4.1 \mathrm{mg}, 4 \mu \mathrm{mol}, 4 \mathrm{~mol} \%)$ and chiral ligand (R)-DTBM-Segphos (10.4 mg, $8.8 \mu \mathrm{mol}, 4.4 \mathrm{~mol} \%)$ were dissolved in dry MTBE $(0.25 \mathrm{M}, 0.8 \mathrm{~mL})$ and stirred at room temperature for $0.5 \mathrm{~h}$. To the solution, substrate sulfinic acid $(0.2 \mathrm{mmol})$ and diene $(0.3 \mathrm{mmol})$ were added sequentially. The reaction mixture was stirred at $30{ }^{\circ} \mathrm{C}$ for $30 \mathrm{~h}$. The volatiles were then removed under reduced pressure. The crude product was used to determine the regioselectivity by ${ }^{1} \mathrm{H}$ NMR analysis. The residue was purified by $\mathrm{SiO}_{2}$ column chromatography (PE/EA $=10: 1$ to 3:1) to give the desired product.

\section{Deuterium-labeling study}

Sodium benzenesulfite $(2 \mathrm{mmol})$ was dissolved in $5 \mathrm{~mL}$ of $\mathrm{D}_{2} \mathrm{O}$ under Ar. Then dry tert-Butylmethyl ether $(5 \mathrm{~mL})$ was added followed by the slow addition of $0.3 \mathrm{~mL}$ of $\mathrm{DCl}\left(35 \mathrm{wt}\right.$. $\%$ in $\mathrm{D}_{2} \mathrm{O}$ ). The mixture was stirred for an additional 5 min, quickly transferred to a separatory funnel, and the aqueous layer was removed. The organic layer was concentrated on a rotary evaporator. Then the white solid obtained was dried under vacuum to give sulfinic acid 2a- $d$. Deuterium rate was determined by ${ }^{1} \mathrm{H}$ NMR analysis in $d^{8}$-THF. ( Figure S2)

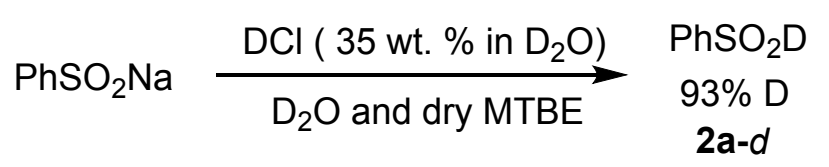

Figure S2 


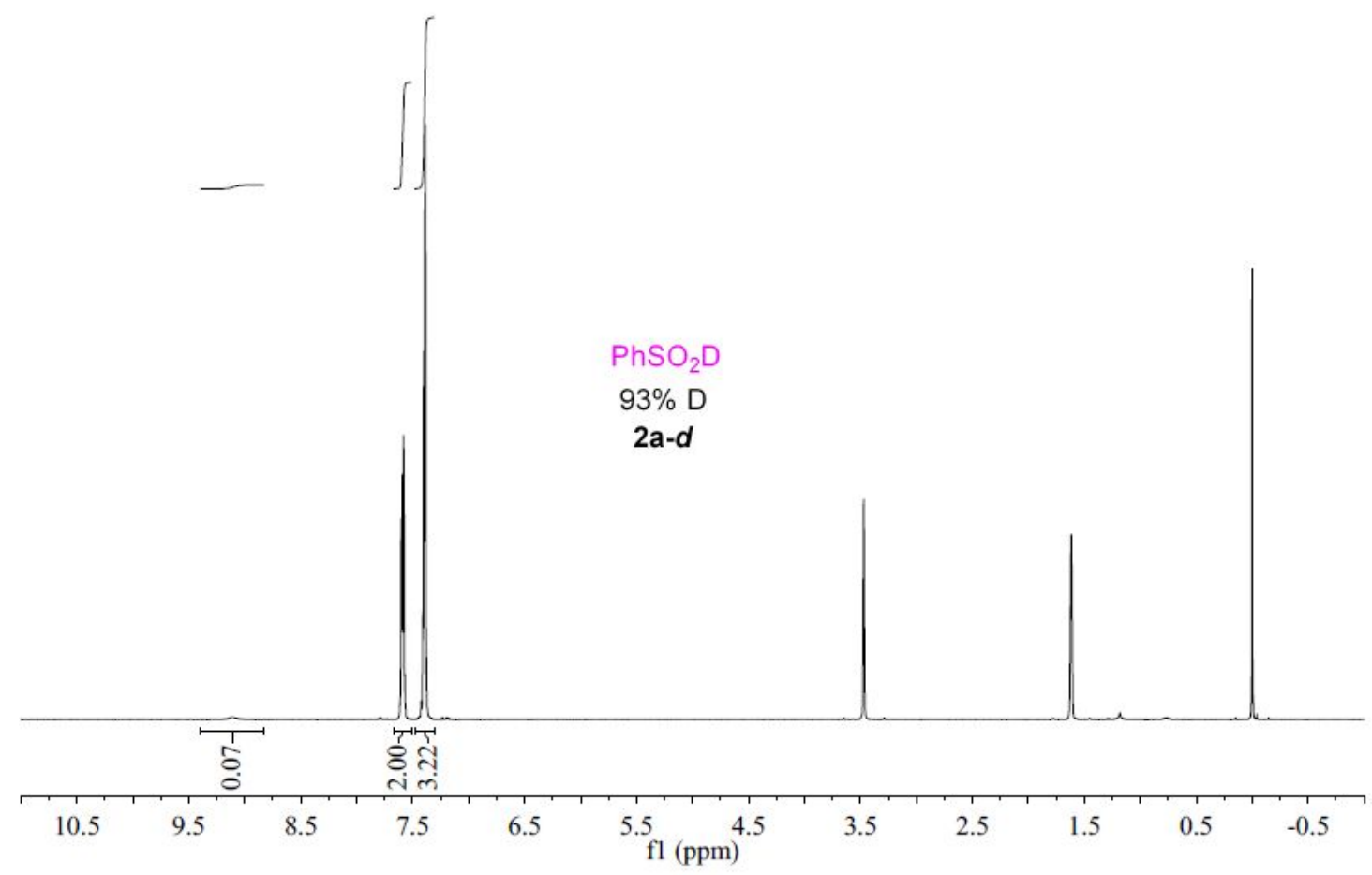

In glovebox, $\mathrm{Pd}_{2}(\mathrm{dba})_{3} \cdot \mathrm{CHCl}_{3}(4.1 \mathrm{mg}, 4 \mu \mathrm{mol}, 4 \mathrm{~mol} \%)$ and chiral ligand $R$-DTBM-Segphos (10.4 mg, $8.8 \mu \mathrm{mol}, 4.4 \mathrm{~mol} \%)$ were dissolved in dry MTBE $(0.25$ $\mathrm{M}, 0.8 \mathrm{~mL}$ ) and stirred at room temperature for $0.5 \mathrm{~h}$. To the solution, substrate sulfinic acid 2a-d $(0.2 \mathrm{mmol})$ and diene 1a $(E, Z / E, E=2.9: 1,0.3 \mathrm{mmol})$ were added sequentially. The reaction mixture was stirred at room temperature for $48 \mathrm{~h}$. After completed conversion, volatiles were removed under reduced pressure. The crude product was used to determine the regioselectivity by ${ }^{1} \mathrm{H}$ NMR analysis $(39 \%$ yield, 1,2-dibromoethane as internal standard). The residue was then purified by $\mathrm{SiO}_{2}$ column chromatography $(\mathrm{PE} / \mathrm{EA}=10: 1$ to $3: 1)$ to give the desired deuterium-labeling product. Deuterium rate was determined by ${ }^{1} \mathrm{H}$ NMR analysis in $\mathrm{CDCl}_{3}$. ( Figure S3)

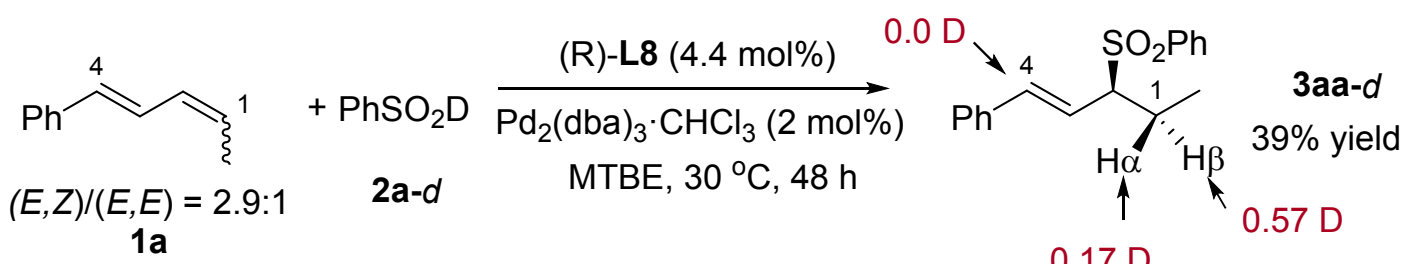

Figure S3 


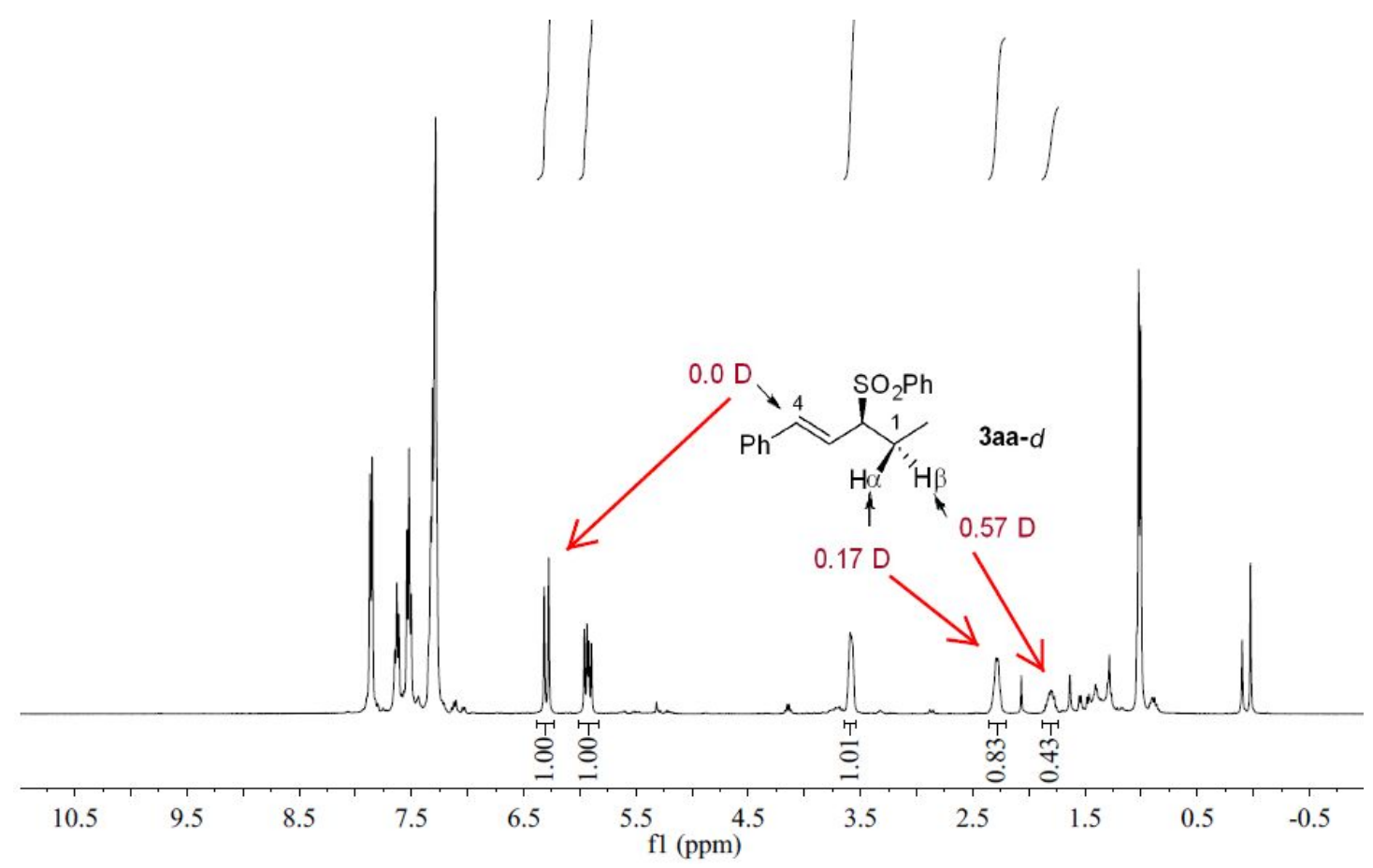

In glovebox, $\mathrm{Pd}_{2}(\mathrm{dba})_{3} \cdot \mathrm{CHCl}_{3}(4.1 \mathrm{mg}, 4 \mu \mathrm{mol}, 4 \mathrm{~mol} \%)$ and chiral ligand $R$-DTBM-Segphos (10.4 mg, $8.8 \mu \mathrm{mol}, 4.4 \mathrm{~mol} \%)$ were dissolved in dry MTBE $(0.25$ $\mathrm{M}, 0.8 \mathrm{~mL}$ ) and stirred at room temperature for $0.5 \mathrm{~h}$. To the solution, substrate sulfinic acid 2a-d $(0.2 \mathrm{mmol})$ and diene $E E-1 \mathbf{a}(0.3 \mathrm{mmol})$ were added sequentially. The reaction mixture was stirred at room temperature for $48 \mathrm{~h}$. After completed conversion, volatiles were removed under reduced pressure. The crude product was used to determine the regioselectivity by ${ }^{1} \mathrm{H}$ NMR analysis $(42 \%$ yield, 1,2-dibromoethane as internal standard). The residue was then purified by $\mathrm{SiO}_{2}$ column chromatography $(\mathrm{PE} / \mathrm{EA}=10: 1$ to $3: 1)$ to give the desired deuterium-labeling product. Deuterium rate was determined by ${ }^{1} \mathrm{H}$ NMR analysis in $\mathrm{CDCl}_{3}$. ( Figure S4)

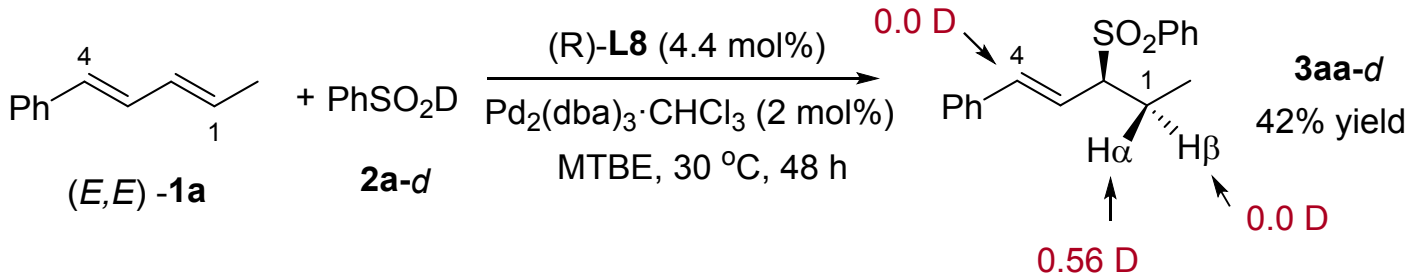

Figure S4 


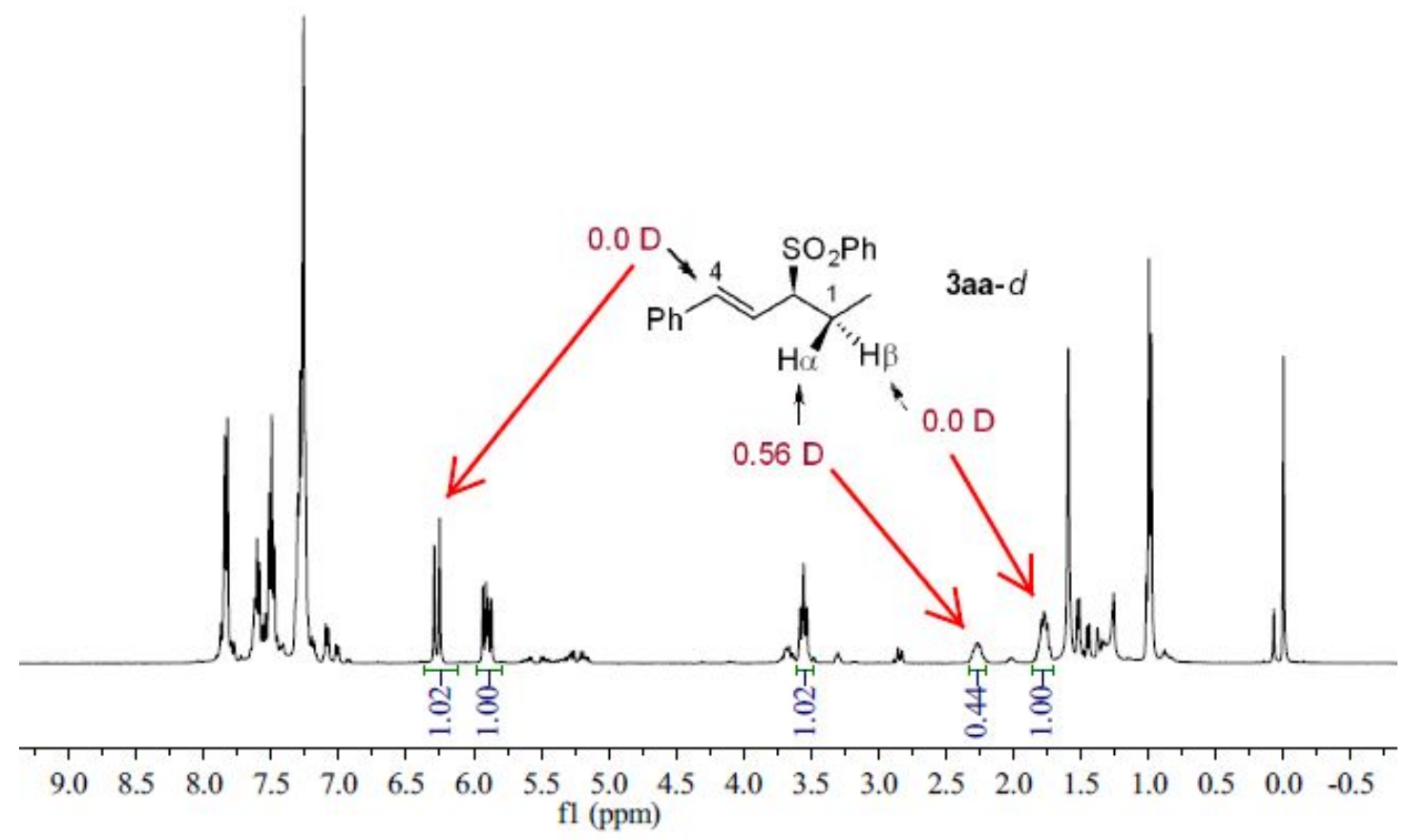

\section{Initial rate KIE study}

In glovebox, $\mathrm{Pd}_{2}(\mathrm{dba})_{3} \cdot \mathrm{CHCl}_{3}(4.1 \mathrm{mg}, 4 \mu \mathrm{mol}, 4 \mathrm{~mol} \%)$ and chiral ligand $R$-DTBM-Segphos (10.4 mg, $8.8 \mu \mathrm{mol}, 4.4 \mathrm{~mol} \%)$ were dissolved in dry MTBE $(0.25 \mathrm{M}, 0.8 \mathrm{~mL})$ and stirred at room temperature for $0.5 \mathrm{~h}$. To the solution, substrate sulfinic acid 2a or $2 \mathbf{a}-\boldsymbol{d}(0.2 \mathrm{mmol})$, diene $(0.3 \mathrm{mmol})$ and 1,3,5-trimethylbenzene (8.4 $\mathrm{mg}, 0.05 \mathrm{mmol}$ ) were added sequentially. The reaction mixture was stirred at $30^{\circ} \mathrm{C}$. Aliquots $(5 \mu \mathrm{l})$ were taken every 1 hour and quenched in $1 \mathrm{~mL}$ of EtOAc. The reaction halts in EtOAc. The amount of 3aa was monitored by GC-FID analysis:
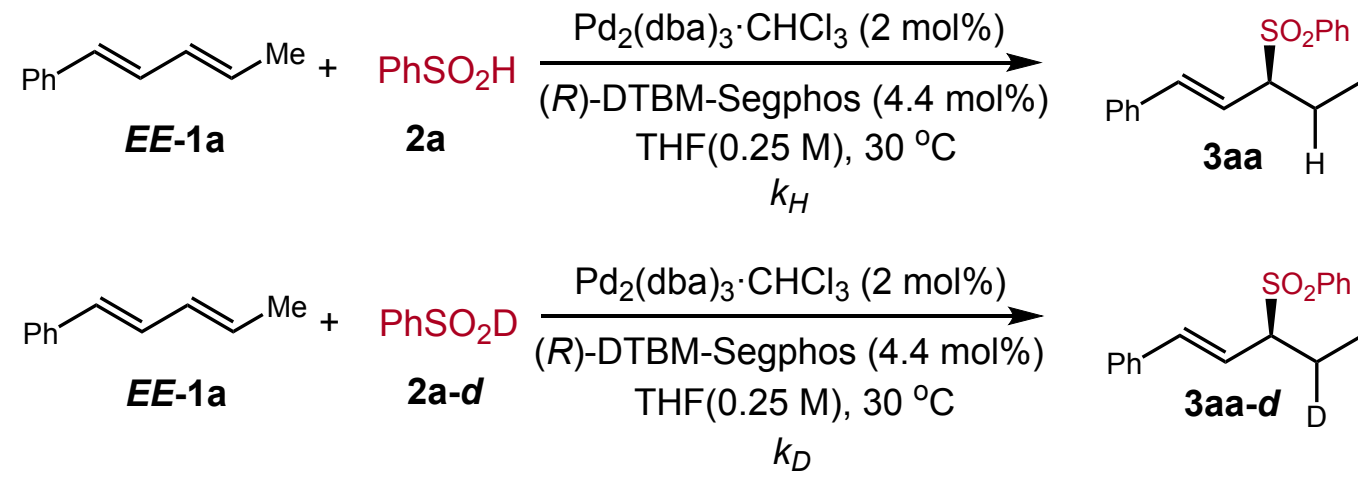<smiles>[2H]C(C)[C@H](/C=C/c1ccccc1)[Se](=O)(=O)c1ccccc1</smiles> 


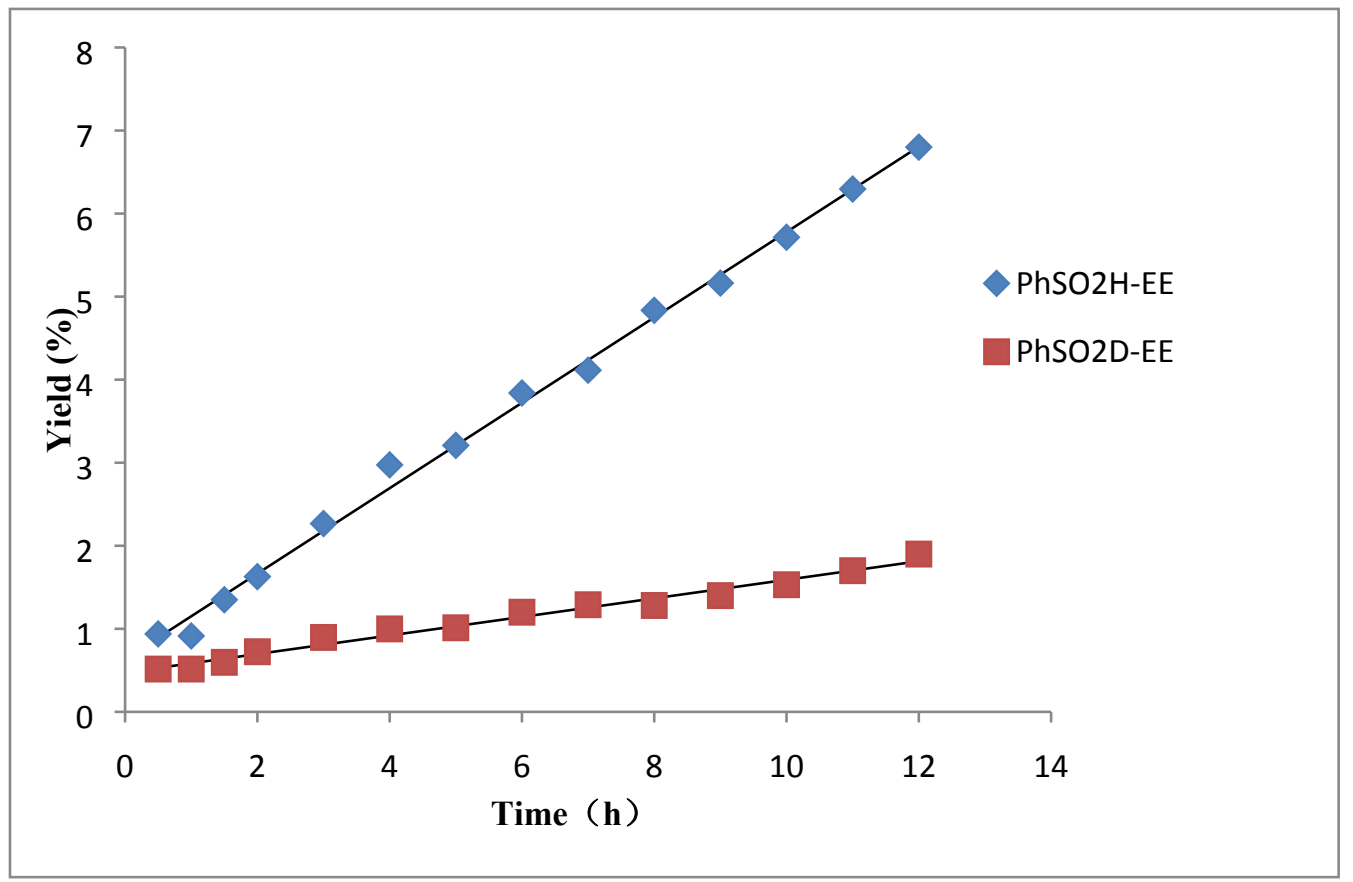

adjusted initial rate of Deuterium species (considering 7\% 2a)

$$
\begin{gathered}
0.112=0.93 k_{D}+0.07 \times 0.514 \\
k_{D}=0.082
\end{gathered}
$$

Calculation of KIE: $k_{H} / k_{D}=0.514 / 0.082=6.3$
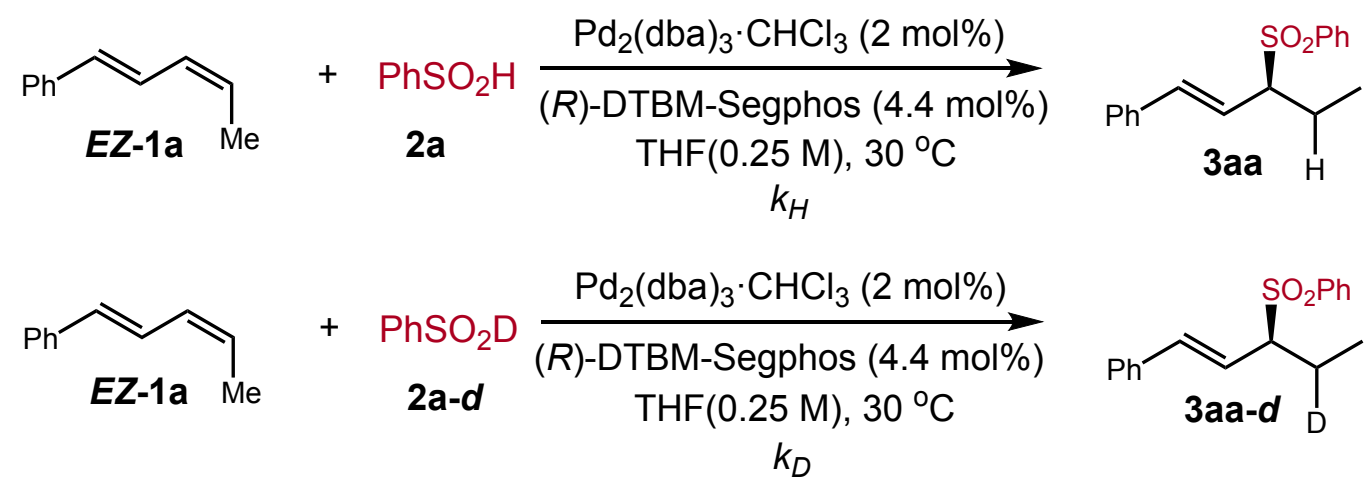


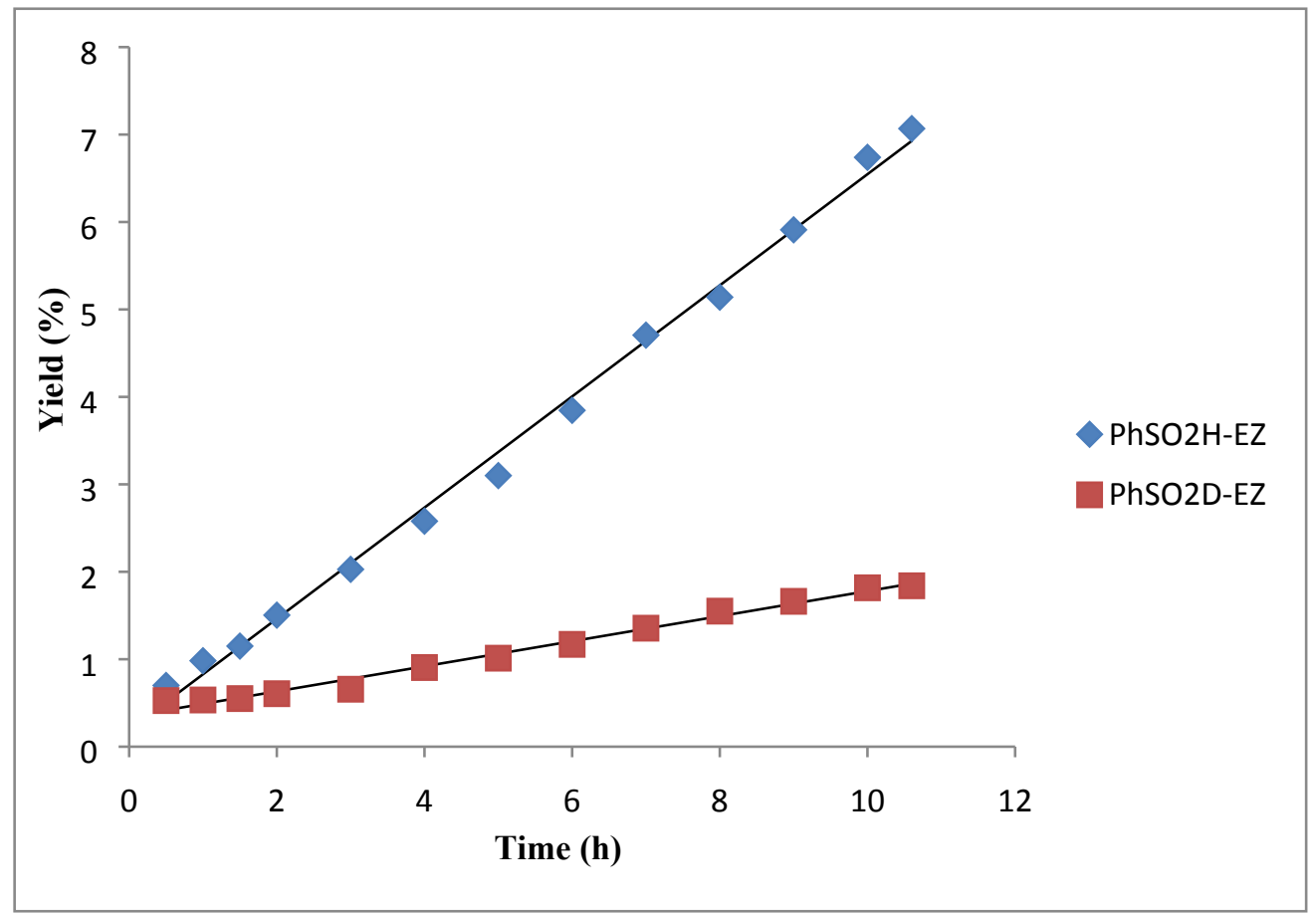

adjusted initial rate of Deuterium species (considering 7\% 2a)

$$
0.143=0.93 k_{D}+0.07 \times 0.635
$$

$$
k_{D}=0.106
$$

Calculation of KIE: $k_{H} / k_{D}=0.635 / 0.106=6.0$

\section{Olefin isomerization}

In glovebox, $\mathrm{Pd}_{2}(\mathrm{dba})_{3} \cdot \mathrm{CHCl}_{3}(4.1 \mathrm{mg}, 4 \mu \mathrm{mol}, 4 \mathrm{~mol} \%)$ and chiral ligand $R$-DTBM-Segphos (10.4 mg, $8.8 \mu \mathrm{mol}, 4.4 \mathrm{~mol} \%$ ) were dissolved in dry MTBE $(0.25 \mathrm{M}, 0.8 \mathrm{~mL})$ and stirred at room temperature for $0.5 \mathrm{~h}$. To the solution, substrate sulfinicacid 2a $(0.2 \mathrm{mmol})$ and diene $E E$-1a or $E Z-1 \mathbf{a}(0.3 \mathrm{mmol})$ were added sequentially. The reaction mixture was stirred at room temperature for $5.5 \mathrm{~h}$. Volatiles were removed under reduced pressure. The crude product was used to determine the ratio of $E E$ or $E Z$ of the remaining substrate diene by ${ }^{1} \mathrm{H}$ NMR analysis. The residue was then purified by $\mathrm{SiO}_{2}$ column chromatography $(\mathrm{PE} / \mathrm{EA}=10: 1$ to $3: 1)$ to give the desired product 3aa and the recovering of diene. ( Figure S5) 

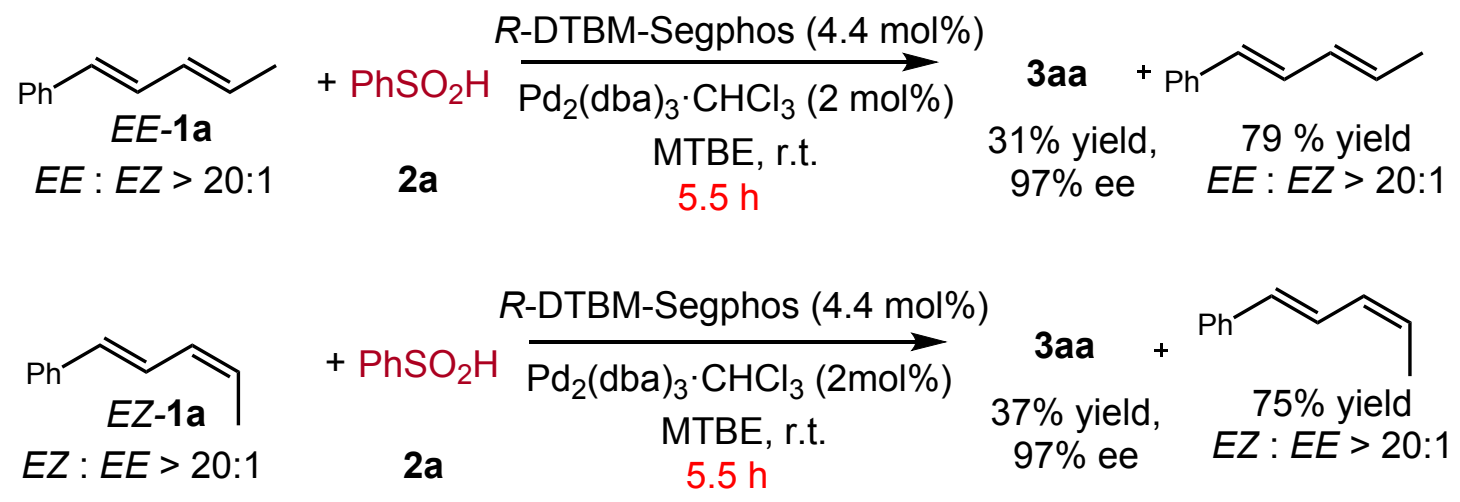

Figure S5

\section{Reversibility of the Coupling}

In glovebox, $\mathrm{Pd}_{2}(\mathrm{dba})_{3} \cdot \mathrm{CHCl}_{3}(4.1 \mathrm{mg}, 4 \mu \mathrm{mol}, 4 \mathrm{~mol} \%)$ and chiral ligand $R$-DTBM-Segphos (10.4 mg, $8.8 \mu \mathrm{mol}, 4.4 \mathrm{~mol} \%$ ) were dissolved in dry MTBE $(0.25 \mathrm{M}, 0.8 \mathrm{~mL})$ and stirred at room temperature for $0.5 \mathrm{~h}$. To the solution, $3 \mathbf{b a}$ $(0.1 \mathrm{mmol})$ and $\mathbf{3 a d}(0.1 \mathrm{mmol})$ were added sequentially. The reaction mixture was stirred at room temperature for $48 \mathrm{~h}$. After complete conversion, volatiles were removed under reduced pressure. The crude product was used to determine by ${ }^{1} \mathrm{H}$ NMR analysis. The residue was then purified by $\mathrm{SiO}_{2}$ column chromatography $(\mathrm{PE} / \mathrm{EA}=10: 1$ to $3: 1)$ to give the recovering of 3ba and 3ad. ( Figure S6)<smiles>CCC(/C=C/c1ccc(F)cc1)S(=O)(=O)c1ccc(F)cc1</smiles>

$R$-DTBM-Segphos (4.4 mol\%) $\overrightarrow{\mathrm{Pd}_{2}(\mathrm{dba})_{3} \cdot \mathrm{CHCl}_{3}(2 \mathrm{~mol} \%)}$ $\operatorname{MTBE}(0.25 \mathrm{M})$, r.t. $48 \mathrm{~h}$

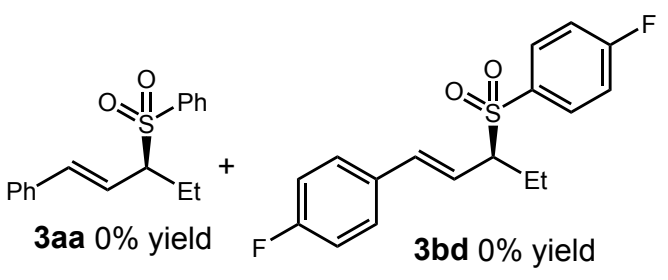

recovered 3 ba $98 \%$ yield and 3 ad $99 \%$ yield

Figure S6 


\section{Scale-up Experiment}

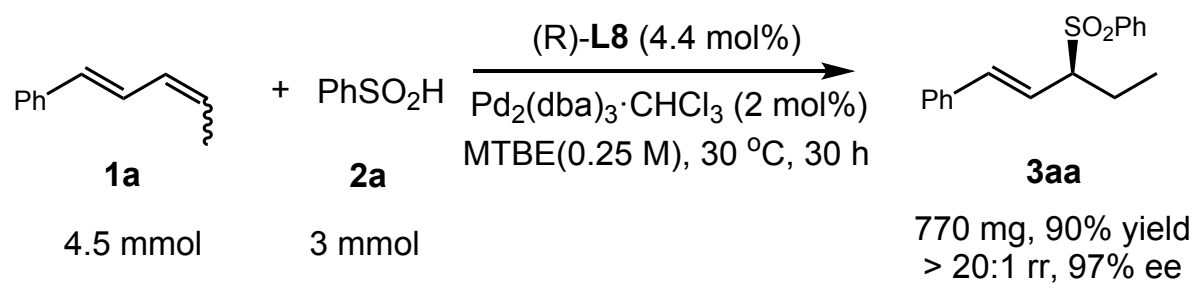

In glovebox, $\mathrm{Pd}_{2}(\mathrm{dba})_{3} \cdot \mathrm{CHCl}_{3}(62.1 \mathrm{mg}, 60 \mu \mathrm{mol}, 4 \mathrm{~mol} \%)$ and chiral ligand (R)-DTBM-Segphos (155.7 mg, $132 \mu \mathrm{mol}, 4.4 \mathrm{~mol} \%)$ were dissolved in dry MTBE $(0.25 \mathrm{M}, 12 \mathrm{~mL})$ and stirred at room temperature for $0.5 \mathrm{~h}$. To the solution, substrate sulfinic acid $(3 \mathrm{mmol})$ and diene $(4.5 \mathrm{mmol})$ were added sequentially. The reaction mixture was stirred at $30{ }^{\circ} \mathrm{C}$ for $30 \mathrm{~h}$. The volatiles were then removed under reduced pressure. The crude product was used to determine the regioselectivity by ${ }^{1} \mathrm{H}$ NMR analysis. The residue was purified by $\mathrm{SiO}_{2}$ column chromatography $(\mathrm{PE} / \mathrm{EA}=10: 1$ to $3: 1)$ to give the desired product 3aa $(0.77 \mathrm{~g}, 90 \%,>20: 1 \mathrm{rr}, 97 \%$ ee $)$.

\section{Synthetic Transformations of allylic Sulfones}<smiles>O=C(/C=C/c1ccccc1)CCCCO</smiles>

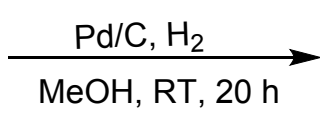<smiles>CCC(CCc1ccccc1)[Se][18O][Ba]</smiles>

$12 \mathrm{mg}$ of $\mathrm{Pd} / \mathrm{C}$ was added to a solution of 3aa $(57.2 \mathrm{mg}, 0.2 \mathrm{mmol})$ in $\mathrm{MeOH}(2.0$ $\mathrm{mL}$ ). The mixture was hydrogenated under a balloon pressure of hydrogen for $20 \mathrm{~h}$. The reaction progress was monitored by ${ }^{1} \mathrm{H}$ NMR analysis. The reaction mixture was filtered through celite and the filtrate was evaporated to give the crude product. The residue was purified by $\mathrm{SiO}_{2}$ column chromatography (PE/EA = 10:1 to 3:1) to give the desired product $4 \mathbf{a a}$ as a colorless oil (50 mg, 87\% yield, $96 \%$ ee). 


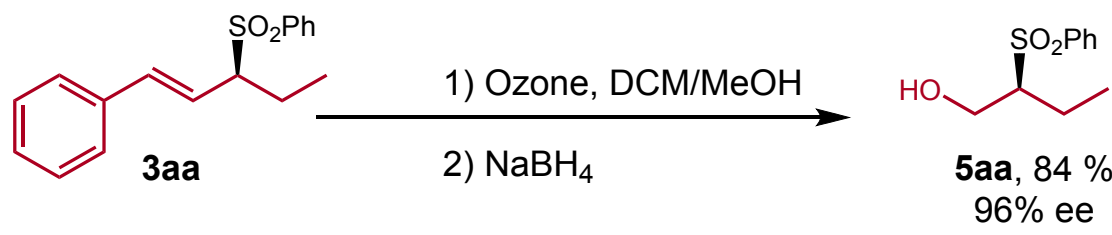

Ozone gas was bubbled through a solution of 3aa $(57.2 \mathrm{mg}, 0.2 \mathrm{mmol})$ in DCM:MeOH $(2 \mathrm{~mL}, 3: 1)$ at $-78{ }^{\circ} \mathrm{C}$. The reaction progress was monitored by TLC. After stirring for $10 \mathrm{~min}$ (appearance of blue color), $\mathrm{N}_{2}$ was bubbled through the reaction mixture for $10 \mathrm{~min}$ at $-78{ }^{\circ} \mathrm{C} . \mathrm{NaBH}_{4}(77 \mathrm{mg}, 2 \mathrm{mmol})$ was added to the reaction mixture, slowly warmed to $0{ }^{\circ} \mathrm{C}$ over a period of $1 \mathrm{~h}$, and the stirring was continued at room temperature for additional $1 \mathrm{~h}$. The reaction was quenched with saturated $\mathrm{NH}_{4} \mathrm{Cl}$ aqueous $(3 \mathrm{ml})$ at $0{ }^{\circ} \mathrm{C}$ and extracted with $\mathrm{DCM}(3 \times 5 \mathrm{~mL})$. The combined organic layers were washed with brine, dried over $\mathrm{Na}_{2} \mathrm{SO}_{4}$, and concentrated. The residue was purified by $\mathrm{SiO}_{2}$ column chromatography $(\mathrm{PE} / \mathrm{EA}=$ $3: 1$ to $1: 1$ ) to give the desired product 5 aa as a colorless oil (36 mg, $84 \%$ yield, $96 \%$ ee).

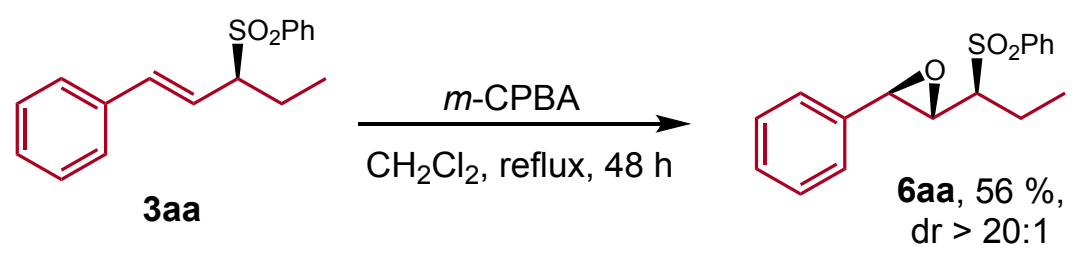

Under an argon atmosphere, $m$-CPBA (69 $\mathrm{mg}, 0.4 \mathrm{mmol})$ was added to a vial was charged with 3aa $(57.2 \mathrm{mg}, 0.2 \mathrm{mmol})$ in dry DCM $(2.0 \mathrm{~mL})$. The mixture is stirred vigorously for $48 \mathrm{~h}$ at $50{ }^{\circ} \mathrm{C}$, and then quenched by addition of saturated $\mathrm{NH}_{4} \mathrm{Cl}$ aqueous. The layers were separated and the aqueous phase was extracted with DCM (3 x $5 \mathrm{~mL}$ ). The combined organic layers were dried over $\mathrm{MgSO}_{4}$, filtered and concentrated in vacuo. The crude product was used to determine the diastereoselectivity by $1 \mathrm{H}$ NMR analysis. The residue was purified by $\mathrm{SiO}_{2}$ column chromatography $(\mathrm{PE} / \mathrm{EA}=10: 1$ to $3: 1)$ to give the desired product 6aa as a colorless oil (34 mg, 56\% yield, >20:1 dr). 


\section{Characterization of Products}<smiles>CCC(/C=C/c1ccccc1)[O+]c1ccccc1</smiles>

\section{(S,E)-((1-phenylpent-1-en-3-yl)sulfonyl)benzene}

Prepared according to General Procedure, white solid, $52 \mathrm{mg}, 91 \%$ yield, m.p. $63-65^{\circ} \mathrm{C},>20: 1 \mathrm{rr}$, $97 \%$ ee

$[\alpha]^{25}{ }_{\mathrm{D}}=-96.8\left(\mathrm{c} 1, \mathrm{CHCl}_{3}\right)$

${ }^{1} \mathrm{H}$ NMR $\left(400 \mathrm{MHz}, \mathrm{CDCl}_{3}\right) \delta 7.83(\mathrm{~d}, J=7.6 \mathrm{~Hz}, 2 \mathrm{H}), 7.61(\mathrm{t}, J=7.4 \mathrm{~Hz}, 1 \mathrm{H}), 7.50(\mathrm{t}, J=7.7 \mathrm{~Hz}$, 2H), $7.34-7.22(\mathrm{~m}, 5 \mathrm{H}), 6.27$ (d, $J=15.9 \mathrm{~Hz}, 1 \mathrm{H}), 5.90(\mathrm{dd}, J=15.9,9.5 \mathrm{~Hz}, 1 \mathrm{H}), 3.57$ (td, $J=10.6$, $3.2 \mathrm{~Hz}, 1 \mathrm{H}), 2.28$ (dqd, $J=15.0,7.5,3.4 \mathrm{~Hz}, 1 \mathrm{H}), 1.79$ (ddq, $J=14.6,11.1,7.4 \mathrm{~Hz}, 1 \mathrm{H}), 0.99$ (t, $J=$ $7.5 \mathrm{~Hz}, 3 \mathrm{H})$.

${ }^{13} \mathrm{C}$ NMR $\left(101 \mathrm{MHz}, \mathrm{CDCl}_{3}\right) \delta 138.3,137.6,135.8,133.6,129.2,128.8,128.7,128.4,126.6,121.0$, $71.2,20.9,11.4$.

HRMS (ESI) calcd. for $\mathrm{C}_{17} \mathrm{H}_{18} \mathrm{NaO}_{2} \mathrm{~S}^{+}(\mathrm{M}+\mathrm{Na})^{+}: 309.0920$, Found: 309.0918

the ee value was $97 \%, \mathrm{t}_{\mathrm{r}}($ minor $)=14.964 \min , \mathrm{t}_{\mathrm{r}}($ major $)=17.686 \min ($ Chiralcel IC, $\lambda=220 \mathrm{~nm}$, hexanes $:{ }^{i} \mathrm{PrOH}=80: 20$, flow rate $=1.0 \mathrm{~mL} / \mathrm{min}$ ).

$\mathrm{mV}$

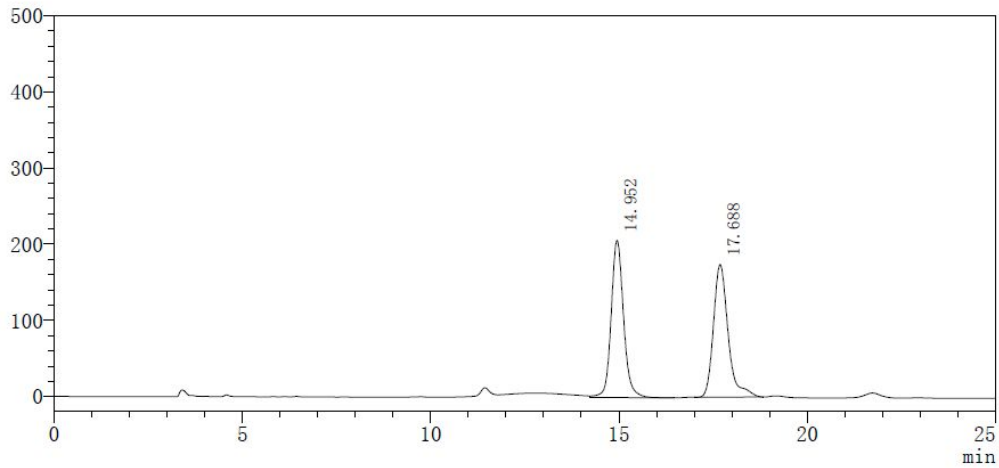

\begin{tabular}{|c|c|c|c|c|c|}
\hline & & & & & \\
\hline Peak\# & Time & $\begin{array}{c}\text { Area } \\
7709713\end{array}$ & Height & Height\% & Area\% \\
\hline 2 & 17.688 & 4727380 & 174114 & 45.728 & $\frac{50.001}{49.993}$ \\
\hline 总计 & & 9456092 & 380758 & 100.000 & 100.000 \\
\hline
\end{tabular}

(检测器 $A=$ detector $A$, 总计 $=$ total) 
$\mathrm{mV}$

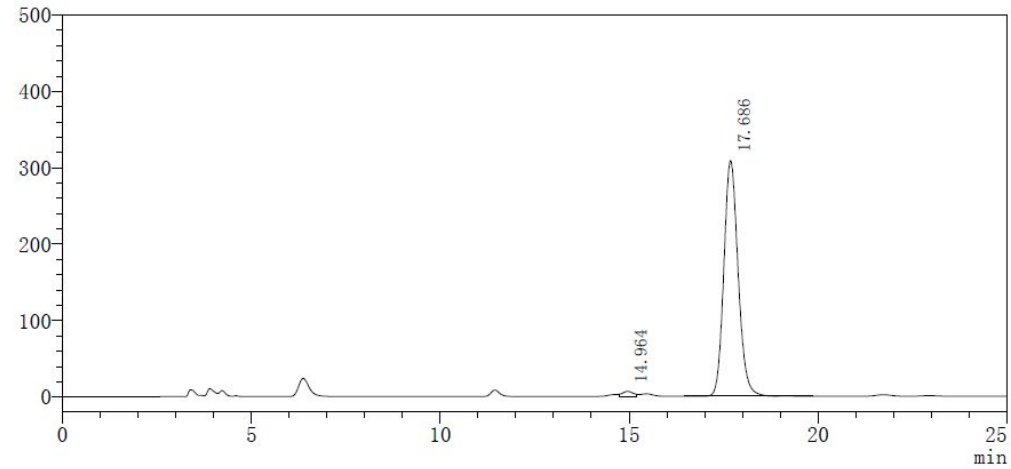

检测器A $220 \mathrm{~nm}$
\begin{tabular}{|c|c|c|c|c|c|}
\hline Peak\# & Time & Area & Height & Height\% & Area $\%$ \\
\hline 1 & 14.964 & 135306 & 6576 & 2.086 & 1.638 \\
\hline 2 & 17.686 & 8125079 & 308614 & 97.914 & 98.362 \\
\hline 总计 & & 8260385 & 315190 & 100.000 & 100.000 \\
\hline
\end{tabular}<smiles>CC[C@H](/C=C/c1ccc(F)cc1)S(=O)(=O)c1ccccc1</smiles>

\section{(S,E)-1-fluoro-4-(3-(phenylsulfonyl)pent-1-en-1-yl)benzene}

Prepared according to General Procedure, white solid, $55 \mathrm{mg}$, $90 \%$ yield, m.p. $75-76{ }^{\circ} \mathrm{C}, 15: 1 \mathrm{rr}$, $95 \%$ ee

$[\alpha]^{25}=-80.9\left(\mathrm{c} 1, \mathrm{CHCl}_{3}\right)$

${ }^{1} \mathrm{H}$ NMR $\left(400 \mathrm{MHz}, \mathrm{CDCl}_{3}\right) \delta 7.88-7.79(\mathrm{~m}, 2 \mathrm{H}), 7.61(\mathrm{dd}, J=10.7,4.2 \mathrm{~Hz}, 1 \mathrm{H}), 7.50(\mathrm{t}, J=7.7 \mathrm{~Hz}$, 2H), $7.26-7.19$ (m, 2H), 6.99 (dd, $J=12.0,5.3 \mathrm{~Hz}, 2 \mathrm{H}), 6.25(\mathrm{~d}, J=15.9 \mathrm{~Hz}, 1 \mathrm{H}), 5.82(\mathrm{dd}, J=15.9$, $9.4 \mathrm{~Hz}, 1 \mathrm{H}), 3.56$ (td, $J=10.6,3.3 \mathrm{~Hz}, 1 \mathrm{H}), 2.25$ (dqd, $J=15.0,7.5,3.4 \mathrm{~Hz}, 1 \mathrm{H}), 1.78$ (ddt, $J=21.0$, $11.1,7.4 \mathrm{~Hz}, 1 \mathrm{H}), 0.99$ (t, $J=7.5 \mathrm{~Hz}, 3 \mathrm{H})$.

${ }^{13} \mathrm{C}$ NMR $\left(101 \mathrm{MHz}, \mathrm{CDCl}_{3}\right) \delta 162.7(\mathrm{~d}, J=248.3 \mathrm{~Hz}), 137.6,137.1,133.6,132.0(\mathrm{~d}, J=3.3 \mathrm{~Hz})$, 129.2, 128.9, $128.2(\mathrm{~d}, J=8.1 \mathrm{~Hz}), 120.7(\mathrm{~d}, J=2.0 \mathrm{~Hz}), 115.6(\mathrm{~d}, J=21.7 \mathrm{~Hz}), 71.0,21.0,11.4$.

${ }^{19} \mathrm{~F}$ NMR $\left(377 \mathrm{MHz}, \mathrm{CDCl}_{3}\right) \delta-112.9$.

HRMS (ESI) calcd. for $\mathrm{C}_{17} \mathrm{H}_{17} \mathrm{FNaO}_{2} \mathrm{~S}^{+}(\mathrm{M}+\mathrm{Na})^{+}: 327.0825$, Found: 327.0826

the ee value was $95 \%, \mathrm{t}_{\mathrm{r}}($ minor $)=14.475 \mathrm{~min}, \mathrm{t}_{\mathrm{r}}($ major $)=17.423 \min ($ Chiralcel IC, $\lambda=220 \mathrm{~nm}$, hexanes : ${ }^{i} \mathrm{PrOH}=80: 20$, flow rate $=1.0 \mathrm{~mL} / \mathrm{min}$ ). 
$\mathrm{mV}$
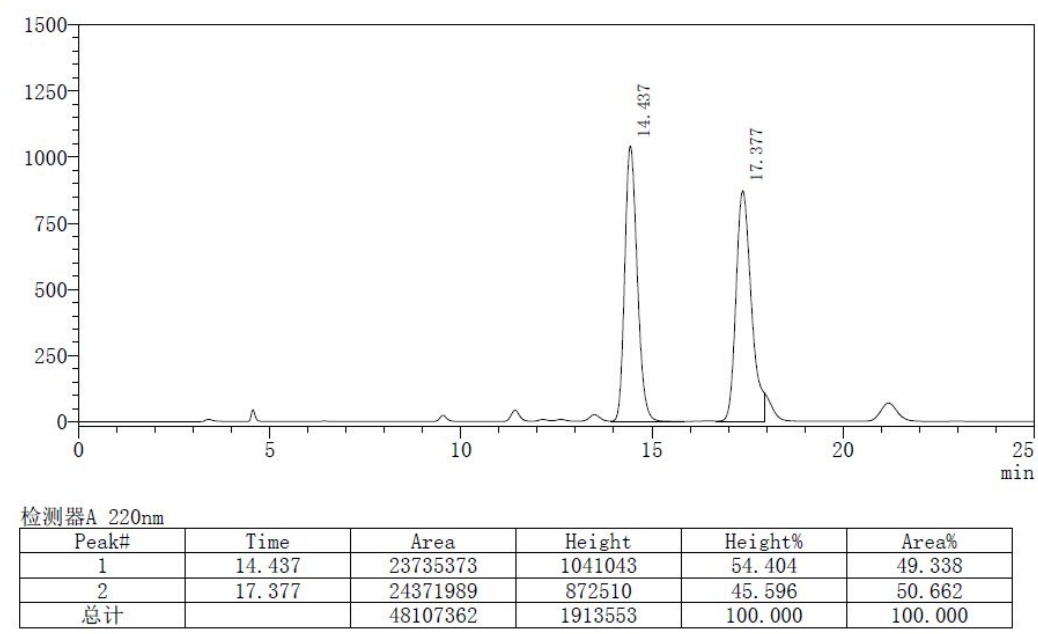

$\mathrm{mV}$

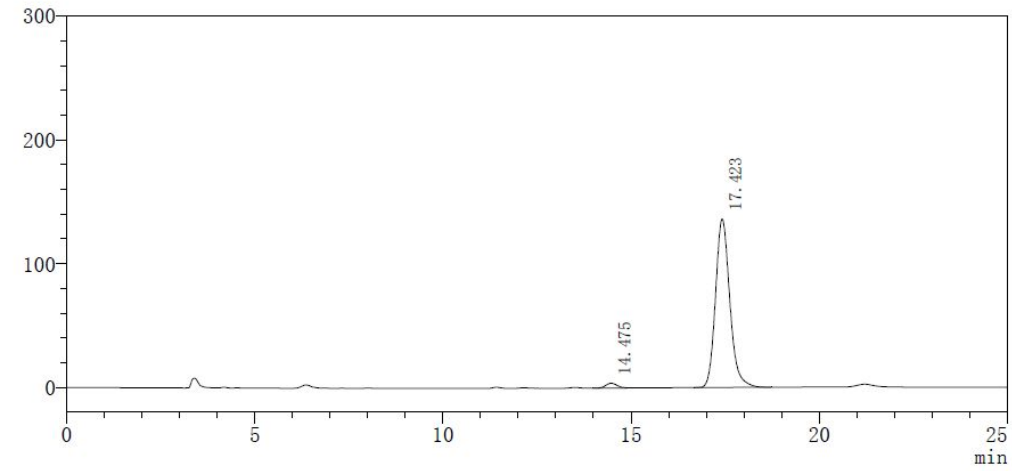

检测器A $220 \mathrm{~nm}$
\begin{tabular}{|c|c|c|c|c|c|}
\hline Peak\# & Time & Area & Height & Height\% & Area $\%$ \\
\hline 1 & 14.475 & 85704 & 4028 & 2.875 & 2.327 \\
\hline 2 & 17.423 & 3597931 & 136039 & 97.125 & 97.673 \\
\hline 总计 & & 3683634 & 140066 & 100.000 & 100.000 \\
\hline
\end{tabular}<smiles>CC[C@H](/C=C/c1cccc(F)c1)S(=O)(=O)c1ccccc1</smiles>

\section{(S,E)-1-fluoro-3-(3-(phenylsulfonyl)pent-1-en-1-yl)benzene}

Prepared according to General Procedure, pale yellow oil, $54 \mathrm{mg}, 89 \%$ yield, $14: 1 \mathrm{rr}, 98 \%$ ee $[\alpha]^{25}{ }_{\mathrm{D}}=-76.4\left(\mathrm{c} 1, \mathrm{CHCl}_{3}\right)$

${ }^{1} \mathrm{H}$ NMR $\left(400 \mathrm{MHz}, \mathrm{CDCl}_{3}\right) \delta 7.85(\mathrm{~d}, J=7.6 \mathrm{~Hz}, 2 \mathrm{H}), 7.64(\mathrm{t}, J=7.4 \mathrm{~Hz}, 1 \mathrm{H}), 7.53(\mathrm{t}, J=7.6 \mathrm{~Hz}$, 2H), $7.33-7.24$ (m, 1H), 7.05 (d, $J=7.7 \mathrm{~Hz}, 1 \mathrm{H}), 7.02-6.94(\mathrm{~m}, 2 \mathrm{H}), 6.27$ (d, $J=15.9 \mathrm{~Hz}, 1 \mathrm{H}), 5.94$ (dd, $J=15.8,9.5 \mathrm{~Hz}, 1 \mathrm{H}), 3.59$ (td, $J=10.9,2.7 \mathrm{~Hz}, 1 \mathrm{H}), 2.28$ (dqd, $J=15.0,7.6,3.4 \mathrm{~Hz}, 1 \mathrm{H}), 1.89-$ $1.73(\mathrm{~m}, 1 \mathrm{H}), 1.01(\mathrm{t}, J=7.4 \mathrm{~Hz}, 3 \mathrm{H})$.

${ }^{13} \mathrm{C}$ NMR $\left(101 \mathrm{MHz}, \mathrm{CDCl}_{3}\right) \delta 163.0(\mathrm{~d}, J=245.8 \mathrm{~Hz}), 138.1(\mathrm{~d}, J=7.9 \mathrm{~Hz}), 137.6,137.1(\mathrm{~d}, J=1.4$ Hz), 133.7, 130.2 (d, $J=8.4 \mathrm{~Hz}), 129.2,128.9,122.6,122.4(\mathrm{~d}, J=2.7 \mathrm{~Hz}), 115.2(\mathrm{~d}, J=21.5 \mathrm{~Hz})$, $113.0(\mathrm{~d}, J=21.9 \mathrm{~Hz}), 71.0,20.9,11.4$. 
${ }^{19} \mathrm{~F}$ NMR $\left(377 \mathrm{MHz}, \mathrm{CDCl}_{3}\right) \delta-113.1$.

HRMS (ESI) calcd. for $\mathrm{C}_{17} \mathrm{H}_{17} \mathrm{FNaO}_{2} \mathrm{~S}^{+}(\mathrm{M}+\mathrm{Na})^{+}: 327.0825$, Found: 327.0825

the ee value was $98 \%, \mathrm{t}_{\mathrm{r}}($ minor $)=13.313 \mathrm{~min}, \mathrm{t}_{\mathrm{r}}($ major $)=16.280 \mathrm{~min}($ Chiralcel $\mathrm{IC}, \lambda=220 \mathrm{~nm}$, hexanes : ${ }^{i} \mathrm{PrOH}=80: 20$, flow rate $=1.0 \mathrm{~mL} / \mathrm{min}$ ).

$\mathrm{mV}$
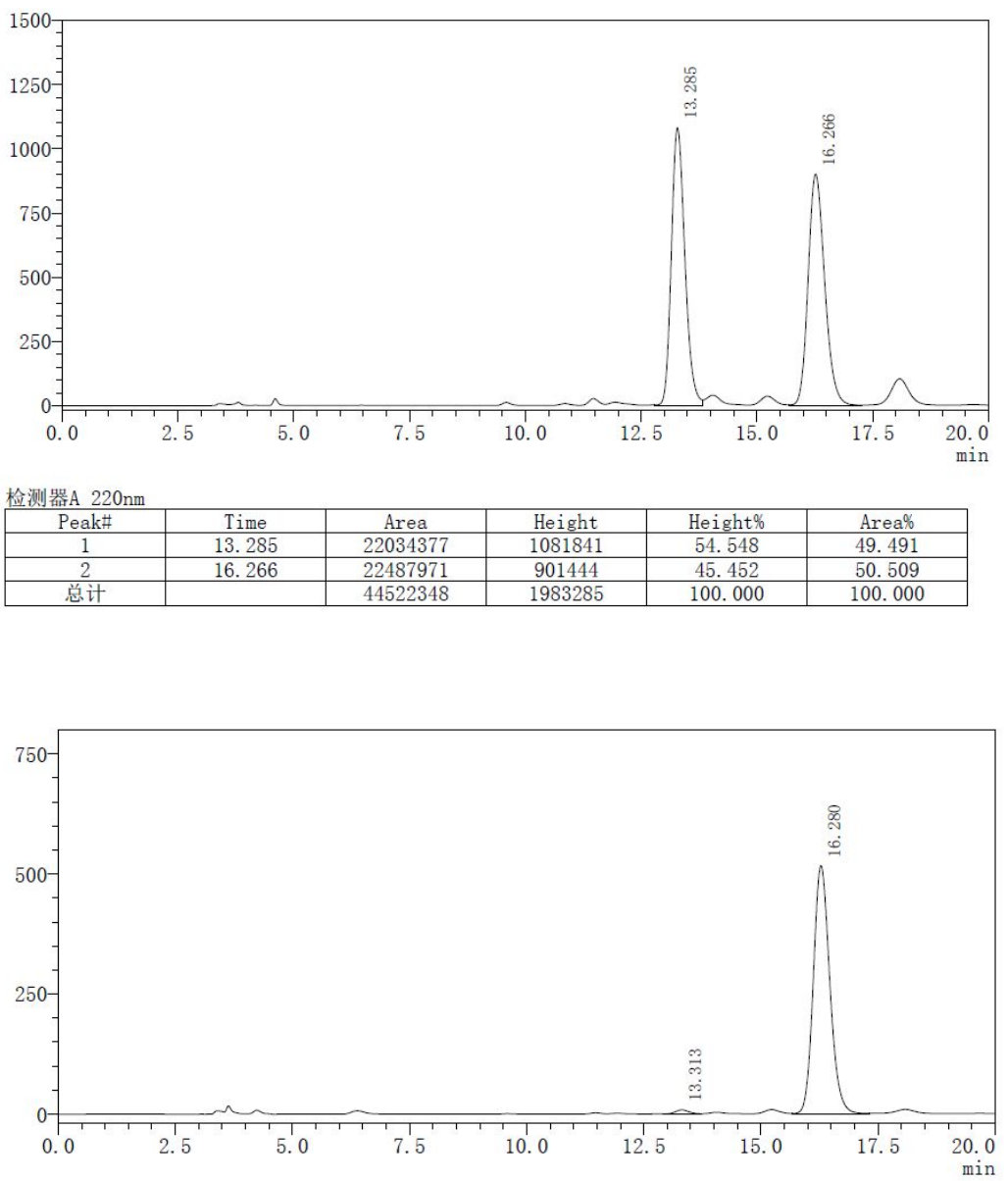

检测器A $220 \mathrm{~nm}$
\begin{tabular}{|c|c|c|c|c|c|}
\hline Peak\# & Time & Area & Height & Height\% & Area $\%$ \\
\hline 1 & 13.313 & 150444 & 8091 & 1.542 & 1.178 \\
\hline 2 & 16.280 & 12624837 & 516572 & 98.458 & 98.822 \\
\hline 总计 & & 12775281 & 524663 & 100.000 & 100.000 \\
\hline
\end{tabular}<smiles>CC[C@H](/C=C/c1ccccc1F)S(=O)(=O)c1ccccc1</smiles>

\section{(S,E)-1-fluoro-2-(3-(phenylsulfonyl)pent-1-en-1-yl)benzene}

Prepared according to General Procedure, white solid, $49 \mathrm{mg}, 80 \%$ yield, m.p. $56-57{ }^{\circ} \mathrm{C}, 17: 1 \mathrm{rr}$, $96 \%$ ee

$[\alpha]^{25}=-91.6\left(\mathrm{c} 0.5, \mathrm{CHCl}_{3}\right)$

${ }^{1} \mathrm{H}$ NMR $\left(400 \mathrm{MHz}, \mathrm{CDCl}_{3}\right) \delta 7.89-7.80(\mathrm{~m}, 2 \mathrm{H}), 7.66-7.58(\mathrm{~m}, 1 \mathrm{H}), 7.55-7.47(\mathrm{~m}, 2 \mathrm{H}), 7.36(\mathrm{td}$, $J=7.6,1.7 \mathrm{~Hz}, 1 \mathrm{H}), 7.25-7.20(\mathrm{~m}, 1 \mathrm{H}), 7.09$ (td, $J=7.6,1.0 \mathrm{~Hz}, 1 \mathrm{H}), 7.00$ (ddd, $J=10.6,8.2,1.0$ 
$\mathrm{Hz}, 1 \mathrm{H}), 6.41(\mathrm{~d}, J=16.0 \mathrm{~Hz}, 1 \mathrm{H}), 6.01(\mathrm{dd}, J=16.0,9.5 \mathrm{~Hz}, 1 \mathrm{H}), 3.64-3.54(\mathrm{~m}, 1 \mathrm{H}), 2.27(\mathrm{dqd}, J=$ 13.7, 7.5, $3.4 \mathrm{~Hz}, 1 \mathrm{H}), 1.79$ (ddq, $J=14.7,10.9,7.4 \mathrm{~Hz}, 1 \mathrm{H}), 1.00$ (t, $J=7.5 \mathrm{~Hz}, 3 \mathrm{H})$.

${ }^{13} \mathrm{C} \mathrm{NMR}\left(101 \mathrm{MHz}, \mathrm{CDCl}_{3}\right) \delta 160.2(\mathrm{~d}, J=250.5 \mathrm{~Hz}), 137.5,133.7,130.7(\mathrm{~d}, J=3.4 \mathrm{~Hz}), 129.8(\mathrm{~d}, J$ $=8.5 \mathrm{~Hz}), 129.2,128.9,127.6(\mathrm{~d}, J=3.4 \mathrm{~Hz}), 124.2(\mathrm{~d}, J=3.5 \mathrm{~Hz}), 123.8(\mathrm{~d}, J=5.0 \mathrm{~Hz}), 123.7(\mathrm{~d}, J=$ $12.1 \mathrm{~Hz}), 115.8(\mathrm{~d}, J=22.0 \mathrm{~Hz}), 71.5,20.9,11.4$.

${ }^{19} \mathrm{~F}$ NMR $\left(377 \mathrm{MHz}, \mathrm{CDCl}_{3}\right) \delta-117.6$.

HRMS (ESI) calcd. for $\mathrm{C}_{17} \mathrm{H}_{17} \mathrm{FNaO}_{2} \mathrm{~S}^{+}(\mathrm{M}+\mathrm{Na})^{+}: 327.0825$, Found: 327.0824

the ee value was $96 \%, \mathrm{t}_{\mathrm{r}}($ minor $)=13.474 \mathrm{~min}, \mathrm{t}_{\mathrm{r}}($ major $)=16.499 \min ($ Chiralcel $\mathrm{IC}, \lambda=220 \mathrm{~nm}$, hexanes : ${ }^{i} \mathrm{PrOH}=80: 20$, flow rate $=1.0 \mathrm{~mL} / \mathrm{min}$ ).
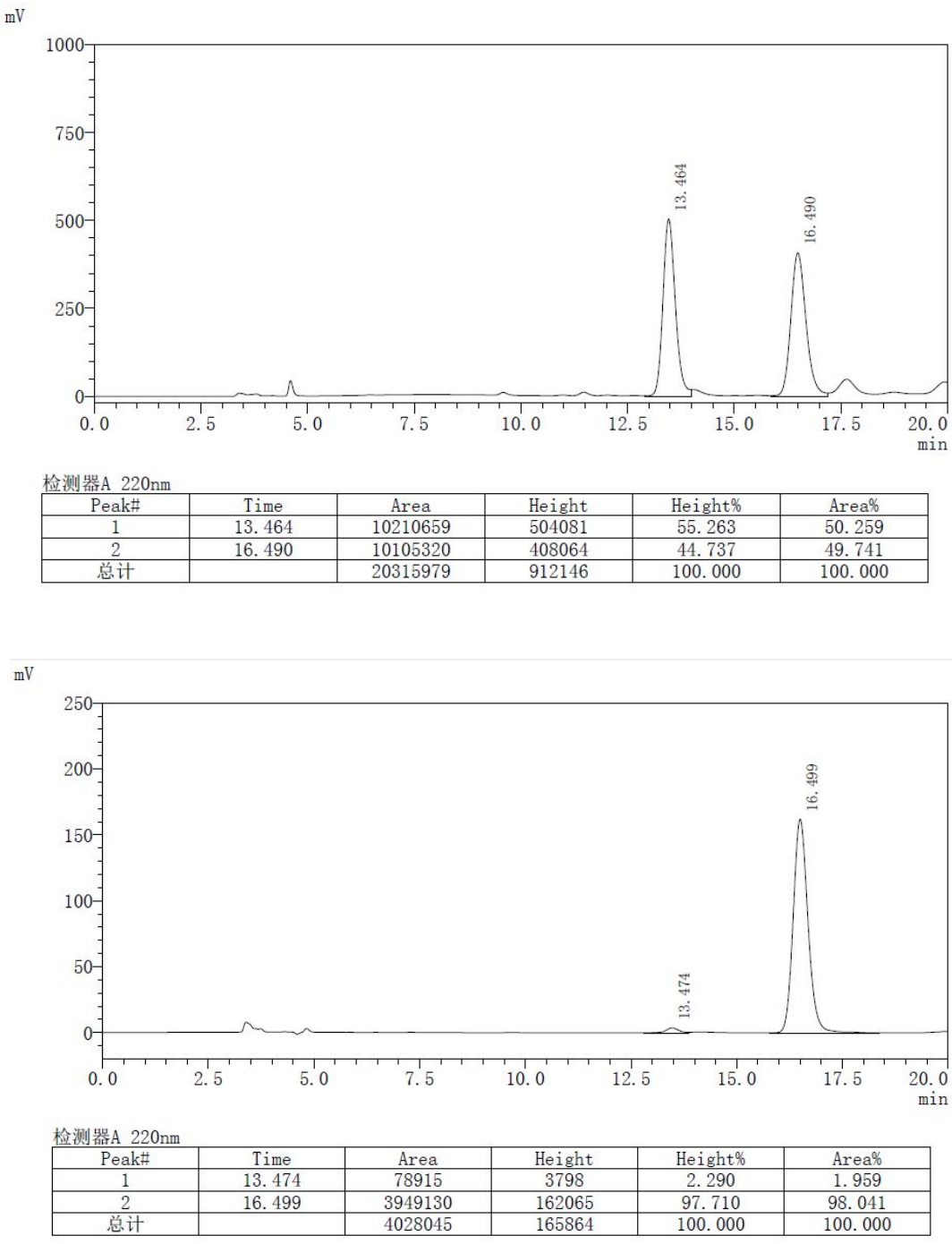<smiles>CC[C@H](/C=C/c1ccc(Cl)cc1)S(=O)(=O)c1ccccc1</smiles>

(S,E)-1-chloro-4-(3-(phenylsulfonyl)pent-1-en-1-yl)benzene 
Prepared according to General Procedure, white solid, $56 \mathrm{mg}$, 88\% yield, m.p. $66-67{ }^{\circ} \mathrm{C}, 17: 1 \mathrm{rr}$, $95 \%$ ee

$[\alpha]^{25}=-121.1\left(\mathrm{c} 1, \mathrm{CHCl}_{3}\right)$

${ }^{1} \mathrm{H}$ NMR $\left(400 \mathrm{MHz}, \mathrm{CDCl}_{3}\right) \delta 7.82(\mathrm{~d}, J=7.5 \mathrm{~Hz}, 2 \mathrm{H}), 7.61(\mathrm{t}, J=7.4 \mathrm{~Hz}, 1 \mathrm{H}), 7.50(\mathrm{t}, J=7.7 \mathrm{~Hz}$, 2H), $7.31-7.23(\mathrm{~m}, 2 \mathrm{H}), 7.19$ (d, $J=8.4 \mathrm{~Hz}, 2 \mathrm{H}), 6.24(\mathrm{~d}, J=15.9 \mathrm{~Hz}, 1 \mathrm{H}), 5.89$ (dd, $J=15.9,9.4$ $\mathrm{Hz}, 1 \mathrm{H}), 3.56(\mathrm{td}, J=10.6,3.2 \mathrm{~Hz}, 1 \mathrm{H}), 2.25(\mathrm{dqd}, J=14.9,7.4,3.4 \mathrm{~Hz}, 1 \mathrm{H}), 1.78(\mathrm{ddq}, J=14.6,11.3$, $7.4 \mathrm{~Hz}, 1 \mathrm{H}), 0.98(\mathrm{t}, J=7.4 \mathrm{~Hz}, 3 \mathrm{H})$.

${ }^{13} \mathrm{C}$ NMR $\left(101 \mathrm{MHz}, \mathrm{CDCl}_{3}\right) \delta 137.6,137.0,134.3,134.1,133.7,129.2,128.9,128.9,127.8,121.7$, 71.0, 20.9, 11.4 .

HRMS (ESI) calcd. for $\mathrm{C}_{17} \mathrm{H}_{17} \mathrm{ClNaO}_{2} \mathrm{~S}^{+}(\mathrm{M}+\mathrm{Na})^{+}: 343.0530$, Found: 343.0529

the ee value was $95 \%, \mathrm{t}_{\mathrm{r}}($ minor $)=14.523 \mathrm{~min}, \mathrm{t}_{\mathrm{r}}($ major $)=17.491 \min ($ Chiralcel IC, $\lambda=220 \mathrm{~nm}$, hexanes $:{ }^{i} \mathrm{PrOH}=80: 20$, flow rate $=1.0 \mathrm{~mL} / \mathrm{min}$ ).

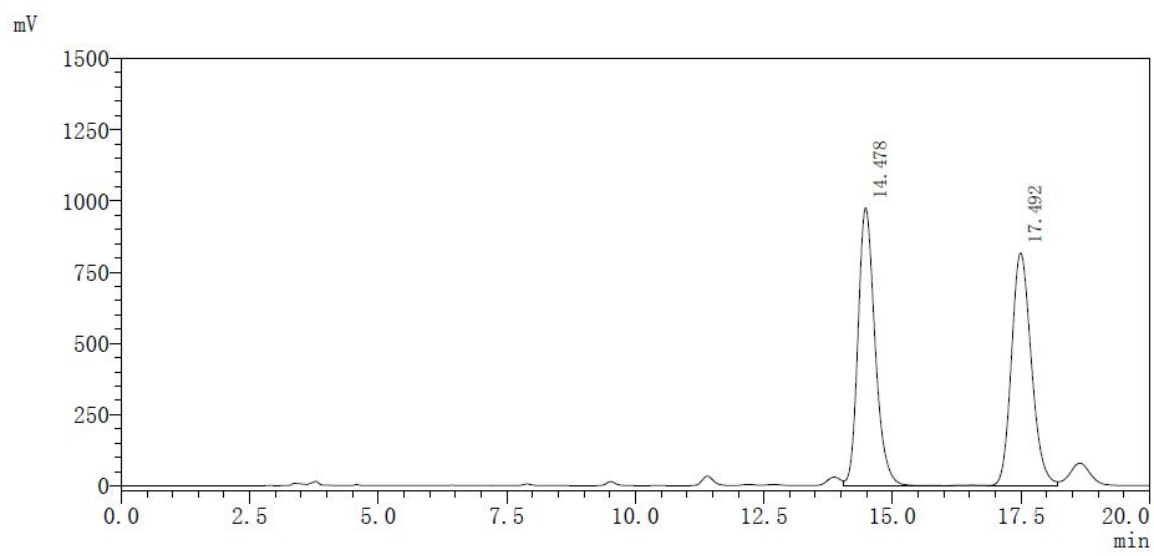

检测器A $220 \mathrm{~nm}$
\begin{tabular}{|c|c|c|c|c|c|}
\hline Peak\# & Time & Area & Height & Height $\%$ & Area $\%$ \\
\hline 1 & 14.478 & 22475737 & 975484 & 54.404 & 50.553 \\
\hline 2 & 17.492 & 21984269 & 817539 & 45.596 & 49.447 \\
\hline 总计 & & 44460005 & 1793022 & 100.000 & 100.000 \\
\hline
\end{tabular}
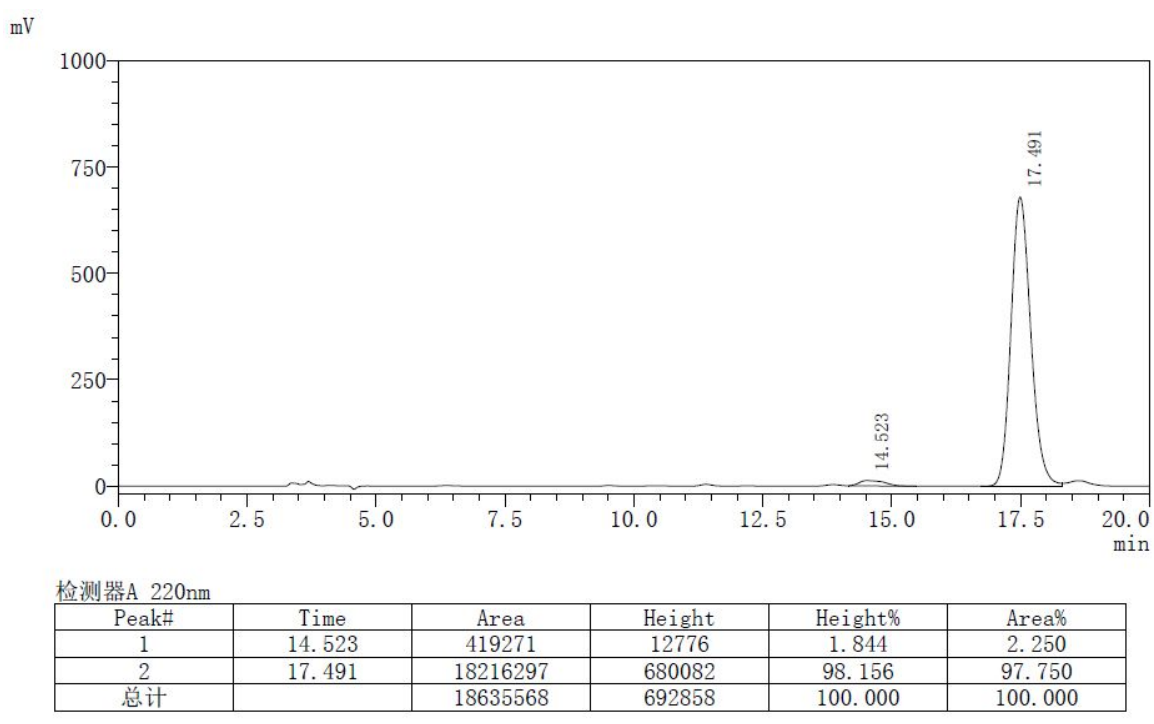
<smiles></smiles>

\section{(S,E)-1-(3-(phenylsulfonyl)pent-1-en-1-yl)-4-(trifluoromethyl)benzene}

Prepared according to General Procedure, white solid, $57 \mathrm{mg}, 80 \%$ yield, m.p. $78-80{ }^{\circ} \mathrm{C}, 6: 1 \mathrm{rr}$, $91 \%$ ee

$[\alpha]^{25}=-91.4\left(\mathrm{c} 1, \mathrm{CHCl}_{3}\right)$

${ }^{1} \mathrm{H}$ NMR (400 MHz, $\left.\mathrm{CDCl}_{3}\right) \delta 7.87-7.81(\mathrm{~m}, 2 \mathrm{H}), 7.63(\mathrm{t}, J=7.4 \mathrm{~Hz}, 1 \mathrm{H}), 7.59-7.47(\mathrm{~m}, 4 \mathrm{H}), 7.37$ $(\mathrm{d}, J=8.1 \mathrm{~Hz}, 2 \mathrm{H}), 6.33(\mathrm{~d}, J=15.9 \mathrm{~Hz}, 1 \mathrm{H}), 6.03(\mathrm{dd}, J=15.9,9.5 \mathrm{~Hz}, 1 \mathrm{H}), 3.65-3.55(\mathrm{~m}, 1 \mathrm{H})$, 2.27 (dqd, $J=15.0,7.5,3.4 \mathrm{~Hz}, 1 \mathrm{H}), 1.81$ (ddq, $J=14.7,11.0,7.4 \mathrm{~Hz}, 1 \mathrm{H}), 1.00$ (t, $J=7.5 \mathrm{~Hz}, 3 \mathrm{H}$ ).

${ }^{13} \mathrm{C}$ NMR $\left(101 \mathrm{MHz}, \mathrm{CDCl}_{3}\right) \delta 139.2,137.5,136.8,133.8,130.1$ (q, $\left.J=32.5 \mathrm{~Hz}\right), 129.1,129.0,126.7$, $125.6(\mathrm{q}, J=3.8 \mathrm{~Hz}), 124.0$ (q, $J=271.9 \mathrm{~Hz}), 123.8,71.0,20.9,11.4$.

${ }^{19} \mathrm{~F}$ NMR $\left(377 \mathrm{MHz}, \mathrm{CDCl}_{3}\right) \delta-62.6$.

HRMS (ESI) calcd.for $\mathrm{C}_{18} \mathrm{H}_{17} \mathrm{~F}_{3} \mathrm{NaO}_{2} \mathrm{~S}^{+}(\mathrm{M}+\mathrm{Na})^{+}: 377.0794$, Found: 377.0793

The ee value was $91 \%, \mathrm{t}_{\mathrm{r}}($ minor $)=10.852 \mathrm{~min}, \mathrm{t}_{\mathrm{r}}$ (major) $=13.223 \mathrm{~min}($ Chiralcel $\mathrm{IC}, \lambda=220$ $\mathrm{nm}$, hexanes $:{ }^{i} \mathrm{PrOH}=80: 20$, flow rate $=1.0 \mathrm{~mL} / \mathrm{min}$ ).

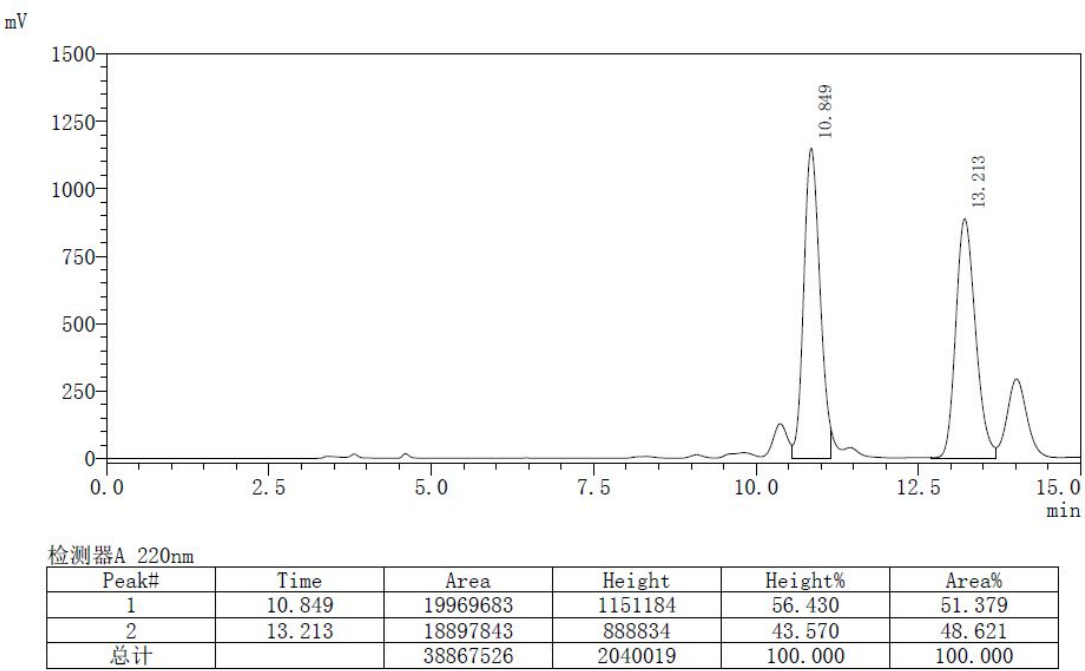




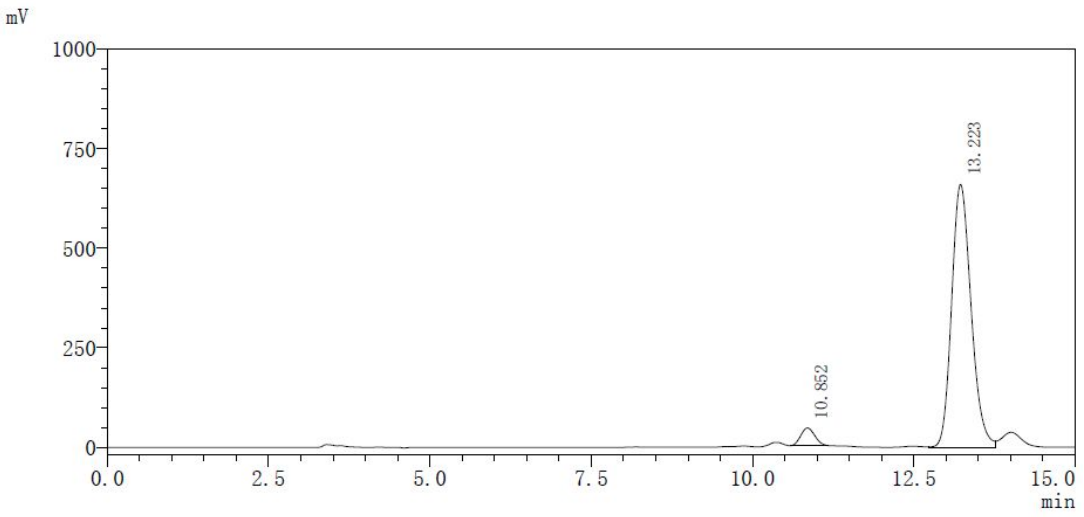

检测器A $220 \mathrm{~nm}$
\begin{tabular}{|c|c|c|c|c|c|}
\hline Peak\# & Time & Area & Height & Height $\%$ & Area $\%$ \\
\hline 1 & 10.852 & 668511 & 44226 & 6.276 & 4.599 \\
\hline 2 & 13.223 & 13867198 & 660492 & 93.724 & 95.401 \\
\hline 总计 & & 14535709 & 704718 & 100.000 & 100.000 \\
\hline
\end{tabular}<smiles>CC[C@H](/C=C/c1ccc(Br)cc1)S(=O)(=O)c1ccccc1</smiles>

\section{(S,E)-1-bromo-4-(3-(phenylsulfonyl)pent-1-en-1-yl)benzene}

Prepared according to General Procedure, white solid, $65 \mathrm{mg}, 89 \%$ yield, m.p. $100-102^{\circ} \mathrm{C}, 14: 1 \mathrm{rr}$, $94 \%$ ee

$[\alpha]^{25}=-115.8\left(\mathrm{c} 1, \mathrm{CHCl}_{3}\right)$

${ }^{1} \mathrm{H}$ NMR (400 MHz, $\left.\mathrm{CDCl}_{3}\right) \delta 7.85-7.78(\mathrm{~m}, 2 \mathrm{H}), 7.61(\mathrm{t}, J=7.4 \mathrm{~Hz}, 1 \mathrm{H}), 7.50(\mathrm{t}, J=7.7 \mathrm{~Hz}, 2 \mathrm{H})$, $7.43(\mathrm{~d}, J=8.4 \mathrm{~Hz}, 2 \mathrm{H}), 7.12(\mathrm{~d}, J=8.4 \mathrm{~Hz}, 2 \mathrm{H}), 6.22(\mathrm{~d}, J=15.9 \mathrm{~Hz}, 1 \mathrm{H}), 5.90(\mathrm{dd}, J=15.9,9.4 \mathrm{~Hz}$, $1 \mathrm{H}), 3.55(\mathrm{td}, J=10.6,3.3 \mathrm{~Hz}, 1 \mathrm{H}), 2.25(\mathrm{dqd}, J=15.0,7.5,3.4 \mathrm{~Hz}, 1 \mathrm{H}), 1.78(\mathrm{ddq}, J=14.6,11.0,7.4$ $\mathrm{Hz}, 1 \mathrm{H}), 0.98(\mathrm{t}, J=7.5 \mathrm{~Hz}, 3 \mathrm{H})$.

${ }^{13} \mathrm{C}$ NMR $\left(101 \mathrm{MHz}, \mathrm{CDCl}_{3}\right) \delta 137.5,137.1,134.7,133.7,131.8,129.2,128.9,128.0,122.4,121.8$, $71.1,20.9,11.4$.

HRMS (ESI) calcd. for $\mathrm{C}_{17} \mathrm{H}_{17} \mathrm{BrNaO}_{2} \mathrm{~S}^{+}(\mathrm{M}+\mathrm{Na})^{+}: 387.0025$, Found: 387.0023

the ee value was $94 \%, \mathrm{t}_{\mathrm{r}}($ minor $)=15.057 \min , \mathrm{t}_{\mathrm{r}}($ major $)=17.806 \min ($ Chiralcel IC, $\lambda=220 \mathrm{~nm}$, hexanes : ${ }^{i} \mathrm{PrOH}=80: 20$, flow rate $=1.0 \mathrm{~mL} / \mathrm{min}$ ) 
$\mathrm{mV}$

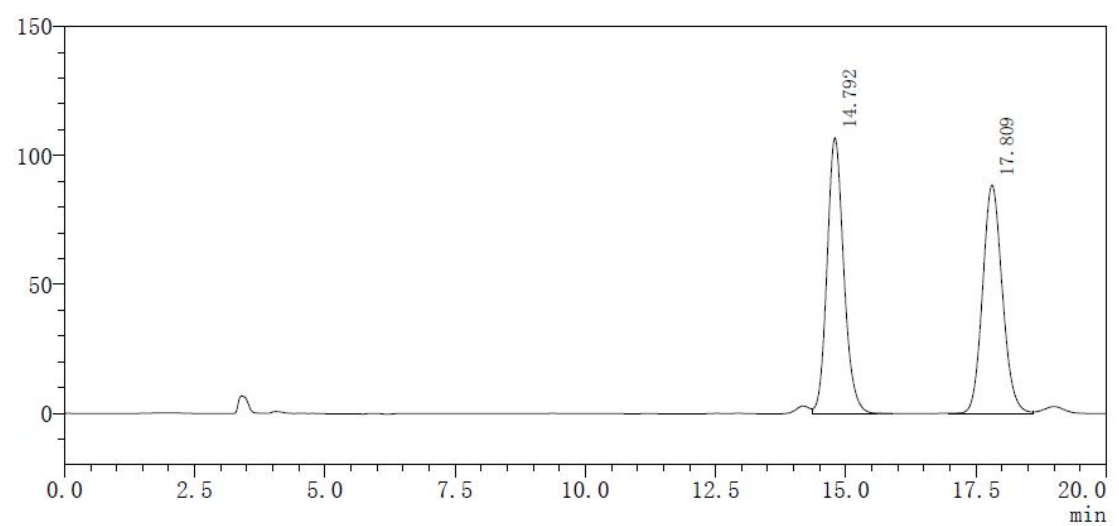

检测器A $220 \mathrm{~nm}$
\begin{tabular}{|c|c|c|c|c|c|}
\hline Peak\# & Time & Area & Height & Height $\%$ & Area $\%$ \\
\hline 1 & 14.792 & 2397480 & 106991 & 54.691 & 50.593 \\
\hline 2 & 17.809 & 2341233 & 88638 & 45.309 & 49.407 \\
\hline 总计 & & 4738712 & 195630 & 100.000 & 100.000 \\
\hline
\end{tabular}

$\mathrm{mV}$

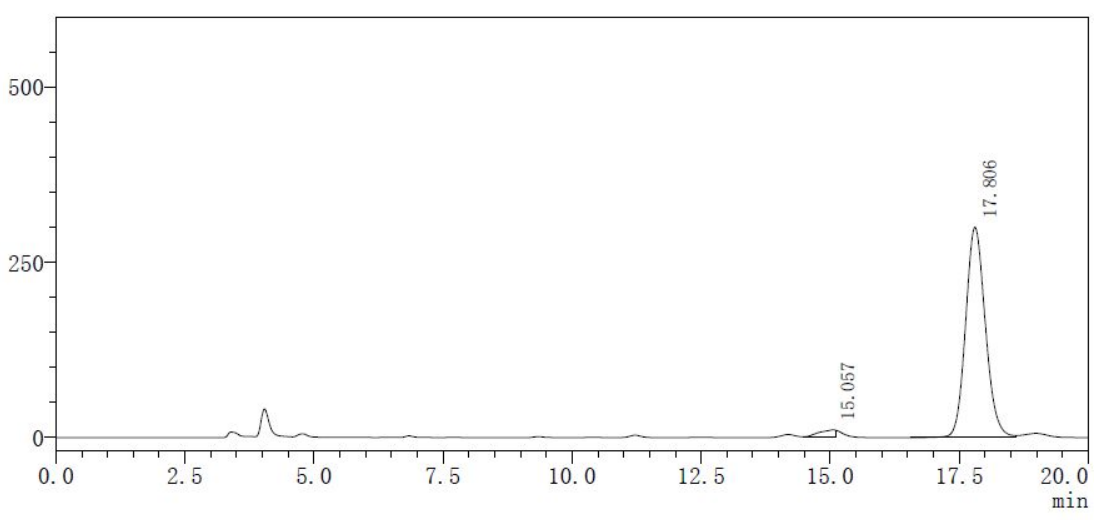

检测器A $220 \mathrm{~nm}$
\begin{tabular}{|c|c|c|c|c|c|}
\hline Peak\# & Time & Area & Height & Height\% & Area $\%$ \\
\hline 1 & 15.057 & 254603 & 10881 & 3.496 & 3.088 \\
\hline 2 & 17.806 & 7990627 & 300321 & 96.504 & 96.912 \\
\hline 总计 & & 8245230 & 311201 & 100.000 & 100.000
\end{tabular}<smiles>CC[C@H](/C=C/c1ccc(C(C)=O)cc1)S(=O)(=O)c1ccccc1</smiles>

\section{methyl (S,E)-4-(3-(phenylsulfonyl)pent-1-en-1-yl)benzoate}

Prepared according to General Procedure, white solid, $54 \mathrm{mg}$, 78\% yield, m.p. $97-98{ }^{\circ} \mathrm{C}, 11: 1 \mathrm{rr}$, $96 \%$ ee

$[\alpha]^{25}=-95.4\left(\mathrm{c} 1, \mathrm{CHCl}_{3}\right)$

${ }^{1} \mathrm{H}$ NMR $\left(400 \mathrm{MHz}, \mathrm{CDCl}_{3}\right) \delta 7.99(\mathrm{~d}, J=8.1 \mathrm{~Hz}, 2 \mathrm{H}), 7.84(\mathrm{~d}, J=7.8 \mathrm{~Hz}, 2 \mathrm{H}), 7.63(\mathrm{t}, J=7.3 \mathrm{~Hz}$, $1 \mathrm{H}), 7.52$ (t, $J=7.6 \mathrm{~Hz}, 2 \mathrm{H}), 7.33(\mathrm{~d}, J=8.1 \mathrm{~Hz}, 2 \mathrm{H}), 6.34$ (d, $J=15.9 \mathrm{~Hz}, 1 \mathrm{H}), 6.04$ (dd, $J=15.9,9.4$ Hz, 1H), 3.93 (s, 3H), $3.61(\mathrm{td}, J=10.4,3.2 \mathrm{~Hz}, 1 \mathrm{H}), 2.28(\mathrm{dqd}, J=14.9,7.5,3.5 \mathrm{~Hz}, 1 \mathrm{H}), 1.89-1.76$ 
(m, 1H), $1.01(\mathrm{t}, J=7.4 \mathrm{~Hz}, 3 \mathrm{H})$.

${ }^{13} \mathrm{C}$ NMR $\left(101 \mathrm{MHz}, \mathrm{CDCl}_{3}\right) \delta 166.6,140.1,137.6,137.3,133.7,130.0,129.8,129.1,128.9,126.4$, $123.8,71.1,52.1,21.0,11.4$.

HRMS (ESI) calcd. for $\mathrm{C}_{19} \mathrm{H}_{20} \mathrm{NaO}_{4} \mathrm{~S}^{+}(\mathrm{M}+\mathrm{Na})^{+}: 367.0975$, Found: 367.0969

the ee value was $96 \%, \mathrm{t}_{\mathrm{r}}$ (major) $=35.654 \mathrm{~min}, \mathrm{t}_{\mathrm{r}}($ minor $)=49.347 \min ($ Chiralcel IC, $\lambda=220 \mathrm{~nm}$, hexanes : ${ }^{i} \mathrm{PrOH}=70: 30$, flow rate $=1.0 \mathrm{~mL} / \mathrm{min}$ )

$\mathrm{mV}$

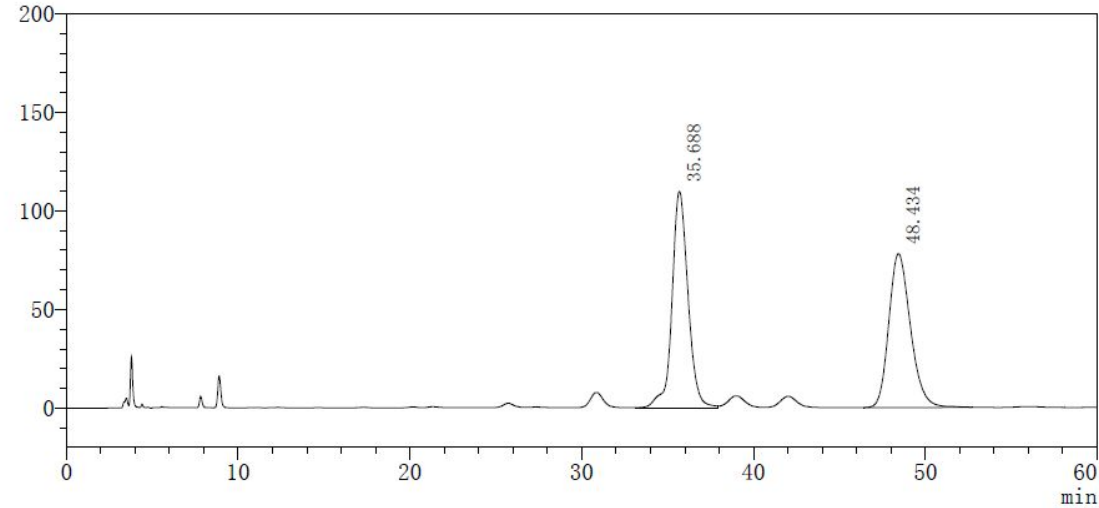

检测器A $220 \mathrm{~nm}$
\begin{tabular}{|c|c|c|c|c|c|}
\hline Peak\# & Time & Area & Height & Height $\%$ & Area\% \\
\hline 1 & 35.688 & 7080409 & 109732 & 58.432 & 50.952 \\
\hline 2 & 48.434 & 6815919 & 78061 & 41.568 & 49.048 \\
\hline 总计 & & 13896328 & 187792 & 100.000 & 100.000 \\
\hline
\end{tabular}

$\mathrm{mV}$

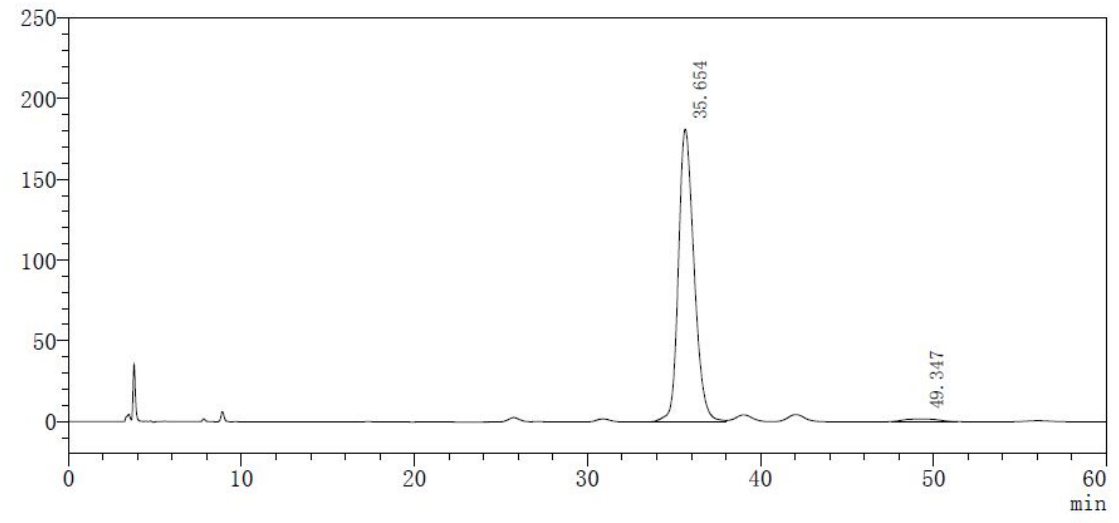

检测器A $220 \mathrm{~nm}$
\begin{tabular}{|c|c|c|c|c|c|}
\hline Peak\# & Time & Area & Height & Height\% & Area\% \\
\hline 1 & 35.654 & 11357732 & 181272 & 99.065 & 98.144 \\
\hline 2 & 49.347 & 214779 & 1711 & 0.935 & 1.856 \\
\hline 总计 & & 11572512 & 182984 & 100.000 & 100.000 \\
\hline
\end{tabular}<smiles>CC[C@H](/C=C/c1ccc(OC)cc1)S(=O)(=O)c1ccccc1</smiles> 


\section{(S,E)-1-methoxy-4-(3-(phenylsulfonyl)pent-1-en-1-yl)benzene}

Prepared according to General Procedure, white solid, $56 \mathrm{mg}, 88 \%$ yield, m.p. $116-117^{\circ} \mathrm{C},>20: 1$ rr, $95 \%$ ee

$[\alpha]^{25}{ }_{\mathrm{D}}=-136.4\left(\mathrm{c} 1, \mathrm{CHCl}_{3}\right)$

${ }^{1} \mathrm{H} \mathrm{NMR}\left(400 \mathrm{MHz}, \mathrm{CDCl}_{3}\right) \delta 7.83(\mathrm{~d}, J=7.4 \mathrm{~Hz}, 2 \mathrm{H}), 7.60(\mathrm{t}, J=7.4 \mathrm{~Hz}, 1 \mathrm{H}), 7.49(\mathrm{t}, J=7.7 \mathrm{~Hz}$, 2H), 7.20 (d, $J=8.7 \mathrm{~Hz}, 2 \mathrm{H}), 6.83(\mathrm{~d}, J=8.7 \mathrm{~Hz}, 2 \mathrm{H}), 6.21$ (d, $J=15.8 \mathrm{~Hz}, 1 \mathrm{H}), 5.74$ (dd, $J=15.8$, $9.4 \mathrm{~Hz}, 1 \mathrm{H}), 3.80$ (s, 3H), 3.53 (td, $J=10.7,3.3 \mathrm{~Hz}, 1 \mathrm{H}), 2.26$ (dqd, $J=15.0,7.5,3.4 \mathrm{~Hz}, 1 \mathrm{H}), 1.76$ (ddq, $J=14.6,11.1,7.4 \mathrm{~Hz}, 1 \mathrm{H}), 0.99(\mathrm{t}, J=7.4 \mathrm{~Hz}, 3 \mathrm{H})$.

${ }^{13} \mathrm{C}$ NMR $\left(101 \mathrm{MHz}, \mathrm{CDCl}_{3}\right) \delta 159.8,137.8,137.7,133.5,129.2,128.8,128.7,127.8,118.5,114.0$, $71.3,55.3,21.0,11.4$.

HRMS (ESI) calcd.for $\mathrm{C}_{18} \mathrm{H}_{20} \mathrm{NaO}_{3} \mathrm{~S}^{+}(\mathrm{M}+\mathrm{Na})^{+}: 339.1025$, Found: 339.1024

theee value was $95 \%, \mathrm{t}_{\mathrm{r}}($ minor $)=23.560 \mathrm{~min}, \mathrm{t}_{\mathrm{r}}$ (major) $=26.729 \min ($ Chiralcel IC, $\lambda=220 \mathrm{~nm}$, hexanes $:{ }^{i} \mathrm{PrOH}=80: 20$, flow rate $=1.0 \mathrm{~mL} / \mathrm{min}$ ).

$\mathrm{mV}$

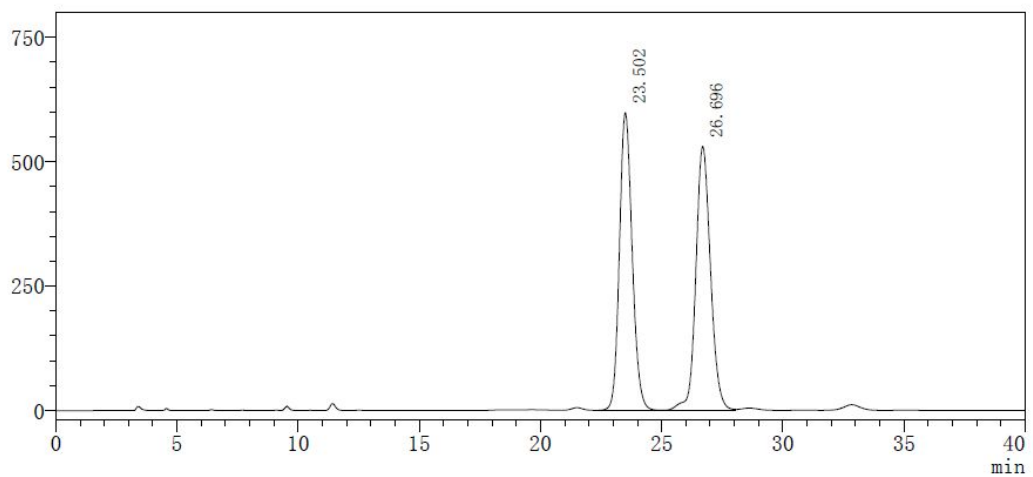

检测器A $220 \mathrm{~nm}$
\begin{tabular}{|c|c|c|c|c|c|}
\hline Peak\# & Time & Area & Height & Height\% & Area $\%$ \\
\hline 1 & 23.502 & 21911901 & 598301 & 53.007 & 49.400 \\
\hline 2 & 26.696 & 22444284 & 530420 & 46.993 & 50.600 \\
\hline 总计 & & 44356185 & 1128721 & 100.000 & 100.000 \\
\hline
\end{tabular}

$\mathrm{mV}$

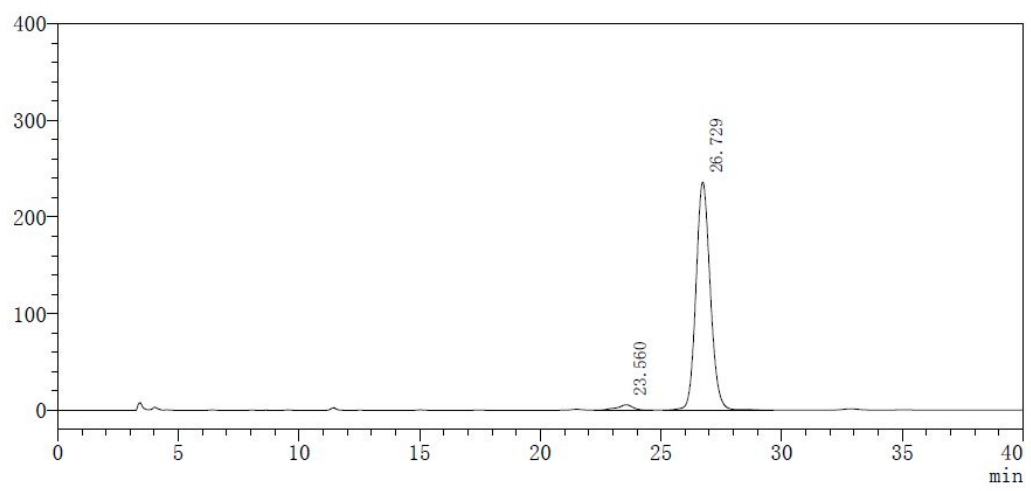

检测器A $220 \mathrm{~nm}$
\begin{tabular}{|c|c|c|c|c|c|}
\hline Peak\# & Time & Area & Height & Height $\%$ & Area $\%$ \\
\hline 1 & 23.560 & 246205 & 5491 & 2.275 & 2.474 \\
\hline 2 & 26.729 & 9706268 & 235910 & 97.725 & 97.526 \\
\hline 总计 & & 9952473 & 241402 & 100.000 & 100.000 \\
\hline
\end{tabular}


<smiles>CC[C@H](/C=C/c1ccc(C)cc1)[Sb](=O)(=O)c1ccccc1</smiles>

\section{(S,E)-1-methyl-4-(3-(phenylsulfonyl)pent-1-en-1-yl)benzene}

Prepared according to General Procedure, white solid, $54 \mathrm{mg}, 90 \%$ yield, m.p. $70-71{ }^{\circ} \mathrm{C},>20: 1 \mathrm{rr}$, $96 \%$ ee

$[\alpha]^{25} \mathrm{D}=-106.2\left(\mathrm{c} 1, \mathrm{CHCl}_{3}\right)$

${ }^{1} \mathrm{H}$ NMR $\left(400 \mathrm{MHz}, \mathrm{CDCl}_{3}\right) \delta 7.89-7.76(\mathrm{~m}, 2 \mathrm{H}), 7.66-7.54(\mathrm{~m}, 1 \mathrm{H}), 7.54-7.45(\mathrm{~m}, 2 \mathrm{H}), 7.22-$ $7.04(\mathrm{~m}, 4 \mathrm{H}), 6.23(\mathrm{~d}, J=15.9 \mathrm{~Hz}, 1 \mathrm{H}), 5.92-5.76(\mathrm{~m}, 1 \mathrm{H}), 3.61-3.48(\mathrm{~m}, 1 \mathrm{H}), 2.33(\mathrm{~s}, 3 \mathrm{H}), 2.30-$ $2.21(\mathrm{~m}, 1 \mathrm{H}), 1.86-1.69(\mathrm{~m}, 1 \mathrm{H}), 0.99(\mathrm{t}, J=9.7 \mathrm{~Hz}, 3 \mathrm{H})$.

${ }^{13} \mathrm{C}$ NMR $\left(101 \mathrm{MHz}, \mathrm{CDCl}_{3}\right) \delta 138.4,138.2,137.7,133.5,133.1,129.4,129.2,128.8,126.5,119.9$, $71.2,21.2,20.9,11.4$.

HRMS (ESI) calcd. for $\mathrm{C}_{18} \mathrm{H}_{20} \mathrm{NaO}_{2} \mathrm{~S}^{+}(\mathrm{M}+\mathrm{Na})^{+}: 323.1076$, Found: 323.1076

the ee value was $96 \%, \mathrm{t}_{\mathrm{r}}($ minor $)=15.772 \mathrm{~min}, \mathrm{t}_{\mathrm{r}}($ major $)=18.476 \min ($ Chiralcel IC, $\lambda=220 \mathrm{~nm}$, hexanes : ${ }^{i} \mathrm{PrOH}=80: 20$, flow rate $=1.0 \mathrm{~mL} / \mathrm{min}$ ).

$\mathrm{mV}$

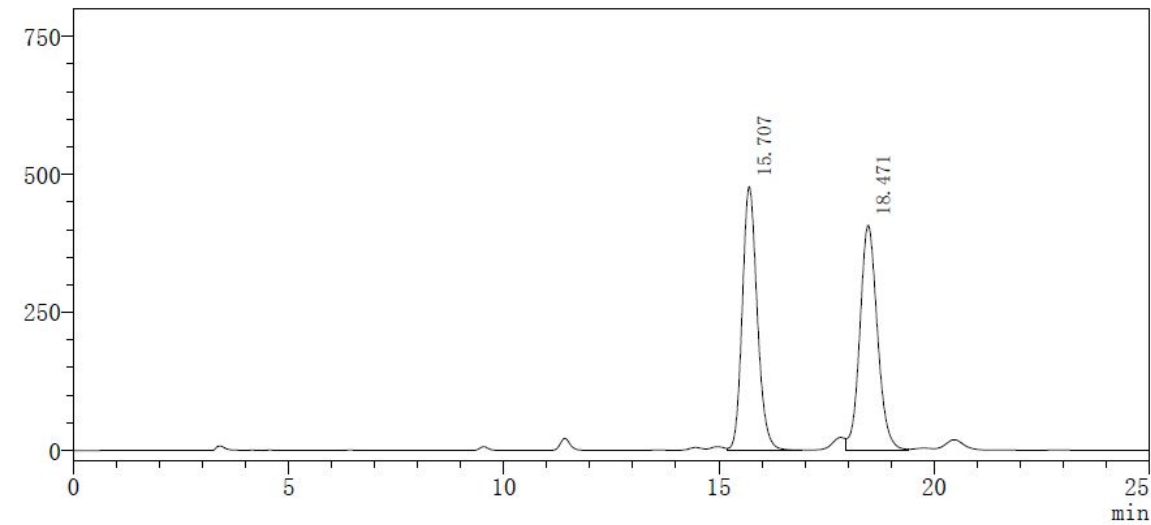

检测器A $220 \mathrm{~nm}$
\begin{tabular}{|c|c|c|c|c|c|}
\hline Peak\# & Time & Area & Height & Height\% & Area $\%$ \\
\hline 1 & 15.707 & 11554992 & 477639 & 53.969 & 49.943 \\
\hline 2 & 18.471 & 11581278 & 407377 & 46.031 & 50.057 \\
\hline 总计 & & 23136270 & 885016 & 100.000 & 100.000 \\
\hline
\end{tabular}


$\mathrm{mV}$

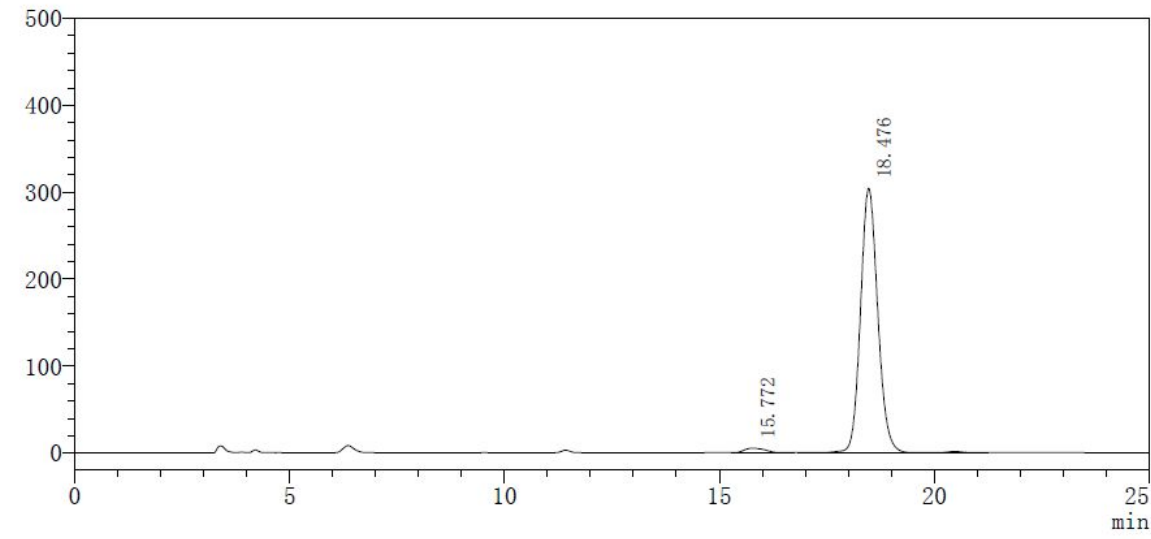

检测器A $220 \mathrm{~nm}$
\begin{tabular}{|c|c|c|c|c|c|}
\hline Peak\# & Time & Area & Height & Height\% & Area\% \\
\hline 1 & 15.772 & 181432 & 5466 & 1.765 & 2.075 \\
\hline 2 & 18.476 & 8561717 & 304266 & 98.235 & 97.925 \\
\hline 总计 & & 8743149 & 309732 & 100.000 & 100.000 \\
\hline
\end{tabular}<smiles>CCC(/C=C/c1cccs1)S(=O)(=O)c1ccccc1</smiles>

\section{(S,E)-2-(3-(phenylsulfonyl)pent-1-en-1-yl)thiophene}

Prepared according to General Procedure, white solid, $55 \mathrm{mg}, 94 \%$ yield, m.p. $84-85{ }^{\circ} \mathrm{C},>20: 1 \mathrm{rr}$, $98 \%$ ee

$[\alpha]^{25}{ }_{\mathrm{D}}=-142.6\left(\mathrm{c} 1, \mathrm{CHCl}_{3}\right)$

${ }^{1} \mathrm{H}$ NMR $\left(400 \mathrm{MHz}, \mathrm{CDCl}_{3}\right) \delta 7.83(\mathrm{~d}, J=7.6 \mathrm{~Hz}, 2 \mathrm{H}), 7.61(\mathrm{t}, J=7.4 \mathrm{~Hz}, 1 \mathrm{H}), 7.50(\mathrm{t}, J=7.6 \mathrm{~Hz}$, $2 \mathrm{H}), 7.18(\mathrm{~d}, J=4.9 \mathrm{~Hz}, 1 \mathrm{H}), 7.00-6.91(\mathrm{~m}, 1 \mathrm{H}), 6.88(\mathrm{~s}, 1 \mathrm{H}), 6.43(\mathrm{~d}, J=15.7 \mathrm{~Hz}, 1 \mathrm{H}), 5.70(\mathrm{dd}, J=$ 15.7, $9.4 \mathrm{~Hz}, 1 \mathrm{H}), 3.58-3.44$ (m, 1H), $2.32-2.18(\mathrm{~m}, 1 \mathrm{H}), 1.82-1.67$ (m, 1H), 0.99 (t, $J=7.4 \mathrm{~Hz}$, $3 \mathrm{H})$.

${ }^{13} \mathrm{C}$ NMR $\left(101 \mathrm{MHz}, \mathrm{CDCl}_{3}\right) \delta 140.8,137.6,133.6,131.1,129.2,128.9,127.5,126.8,125.4,120.2$, $71.1,21.0,11.4$.

HRMS (ESI) calcd.for $\mathrm{C}_{15} \mathrm{H}_{16} \mathrm{NaO}_{2} \mathrm{~S}_{2}^{+}(\mathrm{M}+\mathrm{Na})^{+}: 315.0484$, Found: 315.0482

theee value was $98 \%, \mathrm{t}_{\mathrm{r}}($ minor $)=16.319 \min , \mathrm{t}_{\mathrm{r}}$ (major) $=19.640 \min ($ Chiralcel IC, $\lambda=220 \mathrm{~nm}$, hexanes : ${ }^{i} \mathrm{PrOH}=80: 20$, flow rate $=1.0 \mathrm{~mL} / \mathrm{min}$ ) 

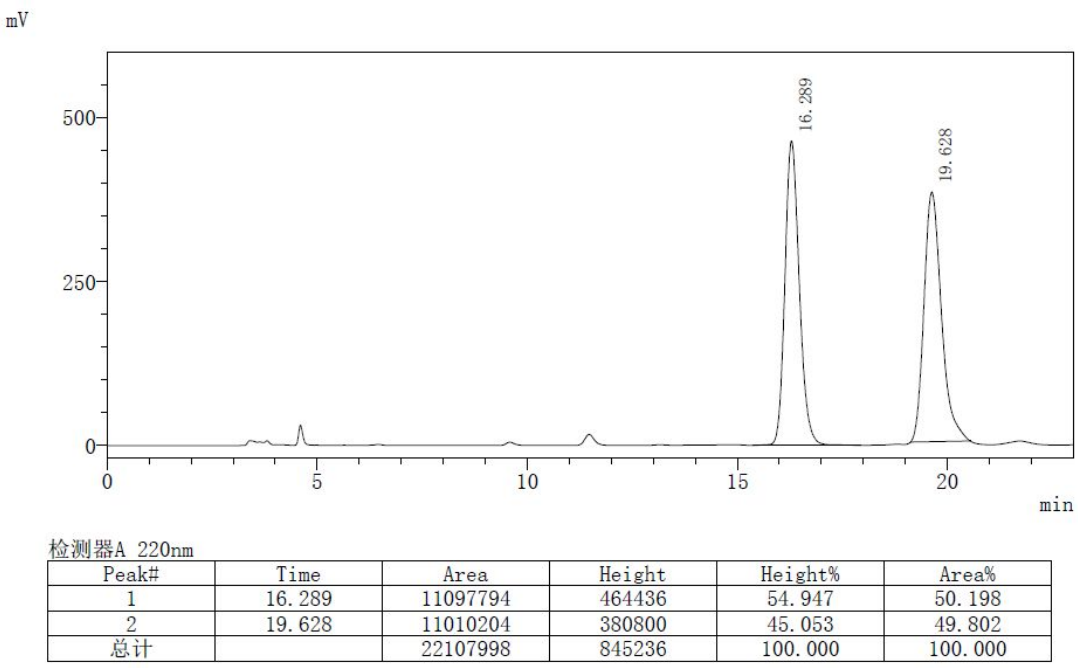

$\mathrm{mV}$
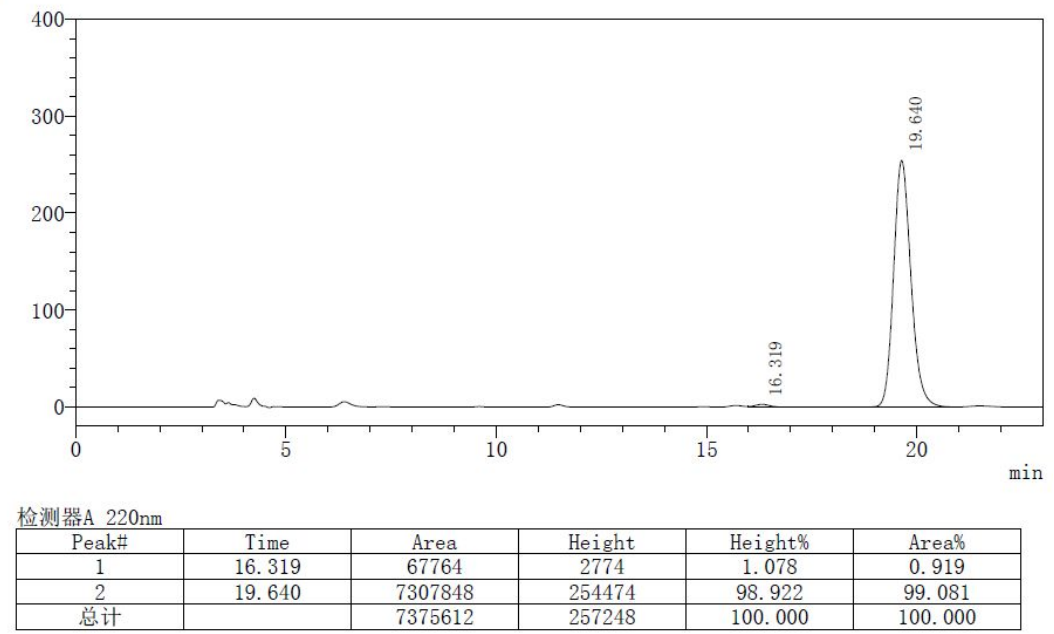<smiles>CCC(/C=C/c1ccco1)[Sb](=O)(=O)c1ccccc1</smiles>

\section{(S,E)-2-(3-(phenylsulfonyl)pent-1-en-1-yl)furan}

Prepared according to General Procedure, pale yellow oil, $52 \mathrm{mg}, 93 \%$ yield, $>20: 1 \mathrm{rr}, 94 \%$ ee $[\alpha]^{25}=-82.8\left(\mathrm{c} 1, \mathrm{CHCl}_{3}\right)$

${ }^{1} \mathrm{H}$ NMR $\left(400 \mathrm{MHz}, \mathrm{CDCl}_{3}\right) \delta 7.83(\mathrm{~d}, J=6.9 \mathrm{~Hz}, 2 \mathrm{H}), 7.61(\mathrm{~d}, J=7.3 \mathrm{~Hz}, 1 \mathrm{H}), 7.51(\mathrm{t}, J=7.3 \mathrm{~Hz}$, 2H), $7.34(\mathrm{~s}, 1 \mathrm{H}), 6.35(\mathrm{~s}, 1 \mathrm{H}), 6.20(\mathrm{~s}, 1 \mathrm{H}), 6.15(\mathrm{~d}, J=15.8 \mathrm{~Hz}, 1 \mathrm{H}), 5.83(\mathrm{dd}, J=15.6,9.6 \mathrm{~Hz}, 1 \mathrm{H})$, $3.59-3.44(\mathrm{~m}, 1 \mathrm{H}), 2.30-2.16(\mathrm{~m}, 1 \mathrm{H}), 1.80-1.66(\mathrm{~m}, 1 \mathrm{H}), 0.97(\mathrm{t}, J=7.2 \mathrm{~Hz}, 3 \mathrm{H})$.

${ }^{13} \mathrm{C}$ NMR $\left(101 \mathrm{MHz}, \mathrm{CDCl}_{3}\right) \delta 151.5,142.7,137.6,137.1,133.6,129.2,128.9,126.0,119.1,111.4$, 109.4, 71.0, 21.2, 11.4 .

HRMS (ESI) calcd. for $\mathrm{C}_{15} \mathrm{H}_{16} \mathrm{NaO}_{3} \mathrm{~S}^{+}(\mathrm{M}+\mathrm{Na})^{+}:$299.0712, Found: 299.0709 
the ee value was $94 \%, \mathrm{t}_{\mathrm{r}}($ minor $)=16.359 \min , \mathrm{t}_{\mathrm{r}}($ major $)=18.970 \min ($ Chiralcel IC, $\lambda=220 \mathrm{~nm}$, hexanes : ${ }^{i} \mathrm{PrOH}=80: 20$, flow rate $=1.0 \mathrm{~mL} / \mathrm{min}$ )

$\mathrm{mV}$

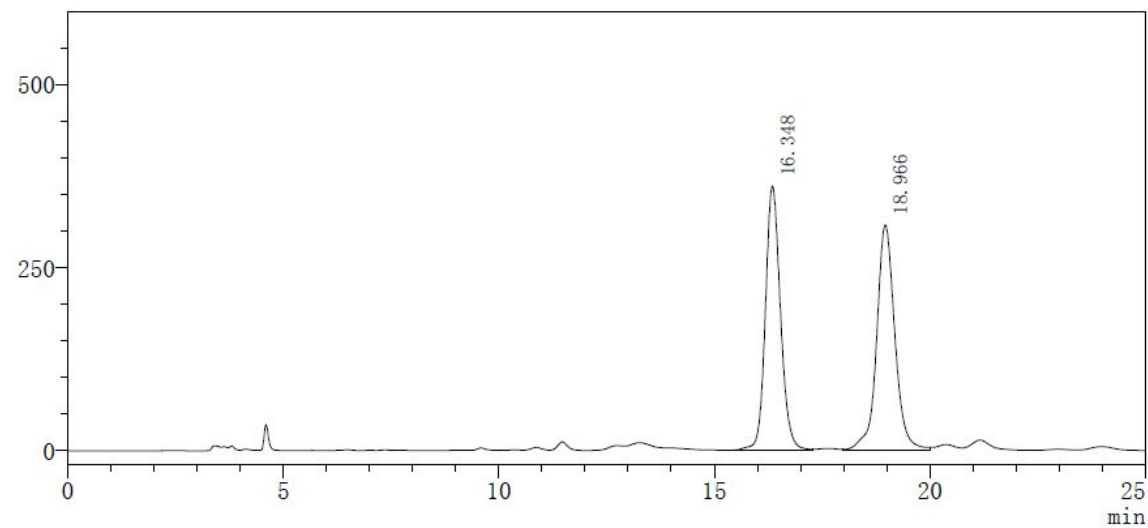

检测器A $220 \mathrm{~nm}$
\begin{tabular}{|c|c|c|c|c|c|}
\hline Peak\# & Time & Area & Height & Height\% & Area $\%$ \\
\hline 1 & 16.348 & 8846724 & 361582 & 53.988 & 49.302 \\
\hline 2 & 18.966 & 9097116 & 308163 & 46.012 & 50.698 \\
\hline 总计 & & 17943840 & 669745 & 100.000 & 100.000 \\
\hline
\end{tabular}

$\mathrm{mV}$

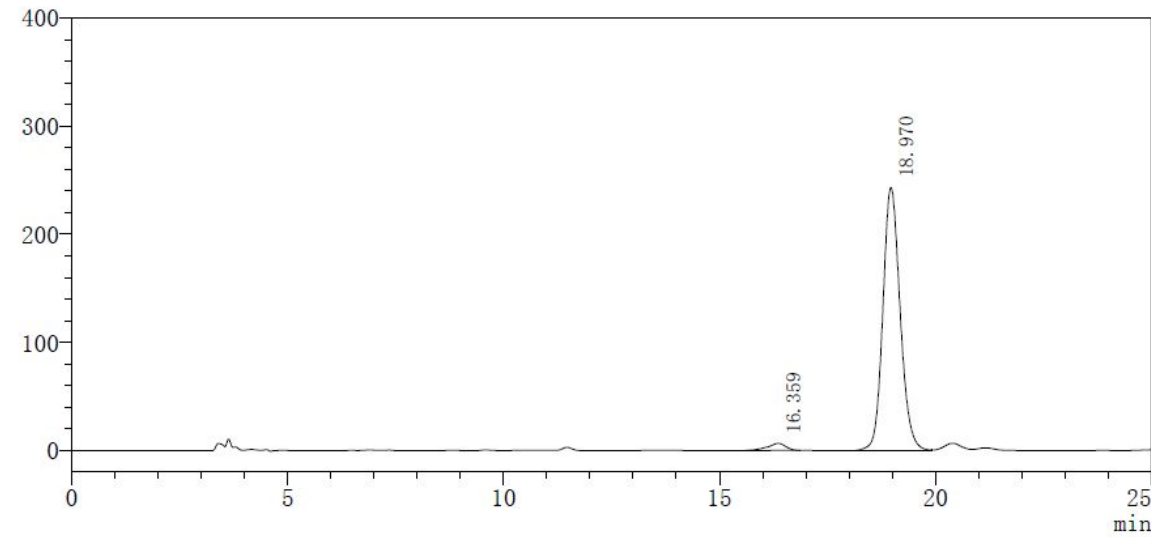

检测器A $220 \mathrm{~nm}$
\begin{tabular}{|c|c|c|c|c|c|}
\hline Peak\# & Time & Area & Height & Height\% & Area\% \\
\hline 1 & 16.359 & 193618 & 6479 & 2.591 & 2.780 \\
\hline 2 & 18.970 & 6772001 & 243522 & 97.409 & 97.220 \\
\hline 总计 & & 6965619 & 250001 & 100.000 & 100.000 \\
\hline
\end{tabular}<smiles>CC[C@@H](/C=C/c1cccnc1)[Sb](=O)(=O)c1ccccc1</smiles>

\section{(S,E)-3-(3-(phenylsulfonyl)pent-1-en-1-yl)pyridine}

Prepared according to General Procedure, pale yellow oil, $42 \mathrm{mg}, 73 \%$ yield, 7.5:1 rr, 97\% ee $[\alpha]^{25}=-58.9\left(\mathrm{c} 1, \mathrm{CHCl}_{3}\right)$

${ }^{1} \mathrm{H}$ NMR $\left(400 \mathrm{MHz}, \mathrm{CDCl}_{3}\right) \delta 8.51(\mathrm{~d}, J=4.5 \mathrm{~Hz}, 1 \mathrm{H}), 8.46(\mathrm{~s}, 1 \mathrm{H}), 7.85(\mathrm{~d}, J=7.7 \mathrm{~Hz}, 2 \mathrm{H}), 7.68-$ $7.60(\mathrm{~m}, 2 \mathrm{H}), 7.53(\mathrm{t}, J=7.7 \mathrm{~Hz}, 2 \mathrm{H}), 7.28-7.24(\mathrm{~m}, 1 \mathrm{H}), 6.30(\mathrm{~d}, J=16.0 \mathrm{~Hz}, 1 \mathrm{H}), 6.01(\mathrm{dd}, J=$ $16.0,9.4 \mathrm{~Hz}, 1 \mathrm{H}), 3.61(\mathrm{td}, J=10.5,3.3 \mathrm{~Hz}, 1 \mathrm{H}), 2.28(\mathrm{dqd}, J=15.0,7.5,3.5 \mathrm{~Hz}, 1 \mathrm{H}), 1.89-1.75(\mathrm{~m}$, $1 \mathrm{H}), 1.01(\mathrm{t}, J=7.5 \mathrm{~Hz}, 3 \mathrm{H})$. 
${ }^{13} \mathrm{C}$ NMR $\left(101 \mathrm{MHz}, \mathrm{CDCl}_{3}\right) \delta 149.4,148.4,137.5,134.7,133.8,133.0,131.5,129.1,129.0,123.6$, $71.1,20.9,11.4$.

HRMS (ESI) calcd. for $\mathrm{C}_{16} \mathrm{H}_{18} \mathrm{NO}_{2} \mathrm{~S}^{+}(\mathrm{M}+\mathrm{H})^{+}:$288.1053, Found: 288.1049

the ee value was $97 \%, \mathrm{t}_{\mathrm{r}}($ minor $)=36.594 \min , \mathrm{t}_{\mathrm{r}}($ major $)=44.777 \min ($ Chiralcel IC, $\lambda=220 \mathrm{~nm}$, hexanes $:{ }^{i} \mathrm{PrOH}=70: 30$, flow rate $=1.0 \mathrm{~mL} / \mathrm{min}$ )

$\mathrm{m} V$

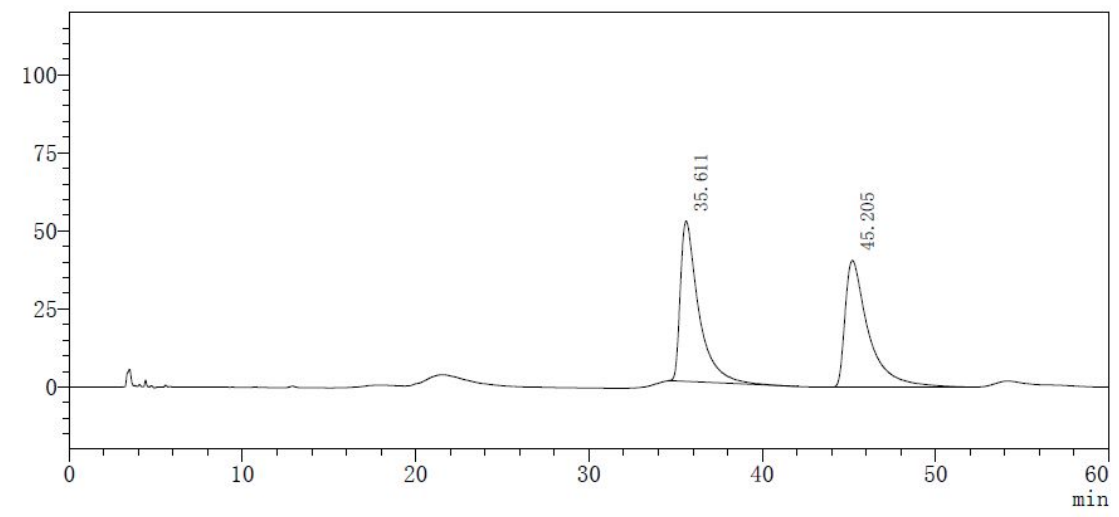

检测器A $220 \mathrm{~nm}$
\begin{tabular}{|c|c|c|c|c|c|}
\hline Peak\# & Time & Area & Height & Height\% & Area\% \\
\hline 1 & 35.611 & 3777432 & 51344 & 55.839 & 49.717 \\
\hline 2 & 45.205 & 3820369 & 40606 & 44.161 & 50.283 \\
\hline 总计 & & 7597801 & 91950 & 100.000 & 100.000 \\
\hline
\end{tabular}

$\mathrm{mV}$

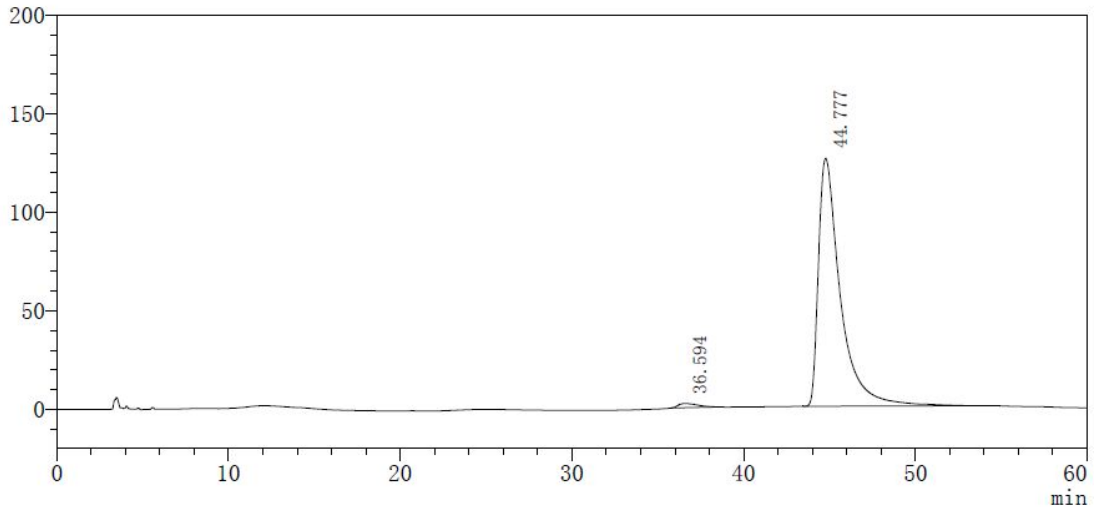

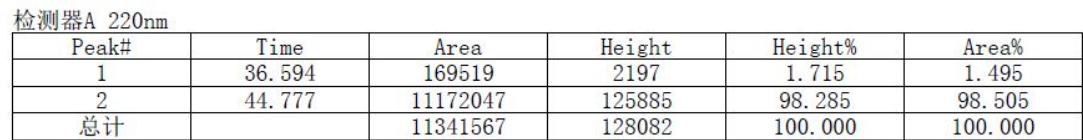<smiles>CC[C@@H](/C=C/C1CCCCC1)S(=O)(=O)c1ccccc1</smiles>

\section{(S,E)-((1-cyclohexylpent-1-en-3-yl)sulfonyl)benzene}

Prepared according to General Procedure, white oil, $30 \mathrm{mg}, 52 \%$ yield, $>20: 1 \mathrm{rr}, 96 \%$ ee 
$[\alpha]^{25} \mathrm{D}=21.2\left(\mathrm{c} 1, \mathrm{CHCl}_{3}\right)$

${ }^{1} \mathrm{H}$ NMR (400 MHz, $\left.\mathrm{CDCl}_{3}\right) \delta 7.81(\mathrm{~d}, J=7.4 \mathrm{~Hz}, 2 \mathrm{H}), 7.61(\mathrm{t}, J=7.4 \mathrm{~Hz}, 1 \mathrm{H}), 7.51$ (t, $J=7.6 \mathrm{~Hz}$, 2H), $5.28(\mathrm{dd}, J=15.5,6.7 \mathrm{~Hz}, 1 \mathrm{H}), 5.14(\mathrm{dd}, J=15.6,9.2 \mathrm{~Hz}, 1 \mathrm{H}), 3.32(\mathrm{td}, J=10.9,3.3 \mathrm{~Hz}, 1 \mathrm{H})$, $2.20(\mathrm{dqd}, J=15.0,7.5,3.4 \mathrm{~Hz}, 1 \mathrm{H}), 1.95-1.81(\mathrm{~m}, 1 \mathrm{H}), 1.76-1.46(\mathrm{~m}, 7 \mathrm{H}), 1.33-1.02(\mathrm{~m}, 4 \mathrm{H})$, $0.94(\mathrm{t}, J=7.4 \mathrm{~Hz}, 3 \mathrm{H})$.

${ }^{13} \mathrm{C}$ NMR $\left(101 \mathrm{MHz}, \mathrm{CDCl}_{3}\right) \delta 146.2,137.8,133.3,129.2,128.6,119.4,70.8,40.7,32.5,26.0,25.7$, 20.3, 11.2.

HRMS (ESI) calcd.for $\mathrm{C}_{17} \mathrm{H}_{24} \mathrm{NaO}_{2} \mathrm{~S}^{+}(\mathrm{M}+\mathrm{Na})^{+}: 315.1389$, Found: 315.1387

theee value was $96 \%, \mathrm{t}_{\mathrm{r}}($ minor $)=14.311$ min, $\mathrm{t}_{\mathrm{r}}$ (major) $=16.493 \min ($ Chiralcel IC, $\lambda=220 \mathrm{~nm}$, hexanes : ${ }^{i} \mathrm{PrOH}=80: 20$, flow rate $=1.0 \mathrm{~mL} / \mathrm{min}$ ).

$\mathrm{mV}$

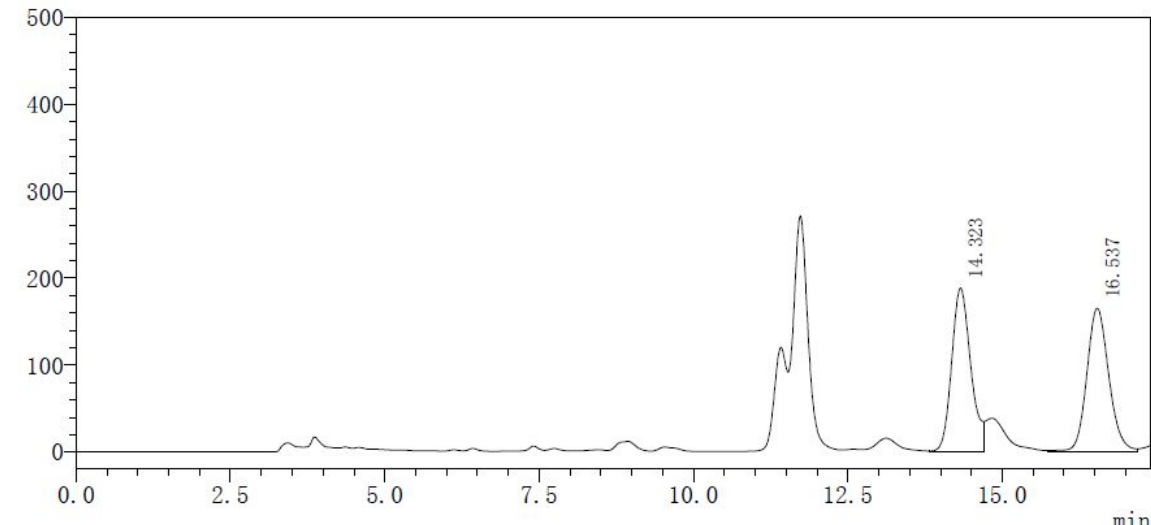

检测器A $220 \mathrm{~nm}$
\begin{tabular}{|c|c|c|c|c|c|}
\hline Peak\# & Time & Area & Height & Height\% & Area\% \\
\hline 1 & 14.323 & 4066009 & 188408 & 53.279 & 49.740 \\
\hline 2 & 16.537 & 4108562 & 165218 & 46.721 & 50.260 \\
\hline 总计 & & 8174571 & 353627 & 100.000 & 100.000 \\
\hline
\end{tabular}

$\mathrm{mV}$

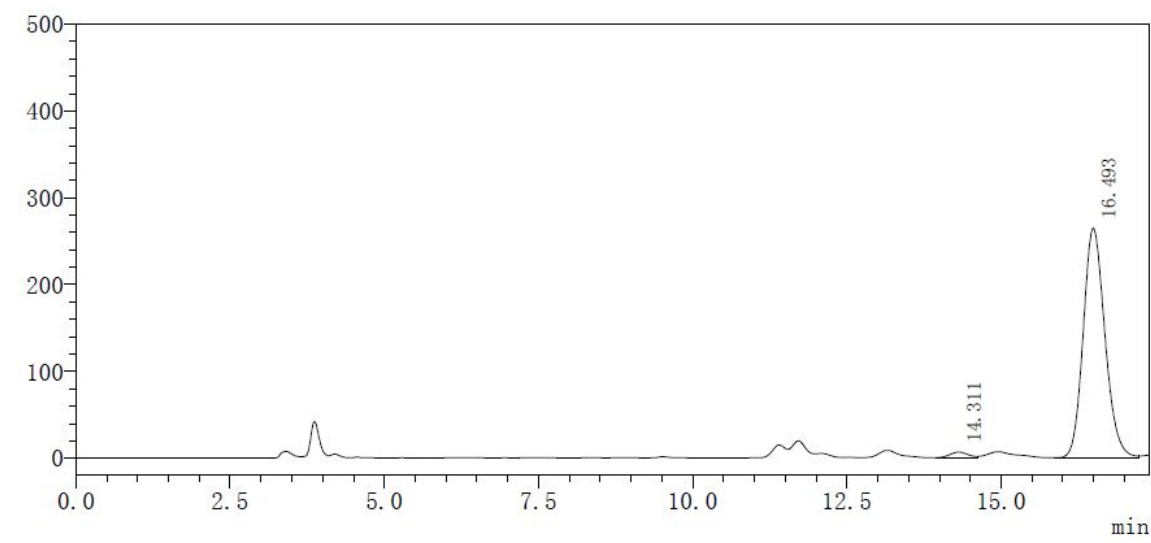

检测器A $220 \mathrm{~nm}$
\begin{tabular}{|c|c|c|c|c|c|}
\hline Peak\# & Time & Area & Height & Height\% & Area\% \\
\hline 1 & 14.311 & 133330 & 6485 & 2.391 & 2.008 \\
\hline 2 & 16.493 & 6506066 & 264774 & 97.609 & 97.992 \\
\hline 总计 & & 6639396 & 271259 & 100.000 & 100.000 \\
\hline
\end{tabular}


<smiles></smiles>

\section{(S,E)-((1-phenylhex-1-en-3-yl)sulfonyl)benzene}

Prepared according to General Procedure, white solid, $44 \mathrm{mg}, 73 \%$ yield, m.p. $54-56{ }^{\circ} \mathrm{C},>20: 1 \mathrm{rr}$, $96 \%$ ee

$[\alpha]^{25}=-69.9\left(\mathrm{c} 1, \mathrm{CHCl}_{3}\right)$

${ }^{1} \mathrm{H}$ NMR (400 MHz, $\left.\mathrm{CDCl}_{3}\right) \delta 7.89-7.79(\mathrm{~m}, 2 \mathrm{H}), 7.61(\mathrm{t}, J=7.4 \mathrm{~Hz}, 1 \mathrm{H}), 7.50(\mathrm{t}, J=7.7 \mathrm{~Hz}, 2 \mathrm{H})$, $7.34-7.22(\mathrm{~m}, 5 \mathrm{H}), 6.25(\mathrm{~d}, J=15.9 \mathrm{~Hz}, 1 \mathrm{H}), 5.90(\mathrm{dd}, J=15.9,9.5 \mathrm{~Hz}, 1 \mathrm{H}), 3.70-3.62(\mathrm{~m}, 1 \mathrm{H})$, 2.17 (dddd, $J=13.3,10.0,6.7,3.3 \mathrm{~Hz}, 1 \mathrm{H}), 1.83-1.71(\mathrm{~m}, 1 \mathrm{H}), 1.56-1.43(\mathrm{~m}, 1 \mathrm{H}), 1.35-1.27(\mathrm{~m}$, $1 \mathrm{H}), 0.93(\mathrm{t}, J=7.3 \mathrm{~Hz}, 3 \mathrm{H})$.

${ }^{13} \mathrm{C}$ NMR $\left(101 \mathrm{MHz}, \mathrm{CDCl}_{3}\right) \delta 138.1,137.5,135.8,133.6,129.2,128.8,128.7,128.4,126.6,121.3$, $69.5,29.2,20.0,13.6$.

HRMS (ESI) calcd. for $\mathrm{C}_{18} \mathrm{H}_{20} \mathrm{NaO}_{2} \mathrm{~S}^{+}(\mathrm{M}+\mathrm{Na})^{+}: 323.1076$, Found: 323.1073

the ee value was $96 \%, \mathrm{t}_{\mathrm{r}}($ minor $)=13.025 \mathrm{~min}, \mathrm{t}_{\mathrm{r}}$ (major) $=15.014 \min ($ Chiralcel IC, $\lambda=220 \mathrm{~nm}$, hexanes : ${ }^{i} \mathrm{PrOH}=80: 20$, flow rate $=1.0 \mathrm{~mL} / \mathrm{min}$ )
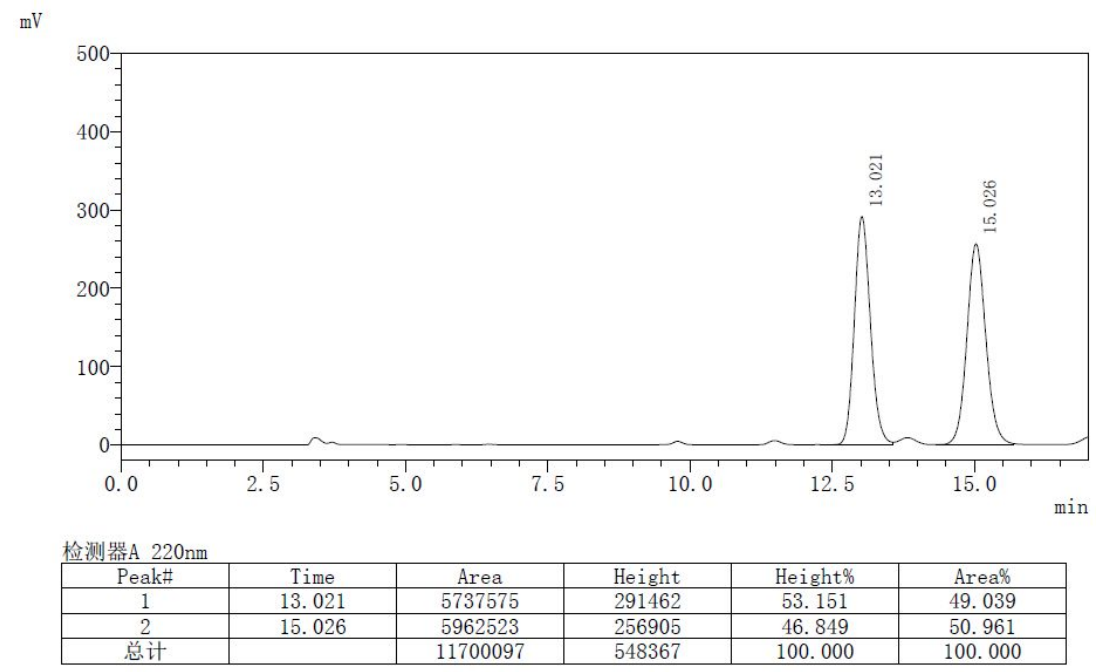
$\mathrm{mV}$

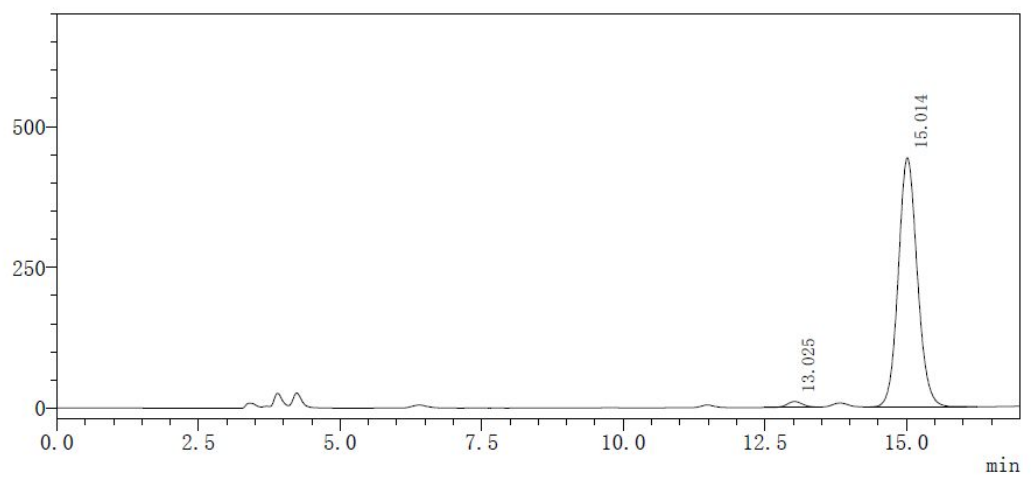

检测器A $220 \mathrm{~nm}$
\begin{tabular}{|c|c|c|c|c|c|}
\hline Peak\# & Time & Area & Height & Height\% & Area $\%$ \\
\hline 1 & 13.025 & 204021 & 10535 & 2.323 & 1.929 \\
\hline 2 & 15.014 & 10372065 & 442900 & 97.677 & 98.071 \\
\hline 总计 & & 10576086 & 453435 & 100.000 & 100.000 \\
\hline
\end{tabular}

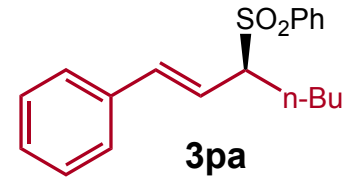

\section{(S,E)-((1-phenylhept-1-en-3-yl)sulfonyl)benzene}

Prepared according to General Procedure, white solid, $49 \mathrm{mg}$, 78\% yield, m.p. $45-46{ }^{\circ} \mathrm{C}, 17: 1 \mathrm{rr}$, $94 \%$ ee

$[\alpha]^{25}=-77.7\left(\mathrm{c} 1, \mathrm{CHCl}_{3}\right)$

${ }^{1} \mathrm{H}$ NMR $\left(400 \mathrm{MHz}, \mathrm{CDCl}_{3}\right) \delta 7.89-7.79(\mathrm{~m}, 2 \mathrm{H}), 7.60(\mathrm{t}, J=7.4 \mathrm{~Hz}, 1 \mathrm{H}), 7.49(\mathrm{t}, J=7.7 \mathrm{~Hz}, 2 \mathrm{H})$, $7.31-7.21(\mathrm{~m}, 5 \mathrm{H}), 6.24(\mathrm{~d}, J=15.9 \mathrm{~Hz}, 1 \mathrm{H}), 5.90(\mathrm{dd}, J=15.9,9.5 \mathrm{~Hz}, 1 \mathrm{H}), 3.68-3.58(\mathrm{~m}, 1 \mathrm{H})$, $2.29-2.16(\mathrm{~m}, 1 \mathrm{H}), 1.84-1.71(\mathrm{~m}, 1 \mathrm{H}), 1.47-1.21(\mathrm{~m}, 4 \mathrm{H}), 0.87(\mathrm{t}, J=6.9 \mathrm{~Hz}, 3 \mathrm{H})$.

${ }^{13} \mathrm{C}$ NMR $\left(101 \mathrm{MHz}, \mathrm{CDCl}_{3}\right) \delta 138.1,137.6,135.9,133.6,129.2,128.8,128.7,128.4,126.6,121.4$, $69.7,28.9,27.0,22.3,13.8$.

HRMS (ESI) calcd. for $\mathrm{C}_{19} \mathrm{H}_{22} \mathrm{NaO}_{2} \mathrm{~S}^{+}(\mathrm{M}+\mathrm{Na})^{+}: 337.1233$, Found: 337.1231

the ee value was $94 \%, \mathrm{t}_{\mathrm{r}}($ minor $)=12.286 \mathrm{~min}, \mathrm{t}_{\mathrm{r}}($ major $)=13.835 \min ($ Chiralcel IC, $\lambda=220 \mathrm{~nm}$, hexanes : ${ }^{i} \mathrm{PrOH}=80: 20$, flow rate $=1.0 \mathrm{~mL} / \mathrm{min}$ ) 
$\mathrm{mV}$

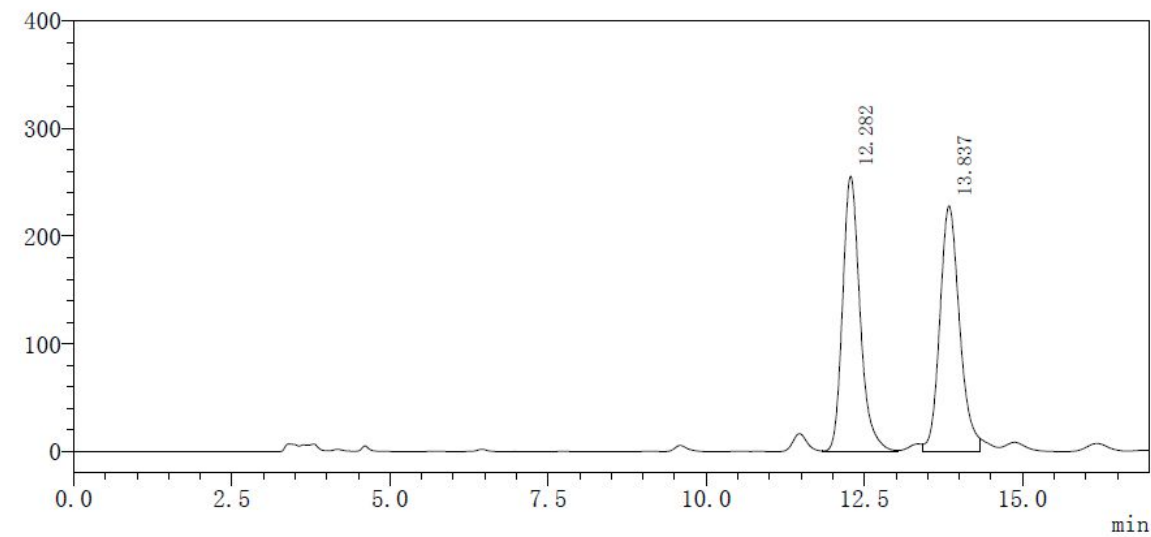

检测器A $220 \mathrm{~nm}$
\begin{tabular}{|c|c|c|c|c|c|}
\hline Peak\# & Time & Area & Height & Height\% & Area\% \\
\hline 1 & 12.282 & 4912349 & 255757 & 52.876 & 49.748 \\
\hline 2 & 13.837 & 4962071 & 227934 & 47.124 & 50.252 \\
\hline 总计 & & 9874420 & 483690 & 100.000 & 100.000 \\
\hline
\end{tabular}

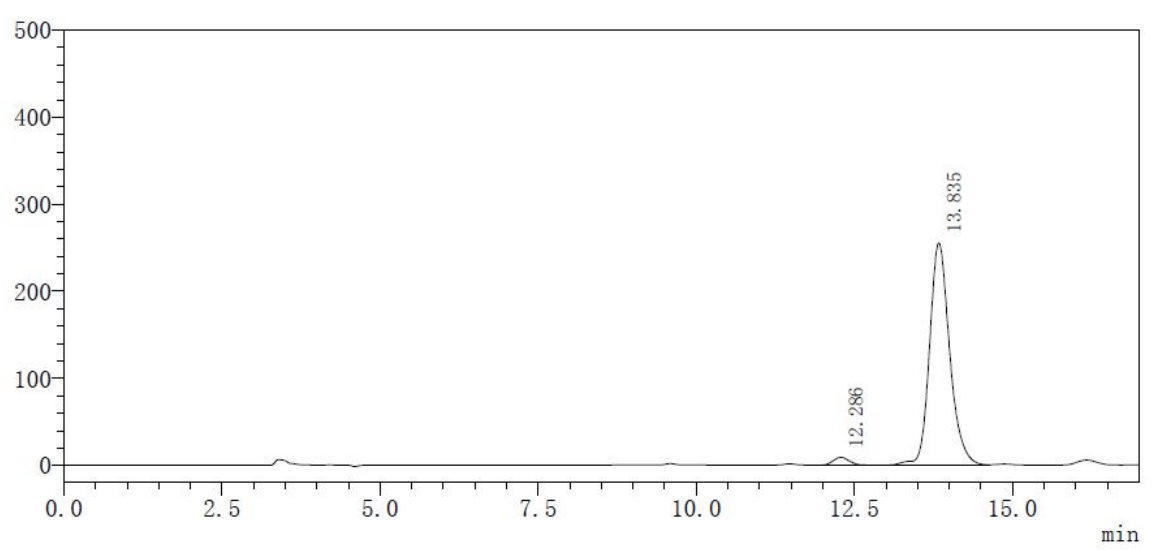

检测器A $220 \mathrm{~nm}$
\begin{tabular}{|c|c|c|c|c|c|}
\hline Peak\# & Time & Area & Height & Height\% & Area\% \\
\hline 1 & 12.286 & 168921 & 9241 & 3.489 & 2.888 \\
\hline 2 & 13.835 & 5681120 & 255628 & 96.511 & 97.112 \\
\hline 总计 & & 5850040 & 264868 & 100.000 & 100.000 \\
\hline
\end{tabular}<smiles>CC(C)CC[C@H](/C=C/c1ccccc1)S(=O)(=O)c1ccccc1</smiles>

\section{(S,E)-((6-methyl-1-phenylhept-1-en-3-yl)sulfonyl)benzene}

Prepared according to General Procedure, white solid, $46 \mathrm{mg}, 70 \%$ yield, m.p. $56-58{ }^{\circ} \mathrm{C}, 12: 1 \mathrm{rr}$, 93\% ee

$[\alpha]^{25}=-69.5\left(\mathrm{c} 1, \mathrm{CHCl}_{3}\right)$

${ }^{1} \mathrm{H}$ NMR $\left(400 \mathrm{MHz}, \mathrm{CDCl}_{3}\right) \delta 7.81(\mathrm{~d}, J=7.6 \mathrm{~Hz}, 2 \mathrm{H}), 7.58(\mathrm{t}, J=7.4 \mathrm{~Hz}, 1 \mathrm{H}), 7.47(\mathrm{t}, J=7.6 \mathrm{~Hz}$, 2H), $7.31-7.20(\mathrm{~m}, 6 \mathrm{H}), 6.20(\mathrm{~d}, J=15.9 \mathrm{~Hz}, 1 \mathrm{H}), 5.88(\mathrm{dd}, J=15.8,9.5 \mathrm{~Hz}, 1 \mathrm{H}), 3.64-3.53(\mathrm{~m}$, $1 \mathrm{H}), 2.29-2.17(\mathrm{~m}, 1 \mathrm{H}), 1.81-1.67(\mathrm{~m}, 1 \mathrm{H}), 1.62-1.49(\mathrm{~m}, 1 \mathrm{H}), 1.36-1.14(\mathrm{~m}, 2 \mathrm{H}), 0.85(\mathrm{dd}, J=$ $13.6,7.6 \mathrm{~Hz}, 6 \mathrm{H})$.

${ }^{13} \mathrm{C}$ NMR $\left(101 \mathrm{MHz}, \mathrm{CDCl}_{3}\right) \delta 138.1,137.6,135.9,133.6,129.2,128.8,128.7,128.4,126.6,121.5$, 
$70.0,35.8,27.9,25.2,22.1$

HRMS (ESI) calcd. for $\mathrm{C}_{20} \mathrm{H}_{24} \mathrm{NaO}_{2} \mathrm{~S}^{+}(\mathrm{M}+\mathrm{Na})^{+}: 351.1389$, Found: 351.1389

the ee value was $93 \%, \mathrm{t}_{\mathrm{r}}($ minor $)=10.758 \mathrm{~min}, \mathrm{t}_{\mathrm{r}}($ major $)=11.815 \mathrm{~min}($ Chiralcel $\mathrm{IC}, \lambda=220 \mathrm{~nm}$, hexanes : ${ }^{i} \mathrm{PrOH}=80: 20$, flow rate $=1.0 \mathrm{~mL} / \mathrm{min}$ )

$\mathrm{mV}$
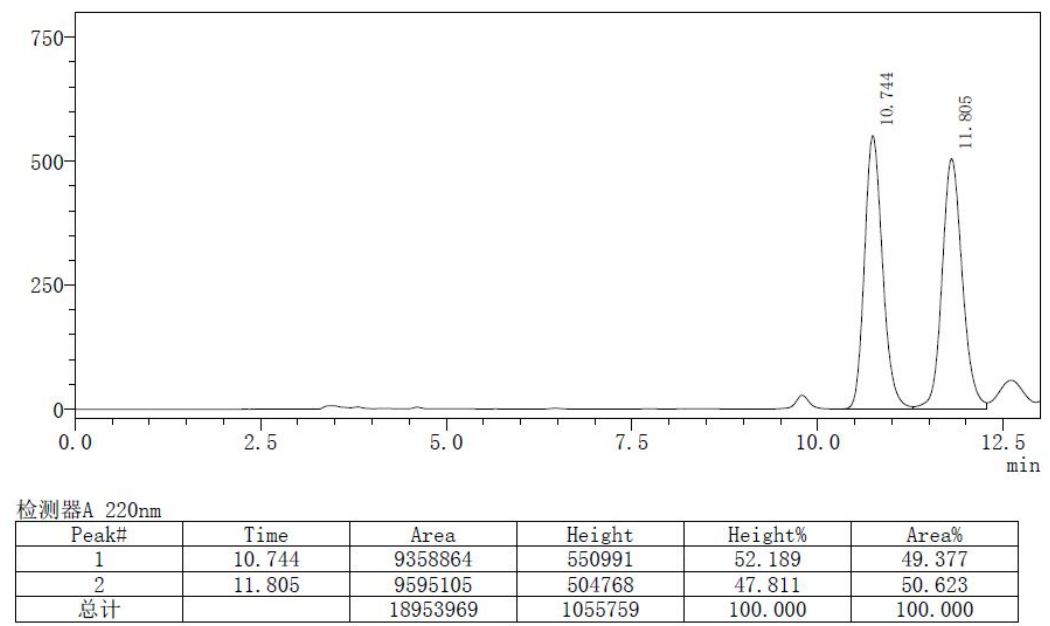

$\mathrm{m} V$

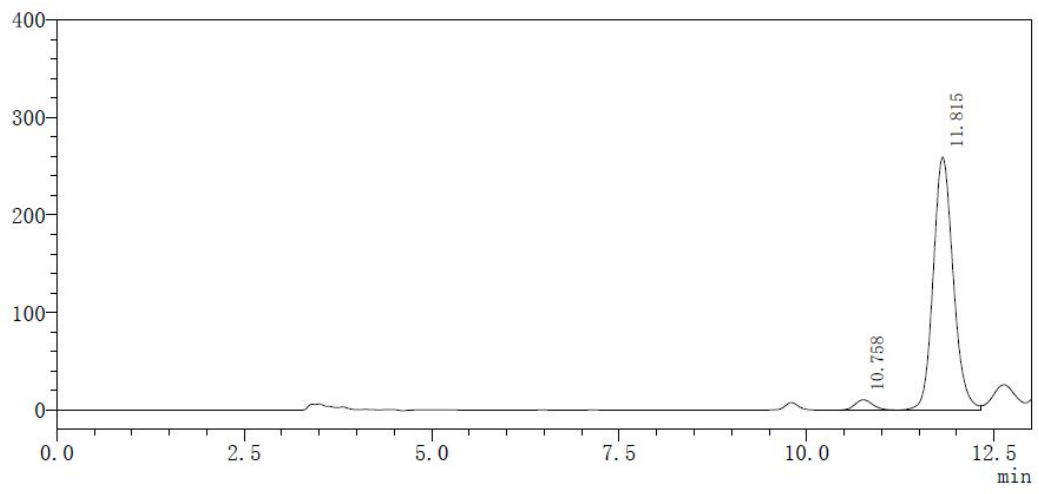

检测器A $220 \mathrm{~nm}$
\begin{tabular}{|c|c|c|c|c|c|}
\hline Peak\# & Time & Area & Height & Height\% & Area\% \\
\hline 1 & 10.758 & 177928 & 10690 & 3.959 & 3.561 \\
\hline 2 & 11.815 & 4818821 & 259302 & 96.041 & 96.439 \\
\hline 总计 & & 4996749 & 269992 & 100.000 & 100.000 \\
\hline
\end{tabular}<smiles>COCCC[C@H](/C=C/c1ccccc1)S(=O)(=O)c1ccccc1</smiles>

\section{(S,E)-((6-methoxy-1-phenylhex-1-en-3-yl)sulfonyl)benzene}

Prepared according to General Procedure, pale yellow oil, $53 \mathrm{mg}, 80 \%$ yield, $17: 1 \mathrm{rr}, 96 \%$ ee $[\alpha]^{25}=-59.2\left(\mathrm{c} 1, \mathrm{CHCl}_{3}\right)$

${ }^{1} \mathrm{H} \mathrm{NMR}\left(400 \mathrm{MHz}, \mathrm{CDCl}_{3}\right) \delta 7.86-7.79(\mathrm{~m}, 2 \mathrm{H}), 7.60(\mathrm{t}, J=7.4 \mathrm{~Hz}, 1 \mathrm{H}), 7.49(\mathrm{t}, J=7.7 \mathrm{~Hz}, 2 \mathrm{H})$, 
$7.33-7.21(\mathrm{~m}, 5 \mathrm{H}), 6.27(\mathrm{~d}, J=15.9 \mathrm{~Hz}, 1 \mathrm{H}), 5.90(\mathrm{dd}, J=15.9,9.4 \mathrm{~Hz}, 1 \mathrm{H}), 3.75-3.66(\mathrm{~m}, 1 \mathrm{H})$, $3.38(\mathrm{t}, J=6.2 \mathrm{~Hz}, 2 \mathrm{H}), 3.29(\mathrm{~s}, 3 \mathrm{H}), 2.36-2.24(\mathrm{~m}, 1 \mathrm{H}), 1.92-1.79(\mathrm{~m}, 1 \mathrm{H}), 1.78-1.65(\mathrm{~m}, 1 \mathrm{H})$, $1.64-1.51(\mathrm{~m}, 1 \mathrm{H})$.

${ }^{13} \mathrm{C}$ NMR $\left(101 \mathrm{MHz}, \mathrm{CDCl}_{3}\right) \delta 138.3,137.5,135.8,133.6,129.2,128.8,128.6,128.4,126.6,121.1$, $72.0,69.5,58.6,26.9,24.5$.

HRMS (ESI) calcd. for $\mathrm{C}_{19} \mathrm{H}_{22} \mathrm{NaO}_{3} \mathrm{~S}^{+}(\mathrm{M}+\mathrm{Na})^{+}:$353.1182, Found: 353.1180

the ee value was $96 \%, \mathrm{t}_{\mathrm{r}}($ minor $)=23.503 \mathrm{~min}, \mathrm{t}_{\mathrm{r}}($ major $)=27.279 \min ($ Chiralcel IC, $\lambda=220 \mathrm{~nm}$, hexanes : ${ }^{i} \mathrm{PrOH}=80: 20$, flow rate $=1.0 \mathrm{~mL} / \mathrm{min}$ )

$\mathrm{mV}$

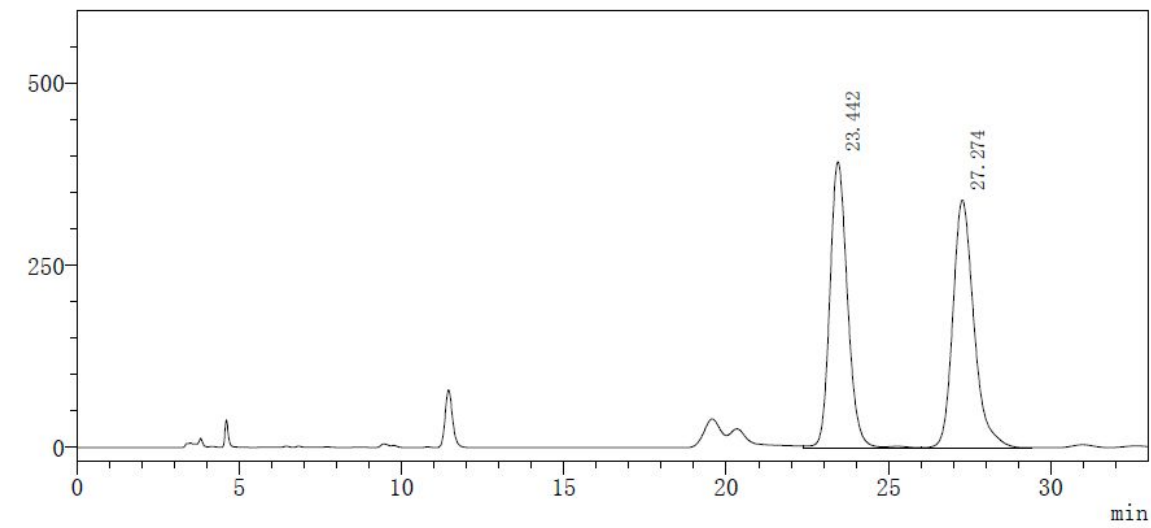

检测器A $220 \mathrm{~nm}$
\begin{tabular}{|c|c|c|c|c|c|}
\hline Peak\# & Time & Area & Height & Height $\%$ & Area\% \\
\hline 1 & 23.442 & 14837173 & 393256 & 53.592 & 49.149 \\
\hline 2 & 27.274 & 15350712 & 340546 & 46.408 & 50.851 \\
\hline 总计 & & 30187885 & 733802 & 100.000 & 100.000 \\
\hline
\end{tabular}

$\mathrm{mV}$

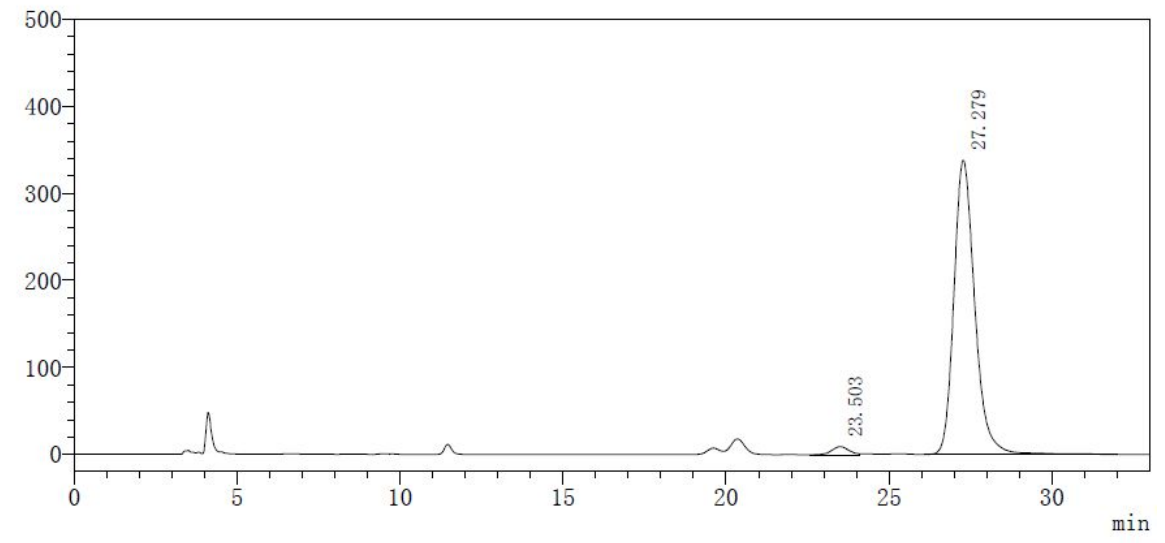

检测器A $220 \mathrm{~nm}$
\begin{tabular}{|c|c|c|c|c|c|}
\hline Peak\# & Time & Area & Height & Height $\%$ & Area\% \\
\hline 1 & 23.503 & 334549 & 9204 & 2.647 & 2.155 \\
\hline 2 & 27.279 & 15186479 & 338471 & 97.353 & 97.845 \\
\hline 总计 & & 15521028 & 347675 & 100.000 & 100.000 \\
\hline
\end{tabular}<smiles>CC(=O)C[C@H](/C=C/c1ccccc1)S(=O)(=O)c1ccccc1</smiles>

methyl (S,E)-5-phenyl-3-(phenylsulfonyl)pent-4-enoate 
Prepared according to General Procedure, white solid, $61 \mathrm{mg}, 92 \%$ yield, m.p. $19-120{ }^{\circ} \mathrm{C},>20: 1$ rr, $94 \%$ ee

$[\alpha]^{25}=-85.7\left(\mathrm{c} 1, \mathrm{CHCl}_{3}\right)$

${ }^{1} \mathrm{H}$ NMR $\left(400 \mathrm{MHz}, \mathrm{CDCl}_{3}\right) \delta 7.85(\mathrm{~d}, J=7.8 \mathrm{~Hz}, 2 \mathrm{H}), 7.63(\mathrm{t}, J=7.4 \mathrm{~Hz}, 1 \mathrm{H}), 7.51$ (t, $J=7.6 \mathrm{~Hz}$, 2H), 7.27 (dd, $J=16.2,8.1 \mathrm{~Hz}, 5 \mathrm{H}), 6.33(\mathrm{~d}, J=15.9 \mathrm{~Hz}, 1 \mathrm{H}), 5.97(\mathrm{dd}, J=15.8,9.1 \mathrm{~Hz}, 1 \mathrm{H}), 4.26$ (td, $J=9.4,4.3 \mathrm{~Hz}, 1 \mathrm{H}), 3.66$ (s, 3H), 3.25 (dd, $J=16.1,4.3 \mathrm{~Hz}, 1 \mathrm{H}), 2.77$ (dd, $J=16.1,9.8 \mathrm{~Hz}, 1 \mathrm{H}$ ).

${ }^{13} \mathrm{C}$ NMR $\left(101 \mathrm{MHz}, \mathrm{CDCl}_{3}\right) \delta 170.0,138.4,136.8,135.6,134.0,129.3,129.0,128.7,128.6,126.7$, 119.6, 65.4, 52.3, 33.0.

HRMS (ESI) calcd. for $\mathrm{C}_{18} \mathrm{H}_{18} \mathrm{NaO}_{4} \mathrm{~S}^{+}(\mathrm{M}+\mathrm{Na})^{+}: 353.0818$, Found: 353.0811

the ee value was $94 \%, \mathrm{t}_{\mathrm{r}}($ major $)=39.851 \mathrm{~min}, \mathrm{t}_{\mathrm{r}}($ minor $)=43.263 \min ($ Chiralcel IC, $\lambda=220 \mathrm{~nm}$, hexanes : ${ }^{i} \mathrm{PrOH}=85: 15$, flow rate $=1.0 \mathrm{~mL} / \mathrm{min}$ )

$\mathrm{mV}$

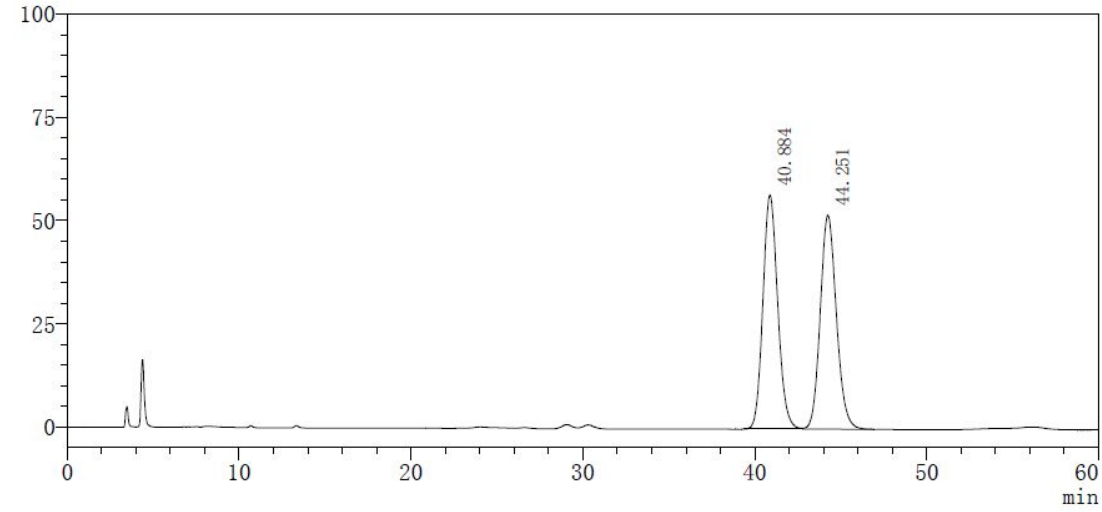

检测器A $220 \mathrm{~nm}$
\begin{tabular}{|c|c|c|c|c|c|}
\hline Peak\# & Time & Area & Height & Height $\%$ & Area\% \\
\hline 1 & 40.884 & 3397684 & 56647 & 52.227 & 50.043 \\
\hline 2 & 44.251 & 3391852 & 51817 & 47.773 & 49.957 \\
\hline 总计 & & 6789536 & 108464 & 100.000 & 100.000 \\
\hline
\end{tabular}

$\mathrm{mV}$

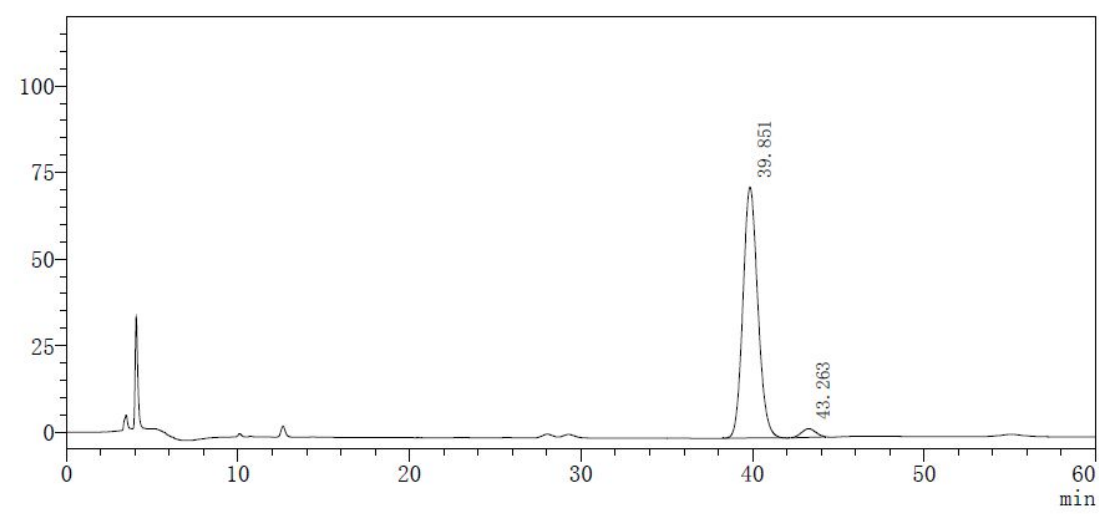

检测器A $220 \mathrm{~nm}$
\begin{tabular}{|c|c|c|c|c|c|}
\hline Peak\# & Time & Area & Height & Height\% & Area $\%$ \\
\hline 1 & 39.851 & 4381732 & 72538 & 96.749 & 96.908 \\
\hline 2 & 43.263 & 139827 & 2437 & 3.251 & 3.092 \\
\hline 总计 & & 4521559 & 74975 & 100.000 & 100.000 \\
\hline
\end{tabular}


<smiles>C[C@H](/C=C/c1ccccc1)[Se]c1ccccc1</smiles>

\section{(S,E)-((4-phenylbut-3-en-2-yl)sulfonyl)benzene}

Prepared according to General Procedure, but R-DTBM-Segphos was replaced by Ligand L6. white solid, $27 \mathrm{mg}, 50 \%$ yield, m.p. $78-80{ }^{\circ} \mathrm{C}, 5: 1 \mathrm{rr}, 91 \%$ ee

$[\alpha]^{25}=-59.7\left(\mathrm{c} 1, \mathrm{CHCl}_{3}\right)$

${ }^{1} \mathrm{H}$ NMR (400 MHz, $\left.\mathrm{CDCl}_{3}\right) \delta 7.84(\mathrm{~d}, J=7.4 \mathrm{~Hz}, 2 \mathrm{H}), 7.61(\mathrm{t}, J=7.4 \mathrm{~Hz}, 1 \mathrm{H}), 7.50$ (t, $J=7.7 \mathrm{~Hz}$, 2H), $7.35-7.23$ (m, 5H), 6.31 (d, $J=15.9 \mathrm{~Hz}, 1 \mathrm{H}), 6.07$ (dd, $J=15.9,8.3 \mathrm{~Hz}, 1 \mathrm{H}), 3.86(\mathrm{p}, J=7.2$ $\mathrm{Hz}, 1 \mathrm{H}), 1.55(\mathrm{~d}, J=6.9 \mathrm{~Hz}, 3 \mathrm{H})$.

${ }^{13} \mathrm{C}$ NMR $\left(101 \mathrm{MHz}, \mathrm{CDCl}_{3}\right) \delta 136.9,136.6,135.8,133.7,129.3,128.9,128.7,128.4,126.6,122.1$, $64.1,13.6$

HRMS (ESI) calcd. for $\mathrm{C}_{16} \mathrm{H}_{16} \mathrm{NaO}_{2} \mathrm{~S}^{+}(\mathrm{M}+\mathrm{Na})^{+}: 295.0763$, Found: 295.0763

the ee value was $91 \%, \mathrm{t}_{\mathrm{r}}($ minor $)=16.907 \mathrm{~min}, \mathrm{t}_{\mathrm{r}}($ major $)=22.864 \min ($ Chiralcel IC, $\lambda=220 \mathrm{~nm}$, hexanes : ${ }^{i} \mathrm{PrOH}=80: 20$, flow rate $=1.0 \mathrm{~mL} / \mathrm{min}$ )

$\mathrm{mV}$

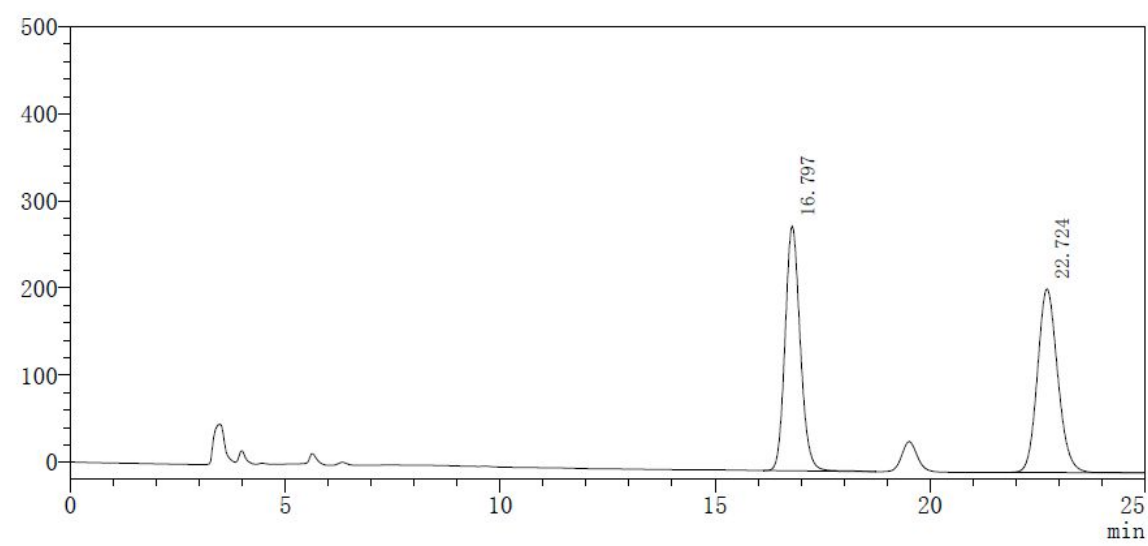

检测器A $220 \mathrm{~nm}$
\begin{tabular}{|c|c|c|c|c|c|}
\hline Peak\# & Time & Area & Height & Height\% & Area $\%$ \\
\hline 1 & 16.797 & 6886524 & 280861 & 57.126 & 49.856 \\
\hline 2 & 22.724 & 6926412 & 210792 & 42.874 & 50.144 \\
\hline 总计 & & 13812936 & 491653 & 100.000 & 100.000 \\
\hline
\end{tabular}


$\mathrm{mV}$

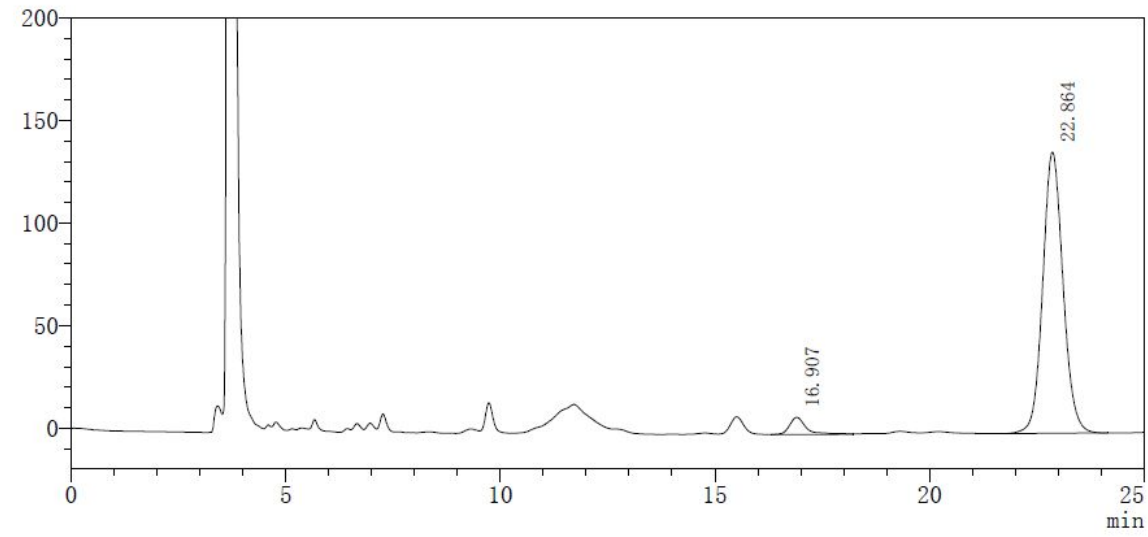

检测器A $220 \mathrm{~nm}$
\begin{tabular}{|c|c|c|c|c|c|}
\hline Peak\# & Time & Area & Height & Height\% & Area\% \\
\hline 1 & 16.907 & 222052 & 8286 & 5.699 & 4.700 \\
\hline 2 & 22.864 & 4502422 & 137119 & 94.301 & 95.300 \\
\hline 总计 & & 4724474 & 145405 & 100.000 & 100.000 \\
\hline
\end{tabular}<smiles>CCCCC[C@H]1C=CCCC1</smiles>

\section{(S)-(cyclohex-2-en-1-ylsulfonyl)benzene}

Prepared according to General Procedure, white oil, $42 \mathrm{mg}, 94 \%$ yield, 28\% ee $[\alpha]^{25}=27.6\left(\mathrm{c} 1, \mathrm{CHCl}_{3}\right)$

${ }^{1} \mathrm{H}$ NMR (400 MHz, $\left.\mathrm{CDCl}_{3}\right) \delta 7.93-7.82(\mathrm{~m}, 2 \mathrm{H}), 7.66(\mathrm{t}, J=7.4 \mathrm{~Hz}, 1 \mathrm{H}), 7.56(\mathrm{t}, J=7.6 \mathrm{~Hz}, 2 \mathrm{H})$, 6.09 (ddd, $J=10.0,6.0,3.7 \mathrm{~Hz}, 1 \mathrm{H}), 5.78$ (dd, $J=10.2,2.3 \mathrm{~Hz}, 1 \mathrm{H}), 3.76$ (ddd, $J=8.9,5.8,2.7 \mathrm{~Hz}$, $1 \mathrm{H}), 2.03-1.93(\mathrm{~m}, 3 \mathrm{H}), 1.92-1.82(\mathrm{~m}, 1 \mathrm{H}), 1.82-1.71(\mathrm{~m}, 1 \mathrm{H}), 1.56-1.44(\mathrm{~m}, 1 \mathrm{H})$.

${ }^{13} \mathrm{C}$ NMR $\left(101 \mathrm{MHz}, \mathrm{CDCl}_{3}\right) \delta 137.4,135.4,133.6,129.2,129.0,118.5,61.8,24.4,22.7,19.5$.

HRMS (ESI) calcd. for $\mathrm{C}_{12} \mathrm{H}_{14} \mathrm{NaO}_{2} \mathrm{~S}+(\mathrm{M}+\mathrm{Na})^{+}: 245.0607$, Found: 245.0602

the ee value was $28 \%, \mathrm{t}_{\mathrm{r}}($ minor $)=23.684 \mathrm{~min}, \mathrm{t}_{\mathrm{r}}($ major $)=24.807 \mathrm{~min}($ Chiralcel $\mathrm{IC}, \lambda=220 \mathrm{~nm}$, hexanes $:{ }^{i} \mathrm{PrOH}=80: 20$, flow rate $=1.0 \mathrm{~mL} / \mathrm{min}$ ) 
$\mathrm{mV}$

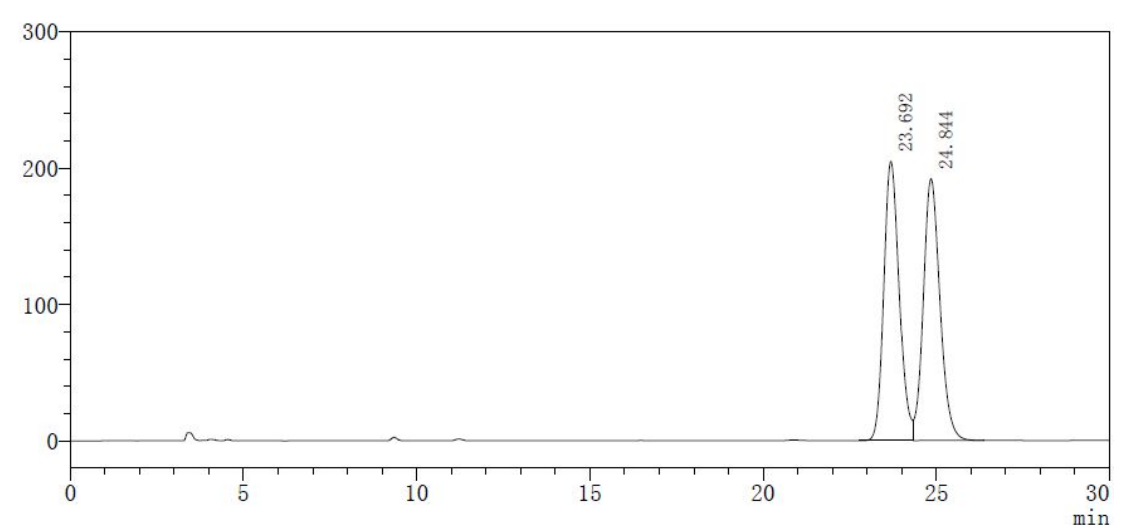

检测器A $220 \mathrm{~nm}$
\begin{tabular}{|c|c|c|c|c|c|}
\hline Peak\# & Time & Area & Height & Height\% & Area\% \\
\hline 1 & 23.692 & 6315183 & 204656 & 51.613 & 49.560 \\
\hline 2 & 24.844 & 6427299 & 191862 & 48.387 & 50.440 \\
\hline 总计 & & 12742482 & 396518 & 100.000 & 100.000 \\
\hline
\end{tabular}

$\mathrm{mV}$

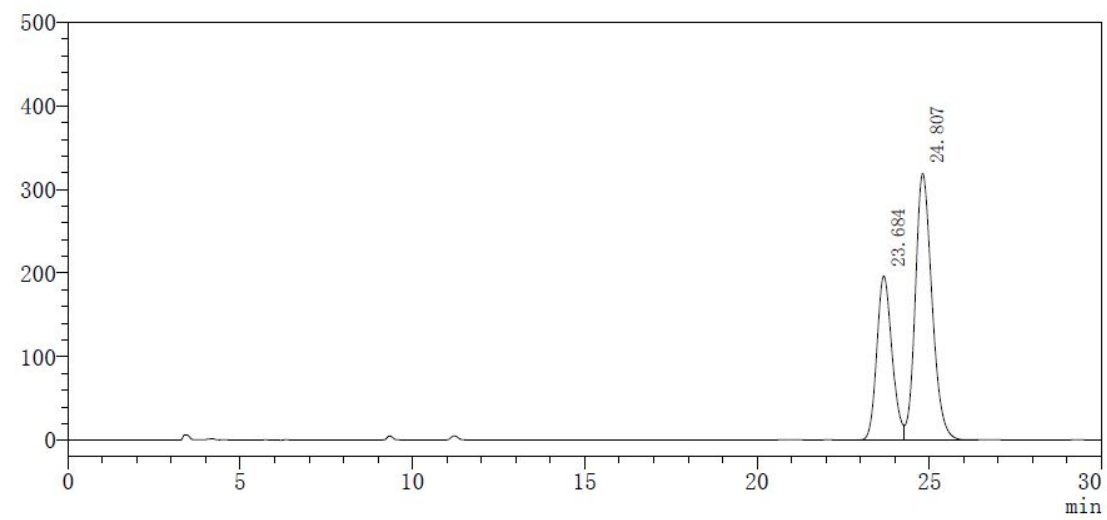

检测器A $220 \mathrm{~nm}$
\begin{tabular}{|c|c|c|c|c|c|}
\hline Peak\# & Time & Area & Height & Height\% & Area\% \\
\hline 1 & 23.684 & 6012133 & 196572 & 38.106 & 35.829 \\
\hline 2 & 24.807 & 10768010 & 319286 & 61.894 & 64.171 \\
\hline 总计 & & 16780143 & 515857 & 100.000 & 100.000 \\
\hline
\end{tabular}<smiles>CC[C@](C)(/C=C/c1ccccc1)S(=O)(=O)c1ccccc1</smiles>

\section{(E)-((3-methyl-1-phenylpent-1-en-3-yl)sulfonyl)benzene}

Prepared according to General Procedure, pale yellow oil, $52 \mathrm{mg}, 86 \%$ yield, $>20: 1 \mathrm{rr}, 40 \%$ ee $[\alpha]^{25} \mathrm{D}=37.6\left(\mathrm{c} 1, \mathrm{CHCl}_{3}\right)$

${ }^{1} \mathrm{H}$ NMR $\left(400 \mathrm{MHz}, \mathrm{CDCl}_{3}\right) \delta 7.79(\mathrm{~d}, J=7.7 \mathrm{~Hz}, 2 \mathrm{H}), 7.59(\mathrm{t}, J=7.4 \mathrm{~Hz}, 1 \mathrm{H}), 7.45(\mathrm{t}, J=7.7 \mathrm{~Hz}$, 2H), $7.31(\mathrm{~s}, 4 \mathrm{H}), 6.27(\mathrm{~d}, J=16.3 \mathrm{~Hz}, 1 \mathrm{H}), 6.14(\mathrm{~d}, J=16.3 \mathrm{~Hz}, 1 \mathrm{H}), 2.18-1.96(\mathrm{~m}, 2 \mathrm{H}), 1.46(\mathrm{~s}$, $3 \mathrm{H}), 0.92(\mathrm{t}, J=7.5 \mathrm{~Hz}, 3 \mathrm{H})$.

${ }^{13} \mathrm{C}$ NMR $\left(101 \mathrm{MHz}, \mathrm{CDCl}_{3}\right) \delta 136.1,135.7,135.0,133.5,130.7,128.7,128.4,128.3,126.6,126.4$, $68.7,26.3,16.5,8.2$.

HRMS (ESI) calcd. for $\mathrm{C}_{18} \mathrm{H}_{20} \mathrm{NaO}_{2} \mathrm{~S}^{+}(\mathrm{M}+\mathrm{Na})^{+}: 323.1076$, Found: 323.1070 
the ee value was $40 \%, \mathrm{t}_{\mathrm{r}}$ (minor) $=14.297 \mathrm{~min}, \mathrm{t}_{\mathrm{r}}($ major $)=14.860 \min ($ Chiralcel IC, $\lambda=220 \mathrm{~nm}$, hexanes $:{ }^{i} \mathrm{PrOH}=80: 20$, flow rate $=1.0 \mathrm{~mL} / \mathrm{min}$ )
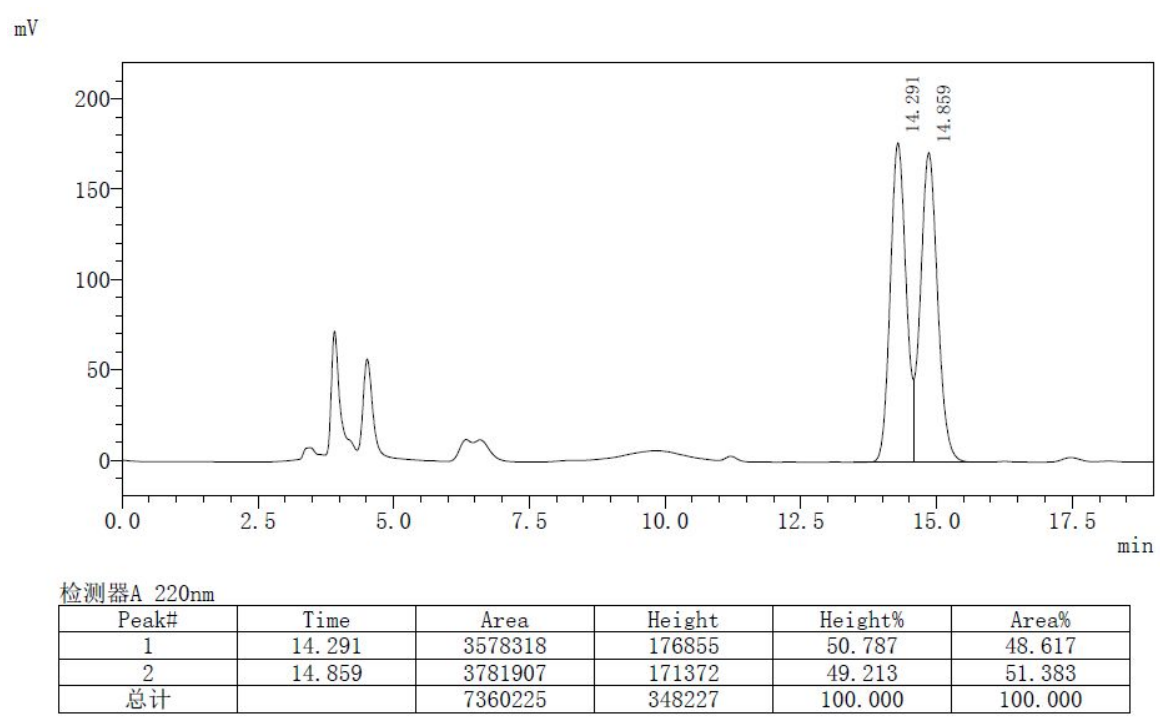

$\mathrm{mV}$

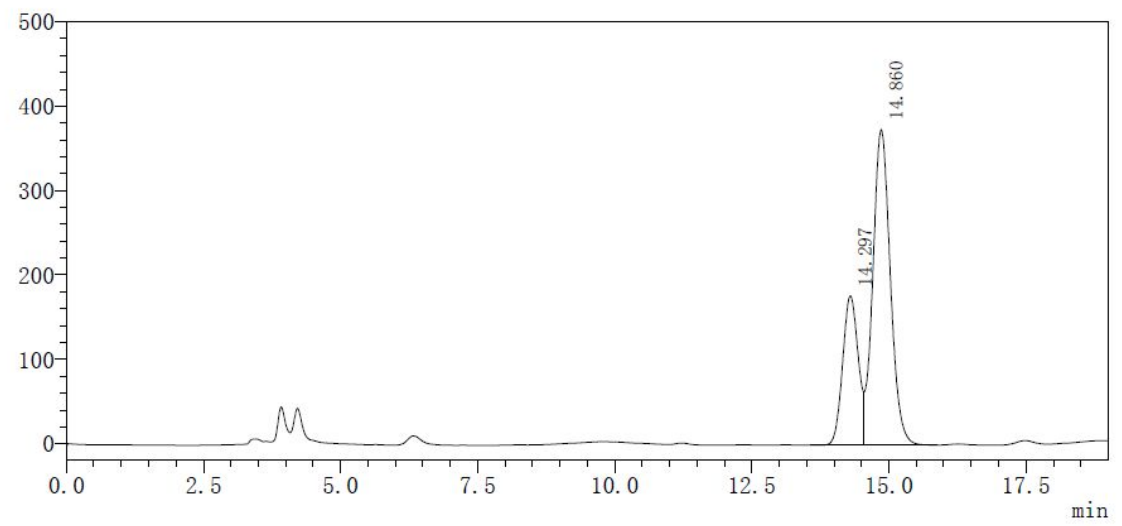

检测器A $220 \mathrm{~nm}$
\begin{tabular}{|c|c|c|c|c|c|}
\hline Peak\# & Time & Area & Height & Height\% & Area $\%$ \\
\hline 1 & 14.297 & 3529645 & 176982 & 32.126 & 29.860 \\
\hline 2 & 14.860 & 8290813 & 373921 & 67.874 & 70.140 \\
\hline 总计 & & 11820458 & 550903 & 100.000 & 100.000 \\
\hline
\end{tabular}<smiles>COc1ccc(/C=C/[C@H](Cc2ccc(C(F)(F)F)cc2)[Pb]([O-])([O-])c2ccccc2)cc1</smiles>

(S,E)-(4-(4-methoxyphenyl)-1-(4-(trifluoromethyl)phenyl)but-3-en-2-yl)(phenylperoxy)sulfan e

${ }^{1} \mathrm{H}$ NMR $\left(400 \mathrm{MHz}, \mathrm{CDCl}_{3}\right) \delta 7.86(\mathrm{~d}, J=7.5 \mathrm{~Hz}, 2 \mathrm{H}), 7.62(\mathrm{t}, J=6.9 \mathrm{~Hz}, 1 \mathrm{H}), 7.50(\mathrm{t}, J=8.4 \mathrm{~Hz}$, 4H), 7.27 (d, $J=7.1 \mathrm{~Hz}, 2 \mathrm{H}), 7.08$ (d, $J=8.6 \mathrm{~Hz}, 2 \mathrm{H}), 6.79$ (d, $J=8.8 \mathrm{~Hz}, 2 \mathrm{H}), 5.96(\mathrm{~d}, J=15.8 \mathrm{~Hz}$, $1 \mathrm{H}), 5.78(\mathrm{dd}, J=15.8,9.4 \mathrm{~Hz}, 1 \mathrm{H}), 3.88-3.80(\mathrm{~m}, 1 \mathrm{H}), 3.78(\mathrm{~s}, 3 \mathrm{H}), 3.69(\mathrm{dd}, J=13.8,2.7 \mathrm{~Hz}, 1 \mathrm{H})$, $3.08(\mathrm{dd}, J=13.6,11.3 \mathrm{~Hz}, 1 \mathrm{H})$.

${ }^{19} \mathrm{~F} \operatorname{NMR}\left(377 \mathrm{MHz}, \mathrm{CDCl}_{3}\right) \delta-62.5$.

HRMS (ESI) calcd. for $\mathrm{C}_{24} \mathrm{H}_{21} \mathrm{~F}_{3} \mathrm{NaO}_{3} \mathrm{~S}^{+}(\mathrm{M}+\mathrm{Na})^{+}:$469.1056, Found: 469.1047 
the ee value was $72 \%, \mathrm{t}_{\mathrm{r}}($ major $)=14.709 \min , \mathrm{t}_{\mathrm{r}}($ minor $)=15.891 \min$ (Chiralcel IE, $\lambda=220 \mathrm{~nm}$, hexanes : ${ }^{i} \mathrm{PrOH}=80: 20$, flow rate $=1.0 \mathrm{~mL} / \mathrm{min}$ )
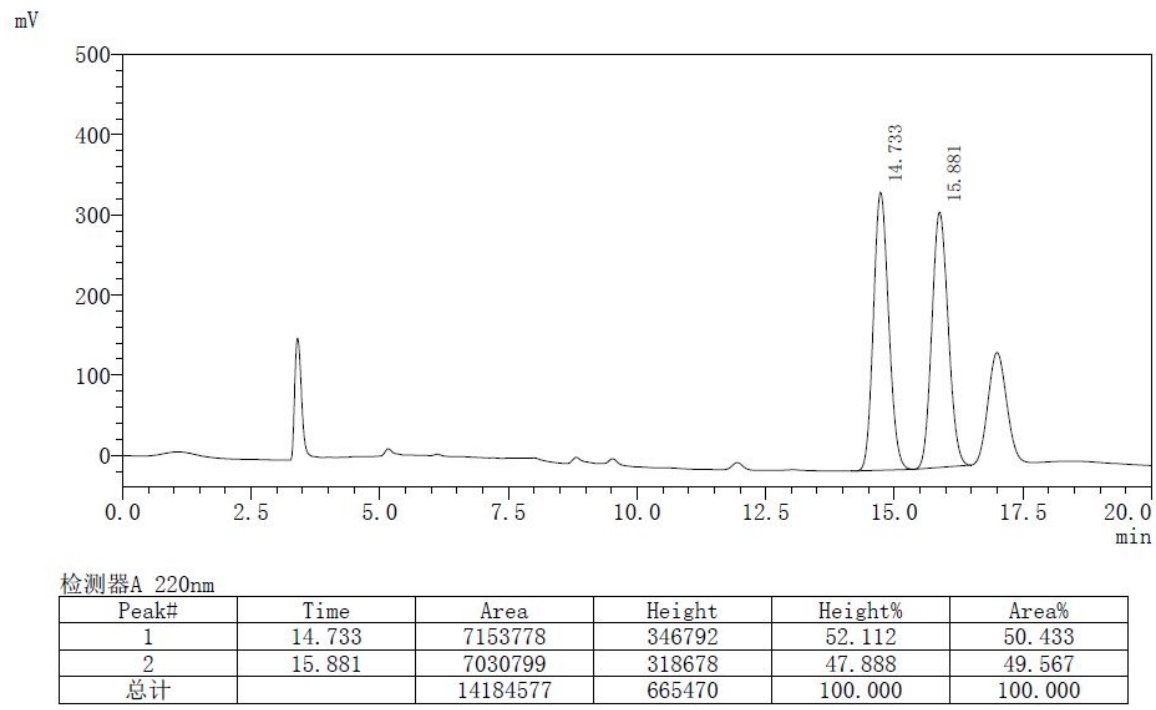

$\mathrm{mV}$

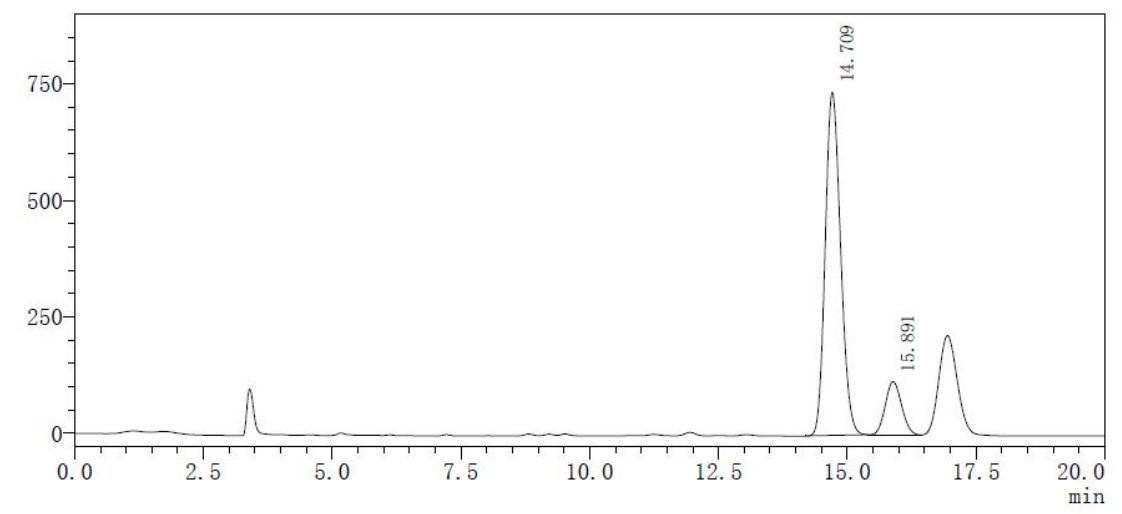

检测器A $220 \mathrm{~nm}$
\begin{tabular}{|c|c|c|c|c|c|}
\hline Peak\# & Time & Area & Height & Height\% & Area\% \\
\hline 1 & 14.709 & 15327294 & 736955 & 86.541 & 85.934 \\
\hline 2 & 15.891 & 2508849 & 114608 & 13.459 & 14.066 \\
\hline 总计 & & 17836143 & 851563 & 100.000 & 100.000 \\
\hline
\end{tabular}<smiles>COc1ccc(CC(/C=C/c2ccc(C(F)(F)F)cc2)[Se]c2ccccc2)cc1</smiles>

(S,E)-1-methoxy-4-(2-(phenylsulfonyl)-4-(4-(trifluoromethyl)phenyl)but-3-en-1-yl)benzene

${ }^{1} \mathrm{H}$ NMR $\left(400 \mathrm{MHz}, \mathrm{CDCl}_{3}\right) \delta 7.86(\mathrm{~d}, J=7.5 \mathrm{~Hz}, 2 \mathrm{H}), 7.68-7.62(\mathrm{~m}, 2 \mathrm{H}), 7.57-7.52(\mathrm{~m}, 3 \mathrm{H}), 7.23$ (s, 2H), 7.03 (d, $J=8.5 \mathrm{~Hz}, 2 \mathrm{H}), 6.77$ (d, $J=9.9 \mathrm{~Hz}, 2 \mathrm{H}), 6.05$ (s, 1H), $6.04(\mathrm{~s}, 1 \mathrm{H}), 3.91-3.86(\mathrm{~m}$, 1H), $3.73(\mathrm{~s}, 3 \mathrm{H}), 3.58(\mathrm{dd}, J=13.8,2.9 \mathrm{~Hz}, 1 \mathrm{H}), 2.98(\mathrm{dd}, J=13.7,11.4 \mathrm{~Hz}, 1 \mathrm{H})$.

${ }^{19} \mathrm{~F}$ NMR $\left(377 \mathrm{MHz}, \mathrm{CDCl}_{3}\right) \delta-62.6$.

HRMS (ESI) calcd. for $\mathrm{C}_{24} \mathrm{H}_{21} \mathrm{~F}_{3} \mathrm{NaO}_{3} \mathrm{~S}^{+}(\mathrm{M}+\mathrm{Na})^{+}:$469.1056, Found: 469.1047

the ee value was $55 \%, \mathrm{t}_{\mathrm{r}}($ minor $)=13.000 \mathrm{~min}, \mathrm{t}_{\mathrm{r}}($ major $)=14.512 \mathrm{~min}($ Chiralcel ID, $\lambda=220 \mathrm{~nm}$, hexanes : ${ }^{i} \mathrm{PrOH}=80: 20$, flow rate $=1.0 \mathrm{~mL} / \mathrm{min}$ ) 
$\mathrm{mV}$

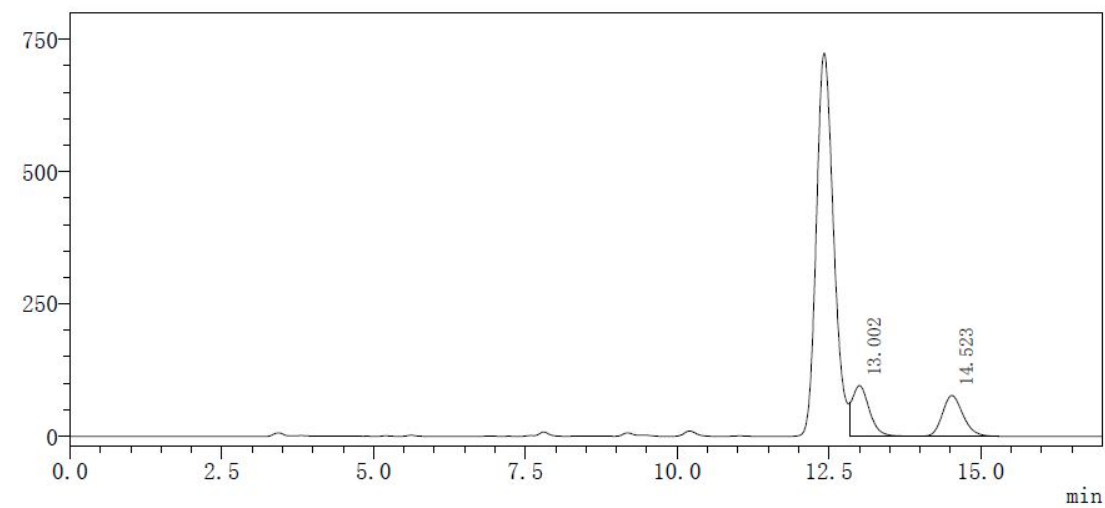

检测器A $220 \mathrm{~nm}$
\begin{tabular}{|c|c|c|c|c|c|}
\hline Peak\# & Time & Area & Height & Height\% & Area\% \\
\hline 1 & 13.002 & 1850706 & 95835 & 55.394 & 50.321 \\
\hline 2 & 14.523 & 1827078 & 77171 & 44.606 & 49.679 \\
\hline 总计 & & 3677784 & 173005 & 100.000 & 100.000 \\
\hline
\end{tabular}

$\mathrm{mV}$

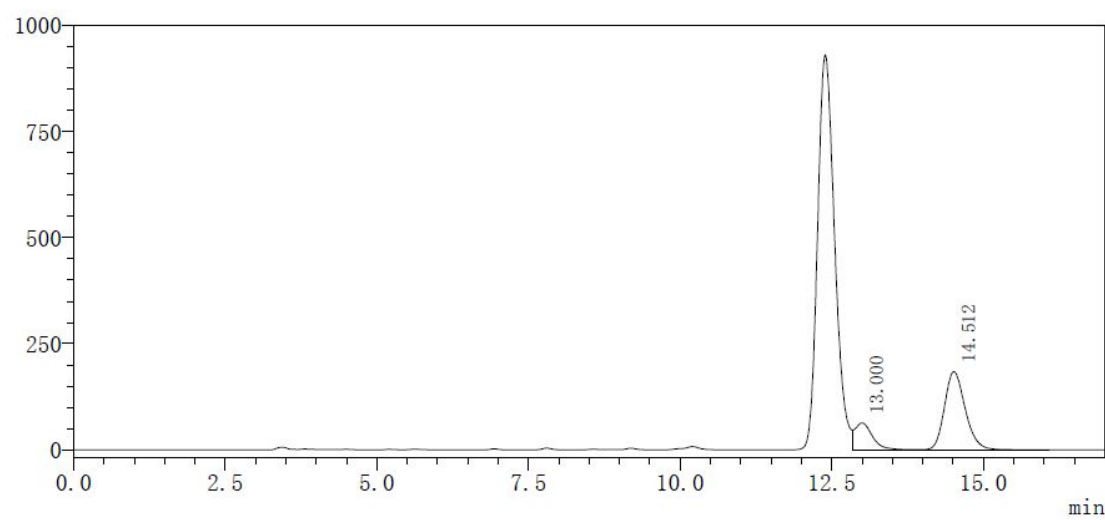

检测器A $220 \mathrm{~nm}$
\begin{tabular}{|c|c|c|c|c|c|}
\hline Peak\# & Time & Area & Height & Height\% & Area\% \\
\hline 1 & 13.000 & 1264019 & 63568 & 25.622 & 22.369 \\
\hline 2 & 14.512 & 4386862 & 184527 & 74.378 & 77.631 \\
\hline 总计 & & 5650881 & 248095 & 100.000 & 100.000 \\
\hline
\end{tabular}<smiles>CC[C@H](/C=C/c1ccccc1)S(=O)(=O)c1ccc(Cl)cc1</smiles>

\section{(S,E)-1-chloro-4-((1-phenylpent-1-en-3-yl)sulfonyl)benzene}

Prepared according to General Procedure, white solid, $57 \mathrm{mg}, 90 \%$ yield, m.p. $105-106{ }^{\circ} \mathrm{C},>20: 1$ rr, $96 \%$ ee

$[\alpha]^{25} \mathrm{D}=-73.7\left(\mathrm{c} 1, \mathrm{CHCl}_{3}\right)$

${ }^{1} \mathrm{H}$ NMR $\left(400 \mathrm{MHz}, \mathrm{CDCl}_{3}\right) \delta 7.76(\mathrm{~d}, J=8.4 \mathrm{~Hz}, 2 \mathrm{H}), 7.47(\mathrm{~d}, J=8.4 \mathrm{~Hz}, 2 \mathrm{H}), 7.35-7.23(\mathrm{~m}, 5 \mathrm{H})$, $6.31(\mathrm{~d}, J=15.9 \mathrm{~Hz}, 1 \mathrm{H}), 5.90(\mathrm{dd}, J=15.9,9.5 \mathrm{~Hz}, 1 \mathrm{H}), 3.55(\mathrm{td}, J=11.0,3.1 \mathrm{~Hz}, 1 \mathrm{H}), 2.27$ (dqd, $J$ $=14.7,7.4,3.5 \mathrm{~Hz}, 1 \mathrm{H}), 1.86-1.71(\mathrm{~m}, 1 \mathrm{H}), 1.00(\mathrm{t}, J=7.4 \mathrm{~Hz}, 3 \mathrm{H})$.

${ }^{13} \mathrm{C}$ NMR $\left(101 \mathrm{MHz}, \mathrm{CDCl}_{3}\right) \delta 140.4,138.6,136.1,135.7,130.7,129.2,128.8,128.6,126.6,120.7$, $71.2,20.9,11.4$. 
HRMS (ESI) calcd. for $\mathrm{C}_{17} \mathrm{H}_{17} \mathrm{ClNaO}_{2} \mathrm{~S}^{+}(\mathrm{M}+\mathrm{Na})^{+}:$343.0530, Found: 343.0530

the ee value was $96 \%, \mathrm{t}_{\mathrm{r}}($ minor $)=11.234 \mathrm{~min}, \mathrm{t}_{\mathrm{r}}($ major $)=12.553 \min ($ Chiralcel IC, $\lambda=220 \mathrm{~nm}$, hexanes : ${ }^{i} \mathrm{PrOH}=80: 20$, flow rate $=1.0 \mathrm{~mL} / \mathrm{min}$ )

$\mathrm{mV}$

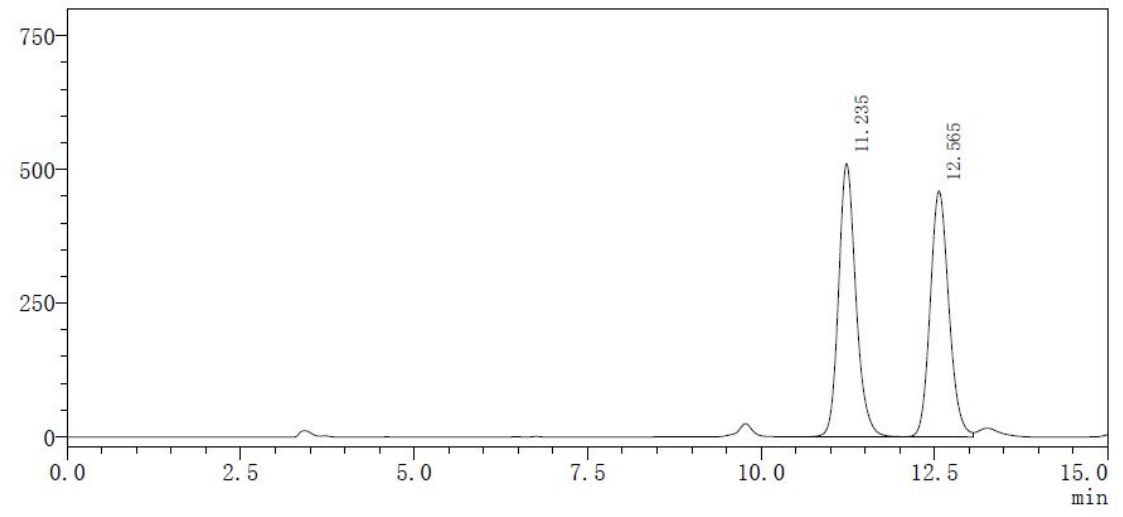

检测器A $220 \mathrm{~nm}$
\begin{tabular}{|c|c|c|c|c|c|}
\hline Peak\# & Time & Area & Height & Height $\%$ & Area\% \\
\hline 1 & 11.235 & 8882097 & 510850 & 52.629 & 50.749 \\
\hline 2 & 12.565 & 8619949 & 459812 & 47.371 & 49.251 \\
\hline 总计 & & 17502046 & 970662 & 100.000 & 100.000 \\
\hline
\end{tabular}

$\mathrm{mV}$

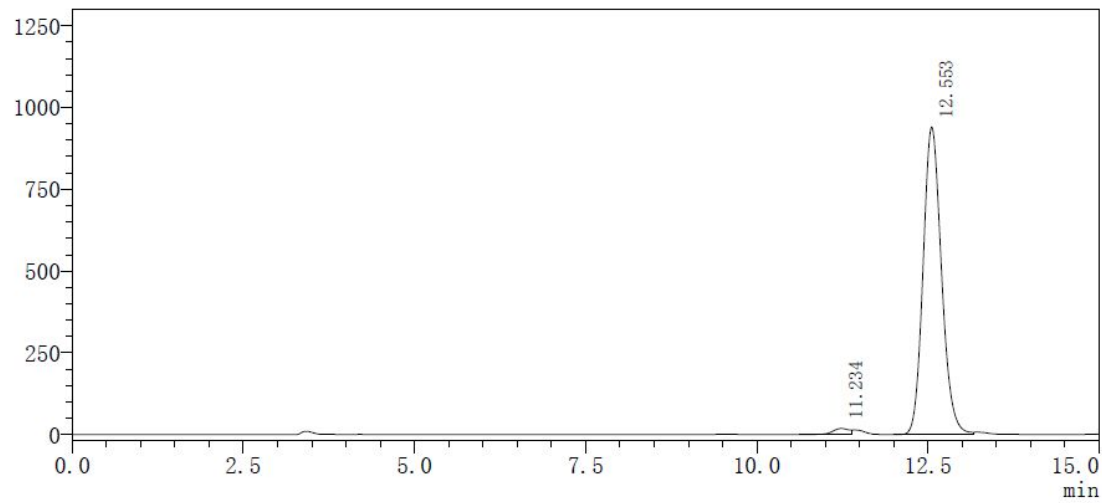

检测器A 220nm

\begin{tabular}{|c|c|c|c|c|c|}
\hline Peak\# & Time & Area & Height & Height\% & Area\% \\
\hline 1 & 11.234 & 334622 & 19159 & 1.996 & 1.860 \\
\hline 2 & 12.553 & 17653660 & 940575 & 98.004 & 98.140 \\
\hline 总计 & & 17988282 & 959734 & 100.000 & 100.000 \\
\hline
\end{tabular}<smiles>CC[C@H](/C=C/c1ccccc1)S(=O)(=O)c1ccc([N+](=O)[O-])cc1</smiles>

\section{(S,E)-1-methyl-4-((1-phenylpent-1-en-3-yl)sulfonyl)benzene}

Prepared according to General Procedure, white solid, $56 \mathrm{mg}, 93 \%$ yield, m.p. $102-103{ }^{\circ} \mathrm{C},>20: 1$ rr, $97 \%$ ee

$[\alpha]^{25}=-65.9\left(\mathrm{c} 1, \mathrm{CHCl}_{3}\right)$ 
${ }^{1} \mathrm{H}$ NMR $\left(400 \mathrm{MHz}, \mathrm{CDCl}_{3}\right) \delta 7.71(\mathrm{~d}, J=8.1 \mathrm{~Hz}, 2 \mathrm{H}), 7.37-7.19(\mathrm{~m}, 7 \mathrm{H}), 6.31(\mathrm{~d}, J=15.9 \mathrm{~Hz}, 1 \mathrm{H})$, $5.90(\mathrm{dd}, J=15.9,9.4 \mathrm{~Hz}, 1 \mathrm{H}), 3.62-3.49(\mathrm{~m}, 1 \mathrm{H}), 2.41(\mathrm{~s}, 3 \mathrm{H}), 2.32-2.17(\mathrm{~m}, 1 \mathrm{H}), 1.87-1.67(\mathrm{~m}$, $1 \mathrm{H}), 0.98(\mathrm{t}, J=7.4 \mathrm{~Hz}, 3 \mathrm{H})$.

${ }^{13} \mathrm{C}$ NMR $\left(101 \mathrm{MHz}, \mathrm{CDCl}_{3}\right) \delta 144.5,138.1,136.0,134.7,129.5,129.2,128.6,128.3,126.6,121.2$, $71.1,21.6,21.1,11.4$.

HRMS (ESI) calcd. for $\mathrm{C}_{18} \mathrm{H}_{20} \mathrm{NaO}_{2} \mathrm{~S}^{+}(\mathrm{M}+\mathrm{Na})^{+}: 323.1076$, Found: 323.1076

the ee value was $97 \%, \mathrm{t}_{\mathrm{r}}($ minor $)=18.642 \mathrm{~min}, \mathrm{t}_{\mathrm{r}}($ major $)=22.063 \mathrm{~min}($ Chiralcel $\mathrm{IC}, \lambda=220 \mathrm{~nm}$, hexanes : ${ }^{i} \mathrm{PrOH}=80: 20$, flow rate $=1.0 \mathrm{~mL} / \mathrm{min}$ )

$\mathrm{mV}$

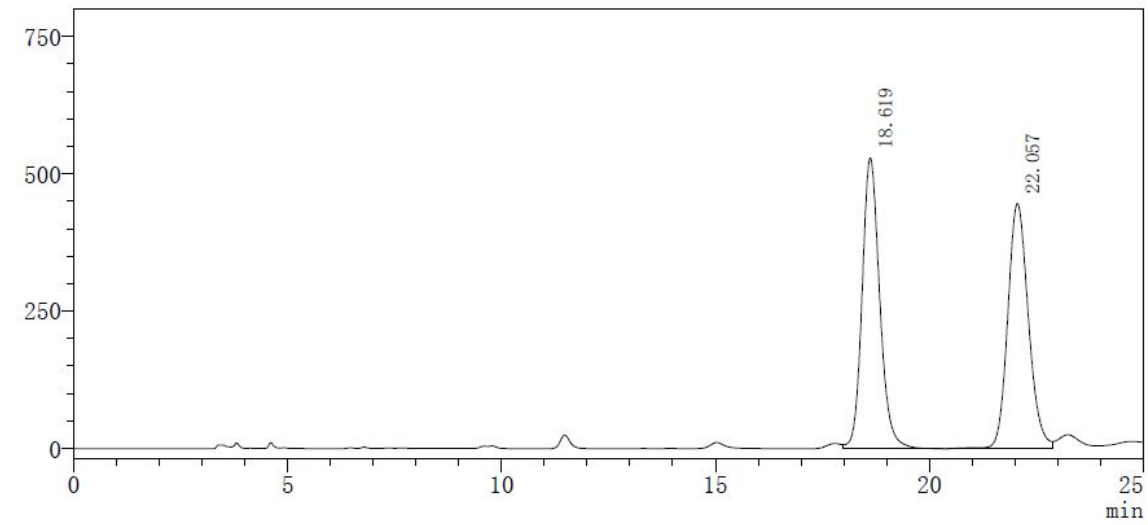

检测器A $220 \mathrm{~nm}$
\begin{tabular}{|c|c|c|c|c|c|}
\hline Peak\# & Time & Area & Height & Height $\%$ & Area $\%$ \\
\hline 1 & 18.619 & 15101698 & 528980 & 54.245 & 50.293 \\
\hline 2 & 22.057 & 14925465 & 446183 & 45.755 & 49.707 \\
\hline 总计 & & 30027163 & 975163 & 100.000 & 100.000 \\
\hline
\end{tabular}

$\mathrm{mV}$

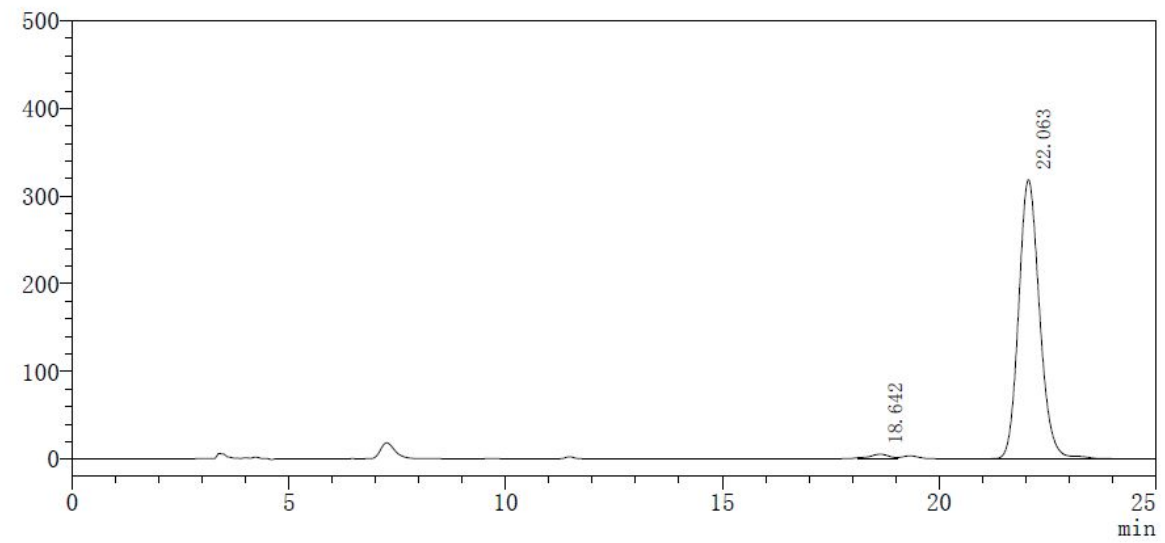

检测器A $220 \mathrm{~nm}$
\begin{tabular}{|c|c|c|c|c|c|}
\hline Peak\# & Time & Area & Height & Height $\%$ & Area\% \\
\hline 1 & 18.642 & 173885 & 5513 & 1.701 & 1.603 \\
\hline 2 & 22.063 & 10673577 & 318507 & 98.299 & 98.397 \\
\hline 总计 & & 10847461 & 324021 & 100.000 & 100.000 \\
\hline
\end{tabular}<smiles>CC[C@H](/C=C/c1ccccc1)S(=O)(=O)c1ccc(F)cc1</smiles>

(S,E)-1-fluoro-4-((1-phenylpent-1-en-3-yl)sulfonyl)benzene 
Prepared according to General Procedure, white solid, $55 \mathrm{mg}, 90 \%$ yield, m.p. $69-71{ }^{\circ} \mathrm{C},>20: 1 \mathrm{rr}$, $95 \%$ ee

$[\alpha]^{25}=-91.7\left(\mathrm{c} 1, \mathrm{CHCl}_{3}\right)$

${ }^{1} \mathrm{H}$ NMR $\left(400 \mathrm{MHz}, \mathrm{CDCl}_{3}\right) \delta 7.84(\mathrm{dd}, J=8.6,5.1 \mathrm{~Hz}, 2 \mathrm{H}), 7.29(\mathrm{dd}, J=14.8,7.1 \mathrm{~Hz}, 6 \mathrm{H}), 7.17(\mathrm{t}, J$ $=8.5 \mathrm{~Hz}, 2 \mathrm{H}), 6.28(\mathrm{~d}, J=15.9 \mathrm{~Hz}, 1 \mathrm{H}), 5.90(\mathrm{dd}, J=15.9,9.5 \mathrm{~Hz}, 1 \mathrm{H}), 3.55(\mathrm{td}, J=10.8,3.1 \mathrm{~Hz}$, $1 \mathrm{H}), 2.28$ (dqd, $J=14.9,7.4,3.5 \mathrm{~Hz}, 1 \mathrm{H}), 1.87-1.71(\mathrm{~m}, 1 \mathrm{H}), 1.00(\mathrm{t}, J=7.4 \mathrm{~Hz}, 3 \mathrm{H})$.

${ }^{13} \mathrm{C}$ NMR $\left(101 \mathrm{MHz}, \mathrm{CDCl}_{3}\right) \delta 165.8(\mathrm{~d}, J=256.2 \mathrm{~Hz}), 138.5,135.7,133.7$ (d, $\left.J=2.6 \mathrm{~Hz}\right), 132.0(\mathrm{~d}, J$ $=9.4 \mathrm{~Hz}), 128.7,128.6,126.6,120.9,116.2(\mathrm{~d}, J=22.6 \mathrm{~Hz}), 71.3,20.9,11.4$.

${ }^{19} \mathrm{~F}$ NMR (377 MHz, $\left.\mathrm{CDCl}_{3}\right) \delta-103.7$.

HRMS (ESI) calcd. for $\mathrm{C}_{17} \mathrm{H}_{17} \mathrm{FNaO}_{2} \mathrm{~S}^{+}(\mathrm{M}+\mathrm{Na})^{+}: 327.0825$, Found: 327.0826

the ee value was $95 \%, \mathrm{t}_{\mathrm{r}}($ minor $)=11.153 \mathrm{~min}, \mathrm{t}_{\mathrm{r}}($ major $)=12.618 \mathrm{~min}($ Chiralcel IC, $\lambda=220 \mathrm{~nm}$, hexanes $:{ }^{i} \mathrm{PrOH}=80: 20$, flow rate $=1.0 \mathrm{~mL} / \mathrm{min}$ )
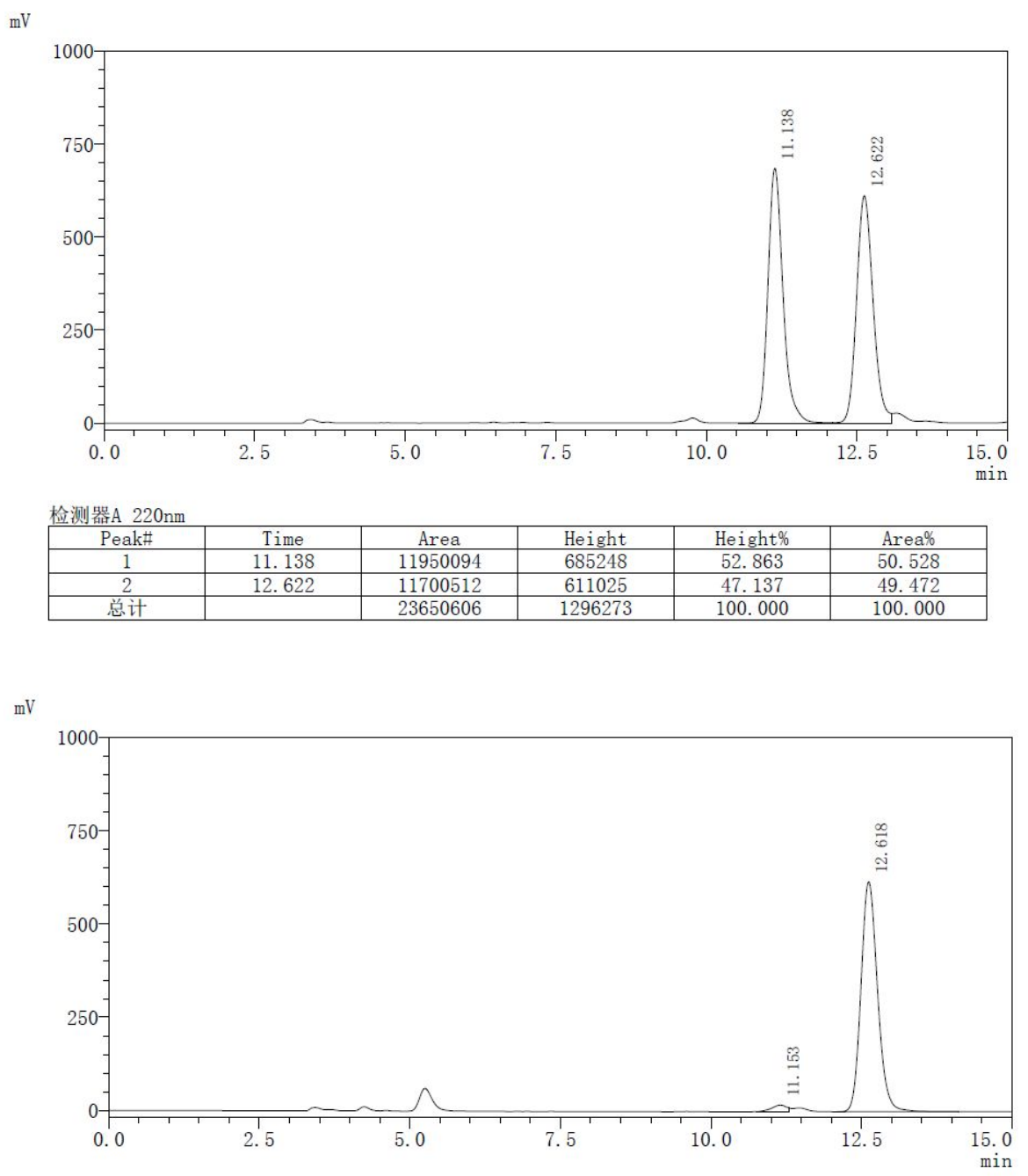

检测器A $220 \mathrm{~nm}$
\begin{tabular}{|c|c|c|c|c|c|}
\hline Peak\# & Time & Area & Height & Height\% & Area $\%$ \\
\hline 1 & 11.153 & 289781 & 17319 & 2.732 & 2.403 \\
\hline 2 & 12.618 & 11769509 & 616709 & 97.268 & 97.597 \\
\hline 总计 & & 12059290 & 634028 & 100.000 & 100.000 \\
\hline
\end{tabular}


<smiles>CCC(/C=C/c1ccccc1)S(=O)(=O)c1cccc(F)c1</smiles>

\section{(S,E)-1-fluoro-3-((1-phenylpent-1-en-3-yl)sulfonyl)benzene}

Prepared according to General Procedure, pale yellow oil, $50 \mathrm{mg}, 83 \%$ yield, $>20: 1 \mathrm{rr}, 95 \%$ ee $[\alpha]^{25} \mathrm{D}=-105.4\left(\mathrm{c} 1, \mathrm{CHCl}_{3}\right)$

${ }^{1} \mathrm{H}$ NMR $\left(400 \mathrm{MHz}, \mathrm{CDCl}_{3}\right) \delta 7.63(\mathrm{~d}, J=7.8 \mathrm{~Hz}, 1 \mathrm{H}), 7.59-7.53(\mathrm{~m}, 1 \mathrm{H}), 7.48(\mathrm{td}, J=8.0,5.2 \mathrm{~Hz}$, $1 \mathrm{H}), 7.32-7.23(\mathrm{~m}, 6 \mathrm{H}), 6.31(\mathrm{~d}, J=15.9 \mathrm{~Hz}, 1 \mathrm{H}), 5.90(\mathrm{dd}, J=15.9,9.5 \mathrm{~Hz}, 1 \mathrm{H}), 3.58(\mathrm{td}, J=10.5$, $3.3 \mathrm{~Hz}, 1 \mathrm{H}), 2.26(\mathrm{dqd}, J=15.0,7.5,3.4 \mathrm{~Hz}, 1 \mathrm{H}), 1.80(\mathrm{ddq}, J=14.7,11.0,7.4 \mathrm{~Hz}, 1 \mathrm{H}), 1.01$ (t, $J=$ $7.5 \mathrm{~Hz}, 3 \mathrm{H})$.

${ }^{13} \mathrm{C}$ NMR $\left(101 \mathrm{MHz}, \mathrm{CDCl}_{3}\right) \delta 162.3(\mathrm{~d}, J=252.2 \mathrm{~Hz}), 161.0,139.8(\mathrm{~d}, J=6.3 \mathrm{~Hz}), 138.7,135.6$, $130.6(\mathrm{~d}, J=7.6 \mathrm{~Hz}), 128.7,128.6,126.6,125.1$ (d, $J=3.3 \mathrm{~Hz}), 120.9$ (d, $J=21.3 \mathrm{~Hz}), 120.6,116.4$ (d, $J=24.2 \mathrm{~Hz}), 71.2,20.9,11.4$.

${ }^{19} \mathrm{~F}$ NMR (377 MHz, $\left.\mathrm{CDCl}_{3}\right) \delta-109.7$.

HRMS (ESI) calcd. for $\mathrm{C}_{17} \mathrm{H}_{17} \mathrm{FNaO}_{2} \mathrm{~S}^{+}(\mathrm{M}+\mathrm{Na})^{+}: 327.0825$, Found: 327.0825

the ee value was $95 \%, \mathrm{t}_{\mathrm{r}}($ minor $)=9.420 \min , \mathrm{t}_{\mathrm{r}}($ major $)=10.533 \min ($ Chiralcel IC, $\lambda=220 \mathrm{~nm}$, hexanes $:{ }^{i} \mathrm{PrOH}=80: 20$, flow rate $=1.0 \mathrm{~mL} / \mathrm{min}$ )

$\mathrm{mV}$

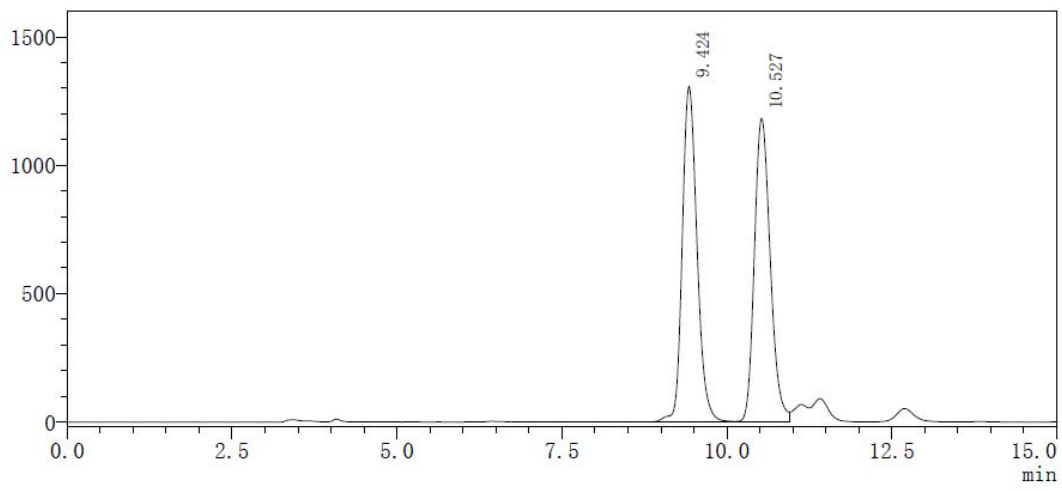

检测器A $220 \mathrm{~nm}$
\begin{tabular}{|c|c|c|c|c|c|}
\hline Peak\# & Time & Area & Height & Height $\%$ & Area\% \\
\hline 1 & 9.424 & 20442037 & 1306519 & 52.491 & 50.717 \\
\hline 2 & 10.527 & 19863937 & 1182535 & 47.509 & 49.283 \\
\hline 总计 & & 40305974 & 2489054 & 100.000 & 100.000 \\
\hline
\end{tabular}




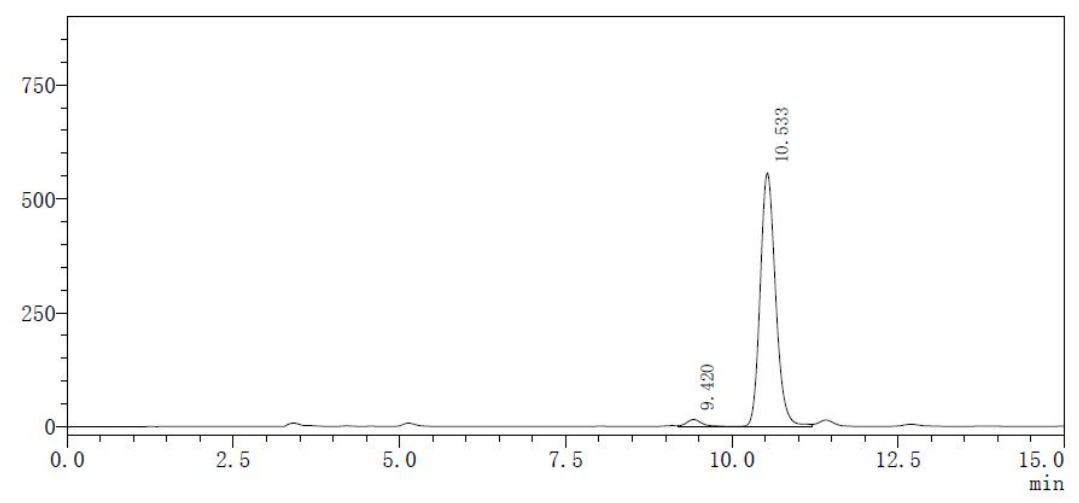

检测器A $220 \mathrm{~nm}$
\begin{tabular}{|c|c|c|c|c|c|}
\hline Peak\# & Time & Area & Height & Height\% & Area\% \\
\hline 1 & 9.420 & 246496 & 15168 & 2.654 & 2.673 \\
\hline 2 & 10.533 & 8976284 & 556345 & 97.346 & 97.327 \\
\hline 总计 & & 9222780 & 571513 & 100.000 & 100.000 \\
\hline
\end{tabular}<smiles>CCC(/C=C/c1ccccc1)S(=O)(=O)c1ccccc1F</smiles>

3af

\section{(S,E)-1-fluoro-2-((1-phenylpent-1-en-3-yl)sulfonyl)benzene}

Prepared according to General Procedure, white solid, $46 \mathrm{mg}, 75 \%$ yield, m.p. $48-50{ }^{\circ} \mathrm{C},>20: 1 \mathrm{rr}$, $96 \%$ ee

$[\alpha]^{25}{ }_{D}=-154.5\left(\mathrm{c} 1, \mathrm{CHCl}_{3}\right)$

${ }^{1} \mathrm{H}$ NMR $\left(400 \mathrm{MHz}, \mathrm{CDCl}_{3}\right) \delta 7.91-7.80(\mathrm{~m}, 1 \mathrm{H}), 7.57(\mathrm{td}, J=7.6,1.5 \mathrm{~Hz}, 1 \mathrm{H}), 7.31-7.15(\mathrm{~m}, 7 \mathrm{H})$, $6.38(\mathrm{~d}, J=15.8 \mathrm{~Hz}, 1 \mathrm{H}), 5.96(\mathrm{dd}, J=15.7,9.8 \mathrm{~Hz}, 1 \mathrm{H}), 3.87$ (td, $J=10.3,3.2 \mathrm{~Hz}, 1 \mathrm{H}), 2.27$ (dqd, $J$ $=14.9,7.5,3.5 \mathrm{~Hz}, 1 \mathrm{H}), 2.01-1.82(\mathrm{~m}, 1 \mathrm{H}), 1.04(\mathrm{t}, J=7.5 \mathrm{~Hz}, 3 \mathrm{H})$.

${ }^{13} \mathrm{C}$ NMR $\left(101 \mathrm{MHz}, \mathrm{CDCl}_{3}\right) \delta 159.5(\mathrm{~d}, J=255.1 \mathrm{~Hz}), 138.4,136.0(\mathrm{~d}, J=8.5 \mathrm{~Hz}), 135.7,131.4$, 128.6, 128.4, 126.6, 126.0 (d, $J=14.8 \mathrm{~Hz}), 124.7$ (d, $J=3.7 \mathrm{~Hz}), 120.8,116.9(\mathrm{~d}, J=21.7 \mathrm{~Hz}), 70.8$, 20.3, 11.3.

${ }^{19} \mathrm{~F}$ NMR (377 MHz, $\left.\mathrm{CDCl}_{3}\right) \delta-108.2$.

HRMS (ESI) calcd. for $\mathrm{C}_{17} \mathrm{H}_{17} \mathrm{FNaO}_{2} \mathrm{~S}^{+}(\mathrm{M}+\mathrm{Na})^{+}: 327.0825$, Found: 327.0825

the ee value was $96 \%, \mathrm{t}_{\mathrm{r}}($ minor $)=13.690 \mathrm{~min}, \mathrm{t}_{\mathrm{r}}($ major $)=15.162 \min ($ Chiralcel IC, $\lambda=220 \mathrm{~nm}$, hexanes : ${ }^{i} \mathrm{PrOH}=80: 20$, flow rate $=1.0 \mathrm{~mL} / \mathrm{min}$ ) 
$\mathrm{mV}$
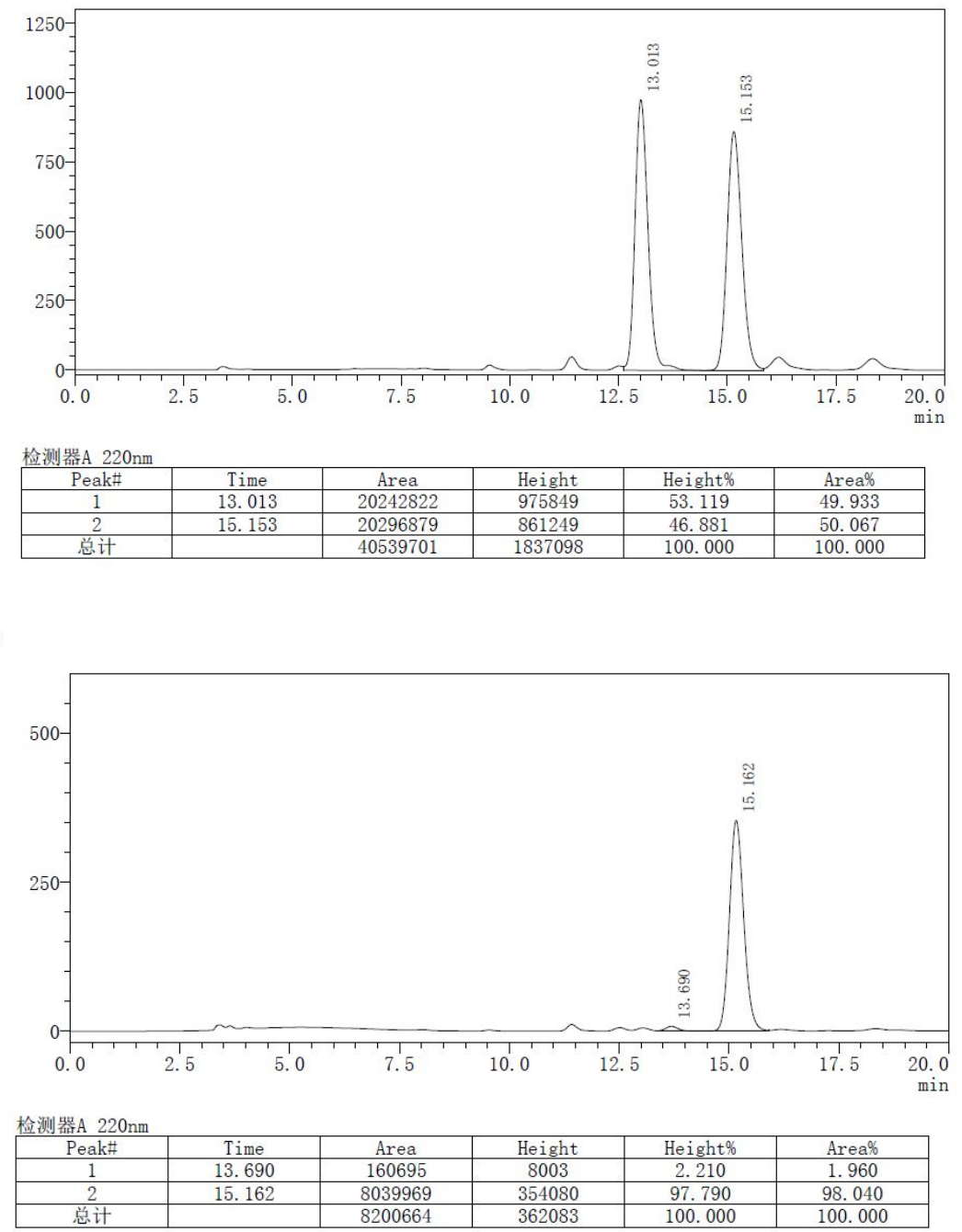<smiles>CCC(/C=C/c1ccccc1)S(=O)(=O)c1ccc(C(F)(F)F)cc1</smiles>

\section{(S,E)-1-((1-phenylpent-1-en-3-yl)sulfonyl)-4-(trifluoromethyl)benzene}

Prepared according to General Procedure, white solid, $61 \mathrm{mg}, 86 \%$ yield, m.p. $82-84{ }^{\circ} \mathrm{C},>20: 1 \mathrm{rr}$, $97 \%$ ee

$[\alpha]^{25}=-56.0\left(\mathrm{c} 1, \mathrm{CHCl}_{3}\right)$

${ }^{1} \mathrm{H}$ NMR $\left(400 \mathrm{MHz}, \mathrm{CDCl}_{3}\right) \delta 7.97(\mathrm{~d}, J=8.0 \mathrm{~Hz}, 2 \mathrm{H}), 7.76(\mathrm{~d}, J=8.1 \mathrm{~Hz}, 2 \mathrm{H}), 7.38-7.20(\mathrm{~m}, 5 \mathrm{H})$, $6.28(\mathrm{~d}, J=15.9 \mathrm{~Hz}, 1 \mathrm{H}), 5.91(\mathrm{dd}, J=15.9,9.5 \mathrm{~Hz}, 1 \mathrm{H}), 3.65-3.55(\mathrm{~m}, 1 \mathrm{H}), 2.36-2.21(\mathrm{~m}, 1 \mathrm{H})$, $1.89-1.72(\mathrm{~m}, 1 \mathrm{H}), 1.02(\mathrm{t}, J=7.4 \mathrm{~Hz}, 3 \mathrm{H})$.

${ }^{13} \mathrm{C}$ NMR $\left(101 \mathrm{MHz}, \mathrm{CDCl}_{3}\right) \delta 141.3,138.9,135.5,135.3(\mathrm{q}, J=32.9 \mathrm{~Hz}), 129.9,128.8,128.7,126.6$, $125.9(\mathrm{q}, J=3.4 \mathrm{~Hz}), \delta 123.2(\mathrm{q}, J=273.1 \mathrm{~Hz}), 120.4,71.2,20.8,11.3$.

${ }^{19} \mathrm{~F}$ NMR $\left(377 \mathrm{MHz}, \mathrm{CDCl}_{3}\right) \delta-63.1$. 
HRMS (ESI) calcd. for $\mathrm{C}_{18} \mathrm{H}_{17} \mathrm{~F}_{3} \mathrm{NaO}_{2} \mathrm{~S}^{+}(\mathrm{M}+\mathrm{Na})^{+}:$377.0794, Found: 377.0794

the ee value was $96 \%, \mathrm{t}_{\mathrm{r}}($ minor $)=18.841 \mathrm{~min}, \mathrm{t}_{\mathrm{r}}($ major $)=19.680 \min ($ Chiralcel IC, $\lambda=220 \mathrm{~nm}$, hexanes : ${ }^{i} \mathrm{PrOH}=95: 5$, flow rate $=1.0 \mathrm{~mL} / \mathrm{min}$ )
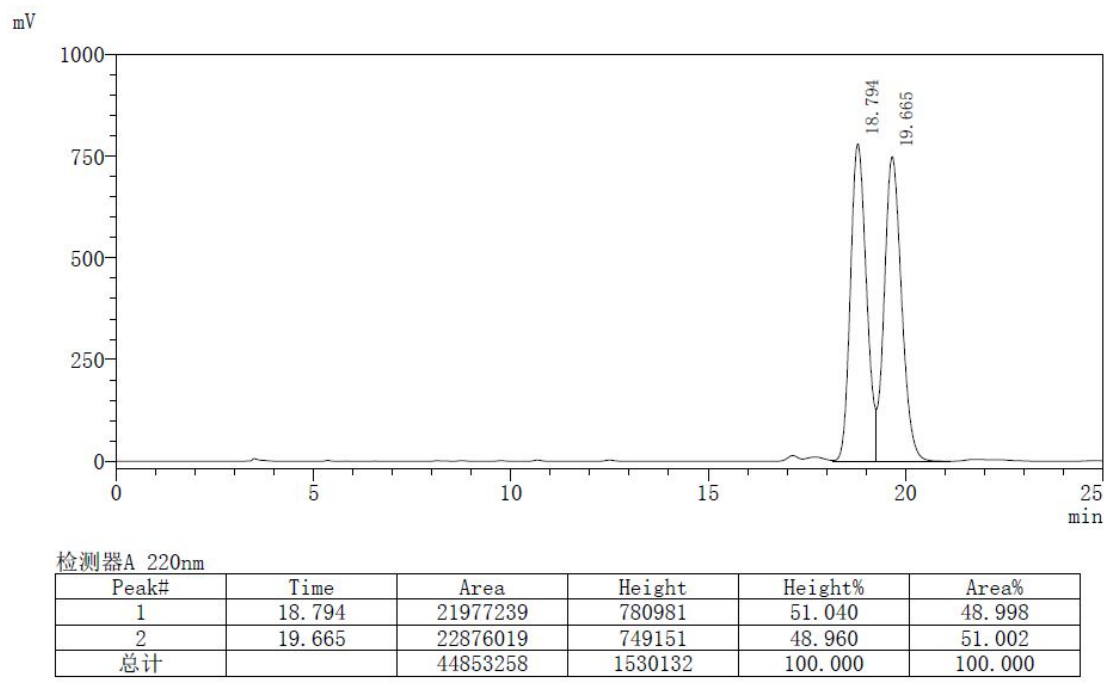

$\mathrm{mV}$

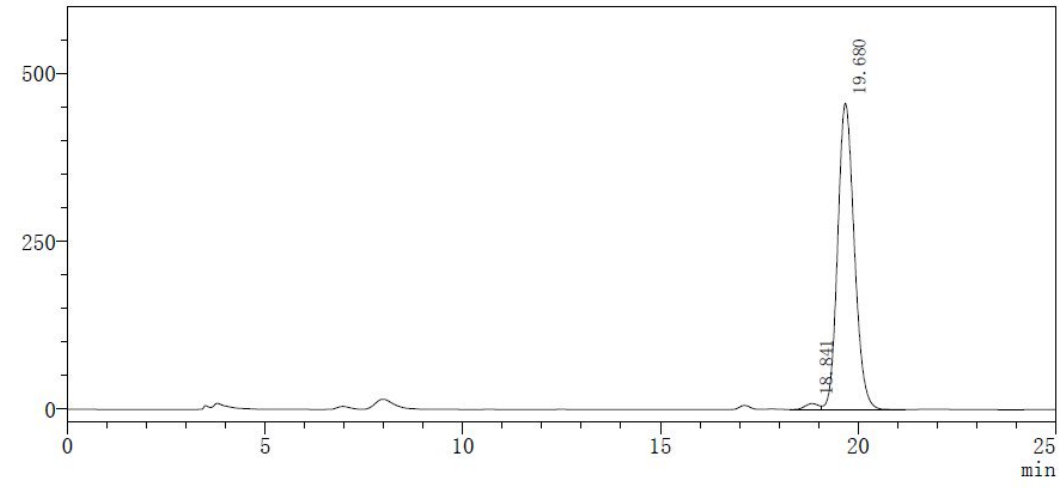

检测器A $220 \mathrm{~nm}$
\begin{tabular}{|c|c|c|c|c|c|}
\hline Peak\# & Time & Area & Height & Height\% & Area $\%$ \\
\hline 1 & 18.841 & 226805 & 9151 & 1.963 & 1.665 \\
\hline 2 & 19.680 & 13395509 & 456979 & 98.037 & 98.335 \\
\hline 总计 & & 13622314 & 466130 & 100.000 & 100.000 \\
\hline
\end{tabular}<smiles>CC[C@H](/C=C/c1ccccc1)S(=O)(=O)c1ccc(C#N)cc1</smiles>

\section{(S,E)-4-((1-phenylpent-1-en-3-yl)sulfonyl)benzonitrile}

Prepared according to General Procedure, white solid, $47 \mathrm{mg}, 75 \%$ yield, m.p. $112-114{ }^{\circ} \mathrm{C},>20: 1$ rr, $96 \%$ ee

$[\alpha]^{25}{ }_{\mathrm{D}}=-146.1\left(\mathrm{c} 1, \mathrm{CHCl}_{3}\right)$

${ }^{1} \mathrm{H}$ NMR $\left(400 \mathrm{MHz}, \mathrm{CDCl}_{3}\right) \delta 7.95(\mathrm{~d}, J=8.3 \mathrm{~Hz}, 2 \mathrm{H}), 7.79(\mathrm{~d}, J=8.3 \mathrm{~Hz}, 2 \mathrm{H}), 7.37-7.19(\mathrm{~m}, 6 \mathrm{H})$, $6.26(\mathrm{~d}, J=15.9 \mathrm{~Hz}, 1 \mathrm{H}), 5.89$ (dd, $J=15.9,9.5 \mathrm{~Hz}, 1 \mathrm{H}), 3.59$ (td, $J=10.4,3.2 \mathrm{~Hz}, 1 \mathrm{H}), 2.29$ (dqd, $J$ $=14.9,7.5,3.5 \mathrm{~Hz}, 1 \mathrm{H}), 1.91-1.73(\mathrm{~m}, 1 \mathrm{H}), 1.02(\mathrm{t}, J=7.4 \mathrm{~Hz}, 3 \mathrm{H})$. 
${ }^{13} \mathrm{C}$ NMR $\left(101 \mathrm{MHz}, \mathrm{CDCl}_{3}\right) \delta 142.0,139.0,135.3,132.6,129.9,128.8,126.6,120.2,117.4,117.2$, $71.2,20.7,11.3$.

HRMS (ESI) calcd. for $\mathrm{C}_{18} \mathrm{H}_{17} \mathrm{NNaO}_{2} \mathrm{~S}^{+}(\mathrm{M}+\mathrm{Na})^{+}: 334.0872$, Found: 334.0872

the ee value was $96 \%, \mathrm{t}_{\mathrm{r}}($ major $)=9.242 \min , \mathrm{t}_{\mathrm{r}}($ minor $)=10.357 \min$ (Chiralcel IA, $\lambda=220 \mathrm{~nm}$, hexanes $:{ }^{i} \mathrm{PrOH}=80: 20$, flow rate $=1.0 \mathrm{~mL} / \mathrm{min}$ )

$\mathrm{mV}$

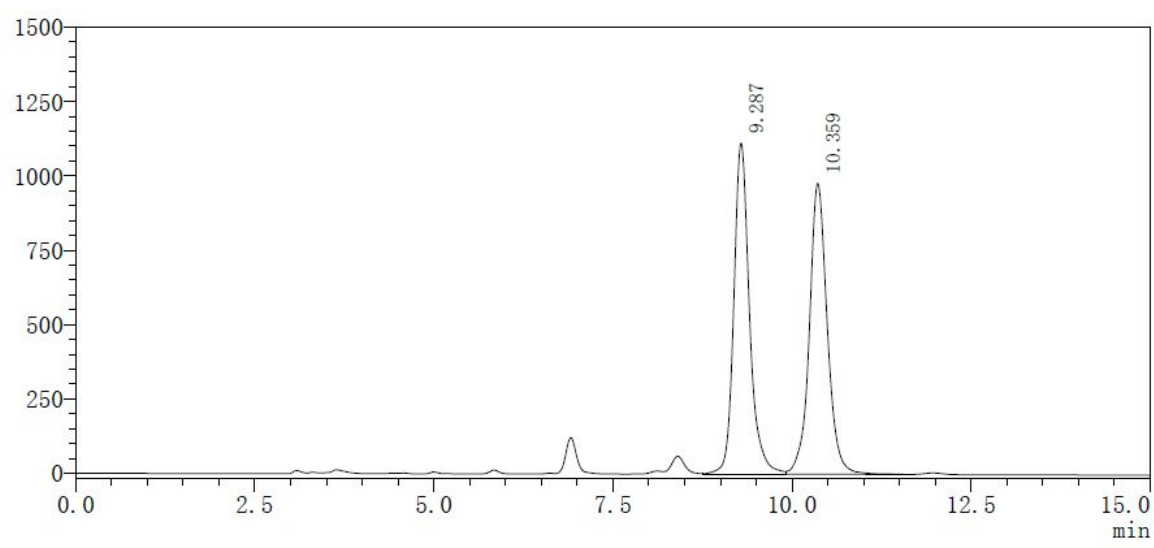

检测器A $220 \mathrm{~nm}$
\begin{tabular}{|c|c|c|c|c|c|}
\hline Peak\# & Time & Area & Height & Height\% & Area\% \\
\hline 1 & 9.287 & 16981793 & 1113357 & 53.216 & 49.884 \\
\hline 2 & 10.359 & 17060908 & 978780 & 46.784 & 50.116 \\
\hline 总计 & & 34042701 & 2092137 & 100.000 & 100.000 \\
\hline
\end{tabular}

$\mathrm{mV}$

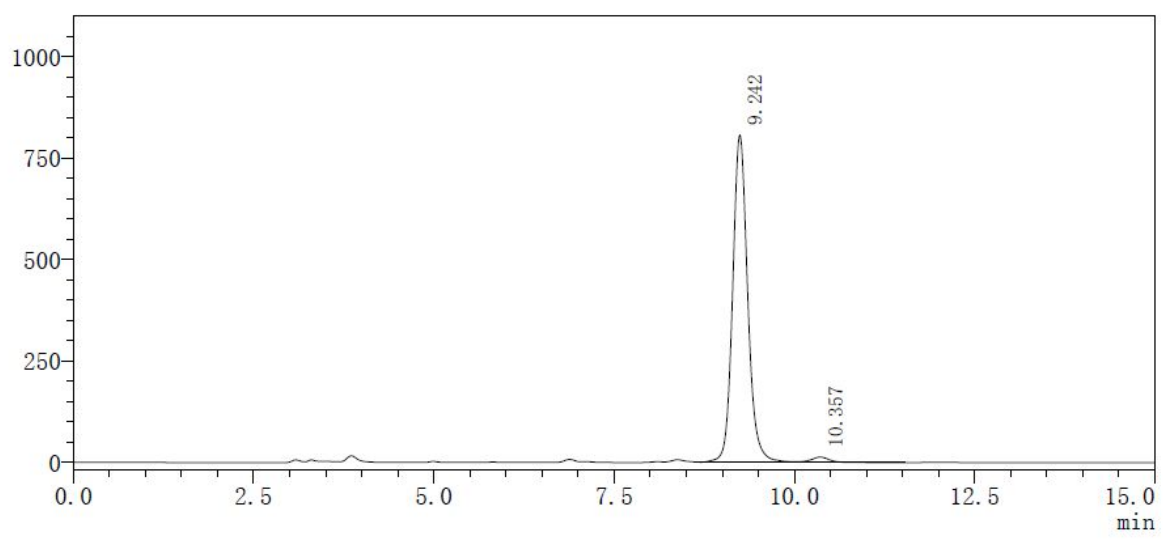

检测器A $220 \mathrm{~nm}$
\begin{tabular}{|c|c|c|c|c|c|}
\hline Peak\# & Time & Area & Height & Height\% & Area\% \\
\hline 1 & 9.242 & 11752720 & 806780 & 98.392 & 97.965 \\
\hline 2 & 10.357 & 244159 & 13186 & 1.608 & 2.035 \\
\hline 总计 & & 11996879 & 819965 & 100.000 & 100.000 \\
\hline
\end{tabular}<smiles>CCC(/C=C/c1ccccc1)S(=O)(=O)c1cc(C(F)(F)F)cc(C(F)(F)F)c1</smiles>

\section{(S,E)-1-((1-phenylpent-1-en-3-yl)sulfonyl)-3,5-bis(trifluoromethyl)benzene}

Prepared according to General Procedure, white solid, $45 \mathrm{mg}$, 53\% yield, m.p. $105-107^{\circ} \mathrm{C},>20: 1$ 
rr, $93 \%$ ee

$[\alpha]^{25}=-94.5\left(\mathrm{c} 1, \mathrm{CHCl}_{3}\right)$

${ }^{1} \mathrm{H}$ NMR $\left(400 \mathrm{MHz}, \mathrm{CDCl}_{3}\right) \delta 8.28(\mathrm{~s}, 2 \mathrm{H}), 8.09(\mathrm{~s}, 1 \mathrm{H}), 7.27(\mathrm{dd}, J=26.4,7.0 \mathrm{~Hz}, 5 \mathrm{H}), 6.26(\mathrm{~d}, J=$ $15.9 \mathrm{~Hz}, 1 \mathrm{H}), 5.91$ (dd, $J=15.9,9.6 \mathrm{~Hz}, 1 \mathrm{H}), 3.62$ (td, $J=10.4,3.1 \mathrm{~Hz}, 1 \mathrm{H}), 2.32$ (ddd, $J=13.3,7.4$, $3.4 \mathrm{~Hz}, 1 \mathrm{H}), 1.94-1.77(\mathrm{~m}, 1 \mathrm{H}), 1.05$ (t, $J=7.4 \mathrm{~Hz}, 3 \mathrm{H})$.

${ }^{13} \mathrm{C}$ NMR $\left(101 \mathrm{MHz}, \mathrm{CDCl}_{3}\right) \delta 140.5,139.6,135.0,132.7$ (q, $\left.J=34.7 \mathrm{~Hz}\right), 129.6(\mathrm{~d}, J=3.2 \mathrm{~Hz}), 129.0$, $128.8,127.1(\mathrm{dd}, J=7.0,3.6 \mathrm{~Hz}), 126.5,122.3$ (q, $J=273.6 \mathrm{~Hz}), 119.9,71.5,20.5,11.3$.

${ }^{19} \mathrm{~F}$ NMR $\left(377 \mathrm{MHz}, \mathrm{CDCl}_{3}\right) \delta-62.9$.

HRMS (ESI) calcd. for $\mathrm{C}_{19} \mathrm{H}_{16} \mathrm{~F}_{6} \mathrm{NaO}_{2} \mathrm{~S}^{+}(\mathrm{M}+\mathrm{Na})^{+}: 445.0667$, Found: 445.0667

the ee value was $93 \%, \mathrm{t}_{\mathrm{r}}$ (minor) $=5.379 \mathrm{~min}, \mathrm{t}_{\mathrm{r}}$ (major) $=5.697 \mathrm{~min}($ Chiralcel $\mathrm{IC}, \lambda=220 \mathrm{~nm}$, hexanes : ${ }^{i} \mathrm{PrOH}=95: 5$, flow rate $=1.0 \mathrm{~mL} / \mathrm{min}$ )

$\mathrm{m} V$
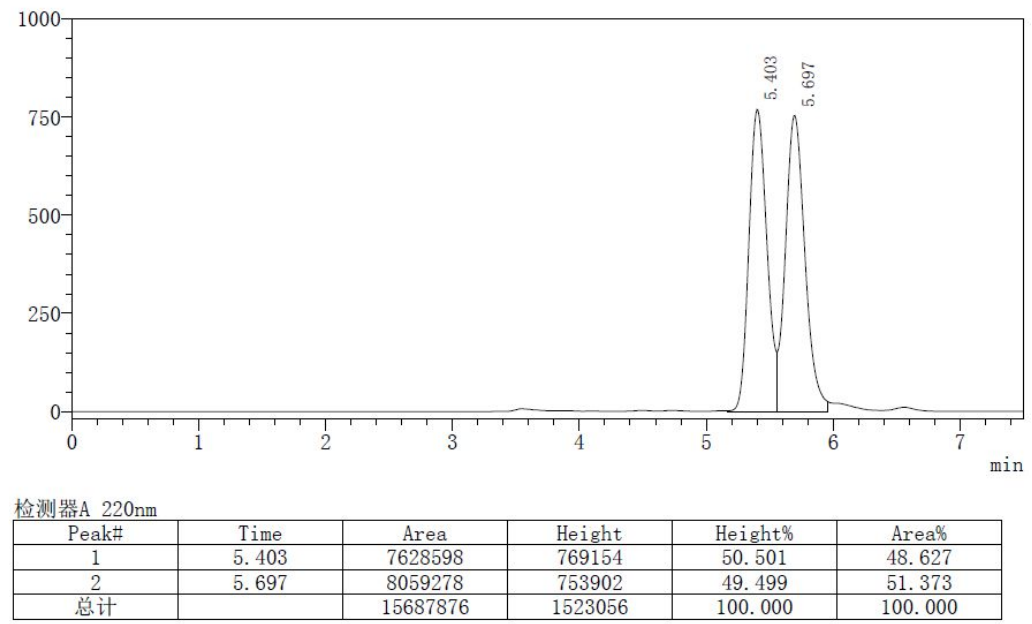

$\mathrm{mV}$

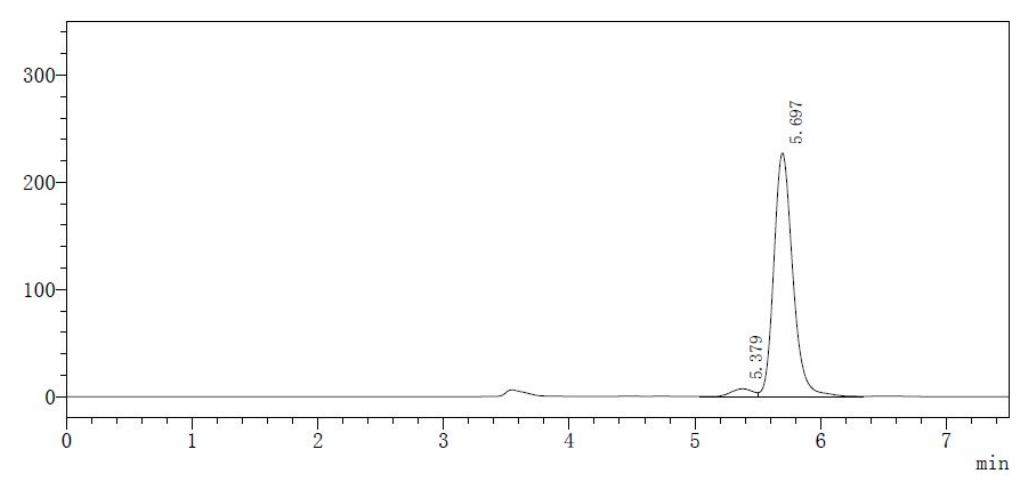

检测器 $\mathrm{A} 220 \mathrm{~nm}$

\begin{tabular}{|c|c|c|c|c|c|}
\hline Peak\# & Time & Area & Height & Height\% & Area\% \\
\hline 1 & 5.379 & 89541 & 7432 & 3.164 & 3.622 \\
\hline 2 & 5.697 & 2382905 & 227490 & 96.836 & 96.378 \\
\hline 总计 & & 2472446 & 234923 & 100.000 & 100.000 \\
\hline
\end{tabular}


<smiles>CCC(/C=C/c1ccccc1)S(=O)(=O)c1cc(F)cc(F)c1</smiles>

\section{(S,E)-1,3-difluoro-5-((1-phenylpent-1-en-3-yl)sulfonyl)benzene}

Prepared according to General Procedure, white solid, $55 \mathrm{mg}, 85 \%$ yield, m.p. $61-62{ }^{\circ} \mathrm{C},>20: 1 \mathrm{rr}$, $97 \%$ ee

$[\alpha]^{25}=-58.5\left(\mathrm{c} 1, \mathrm{CHCl}_{3}\right)$

${ }^{1} \mathrm{H}$ NMR $\left(400 \mathrm{MHz}, \mathrm{CDCl}_{3}\right) \delta 7.42(\mathrm{~d}, J=4.0 \mathrm{~Hz}, 2 \mathrm{H}), 7.38-7.29(\mathrm{~m}, 5 \mathrm{H}), 7.08(\mathrm{tt}, J=8.3,2.2 \mathrm{~Hz}$, 1H), 6.39 (d, $J=15.9 \mathrm{~Hz}, 1 \mathrm{H}), 5.92(\mathrm{dd}, J=15.8,9.5 \mathrm{~Hz}, 1 \mathrm{H}), 3.63$ (td, $J=10.4,3.3 \mathrm{~Hz}, 1 \mathrm{H}), 2.28$ (dqd, $J=15.0,7.5,3.5 \mathrm{~Hz}, 1 \mathrm{H}), 1.84$ (ddq, $J=14.6,11.0,7.4 \mathrm{~Hz}, 1 \mathrm{H}), 1.04(\mathrm{t}, J=7.5 \mathrm{~Hz}, 3 \mathrm{H})$.

${ }^{13} \mathrm{C}$ NMR $\left(101 \mathrm{MHz}, \mathrm{CDCl}_{3}\right) \delta 162.6(\mathrm{dd}, J=255.5,11.3 \mathrm{~Hz}), 141.2$ (t, $\left.J=7.9 \mathrm{~Hz}\right), 139.0,135.5$, $128.8,128.7,126.6,120.1,113.0-112.5(\mathrm{~m}), 109.3(\mathrm{t}, J=25.0 \mathrm{~Hz}), 71.2,20.9,11.3$.

${ }^{19} \mathrm{~F}$ NMR $\left(377 \mathrm{MHz}, \mathrm{CDCl}_{3}\right) \delta-105.5$

HRMS (ESI) calcd. for $\mathrm{C}_{17} \mathrm{H}_{16} \mathrm{~F}_{2} \mathrm{NaO}_{2} \mathrm{~S}^{+}(\mathrm{M}+\mathrm{Na})^{+}: 345.0731$, Found: 345.0731

the ee value was $97 \%, \mathrm{t}_{\mathrm{r}}($ minor $)=12.487 \mathrm{~min}, \mathrm{t}_{\mathrm{r}}($ major $)=13.426 \min$ (Chiralcel IC, $\lambda=220 \mathrm{~nm}$, hexanes : ${ }^{i} \mathrm{PrOH}=95: 5$, flow rate $=1.0 \mathrm{~mL} / \mathrm{min}$ )

mV

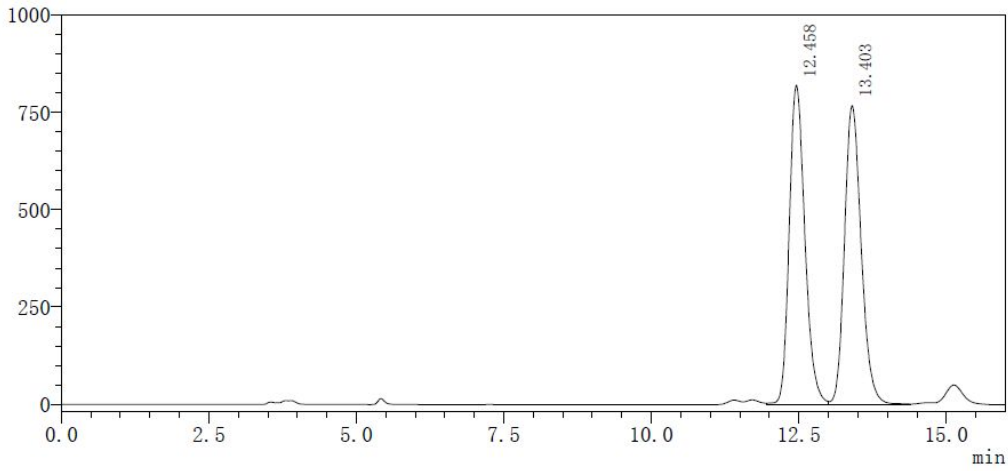

检测器A $220 \mathrm{~nm}$
\begin{tabular}{|c|c|c|c|c|c|}
\hline Peak\# & Time & Area & Height & Height $\%$ & Area $\%$ \\
\hline 1 & 12.458 & 15215728 & 821636 & 51.662 & 49.892 \\
\hline 2 & 13.403 & 15281863 & 768757 & 48.338 & 50.108 \\
\hline 总计 & & 30497591 & 1590393 & 100.000 & 100.000 \\
\hline
\end{tabular}



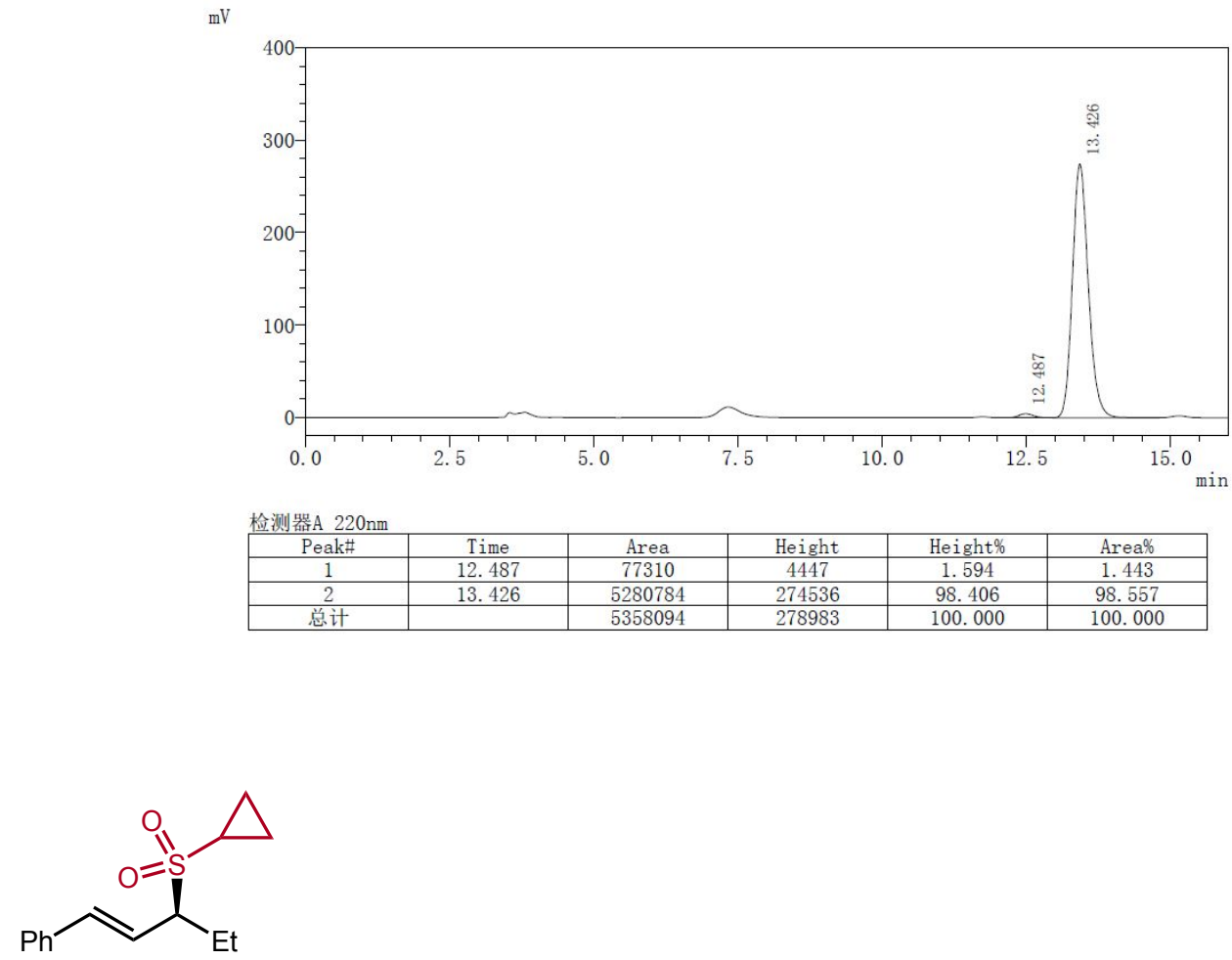

3ak

\section{(S,E)-(3-(cyclopropylsulfonyl)pent-1-en-1-yl)benzene}

Prepared according to General Procedure but in MTBE $0.5 \mathrm{~mL}$ with $\mathrm{C}$ (sulfinic acid) $=0.4 \mathrm{M}$ for $72 \mathrm{~h}$, white solid, $33 \mathrm{mg}, 65 \%$ yield, m.p. $67-68{ }^{\circ} \mathrm{C},>20: 1 \mathrm{rr}, 96 \%$ ee

$[\alpha]^{25}=-72.4\left(\mathrm{c} 1, \mathrm{CHCl}_{3}\right)$

${ }^{1} \mathrm{H}$ NMR (400 MHz, $\left.\mathrm{CDCl}_{3}\right) \delta 7.43(\mathrm{~d}, J=7.3 \mathrm{~Hz}, 2 \mathrm{H}), 7.35(\mathrm{t}, J=7.3 \mathrm{~Hz}, 2 \mathrm{H}), 7.30(\mathrm{~d}, J=7.0 \mathrm{~Hz}$, $1 \mathrm{H}), 6.70(\mathrm{~d}, J=15.9 \mathrm{~Hz}, 1 \mathrm{H}), 6.12(\mathrm{dd}, J=15.8,9.8 \mathrm{~Hz}, 1 \mathrm{H}), 3.56(\mathrm{td}, J=10.3,3.0 \mathrm{~Hz}, 1 \mathrm{H}), 2.40$ (ddd, $J=12.7,8.0,4.9 \mathrm{~Hz}, 1 \mathrm{H}), 2.34-2.21(\mathrm{~m}, 1 \mathrm{H}), 1.94-1.79(\mathrm{~m}, 1 \mathrm{H}), 1.30$ (dd, $J=10.0,4.6 \mathrm{~Hz}$, 1H), $1.19-1.09$ (m, 1H), 1.04 (t, $J=7.4 \mathrm{~Hz}, 3 \mathrm{H}), 0.99$ (d, $J=7.8 \mathrm{~Hz}, 2 \mathrm{H})$.

${ }^{13} \mathrm{C}$ NMR $\left(101 \mathrm{MHz}, \mathrm{CDCl}_{3}\right) \delta 137.7,135.8,128.8,128.6,126.7,121.9,69.4,27.6,20.1,11.3,4.8,4.6$. HRMS (ESI) calcd. for $\mathrm{C}_{14} \mathrm{H}_{18} \mathrm{NaO}_{2} \mathrm{~S}^{+}(\mathrm{M}+\mathrm{Na})^{+}:$273.0920, Found: 273.0918

the ee value was $96 \%, \mathrm{t}_{\mathrm{r}}($ minor $)=18.790 \mathrm{~min}, \mathrm{t}_{\mathrm{r}}($ major $)=20.006 \min ($ Chiralcel IC, $\lambda=220 \mathrm{~nm}$, hexanes : ${ }^{i} \mathrm{PrOH}=80: 20$, flow rate $=1.0 \mathrm{~mL} / \mathrm{min}$ ) 


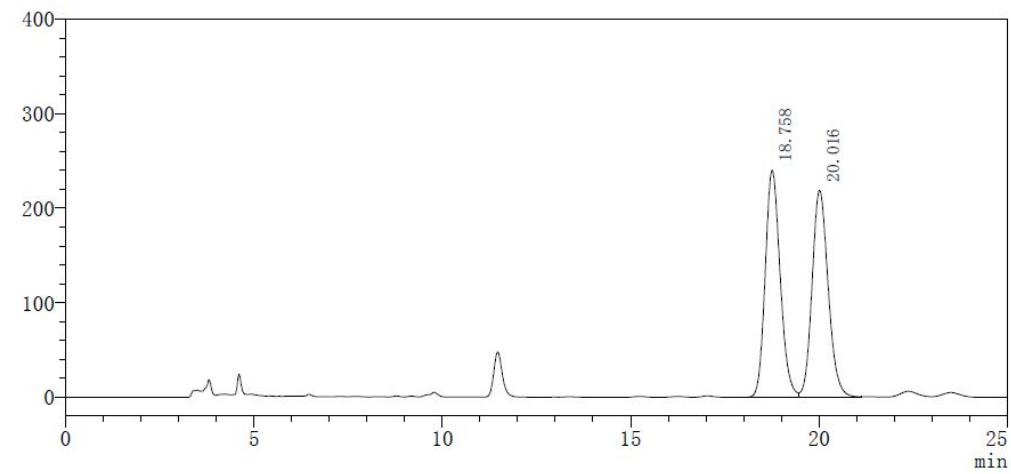

检测器A $220 \mathrm{~nm}$
\begin{tabular}{|c|c|c|c|c|c|}
\hline Peak\# & Time & Area & Height & Height\% & Area $\%$ \\
\hline 1 & 18.758 & 6633117 & 240139 & 52.301 & 50.441 \\
\hline 2 & 20.016 & 6517105 & 219009 & 47.699 & 49.559 \\
\hline 总计 & & 13150223 & 459148 & 100.000 & 100.000 \\
\hline
\end{tabular}

$\mathrm{mV}$

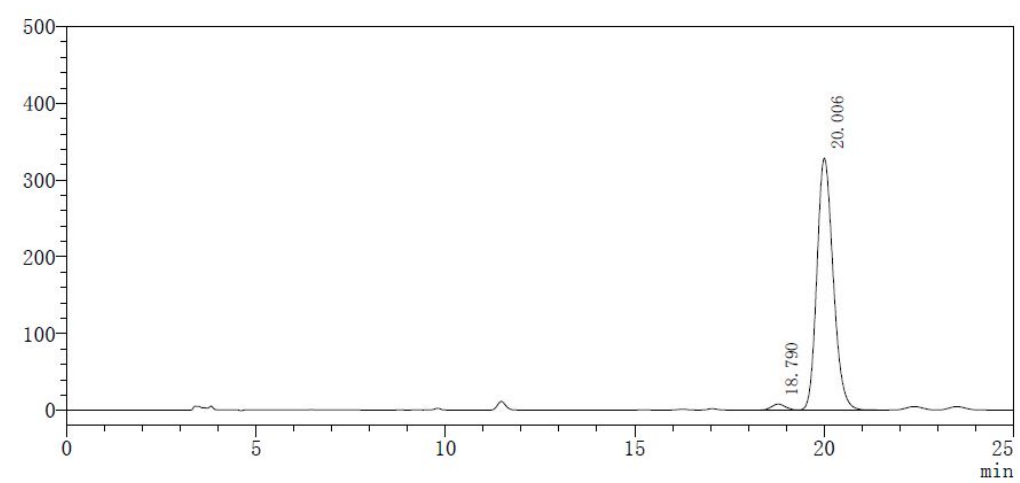

检测器A $220 \mathrm{~nm}$
\begin{tabular}{|c|c|c|c|c|c|}
\hline Peak\# & Time & Area & Height & Height\% & Area\% \\
\hline 1 & 18.790 & 215704 & 8025 & 2.385 & 2.147 \\
\hline 2 & 20.006 & 9833205 & 328501 & 97.615 & 97.853 \\
\hline 总计 & & 10048909 & 336526 & 100.000 & 100.000 \\
\hline
\end{tabular}<smiles>CCC(/C=C/c1ccccc1)S(=O)(=O)C(C)C</smiles>

3al

\section{$(S, E)$-(3-(isopropylsulfonyl)pent-1-en-1-yl)benzene}

Prepared according to General Procedure but in MTBE $0.5 \mathrm{~mL}$ with $\mathrm{C}$ (sulfinic acid) $=0.4 \mathrm{M}$ for $72 \mathrm{~h}$, pale yellow oil, $31 \mathrm{mg}, 61 \%$ yield, $>20: 1 \mathrm{rr}, 98 \%$ ee

$[\alpha]^{25}=-57.1\left(\mathrm{c} 0.5, \mathrm{CHCl}_{3}\right)$

${ }^{1} \mathrm{H}$ NMR $\left(400 \mathrm{MHz}, \mathrm{CDCl}_{3}\right) \delta 7.34-7.27(\mathrm{~m}, 2 \mathrm{H}), 7.27-7.15(\mathrm{~m}, 3 \mathrm{H}), 6.52(\mathrm{~d}, J=16.0 \mathrm{~Hz}, 1 \mathrm{H})$, 5.99 (dd, $J=16.0,10.1 \mathrm{~Hz}, 1 \mathrm{H}$ ), 3.53 (td, $J=10.4,3.3 \mathrm{~Hz}, 1 \mathrm{H}$ ), 3.18 (hept, $J=6.9 \mathrm{~Hz}, 1 \mathrm{H}$ ), 2.15 (dqd, $J=15.0,7.5,3.4 \mathrm{~Hz}, 1 \mathrm{H}), 1.72(\mathrm{ddq}, J=14.7,10.7,7.4 \mathrm{~Hz}, 1 \mathrm{H}), 1.25$ (s, 3H), 1.23 (d, $J=0.8 \mathrm{~Hz}, 3 \mathrm{H}$ ), $0.90(\mathrm{t}, J=7.5 \mathrm{~Hz}, 3 \mathrm{H})$.

${ }^{13} \mathrm{C}$ NMR (101 MHz, $\left.\mathrm{CDCl}_{3}\right) \delta 137.1,135.6,128.8,128.7,126.7,122.2,65.2,50.1,19.4,16.4,13.7$, 11.2 . 
HRMS (ESI) calcd. for $\mathrm{C}_{14} \mathrm{H}_{20} \mathrm{NaO}_{2} \mathrm{~S}^{+}(\mathrm{M}+\mathrm{Na})^{+}:$275.1076, Found: 275.1077

the ee value was $98 \%, \mathrm{t}_{\mathrm{r}}($ minor $)=26.463 \mathrm{~min}, \mathrm{t}_{\mathrm{r}}($ major $)=27.169 \min ($ Chiralcel IC, $\lambda=220 \mathrm{~nm}$, hexanes : ${ }^{i} \mathrm{PrOH}=90: 10$, flow rate $=1.0 \mathrm{~mL} / \mathrm{min}$ )

$\mathrm{m} V$

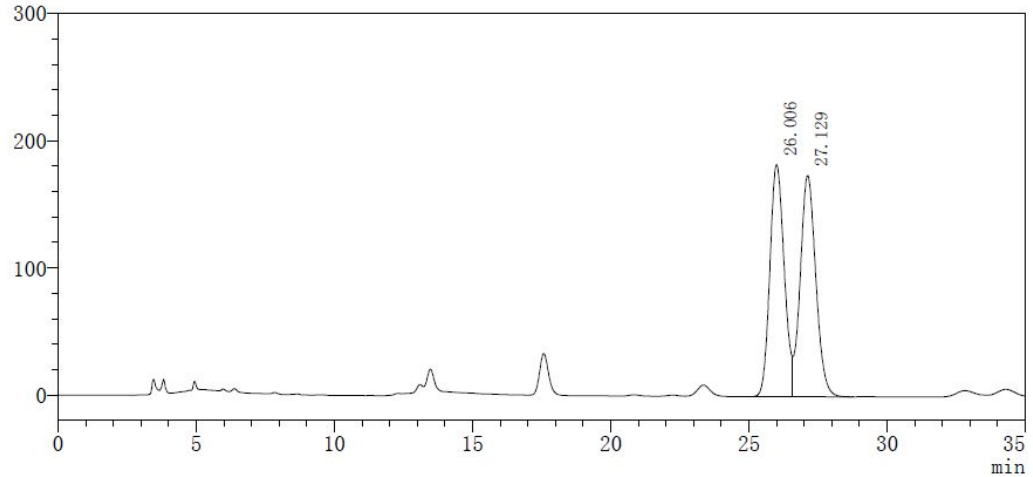

检测器A $220 \mathrm{~nm}$
\begin{tabular}{|c|c|c|c|c|c|}
\hline Peak\# & Time & Area & Height & Height\% & Area\% \\
\hline 1 & 26.006 & 6598269 & 182480 & 51.202 & 49.177 \\
\hline 2 & 27.129 & 6819121 & 173911 & 48.798 & 50.823 \\
\hline 总计 & & 13417390 & 356390 & 100.000 & 100.000 \\
\hline
\end{tabular}

$\mathrm{mV}$

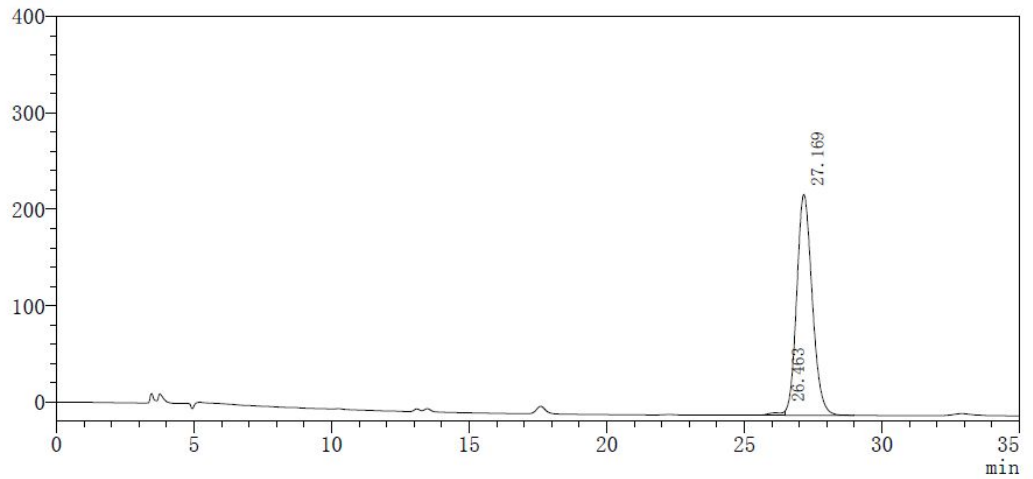

检测器A $220 \mathrm{~nm}$
\begin{tabular}{|c|c|c|c|c|c|}
\hline Peak\# & Time & Area & Height & Height \% & Area\% \\
\hline 1 & 26.463 & 97284 & 3641 & 1.564 & 1.071 \\
\hline 2 & 27.169 & 8984268 & 229074 & 98.436 & 98.929 \\
\hline 总计 & & 9081552 & 232715 & 100.000 & 100.000 \\
\hline
\end{tabular}<smiles>CC[C@H](/C=C/c1ccccc1)S(=O)(=O)Br</smiles>

3am

\section{(S,E)-(3-(benzylsulfonyl)pent-1-en-1-yl)benzene}

Prepared according to General Procedure but in MTBE $0.5 \mathrm{~mL}$ with $\mathrm{C}$ (sulfinic acid) $=0.4 \mathrm{M}$ for $72 \mathrm{~h}$, white solid, $25 \mathrm{mg}, 42 \%$ yield, m.p. $85-87^{\circ} \mathrm{C},>20: 1 \mathrm{rr}, 96 \%$ ee

$[\alpha]^{25}=-148.6\left(\mathrm{c} 0.5, \mathrm{CHCl}_{3}\right)$

${ }^{1} \mathrm{H}$ NMR (400 MHz, $\left.\mathrm{CDCl}_{3}\right) \delta 7.48-7.42(\mathrm{~m}, 2 \mathrm{H}), 7.42-7.37$ (m, 6H), $7.37-7.31(\mathrm{~m}, 2 \mathrm{H}), 6.58(\mathrm{~d}, J$

$=15.9 \mathrm{~Hz}, 1 \mathrm{H}), 6.14(\mathrm{dd}, J=15.9,9.9 \mathrm{~Hz}, 1 \mathrm{H}), 4.25(\mathrm{dd}, J=36.9,14.0 \mathrm{~Hz}, 2 \mathrm{H}), 3.44(\mathrm{td}, J=10.4,3.3$ 
$\mathrm{Hz}, 1 \mathrm{H}), 2.22(\mathrm{dqd}, J=15.0,7.5,3.4 \mathrm{~Hz}, 1 \mathrm{H}), 1.81(\mathrm{ddq}, J=14.7,10.8,7.4 \mathrm{~Hz}, 1 \mathrm{H}), 0.96(\mathrm{t}, J=7.5$ $\mathrm{Hz}, 3 \mathrm{H})$.

${ }^{13} \mathrm{C}$ NMR $\left(101 \mathrm{MHz}, \mathrm{CDCl}_{3}\right) \delta 138.3,135.5,130.9,128.9,128.9,128.8,127.7,126.8,121.7,66.9$, $57.0,19.6,11.1$.

HRMS (ESI) calcd. for $\mathrm{C}_{18} \mathrm{H}_{20} \mathrm{NaO}_{2} \mathrm{~S}^{+}(\mathrm{M}+\mathrm{Na})^{+}: 323.1076$, Found: 323.1076

the ee value was $96 \%, \mathrm{t}_{\mathrm{r}}$ (major) $=20.454 \mathrm{~min}, \mathrm{t}_{\mathrm{r}}($ minor $)=22.248 \mathrm{~min}($ Chiralcel $\mathrm{IC}, \lambda=220 \mathrm{~nm}$, hexanes : ${ }^{i} \mathrm{PrOH}=80: 20$, flow rate $=1.0 \mathrm{~mL} / \mathrm{min}$ )

$\mathrm{mV}$
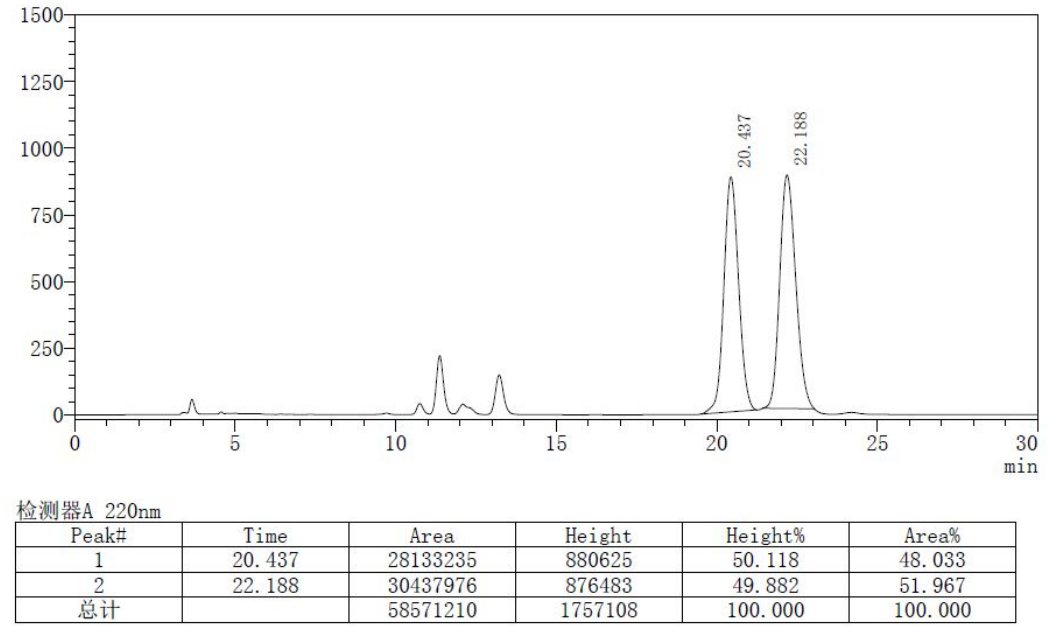

$\mathrm{mV}$

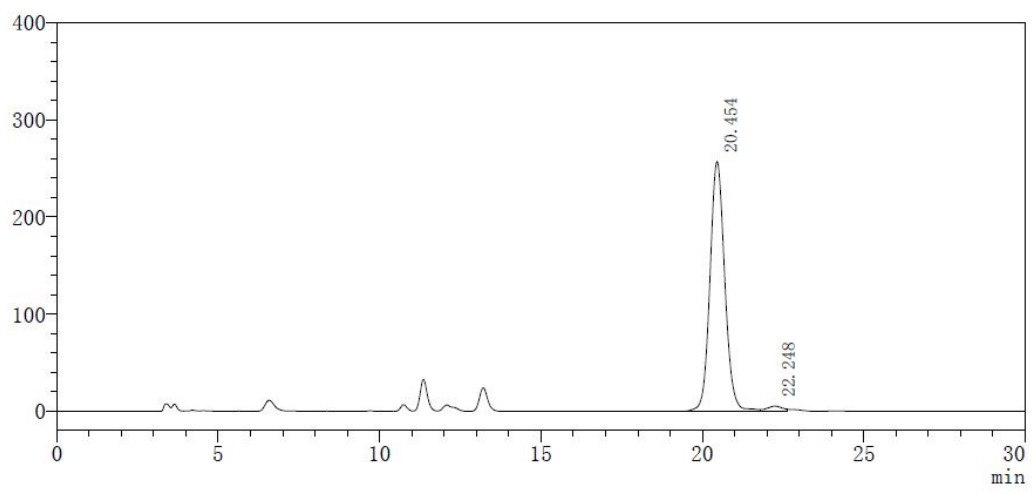

检测器A $220 \mathrm{~nm}$
\begin{tabular}{|c|c|c|c|c|c|}
\hline Peak\# & Time & Area & Height & Height\% & Area\% \\
\hline 1 & 20.454 & 8235048 & 257271 & 97.972 & 97.882 \\
\hline 2 & 22.248 & 178218 & 5325 & 2.028 & 2.118 \\
\hline 总计 & & 8413266 & 262596 & 100.000 & 100.000 \\
\hline
\end{tabular}<smiles>CC[C@H](/C=C/c1ccccc1)S(C)(=O)=O</smiles>

3an

\section{(S,E)-(3-(methylsulfonyl)pent-1-en-1-yl)benzene}

Prepared according to General Procedure but in MTBE $0.5 \mathrm{~mL}$ with $\mathrm{C}$ (sulfinic acid) $=0.4 \mathrm{M}$ for 
$72 \mathrm{~h}$, white solid, $20 \mathrm{mg}, 45 \%$ yield, m.p. $76-78^{\circ} \mathrm{C},>20: 1 \mathrm{rr}, 97 \%$ ee $[\alpha]^{25}=9.1\left(\mathrm{c} 1, \mathrm{CHCl}_{3}\right)$

${ }^{1} \mathrm{H}$ NMR (400 MHz, $\left.\mathrm{CDCl}_{3}\right) \delta 7.43(\mathrm{~d}, J=7.2 \mathrm{~Hz}, 2 \mathrm{H}), 7.33(\mathrm{dt}, J=20.5,7.0 \mathrm{~Hz}, 3 \mathrm{H}), 6.70(\mathrm{~d}, J=$ $15.9 \mathrm{~Hz}, 1 \mathrm{H}), 6.13(\mathrm{dd}, J=15.9,9.7 \mathrm{~Hz}, 1 \mathrm{H}), 3.53(\mathrm{td}, J=10.3,3.3 \mathrm{~Hz}, 1 \mathrm{H}), 2.86$ (s, 3H), 2.29 (dqd, $J$ $=15.0,7.5,3.5 \mathrm{~Hz}, 1 \mathrm{H}), 1.83(\mathrm{ddq}, J=14.6,10.8,7.4 \mathrm{~Hz}, 1 \mathrm{H}), 1.04(\mathrm{t}, J=7.5 \mathrm{~Hz}, 3 \mathrm{H})$.

${ }^{13} \mathrm{C}$ NMR $\left(101 \mathrm{MHz}, \mathrm{CDCl}_{3}\right) \delta 138.1,135.5,128.8,128.8,126.8,121.8,70.0,38.5,19.7,11.3$.

HRMS (ESI) calcd. for $\mathrm{C}_{12} \mathrm{H}_{16} \mathrm{NaO}_{2} \mathrm{~S}^{+}(\mathrm{M}+\mathrm{Na})^{+}$: 247.0763, Found: 247.0763

the ee value was $97 \%, \mathrm{t}_{\mathrm{r}}($ minor $)=20.798 \mathrm{~min}, \mathrm{t}_{\mathrm{r}}($ major $)=22.122 \mathrm{~min}($ Chiralcel $\mathrm{IC}, \lambda=220 \mathrm{~nm}$, hexanes : ${ }^{i} \mathrm{PrOH}=80: 20$, flow rate $=1.0 \mathrm{~mL} / \mathrm{min}$ )

$\mathrm{mV}$

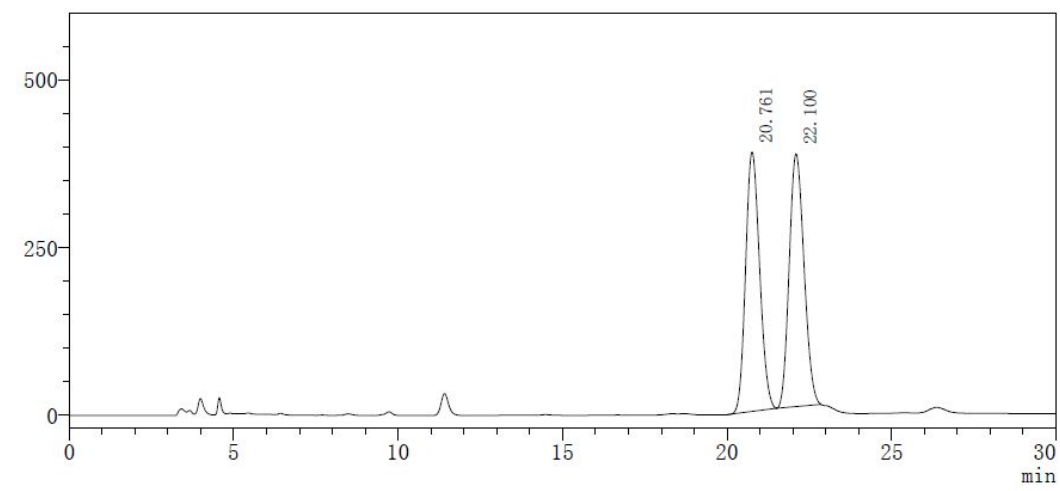

检测器A $220 \mathrm{~nm}$
\begin{tabular}{|c|c|c|c|c|c|}
\hline Peak\# & Time & Area & Height & Height $\%$ & Area $\%$ \\
\hline 1 & 20.761 & 11495969 & 387490 & 50.665 & 49.305 \\
\hline 2 & 22.100 & 11819947 & 377323 & 49.335 & 50.695 \\
\hline 总计 & & 23315916 & 764813 & 100.000 & 100.000 \\
\hline
\end{tabular}

$\mathrm{mV}$

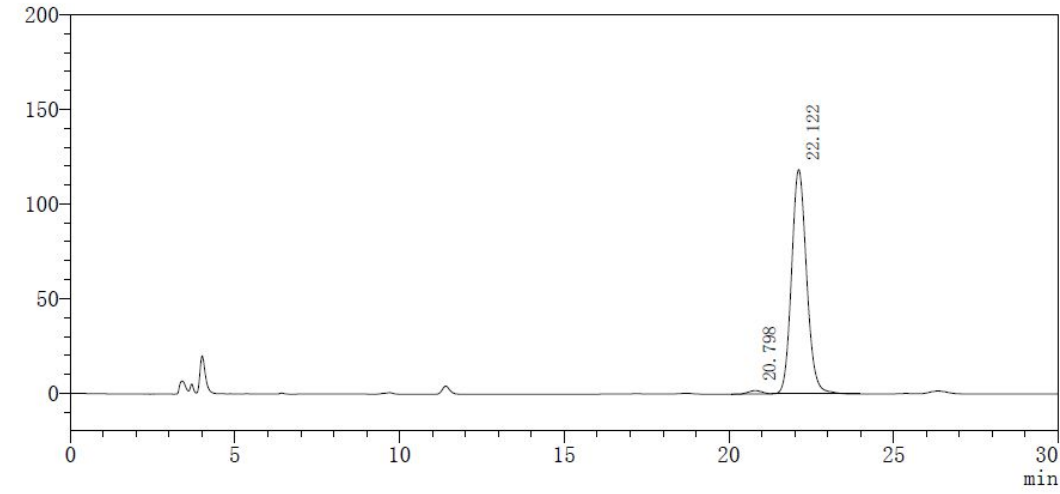

检测器A $220 \mathrm{~nm}$
\begin{tabular}{|c|c|c|c|c|c|}
\hline Peak\# & Time & Area & Height & Height $\%$ & Area $\%$ \\
\hline 1 & 20.798 & 55242 & 1885 & 1.564 & 1.439 \\
\hline 2 & 22.122 & 3784743 & 118654 & 98.436 & 98.561 \\
\hline 总计 & & 3839984 & 120539 & 100.000 & 100.000 \\
\hline
\end{tabular}


<smiles>CC[C@H](CCc1ccccc1)[Sb](=O)(=O)c1ccccc1</smiles>

\section{(S)-((1-phenylpentan-3-yl)sulfonyl)benzene}

colouless oil, $50 \mathrm{mg}, 87 \%$ yield, $96 \%$ ee

$[\alpha]^{25} \mathrm{D}=8.2\left(\mathrm{c} 1, \mathrm{CHCl}_{3}\right)$

${ }^{1} \mathrm{H}$ NMR $\left(400 \mathrm{MHz}, \mathrm{CDCl}_{3}\right) \delta 7.86(\mathrm{~d}, J=8.0 \mathrm{~Hz}, 2 \mathrm{H}), 7.64(\mathrm{t}, J=7.0 \mathrm{~Hz}, 1 \mathrm{H}), 7.55(\mathrm{t}, J=7.4 \mathrm{~Hz}$, 2H), 7.24 (d, $J=7.4 \mathrm{~Hz}, 2 \mathrm{H}), 7.18(\mathrm{t}, J=6.9 \mathrm{~Hz}, 1 \mathrm{H}), 7.09$ (d, $J=7.4 \mathrm{~Hz}, 2 \mathrm{H}), 2.93-2.84(\mathrm{~m}, 1 \mathrm{H})$, $2.84-2.73(\mathrm{~m}, 1 \mathrm{H}), 2.72-2.60(\mathrm{~m}, 1 \mathrm{H}), 2.23-2.09(\mathrm{~m}, 1 \mathrm{H}), 1.98-1.84(\mathrm{~m}, 2 \mathrm{H}), 1.76-1.64(\mathrm{~m}$, $1 \mathrm{H}), 0.99(\mathrm{t}, J=7.3 \mathrm{~Hz}, 3 \mathrm{H})$.

${ }^{13} \mathrm{C}$ NMR $\left(101 \mathrm{MHz}, \mathrm{CDCl}_{3}\right) \delta 140.6,138.2,133.6,129.1,128.8,128.6,128.4,126.3,64.7,32.8$, 28.80, 21.0, 11.1.

HRMS (ESI) calcd. for $\mathrm{C}_{17} \mathrm{H}_{21} \mathrm{O}_{2} \mathrm{~S}^{+}(\mathrm{M}+\mathrm{H})^{+}:$289.1257, Found: 289.1252

the ee value was $96 \%, \mathrm{t}_{\mathrm{r}}($ minor $)=15.135 \mathrm{~min}, \mathrm{t}_{\mathrm{r}}($ major $)=15.925 \min ($ Chiralcel IC, $\lambda=220 \mathrm{~nm}$, hexanes : ${ }^{i} \mathrm{PrOH}=80: 20$, flow rate $=1.0 \mathrm{~mL} / \mathrm{min}$ )

$\mathrm{mV}$

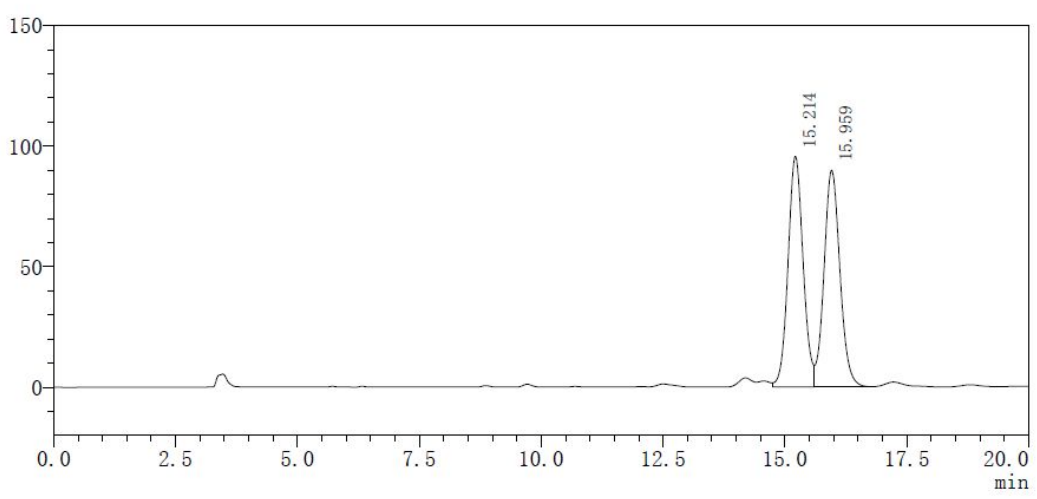

检测器 $\mathrm{A} 220 \mathrm{~nm}$

检测器A $220 \mathrm{~nm}$
\begin{tabular}{|c|c|c|c|c|c|}
\hline Peak\# & Time & Area & Height & Height\% & Area\% \\
\hline 1 & 15.214 & 2059684 & 95621 & 51.544 & 50.353 \\
\hline 2 & 15.959 & 2030791 & 89893 & 48.456 & 49.647 \\
\hline 总计 & & 4090475 & 185514 & 100.000 & 100.000 \\
\hline
\end{tabular}

$\mathrm{mV}$
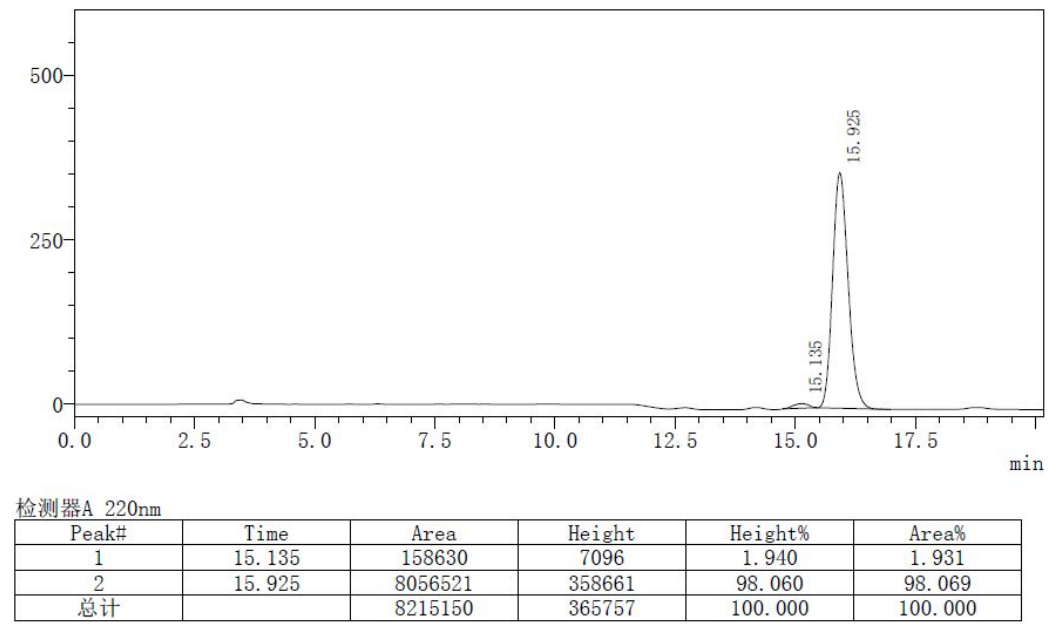
<smiles>CCC(CO)[Sb](=O)(=O)c1ccccc1</smiles>

5 aa

\section{(S)-2-(phenylsulfonyl)butan-1-ol}

colorless oil, 36mg, $84 \%$ yield, $96 \%$ ee

$[\alpha]^{25} \mathrm{D}=-0.8\left(\mathrm{c} 1, \mathrm{CHCl}_{3}\right)$

${ }^{1} \mathrm{H}$ NMR $\left(400 \mathrm{MHz}, \mathrm{CDCl}_{3}\right) \delta 7.91(\mathrm{~d}, J=7.8 \mathrm{~Hz}, 2 \mathrm{H}), 7.69(\mathrm{t}, J=7.4 \mathrm{~Hz}, 1 \mathrm{H}), 7.60$ (t, $J=7.5 \mathrm{~Hz}$, $2 \mathrm{H}), 4.00-3.88(\mathrm{~m}, 2 \mathrm{H}), 3.06-3.00(\mathrm{~m}, 1 \mathrm{H}), 2.93(\mathrm{~s}, 1 \mathrm{H}), 1.90-1.77(\mathrm{~m}, 1 \mathrm{H}), 1.63(\mathrm{qd}, J=14.8,7.5$ $\mathrm{Hz}, 1 \mathrm{H}), 1.00(\mathrm{t}, J=7.4 \mathrm{~Hz}, 3 \mathrm{H})$.

${ }^{13} \mathrm{C}$ NMR (101 MHz, $\left.\mathrm{CDCl}_{3}\right) \delta$ 137.4, 134.0, 129.3, 128.8, 67.6, 58.8, 18.3, 11.2.

HRMS (ESI) calcd. for $\mathrm{C}_{10} \mathrm{H}_{15} \mathrm{O}_{3} \mathrm{~S}^{+}(\mathrm{M}+\mathrm{H})^{+}:$215.0736, Found: 215.0732

the ee value was $96 \%, \mathrm{t}_{\mathrm{r}}$ (major) $=17.430 \mathrm{~min}, \mathrm{t}_{\mathrm{r}}($ minor $)=20.468 \min ($ Chiralcel IC, $\lambda=220 \mathrm{~nm}$, hexanes : ${ }^{i} \mathrm{PrOH}=70: 30$, flow rate $\left.=1.0 \mathrm{~mL} / \mathrm{min}\right)$

$\mathrm{m} V$
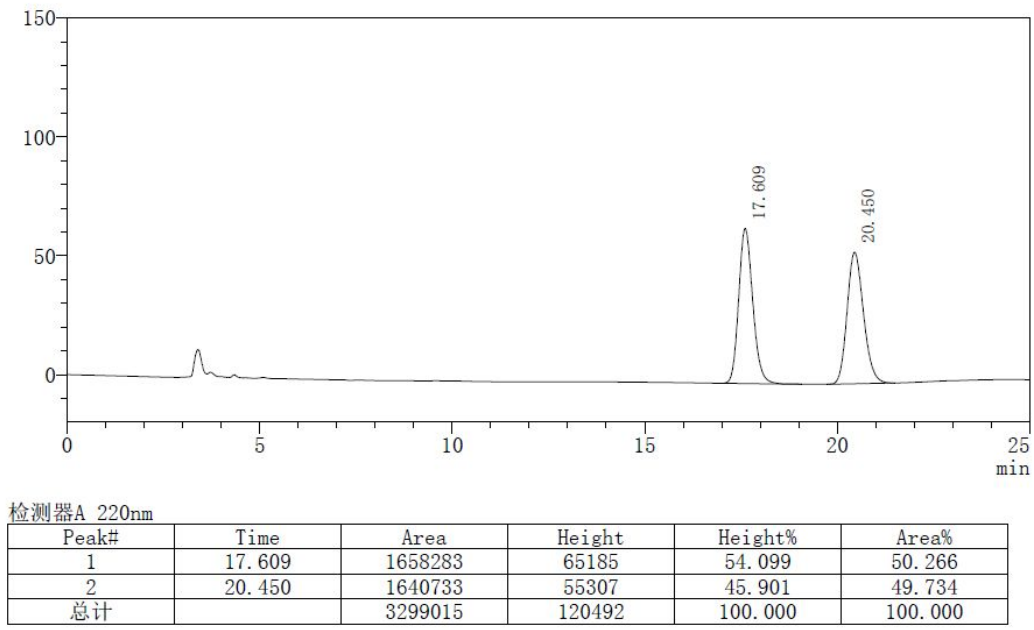

$\mathrm{mV}$

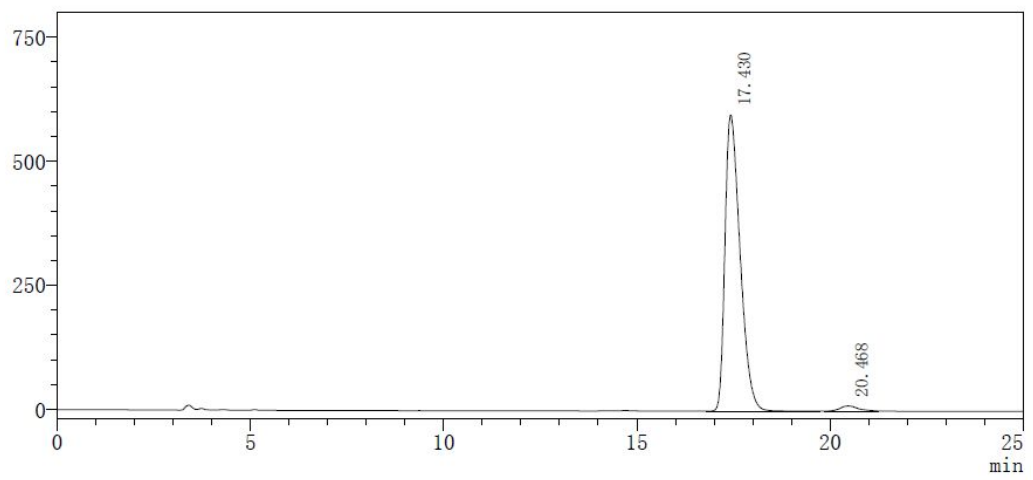

\begin{tabular}{|c|c|c|c|c|c|}
\hline & & & & & \\
\hline Peak\# & $\begin{array}{c}\text { Time } \\
\end{array}$ & $\begin{array}{c}\text { Area } \\
1620746\end{array}$ & $\begin{array}{l}\text { Height } \\
596507\end{array}$ & $\frac{\text { Height } \%}{98.266}$ & $\begin{array}{l}\text { Area\% } \\
97.989\end{array}$ \\
\hline 2 & 20.468 & 333814 & 10525 & 1.734 & 2.011 \\
\hline 总计 & & 16596559 & 607033 & 100.000 & 100.000 \\
\hline
\end{tabular}


<smiles>CC[C@@H](c1ccccc1)[C@@H]1OC1c1ccccc1</smiles>

\section{(2R,3S)-2-phenyl-3-((S)-1-(phenylsulfonyl)propyl)oxirane}

colorless oil, $34 \mathrm{mg}, 56 \%$ yield, $>20: 1 \mathrm{dr}$

$[\alpha]^{25}=-26.8\left(\mathrm{c} 0.5, \mathrm{CHCl}_{3}\right)$

${ }^{1} \mathrm{H}$ NMR $\left(400 \mathrm{MHz}, \mathrm{CDCl}_{3}\right) \delta 8.00(\mathrm{t}, J=18.9 \mathrm{~Hz}, 2 \mathrm{H}), 7.68(\mathrm{t}, J=7.4 \mathrm{~Hz}, 1 \mathrm{H}), 7.58(\mathrm{t}, J=7.7 \mathrm{~Hz}$, 2H), 7.30 (dd, $J=18.4,5.9 \mathrm{~Hz}, 3 \mathrm{H}), 7.17$ (dd, $J=13.4,8.0 \mathrm{~Hz}, 2 \mathrm{H}), 3.57$ (d, $J=0.7 \mathrm{~Hz}, 1 \mathrm{H}), 3.15$ (dd, $J=8.3,1.3 \mathrm{~Hz}, 1 \mathrm{H}), 2.88(\mathrm{td}, J=9.2,4.4 \mathrm{~Hz}, 1 \mathrm{H}), 2.29-2.14(\mathrm{~m}, 1 \mathrm{H}), 1.89-1.73(\mathrm{~m}, 1 \mathrm{H}), 1.12(\mathrm{t}, J$ $=7.5 \mathrm{~Hz}, 3 \mathrm{H})$.

${ }^{13} \mathrm{C}$ NMR $\left(101 \mathrm{MHz}, \mathrm{CDCl}_{3}\right) \delta 138.8,135.5,133.9,129.1,129.0,128.7,128.7,125.5,68.9,59.3,57.0$, 18.6, 11.8.

HRMS (ESI) calcd. for $\mathrm{C}_{17} \mathrm{H}_{19} \mathrm{O}_{3} \mathrm{~S}^{+}(\mathrm{M}+\mathrm{H})^{+}$: 303.1049, Found: 303.1044 


\section{General Information for Computation}

All calculations were performed with the Gaussian 16 program. (Full citation of Gaussian 16: M. J. Frisch, G. W. Trucks, H. B. Schlegel, G. E. Scuseria, M. A. Robb, J. R. Cheeseman, G. Scalmani, V. Barone, G. A. Petersson, H. Nakatsuji, X. Li, M. Caricato, A. V. Marenich, J. Bloino, B. G. Janesko, R. Gomperts, B. Mennucci, H. P. Hratchian, J. V. Ortiz, A. F. Izmaylov, J. L. Sonnenberg, D. Williams-Young, F. Ding, F. Lipparini, F. Egidi, J. Goings, B. Peng, A. Petrone, T. Henderson, D. Ranasinghe, V. G. Zakrzewski, J. Gao, N. Rega, G. Zheng, W. Liang, M. Hada, M. Ehara, K. Toyota, R. Fukuda, J. Hasegawa, M. Ishida, T. Nakajima, Y. Honda, O. Kitao, H. Nakai, T. Vreven, K. Throssell, J. A. Montgomery, Jr., J. E. Peralta, F. Ogliaro, M. J. Bearpark, J. J. Heyd, E. N. Brothers, K. N. Kudin, V. N. Staroverov, T. A. Keith, R. Kobayashi, J. Normand, K. Raghavachari, A. P. Rendell, J. C. Burant, S. S. Iyengar, J. Tomasi, M. Cossi, J. M. Millam, M. Klene, C. Adamo, R. Cammi, J. W. Ochterski, R. L. Martin, K. Morokuma, O. Farkas, J. B. Foresman, and D. J. Fox, Gaussian, Inc., Wallingford CT, 2016. Gaussian 16, Revision A.03). Density functional theory calculations using the M06 functional ((a) Zhao, Y.; Truhlar, D. G. Theor. Chem. Acc. 2008, 120, 215-241. (b) Zhao, Y.; Truhlar, D. G. Acc. Chem. Res. 2008, 41, 157-167.) Geometry optimizations were conducted with the Gaussian 16 software package, B3LYP functional (with gd3(BJ) dispersion correction), and LANL2DZ basis set for Pd and 6-31g(d) basis set for all other atoms. Single-point energy calculations were conducted with the M06 functional (with gd3 dispersion correction) and SDD basis set for Pd and the 6-311++g(d,p) basis set for all other atoms, along with the SMD 1,4-dioxane solvent correction. Concentration was corrected from 1 atm to $1 \mathrm{~mol} / \mathrm{L}$ by addition $1.89 \mathrm{kcal} / \mathrm{mol}$ to the Gibbs energy of each species. The calculated structures were displayed with the CYLview software (CYLview, b. L., C. Y.; Université de Sherbrooke. 2009.)

Different (E,Z)-1a-Pd complexes were considered and among them complex $\mathbf{4}$ has the lowest free energy. Similarly, complex $\mathbf{4 b}$ has the lowest free energy for $(E, E)-\mathbf{1} \mathbf{a}-\mathrm{Pd}$ complex. The structures and energies of these complexes were summarized in Figure S7. Energy profiles for pathways involving Pd-hydride migratory insertion was summarized in Figure S8. Energy profile for hydrosulfonylation of (E,Z)-1,3-diene 1a with sulfonic acid 2a was summarized in Figure S9. Energy profile for hydrosulfonylation of (E,E)-1,3-diene 1a with sulfonic acid 2a was summarized in Figure S10. 
From $(E, Z)-1 \mathbf{a}$

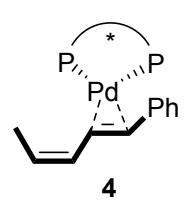

$0.0 \mathrm{kcal} / \mathrm{mol}$

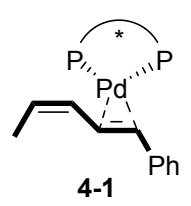

$7.2 \mathrm{kcal} / \mathrm{mol}$

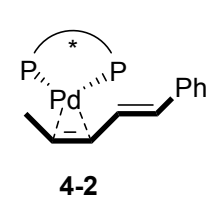

$5.7 \mathrm{kcal} / \mathrm{mol}$

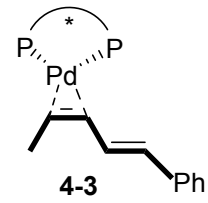

$8.4 \mathrm{kcal} / \mathrm{mol}$

From $(E, E)-\mathbf{1 a}$

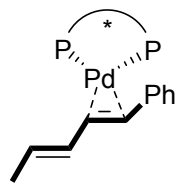

$4 b$

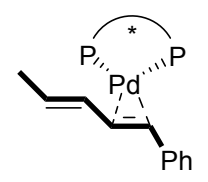

4b-1

$11.8 \mathrm{kcal} / \mathrm{mol}$

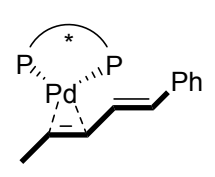

4b-2

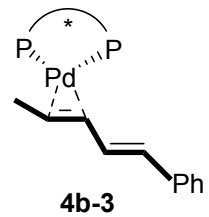

$9.9 \mathrm{kcal} / \mathrm{mol}$

Figure S7

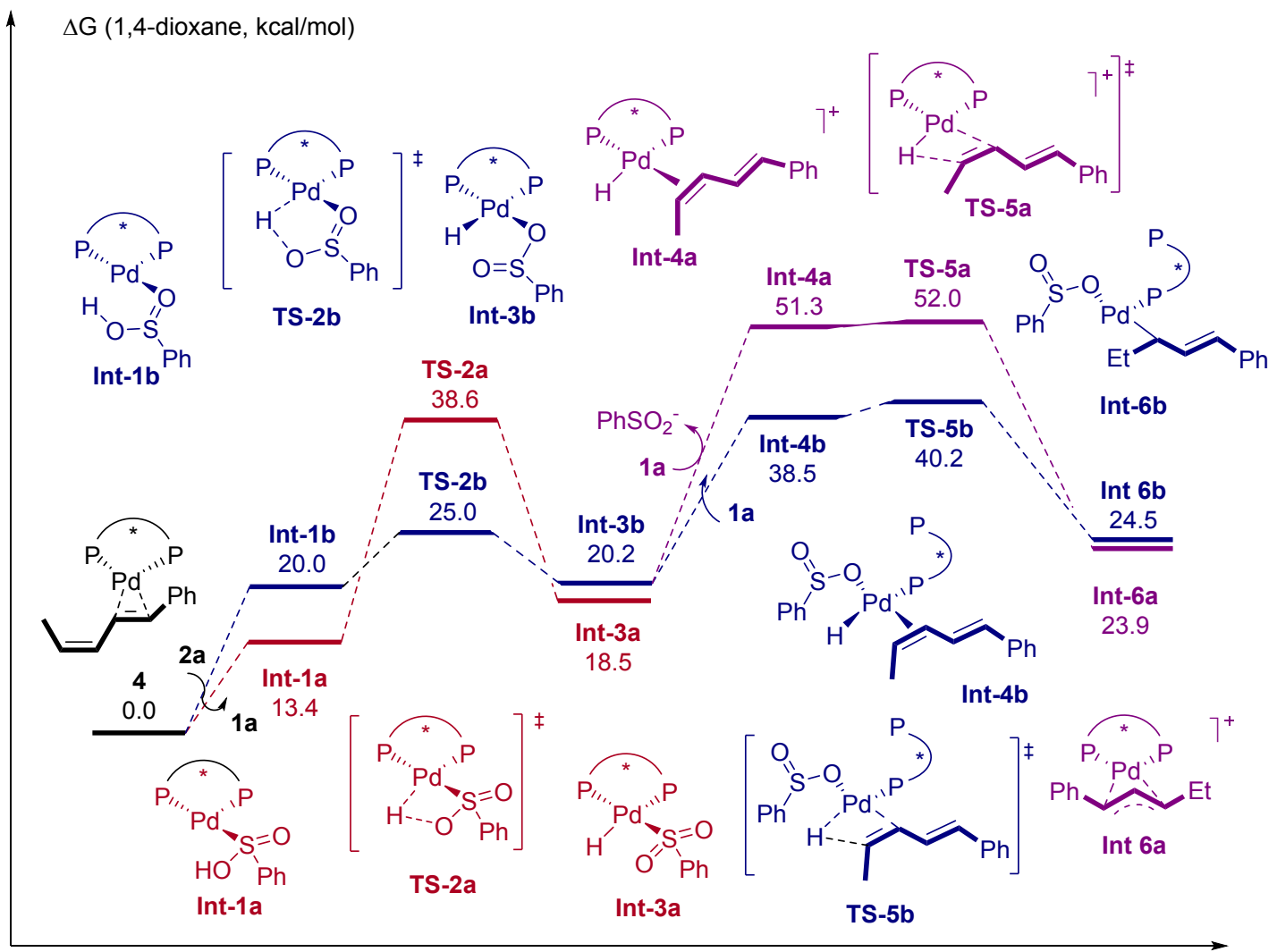

Figure S8. Energy profiles for pathways involving Pd-hydride migratory insertion. 


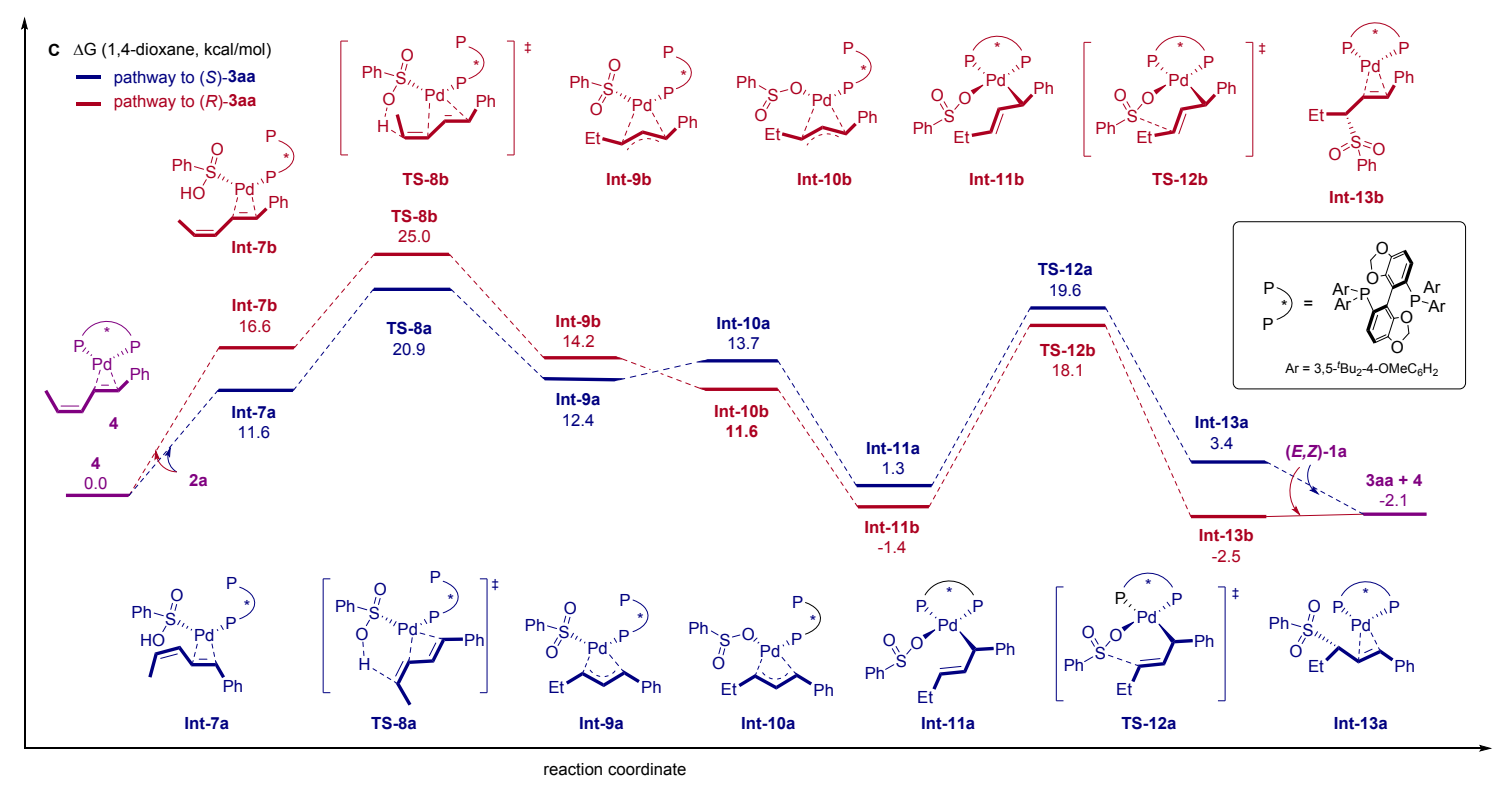

Figure S9. Energy profile for hydrosulfonylation of (E,Z)-1,3-diene 1a with sulfonic acid $\mathbf{2 a}$.

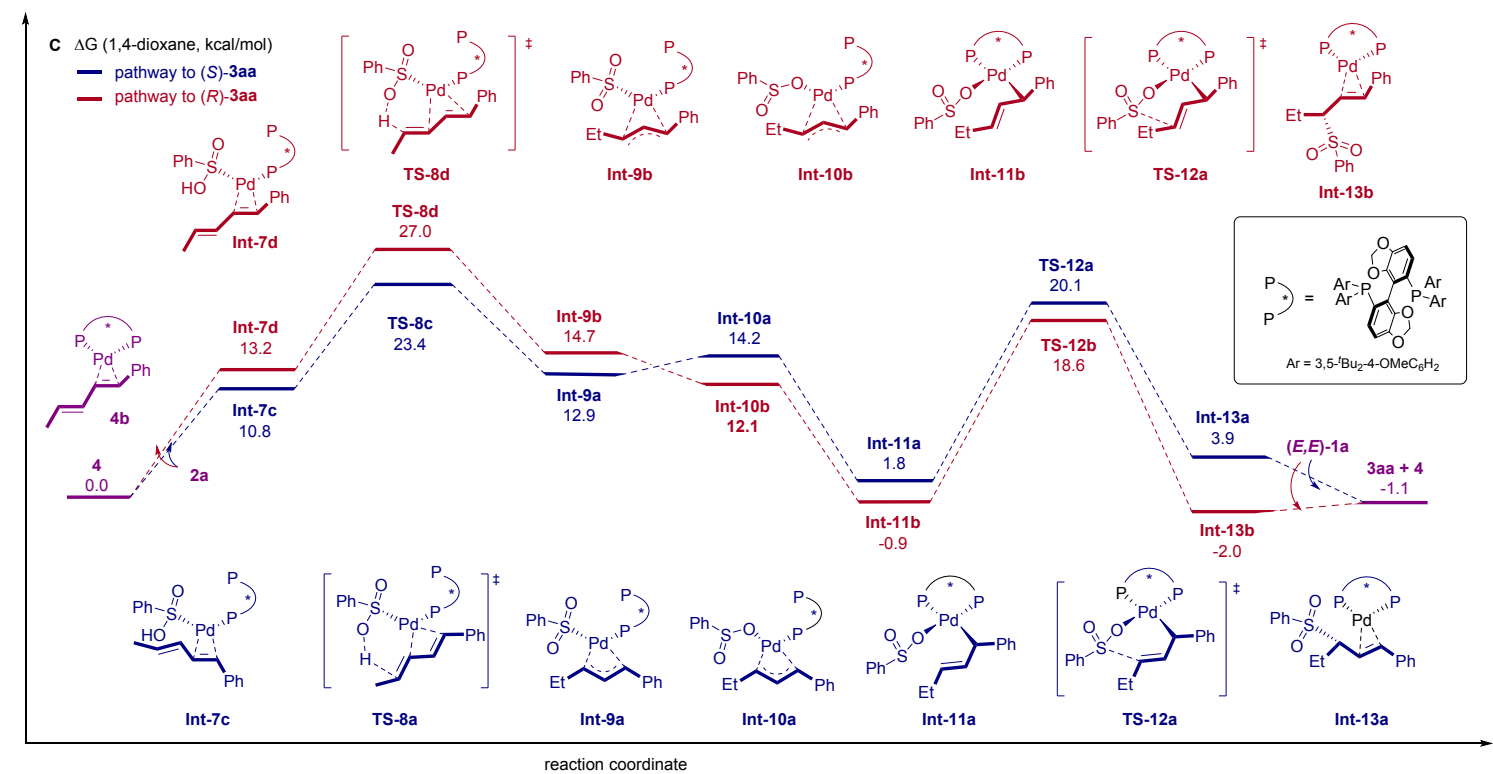

Figure S10. Energy profile for hydrosulfonylation of $(E, E)$-1,3-diene 1a with sulfonic acid 2a.

\section{Coordinates and Energies of Stationary Points}<smiles>C/C=C\C=C\c1ccccc1</smiles>

$(E, Z)-1 \mathbf{a}$

Total SCF energy (M06/SDD-6-311++G(d,p)/SMD(1,4-Dioxane)): -426.132866a.u.

Thermal correction to Gibbs Free Energy at 298.15 K: 0.158529a.u 
Gibbs free energy at 298.15 K (M06/SDD-6-311++G(d,p)/SMD(1,4-Dioxane)): -425.974337a.u.

$\begin{array}{lrrr}\mathrm{C} & 3.44650400 & -0.74761900 & -0.00000900 \\ \mathrm{C} & 2.13925500 & -1.22788300 & -0.00000500 \\ \mathrm{C} & 1.03853100 & -0.35198000 & 0.00000300 \\ \mathrm{C} & 1.30039200 & 1.03190400 & 0.00000800 \\ \mathrm{C} & 2.60499700 & 1.51216000 & 0.00000500 \\ \mathrm{C} & 3.68674600 & 0.62640800 & -0.00000400 \\ \mathrm{H} & 4.27787900 & -1.44716900 & -0.00001500 \\ \mathrm{H} & 1.95786700 & -2.29998400 & -0.00000900 \\ \mathrm{H} & 0.47548400 & 1.73770100 & 0.00001600 \\ \mathrm{H} & 2.78173100 & 2.58434000 & 0.00000900 \\ \mathrm{H} & 4.70456300 & 1.00565900 & -0.00000600 \\ \mathrm{C} & -0.31086200 & -0.91407900 & 0.00000600 \\ \mathrm{C} & -1.48146100 & -0.24046600 & 0.00000300 \\ \mathrm{H} & -0.35434200 & -2.00367300 & 0.00000900 \\ \mathrm{H} & -1.47322900 & 0.84657300 & -0.00000400 \\ \mathrm{C} & -2.77124500 & -0.89924600 & 0.00000600 \\ \mathrm{C} & -3.98217300 & -0.30768100 & 0.00000100 \\ \mathrm{H} & -2.73890800 & -1.98876100 & 0.00001100 \\ \mathrm{H} & -4.85695600 & -0.95690300 & 0.00000000 \\ \mathrm{C} & -4.27858600 & 1.16238500 & -0.00001000 \\ \mathrm{H} & -4.87489700 & 1.43939300 & 0.87998600 \\ \mathrm{H} & -3.37693100 & 1.78001700 & 0.00001700 \\ \mathrm{H} & -4.87484400 & 1.43939200 & -0.88004200\end{array}$

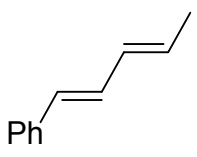

$(E, E)-1 \mathbf{a}$

Total SCF energy (M06/SDD-6-311++G(d,p)/SMD(1,4-Dioxane)): -426.134493a.u.

Thermal correction to Gibbs Free Energy at 298.15 K: 0.158605a.u

Gibbs free energy at 298.15 K (M06/SDD-6-311++G(d,p)/SMD(1,4-Dioxane)): -425.975888a.u.

$\mathrm{C}$

$3.47103900 \quad-0.95574000 \quad 0.00000100$

$\mathrm{C}$

$2.11482100 \quad-1.27241500 \quad 0.00000000$

$\mathrm{C}$

$\begin{array}{lll}1.12951600 & -0.26845800 & 0.00000000\end{array}$

$\mathrm{C}$

$\begin{array}{lll}1.55878500 & 1.07294100 & -0.00000100\end{array}$

$\mathrm{C}$

$\begin{array}{lll}2.91241800 & 1.38988300 & 0.00000000\end{array}$

$\mathrm{C}$

3.87772700

$0.37852700 \quad 0.00000100$

$\mathrm{H}$

4.21051500

$-1.75183800$

0.00000200

$\mathrm{H}$

1.80368800

$-2.31430900$

0.00000000

$\mathrm{H}$

0.82633100

1.87425700

$-0.00000100$

$\mathrm{H}$

3.21893700

2.43242000

0.00000000

$\mathrm{H}$

4.93431100

0.63037100

0.00000100

C

$-0.27871200$

$-0.66080200$

$-0.00000100$ 


$\begin{array}{lccc}\mathrm{C} & -1.35737000 & 0.15109500 & -0.00000100 \\ \mathrm{H} & -0.45610100 & -1.73663800 & -0.00000200 \\ \mathrm{H} & -1.22675500 & 1.23258100 & 0.00000100 \\ \mathrm{C} & -2.72391200 & -0.32605200 & -0.00000100 \\ \mathrm{C} & -3.80634200 & 0.47214800 & 0.00000000 \\ \mathrm{H} & -2.86405000 & -1.40818100 & -0.00000300 \\ \mathrm{H} & -3.65672600 & 1.55292300 & 0.00000000 \\ \mathrm{C} & -5.22578200 & -0.00469000 & 0.00000200 \\ \mathrm{H} & -5.77067500 & 0.36403100 & 0.88010000 \\ \mathrm{H} & -5.77069300 & 0.36407500 & -0.88006700 \\ \mathrm{H} & -5.28191000 & -1.09831800 & -0.00002500 \\ & & & \\ \mathrm{PhSO}_{2} \mathrm{H} & & & \\ \mathbf{2 a} & & & \end{array}$

Total SCF energy (M06/SDD-6-311++G(d,p)/SMD(1,4-Dioxane)): -780.687396a.u.

Thermal correction to Gibbs Free Energy at 298.15 K: 0.076262a.u

Gibbs free energy at 298.15 K (M06/SDD-6-311++G(d,p)/SMD(1,4-Dioxane)): -780.611134a.u.

$\begin{array}{lrrc}\mathrm{S} & -1.68846800 & -0.03231800 & -0.41604000 \\ \mathrm{O} & -2.17519100 & 1.32135300 & -0.02500300 \\ \mathrm{O} & -1.93461800 & -1.15829400 & 0.83744400 \\ \mathrm{H} & -2.84656500 & -1.48515500 & 0.72633700 \\ \mathrm{C} & 0.10014800 & -0.02641700 & -0.15366300 \\ \mathrm{C} & 0.83358300 & -1.20514000 & -0.29390900 \\ \mathrm{C} & 0.71745800 & 1.19326500 & 0.10779000 \\ \mathrm{C} & 2.21894800 & -1.15691800 & -0.15178500 \\ \mathrm{H} & 0.32793000 & -2.14572700 & -0.48894600 \\ \mathrm{C} & 2.10498800 & 1.23002000 & 0.25059400 \\ \mathrm{H} & 0.10558400 & 2.08417300 & 0.20669600 \\ \mathrm{C} & 2.85291000 & 0.05887200 & 0.11947100 \\ \mathrm{H} & 2.80409600 & -2.06676600 & -0.24846000 \\ \mathrm{H} & 2.60139000 & 2.17225600 & 0.46364800 \\ \mathrm{H} & 3.93331600 & 0.09174300 & 0.22683900\end{array}$

$\mathrm{PhSO}_{2}^{-}$

Total SCF energy (M06/SDD-6-311++G(d,p)/SMD(1,4-Dioxane)): -780.188371a.u.

Thermal correction to Gibbs Free Energy at 298.15 K: 0.064031a.u

Gibbs free energy at 298.15 K (M06/SDD-6-311++G(d,p)/SMD(1,4-Dioxane)): -780.124340a.u.

$\begin{array}{cccc}\mathrm{C} & 2.17559700 & 1.20911600 & 0.03431800 \\ \mathrm{C} & 0.78163900 & 1.20582800 & -0.05185000 \\ \mathrm{C} & 0.08206800 & 0.00000200 & -0.10015200 \\ \mathrm{C} & 0.78163400 & -1.20582800 & -0.05184600 \\ \mathrm{C} & 2.17559200 & -1.20912100 & 0.03431900 \\ \mathrm{C} & 2.87716600 & -0.00000400 & 0.07303200 \\ \mathrm{H} & 2.72028200 & 2.15189800 & 0.08125800\end{array}$




$\begin{array}{cccc}\mathrm{H} & 0.20626400 & 2.12866900 & -0.05777000 \\ \mathrm{H} & 0.20625500 & -2.12866500 & -0.05775400 \\ \mathrm{H} & 2.72027200 & -2.15190500 & 0.08125900 \\ \mathrm{H} & 3.96396200 & -0.00000600 & 0.13906200 \\ \mathrm{~S} & -1.77496100 & 0.00000300 & -0.34908500 \\ \mathrm{O} & -2.16623300 & 1.29370200 & 0.36077900 \\ \mathrm{O} & -2.16624800 & -1.29370200 & 0.36076900\end{array}$

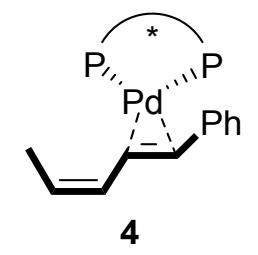

Total SCF energy (M06/SDD-6-311++G(d,p)/SMD(1,4-Dioxane)): -4717.155320a.u.

Thermal correction to Gibbs Free Energy at 298.15 K: 1.662765a.u

Gibbs free energy at 298.15 K (M06/SDD-6-311++G(d,p)/SMD(1,4-Dioxane)): -4715.492555a.u.

$\begin{array}{cccc}\mathrm{Pd} & 0.03701800 & -0.35000700 & -1.51832500 \\ \mathrm{P} & -1.76487100 & -0.24639900 & -0.03316500 \\ \mathrm{P} & 1.60619700 & 0.49656100 & -0.01381400 \\ \mathrm{O} & -6.05093600 & -3.98995500 & -1.85102400 \\ \mathrm{O} & -3.75753800 & 5.37953500 & 0.26453600 \\ \mathrm{O} & 3.29086100 & -3.48297100 & 4.13012500 \\ \mathrm{O} & 6.36731900 & 1.74521900 & -3.35444100 \\ \mathrm{O} & -0.18662300 & -1.26699300 & 5.64653600 \\ \mathrm{O} & 0.54147200 & 0.68333400 & 4.66690400 \\ \mathrm{O} & -1.36022000 & 2.82697200 & 3.72330200 \\ \mathrm{O} & -0.59941300 & 4.95551400 & 3.30199800 \\ \mathrm{C} & -3.13628700 & -1.40325400 & -0.39243800 \\ \mathrm{C} & -4.49026400 & -1.09124400 & -0.25733900 \\ \mathrm{H} & -4.75980200 & -0.13233600 & 0.15949800 \\ \mathrm{C} & -5.48938300 & -1.96824300 & -0.68767100 \\ \mathrm{C} & -5.07676200 & -3.19479400 & -1.27018300 \\ \mathrm{C} & -3.72770400 & -3.61416400 & -1.26613900 \\ \mathrm{C} & -2.77930700 & -2.66081200 & -0.87400200 \\ \mathrm{H} & -1.72144300 & -2.88663900 & -0.96410200 \\ \mathrm{C} & -6.97580100 & -1.62244500 & -0.45443900 \\ \mathrm{C} & -7.12790900 & -0.30056000 & 0.32512700 \\ \mathrm{H} & -6.61672300 & -0.33481100 & 1.29314100 \\ \mathrm{H} & -8.19041700 & -0.11956500 & 0.51859400 \\ \mathrm{H} & -6.74313600 & 0.55809300 & -0.23641900 \\ \mathrm{C} & -7.75702200 & -1.45924200 & -1.77641400 \\ \mathrm{H} & -7.24068500 & -0.77242700 & -2.45690100 \\ \mathrm{H} & -8.74970900 & -1.04227800 & -1.56911200 \\ \mathrm{H} & -7.90034200 & -2.41417500 & -2.28099700 \\ \mathrm{C} & -7.62516600 & -2.73832500 & 0.39550100\end{array}$




\begin{tabular}{|c|c|c|c|}
\hline $\mathrm{H}$ & -7.57090200 & -3.70284300 & -0.11232400 \\
\hline $\mathrm{H}$ & -8.67991700 & -2.50194800 & 0.58201400 \\
\hline $\mathrm{H}$ & -7.12170100 & -2.82556300 & 1.36516600 \\
\hline $\mathrm{C}$ & -3.19372700 & -5.01859400 & -1.63952400 \\
\hline $\mathrm{C}$ & -2.42726200 & -4.94987600 & -2.97863300 \\
\hline $\mathrm{H}$ & -3.07488400 & -4.63738200 & -3.80406200 \\
\hline $\mathrm{H}$ & -2.01022300 & -5.93254800 & -3.22936600 \\
\hline $\mathrm{H}$ & -1.59719300 & -4.23793900 & -2.91914200 \\
\hline $\mathrm{C}$ & -2.19601200 & -5.46348800 & -0.53903700 \\
\hline $\mathrm{H}$ & -1.32095000 & -4.81397000 & -0.47322400 \\
\hline $\mathrm{H}$ & -1.83335500 & -6.47429700 & -0.75697900 \\
\hline $\mathrm{H}$ & -2.67934900 & -5.47934200 & 0.44405900 \\
\hline $\mathrm{C}$ & -4.26181800 & -6.12949100 & -1.71113500 \\
\hline $\mathrm{H}$ & -4.88822500 & -6.14061200 & -0.81500300 \\
\hline $\mathrm{H}$ & -3.75003500 & -7.09643800 & -1.78115600 \\
\hline $\mathrm{H}$ & -4.91730000 & -6.04687900 & -2.57723700 \\
\hline $\mathrm{C}$ & -6.07816900 & -3.89813300 & -3.27561400 \\
\hline $\mathrm{H}$ & -5.15923400 & -4.28926300 & -3.72314700 \\
\hline $\mathrm{H}$ & -6.21081900 & -2.86157900 & -3.60260200 \\
\hline $\mathrm{H}$ & -6.92732200 & -4.50071900 & -3.60679800 \\
\hline $\mathrm{C}$ & -2.51100800 & 1.41767100 & 0.04452700 \\
\hline $\mathrm{C}$ & -2.17972600 & 2.30996400 & -0.96814300 \\
\hline $\mathrm{H}$ & -1.52707600 & 1.95259100 & -1.75687300 \\
\hline $\mathrm{C}$ & -2.63546800 & 3.63601800 & -0.97730600 \\
\hline $\mathrm{C}$ & -3.41151600 & 4.04629300 & 0.12527500 \\
\hline $\mathrm{C}$ & -3.87154900 & 3.13508800 & 1.11453800 \\
\hline $\mathrm{C}$ & -3.37594100 & 1.83143100 & 1.06302300 \\
\hline $\mathrm{H}$ & -3.64110200 & 1.12076000 & 1.83390600 \\
\hline $\mathrm{C}$ & -4.98259700 & 3.50149800 & 2.12265800 \\
\hline $\mathrm{C}$ & -4.66933500 & 4.74136800 & 2.98557200 \\
\hline $\mathrm{H}$ & -3.68859600 & 4.64643500 & 3.45788200 \\
\hline $\mathrm{H}$ & -5.41815500 & 4.83182200 & 3.78119600 \\
\hline $\mathrm{H}$ & -4.68950600 & 5.65883900 & 2.40013400 \\
\hline $\mathrm{C}$ & -6.27124900 & 3.77389900 & 1.31274500 \\
\hline $\mathrm{H}$ & -6.12668600 & 4.61105300 & 0.62468100 \\
\hline $\mathrm{H}$ & -7.09971300 & 4.02025900 & 1.98852000 \\
\hline $\mathrm{H}$ & -6.55780000 & 2.89071600 & 0.73104800 \\
\hline $\mathrm{C}$ & -5.25886900 & 2.33953900 & 3.09687600 \\
\hline $\mathrm{H}$ & -5.59354400 & 1.43503700 & 2.57926500 \\
\hline $\mathrm{H}$ & -6.05584200 & 2.63204200 & 3.78870000 \\
\hline $\mathrm{H}$ & -4.36974100 & 2.09224000 & 3.68814700 \\
\hline $\mathrm{C}$ & -2.24600700 & 4.48421000 & -2.21210700 \\
\hline $\mathrm{C}$ & -0.71650400 & 4.70219300 & -2.20669300 \\
\hline $\mathrm{H}$ & -0.39661900 & 5.26137400 & -1.32074600 \\
\hline
\end{tabular}


H

$\mathrm{H}$

C

$\mathrm{H}$

$\mathrm{H}$

$\mathrm{H}$

C

$\mathrm{H}$

$\mathrm{H}$

$\mathrm{H}$

C

$\mathrm{H}$

$\mathrm{H}$

$\mathrm{H}$

C

C

$\mathrm{H}$

C

C

C

C

$\mathrm{H}$

C

C

$\mathrm{H}$

C

C

C

C

$\mathrm{H}$

C

C

$\mathrm{H}$

$\mathrm{H}$

$\mathrm{H}$

C

$\mathrm{H}$

$\mathrm{H}$

$\mathrm{H}$

C

$\mathrm{H}$

$\mathrm{H}$

$\mathrm{H}$

C

\begin{tabular}{|c|c|c|}
\hline & & \\
\hline 0.17890700 & 3.74883700 & -2.20 \\
\hline 63178500 & 3.68267000 & -3.48127900 \\
\hline 1200 & 2.70070300 & 14200 \\
\hline 5100 & 4.24108600 & -4.3754410 \\
\hline 3400 & 5800 & 9440 \\
\hline .95 & & \\
\hline 00 & & \\
\hline .72888200 & 24364600 & \\
\hline 2900 & 300 & -1.6 \\
\hline-2.70 & & \\
\hline-2.45789100 & & \\
\hline-3.076 & 6100 & \\
\hline .80 & 69800 & \\
\hline 2.17237100 & 200 & \\
\hline 2.908 & & \\
\hline 3.143 & 6200 & \\
\hline 3.33287600 & -1.23236400 & \\
\hline 2.93 & & \\
\hline 2.26345000 & -3.02079 & 530 \\
\hline 1.87789900 & -2.02843400 & 970 \\
\hline 1.32498100 & -2.2 & \\
\hline 3.13 & 8200 & \\
\hline 4.41 & 0.70 & -0.4274720 \\
\hline 4.522 & 0.12671500 & 0.479475 \\
\hline 5.54972700 & & \\
\hline 5.33 & 1.63 & \\
\hline 4.07477100 & 2.18139700 & -2.7 \\
\hline 2.99451100 & 200 & \\
\hline & & \\
\hline 4.3111 & -0.80278800 & 4.4433580 \\
\hline 5.68124400 & -1.45261900 & 4.1415190 \\
\hline .59874600 & 49800 & 120 \\
\hline 6.415 & & \\
\hline 6.06028900 & -1.12768600 & 3.1661690 \\
\hline 49460000 & 0.72826100 & 795 \\
\hline 4.918 & 1.11216400 & \\
\hline 5.18872900 & 0.99512100 & \\
\hline 3.54461300 & 1.24288300 & 4.650690 \\
\hline 3.867208 & -1.21982 & 5.8625830 \\
\hline 2.81469200 & -0.97959700 & 6.0329840 \\
\hline 4.46188400 & -0.67871400 & 6.6076570 \\
\hline 4.00845600 & -2.28511800 & 6.034603 \\
\hline 1.92055300 & -4.48739500 & 1.618132 \\
\hline
\end{tabular}




\begin{tabular}{|c|c|c|c|}
\hline $\mathrm{C}$ & 2.00329800 & -4.68050600 & 0.08420600 \\
\hline $\mathrm{H}$ & 1.23273900 & -4.13284900 & -0.46319000 \\
\hline $\mathrm{H}$ & 1.86864000 & -5.74128300 & -0.15467900 \\
\hline $\mathrm{H}$ & 2.97175800 & -4.36194000 & -0.31145400 \\
\hline $\mathrm{C}$ & 0.46846500 & -4.79716800 & 2.04345500 \\
\hline $\mathrm{H}$ & 0.32342800 & -4.71187600 & 3.12352000 \\
\hline $\mathrm{H}$ & 0.19400300 & -5.81529500 & 1.74149200 \\
\hline $\mathrm{H}$ & -0.22642500 & -4.09899300 & 1.56748600 \\
\hline $\mathrm{C}$ & 2.89349900 & -5.52312100 & 2.22155900 \\
\hline $\mathrm{H}$ & 3.93090800 & -5.26767700 & 1.98203400 \\
\hline $\mathrm{H}$ & 2.67770800 & -6.50463400 & 1.78378800 \\
\hline $\mathrm{H}$ & 2.81894400 & -5.61842900 & 3.30267000 \\
\hline $\mathrm{C}$ & 2.24446400 & -3.81576200 & 5.04525400 \\
\hline $\mathrm{H}$ & 2.72857600 & -4.18833100 & 5.95166000 \\
\hline $\mathrm{H}$ & 1.60030000 & -4.60330500 & 4.64264100 \\
\hline $\mathrm{H}$ & 1.63254800 & -2.94284100 & 5.28971900 \\
\hline $\mathrm{C}$ & 6.91571400 & 0.60209500 & -0.58387000 \\
\hline $\mathrm{C}$ & 7.06830500 & -0.93448800 & -0.57872800 \\
\hline $\mathrm{H}$ & 6.23398300 & -1.41689400 & -0.05886700 \\
\hline $\mathrm{H}$ & 7.99798900 & -1.22224400 & -0.07321200 \\
\hline $\mathrm{H}$ & 7.10148100 & -1.33827900 & -1.59599500 \\
\hline $\mathrm{C}$ & 6.94206200 & 1.09866500 & 0.88587900 \\
\hline $\mathrm{H}$ & 6.79782600 & 2.18367400 & 0.93220300 \\
\hline $\mathrm{H}$ & 7.91257300 & 0.86295700 & 1.33659600 \\
\hline $\mathrm{H}$ & 6.17352100 & 0.62992500 & 1.50477500 \\
\hline $\mathrm{C}$ & 8.14714400 & 1.24089400 & -1.25872500 \\
\hline $\mathrm{H}$ & 8.36263600 & 0.83236500 & -2.24531400 \\
\hline $\mathrm{H}$ & 9.02410300 & 1.05070100 & -0.62887200 \\
\hline $\mathrm{H}$ & 8.03083500 & 2.32329500 & -1.36117500 \\
\hline $\mathrm{C}$ & 3.88177900 & 3.08658100 & -4.02794500 \\
\hline $\mathrm{C}$ & 4.11661300 & 2.32381200 & -5.35013600 \\
\hline $\mathrm{H}$ & 5.16931000 & 2.08443600 & -5.49758000 \\
\hline $\mathrm{H}$ & 3.79659900 & 2.94426700 & -6.19559900 \\
\hline $\mathrm{H}$ & 3.53561500 & 1.39465600 & -5.37743100 \\
\hline $\mathrm{C}$ & 2.45089600 & 3.65810900 & -4.08380900 \\
\hline $\mathrm{H}$ & 1.69658400 & 2.87715500 & -4.23409800 \\
\hline $\mathrm{H}$ & 2.37612700 & 4.35144000 & -4.92818200 \\
\hline $\mathrm{H}$ & 2.19373600 & 4.21215400 & -3.17518400 \\
\hline $\mathrm{C}$ & 4.85518300 & 4.28332900 & -3.93671900 \\
\hline $\mathrm{H}$ & 4.72269200 & 4.94092100 & -4.80448800 \\
\hline $\mathrm{H}$ & 5.89302700 & 3.94690500 & -3.91186600 \\
\hline $\mathrm{H}$ & 4.65752100 & 4.87207900 & -3.03341300 \\
\hline $\mathrm{C}$ & 6.60662200 & 0.53452200 & -4.07386900 \\
\hline $\mathrm{H}$ & 7.02304500 & -0.24327700 & -3.42561900 \\
\hline
\end{tabular}




\begin{tabular}{|c|c|c|c|}
\hline $\mathrm{H}$ & 7.33032700 & 0.77752700 & -4.85555900 \\
\hline $\mathrm{H}$ & 5.68558200 & 0.15373300 & -4.52774700 \\
\hline $\mathrm{C}$ & -0.51534100 & 0.33669900 & 2.45179200 \\
\hline $\mathrm{C}$ & -1.34218600 & -0.57786400 & 1.72971600 \\
\hline $\mathrm{C}$ & -1.76316000 & -1.76799600 & 2.33597600 \\
\hline $\mathrm{H}$ & -2.39407400 & -2.44664900 & 1.77425400 \\
\hline $\mathrm{C}$ & -1.42396300 & -2.11324200 & 3.65558600 \\
\hline $\mathrm{H}$ & -1.76743500 & -3.03644800 & 4.10805700 \\
\hline $\mathrm{C}$ & -0.65467900 & -1.20497200 & 4.34829100 \\
\hline $\mathrm{C}$ & -0.21778300 & -0.02389900 & 3.75579000 \\
\hline $\mathrm{C}$ & 0.23805900 & 0.07373400 & 5.92071300 \\
\hline $\mathrm{H}$ & -0.58578900 & 0.62130700 & 6.40236800 \\
\hline $\mathrm{H}$ & 1.13100100 & 0.05396200 & 6.54315600 \\
\hline $\mathrm{C}$ & 0.96691600 & 1.90572200 & 0.98319100 \\
\hline $\mathrm{C}$ & 1.39205100 & 3.21272000 & 0.71521800 \\
\hline $\mathrm{H}$ & 2.14719700 & 3.37323100 & -0.04493800 \\
\hline $\mathrm{C}$ & 0.91290300 & 4.32869100 & 1.42264700 \\
\hline $\mathrm{H}$ & 1.27314900 & 5.33054300 & 1.21728200 \\
\hline $\mathrm{C}$ & -0.00150400 & 4.07515200 & 2.42186100 \\
\hline $\mathrm{C}$ & -0.45435600 & 2.78449200 & 2.68073600 \\
\hline $\mathrm{C}$ & -0.01982600 & 1.66600500 & 1.98828600 \\
\hline $\mathrm{C}$ & -1.13443600 & 4.10283500 & 4.32114200 \\
\hline $\mathrm{H}$ & -2.07694300 & 4.51179000 & 4.68077300 \\
\hline $\mathrm{H}$ & -0.39591200 & 3.99959900 & 5.13029000 \\
\hline $\mathrm{C}$ & -2.67754200 & 0.01030100 & -4.42296400 \\
\hline $\mathrm{C}$ & -1.34979500 & 0.07277500 & -4.20281500 \\
\hline $\mathrm{H}$ & -0.83536300 & 0.99517000 & -4.47703600 \\
\hline $\mathrm{C}$ & -0.48977700 & -0.96189700 & -3.63314400 \\
\hline $\mathrm{C}$ & 0.90625300 & -0.78519400 & -3.52958800 \\
\hline $\mathrm{H}$ & -0.90604900 & -1.96189300 & -3.56005200 \\
\hline $\mathrm{H}$ & 1.32057100 & 0.16483300 & -3.86608200 \\
\hline $\mathrm{C}$ & 1.89631400 & -1.86372600 & -3.36362400 \\
\hline $\mathrm{C}$ & 3.25156600 & -1.53333800 & -3.16181100 \\
\hline $\mathrm{C}$ & 1.55649500 & -3.22886500 & -3.40774100 \\
\hline $\mathrm{C}$ & 4.21683300 & -2.52024700 & -2.99333900 \\
\hline $\mathrm{H}$ & 3.53433900 & -0.48764000 & -3.11538500 \\
\hline $\mathrm{C}$ & 2.52664200 & -4.21632200 & -3.24895700 \\
\hline $\mathrm{H}$ & 0.52711400 & -3.52055100 & -3.59019700 \\
\hline $\mathrm{C}$ & 3.86298600 & -3.87207100 & -3.03561800 \\
\hline $\mathrm{H}$ & 5.25090800 & -2.23194600 & -2.82636000 \\
\hline $\mathrm{H}$ & 2.23523900 & -5.26274600 & -3.29055900 \\
\hline $\mathrm{H}$ & 4.61651200 & -4.64411500 & -2.90813500 \\
\hline $\mathrm{H}$ & -3.15760400 & 0.88207000 & -4.86459500 \\
\hline $\mathrm{C}$ & -3.58729700 & -1.12982100 & -4.07233700 \\
\hline
\end{tabular}




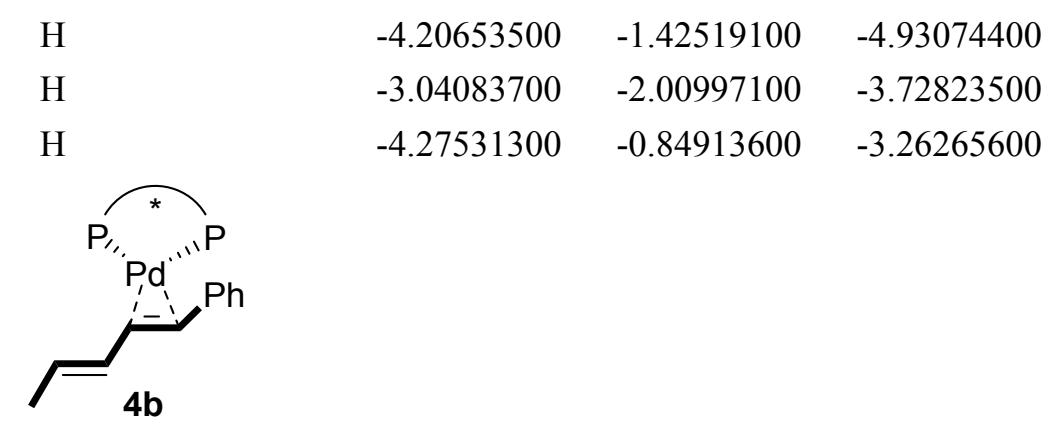

Total SCF energy (M06/SDD-6-311++G(d,p)/SMD(1,4-Dioxane)): -4717.156437a.u.

Thermal correction to Gibbs Free Energy at $298.15 \mathrm{~K}$ : 1.663033a.u

Gibbs free energy at 298.15 K (M06/SDD-6-311++G(d,p)/SMD(1,4-Dioxane)): -4715.493404a.u.

\begin{tabular}{cccc}
$\mathrm{Pd}$ & -0.01850300 & -0.38317300 & -1.43637500 \\
$\mathrm{P}$ & -1.74106100 & -0.31368300 & 0.13429300 \\
$\mathrm{P}$ & 1.61143300 & 0.53580300 & -0.04975200 \\
$\mathrm{O}$ & -5.89856200 & -4.00867700 & -1.99847100 \\
$\mathrm{O}$ & -3.91998600 & 5.23414300 & 0.55227700 \\
$\mathrm{O}$ & 3.61767900 & -3.53395100 & 3.86449500 \\
$\mathrm{O}$ & 6.17165200 & 1.98999000 & -3.58526400 \\
$\mathrm{O}$ & 0.24920500 & -1.35443100 & 5.66808700 \\
$\mathrm{O}$ & 0.82806800 & 0.63790100 & 4.66938600 \\
$\mathrm{O}$ & -1.26503400 & 2.74342700 & 3.83543600 \\
$\mathrm{O}$ & -0.57488600 & 4.89661000 & 3.40528200 \\
$\mathrm{C}$ & -3.10145500 & -1.47817700 & -0.23716500 \\
$\mathrm{C}$ & -4.44504400 & -1.10626700 & -0.31250200 \\
$\mathrm{H}$ & -4.72430500 & -0.11505000 & 0.01141600 \\
$\mathrm{C}$ & -5.41657900 & -1.96310300 & -0.83802000 \\
$\mathrm{C}$ & -4.98508300 & -3.22878300 & -1.30798400 \\
$\mathrm{C}$ & -3.67025500 & -3.70195400 & -1.09536900 \\
$\mathrm{C}$ & -2.74238400 & -2.77322900 & -0.60820100 \\
$\mathrm{H}$ & -1.69527600 & -3.05173300 & -0.54407500 \\
$\mathrm{C}$ & -6.90549500 & -1.55439100 & -0.81450000 \\
$\mathrm{C}$ & -7.09897500 & -0.18013200 & -0.14231000 \\
$\mathrm{H}$ & -6.71410300 & -0.16718200 & 0.88273300 \\
$\mathrm{H}$ & -8.16898200 & 0.04800900 & -0.09601200 \\
$\mathrm{H}$ & -6.61215700 & 0.62715400 & -0.70136400 \\
$\mathrm{C}$ & -7.52718200 & -1.45854900 & -2.22483000 \\
$\mathrm{H}$ & -6.91702400 & -0.83342800 & -2.88533800 \\
$\mathrm{H}$ & -8.52174700 & -1.00198200 & -2.15644700 \\
$\mathrm{H}$ & -7.64741300 & -2.44129400 & -2.67949200 \\
$\mathrm{C}$ & -7.68771500 & -2.59544900 & 0.01878300 \\
$\mathrm{H}$ & -7.60864400 & -3.59082300 & -0.42292700 \\
$\mathrm{H}$ & -8.74832600 & -2.31950600 & 0.06551500 \\
$\mathrm{H}$ & -7.30274200 & -2.63698500 & 1.04410400 \\
& & & \\
\hline
\end{tabular}




\begin{tabular}{|c|c|c|c|}
\hline $\mathrm{C}$ & -3.15412500 & -5.14262000 & -1.32887200 \\
\hline $\mathrm{C}$ & -2.14176300 & -5.15100100 & -2.49578200 \\
\hline $\mathrm{H}$ & -2.60108700 & -4.81032200 & -3.43002300 \\
\hline $\mathrm{H}$ & -1.75649000 & -6.16484400 & -2.65744600 \\
\hline $\mathrm{H}$ & -1.28739300 & -4.49860100 & -2.28718400 \\
\hline $\mathrm{C}$ & -2.41932400 & -5.59737200 & -0.04175900 \\
\hline $\mathrm{H}$ & -1.56207000 & -4.96634800 & 0.19968800 \\
\hline $\mathrm{H}$ & -2.04611000 & -6.61982900 & -0.17006300 \\
\hline $\mathrm{H}$ & -3.09965500 & -5.58805800 & 0.81717600 \\
\hline $\mathrm{C}$ & -4.24005300 & -6.20669000 & -1.58887100 \\
\hline $\mathrm{H}$ & -5.03084000 & -6.16820800 & -0.83545900 \\
\hline $\mathrm{H}$ & -3.76943300 & -7.19569600 & -1.53983300 \\
\hline $\mathrm{H}$ & -4.70528700 & -6.11998400 & -2.57050600 \\
\hline $\mathrm{C}$ & -5.71554700 & -3.95339700 & -3.41374200 \\
\hline $\mathrm{H}$ & -4.73643600 & -4.34490500 & -3.70864600 \\
\hline $\mathrm{H}$ & -5.80598700 & -2.92643900 & -3.78384400 \\
\hline $\mathrm{H}$ & -6.50156700 & -4.57303900 & -3.85189800 \\
\hline $\mathrm{C}$ & -2.52030600 & 1.33108400 & 0.24630500 \\
\hline $\mathrm{C}$ & -2.19578400 & 2.26317300 & -0.73178000 \\
\hline $\mathrm{H}$ & -1.50847500 & 1.95093000 & -1.50986500 \\
\hline $\mathrm{C}$ & -2.70174700 & 3.57048000 & -0.71901800 \\
\hline $\mathrm{C}$ & -3.51767500 & 3.92096600 & 0.37662200 \\
\hline $\mathrm{C}$ & -3.97193800 & 2.96369200 & 1.32463400 \\
\hline $\mathrm{C}$ & -3.42727200 & 1.68162100 & 1.25108500 \\
\hline $\mathrm{H}$ & -3.69555100 & 0.93430800 & 1.98578900 \\
\hline $\mathrm{C}$ & -5.11090600 & 3.26783900 & 2.32148100 \\
\hline $\mathrm{C}$ & -4.77163200 & 4.40318200 & 3.30832300 \\
\hline $\mathrm{H}$ & -3.82377800 & 4.19571200 & 3.81309700 \\
\hline $\mathrm{H}$ & -5.55263400 & 4.47115200 & 4.07490200 \\
\hline $\mathrm{H}$ & -4.70658800 & 5.36990800 & 2.81202100 \\
\hline $\mathrm{C}$ & -6.36939800 & 3.65345600 & 1.51054800 \\
\hline $\mathrm{H}$ & -6.18768400 & 4.54567700 & 0.90733400 \\
\hline $\mathrm{H}$ & -7.20736900 & 3.85726100 & 2.18859100 \\
\hline $\mathrm{H}$ & -6.66403300 & 2.83510800 & 0.84387300 \\
\hline $\mathrm{C}$ & -5.45645800 & 2.03002700 & 3.17216200 \\
\hline $\mathrm{H}$ & -5.76487300 & 1.17911300 & 2.55635800 \\
\hline $\mathrm{H}$ & -6.29187700 & 2.27426700 & 3.83685200 \\
\hline $\mathrm{H}$ & -4.61188300 & 1.71722400 & 3.79656800 \\
\hline $\mathrm{C}$ & -2.32189700 & 4.47079900 & -1.91947600 \\
\hline $\mathrm{C}$ & -0.82723500 & 4.84605200 & -1.82211400 \\
\hline $\mathrm{H}$ & -0.61700400 & 5.43479600 & -0.92362200 \\
\hline $\mathrm{H}$ & -0.52411200 & 5.43403100 & -2.69686000 \\
\hline $\mathrm{H}$ & -0.19945200 & 3.95058500 & -1.77634800 \\
\hline $\mathrm{C}$ & -2.53642700 & 3.66030500 & -3.22374200 \\
\hline
\end{tabular}




\begin{tabular}{|c|c|c|c|}
\hline $\mathrm{H}$ & -1.91290300 & 2.76550400 & -3.27620100 \\
\hline $\mathrm{H}$ & -2.28865800 & 4.28561000 & -4.08882600 \\
\hline $\mathrm{H}$ & -3.58118400 & 3.34535900 & -3.31616900 \\
\hline $\mathrm{C}$ & -3.16788700 & 5.75028900 & -2.08472200 \\
\hline $\mathrm{H}$ & -4.23821400 & 5.52929500 & -2.05545600 \\
\hline $\mathrm{H}$ & -2.93785100 & 6.19140400 & -3.06174800 \\
\hline $\mathrm{H}$ & -2.96078200 & 6.50933500 & -1.33139800 \\
\hline $\mathrm{C}$ & -2.91274000 & 6.05053200 & 1.15449600 \\
\hline $\mathrm{H}$ & -2.57801300 & 5.63489600 & 2.10888900 \\
\hline $\mathrm{H}$ & -3.36675500 & 7.03166000 & 1.31361500 \\
\hline $\mathrm{H}$ & -2.03984100 & 6.15682500 & 0.50259300 \\
\hline $\mathrm{C}$ & 2.26429000 & -0.66792400 & 1.15765600 \\
\hline $\mathrm{C}$ & 3.03975400 & -0.30359700 & 2.25912800 \\
\hline $\mathrm{H}$ & 3.25367500 & 0.74543000 & 2.41194900 \\
\hline $\mathrm{C}$ & 3.52777100 & -1.25840600 & 3.15399200 \\
\hline $\mathrm{C}$ & 3.15802900 & -2.61282700 & 2.93844100 \\
\hline $\mathrm{C}$ & 2.42034800 & -3.02371700 & 1.80254000 \\
\hline $\mathrm{C}$ & 1.98250000 & -2.01233500 & 0.93762500 \\
\hline $\mathrm{H}$ & 1.39719100 & -2.25736800 & 0.06076900 \\
\hline $\mathrm{C}$ & 3.08440200 & 1.19904100 & -0.90717800 \\
\hline $\mathrm{C}$ & 4.38656000 & 0.83522600 & -0.59191600 \\
\hline $\mathrm{H}$ & 4.55945900 & 0.24906300 & 0.30044400 \\
\hline $\mathrm{C}$ & 5.47851700 & 1.17349100 & -1.40864800 \\
\hline $\mathrm{C}$ & 5.18836600 & 1.82759200 & -2.62276100 \\
\hline $\mathrm{C}$ & 3.89248300 & 2.33376700 & -2.91639100 \\
\hline $\mathrm{C}$ & 2.86177500 & 1.98368600 & -2.04712500 \\
\hline $\mathrm{H}$ & 1.84218200 & 2.26682100 & -2.27708900 \\
\hline $\mathrm{C}$ & 4.53424500 & -0.83784100 & 4.24638800 \\
\hline $\mathrm{C}$ & 5.92327200 & -1.38249300 & 3.84097700 \\
\hline $\mathrm{H}$ & 5.90102500 & -2.47255400 & 3.76155000 \\
\hline $\mathrm{H}$ & 6.67499100 & -1.10414900 & 4.59032700 \\
\hline $\mathrm{H}$ & 6.23771400 & -0.97048800 & 2.87562200 \\
\hline $\mathrm{C}$ & 4.62958400 & 0.69788900 & 4.35579500 \\
\hline $\mathrm{H}$ & 4.99681200 & 1.15990800 & 3.43387300 \\
\hline $\mathrm{H}$ & 5.33689100 & 0.95826500 & 5.15013700 \\
\hline $\mathrm{H}$ & 3.65876100 & 1.14389000 & 4.60034000 \\
\hline $\mathrm{C}$ & 4.18333800 & -1.37444700 & 5.65419400 \\
\hline $\mathrm{H}$ & 3.10787800 & -1.33155600 & 5.84385200 \\
\hline $\mathrm{H}$ & 4.68915500 & -0.77288700 & 6.41822500 \\
\hline $\mathrm{H}$ & 4.50575700 & -2.40689300 & 5.78053000 \\
\hline $\mathrm{C}$ & 2.05993100 & -4.48454900 & 1.44249100 \\
\hline $\mathrm{C}$ & 1.83247700 & -4.62600300 & -0.08123200 \\
\hline $\mathrm{H}$ & 0.93569100 & -4.10574100 & -0.43038800 \\
\hline $\mathrm{H}$ & 1.70013300 & -5.68562800 & -0.32625700 \\
\hline
\end{tabular}


H

C

H

H

H

C

$\mathrm{H}$

H

H

C

H

H

H

C

C

H

$\mathrm{H}$

H

C

H

H

$\mathrm{H}$

C

H

$\mathrm{H}$

H

C

C

$\mathrm{H}$

$\mathrm{H}$

H

C

H

$\mathrm{H}$

H

C

H

$\mathrm{H}$

H

C

H

H

H

C
2.68006400

0.73215400

0.78865800

0.44247900

$-0.06393300$

3.18419400

4.11349800

2.90032900

3.39914400

2.67928700

3.21700100

2.27480100

1.86126800

6.88258400

7.07516900

6.28662800

8.04068500

7.05637900

6.96616400

6.80290600

7.96091200

6.23604000

8.06353400

8.24469200

8.97344200

7.91361900

3.61383800

3.82185600

4.87591100

3.44189500

3.27754300

2.16143200

1.43145000

2.02491600

1.92287100

4.54492900

4.35257600

5.59466100

4.36312200

6.41610200

6.88111400

7.10007200

5.48870300

$-0.37993200$
$-4.24682000$

$-4.87729800$

$-4.84148200$

$-5.89401700$

$-4.19173800$

$-5.48329000$

$-5.19932000$

$-6.48144200$

$-5.54441300$

$-4.00179300$

$-4.11873900$

$-4.97598400$

$-3.28970600$

0.81345200

$-0.71861400$

$-1.22839100$

$-0.98673800$

$-1.11117800$

1.29535300

2.37677200

1.07510800

0.80569800

1.49243200

1.09858100

1.32035600

2.57204200

3.25446600

2.52969000

2.33639200

3.15456100

1.57850900

3.77185500

2.96501200

4.47672000

4.29954200

4.48540800

5.15496800

4.18798300

5.04776100

0.80325000

0.02427600

1.08430900

0.40540500

0.28436300
2.12793000

3.21693400

1.83552700

1.82246600

1.79154200

1.28508900

1.43847000

2.85578500

4.83495200

5.77956500

4.54328400

4.97623900

$-0.86599400$

$-0.88824600$

$-0.32494500$

$-0.44263400$

$-1.91036800$

0.60663700

0.67087200

1.00988400

1.25535300

$-1.59010300$

$-2.58954900$

$-1.00322100$

$-1.67597100$

$-4.12270500$

$-5.47057200$

$-5.66746100$

$-6.28751500$

$-5.49504200$

$-4.10737600$

$-4.24047200$

$-4.93433500$

$-3.17834200$

$-4.04502900$

$-4.89224200$

$-4.06957700$

$-3.12180200$

$-4.34126300$

$-3.72820000$

$-5.14568900$

$-4.76677300$

2.53697800 


\begin{tabular}{|c|c|c|c|}
\hline $\mathrm{C}$ & -1.23649500 & -0.64462300 & 1.86955600 \\
\hline $\mathrm{C}$ & -1.58899800 & -1.84767800 & 2.49307300 \\
\hline $\mathrm{H}$ & -2.24868700 & -2.53292300 & 1.97327200 \\
\hline $\mathrm{C}$ & -1.13921800 & -2.19849000 & 3.77789600 \\
\hline $\mathrm{H}$ & -1.42421400 & -3.13462200 & 4.24431000 \\
\hline $\mathrm{C}$ & -0.33425300 & -1.28185200 & 4.41763600 \\
\hline $\mathrm{C}$ & 0.02060500 & -0.08038600 & 3.81146600 \\
\hline $\mathrm{C}$ & 0.66764300 & -0.00956100 & 5.93164300 \\
\hline $\mathrm{H}$ & -0.11513200 & 0.50522200 & 6.50836700 \\
\hline $\mathrm{H}$ & 1.62017300 & -0.01964700 & 6.45882700 \\
\hline $\mathrm{C}$ & 0.99657500 & 1.90546100 & 1.0148910 \\
\hline $\mathrm{C}$ & 1.38516900 & 3.22468800 & 0.7517620 \\
\hline $\mathrm{H}$ & 2.10943500 & 3.41102400 & -0.03217700 \\
\hline $\mathrm{C}$ & 0.90457000 & 4.32154300 & 1.4869350 \\
\hline $\mathrm{H}$ & 1.23514500 & 5.33354900 & 1.2818650 \\
\hline $\mathrm{C}$ & 0.02272000 & 4.03680400 & 2.5059220 \\
\hline $\mathrm{C}$ & -0.38914200 & 2.73277400 & 2.76739500 \\
\hline $\mathrm{C}$ & 0.05322400 & 1.63047100 & 2.0536990 \\
\hline $\mathrm{C}$ & -1.07682000 & 4.02848200 & 4.42788700 \\
\hline $\mathrm{H}$ & -2.03041800 & 4.40945900 & 4.79013100 \\
\hline $\mathrm{H}$ & -0.33299100 & 3.95179900 & 5.23502700 \\
\hline $\mathrm{C}$ & -3.19117700 & -0.66105900 & -3.67658000 \\
\hline $\mathrm{C}$ & -1.88855000 & -0.34610300 & -3.78692600 \\
\hline $\mathrm{H}$ & -3.45836600 & -1.63072700 & -3.25812700 \\
\hline $\mathrm{H}$ & -1.61321500 & 0.63540600 & -4.17733000 \\
\hline $\mathrm{C}$ & -0.78417500 & -1.21277900 & -3.38250700 \\
\hline $\mathrm{C}$ & 0.57419500 & -0.88097900 & -3.55578800 \\
\hline $\mathrm{H}$ & -1.05683000 & -2.24175000 & -3.15953300 \\
\hline $\mathrm{H}$ & 0.81153400 & 0.08556300 & -3.99998400 \\
\hline $\mathrm{C}$ & 1.69491400 & -1.83701100 & -3.49592300 \\
\hline $\mathrm{C}$ & 3.01762800 & -1.35574000 & -3.41693600 \\
\hline $\mathrm{C}$ & 1.50738800 & -3.23119700 & -3.51406500 \\
\hline $\mathrm{C}$ & 4.09705800 & -2.22703400 & -3.32534000 \\
\hline $\mathrm{H}$ & 3.18452300 & -0.28440100 & -3.38884600 \\
\hline $\mathrm{C}$ & 2.59192300 & -4.10351400 & -3.43292600 \\
\hline $\mathrm{H}$ & 0.50508300 & -3.63584700 & -3.61193200 \\
\hline $\mathrm{C}$ & 3.89397900 & -3.61094900 & -3.32950300 \\
\hline $\mathrm{H}$ & 5.10237800 & -1.82358400 & -3.24357500 \\
\hline $\mathrm{H}$ & 2.41614600 & -5.17615700 & -3.44951000 \\
\hline $\mathrm{H}$ & 4.73704900 & -4.29250400 & -3.25977000 \\
\hline $\mathrm{C}$ & -4.32538900 & 0.25716900 & -4.01615600 \\
\hline $\mathrm{H}$ & -4.89241500 & 0.53046000 & -3.11425800 \\
\hline $\mathrm{H}$ & -3.96788700 & 1.18338500 & -4.47850600 \\
\hline $\mathrm{H}$ & -5.04132400 & -0.21055100 & -4.70718800 \\
\hline
\end{tabular}




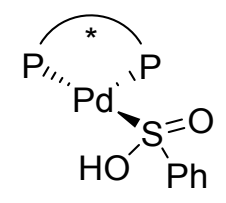

\section{Int-1a}

Total SCF energy (M06/SDD-6-311++G(d,p)/SMD(1,4-Dioxane)): -5071.683625a.u.

Thermal correction to Gibbs Free Energy at $298.15 \mathrm{~K}$ : 1.575603a.u

Gibbs free energy at 298.15 K (M06/SDD-6-311++G(d,p)/SMD(1,4-Dioxane)): -5070.108022a.u.

$\begin{array}{cccc}\mathrm{Pd} & 0.16269000 & -0.40058500 & -1.48114200 \\ \mathrm{P} & 1.54725700 & 0.48546100 & 0.20582000 \\ \mathrm{P} & -1.86158600 & -0.18676200 & -0.32014800 \\ \mathrm{O} & 6.99252700 & 1.75027200 & -1.89361700 \\ \mathrm{O} & 2.06226600 & -4.13071300 & 3.97486700 \\ \mathrm{O} & -3.30803400 & 5.63088800 & -0.59518200 \\ \mathrm{O} & -6.47976000 & -3.42210700 & -2.32127200 \\ \mathrm{O} & -1.14697000 & 4.89103300 & 3.21241900 \\ \mathrm{O} & -1.96657700 & 2.75569700 & 3.48340300 \\ \mathrm{O} & -0.22875200 & 0.52126100 & 4.69837100 \\ \mathrm{O} & -1.13830500 & -1.44276900 & 5.48328000 \\ \mathrm{C} & 3.22290800 & 1.08013300 & -0.24012200 \\ \mathrm{C} & 4.38183800 & 0.72940800 & 0.45213900 \\ \mathrm{H} & 4.28253500 & 0.20702500 & 1.39145000 \\ \mathrm{C} & 5.65424000 & 0.99140300 & -0.06432700 \\ \mathrm{C} & 5.73281500 & 1.61942500 & -1.33441500 \\ \mathrm{C} & 4.58538800 & 2.12017000 & -1.99617900 \\ \mathrm{C} & 3.34604800 & 1.78705800 & -1.43610200 \\ \mathrm{H} & 2.43654000 & 2.03644400 & -1.96984300 \\ \mathrm{C} & 6.90523400 & 0.62436400 & 0.76275300 \\ \mathrm{C} & 6.52134700 & 0.11337400 & 2.16671400 \\ \mathrm{H} & 5.92726300 & 0.84714600 & 2.72220600 \\ \mathrm{H} & 7.43503700 & -0.07641200 & 2.73975200 \\ \mathrm{H} & 5.95766800 & -0.82490600 & 2.12628400 \\ \mathrm{C} & 7.72318100 & -0.49836400 & 0.08948500 \\ \mathrm{H} & 7.09393900 & -1.37419600 & -0.10855400 \\ \mathrm{H} & 8.53487800 & -0.81603700 & 0.75485500 \\ \mathrm{H} & 8.17329500 & -0.17309500 & -0.84697700 \\ \mathrm{C} & 7.78807900 & 1.87752600 & 0.95760600 \\ \mathrm{H} & 8.12232900 & 2.28189800 & 0.00155500 \\ \mathrm{H} & 8.66963900 & 1.62306300 & 1.55848200 \\ \mathrm{C} & 7.23152400 & 2.65786600 & 1.48930600 \\ \mathrm{C} & 4.58271600 & 2.96062600 & -3.29440200 \\ \mathrm{H} & & & -4.51931100\end{array}$


H

$\mathrm{H}$

$\mathrm{H}$

C

$\mathrm{H}$

$\mathrm{H}$

$\mathrm{H}$

C

$\mathrm{H}$

$\mathrm{H}$

$\mathrm{H}$

C

$\mathrm{H}$

$\mathrm{H}$

$\mathrm{H}$

C

C

$\mathrm{H}$

C

C

C

C

$\mathrm{H}$

C

C

$\mathrm{H}$

$\mathrm{H}$

$\mathrm{H}$

C

$\mathrm{H}$

$\mathrm{H}$

$\mathrm{H}$

C

$\mathrm{H}$

$\mathrm{H}$

$\mathrm{H}$

C

C

$\mathrm{H}$

$\mathrm{H}$

$\mathrm{H}$

C

$\mathrm{H}$

$\mathrm{H}$

$\begin{array}{rrr}5.25323400 & 1.33479000 & -4.62725100 \\ 4.36034100 & 2.62820000 & -5.43817800 \\ 3.50554200 & 1.43486800 & -4.43764600 \\ 3.37304100 & 3.92914800 & -3.29145600 \\ 2.41421500 & 3.41377800 & -3.38863900 \\ 3.45439300 & 4.60929000 & -4.14602900 \\ 3.34359700 & 4.53252100 & -2.37768600 \\ 5.83045000 & 3.85776200 & -3.43982000 \\ 5.94606000 & 4.50203400 & -2.56107900 \\ 5.70511900 & 4.50275500 & -4.31694100 \\ 6.75740300 & 3.30224200 & -3.55969300 \\ 7.31395700 & 0.84759400 & -2.95226700 \\ 7.16384900 & 1.31933800 & -3.92828400 \\ 6.70843000 & -0.06286000 & -2.89738400 \\ 8.37168300 & 0.58907100 & -2.84996300 \\ 1.82946500 & -0.86854100 & 1.40000300 \\ 1.56404200 & -2.15158100 & 0.93540900 \\ 1.21832500 & -2.25174700 & -0.08799600 \\ 1.69454100 & -3.29044200 & 1.73844300 \\ 2.06222900 & -3.07344200 & 3.08144500 \\ 2.45356500 & -1.79507000 & 3.56679000 \\ 2.29921000 & -0.70426800 & 2.70711200 \\ 2.52426500 & 0.29573700 & 3.05288700 \\ 3.15774500 & -1.61509900 & 4.93004700 \\ 2.36913400 & -2.18565100 & 6.12969600 \\ 1.32227900 & -1.87580900 & 6.10420400 \\ 2.81152700 & -1.82101700 & 7.06433700 \\ 2.40151200 & -3.27418200 & 6.14886900 \\ 4.51973400 & -2.34293900 & 4.84995500 \\ 4.37734000 & -3.40952600 & 4.65690300 \\ 5.06476300 & -2.23266700 & 5.79580900 \\ 5.13925500 & -1.92351200 & 4.04998700 \\ 3.42357300 & -0.12599200 & 5.22734900 \\ 4.07966600 & 0.33380800 & 4.48096400 \\ 3.92309200 & -0.03630900 & 6.19776400 \\ 2.49172000 & 0.44925600 & 5.26716500 \\ 1.42652400 & -4.64514300 & 1.03977900 \\ -0.07885100 & -4.74557800 & 0.70259200 \\ -0.69433900 & -4.73085800 & 1.60860200 \\ -0.28655000 & -5.67780200 & 0.16349300 \\ -0.40217000 & -3.90965200 & 0.07326200 \\ 2.23090300 & -4.67513000 & -0.28581000 \\ 1.94134200 & -3.88796100 & -0.98373000 \\ 2.06771700 & -5.63294200 & -0.79227400\end{array}$




\begin{tabular}{|c|c|c|c|}
\hline $\mathrm{H}$ & 3.30385200 & -4.56966800 & -0.09422200 \\
\hline $\mathrm{C}$ & 1.86262400 & -5.90084000 & 1.82163500 \\
\hline $\mathrm{H}$ & 2.89711700 & -5.82161900 & 2.16563200 \\
\hline $\mathrm{H}$ & 1.79155500 & -6.76469800 & 1.15029700 \\
\hline $\mathrm{H}$ & 1.23477400 & -6.11384700 & 2.68649200 \\
\hline $\mathrm{C}$ & 0.76012800 & -4.42614000 & 4.48691200 \\
\hline $\mathrm{H}$ & 0.34982900 & -3.57873100 & 5.04345700 \\
\hline $\mathrm{H}$ & 0.87913700 & -5.28384600 & 5.15324300 \\
\hline $\mathrm{H}$ & 0.06506900 & -4.68381000 & 3.68145600 \\
\hline $\mathrm{C}$ & -2.38671700 & 1.56674000 & -0.34816500 \\
\hline $\mathrm{C}$ & -3.35325200 & 2.13543600 & 0.48083000 \\
\hline $\mathrm{H}$ & -3.82090900 & 1.51688300 & 1.23293800 \\
\hline $\mathrm{C}$ & -3.69904400 & 3.48567600 & 0.38054400 \\
\hline $\mathrm{C}$ & -2.98242500 & 4.28541500 & -0.54944600 \\
\hline $\mathrm{C}$ & -2.02458800 & 3.73548900 & -1.43374300 \\
\hline $\mathrm{C}$ & -1.75338200 & 2.37109200 & -1.29055900 \\
\hline $\mathrm{H}$ & -1.00704000 & 1.89505700 & -1.91435700 \\
\hline $\mathrm{C}$ & -3.32071900 & -1.17096500 & -0.82172600 \\
\hline $\mathrm{C}$ & -4.61557400 & -0.94067300 & -0.36715200 \\
\hline $\mathrm{H}$ & -4.78884500 & -0.12712800 & 0.32349100 \\
\hline $\mathrm{C}$ & -5.70075000 & -1.72700700 & -0.77379800 \\
\hline $\mathrm{C}$ & -5.43271800 & -2.73652400 & -1.72688200 \\
\hline $\mathrm{C}$ & -4.11340200 & -3.09115100 & -2.10589700 \\
\hline $\mathrm{C}$ & -3.08005900 & -2.26415300 & -1.65870100 \\
\hline $\mathrm{H}$ & -2.05955700 & -2.44010300 & -1.97612800 \\
\hline $\mathrm{C}$ & -4.91113400 & 4.02222000 & 1.17262000 \\
\hline $\mathrm{C}$ & -6.05474700 & 4.27254500 & 0.16261400 \\
\hline $\mathrm{H}$ & -5.75197900 & 5.00612100 & -0.58919600 \\
\hline $\mathrm{H}$ & -6.94350000 & 4.65370100 & 0.68117800 \\
\hline $\mathrm{H}$ & -6.33089900 & 3.34379900 & -0.34960400 \\
\hline $\mathrm{C}$ & -5.40625800 & 2.99040400 & 2.20756600 \\
\hline $\mathrm{H}$ & -5.76061200 & 2.06727900 & 1.73686800 \\
\hline $\mathrm{H}$ & -6.25208700 & 3.41332200 & 2.75951000 \\
\hline $\mathrm{H}$ & -4.62178200 & 2.72959300 & 2.92660400 \\
\hline $\mathrm{C}$ & -4.62453900 & 5.33722400 & 1.93606800 \\
\hline $\mathrm{H}$ & -3.62799300 & 5.33604800 & 2.38447100 \\
\hline $\mathrm{H}$ & -5.36136900 & 5.46997300 & 2.73684200 \\
\hline $\mathrm{H}$ & -4.69547500 & 6.20275900 & 1.27867900 \\
\hline $\mathrm{C}$ & -1.24737400 & 4.50553700 & -2.52739100 \\
\hline $\mathrm{C}$ & -0.80104400 & 3.54580200 & -3.66023800 \\
\hline $\mathrm{H}$ & -0.03298600 & 2.83847500 & -3.32816600 \\
\hline $\mathrm{H}$ & -0.36448400 & 4.13256000 & -4.47521400 \\
\hline $\mathrm{H}$ & -1.64486000 & 2.97445100 & -4.06167700 \\
\hline $\mathrm{C}$ & 0.04488600 & 5.10105400 & -1.9236900 \\
\hline
\end{tabular}


$\mathrm{H}$

$\mathrm{H}$

$\mathrm{H}$

C

$\mathrm{H}$

$\mathrm{H}$

$\mathrm{H}$

C

$\mathrm{H}$

$\mathrm{H}$

$\mathrm{H}$

C

C

$\mathrm{H}$

$\mathrm{H}$

$\mathrm{H}$

C

$\mathrm{H}$

$\mathrm{H}$

$\mathrm{H}$

C

$\mathrm{H}$

$\mathrm{H}$

$\mathrm{H}$

C

C

$\mathrm{H}$

$\mathrm{H}$

$\mathrm{H}$

C

$\mathrm{H}$

$\mathrm{H}$

$\mathrm{H}$

C

$\mathrm{H}$

$\mathrm{H}$

$\mathrm{H}$

C

$\mathrm{H}$

$\mathrm{H}$

$\mathrm{H}$

C

C

C

\begin{tabular}{rrc}
-0.15518000 & 5.81267900 & -1.12122900 \\
0.62416900 & 5.61591000 & -2.69985400 \\
0.66636200 & 4.30431100 & -1.50344100 \\
-2.09647900 & 5.59563700 & -3.21500100 \\
-2.99805100 & 5.15458500 & -3.65462400 \\
-1.51400700 & 6.05095000 & -4.02428300 \\
-2.41835500 & 6.38389600 & -2.53877100 \\
-2.41489900 & 6.53105400 & 0.06284100 \\
-3.02029000 & 7.32546800 & 0.50674900 \\
-1.72067800 & 6.98179100 & -0.65349000 \\
-1.85180600 & 6.02719500 & 0.85342900 \\
-7.06201700 & -1.43046800 & -0.09730100 \\
-7.59553700 & -0.06534400 & -0.58450000 \\
-6.87835900 & 0.73799100 & -0.38295200 \\
-8.53433500 & 0.18181800 & -0.07426500 \\
-7.78869300 & -0.07231700 & -1.66257700 \\
-6.83395200 & -1.35152600 & 1.43542900 \\
-6.42188600 & -2.29319900 & 1.81429700 \\
-7.78902600 & -1.16831100 & 1.94044000 \\
-6.15252600 & -0.54788000 & 1.72603900 \\
-8.15200600 & -2.50392700 & -0.29735900 \\
-8.55181100 & -2.53089900 & -1.31048400 \\
-8.98738400 & -2.27618400 & 0.37536500 \\
-7.78189000 & -3.50328800 & -0.05438400 \\
-3.78919600 & -4.36976900 & -2.90787800 \\
-4.33789300 & -4.31675700 & -4.34980900 \\
-5.42605600 & -4.38156900 & -4.37253800 \\
-3.94381100 & -5.16382400 & -4.92364400 \\
-4.02333400 & -3.39702900 & -4.85556700 \\
-2.26616200 & -4.59512100 & -3.00737300 \\
-1.75686100 & -3.81220700 & -3.57759200 \\
-2.08497100 & -5.54821600 & -3.51676800 \\
-1.80027400 & -4.65794300 & -2.01732600 \\
-4.39173900 & -5.58680700 & -2.16908500 \\
-4.15585200 & -6.50820600 & -2.71540100 \\
-5.47668000 & -5.50392200 & -2.08187800 \\
-3.96579700 & -5.67387700 & -1.16261400 \\
-7.09129700 & -2.69090100 & -3.38307800 \\
-7.57165000 & -1.77629600 & -3.01852400 \\
-7.84803000 & -3.34954700 & -3.81601000 \\
-6.35746800 & -2.41935900 & -4.14964900 \\
-0.34429700 & 1.60528900 & 2.00997900 \\
0.80767800 & 1.84375100 & 1.19958900 \\
\hline & 3.14770900 & 1.05024700 \\
\hline
\end{tabular}




$\begin{array}{lrrr}\mathrm{H} & 2.17957900 & 3.30799100 & 0.43897100 \\ \mathrm{C} & 0.71271200 & 4.26023100 & 1.67702000 \\ \mathrm{H} & 1.11268900 & 5.25998100 & 1.55102100 \\ \mathrm{C} & -0.37943800 & 4.00786000 & 2.47836400 \\ \mathrm{C} & -0.87690000 & 2.71884000 & 2.63780100 \\ \mathrm{C} & -1.90424400 & 4.04702300 & 4.08924000 \\ \mathrm{H} & -1.38420400 & 3.97183600 & 5.05553800 \\ \mathrm{H} & -2.91162600 & 4.44595700 & 4.19905200 \\ \mathrm{C} & -1.69539600 & -0.57059400 & 1.47220300 \\ \mathrm{C} & -2.21877200 & -1.77706500 & 1.95908100 \\ \mathrm{H} & -2.77057000 & -2.41784800 & 1.28170800 \\ \mathrm{C} & -2.08068200 & -2.18349600 & 3.29649400 \\ \mathrm{H} & -2.50415500 & -3.11515100 & 3.65456000 \\ \mathrm{C} & -1.40838400 & -1.31957200 & 4.13415900 \\ \mathrm{C} & -0.86339200 & -0.13164400 & 3.65998400 \\ \mathrm{C} & -0.95159700 & 0.28312200 & 2.34110200 \\ \mathrm{C} & -0.71265300 & -0.13043100 & 5.87221400 \\ \mathrm{H} & 0.09169600 & -0.20921800 & 6.60242300 \\ \mathrm{H} & -1.57524800 & 0.42485700 & 6.26935600 \\ \mathrm{~S} & 0.82559100 & -1.36048100 & -3.48085900 \\ \mathrm{C} & 2.42276700 & -2.19212800 & -3.31394300 \\ \mathrm{C} & 3.42454800 & -1.59346500 & -2.55034700 \\ \mathrm{C} & 2.57338900 & -3.46747900 & -3.85075100 \\ \mathrm{C} & 4.60933500 & -2.29021900 & -2.32503400 \\ \mathrm{H} & 3.27372200 & -0.60551700 & -2.13682000 \\ \mathrm{C} & 3.76649200 & -4.15683800 & -3.62157300 \\ \mathrm{H} & 1.76072600 & -3.89707800 & -4.42691900 \\ \mathrm{C} & 4.77917400 & -3.57213600 & -2.85819400 \\ \mathrm{H} & 5.38738500 & -1.83596600 & -1.71892100 \\ \mathrm{H} & 3.89999700 & -5.15422400 & -4.03045000 \\ \mathrm{O} & 5.69871600 & -4.11926600 & -2.66990200 \\ \mathrm{H} & 0.00805600 & -2.25206800 & -4.35816200 \\ & 1.43259200 & -0.08461000 & -4.43517300 \\ \mathrm{H} & & & -4.26510000\end{array}$

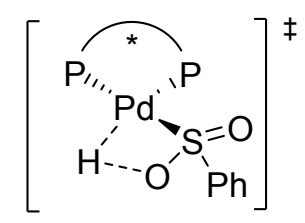

TS-2a

Total SCF energy (M06/SDD-6-311++G(d,p)/SMD(1,4-Dioxane)): -5071.642272a.u.

Thermal correction to Gibbs Free Energy at $298.15 \mathrm{~K}$ : 1.574470a.u

Gibbs free energy at 298.15 K (M06/SDD-6-311++G(d,p)/SMD(1,4-Dioxane)): -5070.067802a.u. 


\begin{tabular}{|c|c|c|c|}
\hline $\mathrm{Pd}$ & 0.14048800 & -0.14863200 & -1.55847600 \\
\hline $\mathrm{P}$ & 1.49246500 & 0.64075300 & 0.27770500 \\
\hline $\mathrm{P}$ & -1.83243100 & -0.12494600 & -0.42459600 \\
\hline $\mathrm{O}$ & 6.85549000 & 1.54116200 & -2.10716000 \\
\hline $\mathrm{O}$ & 2.01991500 & -3.72539300 & 4.32178000 \\
\hline $\mathrm{O}$ & -3.87576900 & 5.47760700 & -0.62080000 \\
\hline $\mathrm{O}$ & -5.60795200 & -4.39102700 & -2.25467700 \\
\hline $\mathrm{O}$ & -1.22002400 & 5.24842800 & 2.90467200 \\
\hline $\mathrm{O}$ & -2.14437000 & 3.15866700 & 3.19023700 \\
\hline $\mathrm{O}$ & -0.41054100 & 0.99887400 & 4.58900000 \\
\hline $\mathrm{O}$ & -1.19451600 & -0.98461100 & 5.46416900 \\
\hline $\mathrm{C}$ & 3.16412300 & 1.14053600 & -0.24104200 \\
\hline $\mathrm{C}$ & 4.31847100 & 0.73174300 & 0.41598800 \\
\hline $\mathrm{H}$ & 4.22296000 & 0.21282100 & 1.36011200 \\
\hline $\mathrm{C}$ & 5.59572200 & 0.93561000 & -0.12796500 \\
\hline $\mathrm{C}$ & 5.65734600 & 1.50980000 & -1.41681100 \\
\hline $\mathrm{C}$ & 4.51535800 & 2.06455600 & -2.05610300 \\
\hline $\mathrm{C}$ & 3.27936300 & 1.83583000 & -1.45248500 \\
\hline $\mathrm{H}$ & 2.37380200 & 2.14994000 & -1.95504700 \\
\hline $\mathrm{C}$ & 6.79730200 & 0.52347100 & 0.75696200 \\
\hline $\mathrm{C}$ & 6.55884000 & 1.07080100 & 2.18925400 \\
\hline $\mathrm{H}$ & 6.43793500 & 2.15938300 & 2.17479900 \\
\hline $\mathrm{H}$ & 7.42063400 & 0.83074100 & 2.82175000 \\
\hline $\mathrm{H}$ & 5.67569500 & 0.63922000 & 2.66771900 \\
\hline $\mathrm{C}$ & 6.88166400 & -1.01662600 & 0.83797400 \\
\hline $\mathrm{H}$ & 5.94213000 & -1.44834000 & 1.19984400 \\
\hline $\mathrm{H}$ & 7.68221900 & -1.31895400 & 1.52383500 \\
\hline $\mathrm{H}$ & 7.09631000 & -1.45806700 & -0.14097200 \\
\hline $\mathrm{C}$ & 8.16176800 & 1.09176800 & 0.31549900 \\
\hline $\mathrm{H}$ & 8.54412100 & 0.63844600 & -0.59800200 \\
\hline $\mathrm{H}$ & 8.89211800 & 0.89544300 & 1.10926500 \\
\hline $\mathrm{H}$ & 8.11258500 & 2.17268900 & 0.15817400 \\
\hline $\mathrm{C}$ & 4.60604700 & 2.91947200 & -3.33770800 \\
\hline $\mathrm{C}$ & 5.09544900 & 2.10558600 & -4.55592100 \\
\hline $\mathrm{H}$ & 6.14902700 & 1.83945200 & -4.46916400 \\
\hline $\mathrm{H}$ & 4.98500400 & 2.71385100 & -5.46153800 \\
\hline $\mathrm{H}$ & 4.49026500 & 1.20364200 & -4.68614700 \\
\hline $\mathrm{C}$ & 3.22817500 & 3.50145400 & -3.71547500 \\
\hline $\mathrm{H}$ & 2.52553500 & 2.71308700 & -4.00253800 \\
\hline $\mathrm{H}$ & 3.35190800 & 4.16629900 & -4.57709500 \\
\hline $\mathrm{H}$ & 2.79493700 & 4.09540300 & -2.90207700 \\
\hline $\mathrm{C}$ & 5.56519100 & 4.10200800 & -3.07372800 \\
\hline $\mathrm{H}$ & 5.19551700 & 4.72535300 & -2.25057000 \\
\hline $\mathrm{H}$ & 5.63247800 & 4.73138500 & -3.96925200 \\
\hline
\end{tabular}




$\begin{array}{lrrr}\mathrm{H} & 6.56823900 & 3.75211500 & -2.82147300 \\ \mathrm{C} & 7.17207700 & 0.29745300 & -2.73583300 \\ \mathrm{H} & 8.03838600 & 0.48594500 & -3.37399800 \\ \mathrm{H} & 6.33672600 & -0.06277600 & -3.34444300 \\ \mathrm{H} & 7.42753900 & -0.47077600 & -1.99775800 \\ \mathrm{C} & 1.72952400 & -0.63353300 & 1.55487900 \\ \mathrm{C} & 1.44428800 & -1.94005300 & 1.17432900 \\ \mathrm{H} & 1.08276800 & -2.10287500 & 0.16520900 \\ \mathrm{C} & 1.59215400 & -3.02702100 & 2.04522800 \\ \mathrm{C} & 1.98517000 & -2.72553600 & 3.36597700 \\ \mathrm{C} & 2.37726600 & -1.41832300 & 3.76734600 \\ \mathrm{C} & 2.21653200 & -0.38563700 & 2.84127700 \\ \mathrm{H} & 2.45743400 & 0.63339900 & 3.11239200 \\ \mathrm{C} & 3.10084900 & -1.14669400 & 5.10482900 \\ \mathrm{C} & 2.34515000 & -1.65850900 & 6.35116200 \\ \mathrm{H} & 1.29614200 & -1.35129200 & 6.33789600 \\ \mathrm{H} & 2.80893100 & -1.24502900 & 7.25430500 \\ \mathrm{H} & 2.38163600 & -2.74420100 & 6.42546900 \\ \mathrm{C} & -2.59006000 & 1.52596100 & -0.54088200 \\ \mathrm{H} & & & \\ \mathrm{H} & & & \end{array}$




\begin{tabular}{|c|c|c|c|}
\hline $\mathrm{C}$ & -3.68688700 & 1.93097500 & 0.22529600 \\
\hline $\mathrm{H}$ & -4.12989100 & 1.22887200 & 0.91716500 \\
\hline $\mathrm{C}$ & -4.19589200 & 3.22593600 & 0.13568600 \\
\hline $\mathrm{C}$ & -3.49428200 & 4.14925200 & -0.68799600 \\
\hline $\mathrm{C}$ & -2.45757200 & 3.74822800 & -1.55675800 \\
\hline $\mathrm{C}$ & -2.01856500 & 2.42386600 & -1.43523100 \\
\hline $\mathrm{H}$ & -1.18975400 & 2.07080100 & -2.03860000 \\
\hline $\mathrm{C}$ & -3.04941600 & -1.39200400 & -0.92476600 \\
\hline $\mathrm{C}$ & -4.39071600 & -1.37390200 & -0.55548300 \\
\hline $\mathrm{H}$ & -4.76305600 & -0.54222500 & 0.02579200 \\
\hline $\mathrm{C}$ & -5.27212600 & -2.40701800 & -0.90237900 \\
\hline $\mathrm{C}$ & -4.75778100 & -3.43769500 & -1.72383900 \\
\hline $\mathrm{C}$ & -3.37608300 & -3.54916500 & -2.02273700 \\
\hline $\mathrm{C}$ & -2.55321000 & -2.49811900 & -1.61873300 \\
\hline $\mathrm{H}$ & -1.49966800 & -2.50702300 & -1.86194400 \\
\hline $\mathrm{C}$ & -5.52878100 & 3.58368400 & 0.82779900 \\
\hline $\mathrm{C}$ & -6.52068400 & 4.08991400 & -0.24440000 \\
\hline $\mathrm{H}$ & -6.14478500 & 4.98842200 & -0.73657300 \\
\hline $\mathrm{H}$ & -7.48638800 & 4.32456000 & 0.21980900 \\
\hline $\mathrm{H}$ & -6.68883900 & 3.31999300 & -1.00600500 \\
\hline $\mathrm{C}$ & -6.16323900 & 2.34735200 & 1.49654800 \\
\hline $\mathrm{H}$ & -6.33890800 & 1.53825900 & 0.77926700 \\
\hline $\mathrm{H}$ & -7.13356600 & 2.62808600 & 1.91954100 \\
\hline $\mathrm{H}$ & -5.54681600 & 1.95868900 & 2.31520100 \\
\hline $\mathrm{C}$ & -5.36504100 & 4.64993600 & 1.92858300 \\
\hline $\mathrm{H}$ & -4.62104800 & 4.32005300 & 2.65944300 \\
\hline $\mathrm{H}$ & -6.31689400 & 4.78957300 & 2.45481300 \\
\hline $\mathrm{H}$ & -5.06556500 & 5.61465600 & 1.52127300 \\
\hline $\mathrm{C}$ & -1.76109900 & 4.60446900 & -2.64218000 \\
\hline $\mathrm{C}$ & -1.67427300 & 3.77064300 & -3.94811400 \\
\hline $\mathrm{H}$ & -1.06142900 & 2.87186200 & -3.84583900 \\
\hline $\mathrm{H}$ & -1.22227800 & 4.37998400 & -4.73813700 \\
\hline $\mathrm{H}$ & -2.67173500 & 3.46424300 & -4.28150100 \\
\hline $\mathrm{C}$ & -0.32247000 & 4.93330400 & -2.18443300 \\
\hline $\mathrm{H}$ & -0.31471500 & 5.53118400 & -1.26725100 \\
\hline $\mathrm{H}$ & 0.20516800 & 5.49435700 & -2.96479100 \\
\hline $\mathrm{H}$ & 0.24448600 & 4.01878900 & -1.98183800 \\
\hline $\mathrm{C}$ & -2.49733600 & 5.90486900 & -3.02514200 \\
\hline $\mathrm{H}$ & -3.54857400 & 5.71515500 & -3.25899700 \\
\hline $\mathrm{H}$ & -2.01992500 & 6.32050300 & -3.91987900 \\
\hline $\mathrm{H}$ & -2.45810100 & 6.67199200 & -2.25265200 \\
\hline $\mathrm{C}$ & -3.00338800 & 6.27339200 & 0.18419200 \\
\hline $\mathrm{H}$ & -3.45019200 & 7.26946700 & 0.22838900 \\
\hline $\mathrm{H}$ & -2.00318600 & 6.34392400 & -0.25412700 \\
\hline
\end{tabular}




\begin{tabular}{|c|c|c|c|}
\hline $\mathrm{H}$ & -2.90532400 & 5.86336500 & 1.19303700 \\
\hline $\mathrm{C}$ & -6.69792800 & -2.33022100 & -0.30150300 \\
\hline $\mathrm{C}$ & -7.48545400 & -1.18527000 & -0.97488300 \\
\hline $\mathrm{H}$ & -6.96553300 & -0.22708300 & -0.86709300 \\
\hline $\mathrm{H}$ & -8.47778400 & -1.08609700 & -0.51892600 \\
\hline $\mathrm{H}$ & -7.62280800 & -1.36696300 & -2.04598000 \\
\hline $\mathrm{C}$ & -6.57023800 & -2.01422900 & 1.21267100 \\
\hline $\mathrm{H}$ & -5.97625300 & -2.78218000 & 1.72030800 \\
\hline $\mathrm{H}$ & -7.56630200 & -1.99613100 & 1.66848300 \\
\hline $\mathrm{H}$ & -6.10623200 & -1.04499200 & 1.41139000 \\
\hline $\mathrm{C}$ & -7.52025500 & -3.63359800 & -0.38154800 \\
\hline $\mathrm{H}$ & -7.85686200 & -3.87279300 & -1.38947200 \\
\hline $\mathrm{H}$ & -8.41567000 & -3.51410600 & 0.23956900 \\
\hline $\mathrm{H}$ & -6.95538900 & -4.48943400 & -0.00281600 \\
\hline $\mathrm{C}$ & -2.75384800 & -4.79455700 & -2.68957800 \\
\hline $\mathrm{C}$ & -3.29520600 & -5.03989600 & -4.11456900 \\
\hline $\mathrm{H}$ & -4.33224200 & -5.37430200 & -4.10239000 \\
\hline $\mathrm{H}$ & -2.70111500 & -5.82235900 & -4.60082800 \\
\hline $\mathrm{H}$ & -3.21703600 & -4.13450200 & -4.72677400 \\
\hline $\mathrm{C}$ & -1.22122800 & -4.65764700 & -2.80895400 \\
\hline $\mathrm{H}$ & -0.92858400 & -3.81580100 & -3.44505200 \\
\hline $\mathrm{H}$ & -0.81855100 & -5.57269100 & -3.25671200 \\
\hline $\mathrm{H}$ & -0.73934000 & -4.53287700 & -1.83385900 \\
\hline $\mathrm{C}$ & -3.05353300 & -6.02529200 & -1.80412800 \\
\hline $\mathrm{H}$ & -2.60903000 & -6.92425700 & -2.24828800 \\
\hline $\mathrm{H}$ & -4.12920700 & -6.18571700 & -1.70230600 \\
\hline $\mathrm{H}$ & -2.62339800 & -5.89403600 & -0.80432100 \\
\hline $\mathrm{C}$ & -6.32182400 & -3.93146900 & -3.40344800 \\
\hline $\mathrm{H}$ & -7.00086200 & -3.10971700 & -3.15356700 \\
\hline $\mathrm{H}$ & -6.90167200 & -4.78313500 & -3.76651100 \\
\hline $\mathrm{H}$ & -5.63438300 & -3.59347800 & -4.18587400 \\
\hline $\mathrm{C}$ & -0.47523700 & 1.90116400 & 1.85375600 \\
\hline $\mathrm{C}$ & 0.73491900 & 2.07992300 & 1.11628200 \\
\hline $\mathrm{C}$ & 1.26777500 & 3.36002000 & 0.92658200 \\
\hline $\mathrm{H}$ & 2.18567100 & 3.46810200 & 0.36022200 \\
\hline $\mathrm{C}$ & 0.67471100 & 4.51137600 & 1.47195500 \\
\hline $\mathrm{H}$ & 1.11122100 & 5.49470400 & 1.33854400 \\
\hline $\mathrm{C}$ & -0.46635900 & 4.31869900 & 2.21925200 \\
\hline $\mathrm{C}$ & -1.02288600 & 3.05275800 & 2.39307500 \\
\hline $\mathrm{C}$ & -2.03821400 & 4.45888300 & 3.77452300 \\
\hline $\mathrm{H}$ & -1.55016000 & 4.37632100 & 4.75683700 \\
\hline $\mathrm{H}$ & -3.02651500 & 4.90983100 & 3.84828900 \\
\hline $\mathrm{C}$ & -1.68869800 & -0.38539900 & 1.39739300 \\
\hline $\mathrm{C}$ & -2.14327500 & -1.59242700 & 1.94415000 \\
\hline
\end{tabular}




$\begin{array}{lrrr}\mathrm{H} & -2.61286800 & -2.31917300 & 1.29414500 \\ \mathrm{C} & -2.02783600 & -1.90587900 & 3.30892400 \\ \mathrm{H} & -2.38708400 & -2.84759800 & 3.70752600 \\ \mathrm{C} & -1.44801900 & -0.94842000 & 4.11095400 \\ \mathrm{C} & -0.98320600 & 0.25139500 & 3.58158300 \\ \mathrm{C} & -1.05848200 & 0.58213600 & 2.23892000 \\ \mathrm{C} & -0.82567900 & 0.36153000 & 5.79681800 \\ \mathrm{H} & 0.00353200 & 0.34744500 & 6.50349100 \\ \mathrm{H} & -1.70244000 & 0.88381500 & 6.20577200 \\ \mathrm{~S} & 1.46032600 & -0.58748000 & -3.54639700 \\ \mathrm{C} & 2.57875700 & -1.96542900 & -3.17033000 \\ \mathrm{C} & 3.73944800 & -1.68497300 & -2.44509200 \\ \mathrm{C} & 2.27529100 & -3.26165500 & -3.57896400 \\ \mathrm{C} & 4.60993500 & -2.72614500 & -2.12771400 \\ \mathrm{H} & 3.95715000 & -0.66993000 & -2.13371100 \\ \mathrm{C} & 3.16156300 & -4.29567400 & -3.26975600 \\ \mathrm{H} & 1.35978300 & -3.43815600 & -4.13254400 \\ \mathrm{C} & 4.32486000 & -4.02981100 & -2.54434700 \\ \mathrm{H} & 5.50788900 & -2.51934100 & -1.55300000 \\ \mathrm{H} & 2.93948900 & -5.31037200 & -3.58868000 \\ \mathrm{H} & 5.00521300 & -4.83946200 & -2.29524000 \\ \mathrm{O} & 0.19162500 & -1.32615200 & -4.15928400 \\ \mathrm{O} & 2.20172700 & 0.27753500 & -4.51719300 \\ \mathrm{H} & -0.57299900 & -0.86768600 & -3.01890200\end{array}$<smiles>O=S(=O)(c1ccccc1)[P+]1PCCCP1</smiles>

Int-3a

Total SCF energy (M06/SDD-6-311++G(d,p)/SMD(1,4-Dioxane)): -5071.679485a.u.

Thermal correction to Gibbs Free Energy at $298.15 \mathrm{~K}: 1.579537 \mathrm{a} . \mathrm{u}$

Gibbs free energy at 298.15 K (M06/SDD-6-311++G(d,p)/SMD(1,4-Dioxane)): -5070.099948a.u.

$\begin{array}{cccc}\mathrm{Pd} & 0.03192900 & 0.04381100 & -1.76505200 \\ \mathrm{P} & 1.53670100 & 0.03290800 & 0.07112400 \\ \mathrm{P} & -1.85346500 & 0.09922500 & -0.42217400 \\ \mathrm{O} & 7.06917300 & 1.88558500 & -1.23975900 \\ \mathrm{O} & 1.18950100 & -4.80575700 & 3.55929100 \\ \mathrm{O} & -3.40509300 & 5.76183200 & 0.64507200 \\ \mathrm{O} & -5.97170800 & -3.17856600 & -3.24368600 \\ \mathrm{O} & -0.32817300 & 4.54403200 & 3.55015300 \\ \mathrm{O} & -1.53104100 & 2.57757200 & 3.62533400 \\ \mathrm{O} & -0.25202900 & -0.03185700 & 4.62439900\end{array}$




\begin{tabular}{|c|c|c|c|}
\hline $\mathrm{O}$ & -1.54071300 & -1.84800100 & 5.21203100 \\
\hline $\mathrm{C}$ & 3.25384000 & 0.46774900 & -0.34818000 \\
\hline $\mathrm{C}$ & 4.37789100 & -0.07646500 & 0.26460700 \\
\hline $\mathrm{H}$ & 4.24394900 & -0.87668700 & 0.97641900 \\
\hline $\mathrm{C}$ & 5.66246200 & 0.38336900 & -0.03909200 \\
\hline $\mathrm{C}$ & 5.78513800 & 1.43749200 & -0.98289900 \\
\hline $\mathrm{C}$ & 4.65564100 & 2.05551800 & -1.57735400 \\
\hline $\mathrm{C}$ & 3.40963900 & 1.49700200 & -1.27580500 \\
\hline $\mathrm{H}$ & 2.52804800 & 1.87921500 & -1.76946500 \\
\hline $\mathrm{C}$ & 6.87672200 & -0.21702300 & 0.70266800 \\
\hline $\mathrm{C}$ & 6.44533900 & -1.31106700 & 1.70109600 \\
\hline $\mathrm{H}$ & 5.76834000 & -0.92314900 & 2.46938100 \\
\hline $\mathrm{H}$ & 7.33316600 & -1.69758100 & 2.21246000 \\
\hline $\mathrm{H}$ & 5.95445900 & -2.15391100 & 1.20161600 \\
\hline $\mathrm{C}$ & 7.87002300 & -0.88270200 & -0.27374600 \\
\hline $\mathrm{H}$ & 7.35531200 & -1.60342600 & -0.91666100 \\
\hline $\mathrm{H}$ & 8.63995600 & -1.42088800 & 0.29192400 \\
\hline $\mathrm{H}$ & 8.37566000 & -0.15345100 & -0.90329900 \\
\hline $\mathrm{C}$ & 7.59036600 & 0.88845600 & 1.5127990 \\
\hline $\mathrm{H}$ & 7.94648600 & 1.68848700 & 0.8626070 \\
\hline $\mathrm{H}$ & 8.44761700 & 0.46514600 & 2.0508380 \\
\hline $\mathrm{H}$ & 6.90746100 & 1.32135600 & 2.2532030 \\
\hline $\mathrm{C}$ & 4.67643700 & 3.30684500 & -2.49040100 \\
\hline $\mathrm{C}$ & 4.88721800 & 2.91027100 & -3.97066700 \\
\hline $\mathrm{H}$ & 5.81387000 & 2.35931000 & -4.13800000 \\
\hline $\mathrm{H}$ & 4.92142000 & 3.81357700 & -4.59231600 \\
\hline $\mathrm{H}$ & 4.05050300 & 2.29397300 & -4.31194500 \\
\hline $\mathrm{C}$ & 3.30907800 & 4.03584000 & -2.44078900 \\
\hline $\mathrm{H}$ & 2.51580600 & 3.45762700 & -2.92150700 \\
\hline $\mathrm{H}$ & 3.39403400 & 4.98232000 & -2.98556800 \\
\hline $\mathrm{H}$ & 3.00911100 & 4.26901100 & -1.41248400 \\
\hline $\mathrm{C}$ & 5.72220700 & 4.34103200 & -2.02003200 \\
\hline $\mathrm{H}$ & 5.51937500 & 4.64275200 & -0.98543200 \\
\hline $\mathrm{H}$ & 5.65254000 & 5.23489600 & -2.65025300 \\
\hline $\mathrm{H}$ & 6.74657700 & 3.97680200 & -2.05427000 \\
\hline $\mathrm{C}$ & 7.65098000 & 1.58950400 & -2.50700900 \\
\hline $\mathrm{H}$ & 7.60459000 & 2.45854100 & -3.16973800 \\
\hline $\mathrm{H}$ & 7.15181000 & 0.74186500 & -2.98411000 \\
\hline $\mathrm{H}$ & 8.70200000 & 1.33969200 & -2.33448600 \\
\hline $\mathrm{C}$ & 1.54310400 & -1.45591000 & 1.11851300 \\
\hline $\mathrm{C}$ & 0.91291000 & -2.60282900 & 0.64397900 \\
\hline $\mathrm{H}$ & 0.45428900 & -2.56127500 & -0.33655800 \\
\hline $\mathrm{C}$ & 0.83498700 & -3.78343400 & 1.39733900 \\
\hline $\mathrm{C}$ & 1.35571600 & -3.72919400 & 2.70897700 \\
\hline
\end{tabular}




\begin{tabular}{|c|c|c|c|}
\hline $\mathrm{C}$ & 2.07695700 & -2.60697800 & 3.20009300 \\
\hline $\mathrm{C}$ & 2.13753100 & -1.47690200 & 2.38351700 \\
\hline $\mathrm{H}$ & 2.63114100 & -0.58107500 & 2.73375000 \\
\hline $\mathrm{C}$ & 2.88919900 & -2.64878800 & 4.51324800 \\
\hline $\mathrm{C}$ & 2.07639000 & -3.10352400 & 5.74456200 \\
\hline $\mathrm{H}$ & 1.13510500 & -2.55294300 & 5.82023400 \\
\hline $\mathrm{H}$ & 2.65501600 & -2.90927000 & 6.65492900 \\
\hline $\mathrm{H}$ & 1.85253800 & -4.16830800 & 5.71190100 \\
\hline $\mathrm{C}$ & 4.06179100 & -3.63364000 & 4.29977700 \\
\hline $\mathrm{H}$ & 3.68827500 & -4.63748100 & 4.07954700 \\
\hline $\mathrm{H}$ & 4.68270300 & -3.68636300 & 5.20256700 \\
\hline $\mathrm{H}$ & 4.69618700 & -3.30797100 & 3.46807700 \\
\hline $\mathrm{C}$ & 3.47145800 & -1.26229100 & 4.85418600 \\
\hline $\mathrm{H}$ & 4.16609900 & -0.90133700 & 4.08958800 \\
\hline $\mathrm{H}$ & 4.03151500 & -1.32872500 & 5.79259300 \\
\hline $\mathrm{H}$ & 2.67928700 & -0.51533700 & 4.98186900 \\
\hline $\mathrm{C}$ & 0.21323800 & -5.02253300 & 0.69935600 \\
\hline $\mathrm{C}$ & -1.30283200 & -4.80449700 & 0.50282100 \\
\hline $\mathrm{H}$ & -1.82255200 & -4.70451300 & 1.46078100 \\
\hline $\mathrm{H}$ & -1.73850700 & -5.65569400 & -0.03382400 \\
\hline $\mathrm{H}$ & -1.50718000 & -3.90033500 & -0.07929600 \\
\hline $\mathrm{C}$ & 0.86206600 & -5.17676400 & -0.69824300 \\
\hline $\mathrm{H}$ & 0.69687300 & -4.31419100 & -1.34459900 \\
\hline $\mathrm{H}$ & 0.43302900 & -6.04563500 & -1.20849600 \\
\hline $\mathrm{H}$ & 1.94135100 & -5.33587100 & -0.60837900 \\
\hline $\mathrm{C}$ & 0.44664400 & -6.37709700 & 1.40231100 \\
\hline $\mathrm{H}$ & 1.49969100 & -6.52509800 & 1.65379600 \\
\hline $\mathrm{H}$ & 0.14596400 & -7.17399200 & 0.71241700 \\
\hline $\mathrm{H}$ & -0.13979100 & -6.50434500 & 2.31126700 \\
\hline $\mathrm{C}$ & -0.10277400 & -4.85666700 & 4.16995600 \\
\hline $\mathrm{H}$ & -0.27811100 & -3.98436500 & 4.80472200 \\
\hline $\mathrm{H}$ & -0.11940600 & -5.76544300 & 4.77587700 \\
\hline $\mathrm{H}$ & -0.89684800 & -4.90548500 & 3.41891600 \\
\hline $\mathrm{C}$ & -2.43285200 & 1.79942500 & -0.15957100 \\
\hline $\mathrm{C}$ & -3.46705800 & 2.11617600 & 0.72252700 \\
\hline $\mathrm{H}$ & -3.95783000 & 1.31727100 & 1.26030800 \\
\hline $\mathrm{C}$ & -3.85524300 & 3.43750400 & 0.93999200 \\
\hline $\mathrm{C}$ & -3.09278100 & 4.45806500 & 0.30762500 \\
\hline $\mathrm{C}$ & -2.10133600 & 4.16917100 & -0.66096200 \\
\hline $\mathrm{C}$ & -1.78892800 & 2.81815400 & -0.85429900 \\
\hline $\mathrm{H}$ & -1.01693200 & 2.53361700 & -1.55713100 \\
\hline $\mathrm{C}$ & -3.16414300 & -0.89309900 & -1.20489000 \\
\hline $\mathrm{C}$ & -4.51078200 & -0.79158900 & -0.87266900 \\
\hline $\mathrm{H}$ & -4.81662200 & -0.06082400 & -0.13628100 \\
\hline
\end{tabular}




\begin{tabular}{|c|c|c|c|}
\hline $\mathrm{C}$ & -5.48299200 & -1.61203700 & -1.46186300 \\
\hline $\mathrm{C}$ & -5.04642600 & -2.48912300 & -2.48215300 \\
\hline $\mathrm{C}$ & -3.67212400 & -2.70322300 & -2.76127900 \\
\hline $\mathrm{C}$ & -2.75775800 & -1.87471600 & -2.11284100 \\
\hline $\mathrm{H}$ & -1.70417000 & -1.94922300 & -2.34482500 \\
\hline $\mathrm{C}$ & -5.10588000 & 3.73627600 & 1.79446300 \\
\hline $\mathrm{C}$ & -6.08501300 & 4.62501200 & 0.99422200 \\
\hline $\mathrm{H}$ & -5.63606100 & 5.58351200 & 0.73435600 \\
\hline $\mathrm{H}$ & -6.98917600 & 4.80862400 & 1.58714200 \\
\hline $\mathrm{H}$ & -6.38558300 & 4.12168900 & 0.06809000 \\
\hline $\mathrm{C}$ & -5.86668700 & 2.44232000 & 2.15293100 \\
\hline $\mathrm{H}$ & -6.16756600 & 1.88464300 & 1.25907300 \\
\hline $\mathrm{H}$ & -6.77811900 & 2.70504800 & 2.70026900 \\
\hline $\mathrm{H}$ & -5.27967000 & 1.77747300 & 2.79632300 \\
\hline $\mathrm{C}$ & -4.73372900 & 4.41371800 & 3.12811500 \\
\hline $\mathrm{H}$ & -4.06937200 & 3.76037300 & 3.70278600 \\
\hline $\mathrm{H}$ & -5.63659300 & 4.58773700 & 3.72573000 \\
\hline $\mathrm{H}$ & -4.24340100 & 5.37518300 & 2.97831200 \\
\hline $\mathrm{C}$ & -1.34138500 & 5.20612800 & -1.52209200 \\
\hline $\mathrm{C}$ & -0.92723800 & 4.57800800 & -2.87761100 \\
\hline $\mathrm{H}$ & -0.15873300 & 3.80638900 & -2.77955200 \\
\hline $\mathrm{H}$ & -0.50802600 & 5.36063800 & -3.51838100 \\
\hline $\mathrm{H}$ & -1.78585500 & 4.13806600 & -3.39584900 \\
\hline $\mathrm{C}$ & -0.03701200 & 5.62412300 & -0.80678200 \\
\hline $\mathrm{H}$ & -0.21802200 & 6.08771100 & 0.16548700 \\
\hline $\mathrm{H}$ & 0.51974300 & 6.33730100 & -1.42645300 \\
\hline $\mathrm{H}$ & 0.59856400 & 4.74903900 & -0.64125200 \\
\hline $\mathrm{C}$ & -2.20149500 & 6.43836100 & -1.87502600 \\
\hline $\mathrm{H}$ & -3.12516700 & 6.12862700 & -2.37628500 \\
\hline $\mathrm{H}$ & -1.64161900 & 7.07727800 & -2.56702100 \\
\hline $\mathrm{H}$ & -2.48405000 & 7.03847800 & -1.01295200 \\
\hline $\mathrm{C}$ & -2.49958300 & 6.44329900 & 1.51418500 \\
\hline $\mathrm{H}$ & -3.10097800 & 7.05599800 & 2.19256400 \\
\hline $\mathrm{H}$ & -1.83860900 & 7.10483300 & 0.94513400 \\
\hline $\mathrm{H}$ & -1.89222900 & 5.74254700 & 2.09406300 \\
\hline $\mathrm{C}$ & -6.91764500 & -1.50094800 & -0.88883600 \\
\hline $\mathrm{C}$ & -7.52946000 & -0.13658300 & -1.27437100 \\
\hline $\mathrm{H}$ & -6.90833100 & 0.69441600 & -0.92245600 \\
\hline $\mathrm{H}$ & -8.52574800 & -0.02716400 & -0.82960500 \\
\hline $\mathrm{H}$ & -7.63122100 & -0.03541300 & -2.36001900 \\
\hline $\mathrm{C}$ & -6.82429200 & -1.58067100 & 0.65830000 \\
\hline $\mathrm{H}$ & -6.36778700 & -2.52699500 & 0.96943700 \\
\hline $\mathrm{H}$ & -7.82976400 & -1.52927200 & 1.09049200 \\
\hline $\mathrm{H}$ & -6.24083200 & -0.76634000 & 1.09531800 \\
\hline
\end{tabular}




\begin{tabular}{|c|c|c|c|}
\hline $\mathrm{C}$ & -7.88608200 & -2.62896900 & -1.30134300 \\
\hline $\mathrm{H}$ & -8.19787100 & -2.57202700 & -2.34359400 \\
\hline $\mathrm{H}$ & -8.79060800 & -2.54703400 & -0.68728700 \\
\hline $\mathrm{H}$ & -7.44897500 & -3.61660400 & -1.13352400 \\
\hline $\mathrm{C}$ & -3.15948300 & -3.83003400 & -3.68277600 \\
\hline $\mathrm{C}$ & -3.60821800 & -3.64213500 & -5.14786000 \\
\hline $\mathrm{H}$ & -4.67800200 & -3.81286900 & -5.27028500 \\
\hline $\mathrm{H}$ & -3.08289500 & -4.36279600 & -5.78490900 \\
\hline $\mathrm{H}$ & -3.35987300 & -2.63802600 & -5.50876700 \\
\hline $\mathrm{C}$ & -1.61714200 & -3.89276900 & -3.68525500 \\
\hline $\mathrm{H}$ & -1.14879100 & -2.99106700 & -4.09494800 \\
\hline $\mathrm{H}$ & -1.30050200 & -4.73823700 & -4.30511100 \\
\hline $\mathrm{H}$ & -1.21933900 & -4.06254500 & -2.67884200 \\
\hline $\mathrm{C}$ & -3.68481000 & -5.18182900 & -3.14797400 \\
\hline $\mathrm{H}$ & -3.31917200 & -6.00024400 & -3.77963600 \\
\hline $\mathrm{H}$ & -4.77646400 & -5.21000900 & -3.14218300 \\
\hline $\mathrm{H}$ & -3.32542500 & -5.35734800 & -2.12703500 \\
\hline $\mathrm{C}$ & -6.55908800 & -2.37907500 & -4.27195100 \\
\hline $\mathrm{H}$ & -7.14493100 & -1.55348000 & -3.85430200 \\
\hline $\mathrm{H}$ & -7.21756000 & -3.04170700 & -4.83807400 \\
\hline $\mathrm{H}$ & -5.79264800 & -1.96569800 & -4.93561900 \\
\hline $\mathrm{C}$ & -0.12977200 & 1.30173900 & 2.02688300 \\
\hline $\mathrm{C}$ & 1.03390600 & 1.41078100 & 1.2074460 \\
\hline $\mathrm{C}$ & 1.75322600 & 2.61244500 & 1.1865340 \\
\hline $\mathrm{H}$ & 2.64614900 & 2.68291500 & 0.5817760 \\
\hline $\mathrm{C}$ & 1.38196000 & 3.74045000 & 1.9365970 \\
\hline $\mathrm{H}$ & 1.95969300 & 4.65683500 & 1.9045220 \\
\hline $\mathrm{C}$ & 0.25884800 & 3.61449300 & 2.7206060 \\
\hline $\mathrm{C}$ & -0.46727800 & 2.42820900 & 2.76055000 \\
\hline $\mathrm{C}$ & -1.25162400 & 3.78469400 & 4.33932800 \\
\hline $\mathrm{H}$ & -0.78590900 & 3.53735000 & 5.30415800 \\
\hline $\mathrm{H}$ & -2.17051900 & 4.35495000 & 4.46611800 \\
\hline $\mathrm{C}$ & -1.77237900 & -0.56944800 & 1.28852100 \\
\hline $\mathrm{C}$ & -2.52190000 & -1.69440300 & 1.64937000 \\
\hline $\mathrm{H}$ & -3.14682800 & -2.17291400 & 0.90692700 \\
\hline $\mathrm{C}$ & -2.51741500 & -2.21947600 & 2.95400100 \\
\hline $\mathrm{H}$ & -3.11878500 & -3.08089800 & 3.22082000 \\
\hline $\mathrm{C}$ & -1.73018500 & -1.56764200 & 3.87734400 \\
\hline $\mathrm{C}$ & -0.95894500 & -0.46327300 & 3.52275600 \\
\hline $\mathrm{C}$ & -0.93929700 & 0.07358900 & 2.24863100 \\
\hline $\mathrm{C}$ & -0.88036600 & -0.68294300 & 5.72854700 \\
\hline $\mathrm{H}$ & -0.12472300 & -0.98397300 & 6.45269700 \\
\hline $\mathrm{H}$ & -1.63015600 & -0.01195400 & 6.17169100 \\
\hline $\mathrm{S}$ & 1.44918800 & -0.21744000 & -3.65462200 \\
\hline
\end{tabular}




$\begin{array}{lrrr}\mathrm{C} & 2.89543700 & -1.19917500 & -3.12326600 \\ \mathrm{C} & 4.18659700 & -0.80757100 & -3.46113200 \\ \mathrm{C} & 2.66927400 & -2.36573600 & -2.39936800 \\ \mathrm{C} & 5.26400400 & -1.59467800 & -3.05209700 \\ \mathrm{H} & 4.33010100 & 0.10394600 & -4.02796000 \\ \mathrm{C} & 3.74769300 & -3.13486400 & -1.96718000 \\ \mathrm{H} & 1.64982700 & -2.65939500 & -2.17882600 \\ \mathrm{C} & 5.04898600 & -2.74880700 & -2.29616400 \\ \mathrm{H} & 6.27730000 & -1.30640900 & -3.31772600 \\ \mathrm{H} & 3.57525700 & -4.03446600 & -1.38513500 \\ \mathrm{H} & 5.89289700 & -3.35028300 & -1.96948600 \\ \mathrm{O} & 0.78555800 & -1.09991700 & -4.66206700 \\ \mathrm{O} & 2.02790400 & 1.08006200 & -4.14036900 \\ \mathrm{H} & -1.03241300 & 0.29156700 & -2.92469300\end{array}$<smiles>O[SH](O[Pb](O)c1ccccc1)P1PCCPP1</smiles>

Total SCF energy (M06/SDD-6-311++G(d,p)/SMD(1,4-Dioxane)): -5071.673759a.u.

Thermal correction to Gibbs Free Energy at $298.15 \mathrm{~K}: 1.576317 \mathrm{a} . \mathrm{u}$

Gibbs free energy at $298.15 \mathrm{~K}$ (M06/SDD-6-311++G(d,p)/SMD(1,4-Dioxane)): -5070.097442a.u.

$\begin{array}{rrrr}\mathrm{Pd} & 0.00231000 & -0.19034800 & -1.60728900 \\ \mathrm{P} & -1.95196500 & -0.12172100 & -0.39915300 \\ \mathrm{P} & 1.62250200 & 0.36844100 & -0.05757900 \\ \mathrm{O} & -6.93285400 & -3.09650300 & -1.93321300 \\ \mathrm{O} & -3.01020100 & 5.77737500 & -0.31001000 \\ \mathrm{O} & 2.15187500 & -4.12480500 & 3.87579000 \\ \mathrm{O} & 7.09823400 & 1.65910700 & -2.10565300 \\ \mathrm{O} & -0.93446300 & -1.45697300 & 5.35219600 \\ \mathrm{O} & 0.05413500 & 0.44220600 & 4.51373200 \\ \mathrm{O} & -1.55822400 & 2.72542500 & 3.51058200 \\ \mathrm{O} & -0.62710000 & 4.81434000 & 3.27030600 \\ \mathrm{C} & -3.51425200 & -1.02191700 & -0.76294700 \\ \mathrm{C} & -4.77687800 & -0.63348800 & -0.30247500 \\ \mathrm{H} & -4.85356000 & 0.24986000 & 0.31244900 \\ \mathrm{C} & -5.93075800 & -1.34271700 & -0.63761200 \\ \mathrm{C} & -5.77892300 & -2.48296900 & -1.47165500 \\ \mathrm{C} & -4.51315900 & -2.99502200 & -1.82218300 \\ \mathrm{C} & -3.40436900 & -2.19726900 & -1.49905700 \\ \mathrm{H} & -2.42255900 & -2.49794100 & -1.84039100 \\ \mathrm{C} & -7.29451100 & -0.93995500 & -0.03590800\end{array}$




\begin{tabular}{|c|c|c|c|}
\hline $\mathrm{C}$ & -7.15028200 & 0.24959900 & 0.93365300 \\
\hline $\mathrm{H}$ & -6.45592200 & 0.03031500 & 1.75181800 \\
\hline $\mathrm{H}$ & -8.12635200 & 0.47015900 & 1.37850800 \\
\hline $\mathrm{H}$ & -6.80751300 & 1.15698900 & 0.42384000 \\
\hline $\mathrm{C}$ & -8.31771100 & -0.51954900 & -1.11358900 \\
\hline $\mathrm{H}$ & -7.89258300 & 0.23228800 & -1.78837200 \\
\hline $\mathrm{H}$ & -9.19871500 & -0.07768200 & -0.63286900 \\
\hline $\mathrm{H}$ & -8.65664600 & -1.37107600 & -1.70248400 \\
\hline $\mathrm{C}$ & -7.85680700 & -2.13149000 & 0.77258300 \\
\hline $\mathrm{H}$ & -8.00501000 & -3.00499500 & 0.13513500 \\
\hline $\mathrm{H}$ & -8.82067600 & -1.86007200 & 1.22061700 \\
\hline $\mathrm{H}$ & -7.17124000 & -2.40322200 & 1.58346200 \\
\hline $\mathrm{C}$ & -4.21746700 & -4.35849600 & -2.49277400 \\
\hline $\mathrm{C}$ & -3.72465600 & -4.13561500 & -3.93973900 \\
\hline $\mathrm{H}$ & -4.48694600 & -3.64660300 & -4.55572300 \\
\hline $\mathrm{H}$ & -3.48000500 & -5.09745600 & -4.40686400 \\
\hline $\mathrm{H}$ & -2.82475000 & -3.51297400 & -3.95659400 \\
\hline $\mathrm{C}$ & -3.07758600 & -5.04953300 & -1.69737000 \\
\hline $\mathrm{H}$ & -2.13444900 & -4.50259200 & -1.74984000 \\
\hline $\mathrm{H}$ & -2.89591800 & -6.04747700 & -2.11250600 \\
\hline $\mathrm{H}$ & -3.35157800 & -5.16478500 & -0.64265500 \\
\hline $\mathrm{C}$ & -5.39355900 & -5.35702200 & -2.49236400 \\
\hline $\mathrm{H}$ & -5.82693900 & -5.46643000 & -1.49428600 \\
\hline $\mathrm{H}$ & -5.01423000 & -6.33606000 & -2.80784900 \\
\hline $\mathrm{H}$ & -6.19560900 & -5.08559800 & -3.17798100 \\
\hline $\mathrm{C}$ & -7.23678900 & -2.77289000 & -3.28940500 \\
\hline $\mathrm{H}$ & -6.47727800 & -3.15886800 & -3.97665500 \\
\hline $\mathrm{H}$ & -7.31697500 & -1.68911000 & -3.42874200 \\
\hline $\mathrm{H}$ & -8.19793900 & -3.24321300 & -3.51103500 \\
\hline $\mathrm{C}$ & -2.41869800 & 1.65166800 & -0.34058700 \\
\hline $\mathrm{C}$ & -1.91147400 & 2.44587500 & -1.36344500 \\
\hline $\mathrm{H}$ & -1.29894000 & 1.96033200 & -2.11627500 \\
\hline $\mathrm{C}$ & -2.12974200 & 3.82684600 & -1.43251700 \\
\hline $\mathrm{C}$ & -2.86843000 & 4.40165500 & -0.37871700 \\
\hline $\mathrm{C}$ & -3.49685500 & 3.61678200 & 0.62579900 \\
\hline $\mathrm{C}$ & -3.22371400 & 2.24585300 & 0.63634300 \\
\hline $\mathrm{H}$ & -3.61429100 & 1.62781800 & 1.43271100 \\
\hline $\mathrm{C}$ & -4.57142200 & 4.19573400 & 1.57463600 \\
\hline $\mathrm{C}$ & -4.14701900 & 5.47439200 & 2.33003600 \\
\hline $\mathrm{H}$ & -3.16770700 & 5.35724400 & 2.79871500 \\
\hline $\mathrm{H}$ & -4.87772000 & 5.68960600 & 3.11866500 \\
\hline $\mathrm{H}$ & -4.10605900 & 6.33775000 & 1.66774000 \\
\hline $\mathrm{C}$ & -5.80475900 & 4.53311600 & 0.70422200 \\
\hline $\mathrm{H}$ & -5.55175000 & 5.28421400 & -0.04951900 \\
\hline
\end{tabular}


$\mathrm{H}$

$\mathrm{H}$

C

$\mathrm{H}$

$\mathrm{H}$

$\mathrm{H}$

C

C

$\mathrm{H}$

$\mathrm{H}$

$\mathrm{H}$

C

$\mathrm{H}$

$\mathrm{H}$

$\mathrm{H}$

C

$\mathrm{H}$

$\mathrm{H}$

$\mathrm{H}$

C

$\mathrm{H}$

$\mathrm{H}$

$\mathrm{H}$

C

C

$\mathrm{H}$

C

C

C

C

$\mathrm{H}$

C

C

$\mathrm{H}$

C

C

C

C

$\mathrm{H}$

C

C

$\mathrm{H}$

$\mathrm{H}$

$\mathrm{H}$

\begin{tabular}{rrr}
-6.61531800 & 4.92921000 & 1.32879000 \\
-6.17496400 & 3.63869400 & 0.19061100 \\
-4.99763300 & 3.16288000 & 2.63757000 \\
-5.45473000 & 2.27436600 & 2.19291700 \\
-5.74655800 & 3.61390700 & 3.29727800 \\
-4.14792700 & 2.84166800 & 3.24932700 \\
-1.53035200 & 4.55110000 & -2.66208500 \\
0.00898000 & 4.56929000 & -2.52475700 \\
0.32630500 & 5.12275300 & -1.63433100 \\
0.46594900 & 5.04072000 & -3.40321000 \\
0.40721700 & 3.55302200 & -2.43728300 \\
-1.89918400 & 3.74777100 & -3.93692200 \\
-1.49491000 & 2.73275100 & -3.93649200 \\
-1.49725600 & 4.25844800 & -4.81924300 \\
-2.98682300 & 3.67862800 & -4.04873700 \\
-2.04207400 & 5.98456700 & -2.91337800 \\
-3.13420500 & 6.02797500 & -2.90945000 \\
-1.69122100 & 6.30637100 & -3.90108300 \\
-1.67338300 & 6.71045200 & -2.18945400 \\
-1.88625800 & 6.42432500 & 0.29174200 \\
-1.73265300 & 6.08416200 & 1.31941000 \\
-2.10722700 & 7.49447800 & 0.28532600 \\
-0.96917800 & 6.23892500 & -0.27676400 \\
1.86837400 & -0.97034400 & 1.16786800 \\
2.40799900 & -0.76816800 & 2.44211400 \\
2.68661000 & 0.23698800 & 2.72790700 \\
2.56795100 & -1.82141700 & 3.34574300 \\
2.10420400 & -3.10334400 & 2.94327800 \\
1.63474500 & -3.36214100 & 1.63836400 \\
1.51385000 & -2.25815800 & 0.78369800 \\
1.11170100 & -2.38745800 & -0.21311500 \\
3.33190100 & 0.91383000 & -0.47377500 \\
4.47821700 & 0.52215000 & 0.20777100 \\
4.36933800 & -0.06513200 & 1.10880500 \\
5.77040300 & 0.83114500 & -0.25013600 \\
5.86713000 & 1.50189800 & -1.48746600 \\
4.72610200 & 2.03313800 & -2.14759500 \\
3.47687100 & 1.69625600 & -1.62721200 \\
2.57511700 & 2.01055800 & -2.13984000 \\
3.37172600 & -1.59849000 & 4.64762100 \\
4.74395100 & -2.28540500 & 4.45328300 \\
5.27359600 & -1.86450700 & 3.59118100 \\
\hline
\end{tabular}




\begin{tabular}{|c|c|c|c|}
\hline $\mathrm{C}$ & 3.60874300 & -0.09690700 & 4.90948500 \\
\hline $\mathrm{H}$ & 4.20834800 & 0.37568000 & 4.1259900 \\
\hline $\mathrm{H}$ & 4.15801100 & 0.02268400 & 5.8492900 \\
\hline $\mathrm{H}$ & 2.66262100 & 0.44903200 & 4.9897120 \\
\hline $\mathrm{C}$ & 2.71720400 & -2.17634700 & 5.92186200 \\
\hline $\mathrm{H}$ & 1.66890500 & -1.88082100 & 6.00347800 \\
\hline $\mathrm{H}$ & 3.24544400 & -1.79695100 & 6.80441300 \\
\hline $\mathrm{H}$ & 2.76753100 & -3.26357700 & 5.94369800 \\
\hline $\mathrm{C}$ & 1.24288600 & -4.74055100 & 1.05163900 \\
\hline $\mathrm{C}$ & 1.81977500 & -4.85252300 & -0.38210500 \\
\hline $\mathrm{H}$ & 1.36471200 & -4.15159300 & -1.08262600 \\
\hline $\mathrm{H}$ & 1.63200400 & -5.85895200 & -0.77324600 \\
\hline $\mathrm{H}$ & 2.90052800 & -4.67909700 & -0.38755400 \\
\hline $\mathrm{C}$ & -0.29669000 & -4.82855700 & 0.95369800 \\
\hline $\mathrm{H}$ & -0.77073900 & -4.77896100 & 1.93904600 \\
\hline $\mathrm{H}$ & -0.59541900 & -5.76996000 & 0.47699600 \\
\hline $\mathrm{H}$ & -0.69945800 & -4.00338800 & 0.35775900 \\
\hline $\mathrm{C}$ & 1.79009600 & -5.96386900 & 1.81721600 \\
\hline $\mathrm{H}$ & 2.86733800 & -5.87921100 & 1.98650400 \\
\hline $\mathrm{H}$ & 1.61057800 & -6.85870800 & 1.20975200 \\
\hline $\mathrm{H}$ & 1.31179000 & -6.12736300 & 2.78144300 \\
\hline $\mathrm{C}$ & 0.89904000 & -4.36657300 & 4.52133200 \\
\hline $\mathrm{H}$ & 1.08702100 & -5.13087700 & 5.27934400 \\
\hline $\mathrm{H}$ & 0.15097900 & -4.73658400 & 3.81348000 \\
\hline $\mathrm{H}$ & 0.51296000 & -3.46119800 & 4.99640300 \\
\hline $\mathrm{C}$ & 6.94648400 & 0.40690600 & 0.6627570 \\
\hline $\mathrm{C}$ & 7.07792900 & -1.13248000 & 0.64934100 \\
\hline $\mathrm{H}$ & 6.14538300 & -1.61411200 & 0.96226300 \\
\hline $\mathrm{H}$ & 7.87294000 & -1.45345300 & 1.33302100 \\
\hline $\mathrm{H}$ & 7.32434900 & -1.50592000 & -0.35097700 \\
\hline $\mathrm{C}$ & 6.62362900 & 0.85660600 & 2.1111220 \\
\hline $\mathrm{H}$ & 6.48391900 & 1.94200500 & 2.1589820 \\
\hline $\mathrm{H}$ & 7.45390300 & 0.58850800 & 2.7738590 \\
\hline $\mathrm{H}$ & 5.72448300 & 0.38314800 & 2.5100270 \\
\hline $\mathrm{C}$ & 8.31127000 & 1.04436700 & 0.3280840 \\
\hline $\mathrm{H}$ & 8.76213800 & 0.65472900 & -0.58380700 \\
\hline $\mathrm{H}$ & 9.00358300 & 0.82716800 & 1.1498980 \\
\hline $\mathrm{H}$ & 8.23280500 & 2.13009400 & 0.2278600 \\
\hline $\mathrm{C}$ & 4.83277600 & 2.98058500 & -3.36103200 \\
\hline $\mathrm{C}$ & 5.42478600 & 2.27913900 & -4.60295100 \\
\hline $\mathrm{H}$ & 6.48990600 & 2.08037600 & -4.48640200 \\
\hline $\mathrm{H}$ & 5.30354300 & 2.92266700 & -5.48219800 \\
\hline $\mathrm{H}$ & 4.90686800 & 1.33449500 & -4.80570400 \\
\hline $\mathrm{C}$ & 3.44930800 & 3.52722000 & -3.76643000 \\
\hline
\end{tabular}


$\mathrm{H}$

$\mathrm{H}$

$\mathrm{H}$

C

$\mathrm{H}$

$\mathrm{H}$

$\mathrm{H}$

$\mathrm{C}$

$\mathrm{H}$

$\mathrm{H}$

$\mathrm{H}$

C

C

C

$\mathrm{H}$

C

$\mathrm{H}$

C

C

C

$\mathrm{H}$

$\mathrm{H}$

C

C

$\mathrm{H}$

C

$\mathrm{H}$

C

C

C

C

$\mathrm{H}$

$\mathrm{H}$

$\mathrm{O}$

$\mathrm{S}$

C

C

C

C

$\mathrm{H}$

C

$\mathrm{H}$

C

$\mathrm{H}$
2.77947400

3.57641400

2.95374100

5.71450900

5.79065600

6.72049300

5.27267200

7.54045200

7.73415800

8.47025700

6.80295400

$-0.80665700$

$-1.65699500$

$-2.22545200$

$-2.86063900$

$-2.03515100$

$-2.49731500$

$-1.26415200$

$-0.67164700$

$-0.42714700$

$-1.24670000$

0.39322500

0.96002800

1.49933600

2.33734800

1.02925800

1.47465600

0.00398200

$-0.55600000$

$-0.14401700$

$-1.32823700$

$-2.28210600$

$-0.69674300$

0.37687500

0.57724900

2.25743700

2.75784600

3.01672800

4.04328800

2.14616900

4.29686200

2.59840000

4.80579900

4.44383100
2.73937200$$
4.24942800 \quad-4.57980700
$$

$4.04433300 \quad-2.93857500$

$4.19092300 \quad-2.97818200$

$4.88124600 \quad-3.82703700$

$3.87470600 \quad-2.69704500$

$4.73760100-2.13711100$

$0.48854200 \quad-2.79103500$

$-0.33663600 \quad-2.09751800$

$0.75909700 \quad-3.29706700$

$0.15775600 \quad-3.52997200$

0.25676200

2.19691600

$-0.54373600$

$-1.71583300$

1.37336200

1.89281200

$-2.31576900$

1.25227300

$-2.13786600$

3.21905100

$-3.04149800$

3.60034200

$-1.32041000$

4.01636400

$-0.16971000$

3.50898300

$-0.16550200$

5.71025600

0.44121700

6.12350000

$-0.27751400$

6.41741200

1.75599800

0.96597100

3.04110400

0.81395100

3.18137600

0.14210600

4.15833500

1.52379100

5.13926200

1.40098100

3.92988800

2.41567500

2.66593000

2.56129600

1.54970600

1.85008100

3.96139500

4.18374800

4.41452500

4.44904900

3.78330400

5.06696800

$-0.71743800$

$-4.02081500$

$-2.20927600$

$-4.13955900$

$-2.50233500$

$-3.53594200$

$-3.80502600$

$-3.55281500$

$-1.43098000$

$-3.07343400$

$-4.03975200$

$-3.06609300$

$-4.62586700$

$-3.91749100$

$-1.67781000$

$-2.57635400$

$-0.43248300$

$-3.08527700$

$-2.97764300$

$-2.57058000$

$-5.04910700$

$-3.06254600$ 


$\begin{array}{lrrr}\mathrm{H} & 4.88463400 & -0.85624600 & -2.18766300 \\ \mathrm{H} & 5.80200800 & -3.16452000 & -2.17904800 \\ \mathrm{O} & -0.26336000 & -2.91870500 & -2.88966700 \\ \mathrm{H} & -0.25896800 & -2.23804900 & -2.13697500\end{array}$

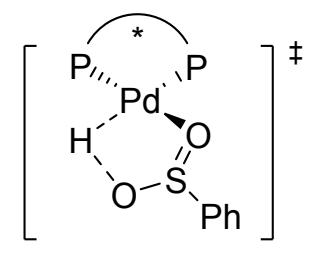

TS-2b

Total SCF energy (M06/SDD-6-311++G(d,p)/SMD(1,4-Dioxane)): -5071.660894a.u.

Thermal correction to Gibbs Free Energy at $298.15 \mathrm{~K}$ : 1.571322a.u

Gibbs free energy at 298.15 K (M06/SDD-6-311++G(d,p)/SMD(1,4-Dioxane)): -5070.089572a.u.

$\mathrm{Pd}$

$P$

$P$

$\mathrm{O}$

$\mathrm{O}$

$\mathrm{O}$

$\mathrm{O}$

$\mathrm{O}$

O

O

O

C

C

$\mathrm{H}$

C

C

C

C

$\mathrm{H}$

C

C

$\mathrm{H}$

$\mathrm{H}$

$\mathrm{H}$

C

$\mathrm{H}$

$\mathrm{H}$

$\mathrm{H}$

C

$\mathrm{H}$

$\mathrm{H}$

$\begin{array}{ccc}-0.08925200 & -0.68818100 & -1.34500200 \\ 1.85179400 & -0.02252600 & -0.08205900 \\ -1.65190800 & 0.16413900 & 0.03837100 \\ 7.07847300 & -0.06355400 & -2.99755300 \\ 2.18569200 & -3.35229300 & 4.88853400 \\ -2.08384900 & 5.88163500 & -1.65840100 \\ -6.96032100 & -2.52389800 & -0.44603900 \\ 0.81846200 & 5.60657100 & 1.65708700 \\ -0.30935800 & 3.92745000 & 2.75473000 \\ 1.08425000 & 1.69762400 & 4.34172600 \\ -0.04910100 & 0.32137200 & 5.80077000 \\ 3.49051400 & 0.02754100 & -0.88980800 \\ 4.70365300 & 0.26997200 & -0.23631600 \\ 4.69491800 & 0.43423900 & 0.83077000 \\ 5.91292000 & 0.29367300 & -0.93062000 \\ 5.86831900 & 0.05616700 & -2.33301700 \\ 4.65462600 & -0.03992700 & -3.04275800 \\ 3.48203800 & -0.10693800 & -2.27448600 \\ 2.53266500 & -0.27527900 & -2.76580700 \\ 7.22156500 & 0.66598500 & -0.20043900 \\ 6.96506400 & 0.98166900 & 1.28677900 \\ 6.24676900 & 1.79806200 & 1.41788900 \\ 7.90575300 & 1.29081700 & 1.75448700 \\ 6.59720900 & 0.10588400 & 1.83372500 \\ 8.26990700 & -0.46731700 & -0.24238000 \\ 7.83552700 & -1.41813800 & 0.08736000 \\ 9.09974200 & -0.22675800 & 0.43291000 \\ 8.68523200 & -0.59653600 & -1.24100300 \\ 7.81109100 & 1.93801800 & -0.85143400 \\ 8.03667000 & 1.77107700 & -1.90630300 \\ 8.73673000 & 2.22868400 & -0.33938300 \\ & & \end{array}$




\begin{tabular}{|c|c|c|c|}
\hline $\mathrm{H}$ & 7.10454300 & 2.77231700 & -0.77356300 \\
\hline $\mathrm{C}$ & 4.46676700 & -0.04668200 & -4.57907200 \\
\hline $\mathrm{C}$ & 4.03596400 & -1.45612000 & -5.04354200 \\
\hline $\mathrm{H}$ & 4.80315300 & -2.20716000 & -4.82507500 \\
\hline $\mathrm{H}$ & 3.86273400 & -1.45838300 & -6.12674700 \\
\hline $\mathrm{H}$ & 3.10803100 & -1.76118100 & -4.54967600 \\
\hline $\mathrm{C}$ & 3.32040900 & 0.94070700 & -4.93076200 \\
\hline $\mathrm{H}$ & 2.35555600 & 0.62392400 & -4.52883600 \\
\hline $\mathrm{H}$ & 3.21601200 & 1.00234400 & -6.01998000 \\
\hline $\mathrm{H}$ & 3.54021900 & 1.94583600 & -4.55321900 \\
\hline $\mathrm{C}$ & 5.69285800 & 0.42279300 & -5.38842500 \\
\hline $\mathrm{H}$ & 6.07608500 & 1.37785800 & -5.01761400 \\
\hline $\mathrm{H}$ & 5.38573000 & 0.56186700 & -6.43152200 \\
\hline $\mathrm{H}$ & 6.51588400 & -0.29132300 & -5.38684200 \\
\hline $\mathrm{C}$ & 7.42532100 & -1.41446400 & -3.29884200 \\
\hline $\mathrm{H}$ & 6.72311000 & -1.86315800 & -4.00818100 \\
\hline $\mathrm{H}$ & 7.44812000 & -2.02830500 & -2.39141400 \\
\hline $\mathrm{H}$ & 8.42193500 & -1.38470900 & -3.74601100 \\
\hline $\mathrm{C}$ & 2.04650700 & -0.98220700 & 1.46125500 \\
\hline $\mathrm{C}$ & 1.36396800 & -2.19421400 & 1.50187600 \\
\hline $\mathrm{H}$ & 0.76341600 & -2.45292700 & 0.63779300 \\
\hline $\mathrm{C}$ & 1.40842700 & -3.05216000 & 2.60766100 \\
\hline $\mathrm{C}$ & 2.14938600 & -2.60450900 & 3.72387800 \\
\hline $\mathrm{C}$ & 2.91673600 & -1.40787700 & 3.69786100 \\
\hline $\mathrm{C}$ & 2.82462400 & -0.60558200 & 2.55780900 \\
\hline $\mathrm{H}$ & 3.34122100 & 0.34298800 & 2.52472300 \\
\hline $\mathrm{C}$ & 3.94156000 & -1.05325100 & 4.79982000 \\
\hline $\mathrm{C}$ & 3.37760100 & -1.09752400 & 6.23810900 \\
\hline $\mathrm{H}$ & 2.41393900 & -0.58734300 & 6.30974900 \\
\hline $\mathrm{H}$ & 4.07730800 & -0.59967100 & 6.91950900 \\
\hline $\mathrm{H}$ & 3.24660700 & -2.12017100 & 6.58782300 \\
\hline $\mathrm{C}$ & 5.09911400 & -2.07295800 & 4.69442600 \\
\hline $\mathrm{H}$ & 4.73268600 & -3.09056400 & 4.85534200 \\
\hline $\mathrm{H}$ & 5.86654200 & -1.85774800 & 5.44856300 \\
\hline $\mathrm{H}$ & 5.56875200 & -2.02565600 & 3.70537000 \\
\hline $\mathrm{C}$ & 4.52279700 & 0.36018700 & 4.58914600 \\
\hline $\mathrm{H}$ & 5.07753900 & 0.44699500 & 3.65012800 \\
\hline $\mathrm{H}$ & 5.22514100 & 0.58469000 & 5.39868300 \\
\hline $\mathrm{H}$ & 3.73703000 & 1.12390000 & 4.59392800 \\
\hline $\mathrm{C}$ & 0.63989100 & -4.39127400 & 2.49008000 \\
\hline $\mathrm{C}$ & -0.85621100 & -4.15403400 & 2.79464300 \\
\hline $\mathrm{H}$ & -1.01875700 & -3.78669900 & 3.81138300 \\
\hline $\mathrm{H}$ & -1.42097500 & -5.08657000 & 2.67431500 \\
\hline $\mathrm{H}$ & -1.27336900 & -3.40915100 & 2.10959200 \\
\hline
\end{tabular}




\begin{tabular}{|c|c|c|c|}
\hline $\mathrm{C}$ & 0.73046000 & -4.91895500 & 1.03464500 \\
\hline $\mathrm{H}$ & 0.19690100 & -4.29228400 & 0.31677100 \\
\hline $\mathrm{H}$ & 0.27495500 & -5.91384800 & 0.98248200 \\
\hline $\mathrm{H}$ & 1.77328700 & -5.00135600 & 0.70797500 \\
\hline $\mathrm{C}$ & 1.19847800 & -5.52124800 & 3.38092300 \\
\hline $\mathrm{H}$ & 2.27619700 & -5.64115000 & 3.23105900 \\
\hline $\mathrm{H}$ & 0.71037400 & -6.46246800 & 3.10239900 \\
\hline $\mathrm{H}$ & 1.02927800 & -5.36434600 & 4.44412700 \\
\hline $\mathrm{C}$ & 1.06364600 & -3.16501500 & 5.75334300 \\
\hline $\mathrm{H}$ & 0.80182700 & -2.10737200 & 5.84462800 \\
\hline $\mathrm{H}$ & 1.35740600 & -3.55372300 & 6.73155400 \\
\hline $\mathrm{H}$ & 0.19017700 & -3.72106000 & 5.39961300 \\
\hline $\mathrm{C}$ & -1.91102600 & 1.91021100 & -0.42836400 \\
\hline $\mathrm{C}$ & -2.51481600 & 2.87407000 & 0.38502300 \\
\hline $\mathrm{H}$ & -2.85455800 & 2.58297300 & 1.36959300 \\
\hline $\mathrm{C}$ & -2.65115500 & 4.19877100 & -0.03775500 \\
\hline $\mathrm{C}$ & -2.09903500 & 4.54422700 & -1.30229000 \\
\hline $\mathrm{C}$ & -1.58271900 & 3.57852000 & -2.18887900 \\
\hline $\mathrm{C}$ & -1.48192000 & 2.27198200 & -1.69969900 \\
\hline $\mathrm{H}$ & -1.03797500 & 1.49996500 & -2.31695600 \\
\hline $\mathrm{C}$ & -3.30252800 & -0.61786000 & 0.04775200 \\
\hline $\mathrm{C}$ & -4.47811100 & 0.05714800 & 0.35299000 \\
\hline $\mathrm{H}$ & -4.42351200 & 1.10352600 & 0.62287700 \\
\hline $\mathrm{C}$ & -5.73143500 & -0.57001700 & 0.29739600 \\
\hline $\mathrm{C}$ & -5.75406900 & -1.90874000 & -0.15265200 \\
\hline $\mathrm{C}$ & -4.56901900 & -2.66909500 & -0.32877600 \\
\hline $\mathrm{C}$ & -3.35815400 & -1.98209500 & -0.24961100 \\
\hline $\mathrm{H}$ & -2.42458100 & -2.49034100 & -0.45534100 \\
\hline $\mathrm{C}$ & -3.51309200 & 5.18411900 & 0.78256900 \\
\hline $\mathrm{C}$ & -4.80805900 & 5.43611000 & -0.02463200 \\
\hline $\mathrm{H}$ & -4.57743000 & 5.88249300 & -0.99590200 \\
\hline $\mathrm{H}$ & -5.47088600 & 6.11911100 & 0.52136300 \\
\hline $\mathrm{H}$ & -5.34929400 & 4.49822100 & -0.19430600 \\
\hline $\mathrm{C}$ & -3.90398100 & 4.58338600 & 2.14778900 \\
\hline $\mathrm{H}$ & -4.51320300 & 3.67935300 & 2.04355600 \\
\hline $\mathrm{H}$ & -4.50231600 & 5.31219500 & 2.70461500 \\
\hline $\mathrm{H}$ & -3.02044400 & 4.33502400 & 2.74624300 \\
\hline $\mathrm{C}$ & -2.83924500 & 6.54540000 & 1.06192900 \\
\hline $\mathrm{H}$ & -1.82652800 & 6.41648700 & 1.45056400 \\
\hline $\mathrm{H}$ & -3.42246500 & 7.09490100 & 1.81019000 \\
\hline $\mathrm{H}$ & -2.78379500 & 7.15795300 & 0.16345800 \\
\hline $\mathrm{C}$ & -1.12251200 & 3.78326100 & -3.65205100 \\
\hline $\mathrm{C}$ & -1.81418800 & 2.70790100 & -4.53208200 \\
\hline $\mathrm{H}$ & -1.58627900 & 1.68283000 & -4.23084400 \\
\hline
\end{tabular}


$\mathrm{H}$

$\mathrm{H}$

C

$\mathrm{H}$

$\mathrm{H}$

$\mathrm{H}$

C

$\mathrm{H}$

$\mathrm{H}$

$\mathrm{H}$

C

$\mathrm{H}$

$\mathrm{H}$

$\mathrm{H}$

C

C

$\mathrm{H}$

$\mathrm{H}$

$\mathrm{H}$

C

$\mathrm{H}$

$\mathrm{H}$

$\mathrm{H}$

C

$\mathrm{H}$

$\mathrm{H}$

$\mathrm{H}$

C

C

$\mathrm{H}$

$\mathrm{H}$

$\mathrm{H}$

C

$\mathrm{H}$

$\mathrm{H}$

$\mathrm{H}$

C

$\mathrm{H}$

$\mathrm{H}$

$\mathrm{H}$

C

$\mathrm{H}$

$\mathrm{H}$

$\mathrm{H}$

\begin{tabular}{rrr}
-1.48968400 & 2.82636400 & -5.57232000 \\
-2.90274200 & 2.82979800 & -4.50045600 \\
0.40791800 & 3.57615500 & -3.72698300 \\
0.94021500 & 4.30830300 & -3.10930800 \\
0.75583600 & 3.68420900 & -4.76134900 \\
0.69438300 & 2.57937500 & -3.37612900 \\
-1.48655300 & 5.13991800 & -4.28826400 \\
-2.54726600 & 5.37199000 & -4.16410300 \\
-1.27330900 & 5.08296800 & -5.36218200 \\
-0.90905000 & 5.97440800 & -3.89038600 \\
-0.81446300 & 6.49842700 & -1.42519900 \\
-0.91316700 & 7.53808200 & -1.74641300 \\
-0.02401300 & 6.01126400 & -2.00548600 \\
-0.54122000 & 6.46007600 & -0.36676900 \\
-6.94426000 & 0.26617700 & 0.77315400 \\
-7.21603000 & 1.40678200 & -0.23201300 \\
-6.33498500 & 2.04529300 & -0.35894100 \\
-8.04297100 & 2.03521000 & 0.12000800 \\
-7.48513600 & 1.01573100 & -1.21899700 \\
-6.58568400 & 0.89446600 & 2.14563000 \\
-6.35780800 & 0.11500900 & 2.88093700 \\
-7.43591300 & 1.47733600 & 2.51721000 \\
-5.72631800 & 1.56786700 & 2.09408800 \\
-8.24603100 & -0.52737500 & 1.00944000 \\
-8.71979600 & -0.86585100 & 0.08848400 \\
-8.96106600 & 0.12827200 & 1.52029500 \\
-8.07483400 & -1.40239900 & 1.64182400 \\
-4.57543000 & -4.19552200 & -0.55388500 \\
-5.14468100 & -4.57411500 & -1.93800600 \\
-6.21802600 & -4.39151000 & -1.99868700 \\
-4.97899500 & -5.64204900 & -2.12501700 \\
-4.64098500 & -4.01291900 & -2.73267300 \\
-3.14687700 & -4.76963700 & -0.47386900 \\
-2.50196800 & -4.40066700 & -1.27514700 \\
-3.19361300 & -5.85921700 & -0.57343400 \\
-2.67244700 & -4.54309800 & 0.48604100 \\
-5.40665300 & -4.87469200 & 0.55757700 \\
-5.39908700 & -5.96236800 & 0.41600600 \\
-6.44191600 & -4.53039100 & 0.54893000 \\
-4.97603400 & -4.66086500 & 1.54289500 \\
-6.47667200 & -2.15249500 & -1.72407000 \\
-7.373376400 & -1.09076300 & -1.75844200 \\
-2.75659700 & -1.88188200 \\
\hline & -2.35557100 & -2.51791100
\end{tabular}

S100 


\begin{tabular}{|c|c|c|c|}
\hline $\mathrm{C}$ & 0.64240800 & 2.02725100 & 1.4809720 \\
\hline $\mathrm{C}$ & 1.59241000 & 1.71571600 & 0.4646770 \\
\hline $\mathrm{C}$ & 2.25327900 & 2.74973200 & -0.21356100 \\
\hline $\mathrm{H}$ & 2.96040600 & 2.49484800 & -0.99426100 \\
\hline $\mathrm{C}$ & 2.05457200 & 4.10716700 & 0.0911150 \\
\hline $\mathrm{H}$ & 2.58294600 & 4.89172000 & -0.43888600 \\
\hline $\mathrm{C}$ & 1.18277500 & 4.38543000 & 1.1223480 \\
\hline $\mathrm{C}$ & 0.50317200 & 3.37052500 & 1.7873550 \\
\hline $\mathrm{C}$ & 0.17938100 & 5.26172100 & 2.8921850 \\
\hline $\mathrm{H}$ & 0.92120700 & 5.29580500 & 3.7039650 \\
\hline $\mathrm{H}$ & -0.65424600 & 5.93770700 & 3.07456900 \\
\hline $\mathrm{C}$ & -1.20812000 & 0.22690500 & 1.82698000 \\
\hline $\mathrm{C}$ & -1.88240400 & -0.61029800 & 2.72531700 \\
\hline $\mathrm{H}$ & -2.69141700 & -1.22983200 & 2.35834100 \\
\hline $\mathrm{C}$ & -1.56874400 & -0.66993000 & 4.09395300 \\
\hline $\mathrm{H}$ & -2.10619300 & -1.32300700 & 4.77199600 \\
\hline $\mathrm{C}$ & -0.56161800 & 0.16298100 & 4.52954200 \\
\hline $\mathrm{C}$ & 0.12106600 & 0.99474900 & 3.6481840 \\
\hline $\mathrm{C}$ & -0.14172100 & 1.05391300 & 2.29081500 \\
\hline $\mathrm{C}$ & 0.74021900 & 1.51610000 & 5.7147280 \\
\hline $\mathrm{H}$ & 1.64816200 & 1.39618400 & 6.3042780 \\
\hline $\mathrm{H}$ & 0.13803700 & 2.37011600 & 6.0573200 \\
\hline $\mathrm{O}$ & 0.72194100 & -1.33618100 & -3.37008600 \\
\hline S & -0.46480800 & -1.82951400 & -4.23917300 \\
\hline $\mathrm{C}$ & -0.66267300 & -3.54515200 & -3.67360400 \\
\hline $\mathrm{C}$ & -1.75130500 & -4.28365000 & -4.14192200 \\
\hline $\mathrm{C}$ & 0.29391800 & -4.11863600 & -2.83751600 \\
\hline $\mathrm{C}$ & -1.87989300 & -5.62090100 & -3.76783200 \\
\hline $\mathrm{H}$ & -2.49354800 & -3.81385500 & -4.78106800 \\
\hline $\mathrm{C}$ & 0.15248800 & -5.45616100 & -2.46095500 \\
\hline $\mathrm{H}$ & 1.12578900 & -3.51309200 & -2.49277000 \\
\hline $\mathrm{C}$ & -0.92715800 & -6.20697000 & -2.92999400 \\
\hline $\mathrm{H}$ & -2.72752500 & -6.20157200 & -4.11981300 \\
\hline $\mathrm{H}$ & 0.88760700 & -5.91098000 & -1.80387100 \\
\hline $\mathrm{H}$ & -1.03060300 & -7.24815800 & -2.63825200 \\
\hline $\mathrm{O}$ & -1.76714400 & -1.15323900 & -3.65469300 \\
\hline $\mathrm{H}$ & -1.47446000 & -0.94582700 & -2.40307500 \\
\hline
\end{tabular}<smiles>O=S(OP1PCCP[PH2]1)c1ccccc1</smiles>

Int-3b 
Total SCF energy (M06/SDD-6-311++G(d,p)/SMD(1,4-Dioxane)): -5071.674505a.u.

Thermal correction to Gibbs Free Energy at 298.15 K: 1.577405a.u

Gibbs free energy at $298.15 \mathrm{~K}$ (M06/SDD-6-311++G(d,p)/SMD(1,4-Dioxane)): -5070.097100a.u.

$\mathrm{Pd}$

$P$

$\mathrm{P}$

$\mathrm{O}$

$\mathrm{O}$

$\mathrm{O}$

$\mathrm{O}$

$\mathrm{O}$

O

O

O

C

C

$\mathrm{H}$

C

C

C

C

$\mathrm{H}$

C

C

$\mathrm{H}$

$\mathrm{H}$

$\mathrm{H}$

C

$\mathrm{H}$

$\mathrm{H}$

$\mathrm{H}$

C

$\mathrm{H}$

$\mathrm{H}$

$\mathrm{H}$

C

C

$\mathrm{H}$

$\mathrm{H}$

$\mathrm{H}$

C

$\mathrm{H}$

$\mathrm{H}$

$\mathrm{H}$ 


\begin{tabular}{|c|c|c|c|}
\hline $\mathrm{C}$ & -5.89089300 & -5.06199600 & -0.40384800 \\
\hline $\mathrm{H}$ & -6.06615800 & -4.74922100 & 0.63185000 \\
\hline $\mathrm{H}$ & -5.73405500 & -6.14663500 & -0.40507900 \\
\hline $\mathrm{H}$ & -6.79868500 & -4.85197700 & -0.96522100 \\
\hline $\mathrm{C}$ & -7.30513700 & -2.89513200 & -2.59957900 \\
\hline $\mathrm{H}$ & -7.10858700 & -3.94950900 & -2.81583100 \\
\hline $\mathrm{H}$ & -6.68315700 & -2.27001600 & -3.24840800 \\
\hline $\mathrm{H}$ & -8.36045300 & -2.68974600 & -2.80136200 \\
\hline $\mathrm{C}$ & -2.05101400 & 1.79857300 & -0.28540200 \\
\hline $\mathrm{C}$ & -1.46246500 & 2.35408700 & -1.41610400 \\
\hline $\mathrm{H}$ & -0.90914700 & 1.69140700 & -2.07174400 \\
\hline $\mathrm{C}$ & -1.53903000 & 3.72001000 & -1.71365200 \\
\hline $\mathrm{C}$ & -2.19912000 & 4.53404200 & -0.76943700 \\
\hline $\mathrm{C}$ & -2.89713300 & 3.99548100 & 0.34644700 \\
\hline $\mathrm{C}$ & -2.78343000 & 2.62185000 & 0.57536100 \\
\hline $\mathrm{H}$ & -3.24956200 & 2.17682500 & 1.44385700 \\
\hline $\mathrm{C}$ & -3.86448700 & 4.84443000 & 1.20203800 \\
\hline $\mathrm{C}$ & -3.23377100 & 6.12733800 & 1.78778700 \\
\hline $\mathrm{H}$ & -2.26463800 & 5.92306400 & 2.24828600 \\
\hline $\mathrm{H}$ & -3.89668700 & 6.54052400 & 2.55699800 \\
\hline $\mathrm{H}$ & -3.09437600 & 6.89180900 & 1.02485200 \\
\hline $\mathrm{C}$ & -5.05004300 & 5.25142600 & 0.29655500 \\
\hline $\mathrm{H}$ & -4.70219200 & 5.84848500 & -0.55067400 \\
\hline $\mathrm{H}$ & -5.77362200 & 5.84806200 & 0.86584200 \\
\hline $\mathrm{H}$ & -5.56749900 & 4.36691100 & -0.08927000 \\
\hline $\mathrm{C}$ & -4.41854800 & 4.03206800 & 2.38964500 \\
\hline $\mathrm{H}$ & -4.99345700 & 3.16020500 & 2.06318300 \\
\hline $\mathrm{H}$ & -5.09529200 & 4.66498200 & 2.97313700 \\
\hline $\mathrm{H}$ & -3.61588100 & 3.68760100 & 3.05147800 \\
\hline $\mathrm{C}$ & -0.89059700 & 4.16959600 & -3.04597400 \\
\hline $\mathrm{C}$ & 0.64713900 & 4.12750300 & -2.89881400 \\
\hline $\mathrm{H}$ & 1.00138600 & 4.82688700 & -2.13456200 \\
\hline $\mathrm{H}$ & 1.12754700 & 4.39047500 & -3.84896300 \\
\hline $\mathrm{H}$ & 0.98696700 & 3.12741100 & -2.60993600 \\
\hline $\mathrm{C}$ & -1.29551100 & 3.16859000 & -4.16040000 \\
\hline $\mathrm{H}$ & -0.92715600 & 2.15518000 & -3.98379800 \\
\hline $\mathrm{H}$ & -0.87865000 & 3.50287700 & -5.11680700 \\
\hline $\mathrm{H}$ & -2.38495400 & 3.11851300 & -4.26150600 \\
\hline $\mathrm{C}$ & -1.32707500 & 5.55885600 & -3.55603700 \\
\hline $\mathrm{H}$ & -2.41610400 & 5.65525900 & -3.56949600 \\
\hline $\mathrm{H}$ & -0.96210500 & 5.68003900 & -4.58253000 \\
\hline $\mathrm{H}$ & -0.92428000 & 6.38451600 & -2.97096600 \\
\hline $\mathrm{C}$ & -1.00749900 & 6.53924400 & -0.46229400 \\
\hline $\mathrm{H}$ & -0.83065400 & 6.33087400 & 0.59660000 \\
\hline
\end{tabular}




\begin{tabular}{|c|c|c|c|}
\hline $\mathrm{H}$ & -1.15085900 & 7.61261800 & -0.60718000 \\
\hline $\mathrm{H}$ & -0.13524600 & 6.21029600 & -1.03541900 \\
\hline $\mathrm{C}$ & 1.88151700 & -1.07306600 & 1.34138400 \\
\hline $\mathrm{C}$ & 2.53308200 & -0.72073000 & 2.52848800 \\
\hline $\mathrm{H}$ & 2.84893500 & 0.30413100 & 2.66515600 \\
\hline $\mathrm{C}$ & 2.74230700 & -1.65285500 & 3.54416500 \\
\hline $\mathrm{C}$ & 2.20994700 & -2.95663000 & 3.35124900 \\
\hline $\mathrm{C}$ & 1.64414000 & -3.37269600 & 2.12869400 \\
\hline $\mathrm{C}$ & 1.46674500 & -2.38695000 & 1.14566900 \\
\hline $\mathrm{H}$ & 0.97872400 & -2.64147800 & 0.21068900 \\
\hline $\mathrm{C}$ & 3.20628700 & 0.47715000 & -0.76520000 \\
\hline $\mathrm{C}$ & 4.36602800 & 0.59087000 & -0.00636300 \\
\hline $\mathrm{H}$ & 4.30729800 & 0.45119700 & 1.06415800 \\
\hline $\mathrm{C}$ & 5.61143500 & 0.86620600 & -0.58660100 \\
\hline $\mathrm{C}$ & 5.65417500 & 0.93760600 & -1.99694100 \\
\hline $\mathrm{C}$ & 4.47779400 & 0.96527000 & -2.78869000 \\
\hline $\mathrm{C}$ & 3.26768700 & 0.71746200 & -2.13982800 \\
\hline $\mathrm{H}$ & 2.34771900 & 0.66585500 & -2.70447200 \\
\hline $\mathrm{C}$ & 3.64714100 & -1.29954800 & 4.74485000 \\
\hline $\mathrm{C}$ & 4.94653200 & -2.12514700 & 4.59861000 \\
\hline $\mathrm{H}$ & 4.72808500 & -3.19621600 & 4.61509200 \\
\hline $\mathrm{H}$ & 5.63510900 & -1.89941300 & 5.42242500 \\
\hline $\mathrm{H}$ & 5.45349500 & -1.88916600 & 3.65606000 \\
\hline $\mathrm{C}$ & 4.02316000 & 0.19571600 & 4.74149200 \\
\hline $\mathrm{H}$ & 4.58929700 & 0.47836000 & 3.84761600 \\
\hline $\mathrm{H}$ & 4.66068700 & 0.40872700 & 5.60595800 \\
\hline $\mathrm{H}$ & 3.13537100 & 0.83472200 & 4.80488800 \\
\hline $\mathrm{C}$ & 3.02212700 & -1.60801800 & 6.12309800 \\
\hline $\mathrm{H}$ & 2.00099200 & -1.22664600 & 6.18985400 \\
\hline $\mathrm{H}$ & 3.61673700 & -1.12998800 & 6.91044800 \\
\hline $\mathrm{H}$ & 2.99948500 & -2.67804400 & 6.32313100 \\
\hline $\mathrm{C}$ & 1.20772400 & -4.80803800 & 1.75091000 \\
\hline $\mathrm{C}$ & 1.75214100 & -5.12282200 & 0.33245900 \\
\hline $\mathrm{H}$ & 1.29915100 & -4.49496700 & -0.43537300 \\
\hline $\mathrm{H}$ & 1.51334900 & -6.16205200 & 0.07888600 \\
\hline $\mathrm{H}$ & 2.84155300 & -5.00494100 & 0.29889600 \\
\hline $\mathrm{C}$ & -0.33504500 & -4.86054600 & 1.69411900 \\
\hline $\mathrm{H}$ & -0.78466900 & -4.62764700 & 2.66618600 \\
\hline $\mathrm{H}$ & -0.66888400 & -5.86113800 & 1.39549200 \\
\hline $\mathrm{H}$ & -0.71995400 & -4.15022600 & 0.95883300 \\
\hline $\mathrm{C}$ & 1.73922400 & -5.92789200 & 2.66889800 \\
\hline $\mathrm{H}$ & 2.82160600 & -5.85577600 & 2.80825300 \\
\hline $\mathrm{H}$ & 1.52205100 & -6.89017300 & 2.19119300 \\
\hline $\mathrm{H}$ & 1.27354000 & -5.94644500 & 3.65382600 \\
\hline
\end{tabular}




\begin{tabular}{|c|c|c|c|}
\hline $\mathrm{C}$ & 1.04561100 & -3.93264800 & 5.13915500 \\
\hline $\mathrm{H}$ & 1.22790400 & -4.62472300 & 5.96466000 \\
\hline $\mathrm{H}$ & 0.24459500 & -4.32571700 & 4.50588400 \\
\hline $\mathrm{H}$ & 0.73609800 & -2.95995300 & 5.53300400 \\
\hline $\mathrm{C}$ & 6.78873900 & 1.10501700 & 0.39013900 \\
\hline $\mathrm{C}$ & 7.14562800 & -0.21436400 & 1.10902900 \\
\hline $\mathrm{H}$ & 6.28129000 & -0.62800200 & 1.63971900 \\
\hline $\mathrm{H}$ & 7.94345400 & -0.04449500 & 1.84179000 \\
\hline $\mathrm{H}$ & 7.49312300 & -0.97472000 & 0.40144900 \\
\hline $\mathrm{C}$ & 6.32468100 & 2.13759400 & 1.45121400 \\
\hline $\mathrm{H}$ & 6.04321700 & 3.08260700 & 0.97372700 \\
\hline $\mathrm{H}$ & 7.14256500 & 2.33895300 & 2.15197600 \\
\hline $\mathrm{H}$ & 5.46972700 & 1.79199700 & 2.03783000 \\
\hline $\mathrm{C}$ & 8.06415200 & 1.70392100 & -0.23806800 \\
\hline $\mathrm{H}$ & 8.60822000 & 1.00025900 & -0.86745600 \\
\hline $\mathrm{H}$ & 8.73908600 & 2.00162500 & 0.57294900 \\
\hline $\mathrm{H}$ & 7.84062800 & 2.59023400 & -0.83729800 \\
\hline $\mathrm{C}$ & 4.49095300 & 1.30521900 & -4.29375600 \\
\hline $\mathrm{C}$ & 5.22639100 & 0.23326700 & -5.12746600 \\
\hline $\mathrm{H}$ & 6.30246900 & 0.25452000 & -4.95537000 \\
\hline $\mathrm{H}$ & 5.05934300 & 0.42103800 & -6.19493500 \\
\hline $\mathrm{H}$ & 4.84448000 & -0.76769100 & -4.89952500 \\
\hline $\mathrm{C}$ & 3.05788700 & 1.40851200 & -4.85456500 \\
\hline $\mathrm{H}$ & 2.52548800 & 0.45348700 & -4.80387100 \\
\hline $\mathrm{H}$ & 3.11069400 & 1.70456700 & -5.90793000 \\
\hline $\mathrm{H}$ & 2.46497900 & 2.16427800 & -4.32925500 \\
\hline $\mathrm{C}$ & 5.17083600 & 2.67850600 & -4.49268200 \\
\hline $\mathrm{H}$ & 5.17796700 & 2.94147800 & -5.55741600 \\
\hline $\mathrm{H}$ & 6.20122900 & 2.66725300 & -4.13228900 \\
\hline $\mathrm{H}$ & 4.62127600 & 3.46077400 & -3.95594700 \\
\hline $\mathrm{C}$ & 7.50098100 & -0.29376200 & -2.75076700 \\
\hline $\mathrm{H}$ & 7.75150100 & -0.69693500 & -1.76402900 \\
\hline $\mathrm{H}$ & 8.41795100 & -0.14233300 & -3.32491500 \\
\hline $\mathrm{H}$ & 6.85515500 & -1.01106700 & -3.26840200 \\
\hline $\mathrm{C}$ & -0.56916700 & 0.58276900 & 2.43121800 \\
\hline $\mathrm{C}$ & -1.50557100 & -0.23675400 & 1.73529500 \\
\hline $\mathrm{C}$ & -2.13566200 & -1.29733600 & 2.39746900 \\
\hline $\mathrm{H}$ & -2.84003900 & -1.91298600 & 1.85145000 \\
\hline $\mathrm{C}$ & -1.91122900 & -1.58518700 & 3.75468600 \\
\hline $\mathrm{H}$ & -2.41377000 & -2.40772200 & 4.25058800 \\
\hline $\mathrm{C}$ & -1.04779200 & -0.74727600 & 4.42641600 \\
\hline $\mathrm{C}$ & -0.39987000 & 0.29827800 & 3.77564500 \\
\hline $\mathrm{C}$ & -0.04344300 & 0.50801500 & 5.95062700 \\
\hline $\mathrm{H}$ & -0.79281300 & 1.21614000 & 6.33371800 \\
\hline
\end{tabular}




\begin{tabular}{|c|c|c|c|}
\hline $\mathrm{H}$ & 0.80287100 & 0.40340900 & 6.62719500 \\
\hline $\mathrm{C}$ & 1.15963600 & 1.69406600 & 0.83784100 \\
\hline $\mathrm{C}$ & 1.79384300 & 2.87003000 & 0.41763100 \\
\hline $\mathrm{H}$ & 2.53455600 & 2.81566200 & -0.37018100 \\
\hline $\mathrm{C}$ & 1.52743300 & 4.12357300 & 0.99335000 \\
\hline $\mathrm{H}$ & 2.03555600 & 5.02132700 & 0.66074100 \\
\hline $\mathrm{C}$ & 0.60845400 & 4.14422100 & 2.01966500 \\
\hline $\mathrm{C}$ & -0.04648400 & 2.98668800 & 2.43056600 \\
\hline $\mathrm{C}$ & 0.17534100 & 1.74140100 & 1.86892200 \\
\hline $\mathrm{C}$ & -0.54884200 & 4.61021600 & 3.85684100 \\
\hline $\mathrm{H}$ & -1.43758900 & 5.19852900 & 4.08064700 \\
\hline $\mathrm{H}$ & 0.12306600 & 4.53189400 & 4.72341500 \\
\hline $\mathrm{O}$ & -1.20571300 & -1.61195400 & -2.89349400 \\
\hline S & -0.93913500 & -3.15690900 & -3.02011600 \\
\hline $\mathrm{C}$ & 0.67096400 & -3.15636400 & -3.88005200 \\
\hline $\mathrm{C}$ & 1.65307000 & -4.07296200 & -3.51216500 \\
\hline $\mathrm{C}$ & 0.88653200 & -2.25288400 & -4.92227000 \\
\hline $\mathrm{C}$ & 2.87854400 & -4.07069400 & -4.18162900 \\
\hline $\mathrm{H}$ & 1.45901500 & -4.75753900 & -2.69384400 \\
\hline $\mathrm{C}$ & 2.10963600 & -2.25934600 & -5.59233300 \\
\hline $\mathrm{H}$ & 0.11274600 & -1.53431600 & -5.17389200 \\
\hline $\mathrm{C}$ & 3.10739800 & -3.16599700 & -5.22051700 \\
\hline $\mathrm{H}$ & 3.65485800 & -4.77370400 & -3.89120700 \\
\hline H & 2.29020900 & -1.55435000 & -6.39926500 \\
\hline $\mathrm{H}$ & 4.05926800 & -3.16874100 & -5.74488200 \\
\hline $\mathrm{O}$ & -0.60239100 & -3.75352700 & -1.65746800 \\
\hline $\mathrm{H}$ & 1.30682500 & -1.18735000 & -2.23739500 \\
\hline
\end{tabular}

Total SCF energy (M06/SDD-6-311++G(d,p)/SMD(1,4-Dioxane)): -4717.571758a.u.

Thermal correction to Gibbs Free Energy at $298.15 \mathrm{~K}$ : 1.674248a.u

Gibbs free energy at 298.15 K (M06/SDD-6-311++G(d,p)/SMD(1,4-Dioxane)): -4715.897510a.u.

$\begin{array}{cccc}\mathrm{P} & 1.46152500 & -0.08936900 & -0.32991900 \\ \mathrm{P} & -1.92244300 & -0.05509000 & -0.34261500 \\ \mathrm{O} & 0.49264500 & 0.04152000 & 4.36948700 \\ \mathrm{O} & -0.47827000 & -1.91357300 & 5.12607900 \\ \mathrm{O} & -1.01677700 & 2.72973700 & 3.43960700 \\ \mathrm{O} & -0.03014200 & 4.72611800 & 2.83184800 \\ \mathrm{O} & -3.78729900 & 5.49312200 & 0.84796300\end{array}$




\begin{tabular}{|c|c|c|c|}
\hline $\mathrm{O}$ & -6.46058500 & -3.40663800 & -2.29157500 \\
\hline $\mathrm{O}$ & 1.62120200 & -5.15585700 & 2.82095900 \\
\hline $\mathrm{O}$ & 7.32460700 & -0.88172100 & -0.74942000 \\
\hline $\mathrm{C}$ & -1.50748000 & -0.65922300 & 1.33767300 \\
\hline $\mathrm{C}$ & -0.60232000 & 0.07749200 & 2.16008400 \\
\hline $\mathrm{C}$ & -0.36715500 & -0.44276800 & 3.41671300 \\
\hline $\mathrm{C}$ & -0.94311000 & -1.62964200 & 3.87074900 \\
\hline $\mathrm{C}$ & -1.80030400 & -2.36390800 & 3.08058600 \\
\hline $\mathrm{C}$ & -2.07424400 & -1.85028800 & 1.80156200 \\
\hline $\mathrm{C}$ & 0.23573200 & -0.73771000 & 5.54397100 \\
\hline $\mathrm{C}$ & 1.06232100 & 1.37425600 & 0.6933570 \\
\hline $\mathrm{C}$ & 1.62184100 & 2.60071400 & 0.3111970 \\
\hline $\mathrm{C}$ & 1.32469000 & 3.80268100 & 0.9717400 \\
\hline $\mathrm{C}$ & 0.44048600 & 3.71944300 & 2.0260310 \\
\hline $\mathrm{C}$ & -0.15379100 & 2.51270800 & 2.39390800 \\
\hline $\mathrm{C}$ & 0.10118700 & 1.31649200 & 1.7421530 \\
\hline $\mathrm{C}$ & -0.71370000 & 4.05408000 & 3.89898600 \\
\hline $\mathrm{C}$ & -2.52475200 & 1.62818000 & -0.02363400 \\
\hline $\mathrm{C}$ & -2.16057200 & 2.67372300 & -0.86599200 \\
\hline $\mathrm{C}$ & -2.57672300 & 3.99230200 & -0.64027900 \\
\hline $\mathrm{C}$ & -3.37495800 & 4.22353800 & 0.50910700 \\
\hline $\mathrm{C}$ & -3.85698100 & 3.16032700 & 1.32140800 \\
\hline $\mathrm{C}$ & -3.36997500 & 1.88134100 & 1.05961800 \\
\hline $\mathrm{C}$ & -4.93167700 & 3.35894300 & 2.41257700 \\
\hline $\mathrm{C}$ & -6.16838600 & 4.05212800 & 1.79590900 \\
\hline $\mathrm{C}$ & -4.39598100 & 4.17531400 & 3.60494800 \\
\hline $\mathrm{C}$ & -5.41161600 & 2.00941500 & 2.98609400 \\
\hline $\mathrm{C}$ & -2.15869900 & 5.07319100 & -1.66630100 \\
\hline $\mathrm{C}$ & -0.85237800 & 5.76523000 & -1.21593800 \\
\hline $\mathrm{C}$ & -1.85762100 & 4.42784800 & -3.04080900 \\
\hline $\mathrm{C}$ & -3.27851100 & 6.10448200 & -1.92457000 \\
\hline $\mathrm{C}$ & -2.84388600 & 6.37426200 & 1.46373800 \\
\hline $\mathrm{C}$ & -3.31280000 & -1.10376100 & -0.87122500 \\
\hline $\mathrm{C}$ & -4.55268400 & -1.04916700 & -0.24069000 \\
\hline $\mathrm{C}$ & -3.09941400 & -2.05555300 & -1.87309200 \\
\hline $\mathrm{C}$ & -4.12569600 & -2.88522000 & -2.32708900 \\
\hline $\mathrm{C}$ & -5.40685700 & -2.71270100 & -1.73982200 \\
\hline $\mathrm{C}$ & -5.61483700 & -1.88241500 & -0.61372800 \\
\hline $\mathrm{C}$ & -6.87563800 & -1.83742900 & 0.28496800 \\
\hline $\mathrm{C}$ & -6.41180500 & -1.95930700 & 1.76138500 \\
\hline $\mathrm{C}$ & -7.88108800 & -2.98857900 & 0.07341300 \\
\hline $\mathrm{C}$ & -7.31980200 & -2.59918300 & -3.10310100 \\
\hline $\mathrm{C}$ & -7.59863800 & -0.48498000 & 0.10361600 \\
\hline $\mathrm{C}$ & -3.83530500 & -3.97843600 & -3.37674100 \\
\hline
\end{tabular}




\begin{tabular}{|c|c|c|c|}
\hline $\mathrm{C}$ & -2.34037900 & -4.01038000 & -3.75285800 \\
\hline $\mathrm{C}$ & -4.19602900 & -5.35674100 & -2.77659400 \\
\hline $\mathrm{C}$ & -4.62709900 & -3.76190800 & -4.68486400 \\
\hline $\mathrm{C}$ & 1.44290500 & -1.59552200 & 0.69568000 \\
\hline $\mathrm{C}$ & 2.23957900 & -1.69439300 & 1.83975600 \\
\hline $\mathrm{C}$ & 2.35237800 & -2.89017600 & 2.54367500 \\
\hline $\mathrm{C}$ & 1.61066200 & -4.00501300 & 2.06021600 \\
\hline $\mathrm{C}$ & 0.91130000 & -3.97413300 & 0.83611100 \\
\hline $\mathrm{C}$ & 0.81201300 & -2.72723700 & 0.19962300 \\
\hline $\mathrm{C}$ & 0.28404300 & -5.17935100 & 0.09477200 \\
\hline $\mathrm{C}$ & -1.25502100 & -5.05526500 & 0.11999700 \\
\hline $\mathrm{C}$ & 0.68860800 & -6.57204700 & 0.62054700 \\
\hline $\mathrm{C}$ & 0.76363700 & -5.13883600 & -1.38047000 \\
\hline $\mathrm{C}$ & 2.77366500 & -3.55824800 & 5.00867300 \\
\hline $\mathrm{C}$ & 0.44409800 & -5.31937700 & 3.61675600 \\
\hline $\mathrm{C}$ & 3.37372700 & -3.01045900 & 3.69506100 \\
\hline $\mathrm{C}$ & 4.00973900 & -1.64310200 & 4.01807900 \\
\hline $\mathrm{C}$ & 5.99337100 & -0.53135900 & -0.81863900 \\
\hline $\mathrm{C}$ & 5.12933200 & -1.17583400 & -1.73501800 \\
\hline $\mathrm{C}$ & 4.76124300 & -1.69769800 & -4.16616200 \\
\hline $\mathrm{C}$ & 3.75849100 & -0.93467700 & -1.57770600 \\
\hline $\mathrm{C}$ & 3.26155900 & -0.02657100 & -0.64580600 \\
\hline $\mathrm{C}$ & 4.16063200 & 0.71062200 & 0.12783900 \\
\hline $\mathrm{C}$ & 5.53926100 & 0.49131900 & 0.05457300 \\
\hline $\mathrm{C}$ & 7.56613100 & -2.09540400 & -0.03059000 \\
\hline $\mathrm{C}$ & 5.17572100 & -3.56985600 & -2.54938300 \\
\hline $\mathrm{C}$ & 7.56581700 & 2.00358600 & -0.03469700 \\
\hline $\mathrm{C}$ & 6.50437100 & 1.36302100 & 0.88886900 \\
\hline $\mathrm{C}$ & 5.75131600 & 2.50972700 & 1.59431400 \\
\hline $\mathrm{C}$ & 7.03935200 & -2.02929000 & -3.28648300 \\
\hline $\mathrm{C}$ & 4.49846600 & -3.96032700 & 3.22128900 \\
\hline $\mathrm{C}$ & 5.54887200 & -2.11088400 & -2.89490500 \\
\hline $\mathrm{C}$ & 7.21658900 & 0.54182600 & 1.98643800 \\
\hline $\mathrm{H}$ & -2.24284400 & -3.29050600 & 3.42605900 \\
\hline $\mathrm{H}$ & -2.75204400 & -2.40114500 & 1.16324300 \\
\hline $\mathrm{H}$ & 1.18180800 & -1.03153300 & 5.99822200 \\
\hline $\mathrm{H}$ & -0.39123500 & -0.16313200 & 6.23735800 \\
\hline $\mathrm{H}$ & 2.31911800 & 2.63597600 & -0.51333500 \\
\hline $\mathrm{H}$ & 1.77735500 & 4.73832700 & 0.66589500 \\
\hline $\mathrm{H}$ & -1.63839300 & 4.58226200 & 4.12316300 \\
\hline $\mathrm{H}$ & -0.05210900 & 3.99316400 & 4.77317100 \\
\hline $\mathrm{H}$ & -1.53228500 & 2.43946200 & -1.71413600 \\
\hline $\mathrm{H}$ & -3.63729400 & 1.06619800 & 1.71612400 \\
\hline $\mathrm{H}$ & -6.57489200 & 3.44900100 & 0.97582500 \\
\hline
\end{tabular}




\begin{tabular}{|c|c|c|c|}
\hline $\mathrm{H}$ & -6.95066900 & 4.15693900 & 2.55634200 \\
\hline $\mathrm{H}$ & -5.92493100 & 5.04107900 & 1.40792800 \\
\hline $\mathrm{H}$ & -4.06823900 & 5.17145800 & 3.31051400 \\
\hline $\mathrm{H}$ & -5.18308400 & 4.29402300 & 4.35817500 \\
\hline $\mathrm{H}$ & -3.56065200 & 3.64634100 & 4.07482200 \\
\hline $\mathrm{H}$ & -4.61282900 & 1.46790000 & 3.50539600 \\
\hline $\mathrm{H}$ & -6.20578300 & 2.19615300 & 3.71577800 \\
\hline $\mathrm{H}$ & -5.82807800 & 1.35867100 & 2.20974400 \\
\hline $\mathrm{H}$ & -0.54345300 & 6.50315900 & -1.96662500 \\
\hline $\mathrm{H}$ & -0.95065500 & 6.28253900 & -0.26147000 \\
\hline $\mathrm{H}$ & -0.05093500 & 5.02826000 & -1.10997600 \\
\hline $\mathrm{H}$ & -1.68776100 & 5.21864300 & -3.77828800 \\
\hline $\mathrm{H}$ & -0.95426200 & 3.80993600 & -3.01947800 \\
\hline $\mathrm{H}$ & -2.69035000 & 3.80876700 & -3.39129900 \\
\hline $\mathrm{H}$ & -4.18848500 & 5.60051900 & -2.26843600 \\
\hline $\mathrm{H}$ & -3.54238600 & 6.68730600 & -1.04553100 \\
\hline $\mathrm{H}$ & -2.95787000 & 6.79508600 & -2.71217200 \\
\hline $\mathrm{H}$ & -2.53479000 & 7.15040800 & 0.75703500 \\
\hline $\mathrm{H}$ & -3.34527200 & 6.85761700 & 2.30704800 \\
\hline $\mathrm{H}$ & -1.96286600 & 5.83344200 & 1.81998400 \\
\hline $\mathrm{H}$ & -4.70125500 & -0.34796700 & 0.56799200 \\
\hline $\mathrm{H}$ & -2.11470800 & -2.11666700 & -2.31660000 \\
\hline $\mathrm{H}$ & -7.28590200 & -1.95287000 & 2.42079000 \\
\hline $\mathrm{H}$ & -5.87323900 & -2.90006500 & 1.92103600 \\
\hline $\mathrm{H}$ & -5.76111000 & -1.14038200 & 2.07863900 \\
\hline $\mathrm{H}$ & -8.43810200 & -2.91281400 & -0.85957800 \\
\hline $\mathrm{H}$ & -7.38888200 & -3.96418300 & 0.09622900 \\
\hline $\mathrm{H}$ & -8.61266400 & -2.95813900 & 0.88836200 \\
\hline $\mathrm{H}$ & -7.77876800 & -1.79281300 & -2.52367600 \\
\hline $\mathrm{H}$ & -6.76810700 & -2.16225700 & -3.94214100 \\
\hline $\mathrm{H}$ & -8.09751600 & -3.26561400 & -3.48049100 \\
\hline $\mathrm{H}$ & -7.96908800 & -0.35997000 & -0.91919600 \\
\hline $\mathrm{H}$ & -8.45687900 & -0.42122900 & 0.78200000 \\
\hline $\mathrm{H}$ & -6.93417700 & 0.35896800 & 0.31935700 \\
\hline $\mathrm{H}$ & -2.01805500 & -3.08249200 & -4.24107300 \\
\hline $\mathrm{H}$ & -1.69963800 & -4.18627900 & -2.88350100 \\
\hline $\mathrm{H}$ & -2.16810500 & -4.82944600 & -4.45801800 \\
\hline $\mathrm{H}$ & -3.98117700 & -6.14818700 & -3.50392800 \\
\hline $\mathrm{H}$ & -3.60328900 & -5.55373600 & -1.87666700 \\
\hline $\mathrm{H}$ & -5.25413800 & -5.40818100 & -2.51423900 \\
\hline $\mathrm{H}$ & -5.68969400 & -3.96027100 & -4.55150600 \\
\hline $\mathrm{H}$ & -4.50078000 & -2.73972600 & -5.06041100 \\
\hline $\mathrm{H}$ & -4.25761100 & -4.44892300 & -5.45446100 \\
\hline $\mathrm{H}$ & 2.78126700 & -0.81833200 & 2.16696900 \\
\hline
\end{tabular}


$\mathrm{H}$

$\mathrm{H}$

$\mathrm{H}$

$\mathrm{H}$

$\mathrm{H}$

$\mathrm{H}$

$\mathrm{H}$

$\mathrm{H}$

$\mathrm{H}$

$\mathrm{H}$

$\mathrm{H}$

$\mathrm{H}$

$\mathrm{H}$

$\mathrm{H}$

$\mathrm{H}$

$\mathrm{H}$

$\mathrm{H}$

$\mathrm{H}$

$\mathrm{H}$

$\mathrm{H}$

$\mathrm{H}$

$\mathrm{H}$

$\mathrm{H}$

$\mathrm{H}$

$\mathrm{H}$

$\mathrm{H}$

$\mathrm{H}$

$\mathrm{H}$

$\mathrm{H}$

$\mathrm{H}$

$\mathrm{H}$

$\mathrm{H}$

$\mathrm{H}$

$\mathrm{H}$

$\mathrm{H}$

$\mathrm{H}$

$\mathrm{H}$

$\mathrm{H}$

$\mathrm{H}$

$\mathrm{H}$

$\mathrm{H}$

$\mathrm{H}$

$\mathrm{H}$

$\mathrm{H}$
0.23205200

$-1.64061600$

$-1.59432200$

$-1.71038100$

0.34017000

1.77281200

0.24232700

0.34033600

0.46028000

1.85581700

3.48963800

1.84722800

2.56223700

0.31648600

$-0.45059200$

0.57946000

4.55514300

3.25956700

4.72772300

4.93747400

5.09239700

3.68250800

3.05535800

3.77751400

7.21191400

8.64800000

7.07720000

5.42406100

5.71294200

4.10326900

8.14570700

7.09648800

8.25420400

6.47839400

5.19559700

5.05371900

7.35548400

7.70749700

7.18037700

4.98225900

5.26134500

4.10150900

7.75111600

6.49750300
$-2.63275300$

$-5.08277600$

$-4.11986600$

$-5.88490700$

$-7.32348500$

$-6.66914800$

$-6.81725900$

$-5.98705600$

$-4.22903200$

$-5.21039400$

$-3.41364800$

$-3.04030900$

$-4.62437300$

$-4.48164600$

$-5.40129600$

$-6.24559500$

$-1.22986500$

$-0.90741700$

$-1.76407500$

$-0.64485600$

$-2.30432500$

$-1.85046100$

$-1.50200800$

1.44349100

$-2.96902400$

$-2.16376400$

$-2.07569400$

$-4.23204300$

$-3.92873900$

$-3.66833300$

1.24603100

2.66064200

2.61281600

3.14966200

3.13510800

2.14072700

$-0.99414600$

$-2.47557800$

$-2.57295500$

$-3.56650700$

$-4.06226600$

$-4.95348400$

1.21960700

$-0.02760300$
$-0.71195800$

1.14482900

$-0.33718000$

$-0.43273600$

$-0.09694900$

0.71482300

1.58363900

$-1.92887100$

$-1.90494100$

$-1.43296000$

5.82544800

5.27317200

4.94014700

4.30946100

2.99060900

4.17866100

3.16288200

4.33193100

4.83518600

$-4.41317500$

$-5.01512500$

$-4.06783600$

$-2.17710000$

0.82074800

$-0.58535600$

0.09878500

0.94935600

$-3.38656200$

$-1.66619100$

$-2.34551800$

$-0.56304600$

$-0.77410600$

0.56222700

2.10434100

0.88791700

2.35486200

$-3.44334800$

$-2.55201200$

$-4.22697900$

2.31964400

4.00221200

2.99679200

2.66160000

2.58628300 


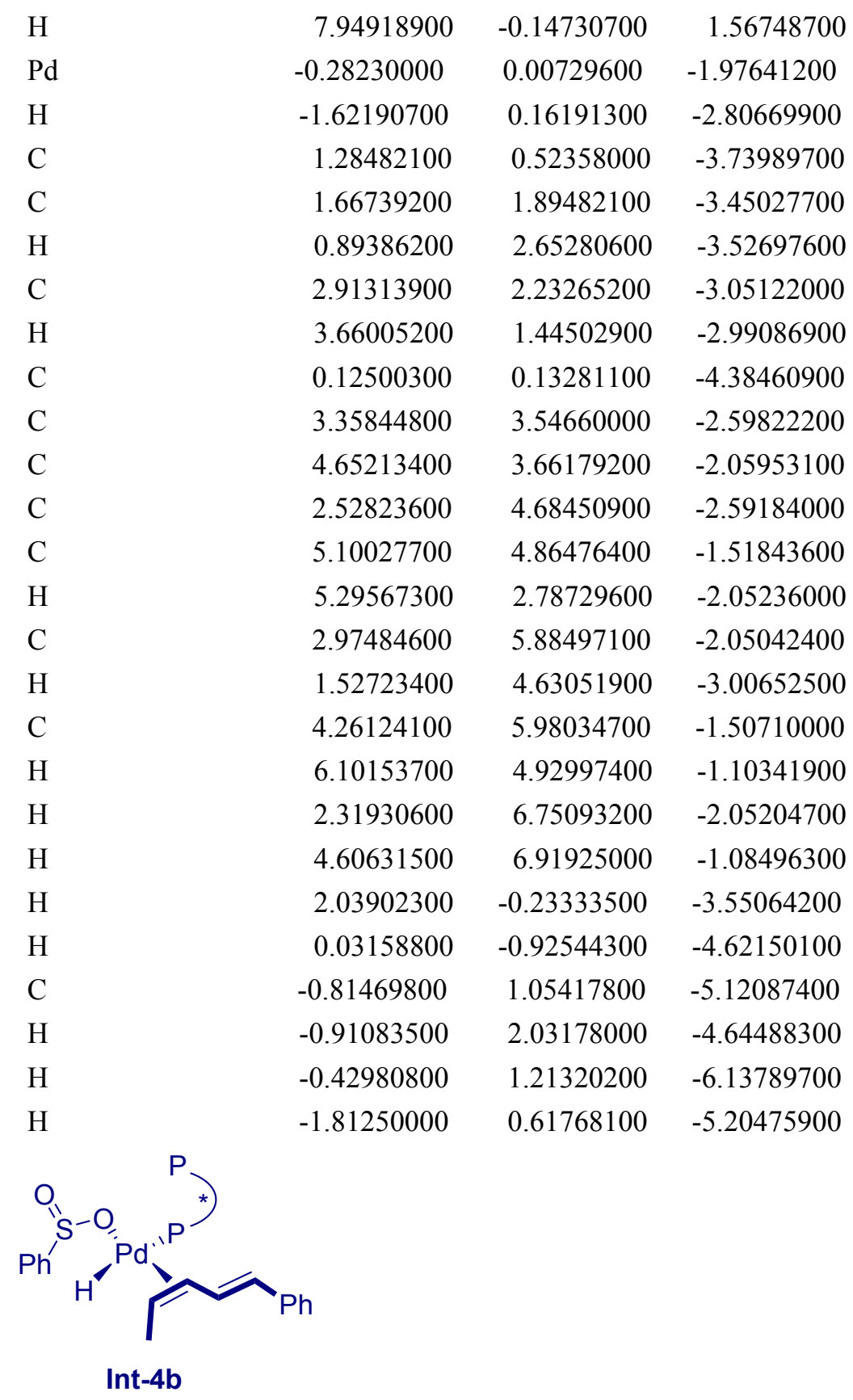

Total SCF energy (M06/SDD-6-311++G(d,p)/SMD(1,4-Dioxane)): -5497.800836a.u.

Thermal correction to Gibbs Free Energy at 298.15 K: 1.761564a.u

Gibbs free energy at 298.15 K (M06/SDD-6-311++G(d,p)/SMD(1,4-Dioxane)): -5496.039272a.u.

$\begin{array}{cccc}\mathrm{Pd} & 2.23819200 & 0.50352100 & -2.64077400 \\ \mathrm{P} & 1.89268600 & -0.58748900 & -0.46656200 \\ \mathrm{P} & -1.96610100 & -0.36241300 & 0.11055800 \\ \mathrm{O} & 7.36443000 & -1.61472800 & 1.62271100 \\ \mathrm{O} & -1.19498400 & -5.59257100 & -1.50342800 \\ \mathrm{O} & -3.72159200 & 4.60549900 & 3.00402500 \\ \mathrm{O} & -7.72075500 & -1.22972800 & -1.28592400 \\ \mathrm{O} & 0.27872800 & 3.27302200 & 3.72433700 \\ \mathrm{O} & -0.35798000 & 1.06774600 & 4.06022500 \\ & & \mathrm{~S} 111 & \end{array}$




\begin{tabular}{|c|c|c|c|}
\hline $\mathrm{O}$ & 1.55193700 & -1.93217600 & 3.69695000 \\
\hline $\mathrm{O}$ & 0.63708100 & -4.02783400 & 4.05663000 \\
\hline $\mathrm{C}$ & 3.42209400 & -1.10282400 & 0.39072600 \\
\hline $\mathrm{C}$ & 4.23350300 & -2.08135100 & -0.20223900 \\
\hline $\mathrm{H}$ & 3.83200600 & -2.64330400 & -1.03512800 \\
\hline $\mathrm{C}$ & 5.53093700 & -2.33009900 & 0.24251300 \\
\hline $\mathrm{C}$ & 6.01917400 & -1.52445000 & 1.30772900 \\
\hline $\mathrm{C}$ & 5.17955200 & -0.65687900 & 2.03360000 \\
\hline $\mathrm{C}$ & 3.89117000 & -0.44063400 & 1.51788500 \\
\hline $\mathrm{H}$ & 3.24077000 & 0.26821600 & 2.0108140 \\
\hline $\mathrm{C}$ & 6.35094600 & -3.48940700 & -0.36136000 \\
\hline $\mathrm{C}$ & 5.53799500 & -4.26639500 & -1.41599600 \\
\hline $\mathrm{H}$ & 4.60514100 & -4.66868700 & -1.00860200 \\
\hline $\mathrm{H}$ & 6.13357700 & -5.11330900 & -1.77178800 \\
\hline $\mathrm{H}$ & 5.29461300 & -3.64755500 & -2.28778500 \\
\hline $\mathrm{C}$ & 7.64266200 & -3.00547700 & -1.05537500 \\
\hline $\mathrm{H}$ & 7.42951100 & -2.19786400 & -1.76579600 \\
\hline $\mathrm{H}$ & 8.09070600 & -3.83385200 & -1.61635300 \\
\hline $\mathrm{H}$ & 8.38193200 & -2.65690300 & -0.33514600 \\
\hline $\mathrm{C}$ & 6.71152800 & -4.47684500 & 0.77213000 \\
\hline $\mathrm{H}$ & 7.30967300 & -3.98783500 & 1.54375700 \\
\hline $\mathrm{H}$ & 7.28706200 & -5.31833000 & 0.36807200 \\
\hline $\mathrm{H}$ & 5.80366600 & -4.87956800 & 1.23552900 \\
\hline $\mathrm{C}$ & 5.50807500 & 0.06514500 & 3.3626870 \\
\hline $\mathrm{C}$ & 5.50242300 & 1.59312900 & 3.1296870 \\
\hline $\mathrm{H}$ & 6.25825100 & 1.88834700 & 2.3928630 \\
\hline $\mathrm{H}$ & 5.71923100 & 2.12110500 & 4.0657540 \\
\hline $\mathrm{H}$ & 4.52787700 & 1.93928800 & 2.7684690 \\
\hline $\mathrm{C}$ & 4.39270100 & -0.28886700 & 4.38023400 \\
\hline $\mathrm{H}$ & 3.39201200 & -0.02325300 & 4.03551000 \\
\hline $\mathrm{H}$ & 4.57788000 & 0.23706300 & 5.3239830 \\
\hline $\mathrm{H}$ & 4.39361900 & -1.36539400 & 4.58433300 \\
\hline $\mathrm{C}$ & 6.83385400 & -0.33478400 & 4.04115400 \\
\hline $\mathrm{H}$ & 6.92725800 & -1.41879900 & 4.13921600 \\
\hline $\mathrm{H}$ & 6.84790800 & 0.10156900 & 5.0467700 \\
\hline $\mathrm{H}$ & 7.71663400 & 0.03551700 & 3.5194970 \\
\hline $\mathrm{C}$ & 8.12819300 & -0.53258000 & 1.08816700 \\
\hline $\mathrm{H}$ & 7.77891900 & 0.43269000 & 1.4696620 \\
\hline $\mathrm{H}$ & 8.07547500 & -0.51505000 & -0.00611300 \\
\hline $\mathrm{H}$ & 9.16105300 & -0.69666200 & 1.40362800 \\
\hline $\mathrm{C}$ & 0.96004400 & -2.13029700 & -0.71337500 \\
\hline $\mathrm{C}$ & 0.05416700 & -2.16114200 & -1.77673400 \\
\hline $\mathrm{H}$ & -0.10283800 & -1.24987900 & -2.33329000 \\
\hline $\mathrm{C}$ & -0.64097100 & -3.32681300 & -2.10989800 \\
\hline
\end{tabular}




\begin{tabular}{|c|c|c|c|}
\hline $\mathrm{C}$ & -0.41574900 & -4.46298200 & -1.29666700 \\
\hline $\mathrm{C}$ & 0.61445000 & -4.51199800 & -0.32524200 \\
\hline $\mathrm{C}$ & 1.25815400 & -3.30768100 & -0.02591300 \\
\hline $\mathrm{H}$ & 2.01408800 & -3.28060700 & 0.74413300 \\
\hline $\mathrm{C}$ & 1.18868600 & -5.84020600 & 0.22439200 \\
\hline $\mathrm{C}$ & 0.17207300 & -6.75961400 & 0.92998600 \\
\hline $\mathrm{H}$ & -0.33897500 & -6.23668000 & 1.74311000 \\
\hline $\mathrm{H}$ & 0.70296600 & -7.61230800 & 1.36920200 \\
\hline $\mathrm{H}$ & -0.56572800 & -7.15485900 & 0.23315700 \\
\hline $\mathrm{C}$ & 1.77748900 & -6.60107500 & -0.98768800 \\
\hline $\mathrm{H}$ & 0.99595300 & -6.83542500 & -1.71527700 \\
\hline $\mathrm{H}$ & 2.23836900 & -7.54089900 & -0.65922900 \\
\hline $\mathrm{H}$ & 2.54553900 & -6.00040800 & -1.48808100 \\
\hline $\mathrm{C}$ & 2.32981800 & -5.58974200 & 1.23221100 \\
\hline $\mathrm{H}$ & 3.15267400 & -5.01255400 & 0.79866500 \\
\hline $\mathrm{H}$ & 2.74013400 & -6.55413900 & 1.55021000 \\
\hline $\mathrm{H}$ & 1.97190100 & -5.06985900 & 2.12702700 \\
\hline $\mathrm{C}$ & -1.45669200 & -3.37110500 & -3.42304700 \\
\hline $\mathrm{C}$ & -2.96041400 & -3.65394500 & -3.21813200 \\
\hline $\mathrm{H}$ & -3.14323800 & -4.69383000 & -2.94496000 \\
\hline $\mathrm{H}$ & -3.49527700 & -3.46719400 & -4.15662700 \\
\hline $\mathrm{H}$ & -3.39291300 & -2.99791300 & -2.45707900 \\
\hline $\mathrm{C}$ & -1.34025300 & -2.03425100 & -4.18516100 \\
\hline $\mathrm{H}$ & -1.76817500 & -1.19402400 & -3.63132000 \\
\hline $\mathrm{H}$ & -1.88450600 & -2.11755600 & -5.13169000 \\
\hline $\mathrm{H}$ & -0.29985300 & -1.78740300 & -4.42319500 \\
\hline $\mathrm{C}$ & -0.85415600 & -4.47246700 & -4.32600300 \\
\hline $\mathrm{H}$ & 0.20089200 & -4.26324800 & -4.53801400 \\
\hline $\mathrm{H}$ & -1.39144900 & -4.50661600 & -5.28160100 \\
\hline $\mathrm{H}$ & -0.92612700 & -5.45431600 & -3.85332600 \\
\hline $\mathrm{C}$ & -2.37857800 & -5.60424500 & -0.69626900 \\
\hline $\mathrm{H}$ & -2.96319100 & -4.69156700 & -0.83594600 \\
\hline $\mathrm{H}$ & -2.13311600 & -5.70636000 & 0.36407700 \\
\hline $\mathrm{H}$ & -2.96318300 & -6.46807000 & -1.02161200 \\
\hline $\mathrm{C}$ & -2.41543800 & 1.12771800 & 1.10533700 \\
\hline $\mathrm{C}$ & -3.04522900 & 1.06265500 & 2.35143800 \\
\hline $\mathrm{H}$ & -3.16552400 & 0.09140600 & 2.80972200 \\
\hline $\mathrm{C}$ & -3.53113200 & 2.20246900 & 2.99571900 \\
\hline $\mathrm{C}$ & -3.32594700 & 3.44952200 & 2.34372900 \\
\hline $\mathrm{C}$ & -2.77771200 & 3.54442000 & 1.04863900 \\
\hline $\mathrm{C}$ & -2.29426600 & 2.35790900 & 0.47045500 \\
\hline $\mathrm{H}$ & -1.84477400 & 2.37914300 & -0.51612900 \\
\hline $\mathrm{C}$ & -3.68823600 & -0.90092500 & -0.27796000 \\
\hline $\mathrm{C}$ & -4.59403300 & -1.47021400 & 0.61388300 \\
\hline
\end{tabular}




\begin{tabular}{|c|c|c|c|}
\hline $\mathrm{H}$ & -4.25897000 & -1.72545000 & 1.61066800 \\
\hline $\mathrm{C}$ & -5.93034000 & -1.72033600 & 0.27208800 \\
\hline $\mathrm{C}$ & -6.36793900 & -1.23238500 & -0.97851600 \\
\hline $\mathrm{C}$ & -5.46755600 & -0.73283700 & -1.95142000 \\
\hline $\mathrm{C}$ & -4.12979000 & -0.58208200 & -1.56940500 \\
\hline $\mathrm{H}$ & -3.41491900 & -0.15174000 & -2.26010800 \\
\hline $\mathrm{C}$ & -4.45239700 & 2.03488500 & 4.22967700 \\
\hline $\mathrm{C}$ & -5.89953300 & 2.24325300 & 3.72306800 \\
\hline $\mathrm{H}$ & -6.02879000 & 3.25819600 & 3.33525200 \\
\hline $\mathrm{H}$ & -6.61858600 & 2.08927100 & 4.53778100 \\
\hline $\mathrm{H}$ & -6.13092300 & 1.53517400 & 2.91996900 \\
\hline $\mathrm{C}$ & -4.34124200 & 0.61527600 & 4.82320900 \\
\hline $\mathrm{H}$ & -4.68921400 & -0.15451000 & 4.12918100 \\
\hline $\mathrm{H}$ & -4.96585600 & 0.54710600 & 5.72024400 \\
\hline $\mathrm{H}$ & -3.30942300 & 0.37646000 & 5.10626900 \\
\hline $\mathrm{C}$ & -4.20667300 & 3.01656800 & 5.39515600 \\
\hline $\mathrm{H}$ & -3.18456500 & 2.94328300 & 5.78389800 \\
\hline $\mathrm{H}$ & -4.88068500 & 2.76327300 & 6.22187700 \\
\hline $\mathrm{H}$ & -4.40289200 & 4.04641600 & 5.10462600 \\
\hline $\mathrm{C}$ & -2.71359500 & 4.80567400 & 0.15403200 \\
\hline $\mathrm{C}$ & -3.38067800 & 4.45175300 & -1.20178500 \\
\hline $\mathrm{H}$ & -2.91557700 & 3.60629600 & -1.71118000 \\
\hline $\mathrm{H}$ & -3.32098100 & 5.31633300 & -1.87297800 \\
\hline $\mathrm{H}$ & -4.43961500 & 4.21252000 & -1.04758700 \\
\hline $\mathrm{C}$ & -1.23432600 & 5.17781200 & -0.08031400 \\
\hline $\mathrm{H}$ & -0.74065700 & 5.42815100 & 0.86698100 \\
\hline $\mathrm{H}$ & -1.15946500 & 6.04553000 & -0.74330400 \\
\hline $\mathrm{H}$ & -0.67828000 & 4.35995700 & -0.54418700 \\
\hline $\mathrm{C}$ & -3.45564900 & 6.05629100 & 0.66714100 \\
\hline $\mathrm{H}$ & -4.48730600 & 5.83512200 & 0.94976600 \\
\hline $\mathrm{H}$ & -3.47403900 & 6.79310600 & -0.14494700 \\
\hline $\mathrm{H}$ & -2.96239500 & 6.53213100 & 1.51592700 \\
\hline $\mathrm{C}$ & -2.64804900 & 5.29471500 & 3.63773300 \\
\hline $\mathrm{H}$ & -3.00852300 & 6.30061400 & 3.86727700 \\
\hline $\mathrm{H}$ & -1.77023700 & 5.35505200 & 2.98689700 \\
\hline $\mathrm{H}$ & -2.35413100 & 4.79978000 & 4.57132300 \\
\hline $\mathrm{C}$ & -6.76898500 & -2.54333700 & 1.28053700 \\
\hline $\mathrm{C}$ & -7.06661600 & -1.70041500 & 2.53877000 \\
\hline $\mathrm{H}$ & -6.14169400 & -1.36068100 & 3.01518600 \\
\hline $\mathrm{H}$ & -7.62700200 & -2.29201700 & 3.27304000 \\
\hline $\mathrm{H}$ & -7.65861600 & -0.81111500 & 2.29909500 \\
\hline $\mathrm{C}$ & -5.92922000 & -3.77690700 & 1.70728000 \\
\hline $\mathrm{H}$ & -5.66504500 & -4.38338100 & 0.83341000 \\
\hline $\mathrm{H}$ & -6.51209800 & -4.40242900 & 2.39299900 \\
\hline
\end{tabular}




\begin{tabular}{|c|c|c|c|}
\hline $\mathrm{H}$ & -5.00390000 & -3.50409500 & 2.22138600 \\
\hline $\mathrm{C}$ & -8.08929300 & -3.11669700 & 0.72402900 \\
\hline $\mathrm{H}$ & -8.85696400 & -2.36067100 & 0.56419800 \\
\hline $\mathrm{H}$ & -8.48660800 & -3.83688600 & 1.44936500 \\
\hline $\mathrm{H}$ & -7.93092700 & -3.63924700 & -0.22319700 \\
\hline $\mathrm{C}$ & -5.90674200 & -0.41649800 & -3.39792200 \\
\hline $\mathrm{C}$ & -6.87773500 & 0.78295400 & -3.45585900 \\
\hline $\mathrm{H}$ & -7.85510500 & 0.53789300 & -3.03975900 \\
\hline $\mathrm{H}$ & -7.02909800 & 1.08226200 & -4.49992000 \\
\hline $\mathrm{H}$ & -6.46695300 & 1.64476500 & -2.91758200 \\
\hline $\mathrm{C}$ & -4.69918200 & -0.06623400 & -4.28895000 \\
\hline $\mathrm{H}$ & -4.16530300 & 0.82970600 & -3.96064300 \\
\hline $\mathrm{H}$ & -5.05613100 & 0.10755500 & -5.31056900 \\
\hline $\mathrm{H}$ & -3.97783100 & -0.88697300 & -4.32845100 \\
\hline $\mathrm{C}$ & -6.58422700 & -1.66375600 & -4.01080700 \\
\hline $\mathrm{H}$ & -6.87896500 & -1.45528900 & -5.04675000 \\
\hline $\mathrm{H}$ & -7.47343600 & -1.95430100 & -3.44892400 \\
\hline $\mathrm{H}$ & -5.88746900 & -2.50973400 & -4.02335400 \\
\hline $\mathrm{C}$ & -8.43358700 & -0.16327200 & -0.66318700 \\
\hline $\mathrm{H}$ & -8.49249400 & -0.29441100 & 0.42269200 \\
\hline $\mathrm{H}$ & -9.44274500 & -0.17901200 & -1.08262800 \\
\hline $\mathrm{H}$ & -7.96321300 & 0.80336500 & -0.87339600 \\
\hline $\mathrm{C}$ & 0.44891800 & 0.19942300 & 1.89992600 \\
\hline $\mathrm{C}$ & 1.14934900 & 0.58608700 & 0.72316100 \\
\hline $\mathrm{C}$ & 1.52462700 & 1.92110200 & 0.53029100 \\
\hline $\mathrm{H}$ & 2.03372700 & 2.19521600 & -0.38457100 \\
\hline $\mathrm{C}$ & 1.27011900 & 2.92181400 & 1.48329500 \\
\hline $\mathrm{H}$ & 1.53978700 & 3.95551700 & 1.30365500 \\
\hline $\mathrm{C}$ & 0.64418000 & 2.51711300 & 2.64360100 \\
\hline $\mathrm{C}$ & 0.25787500 & 1.19198600 & 2.84224600 \\
\hline $\mathrm{C}$ & -0.43444600 & 2.38773300 & 4.60436400 \\
\hline $\mathrm{H}$ & 0.03797100 & 2.40215600 & 5.59163800 \\
\hline $\mathrm{H}$ & -1.48029700 & 2.69604000 & 4.64742200 \\
\hline $\mathrm{C}$ & -1.35278600 & -1.52460200 & 1.41256700 \\
\hline $\mathrm{C}$ & -1.87227100 & -2.80658900 & 1.62041600 \\
\hline $\mathrm{H}$ & -2.74570700 & -3.11924400 & 1.06493000 \\
\hline $\mathrm{C}$ & -1.29819600 & -3.72248300 & 2.52209800 \\
\hline $\mathrm{H}$ & -1.72879200 & -4.70423300 & 2.68058100 \\
\hline $\mathrm{C}$ & -0.16198900 & -3.31704700 & 3.18557900 \\
\hline $\mathrm{C}$ & 0.38717300 & -2.05672600 & 2.97250000 \\
\hline $\mathrm{C}$ & -0.16079400 & -1.13981100 & 2.10156700 \\
\hline $\mathrm{C}$ & 1.60403500 & -3.08686300 & 4.54001200 \\
\hline $\mathrm{H}$ & 2.60065200 & -3.53050300 & 4.48357200 \\
\hline $\mathrm{H}$ & 1.35180600 & -2.80005900 & 5.57028900 \\
\hline
\end{tabular}




\begin{tabular}{|c|c|c|c|}
\hline $\mathrm{C}$ & 4.44143300 & 0.63656400 & -2.30211900 \\
\hline $\mathrm{C}$ & 4.64236200 & 2.07542900 & -2.25739600 \\
\hline $\mathrm{C}$ & 4.73312800 & 2.70648600 & -1.06467000 \\
\hline $\mathrm{H}$ & 4.60759000 & 2.63559300 & -3.18722400 \\
\hline $\mathrm{H}$ & 4.69196500 & 2.08843600 & -0.16777400 \\
\hline $\mathrm{C}$ & 4.76604300 & 4.14608500 & -0.83173400 \\
\hline $\mathrm{C}$ & 5.06154700 & 5.08112200 & -1.84135300 \\
\hline $\mathrm{C}$ & 4.44338600 & 4.62744100 & 0.45075500 \\
\hline $\mathrm{C}$ & 5.01277700 & 6.44688800 & -1.57965600 \\
\hline $\mathrm{H}$ & 5.33510100 & 4.73414900 & -2.83303500 \\
\hline $\mathrm{C}$ & 4.38521800 & 5.99479600 & 0.70901900 \\
\hline $\mathrm{H}$ & 4.21725100 & 3.91490400 & 1.23924000 \\
\hline $\mathrm{C}$ & 4.66706100 & 6.91073600 & -0.30636000 \\
\hline $\mathrm{H}$ & 5.24352300 & 7.15406700 & -2.37122500 \\
\hline $\mathrm{H}$ & 4.12104900 & 6.34551600 & 1.70257400 \\
\hline $\mathrm{H}$ & 4.62497700 & 7.97750600 & -0.10752800 \\
\hline S & -0.57350700 & 1.71514100 & -3.54894800 \\
\hline $\mathrm{C}$ & -0.08023600 & 3.46681000 & -3.30181100 \\
\hline $\mathrm{C}$ & -0.97272000 & 4.43527100 & -3.76614800 \\
\hline $\mathrm{C}$ & 1.13931100 & 3.84726400 & -2.73911000 \\
\hline $\mathrm{C}$ & -0.64396400 & 5.78734400 & -3.66657800 \\
\hline $\mathrm{H}$ & -1.92749000 & 4.12569100 & -4.17871200 \\
\hline $\mathrm{C}$ & 1.45916800 & 5.20184500 & -2.62608000 \\
\hline $\mathrm{H}$ & 1.83391300 & 3.09334600 & -2.39091700 \\
\hline $\mathrm{C}$ & 0.57118700 & 6.17158400 & -3.09569200 \\
\hline $\mathrm{H}$ & -1.34183600 & 6.54014800 & -4.02323500 \\
\hline $\mathrm{H}$ & 2.39913300 & 5.49607300 & -2.17042500 \\
\hline $\mathrm{H}$ & 0.82411400 & 7.22506300 & -3.01120400 \\
\hline $\mathrm{O}$ & 0.26279400 & 0.99993700 & -2.38259700 \\
\hline $\mathrm{O}$ & -2.02368400 & 1.70979400 & -3.12134200 \\
\hline $\mathrm{C}$ & 4.55111200 & 0.19004800 & -4.85094000 \\
\hline $\mathrm{H}$ & 4.50684400 & 1.26751100 & -5.02262900 \\
\hline $\mathrm{H}$ & 5.55966700 & -0.16214500 & -5.11362000 \\
\hline $\mathrm{H}$ & 3.84413300 & -0.28985500 & -5.53257500 \\
\hline $\mathrm{C}$ & 4.26149700 & -0.17780000 & -3.41887400 \\
\hline $\mathrm{H}$ & 4.24948400 & -1.24761200 & -3.21423200 \\
\hline $\mathrm{H}$ & 4.57193000 & 0.13415100 & -1.35016600 \\
\hline $\mathrm{H}$ & 2.25163000 & 1.22268900 & -4.04767800 \\
\hline
\end{tabular}




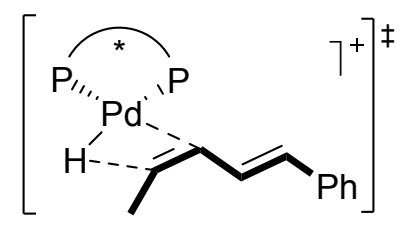

TS-5a

Total SCF energy (M06/SDD-6-311++G(d,p)/SMD(1,4-Dioxane)): -4717.570569a.u.

Thermal correction to Gibbs Free Energy at 298.15 K: 1.673993a.u

Gibbs free energy at 298.15 K (M06/SDD-6-311++G(d,p)/SMD(1,4-Dioxane)): -4715.896576a.u.

$\begin{array}{lrrc}\mathrm{P} & 1.46877800 & -0.33528400 & -0.20368700 \\ \mathrm{P} & -1.88879900 & 0.06250200 & -0.36643400 \\ \mathrm{O} & 0.24384000 & -0.19195800 & 4.47139200 \\ \mathrm{O} & -1.03784600 & -1.98462900 & 5.16249000 \\ \mathrm{O} & -0.88008900 & 2.62763700 & 3.54315400 \\ \mathrm{O} & 0.33091900 & 4.52618700 & 3.03522200 \\ \mathrm{O} & -3.24522900 & 5.73307600 & 0.96152400 \\ \mathrm{O} & -6.64400200 & -2.64463700 & -2.75624400 \\ \mathrm{O} & 0.77733300 & -5.40383900 & 2.86794200 \\ \mathrm{O} & 7.36316300 & -0.94977600 & -0.98982000 \\ \mathrm{C} & -1.65653800 & -0.61191900 & 1.32437200 \\ \mathrm{C} & -0.70523200 & -0.00786300 & 2.20169200 \\ \mathrm{C} & -0.61494000 & -0.55656900 & 3.46521900 \\ \mathrm{C} & -1.38135300 & -1.64577400 & 3.88033700 \\ \mathrm{C} & -2.28913500 & -2.25119500 & 3.03956100 \\ \mathrm{C} & -2.41245000 & -1.70790800 & 1.74852500 \\ \mathrm{C} & -0.20374100 & -0.91048500 & 5.62729100 \\ \mathrm{C} & 1.15888500 & 1.13729600 & 0.83794400 \\ \mathrm{C} & 1.85131700 & 2.31161600 & 0.50848200 \\ \mathrm{C} & 1.65289600 & 3.51825600 & 1.19539800 \\ \mathrm{C} & 0.72519600 & 3.49854300 & 2.21549400 \\ \mathrm{C} & -0.00255600 & 2.35049600 & 2.52387600 \\ \mathrm{C} & 0.15488100 & 1.15038500 & 1.84709800 \\ \mathrm{C} & -0.46338400 & 3.90078400 & 4.05372300 \\ \mathrm{C} & -2.33709900 & 1.79199000 & -0.01570500 \\ \mathrm{C} & -1.82190100 & 2.82613400 & -0.79122600 \\ \mathrm{C} & -2.11435000 & 4.17228900 & -0.52862600 \\ \mathrm{C} & -2.94521100 & 4.44110500 & 0.58916400 \\ \mathrm{C} & -3.57524600 & 3.40367100 & 1.32923000 \\ \mathrm{C} & -3.20951300 & 2.09232700 & 1.03310100 \\ \mathrm{C} & -4.67367200 & 3.66978400 & 2.38147500 \\ \mathrm{C} & -5.80240200 & 4.51449200 & 1.74693200 \\ \mathrm{C} & -4.11007700 & 4.37490100 & 3.63072900 \\ \mathrm{C} & -5.31727100 & 2.35629600 & 2.87245200 \\ & -1.53942000 & 5.24789500 & -1.48225500\end{array}$




\begin{tabular}{|c|c|c|c|}
\hline $\mathrm{C}$ & -0.19855100 & 5.79203800 & -0.93917400 \\
\hline $\mathrm{C}$ & -1.22659000 & 4.63257100 & -2.86629200 \\
\hline $\mathrm{C}$ & -2.54050500 & 6.39267100 & -1.75143700 \\
\hline $\mathrm{C}$ & -2.25409100 & 6.49271700 & 1.65800400 \\
\hline $\mathrm{C}$ & -3.35737300 & -0.79699400 & -1.01510400 \\
\hline $\mathrm{C}$ & -4.62707900 & -0.61181600 & -0.47492800 \\
\hline $\mathrm{C}$ & -3.18610700 & -1.73327400 & -2.03992000 \\
\hline $\mathrm{C}$ & -4.26716000 & -2.41196800 & -2.60455100 \\
\hline $\mathrm{C}$ & -5.55882700 & -2.10542300 & -2.10138000 \\
\hline $\mathrm{C}$ & -5.74960300 & -1.29721800 & -0.95644800 \\
\hline $\mathrm{C}$ & -7.05834700 & -1.13318600 & -0.14443200 \\
\hline $\mathrm{C}$ & -6.72111500 & -1.37541400 & 1.35137000 \\
\hline $\mathrm{C}$ & -8.17879100 & -2.13900000 & -0.48131800 \\
\hline $\mathrm{C}$ & -7.33852000 & -1.70780000 & -3.58580900 \\
\hline $\mathrm{C}$ & -7.59599400 & 0.30553100 & -0.30474800 \\
\hline $\mathrm{C}$ & -4.03185500 & -3.49323400 & -3.68001800 \\
\hline $\mathrm{C}$ & -2.52809700 & -3.69067600 & -3.95670200 \\
\hline $\mathrm{C}$ & -4.59307800 & -4.83926800 & -3.16695000 \\
\hline $\mathrm{C}$ & -4.69718500 & -3.13674200 & -5.02738800 \\
\hline $\mathrm{C}$ & 1.23710400 & -1.85438200 & 0.76877900 \\
\hline $\mathrm{C}$ & 1.94792400 & -2.06895400 & 1.95307300 \\
\hline $\mathrm{C}$ & 1.84301500 & -3.26820500 & 2.65369900 \\
\hline $\mathrm{C}$ & 0.97677300 & -4.26329600 & 2.11881100 \\
\hline $\mathrm{C}$ & 0.36235600 & -4.13237200 & 0.85561600 \\
\hline $\mathrm{C}$ & 0.47915700 & -2.88358800 & 0.22699600 \\
\hline $\mathrm{C}$ & -0.38843600 & -5.23511900 & 0.06970200 \\
\hline $\mathrm{C}$ & -1.89320500 & -4.89365200 & 0.00986400 \\
\hline $\mathrm{C}$ & -0.21558900 & -6.67214500 & 0.60401700 \\
\hline $\mathrm{C}$ & 0.17323300 & -5.26217800 & -1.37627400 \\
\hline $\mathrm{C}$ & 1.98927500 & -3.98020700 & 5.13626300 \\
\hline $\mathrm{C}$ & -0.46063600 & -5.40826900 & 3.58418700 \\
\hline $\mathrm{C}$ & 2.75460000 & -3.53156800 & 3.87159300 \\
\hline $\mathrm{C}$ & 3.55654100 & -2.26911300 & 4.24831200 \\
\hline $\mathrm{C}$ & 6.01763900 & -0.70101200 & -0.82055600 \\
\hline $\mathrm{C}$ & 5.12885900 & -1.31024700 & -1.74977400 \\
\hline $\mathrm{C}$ & 4.45362600 & -2.50277700 & -3.90248300 \\
\hline $\mathrm{C}$ & 3.76073600 & -1.14517900 & -1.53248100 \\
\hline $\mathrm{C}$ & 3.27232800 & -0.37291200 & -0.47570100 \\
\hline $\mathrm{C}$ & 4.17361800 & 0.26479000 & 0.36902100 \\
\hline $\mathrm{C}$ & 5.56124600 & 0.16141800 & 0.20316500 \\
\hline $\mathrm{C}$ & 7.92793200 & -1.91557400 & -0.09817800 \\
\hline $\mathrm{C}$ & 6.44497200 & -3.31293800 & -2.66937400 \\
\hline $\mathrm{C}$ & 7.81989900 & 1.37766100 & 0.55083500 \\
\hline $\mathrm{C}$ & 6.44453500 & 1.01141800 & 1.14914900 \\
\hline
\end{tabular}




\begin{tabular}{|c|c|c|c|}
\hline $\mathrm{C}$ & 5.74322800 & 2.36370200 & 1.44615000 \\
\hline $\mathrm{C}$ & 6.49186300 & -1.07850600 & -3.85167200 \\
\hline $\mathrm{C}$ & 3.75867300 & -4.63869400 & 3.47526100 \\
\hline $\mathrm{C}$ & 5.62784000 & -2.04793000 & -3.01178200 \\
\hline $\mathrm{C}$ & 6.61755700 & 0.27533900 & 2.4969180 \\
\hline $\mathrm{H}$ & -2.88276600 & -3.10037600 & 3.35646000 \\
\hline $\mathrm{H}$ & -3.12648300 & -2.15544000 & 1.06979300 \\
\hline $\mathrm{H}$ & 0.65683500 & -1.32229000 & 6.15381500 \\
\hline $\mathrm{H}$ & -0.79681100 & -0.24459300 & 6.26712700 \\
\hline $\mathrm{H}$ & 2.58396500 & 2.29579800 & -0.28774000 \\
\hline $\mathrm{H}$ & 2.21245400 & 4.40978100 & 0.9386690 \\
\hline $\mathrm{H}$ & -1.33829200 & 4.51562800 & 4.25652900 \\
\hline $\mathrm{H}$ & 0.15063600 & 3.74989800 & 4.9513770 \\
\hline $\mathrm{H}$ & -1.17121200 & 2.56209700 & -1.61419900 \\
\hline $\mathrm{H}$ & -3.59299400 & 1.28458400 & 1.63909000 \\
\hline $\mathrm{H}$ & -6.23156000 & 3.99148000 & 0.88442300 \\
\hline $\mathrm{H}$ & -6.60350200 & 4.67011700 & 2.47855000 \\
\hline $\mathrm{H}$ & -5.44050900 & 5.48718900 & 1.41422000 \\
\hline $\mathrm{H}$ & -3.67389300 & 5.34499700 & 3.39604200 \\
\hline $\mathrm{H}$ & -4.91212300 & 4.53930800 & 4.35920800 \\
\hline $\mathrm{H}$ & -3.35069400 & 3.74487800 & 4.10513200 \\
\hline $\mathrm{H}$ & -4.60440700 & 1.71261400 & 3.40002400 \\
\hline $\mathrm{H}$ & -6.12128000 & 2.59621000 & 3.57533700 \\
\hline $\mathrm{H}$ & -5.76198900 & 1.78520600 & 2.05040100 \\
\hline $\mathrm{H}$ & 0.22188800 & 6.52052700 & -1.64308700 \\
\hline $\mathrm{H}$ & -0.29647300 & 6.28162700 & 0.02962900 \\
\hline $\mathrm{H}$ & 0.51797500 & 4.97330500 & -0.82162800 \\
\hline $\mathrm{H}$ & -0.94433800 & 5.43065600 & -3.55992400 \\
\hline $\mathrm{H}$ & -0.38514500 & 3.93381700 & -2.82492500 \\
\hline $\mathrm{H}$ & -2.09298800 & 4.10853500 & -3.28359400 \\
\hline $\mathrm{H}$ & -3.47284800 & 5.99274300 & -2.16512700 \\
\hline $\mathrm{H}$ & -2.79813200 & 6.96217200 & -0.86204900 \\
\hline $\mathrm{H}$ & -2.11212300 & 7.07996100 & -2.48911700 \\
\hline $\mathrm{H}$ & -1.84261900 & 7.26661500 & 1.00275100 \\
\hline $\mathrm{H}$ & -2.74626900 & 6.98418200 & 2.50213600 \\
\hline $\mathrm{H}$ & -1.44379400 & 5.85635100 & 2.02382000 \\
\hline $\mathrm{H}$ & -4.75220100 & 0.07649500 & 0.34876100 \\
\hline $\mathrm{H}$ & -2.18421300 & -1.90682600 & -2.40934900 \\
\hline $\mathrm{H}$ & -7.63231400 & -1.28289200 & 1.95149200 \\
\hline $\mathrm{H}$ & -6.31923200 & -2.38418200 & 1.49796000 \\
\hline $\mathrm{H}$ & -5.99562900 & -0.66234900 & 1.75113500 \\
\hline $\mathrm{H}$ & -8.65274000 & -1.95449000 & -1.44459700 \\
\hline $\mathrm{H}$ & -7.81283600 & -3.16881300 & -0.47493200 \\
\hline $\mathrm{H}$ & -8.95877600 & -2.05100600 & 0.28299900 \\
\hline
\end{tabular}


H

$\mathrm{H}$

$\mathrm{H}$

$\mathrm{H}$

$\mathrm{H}$

$\mathrm{H}$

$\mathrm{H}$

$\mathrm{H}$

$\mathrm{H}$

$\mathrm{H}$

$\mathrm{H}$

$\mathrm{H}$

$\mathrm{H}$

$\mathrm{H}$

$\mathrm{H}$

$\mathrm{H}$

$\mathrm{H}$

$\mathrm{H}$

$\mathrm{H}$

$\mathrm{H}$

$\mathrm{H}$

$\mathrm{H}$

$\mathrm{H}$

$\mathrm{H}$

$\mathrm{H}$

$\mathrm{H}$

$\mathrm{H}$

$\mathrm{H}$

$\mathrm{H}$

$\mathrm{H}$

$\mathrm{H}$

$\mathrm{H}$

$\mathrm{H}$

$\mathrm{H}$

$\mathrm{H}$

$\mathrm{H}$

$\mathrm{H}$

$\mathrm{H}$

$\mathrm{H}$

$\mathrm{H}$

$\mathrm{H}$

$\mathrm{H}$

$\mathrm{H}$

$\mathrm{H}$

\begin{tabular}{rcc}
-7.73589100 & -0.87183300 & -3.00267200 \\
-6.67930800 & -1.31303500 & -4.36622600 \\
-8.16285400 & -2.25784100 & -4.04336300 \\
-7.87097300 & 0.51909000 & -1.34300100 \\
-8.48855900 & 0.44671000 & 0.31511100 \\
-6.85231600 & 1.05021700 & -0.00025600 \\
-2.06523000 & -2.79038900 & -4.37984100 \\
-1.97576200 & -3.97424600 & -3.05588000 \\
-2.40251600 & -4.49720300 & -4.68570100 \\
-4.42288700 & -5.62375100 & -3.91339800 \\
-4.09174300 & -5.13894300 & -2.24015700 \\
-5.66559900 & -4.77216800 & -2.97625900 \\
-5.78239400 & -3.21171400 & -4.97439800 \\
-4.42653800 & -2.12366800 & -5.34759000 \\
-4.35574800 & -3.83416600 & -5.80057700 \\
2.58795700 & -1.27963600 & 2.32151700 \\
-0.03209200 & -2.70359400 & -0.71241400 \\
-2.33685400 & -4.86575400 & 1.01075200 \\
-2.07026700 & -3.91986500 & -0.45829900 \\
-2.42903500 & -5.65063200 & -0.57428000 \\
-0.6224500 & -7.36541800 & -0.14088700 \\
0.83654000 & -6.92117200 & 0.76124800 \\
-0.74927700 & -6.85456100 & 1.53605600 \\
-0.34143700 & -6.03570200 & -1.95617000 \\
0.03948700 & -4.31544900 & -1.90595300 \\
1.24326900 & -5.49645300 & -1.37016900 \\
2.65650400 & -3.92858700 & 6.00389800 \\
1.12615800 & -3.33809600 & 5.33215800 \\
1.63895600 & -5.00781200 & 5.05077100 \\
-0.51045800 & -4.57474400 & 4.29133400 \\
-1.31488900 & -5.34635600 & 2.90231600 \\
-0.49700100 & -6.35493900 & 4.12677600 \\
4.21774600 & -1.94126500 & 3.43901900 \\
2.89764100 & -1.43374900 & 4.51451100 \\
4.18874800 & -2.49012100 & 5.11389100 \\
3.83704300 & -1.65985200 & -4.23536600 \\
4.85424800 & -2.98319600 & -4.80028600 \\
3.80831000 & -3.23236600 & -3.39985100 \\
3.04951900 & -1.62247000 & -2.19296800 \\
3.79059800 & 0.87327800 & 1.17369800 \\
7.247983000 & -2.26652300 & -0.56663400 \\
\hline .61782900 & -3.89583000 & -3.58091500
\end{tabular}




\begin{tabular}{|c|c|c|c|}
\hline $\mathrm{H}$ & 7.41918900 & -3.06759500 & -2.25013500 \\
\hline $\mathrm{H}$ & 5.90549500 & -3.95189900 & -1.96107900 \\
\hline $\mathrm{H}$ & 8.49660300 & 0.53170500 & 0.45353900 \\
\hline $\mathrm{H}$ & 7.70568200 & 1.82764200 & -0.44014200 \\
\hline $\mathrm{H}$ & 8.30186300 & 2.11450300 & 1.20233700 \\
\hline $\mathrm{H}$ & 6.42551900 & 3.00004400 & 2.01896200 \\
\hline $\mathrm{H}$ & 5.48316800 & 2.88898200 & 0.52299200 \\
\hline $\mathrm{H}$ & 4.83647200 & 2.25436000 & 2.04789000 \\
\hline $\mathrm{H}$ & 5.90116400 & -0.21263400 & -4.17490200 \\
\hline $\mathrm{H}$ & 7.35292200 & -0.72167500 & -3.28463900 \\
\hline $\mathrm{H}$ & 6.85600500 & -1.58662400 & -4.75200800 \\
\hline $\mathrm{H}$ & 4.36046400 & -4.32661500 & 2.61424700 \\
\hline $\mathrm{H}$ & 4.43878200 & -4.84727200 & 4.30962800 \\
\hline $\mathrm{H}$ & 3.23680700 & -5.56380600 & 3.21819300 \\
\hline $\mathrm{H}$ & 7.22153400 & 0.88008200 & 3.18320700 \\
\hline $\mathrm{H}$ & 5.64406200 & 0.09998000 & 2.96901600 \\
\hline $\mathrm{H}$ & 7.10802600 & -0.69496800 & 2.38319800 \\
\hline $\mathrm{Pd}$ & -0.13094000 & -0.02110600 & -1.91803400 \\
\hline $\mathrm{H}$ & -1.26466400 & 0.22833500 & -3.03850400 \\
\hline $\mathrm{C}$ & 1.26482200 & 0.37627900 & -3.65647500 \\
\hline $\mathrm{C}$ & 1.90476200 & 1.63636900 & -3.31298600 \\
\hline $\mathrm{H}$ & 1.26335600 & 2.51067700 & -3.25141200 \\
\hline $\mathrm{C}$ & 3.22237000 & 1.74818600 & -3.03241500 \\
\hline $\mathrm{H}$ & 3.84326300 & 0.86318000 & -3.13225000 \\
\hline $\mathrm{C}$ & 0.00450200 & 0.27506500 & -4.28712500 \\
\hline $\mathrm{C}$ & 3.92009600 & 2.93937500 & -2.56056000 \\
\hline $\mathrm{C}$ & 5.31641200 & 2.88393600 & -2.39862100 \\
\hline $\mathrm{C}$ & 3.25538800 & 4.13378100 & -2.21729600 \\
\hline $\mathrm{C}$ & 6.02646600 & 3.98512400 & -1.92482400 \\
\hline $\mathrm{H}$ & 5.84035000 & 1.96371600 & -2.64130100 \\
\hline $\mathrm{C}$ & 3.96359400 & 5.22849400 & -1.73404100 \\
\hline $\mathrm{H}$ & 2.17753500 & 4.20444900 & -2.32051400 \\
\hline $\mathrm{C}$ & 5.35354200 & 5.16066400 & -1.58706400 \\
\hline $\mathrm{H}$ & 7.10454600 & 3.92307900 & -1.81146500 \\
\hline $\mathrm{H}$ & 3.43201900 & 6.13981300 & -1.47469200 \\
\hline $\mathrm{H}$ & 5.90495400 & 6.01738100 & -1.21196600 \\
\hline $\mathrm{H}$ & 1.89417400 & -0.50862000 & -3.66434300 \\
\hline $\mathrm{H}$ & -0.24539400 & -0.71135400 & -4.67233400 \\
\hline $\mathrm{C}$ & -0.68160900 & 1.42804800 & -4.98537900 \\
\hline $\mathrm{H}$ & -0.61448000 & 2.35964200 & -4.42314900 \\
\hline $\mathrm{H}$ & -0.19509200 & 1.58566000 & -5.95681300 \\
\hline $\mathrm{H}$ & -1.73806900 & 1.21260900 & -5.16405000 \\
\hline
\end{tabular}


<smiles>CC1=C(/C=C/c2ccccc2)[Pb](OS(=O)c2ccccc2)[PH]1PCP</smiles>

TS-5b

Total SCF energy (M06/SDD-6-311++G(d,p)/SMD(1,4-Dioxane)): -5497.799617a.u.

Thermal correction to Gibbs Free Energy at 298.15 K: 1.763011a.u

Gibbs free energy at 298.15 K (M06/SDD-6-311++G(d,p)/SMD(1,4-Dioxane)): -5496.036606a.u.

$\mathrm{Pd}$

$P$

$P$

0

0

0

0

0

O

0

O

C

C

$\mathrm{H}$

C

C

C

C

$\mathrm{H}$

C

C

$\mathrm{H}$

$\mathrm{H}$

$\mathrm{H}$

C

$\mathrm{H}$

$\mathrm{H}$

$\mathrm{H}$

C

$\mathrm{H}$

$\mathrm{H}$

$\mathrm{H}$

C

C

$\mathrm{H}$

$$
-1.72238600
$$

$-1.74479100$

1.97587000

$-7.42780800$

0.69897600

2.70400900

7.86110800

$-0.80868400$

$-0.13683700$

$-1.67518100$

$-0.70899900$

$-3.42215700$

$-4.25354600$

$-3.83475700$

$-5.57957300$

$-6.07021700$

$-5.22003800$

$-3.90684000$

$-3.24831500$

$-6.43020300$

$-5.60242300$

$-4.71928200$

$-6.22281300$

$-5.27216100$

$-7.64557100$

$-7.33879500$

$-8.12204000$

$-8.39493100$

$-6.91730800$

$-7.52902400$

$-7.51759300$

$-6.06526400$

$-5.56340800$

$-5.58196900$

$-6.39663800$

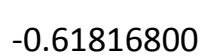

2.57774500 
H

$\mathrm{H}$

C

$\mathrm{H}$

$\mathrm{H}$

$\mathrm{H}$

C

$\mathrm{H}$

$\mathrm{H}$

$\mathrm{H}$

C

$\mathrm{H}$

$\mathrm{H}$

$\mathrm{H}$

C

C

$\mathrm{H}$

C

C

C

C

$\mathrm{H}$

C

C

$\mathrm{H}$

$\mathrm{H}$

$\mathrm{H}$

C

$\mathrm{H}$

$\mathrm{H}$

$\mathrm{H}$

C

$\mathrm{H}$

$\mathrm{H}$

$\mathrm{H}$

C

C

$\mathrm{H}$

$\mathrm{H}$

$\mathrm{H}$

C

$\mathrm{H}$

$\mathrm{H}$

$\mathrm{H}$

\begin{tabular}{ccc}
-5.73550300 & -1.85411800 & 4.21585900 \\
-4.64357200 & -1.96820700 & 2.82325200 \\
-4.44778300 & 0.53654500 & 4.03685000 \\
-3.45746400 & 0.15037700 & 3.79086200 \\
-4.67490500 & 0.24832100 & 5.06996600 \\
-4.39010200 & 1.62854100 & 3.98825600 \\
-6.88499200 & 0.56154600 & 3.67047800 \\
-6.95152100 & 1.64647200 & 3.55619500 \\
-6.91847200 & 0.32915300 & 4.74136600 \\
-7.77252600 & 0.11990700 & 3.21749600 \\
-8.13198000 & 0.04050900 & 0.69944500 \\
-7.79073300 & -0.80655000 & 1.30434700 \\
-8.00752800 & -0.21216300 & -0.35997700 \\
-9.18658700 & 0.23390500 & 0.90873800 \\
-1.01577100 & 2.01632400 & -0.78981500 \\
-0.04288000 & 2.21883300 & -1.77027100 \\
0.28378900 & 1.36482600 & -2.34323100 \\
0.51396700 & 3.48178600 & -1.99736000 \\
\hline .07611600 & 4.53805400 & -1.16280500 \\
-1.01787100 & 4.39969100 & -0.27197900 \\
-1.51575900 & 3.10886300 & -0.07993800 \\
-2.31558500 & 2.94346500 & 0.62520200 \\
-1.79849300 & 5.60507700 & 0.30440000 \\
-0.96434000 & 6.57915200 & 1.16006200 \\
-0.44264400 & 6.05563500 & 1.96596400 \\
-1.63255800 & 7.31475400 & 1.62313400 \\
-0.24416800 & 7.12846200 & 0.55504200 \\
-2.38206200 & 6.38064100 & -0.90016300 \\
-1.58245100 & 6.75747300 & -1.54297300 \\
-2.97765300 & 7.23317900 & -0.55075800 \\
-3.03232400 & 5.73368900 & -1.50035500 \\
-2.97700300 & 5.13936400 & 1.18440100 \\
-3.68401300 & 4.50816700 & 0.63648800 \\
-3.52598900 & 6.01799800 & 1.53929300 \\
-2.62909800 & 4.58564400 & 2.06258800 \\
1.44079300 & 3.68677700 & -3.21904600 \\
2.88325800 & 4.08817100 & -2.84040600 \\
2.94211700 & 5.12041400 & -2.49436000 \\
3.52672000 & 4.00817900 & -3.72415100 \\
\hline .29517800 & 3.42583100 & -2.07166500 \\
\hline
\end{tabular}




\begin{tabular}{ccc}
0.82857700 & 4.77845000 & -4.12624700 \\
-0.17110900 & 4.48112600 & -4.46427200 \\
1.45704100 & 4.92093400 & -5.01379400 \\
0.74797900 & 5.73194100 & -3.60099400 \\
1.81858500 & 5.92529900 & -0.39036800 \\
2.49052400 & 5.06666000 & -0.45208500 \\
1.49500300 & 6.04115900 & 0.64636500 \\
2.34287500 & 6.82764100 & -0.71425100 \\
2.21226200 & -1.09819800 & 1.08117100 \\
2.60016400 & -1.20222400 & 2.42062100 \\
2.71522400 & -0.29232300 & 2.99328800 \\
2.81831300 & -2.44033900 & 3.02719500 \\
2.62048500 & -3.59907300 & 2.22855000 \\
2.35561900 & -3.52738100 & 0.84875400 \\
2.10813900 & -2.25233900 & 0.31465900 \\
1.83347600 & -2.14518300 & -0.72881000 \\
3.74364300 & 0.98419100 & 0.01634300 \\
4.55940000 & 1.33495100 & 1.08934800 \\
4.11435900 & 1.42105900 & 2.07265700 \\
5.92982600 & 1.58186200 & 0.94331800 \\
6.48392400 & 1.34481700 & -0.33467300 \\
5.68034800 & 1.09108000 & -1.47329600 \\
4.30862300 & 0.90686300 & -1.26179100 \\
3.66582600 & 0.63719800 & -2.08980000 \\
3.42406900 & -2.50797300 & 4.44720700 \\
4.89112300 & -2.97330000 & 4.29636300 \\
4.93487000 & -3.96726300 & 3.84222300 \\
5.38179900 & -3.01863600 & 5.27713700 \\
5.45328500 & -2.27805700 & 3.66309000 \\
3.42507000 & -1.12157900 & 5.12268000 \\
4.04823500 & -0.40075500 & 4.58439500 \\
3.83572400 & -1.21165000 & 6.13412200 \\
2.41380100 & -0.70625700 & 5.19633800 \\
2.70191600 & -3.47860600 & 5.40765100 \\
1.62120000 & -3.31422400 & 5.40580600 \\
3.06671000 & -3.32108600 & 6.42970700 \\
2.88686100 & -4.51786000 & 5.14090000 \\
2.35409300 & -4.69692300 & -0.16218800 \\
3.30513200 & -4.30811700 & -1.32501500 \\
3.03284900 & -3.37657700 & -1.82393200 \\
3.29953800 & -5.10075200 & -2.08156200 \\
4.32940500 & -4.20045600 & -0.94929700 \\
0.92300800 & -4.88423500 & -0.70979000 \\
0.23437400 & -5.19247800 & 0.08640400 \\
\hline & &
\end{tabular}


H

$\mathrm{H}$

C

$\mathrm{H}$

$\mathrm{H}$

$\mathrm{H}$

C

$\mathrm{H}$

$\mathrm{H}$

$\mathrm{H}$

C

C

$\mathrm{H}$

$\mathrm{H}$

$\mathrm{H}$

C

$\mathrm{H}$

$\mathrm{H}$

$\mathrm{H}$

C

$\mathrm{H}$

$\mathrm{H}$

$\mathrm{H}$

C

C

$\mathrm{H}$

$\mathrm{H}$

$\mathrm{H}$

C

$\mathrm{H}$

$\mathrm{H}$

$\mathrm{H}$

C

$\mathrm{H}$

$\mathrm{H}$

$\mathrm{H}$

C

$\mathrm{H}$

$\mathrm{H}$

$\mathrm{H}$

C

C

C

$\mathrm{H}$

\begin{tabular}{ccc}
0.90894900 & -5.65028600 & -1.49288000 \\
0.53704400 & -3.95948100 & -1.14460100 \\
2.87184800 & -6.05376300 & 0.35649800 \\
3.84527700 & -5.95732800 & 0.84326900 \\
2.98566800 & -6.72394600 & -0.50428800 \\
2.19099800 & -6.54194800 & 1.05479500 \\
1.42024700 & -5.38009000 & 3.15775800 \\
1.59690000 & -6.34632700 & 3.63759900 \\
0.82286800 & -5.52938200 & 2.25138600 \\
0.86291600 & -4.72521000 & 3.83453200 \\
6.67647900 & 2.12044800 & 2.18831700 \\
6.78548700 & 1.00810500 & 3.25384300 \\
5.79733400 & 0.62803300 & 3.53295000 \\
7.27027500 & 1.39102800 & 4.16021100 \\
7.37291600 & 0.15874600 & 2.88943600 \\
5.84489500 & 3.28925200 & 2.78024400 \\
5.73214800 & 4.09164700 & 2.04235000 \\
6.35895900 & 3.70184200 & 3.65595400 \\
4.84526900 & 2.98469900 & 3.10039400 \\
8.07745700 & 2.70869100 & 1.91905400 \\
8.82698800 & 1.95363600 & 1.68492500 \\
8.41265700 & 3.23023300 & 2.82369700 \\
8.05856900 & 3.42952500 & 1.09743000 \\
6.25657800 & 1.08345900 & -2.90643700 \\
7.24436400 & -0.08110800 & -3.13572500 \\
8.17987700 & 0.06447000 & -2.59550400 \\
7.48689100 & -0.15038500 & -4.20293100 \\
6.80050500 & -1.03593300 & -2.83223700 \\
5.14095300 & 0.94303800 & -3.96055200 \\
4.59156800 & 0.00068500 & -3.88047300 \\
5.59330700 & 0.98926000 & -4.95792200 \\
4.41808200 & 1.76069000 & -3.89098900 \\
6.97235000 & 2.42911900 & -3.16554200 \\
7.37115800 & 2.44867900 & -4.18741700 \\
7.79786700 & 2.58382900 & -2.46822300 \\
6.26761300 & 3.26272200 & -3.06346400 \\
8.48270500 & 0.14671200 & -0.07047200 \\
8.39910400 & 0.01383700 & 1.01357300 \\
9.53794800 & 0.22629000 & -0.34376100 \\
8.03966400 & -0.72491000 & -0.56398200 \\
-1.72921600 & -2.38470400 & -0.25851700 \\
\hline & -0.06141900 & 1.95910000 \\
\hline
\end{tabular}


1.80032400

1.72884700

2.98904700

3.05273500

5.11993700

5.56976700

5.87351200

1.45662500

1.67806100

1.17576700

2.54563000

2.71694900

3.16776000

2.94314200

2.09912300

4.50970200

4.51185600

5.51734800

$-2.97772000$

$-2.46015000$

$-2.34156300$

$-1.09724400$

$-2.89082100$

$-0.55249200$

$-0.36896100$

$-0.69981600$

0.74969500

0.05535700

$-1.51968900$

1.50781700

1.02245700

1.16478300

$-0.21070100$

2.37098600

1.76095200

$-3.83766100$

$-3.98543100$

$-4.46315200$

$-3.72432500$

$-4.67835800$

$-4.63407900$

$-3.94153400$

$-3.32656500$

$-4.42334600$ 


\begin{tabular}{|c|c|c|c|}
\hline $\mathrm{H}$ & 2.35245500 & -5.94831200 & -5.04028900 \\
\hline $\mathrm{H}$ & -1.70399600 & -5.38653400 & -3.72352500 \\
\hline $\mathrm{H}$ & 0.08917500 & -6.87328200 & -4.59372000 \\
\hline 0 & 0.33308100 & -0.88711500 & -2.57755700 \\
\hline 0 & 2.65789900 & -1.33603900 & -3.39553900 \\
\hline C & -3.49703400 & -0.84644500 & -4.33357200 \\
\hline $\mathrm{H}$ & -3.63902600 & 0.15593400 & -4.73095300 \\
\hline $\mathrm{H}$ & -1.66764000 & -0.88252500 & -4.41946700 \\
\hline C & -3.52974600 & -1.95165900 & -5.36900700 \\
\hline $\mathrm{H}$ & -3.09175100 & -2.88417700 & -5.00779000 \\
\hline $\mathrm{H}$ & -4.57493900 & -2.15002000 & -5.64251800 \\
\hline $\mathrm{H}$ & -2.98884700 & -1.65801200 & -6.27214100 \\
\hline
\end{tabular}

Total SCF energy (M06/SDD-6-311++G(d,p)/SMD(1,4-Dioxane)): -4717.620315a.u.

Thermal correction to Gibbs Free Energy at 298.15 K: 1.679083a.u

Gibbs free energy at 298.15 K (M06/SDD-6-311++G(d,p)/SMD(1,4-Dioxane)): -4715.941232a.u.

$\mathrm{Pd}$ $\mathrm{Pd}$ $P$

$\mathrm{P}$

$\mathrm{O}$

$\mathrm{O}$

$\mathrm{O}$

$\mathrm{O}$

$\mathrm{O}$

$\mathrm{O}$

$\mathrm{O}$

$\mathrm{O}$

$\mathrm{C}$

$\mathrm{C}$

$\mathrm{C}$

C

C

C

C

$\mathrm{C}$

$\begin{array}{ccc}-0.02593000 & 0.03289400 & -1.61904800 \\ 1.56085400 & 0.15640200 & 0.10200600\end{array}$

1.56085400

$-1.80568500$

$-0.23454000$

$-0.11883700$

$\begin{array}{lll}0.51103200 & -1.36797500 & 4.50742900\end{array}$

$\begin{array}{lll}-0.23693600 & -3.54753000 & 4.58600800\end{array}$

$\begin{array}{lll}-1.35641900 & 1.16850800 & 4.40605300\end{array}$

$\begin{array}{lll}-0.66117100 & 3.35667800 & 4.61721500\end{array}$

$-3.80808200$

$4.97044600 \quad 2.00415800$

$-6.28356700$

$-2.73926900$

$-3.15328000$

3.04516900

$-5.24483300$

2.18317100

6.39932600

$3.22651900-1.60477600$

$-1.37655400$

$-1.28556900$

1.32460600

$-0.54095800$

$-0.76708000$

2.35580300

$-0.25879100$

$-1.62619900$

3.40076400

$-0.71298200$

$-2.94328100$

3.44933100

$-1.50161800$

$-3.46882100$

2.44897500

$-1.83269500$

$-2.60740800$

1.38818600

$0.25593000 \quad-2.46536700$

5.39246700

0.95224700

1.16288500

1.51232000

C

1.37348700

2.49051400

1.65581700

C

0.88330800

3.33094000

2.67048400

$-0.02442800$

2.77748200

3.54743000

C

$-0.44643700$

1.45547500

3.41889700

C

$-0.01211600$

0.61829600

2.40806500 


\begin{tabular}{|c|c|c|c|}
\hline $\mathrm{C}$ & -1.24051200 & 2.25468000 & 5.33291900 \\
\hline $\mathrm{C}$ & -2.42115400 & 1.32551200 & 0.56695600 \\
\hline $\mathrm{C}$ & -2.03469300 & 2.50568800 & -0.05988300 \\
\hline $\mathrm{C}$ & -2.49551800 & 3.76005800 & 0.35683000 \\
\hline $\mathrm{C}$ & -3.34340700 & 3.78042300 & 1.49074300 \\
\hline $\mathrm{C}$ & -3.80946800 & 2.58965000 & 2.11447600 \\
\hline $\mathrm{C}$ & -3.30758900 & 1.37442200 & 1.64529400 \\
\hline $\mathrm{C}$ & -4.93462100 & 2.59051400 & 3.17232300 \\
\hline $\mathrm{C}$ & -6.24675300 & 2.97235500 & 2.44769400 \\
\hline $\mathrm{C}$ & -4.70521200 & 3.58437600 & 4.33430900 \\
\hline $\mathrm{C}$ & -5.11282200 & 1.19186000 & 3.79840500 \\
\hline $\mathrm{C}$ & -2.04301000 & 4.99618500 & -0.45598600 \\
\hline $\mathrm{C}$ & -3.11115300 & 6.10906000 & -0.51849400 \\
\hline $\mathrm{C}$ & -0.71445900 & 5.53318800 & 0.12159300 \\
\hline $\mathrm{C}$ & -1.76917800 & 4.59863800 & -1.92882400 \\
\hline $\mathrm{C}$ & -2.91341200 & 5.69772100 & 2.85472800 \\
\hline $\mathrm{C}$ & -3.21965300 & -1.08704900 & -0.88847400 \\
\hline $\mathrm{C}$ & -4.53340500 & -0.67485000 & -0.69456000 \\
\hline $\mathrm{C}$ & -2.96586900 & -2.16273300 & -1.74163200 \\
\hline $\mathrm{C}$ & -3.98457000 & -2.80537900 & -2.45154500 \\
\hline $\mathrm{C}$ & -5.29081400 & -2.26556700 & -2.32817800 \\
\hline $\mathrm{C}$ & -5.60204400 & -1.25223900 & -1.39098700 \\
\hline $\mathrm{C}$ & -7.00903500 & -0.69731800 & -1.06257800 \\
\hline $\mathrm{C}$ & -7.20138700 & -0.74936300 & 0.47446300 \\
\hline $\mathrm{C}$ & -8.20186800 & -1.46331800 & -1.66835900 \\
\hline $\mathrm{C}$ & -6.38317000 & -2.02308200 & -4.38185700 \\
\hline $\mathrm{C}$ & -7.08237300 & 0.77448000 & -1.52974800 \\
\hline $\mathrm{C}$ & -3.57436300 & -4.04079100 & -3.29046400 \\
\hline $\mathrm{C}$ & -2.81734000 & -5.01837400 & -2.35704900 \\
\hline $\mathrm{C}$ & -4.72629500 & -4.85125400 & -3.91758700 \\
\hline $\mathrm{C}$ & -2.62288700 & -3.59446000 & -4.42334300 \\
\hline $\mathrm{C}$ & 2.03110100 & -1.46024000 & 0.77873300 \\
\hline $\mathrm{C}$ & 2.77489100 & -1.61294800 & 1.95005500 \\
\hline $\mathrm{C}$ & 3.13453200 & -2.87829400 & 2.42063200 \\
\hline $\mathrm{C}$ & 2.68644800 & -4.01143400 & 1.68733200 \\
\hline $\mathrm{C}$ & 1.97216200 & -3.88947700 & 0.46837000 \\
\hline $\mathrm{C}$ & 1.64199100 & -2.59122600 & 0.06531700 \\
\hline $\mathrm{C}$ & 1.54342900 & -5.06200400 & -0.44693400 \\
\hline $\mathrm{C}$ & 0.18298200 & -5.62847700 & 0.01834400 \\
\hline $\mathrm{C}$ & 2.61641500 & -6.16956300 & -0.52520700 \\
\hline $\mathrm{C}$ & 1.33752400 & -4.57856700 & -1.90443000 \\
\hline $\mathrm{C}$ & 3.59221000 & -4.01578700 & 4.69474200 \\
\hline $\mathrm{C}$ & 2.04162500 & -6.01952300 & 2.84918600 \\
\hline $\mathrm{C}$ & 4.08139600 & -3.00012000 & 3.63372700 \\
\hline
\end{tabular}




\begin{tabular}{|c|c|c|c|}
\hline $\mathrm{C}$ & 4.25417100 & -1.64225000 & 4.34503300 \\
\hline $\mathrm{C}$ & 5.27211800 & 2.52423900 & -1.23862700 \\
\hline $\mathrm{C}$ & 3.98162900 & 2.93127600 & -1.66644500 \\
\hline $\mathrm{C}$ & 3.75069600 & 3.95126300 & -3.98849000 \\
\hline $\mathrm{C}$ & 2.90939900 & 2.11576500 & -1.28638100 \\
\hline $\mathrm{C}$ & 3.06753500 & 1.02150700 & -0.43628400 \\
\hline $\mathrm{C}$ & 4.33810600 & 0.70492800 & 0.0390690 \\
\hline $\mathrm{C}$ & 5.46362900 & 1.43663700 & -0.34539500 \\
\hline $\mathrm{C}$ & 6.89174000 & 3.08349400 & -2.93818400 \\
\hline $\mathrm{C}$ & 4.57544500 & 5.40016800 & -2.05984600 \\
\hline $\mathrm{C}$ & 7.41901800 & 2.28953700 & 1.0113680 \\
\hline $\mathrm{C}$ & 6.84435600 & 1.07700300 & 0.2443880 \\
\hline $\mathrm{C}$ & 6.74239000 & -0.08941500 & 1.24773300 \\
\hline $\mathrm{C}$ & 2.22303000 & 4.69357700 & -2.18449900 \\
\hline $\mathrm{C}$ & 5.46397400 & -3.45349600 & 3.11069400 \\
\hline $\mathrm{C}$ & 3.66846600 & 4.21821700 & -2.46960100 \\
\hline $\mathrm{C}$ & 7.82829500 & 0.62800000 & -0.85745400 \\
\hline $\mathrm{H}$ & -1.86394300 & -4.48945700 & 2.48326900 \\
\hline $\mathrm{H}$ & -2.48288600 & -2.98355600 & 0.60851700 \\
\hline $\mathrm{H}$ & 1.18335100 & -2.76404000 & 5.87690100 \\
\hline $\mathrm{H}$ & -0.51559500 & -2.17695400 & 6.11852600 \\
\hline $\mathrm{H}$ & 2.11626000 & 2.88795500 & 0.97590900 \\
\hline $\mathrm{H}$ & 1.21783400 & 4.35715100 & 2.7666260 \\
\hline $\mathrm{H}$ & -2.22916400 & 2.53160500 & 5.69306500 \\
\hline $\mathrm{H}$ & -0.56971400 & 1.96446000 & 6.15247400 \\
\hline $\mathrm{H}$ & -1.35316400 & 2.42955000 & -0.89710100 \\
\hline $\mathrm{H}$ & -3.59806100 & 0.44729500 & 2.11979200 \\
\hline $\mathrm{H}$ & -6.48211200 & 2.25154500 & 1.65669600 \\
\hline $\mathrm{H}$ & -7.08197100 & 2.98119800 & 3.15795900 \\
\hline $\mathrm{H}$ & -6.16616200 & 3.96606300 & 1.99898100 \\
\hline $\mathrm{H}$ & -4.95515300 & 4.60399200 & 4.04677700 \\
\hline $\mathrm{H}$ & -5.34348600 & 3.31240800 & 5.18172400 \\
\hline $\mathrm{H}$ & -3.66707900 & 3.57392400 & 4.67754300 \\
\hline $\mathrm{H}$ & -4.19133700 & 0.84491500 & 4.28027100 \\
\hline $\mathrm{H}$ & -5.89921300 & 1.23632600 & 4.55788400 \\
\hline $\mathrm{H}$ & -5.42135300 & 0.44002900 & 3.06502400 \\
\hline $\mathrm{H}$ & -2.77112000 & 6.89094700 & -1.20625000 \\
\hline $\mathrm{H}$ & -4.05797100 & 5.71259700 & -0.90067800 \\
\hline $\mathrm{H}$ & -3.31607500 & 6.57272800 & 0.44300300 \\
\hline $\mathrm{H}$ & -0.35244600 & 6.37514000 & -0.47991500 \\
\hline $\mathrm{H}$ & -0.81474800 & 5.87390800 & 1.15389200 \\
\hline $\mathrm{H}$ & 0.04810600 & 4.74815000 & 0.1073870 \\
\hline $\mathrm{H}$ & -0.89501400 & 3.94979200 & -2.03817400 \\
\hline $\mathrm{H}$ & -2.63281300 & 4.09357000 & -2.37455100 \\
\hline
\end{tabular}


H

$\mathrm{H}$

$\mathrm{H}$

$\mathrm{H}$

$\mathrm{H}$

$\mathrm{H}$

$\mathrm{H}$

$\mathrm{H}$

$\mathrm{H}$

$\mathrm{H}$

$\mathrm{H}$

$\mathrm{H}$

$\mathrm{H}$

$\mathrm{H}$

$\mathrm{H}$

$\mathrm{H}$

$\mathrm{H}$

$\mathrm{H}$

$\mathrm{H}$

$\mathrm{H}$

$\mathrm{H}$

$\mathrm{H}$

$\mathrm{H}$

$\mathrm{H}$

$\mathrm{H}$

$\mathrm{H}$

$\mathrm{H}$

$\mathrm{H}$

$\mathrm{H}$

$\mathrm{H}$

$\mathrm{H}$

$\mathrm{H}$

$\mathrm{H}$

$\mathrm{H}$

$\mathrm{H}$

$\mathrm{H}$

$\mathrm{H}$

$\mathrm{H}$

$\mathrm{H}$

$\mathrm{H}$

$\mathrm{H}$

$\mathrm{H}$

$\mathrm{H}$

$\mathrm{H}$

\begin{tabular}{rrr}
-1.56556200 & 5.50263200 & -2.51121000 \\
-2.36912200 & 6.45697100 & 2.28604200 \\
-3.52463100 & 6.19821500 & 3.60865600 \\
-2.20408800 & 5.02842000 & 3.35027100 \\
-4.72840300 & 0.14865400 & -0.02165000 \\
-1.94083400 & -2.49880300 & -1.86178600 \\
-8.18607800 & -0.34785000 & 0.73634000 \\
-7.14711400 & -1.78127700 & 0.83803600 \\
-6.45534600 & -0.16136500 & 1.01551800 \\
-8.28084700 & -1.34171500 & -2.74908400 \\
-8.15764000 & -2.53227000 & -1.44857400 \\
-9.12186600 & -1.06180300 & -1.22856400 \\
-6.60922900 & -0.96404900 & -4.20634100 \\
-5.45243600 & -2.09403700 & -4.95916400 \\
-7.19773200 & -2.48285900 & -4.94457200 \\
-6.94321800 & 0.85091100 & -2.61398300 \\
-8.06203200 & 1.19970400 & -1.28386800 \\
-6.31632200 & 1.39370000 & -1.05072900 \\
-1.92134400 & -4.57370300 & -1.91766100 \\
-3.46446900 & -5.35324700 & -1.53892900 \\
-2.50046100 & -5.90169400 & -2.92216800 \\
-4.30400300 & -5.77166900 & -4.33682300 \\
-5.48230300 & -5.12768900 & -3.17992000 \\
-5.22372700 & -4.32320800 & -4.73171400 \\
-3.10797700 & -2.86852000 & -5.08538300 \\
-1.70845900 & -3.13498200 & -4.03117500 \\
-2.32459900 & -4.45750100 & -5.02905500 \\
3.05688000 & -0.72901300 & 2.50485000 \\
1.05467600 & -2.44058800 & -0.82984900 \\
0.21476100 & -6.03063500 & 1.03084700 \\
-0.57806200 & -4.84242700 & 0.00172700 \\
-0.14007900 & -6.43149800 & -0.65416200 \\
2.29147500 & -6.93776200 & -1.23527600 \\
3.56405700 & -5.75573600 & -0.88765100 \\
2.81617100 & -6.64819800 & 0.43013000 \\
1.14083500 & -5.44609500 & -2.54177300 \\
0.47712500 & -3.90901900 & -2.01204800 \\
2.22553600 & -4.07033800 & -2.29530000 \\
4.04082700 & -3.78178200 & 5.66618200 \\
2.50514000 & -3.99433600 & 4.80787500 \\
1.21549100 & -5.38965600 & 3.19096400 \\
\hline
\end{tabular}


$\mathrm{H}$

$\mathrm{H}$

$\mathrm{H}$

$\mathrm{H}$

$\mathrm{H}$

$\mathrm{H}$

$\mathrm{H}$

$\mathrm{H}$

$\mathrm{H}$

$\mathrm{H}$

$\mathrm{H}$

$\mathrm{H}$

$\mathrm{H}$

$\mathrm{H}$

$\mathrm{H}$

$\mathrm{H}$

$\mathrm{H}$

$\mathrm{H}$

$\mathrm{H}$

$\mathrm{H}$

$\mathrm{H}$

$\mathrm{H}$

$\mathrm{H}$

$\mathrm{H}$

$\mathrm{H}$

$\mathrm{H}$

$\mathrm{H}$

$\mathrm{H}$

$\mathrm{H}$

C

C

C

$\mathrm{H}$

$\mathrm{H}$

$\mathrm{H}$

C

C

C

C

$\mathrm{H}$

C

$\mathrm{H}$

C

$\mathrm{H}$
4.71959400

3.29584300

4.91239400

3.01984900

3.52449300

4.73188400

1.90942100

4.44749800

7.98286600

6.52724400

6.59943100

4.24381300

4.49589300

5.62728100

7.54858000

6.75200600

8.39240200

7.73494400

6.07944700

6.38841200

1.46329600

2.04144200

2.06405300

5.87643100

6.16686200

5.38851900

8.74892300

7.39732800

8.10430700

1.28831800

$-1.05921700$

0.32988300

0.67778200

0.94474400

$-1.45400600$

2.73904400

3.60147000

3.29908800

4.97971600

3.18344600

4.67969900

2.65167700

5.52501900

5.62986200
$-0.88972300$

$-1.24674200$

$-1.77214300$

3.18920300

4.86921500

3.59811400

2.35258500

$-0.13635400$

3.05748900

2.16022000

3.93977200

6.30414700

5.58521400

5.24466900

3.15223500

2.57378100

2.02862700

$-0.29180700$

0.14620200

$-1.01304300$

4.01728900

4.81182100

5.66605100

$-2.71998300$

$-3.55731800$

$-4.41834800$

0.25077700

$-0.17887900$

1.44566300

$-0.64723400$

0.10618000

0.28840000

1.27653100

$-1.66121400$

$-0.90782200$

$-0.50715700$

$-1.29149400$

0.35062000

$-1.21352800$

$-1.94310400$

0.41955600

0.93338900

$-0.36063400$

$-1.81961500$
3.70071900

4.70099100

5.20939100

$-4.27827800$

$-4.54327400$

$-4.30618800$

$-1.62867200$

0.70603400

$-2.87930100$

$-3.39457800$

$-3.55317100$

$-2.58204300$

$-0.98288700$

$-2.28454500$

0.35746000

1.83341900

1.44272100

1.66181700

2.08717800

0.77560500

$-2.58886800$

$-1.11167300$

$-2.66098800$

2.40983500

3.94575100

2.60264400

$-0.39820400$

$-1.45994500$

$-1.52019900$

$-3.41083600$

$-3.62029300$

$-3.82877000$

$-4.12824600$

$-3.20089300$

$-3.63847500$

$-3.56945100$

$-2.78320400$

$-4.52988100$

$-2.94524300$

$-2.02317100$

$-4.69938200$

$-5.17714100$

$-3.90789200$

$-2.32172900$ 


$\begin{array}{rrrr}\mathrm{H} & 5.09402700 & 1.07246400 & -5.46171800 \\ \mathrm{H} & 6.60097300 & -0.31405500 & -4.04645700 \\ \mathrm{C} & -2.02159100 & 1.19446500 & -4.03993900 \\ \mathrm{H} & -1.55177900 & 2.17431200 & -3.88753900 \\ \mathrm{H} & -2.17323400 & 1.09682900 & -5.12689500 \\ \mathrm{C} & -3.38754700 & 1.16614600 & -3.35469800 \\ \mathrm{H} & -3.30226800 & 1.40181100 & -2.29186300 \\ \mathrm{H} & -4.05722500 & 1.90320600 & -3.81049100 \\ \mathrm{H} & -3.85816400 & 0.18194600 & -3.44065100\end{array}$<smiles>CCC(/C=C/c1ccccc1)[Pb]POS(=O)c1ccccc1</smiles>

Int-6b

Total SCF energy (M06/SDD-6-311++G(d,p)/SMD(1,4-Dioxane)): -5497.822481a.u.

Thermal correction to Gibbs Free Energy at $298.15 \mathrm{~K}$ : 1.760886a.u

Gibbs free energy at 298.15 K (M06/SDD-6-311++G(d,p)/SMD(1,4-Dioxane)): -5496.061595a.u.

$\begin{array}{cccc}\mathrm{Pd} & 1.37652600 & 1.09417600 & -2.51277500 \\ \mathrm{P} & 1.74114800 & -0.23294700 & -0.69727100 \\ \mathrm{P} & -1.92277300 & -0.53487700 & 0.20114400 \\ \mathrm{O} & 7.55682200 & -0.88573700 & 0.54921500 \\ \mathrm{O} & 0.00785900 & -5.91862600 & -1.33872600 \\ \mathrm{O} & -2.45133400 & 4.81969300 & 2.87417100 \\ \mathrm{O} & -7.80647600 & -1.70268700 & 0.06695000 \\ \mathrm{O} & 0.98538200 & 2.64228500 & 4.38786700 \\ \mathrm{O} & 0.32359200 & 0.43461900 & 4.38526800 \\ \mathrm{O} & 1.84409300 & -2.20500600 & 3.57042100 \\ \mathrm{O} & 0.94214800 & -4.30534300 & 3.88368700 \\ \mathrm{C} & 3.48638600 & -0.50006600 & -0.21403000 \\ \mathrm{C} & 4.30594700 & -1.24575900 & -1.07286500 \\ \mathrm{H} & 3.85835000 & -1.67769500 & -1.95814100 \\ \mathrm{C} & 5.65967100 & -1.44031200 & -0.81365900 \\ \mathrm{C} & 6.18628100 & -0.84832500 & 0.36784500 \\ \mathrm{C} & 5.35736100 & -0.25643100 & 1.34103100 \\ \mathrm{C} & 4.01561600 & -0.03934100 & 0.98442100 \\ \mathrm{H} & 3.37051000 & 0.47846900 & 1.67839000 \\ \mathrm{C} & 6.50960000 & -2.33050000 & -1.74327700 \\ \mathrm{C} & 5.65034400 & -2.95592200 & -2.86002600 \\ \mathrm{H} & 4.82121500 & -3.54881400 & -2.45926400 \\ \mathrm{H} & 6.27641200 & -3.62550300 & -3.45853800 \\ \mathrm{H} & 5.23755100 & -2.19916000 & -3.53738200 \\ \mathrm{C} & 7.64128800 & -1.53569300 & -2.43012200\end{array}$




\begin{tabular}{|c|c|c|c|}
\hline $\mathrm{H}$ & 7.24935500 & -0.63564600 & -2.91910300 \\
\hline $\mathrm{H}$ & 8.11258000 & -2.15658300 & -3.20085300 \\
\hline $\mathrm{H}$ & 8.41640500 & -1.24275000 & -1.72249800 \\
\hline $\mathrm{C}$ & 7.11322300 & -3.48982500 & -0.91800800 \\
\hline $\mathrm{H}$ & 7.74980300 & -3.11497800 & -0.11455600 \\
\hline $\mathrm{H}$ & 7.71698700 & -4.13545000 & -1.56695800 \\
\hline $\mathrm{H}$ & 6.31912300 & -4.10332700 & -0.47769800 \\
\hline $\mathrm{C}$ & 5.74836500 & 0.10394800 & 2.79570200 \\
\hline $\mathrm{C}$ & 5.69942400 & 1.63278700 & 2.99727300 \\
\hline $\mathrm{H}$ & 6.46449200 & 2.13700900 & 2.39648100 \\
\hline $\mathrm{H}$ & 5.88568800 & 1.88179100 & 4.04884100 \\
\hline $\mathrm{H}$ & 4.72631600 & 2.04910100 & 2.71768500 \\
\hline $\mathrm{C}$ & 4.70120400 & -0.55102500 & 3.73471000 \\
\hline $\mathrm{H}$ & 3.68626500 & -0.18450700 & 3.57155800 \\
\hline $\mathrm{H}$ & 4.96790300 & -0.34555200 & 4.77806000 \\
\hline $\mathrm{H}$ & 4.68023900 & -1.63636900 & 3.59153900 \\
\hline $\mathrm{C}$ & 7.11933400 & -0.42544000 & 3.26292000 \\
\hline $\mathrm{H}$ & 7.22761000 & -1.49475800 & 3.06432700 \\
\hline $\mathrm{H}$ & 7.19607600 & -0.27055100 & 4.34546000 \\
\hline $\mathrm{H}$ & 7.96268300 & 0.09004000 & 2.80341100 \\
\hline $\mathrm{C}$ & 8.18914200 & 0.36602500 & 0.28233400 \\
\hline $\mathrm{H}$ & 7.83642500 & 1.14840600 & 0.96275900 \\
\hline $\mathrm{H}$ & 8.00494000 & 0.68808700 & -0.74906400 \\
\hline $\mathrm{H}$ & 9.25971100 & 0.20967000 & 0.43243300 \\
\hline $\mathrm{C}$ & 1.14910200 & -1.94577400 & -0.87555000 \\
\hline $\mathrm{C}$ & 0.16362500 & -2.26703400 & -1.80759400 \\
\hline $\mathrm{H}$ & -0.29480800 & -1.46619900 & -2.36949300 \\
\hline $\mathrm{C}$ & -0.24253800 & -3.59314900 & -2.02321100 \\
\hline $\mathrm{C}$ & 0.38061400 & -4.59171500 & -1.23352800 \\
\hline $\mathrm{C}$ & 1.46213800 & -4.30734300 & -0.36165800 \\
\hline $\mathrm{C}$ & 1.79698200 & -2.96881900 & -0.17754600 \\
\hline $\mathrm{H}$ & 2.58479800 & -2.70730100 & 0.51036200 \\
\hline $\mathrm{C}$ & 2.35768300 & -5.40562400 & 0.25308200 \\
\hline $\mathrm{C}$ & 1.59074100 & -6.42161100 & 1.12462800 \\
\hline $\mathrm{H}$ & 0.98261800 & -5.92005800 & 1.88100400 \\
\hline $\mathrm{H}$ & 2.30783000 & -7.06461900 & 1.64860900 \\
\hline $\mathrm{H}$ & 0.95379600 & -7.06454800 & 0.52052600 \\
\hline $\mathrm{C}$ & 3.04689900 & -6.15568200 & -0.91043200 \\
\hline $\mathrm{H}$ & 2.30838200 & -6.62508800 & -1.56425000 \\
\hline $\mathrm{H}$ & 3.70940500 & -6.93661000 & -0.51702400 \\
\hline $\mathrm{H}$ & 3.65363700 & -5.46688900 & -1.50996100 \\
\hline $\mathrm{C}$ & 3.46465000 & -4.79514400 & 1.13768400 \\
\hline $\mathrm{H}$ & 4.11973200 & -4.11900000 & 0.57822000 \\
\hline $\mathrm{H}$ & 4.087744500 & -5.60238900 & 1.53681300 \\
\hline
\end{tabular}




\begin{tabular}{|c|c|c|c|}
\hline $\mathrm{H}$ & 3.04769900 & -4.24244600 & 1.98554100 \\
\hline $\mathrm{C}$ & -1.29343000 & -3.86858800 & -3.12633400 \\
\hline $\mathrm{C}$ & -2.71249900 & -3.91773800 & -2.51647000 \\
\hline $\mathrm{H}$ & -2.85099200 & -4.74927400 & -1.82435300 \\
\hline $\mathrm{H}$ & -3.45602200 & -4.02471200 & -3.31556600 \\
\hline $\mathrm{H}$ & -2.93230900 & -2.99251200 & -1.97840500 \\
\hline $\mathrm{C}$ & -1.30060200 & -2.72238200 & -4.16641200 \\
\hline $\mathrm{H}$ & -1.71320700 & -1.78931600 & -3.77571200 \\
\hline $\mathrm{H}$ & -1.93619000 & -3.01708100 & -5.00854500 \\
\hline $\mathrm{H}$ & -0.29438400 & -2.53125200 & -4.55845600 \\
\hline $\mathrm{C}$ & -0.99029900 & -5.16069900 & -3.91643900 \\
\hline $\mathrm{H}$ & 0.01147900 & -5.11118500 & -4.35901900 \\
\hline $\mathrm{H}$ & -1.71362000 & -5.25572300 & -4.73411300 \\
\hline $\mathrm{H}$ & -1.03588000 & -6.06410200 & -3.31179700 \\
\hline $\mathrm{C}$ & -1.19668000 & -6.30774000 & -0.67696600 \\
\hline $\mathrm{H}$ & -1.58118300 & -5.49769900 & -0.05233700 \\
\hline $\mathrm{H}$ & -0.97971900 & -7.17397100 & -0.04459500 \\
\hline $\mathrm{H}$ & -1.95181900 & -6.59112600 & -1.41655300 \\
\hline $\mathrm{C}$ & -2.10086400 & 1.05216900 & 1.12602100 \\
\hline $\mathrm{C}$ & -2.44042400 & 1.17546600 & 2.47747000 \\
\hline $\mathrm{H}$ & -2.55545900 & 0.27471100 & 3.06454200 \\
\hline $\mathrm{C}$ & -2.60998700 & 2.42478400 & 3.07839700 \\
\hline $\mathrm{C}$ & -2.41837300 & 3.57326000 & 2.26191700 \\
\hline $\mathrm{C}$ & -2.20805800 & 3.48325300 & 0.87443700 \\
\hline $\mathrm{C}$ & -2.00389200 & 2.19831100 & 0.34615200 \\
\hline $\mathrm{H}$ & -1.75050400 & 2.08138600 & -0.70174100 \\
\hline $\mathrm{C}$ & -3.69006600 & -1.06872400 & 0.20093600 \\
\hline $\mathrm{C}$ & -4.37873100 & -1.46963600 & 1.34390000 \\
\hline $\mathrm{H}$ & -3.83481900 & -1.54529200 & 2.27695600 \\
\hline $\mathrm{C}$ & -5.73919200 & -1.79987100 & 1.32628800 \\
\hline $\mathrm{C}$ & -6.42332700 & -1.59759300 & 0.10729500 \\
\hline $\mathrm{C}$ & -5.74685100 & -1.29444600 & -1.09923900 \\
\hline $\mathrm{C}$ & -4.37541400 & -1.02065300 & -1.01869000 \\
\hline $\mathrm{H}$ & -3.82209600 & -0.73094700 & -1.90385500 \\
\hline $\mathrm{C}$ & -3.15568000 & 2.52214400 & 4.52105300 \\
\hline $\mathrm{C}$ & -4.61032400 & 3.03854400 & 4.42829800 \\
\hline $\mathrm{H}$ & -4.63847400 & 4.02991100 & 3.96762600 \\
\hline $\mathrm{H}$ & -5.05630300 & 3.10873300 & 5.42874800 \\
\hline $\mathrm{H}$ & -5.22335800 & 2.35868500 & 3.82623200 \\
\hline $\mathrm{C}$ & -3.18061700 & 1.14071500 & 5.20549800 \\
\hline $\mathrm{H}$ & -3.84718100 & 0.44050200 & 4.69332600 \\
\hline $\mathrm{H}$ & -3.55219800 & 1.25056500 & 6.23000600 \\
\hline $\mathrm{H}$ & -2.18339300 & 0.68875700 & 5.24676400 \\
\hline & -2.35826800 & 3.47069600 & 5.44379000 \\
\hline
\end{tabular}




\begin{tabular}{|c|c|c|c|}
\hline $\mathrm{H}$ & -1.28464900 & 3.27313700 & 5.39056300 \\
\hline $\mathrm{H}$ & -2.68092700 & 3.32767000 & 6.48216600 \\
\hline $\mathrm{H}$ & -2.52422600 & 4.51482100 & 5.18287600 \\
\hline $\mathrm{C}$ & -2.22837300 & 4.63817600 & -0.15579100 \\
\hline $\mathrm{C}$ & -3.24960700 & 4.25619200 & -1.25869000 \\
\hline $\mathrm{H}$ & -3.01464100 & 3.30682000 & -1.73900200 \\
\hline $\mathrm{H}$ & -3.26153100 & 5.02382100 & -2.03996300 \\
\hline $\mathrm{H}$ & -4.25686500 & 4.18170200 & -0.83292400 \\
\hline $\mathrm{C}$ & -0.82709300 & 4.77439300 & -0.79332600 \\
\hline $\mathrm{H}$ & -0.07458900 & 5.02352100 & -0.03633800 \\
\hline $\mathrm{H}$ & -0.83296700 & 5.57245700 & -1.54676700 \\
\hline $\mathrm{H}$ & -0.52483300 & 3.84702800 & -1.28734500 \\
\hline $\mathrm{C}$ & -2.67585200 & 6.01683700 & 0.36891900 \\
\hline $\mathrm{H}$ & -3.61964500 & 5.95692000 & 0.91586900 \\
\hline $\mathrm{H}$ & -2.82265300 & 6.67702600 & -0.49461900 \\
\hline $\mathrm{H}$ & -1.93896600 & 6.49478200 & 1.01624800 \\
\hline $\mathrm{C}$ & -1.14667200 & 5.34112100 & 3.12129700 \\
\hline $\mathrm{H}$ & -1.28548000 & 6.33015100 & 3.56577700 \\
\hline $\mathrm{H}$ & -0.57283900 & 5.43803100 & 2.19286000 \\
\hline $\mathrm{H}$ & -0.58604900 & 4.70205500 & 3.81117000 \\
\hline $\mathrm{C}$ & -6.32954100 & -2.39752300 & 2.62734600 \\
\hline $\mathrm{C}$ & -6.40131800 & -1.30747400 & 3.71795300 \\
\hline $\mathrm{H}$ & -5.41455300 & -0.87499600 & 3.91120700 \\
\hline $\mathrm{H}$ & -6.77656600 & -1.72926100 & 4.65840000 \\
\hline $\mathrm{H}$ & -7.06756600 & -0.48924300 & 3.42457000 \\
\hline $\mathrm{C}$ & -5.37756500 & -3.52200000 & 3.11447400 \\
\hline $\mathrm{H}$ & -5.29552900 & -4.30895700 & 2.35619000 \\
\hline $\mathrm{H}$ & -5.77646800 & -3.97244300 & 4.03068900 \\
\hline $\mathrm{H}$ & -4.36898100 & -3.16453300 & 3.33629900 \\
\hline $\mathrm{C}$ & -7.71365900 & -3.06489500 & 2.48527300 \\
\hline $\mathrm{H}$ & -8.52498000 & -2.35255600 & 2.33900300 \\
\hline $\mathrm{H}$ & -7.92715100 & -3.61508600 & 3.40967800 \\
\hline $\mathrm{H}$ & -7.73455600 & -3.77421600 & 1.65393200 \\
\hline $\mathrm{C}$ & -6.45358500 & -1.35198300 & -2.47155300 \\
\hline $\mathrm{C}$ & -7.56194200 & -0.28580100 & -2.61273400 \\
\hline $\mathrm{H}$ & -8.42633300 & -0.51026900 & -1.98804800 \\
\hline $\mathrm{H}$ & -7.90629000 & -0.25082200 & -3.65340200 \\
\hline $\mathrm{H}$ & -7.18365300 & 0.70971900 & -2.35315100 \\
\hline $\mathrm{C}$ & -5.45911100 & -1.12312600 & -3.62480800 \\
\hline $\mathrm{H}$ & -4.99647200 & -0.13410400 & -3.59049600 \\
\hline $\mathrm{H}$ & -5.99413000 & -1.21890900 & -4.57663700 \\
\hline $\mathrm{H}$ & -4.64934200 & -1.85803200 & -3.62343500 \\
\hline $\mathrm{C}$ & -7.06520300 & -2.75984100 & -2.65681400 \\
\hline $\mathrm{H}$ & -7.55563700 & -2.82855000 & -3.63586000 \\
\hline
\end{tabular}




\begin{tabular}{|c|c|c|c|}
\hline $\mathrm{H}$ & -7.80392800 & -2.97909900 & -1.88334300 \\
\hline $\mathrm{H}$ & -6.28076600 & -3.52469700 & -2.61856200 \\
\hline $\mathrm{C}$ & -8.46477700 & -0.53831200 & 0.56091600 \\
\hline $\mathrm{H}$ & -8.26603200 & -0.38256100 & 1.62688300 \\
\hline $\mathrm{H}$ & -9.53538000 & -0.70127100 & 0.41318900 \\
\hline $\mathrm{H}$ & -8.15019400 & 0.35677600 & 0.01321800 \\
\hline $\mathrm{C}$ & 0.66466400 & 0.01409000 & 1.97328700 \\
\hline $\mathrm{C}$ & 1.10884100 & 0.64986500 & 0.77968000 \\
\hline $\mathrm{C}$ & 1.42268100 & 2.01590500 & 0.78186100 \\
\hline $\mathrm{H}$ & 1.72957500 & 2.47989300 & -0.14570700 \\
\hline $\mathrm{C}$ & 1.41145800 & 2.79835400 & 1.94644000 \\
\hline $\mathrm{H}$ & 1.67204600 & 3.84955300 & 1.92223500 \\
\hline $\mathrm{C}$ & 1.08247700 & 2.14049300 & 3.11350900 \\
\hline $\mathrm{C}$ & 0.70363800 & 0.79815700 & 3.11302800 \\
\hline $\mathrm{C}$ & 0.80148000 & 1.48785300 & 5.22170200 \\
\hline $\mathrm{H}$ & 1.77070800 & 1.20155700 & 5.65470700 \\
\hline $\mathrm{H}$ & 0.06057400 & 1.70812000 & 5.98911500 \\
\hline $\mathrm{C}$ & -1.14577400 & -1.69703200 & 1.41744200 \\
\hline $\mathrm{C}$ & -1.64283200 & -2.99931000 & 1.57520700 \\
\hline $\mathrm{H}$ & -2.55653800 & -3.27538100 & 1.06289100 \\
\hline $\mathrm{C}$ & -1.02914000 & -3.95820900 & 2.40056900 \\
\hline $\mathrm{H}$ & -1.44475500 & -4.95226600 & 2.52142400 \\
\hline $\mathrm{C}$ & 0.11973300 & -3.56969500 & 3.05526600 \\
\hline $\mathrm{C}$ & 0.65456300 & -2.30028100 & 2.87684800 \\
\hline $\mathrm{C}$ & 0.07558400 & -1.34279100 & 2.06549300 \\
\hline $\mathrm{C}$ & 1.86125500 & -3.35149300 & 4.42557400 \\
\hline $\mathrm{H}$ & 2.86477300 & -3.77933800 & 4.43628900 \\
\hline $\mathrm{H}$ & 1.53350900 & -3.05890100 & 5.43373200 \\
\hline $\mathrm{C}$ & 3.39069000 & 1.30969100 & -2.99324500 \\
\hline $\mathrm{H}$ & 4.05787000 & 0.57549900 & -2.54791900 \\
\hline $\mathrm{C}$ & 3.50294200 & 2.63694400 & -2.39427100 \\
\hline $\mathrm{C}$ & 4.12460000 & 2.89891700 & -1.21552500 \\
\hline $\mathrm{H}$ & 2.96219800 & 3.44235700 & -2.89640500 \\
\hline $\mathrm{H}$ & 4.72969000 & 2.11025700 & -0.77110100 \\
\hline $\mathrm{C}$ & 3.96500700 & 4.10357200 & -0.40981200 \\
\hline $\mathrm{C}$ & 2.90521600 & 5.01200300 & -0.61002100 \\
\hline $\mathrm{C}$ & 4.82836400 & 4.32811000 & 0.67839600 \\
\hline $\mathrm{C}$ & 2.72461400 & 6.09737700 & 0.24168400 \\
\hline $\mathrm{H}$ & 2.19063400 & 4.84008600 & -1.40843500 \\
\hline $\mathrm{C}$ & 4.64634300 & 5.41197900 & 1.53298100 \\
\hline $\mathrm{H}$ & 5.64271600 & 3.63171600 & 0.85173500 \\
\hline $\mathrm{C}$ & 3.59174400 & 6.30290900 & 1.32015700 \\
\hline $\mathrm{H}$ & 1.89145700 & 6.77413700 & 0.07468900 \\
\hline $\mathrm{H}$ & 5.32324100 & 5.55800000 & 2.37011200 \\
\hline
\end{tabular}




$\begin{array}{lrrr}\mathrm{H} & 3.44075100 & 7.14373800 & 1.99073700 \\ \mathrm{~S} & -1.41110800 & 1.14412800 & -3.81315500 \\ \mathrm{C} & -2.09946000 & 2.75416700 & -4.33132100 \\ \mathrm{C} & -3.43990500 & 2.83179100 & -4.69935600 \\ \mathrm{C} & -1.27673300 & 3.88024600 & -4.37150700 \\ \mathrm{C} & -3.96921900 & 4.06084300 & -5.09847400 \\ \mathrm{H} & -4.05359700 & 1.93846800 & -4.64627400 \\ \mathrm{C} & -1.80832600 & 5.10384100 & -4.77644600 \\ \mathrm{H} & -0.24148600 & 3.80504700 & -4.05308400 \\ \mathrm{C} & -3.15559500 & 5.19474400 & -5.13887200 \\ \mathrm{H} & -5.01782600 & 4.13421000 & -5.37370300 \\ \mathrm{H} & -1.17751700 & 5.98852700 & -4.79869100 \\ \mathrm{H} & -3.56988400 & 6.14980700 & -5.44976000 \\ \mathrm{O} & -0.66985200 & 1.65343500 & -2.50298400 \\ \mathrm{O} & -2.63030200 & 0.32475600 & -3.46686300 \\ \mathrm{C} & 3.33225600 & 1.21780900 & -4.50965600 \\ \mathrm{H} & 3.08557700 & 0.19188700 & -4.80694500 \\ \mathrm{H} & 2.52837000 & 1.86400000 & -4.89990100 \\ \mathrm{C} & 4.66194900 & 1.63625800 & -5.16544200 \\ \mathrm{H} & 4.91564100 & 2.66997100 & -4.90757300 \\ \mathrm{H} & 5.47943200 & 0.99406200 & -4.81713000 \\ \mathrm{H} & 4.60490400 & 1.55521400 & -6.25632900\end{array}$

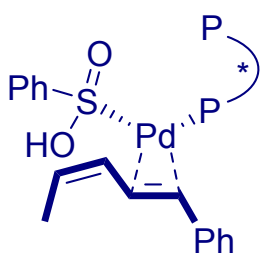

Int-7a

Total SCF energy (M06/SDD-6-311++G(d,p)/SMD(1,4-Dioxane)): -5497.848202a.u.

Thermal correction to Gibbs Free Energy at $298.15 \mathrm{~K}: 1.766067 \mathrm{a} . \mathrm{u}$

Gibbs free energy at 298.15 K (M06/SDD-6-311++G(d,p)/SMD(1,4-Dioxane)): -5496.082135a.u.

$\mathrm{Pd}$

$P$

$\mathrm{P}$

$\mathrm{O}$

$\mathrm{O}$

$\mathrm{O}$

$\mathrm{O}$

O

O

O

O

$\begin{array}{ccc}-1.35547900 & 0.72422900 & 2.66963300 \\ -1.65953500 & -0.12309900 & 0.47396900 \\ 1.91794800 & -0.30051300 & -0.25414300 \\ -7.55357000 & 0.33235600 & -0.62496800 \\ -1.22209400 & -6.01041500 & -0.64758400 \\ 2.89227100 & 5.10137600 & -2.71181800 \\ 7.57364100 & -1.96743700 & 0.89707600 \\ -0.66446200 & 3.24542000 & -4.29825200 \\ -0.02840900 & 1.04380100 & -4.49078500 \\ -1.47536200 & -1.67403800 & -4.08440100 \\ -0.42139500 & -3.65932100 & -4.62210700\end{array}$




\begin{tabular}{|c|c|c|c|}
\hline $\mathrm{C}$ & -3.45477400 & 0.00528700 & 0.05018800 \\
\hline $\mathrm{C}$ & -4.37140900 & -0.55813600 & 0.94374100 \\
\hline $\mathrm{H}$ & -3.98299800 & -1.02963400 & 1.83750000 \\
\hline $\mathrm{C}$ & -5.74560500 & -0.52433200 & 0.71123300 \\
\hline $\mathrm{C}$ & -6.19061900 & 0.12897100 & -0.47130300 \\
\hline $\mathrm{C}$ & -5.29309200 & 0.55235900 & -1.47068000 \\
\hline $\mathrm{C}$ & -3.92713800 & 0.51703500 & -1.15042200 \\
\hline $\mathrm{H}$ & -3.21834500 & 0.88766300 & -1.87565800 \\
\hline $\mathrm{C}$ & -6.71439400 & -1.25662900 & 1.66235200 \\
\hline $\mathrm{C}$ & -5.95175200 & -1.99885200 & 2.77780600 \\
\hline $\mathrm{H}$ & -5.23509800 & -2.72200600 & 2.37439200 \\
\hline $\mathrm{H}$ & -6.66939600 & -2.55054500 & 3.39405100 \\
\hline $\mathrm{H}$ & -5.41170600 & -1.31036200 & 3.43845500 \\
\hline $\mathrm{C}$ & -7.70527600 & -0.29374200 & 2.35128000 \\
\hline $\mathrm{H}$ & -7.17710300 & 0.54189000 & 2.82558600 \\
\hline $\mathrm{H}$ & -8.25569000 & -0.82835800 & 3.13445800 \\
\hline $\mathrm{H}$ & -8.43582800 & 0.10470000 & 1.64782700 \\
\hline $\mathrm{C}$ & -7.49661000 & -2.31526800 & 0.85136800 \\
\hline $\mathrm{H}$ & -8.07960600 & -1.84976300 & 0.05438200 \\
\hline $\mathrm{H}$ & -8.18238900 & -2.86232400 & 1.50979000 \\
\hline $\mathrm{H}$ & -6.80829400 & -3.04069900 & 0.40202000 \\
\hline $\mathrm{C}$ & -5.64355800 & 0.99700400 & -2.91204400 \\
\hline $\mathrm{C}$ & -5.30966900 & 2.49337500 & -3.09334100 \\
\hline $\mathrm{H}$ & -5.94907100 & 3.12099500 & -2.46277700 \\
\hline $\mathrm{H}$ & -5.47069000 & 2.79466900 & -4.13561300 \\
\hline $\mathrm{H}$ & -4.26868400 & 2.70925700 & -2.83163300 \\
\hline $\mathrm{C}$ & -4.76842700 & 0.17158400 & -3.89342400 \\
\hline $\mathrm{H}$ & -3.69527400 & 0.29874700 & -3.73344500 \\
\hline $\mathrm{H}$ & -4.99003400 & 0.47601300 & -4.92321400 \\
\hline $\mathrm{H}$ & -4.99205900 & -0.89585000 & -3.79868900 \\
\hline $\mathrm{C}$ & -7.10124700 & 0.74654000 & -3.34841000 \\
\hline $\mathrm{H}$ & -7.40799300 & -0.28399800 & -3.15253000 \\
\hline $\mathrm{H}$ & -7.17376100 & 0.92491900 & -4.42794900 \\
\hline $\mathrm{H}$ & -7.81777000 & 1.40958200 & -2.86340600 \\
\hline $\mathrm{C}$ & -7.94715200 & 1.67171900 & -0.33230600 \\
\hline $\mathrm{H}$ & -7.47874400 & 2.38770700 & -1.01656500 \\
\hline $\mathrm{H}$ & -7.68213900 & 1.94663300 & 0.69513500 \\
\hline $\mathrm{H}$ & -9.03235000 & 1.71080200 & -0.45395500 \\
\hline $\mathrm{C}$ & -1.44734800 & -1.91495700 & 0.09971200 \\
\hline $\mathrm{C}$ & -0.57110200 & -2.70328300 & 0.84171700 \\
\hline $\mathrm{H}$ & 0.05696100 & -2.22202200 & 1.58066300 \\
\hline $\mathrm{C}$ & -0.48816800 & -4.09646400 & 0.65397200 \\
\hline $\mathrm{C}$ & -1.31412400 & -4.66236300 & -0.34064400 \\
\hline $\mathrm{C}$ & -2.29894800 & -3.90873800 & -1.02719400 \\
\hline
\end{tabular}




\begin{tabular}{|c|c|c|c|}
\hline $\mathrm{C}$ & -2.31247800 & -2.53506600 & -0.80872300 \\
\hline $\mathrm{H}$ & -3.02683700 & -1.92529700 & -1.33619700 \\
\hline $\mathrm{C}$ & -3.43947200 & -4.56223900 & -1.83940500 \\
\hline $\mathrm{C}$ & -2.95918500 & -5.47568000 & -2.98783800 \\
\hline $\mathrm{H}$ & -2.20756300 & -4.98014500 & -3.60887700 \\
\hline $\mathrm{H}$ & -3.81157200 & -5.73684200 & -3.62666100 \\
\hline $\mathrm{H}$ & -2.53556700 & -6.40536000 & -2.60948500 \\
\hline $\mathrm{C}$ & -4.28670700 & -5.39983500 & -0.85350000 \\
\hline $\mathrm{H}$ & -3.68367400 & -6.18765900 & -0.39489500 \\
\hline $\mathrm{H}$ & -5.12846700 & -5.86836300 & -1.37877400 \\
\hline $\mathrm{H}$ & -4.69236700 & -4.76408200 & -0.05783400 \\
\hline $\mathrm{C}$ & -4.36098600 & -3.49391900 & -2.46365300 \\
\hline $\mathrm{H}$ & -4.83932900 & -2.86142000 & -1.70845200 \\
\hline $\mathrm{H}$ & -5.15734800 & -3.99170500 & -3.02741400 \\
\hline $\mathrm{H}$ & -3.81242800 & -2.84125900 & -3.15148400 \\
\hline $\mathrm{C}$ & 0.47802800 & -4.88429000 & 1.57321700 \\
\hline $\mathrm{C}$ & 1.92659700 & -4.65556600 & 1.09102500 \\
\hline $\mathrm{H}$ & 2.07975500 & -5.03372900 & 0.07502600 \\
\hline $\mathrm{H}$ & 2.63491600 & -5.17056700 & 1.75181700 \\
\hline $\mathrm{H}$ & 2.17634400 & -3.59206400 & 1.08570400 \\
\hline $\mathrm{C}$ & 0.35194800 & -4.34516500 & 3.02124400 \\
\hline $\mathrm{H}$ & 0.65986600 & -3.30462500 & 3.12085900 \\
\hline $\mathrm{H}$ & 0.99216900 & -4.93966200 & 3.68358500 \\
\hline $\mathrm{H}$ & -0.68055800 & -4.43712900 & 3.37799100 \\
\hline $\mathrm{C}$ & 0.20770300 & -6.40158400 & 1.67240500 \\
\hline $\mathrm{H}$ & -0.84245700 & -6.60912400 & 1.89587100 \\
\hline $\mathrm{H}$ & 0.81513500 & -6.80332400 & 2.49211000 \\
\hline $\mathrm{H}$ & 0.47464100 & -6.95377000 & 0.77240300 \\
\hline $\mathrm{C}$ & -0.18563100 & -6.31202100 & -1.57986300 \\
\hline $\mathrm{H}$ & -0.31800300 & -5.75815700 & -2.51404000 \\
\hline $\mathrm{H}$ & -0.25107200 & -7.38507800 & -1.77655000 \\
\hline $\mathrm{H}$ & 0.80217100 & -6.07788000 & -1.17040400 \\
\hline $\mathrm{C}$ & 2.27538000 & 1.31524000 & -1.07167200 \\
\hline $\mathrm{C}$ & 2.68546700 & 1.46381300 & -2.40051100 \\
\hline $\mathrm{H}$ & 2.76396200 & 0.57711100 & -3.01407800 \\
\hline $\mathrm{C}$ & 2.95962200 & 2.71649800 & -2.95286800 \\
\hline $\mathrm{C}$ & 2.75503800 & 3.85224600 & -2.12634100 \\
\hline $\mathrm{C}$ & 2.43871500 & 3.74092000 & -0.75764700 \\
\hline $\mathrm{C}$ & 2.17121400 & 2.44999100 & -0.27440400 \\
\hline $\mathrm{H}$ & 1.85684800 & 2.31918300 & 0.7550400 \\
\hline $\mathrm{C}$ & 3.63352200 & -0.94384000 & -0.00181200 \\
\hline $\mathrm{C}$ & 4.62459100 & -0.86183100 & -0.97655100 \\
\hline $\mathrm{H}$ & 4.36737800 & -0.44672400 & -1.94127000 \\
\hline $\mathrm{C}$ & 5.93587400 & -1.30240200 & -0.76113900 \\
\hline
\end{tabular}




\begin{tabular}{|c|c|c|c|}
\hline $\mathrm{C}$ & 6.25263000 & -1.73281000 & 0.54650100 \\
\hline $\mathrm{C}$ & 5.25708900 & -1.94710500 & 1.53088100 \\
\hline $\mathrm{C}$ & 3.95238500 & -1.54266300 & 1.22243900 \\
\hline $\mathrm{H}$ & 3.16099600 & -1.66572800 & 1.94771300 \\
\hline $\mathrm{C}$ & 3.59754700 & 2.82328800 & -4.35607300 \\
\hline $\mathrm{C}$ & 5.04146600 & 3.34635100 & -4.17648300 \\
\hline $\mathrm{H}$ & 5.03841900 & 4.33555300 & -3.71155400 \\
\hline $\mathrm{H}$ & 5.54148000 & 3.42252700 & -5.15027000 \\
\hline $\mathrm{H}$ & 5.62683300 & 2.66856700 & -3.54613500 \\
\hline $\mathrm{C}$ & 3.66518700 & 1.44493900 & -5.04406300 \\
\hline $\mathrm{H}$ & 4.27876000 & 0.73172300 & -4.48347300 \\
\hline $\mathrm{H}$ & 4.12253200 & 1.55788700 & -6.03274000 \\
\hline $\mathrm{H}$ & 2.66793100 & 1.01032600 & -5.17592500 \\
\hline $\mathrm{C}$ & 2.85180500 & 3.77507200 & -5.31769700 \\
\hline $\mathrm{H}$ & 1.77531400 & 3.59261700 & -5.30256100 \\
\hline $\mathrm{H}$ & 3.21109000 & 3.61821500 & -6.34164000 \\
\hline $\mathrm{H}$ & 3.02324000 & 4.81953500 & -5.06120800 \\
\hline $\mathrm{C}$ & 2.36854100 & 4.90261000 & 0.2618960 \\
\hline $\mathrm{C}$ & 3.06668900 & 4.45521100 & 1.5681080 \\
\hline $\mathrm{H}$ & 2.55738400 & 3.62495100 & 2.0570180 \\
\hline $\mathrm{H}$ & 3.08051800 & 5.28629100 & 2.2819520 \\
\hline $\mathrm{H}$ & 4.10158800 & 4.15113300 & 1.3779140 \\
\hline $\mathrm{C}$ & 0.89198600 & 5.19919800 & 0.5969150 \\
\hline $\mathrm{H}$ & 0.33413800 & 5.54077300 & -0.28046900 \\
\hline $\mathrm{H}$ & 0.82573800 & 5.98041000 & 1.3649960 \\
\hline $\mathrm{H}$ & 0.38599800 & 4.30592100 & 0.9748720 \\
\hline $\mathrm{C}$ & 3.08083800 & 6.20259000 & -0.16747800 \\
\hline $\mathrm{H}$ & 4.10183500 & 6.00472000 & -0.50590000 \\
\hline $\mathrm{H}$ & 3.13366200 & 6.86968100 & 0.7013250 \\
\hline $\mathrm{H}$ & 2.56551900 & 6.74300500 & -0.95978900 \\
\hline $\mathrm{C}$ & 1.64376700 & 5.69516500 & -3.07315800 \\
\hline $\mathrm{H}$ & 1.88371600 & 6.59305100 & -3.64834100 \\
\hline $\mathrm{H}$ & 1.06603200 & 5.98081000 & -2.18785200 \\
\hline $\mathrm{H}$ & 1.04005900 & 5.01585400 & -3.68140600 \\
\hline $\mathrm{C}$ & 6.87376800 & -1.31239200 & -1.99305100 \\
\hline $\mathrm{C}$ & 7.19043100 & 0.13737000 & -2.41857500 \\
\hline $\mathrm{H}$ & 6.27340300 & 0.70126800 & -2.61687300 \\
\hline $\mathrm{H}$ & 7.79779500 & 0.14431600 & -3.33163000 \\
\hline $\mathrm{H}$ & 7.74512300 & 0.67294000 & -1.64049300 \\
\hline $\mathrm{C}$ & 6.12418600 & -2.01517000 & -3.15605300 \\
\hline $\mathrm{H}$ & 5.86539700 & -3.04383000 & -2.88170100 \\
\hline $\mathrm{H}$ & 6.76824900 & -2.04964600 & -4.04200100 \\
\hline $\mathrm{H}$ & 5.20111000 & -1.50411200 & -3.44078700 \\
\hline $\mathrm{C}$ & 8.19220700 & -2.09456600 & -1.82005400 \\
\hline
\end{tabular}




\begin{tabular}{|c|c|c|c|}
\hline $\mathrm{H}$ & 8.90880200 & -1.59775800 & -1.16649000 \\
\hline $\mathrm{H}$ & 8.66569200 & -2.19340600 & -2.80403700 \\
\hline $\mathrm{H}$ & 8.01525900 & -3.09844000 & -1.42536600 \\
\hline $\mathrm{C}$ & 5.56003300 & -2.65983500 & 2.86724400 \\
\hline $\mathrm{C}$ & 6.53139000 & -1.85102200 & 3.75479600 \\
\hline $\mathrm{H}$ & 7.54080900 & -1.83525300 & 3.34506200 \\
\hline $\mathrm{H}$ & 6.58531800 & -2.30616700 & 4.75096600 \\
\hline $\mathrm{H}$ & 6.18216700 & -0.81873300 & 3.88012100 \\
\hline $\mathrm{C}$ & 4.27945300 & -2.88450500 & 3.69518200 \\
\hline $\mathrm{H}$ & 3.80674900 & -1.94749700 & 4.00231700 \\
\hline $\mathrm{H}$ & 4.54070600 & -3.44170300 & 4.60180700 \\
\hline $\mathrm{H}$ & 3.53231500 & -3.46748300 & 3.15145700 \\
\hline $\mathrm{C}$ & 6.16672400 & -4.04962600 & 2.56774000 \\
\hline $\mathrm{H}$ & 6.37783500 & -4.57711000 & 3.50613300 \\
\hline $\mathrm{H}$ & 7.09627800 & -3.96396400 & 2.00192700 \\
\hline $\mathrm{H}$ & 5.46068400 & -4.65749500 & 1.99039000 \\
\hline $\mathrm{C}$ & 8.30308700 & -0.76965400 & 1.15391700 \\
\hline $\mathrm{H}$ & 8.37704900 & -0.14081000 & 0.26057100 \\
\hline $\mathrm{H}$ & 9.30465900 & -1.07726500 & 1.46426500 \\
\hline $\mathrm{H}$ & 7.83433600 & -0.18524800 & 1.95402900 \\
\hline $\mathrm{C}$ & -0.49178800 & 0.37957800 & -2.14648800 \\
\hline $\mathrm{C}$ & -0.99356700 & 0.88807900 & -0.91745300 \\
\hline $\mathrm{C}$ & -1.28510000 & 2.25430800 & -0.79075900 \\
\hline $\mathrm{H}$ & -1.65145900 & 2.62626600 & 0.15809300 \\
\hline $\mathrm{C}$ & -1.19968300 & 3.15325400 & -1.86525400 \\
\hline $\mathrm{H}$ & -1.44742500 & 4.20013000 & -1.74108700 \\
\hline $\mathrm{C}$ & -0.82890500 & 2.61687900 & -3.08128200 \\
\hline $\mathrm{C}$ & -0.46654500 & 1.27758500 & -3.20223800 \\
\hline $\mathrm{C}$ & -0.51754200 & 2.16251500 & -5.22781200 \\
\hline $\mathrm{H}$ & -1.50324800 & 1.91926400 & -5.65173400 \\
\hline $\mathrm{H}$ & 0.20207700 & 2.43568100 & -5.99754500 \\
\hline $\mathrm{C}$ & 1.32760600 & -1.33649100 & -1.67115300 \\
\hline $\mathrm{C}$ & 1.94137900 & -2.55153500 & -1.99431900 \\
\hline $\mathrm{H}$ & 2.84434000 & -2.84124700 & -1.47137800 \\
\hline $\mathrm{C}$ & 1.44097900 & -3.41180800 & -2.98944500 \\
\hline $\mathrm{H}$ & 1.93604900 & -4.34488700 & -3.23350700 \\
\hline $\mathrm{C}$ & 0.29629000 & -3.00754000 & -3.64097700 \\
\hline $\mathrm{C}$ & -0.33274000 & -1.80850100 & -3.32475000 \\
\hline $\mathrm{C}$ & 0.13478400 & -0.94783700 & -2.35255600 \\
\hline $\mathrm{C}$ & -1.38459900 & -2.69924300 & -5.07643700 \\
\hline $\mathrm{H}$ & -2.35551800 & -3.18496000 & -5.18410400 \\
\hline $\mathrm{H}$ & -1.04059400 & -2.26158500 & -6.02424000 \\
\hline $\mathrm{C}$ & -2.84646000 & 0.89734900 & 5.30156000 \\
\hline $\mathrm{C}$ & -2.40678400 & 0.88578400 & 6.57258000 \\
\hline
\end{tabular}




$\begin{array}{lrrr}\mathrm{H} & -3.53561000 & 0.11166500 & 4.99184300 \\ \mathrm{C} & -2.50313400 & 1.86116300 & 4.25986600 \\ \mathrm{C} & -3.21000700 & 1.90976700 & 3.04931700 \\ \mathrm{H} & -1.91264700 & 2.72458800 & 4.55481400 \\ \mathrm{H} & -4.01256300 & 1.18842300 & 2.91190300 \\ \mathrm{C} & -3.27166100 & 3.05863200 & 2.13265900 \\ \mathrm{C} & -2.37819000 & 4.14514200 & 2.19484600 \\ \mathrm{C} & -4.26153100 & 3.07660700 & 1.13290100 \\ \mathrm{C} & -2.47517500 & 5.20335400 & 1.29491900 \\ \mathrm{H} & -1.58862700 & 4.15108700 & 2.94018700 \\ \mathrm{C} & -4.35068400 & 4.13075500 & 0.22767100 \\ \mathrm{H} & -4.95280200 & 2.24357500 & 1.06154700 \\ \mathrm{C} & -3.45915400 & 5.20232000 & 0.30147100 \\ \mathrm{H} & -1.77425300 & 6.02968300 & 1.36467200 \\ \mathrm{H} & -5.11797300 & 4.11201400 & -0.53992700 \\ \mathrm{H} & -3.53058800 & 6.02772300 & -0.40132400 \\ \mathrm{~S} & 0.64934700 & -0.03520900 & 3.66370400 \\ \mathrm{C} & 1.85989000 & 1.27679700 & 3.97981300 \\ \mathrm{C} & 1.45837700 & 2.40972900 & 4.69109500 \\ \mathrm{C} & 3.17681100 & 1.10732600 & 3.55681800 \\ \mathrm{C} & 2.41089400 & 3.37262700 & 5.01872900 \\ \mathrm{H} & 0.42448400 & 2.51553200 & 5.00006000 \\ \mathrm{C} & -1.12130600 & 2.08012800 & 3.89003400 \\ \mathrm{H} & 3.45232700 & 0.23274100 & 2.98156000 \\ \mathrm{C} & 3.74301800 & 3.20238100 & 4.62815500 \\ \mathrm{H} & 2.11620400 & 4.25322500 & 5.58189000 \\ \mathrm{H} & 5.15055700 & 1.95945000 & 3.56507400 \\ \mathrm{H} & 4.48163600 & 3.95560800 & 4.88583800 \\ \mathrm{O} & 1.46782300 & -1.18143100 & 3.15537200 \\ \mathrm{O} & 0.31927100 & -0.39956800 & 5.27191700 \\ \mathrm{H} & -2.75404900 & 0.08181600 & 7.21921400 \\ \mathrm{C} & -1.42780700 & 1.84099100 & 7.18930000 \\ \mathrm{H} & -0.42916600 & 1.38589000 & 7.24843700 \\ \mathrm{H} & -1.33285400 & 2.77040700 & 6.61812400 \\ \mathrm{H} & 2.11078000 & 8.21054000 \\ & & & \\ \mathrm{H} & -1.72387700 & .80012900 & 5.63082300\end{array}$<smiles>C/C=C\C1=C(P(O)[SH](=O)(O)c2ccccc2)[P+]1[Pb]CP</smiles>

Total SCF energy (M06/SDD-6-311++G(d,p)/SMD(1,4-Dioxane)): -5497.841341a.u. 
Thermal correction to Gibbs Free Energy at 298.15 K: 1.767171a.u

Gibbs free energy at 298.15 K (M06/SDD-6-311++G(d,p)/SMD(1,4-Dioxane)): -5496.074170a.u.

$\mathrm{Pd}$

$P$

$P$

$\mathrm{O}$

$\mathrm{O}$

$\mathrm{O}$

$\mathrm{O}$

$\mathrm{O}$

$\mathrm{O}$

$\mathrm{O}$

$\mathrm{O}$

C

C

$\mathrm{H}$

C

C

C

C

$\mathrm{H}$

C

C

$\mathrm{H}$

$\mathrm{H}$

$\mathrm{H}$

C

$\mathrm{H}$

$\mathrm{H}$

$\mathrm{H}$

C

$\mathrm{H}$

$\mathrm{H}$

$\mathrm{H}$

C

C

$\mathrm{H}$

$\mathrm{H}$

$\mathrm{H}$

C

$\mathrm{H}$

$\mathrm{H}$

$\mathrm{H}$

C

$$
0.92840200
$$

1.49101500

$-2.03909200$

7.27823300

1.08680900

$-3.83564200$

$-7.22148400$

$-0.51063700$

$-1.14696000$

0.34604700

$-0.84689000$

3.26652500

4.22378900

3.89592500

5.55647300

5.93435700

4.98909500

3.66392200

2.91893300

6.51516200

5.83291000

4.93844500

6.53115500

5.54855900

7.82351400

7.61760800

8.37327000

8.47182800

6.86825700

7.38800200

7.51766000

5.96140600

5.24024100

5.13344500

5.89147300

5.27435900

4.15056700

4.14340800

3.13266500

4.32974700

4.16309000

6.58088600
$-1.68540600$

$-0.00185800$

$-1.75670600$

$-0.09481400$

$-0.21218100$

1.61616100

0.11139000

$-0.27650800$

0.16571100

3.72646100

5.78494900

$-2.87080700$

$-4.17677400$

5.36004500

1.37928300

4.67755300

3.91345400

$-1.90981300$

3.69386000

0.52933600

0.19044400

2.64344500

4.60424400

$-0.40546700$

$-0.26039900$

$-1.41838100$

0.15012000

$-0.05142700$

0.34302800

1.28622000

0.36305900

0.06621800

2.27174400

$-0.27557500$

1.84514300

$-0.45199900$

2.57900900

$-0.71564000$

$-1.03232900$

1.07685800

$-2.39074500$

1.32241200

$-2.29719400$

1.94759100

$-3.05558300$

1.84287600

$-2.86838000$

0.37946100

$-1.32642900$

0.31414200

$-1.67238900$

$-0.70165600$

$-2.11992300$

0.83439700

$-0.45272900$

0.27118100

$-0.41776200$

2.45057700

0.53566800

2.33029800

$-1.09857000$

3.01493500

$-0.24645700$

3.03916300

3.79178800

$-0.43618900$

4.16000400

$-1.93270200$

3.63932700

$-2.52831700$

$5.23863800-2.07378100$

3.88952700

$-2.33413600$

4.57108800

0.33799300

4.38436100

$-0.03326500$

5.64714200

0.24526300

4.31210200

1.40120600

4.30815300

0.12672000 


\begin{tabular}{|c|c|c|c|}
\hline $\mathrm{H}$ & 6.73947300 & 3.97247200 & 1.15470700 \\
\hline $\mathrm{H}$ & 6.55695500 & 5.40439900 & 0.12498700 \\
\hline $\mathrm{H}$ & 7.44468800 & 4.00527500 & -0.46385000 \\
\hline $\mathrm{C}$ & 7.95428000 & 1.63224500 & -1.08855300 \\
\hline $\mathrm{H}$ & 7.50626300 & 2.35604300 & -1.77806600 \\
\hline $\mathrm{H}$ & 7.93710900 & 0.64535100 & -1.56002100 \\
\hline $\mathrm{H}$ & 8.98700200 & 1.92301300 & -0.88035600 \\
\hline $\mathrm{C}$ & 1.35307800 & -0.12569600 & 1.62740000 \\
\hline $\mathrm{C}$ & 0.68351500 & -1.18104900 & 2.24009200 \\
\hline $\mathrm{H}$ & 0.19166200 & -1.91906900 & 1.61872300 \\
\hline $\mathrm{C}$ & 0.61746900 & -1.30599400 & 3.64393300 \\
\hline $\mathrm{C}$ & 1.22382000 & -0.28885500 & 4.40661700 \\
\hline $\mathrm{C}$ & 2.02549400 & 0.72375100 & 3.81866700 \\
\hline $\mathrm{C}$ & 2.04632200 & 0.79145200 & 2.42891400 \\
\hline $\mathrm{H}$ & 2.62197600 & 1.56444100 & 1.94424900 \\
\hline $\mathrm{C}$ & 2.97329200 & 1.61459500 & 4.65162300 \\
\hline $\mathrm{C}$ & 2.26159400 & 2.44312300 & 5.74281600 \\
\hline $\mathrm{H}$ & 1.37888600 & 2.95171800 & 5.34928600 \\
\hline $\mathrm{H}$ & 2.95000000 & 3.20291400 & 6.13213400 \\
\hline $\mathrm{H}$ & 1.95193000 & 1.81872300 & 6.58029900 \\
\hline $\mathrm{C}$ & 4.00655100 & 0.68950500 & 5.33452000 \\
\hline $\mathrm{H}$ & 3.51175300 & -0.00851400 & 6.01432600 \\
\hline $\mathrm{H}$ & 4.72623200 & 1.28442100 & 5.91057500 \\
\hline $\mathrm{H}$ & 4.56282000 & 0.11214600 & 4.58919600 \\
\hline $\mathrm{C}$ & 3.73579000 & 2.60628000 & 3.75015200 \\
\hline $\mathrm{H}$ & 4.34344100 & 2.10026700 & 2.99277800 \\
\hline $\mathrm{H}$ & 4.41281000 & 3.20605600 & 4.36800800 \\
\hline $\mathrm{H}$ & 3.04727900 & 3.28845700 & 3.23855000 \\
\hline $\mathrm{C}$ & -0.05640500 & -2.58600300 & 4.20340500 \\
\hline $\mathrm{C}$ & -1.54635900 & -2.59541600 & 3.79759700 \\
\hline $\mathrm{H}$ & -2.08225400 & -1.75464700 & 4.25211700 \\
\hline $\mathrm{H}$ & -2.02440700 & -3.52379200 & 4.13458600 \\
\hline $\mathrm{H}$ & -1.67192100 & -2.52820900 & 2.71503300 \\
\hline $\mathrm{C}$ & 0.65179700 & -3.80655700 & 3.56376600 \\
\hline $\mathrm{H}$ & 0.51300000 & -3.84577900 & 2.48537800 \\
\hline $\mathrm{H}$ & 0.23547000 & -4.73282300 & 3.97748900 \\
\hline $\mathrm{H}$ & 1.72475300 & -3.78886800 & 3.79017200 \\
\hline $\mathrm{C}$ & 0.03590100 & -2.81174600 & 5.72709300 \\
\hline $\mathrm{H}$ & 1.06372400 & -2.74964400 & 6.09084500 \\
\hline $\mathrm{H}$ & -0.34098600 & -3.81965300 & 5.93794200 \\
\hline $\mathrm{H}$ & -0.57448400 & -2.11885700 & 6.30629900 \\
\hline $\mathrm{C}$ & -0.13700700 & 0.31117200 & 6.22589900 \\
\hline $\mathrm{H}$ & -0.15840200 & 1.38558700 & 6.02186700 \\
\hline $\mathrm{H}$ & -0.19025800 & 0.14006100 & 7.3039220 \\
\hline
\end{tabular}




\begin{tabular}{|c|c|c|c|}
\hline $\mathrm{H}$ & -0.99929600 & -0.15239900 & 5.73669800 \\
\hline $\mathrm{C}$ & -2.63365800 & 1.10675400 & -1.15989300 \\
\hline $\mathrm{C}$ & -3.34750900 & 2.27191800 & -0.85850200 \\
\hline $\mathrm{H}$ & -3.51501300 & 2.51508800 & 0.18086500 \\
\hline $\mathrm{C}$ & -3.81433000 & 3.13243000 & -1.85234400 \\
\hline $\mathrm{C}$ & -3.49589600 & 2.80658400 & -3.19662600 \\
\hline $\mathrm{C}$ & -2.86797600 & 1.59604000 & -3.54860200 \\
\hline $\mathrm{C}$ & -2.41246200 & 0.78620500 & -2.49418100 \\
\hline $\mathrm{H}$ & -1.85571200 & -0.11949300 & -2.71245800 \\
\hline $\mathrm{C}$ & -3.62782100 & -0.90837800 & 0.58874500 \\
\hline $\mathrm{C}$ & -4.77791800 & -0.18614000 & 0.89943100 \\
\hline $\mathrm{H}$ & -4.73317900 & 0.89344400 & 0.86999600 \\
\hline $\mathrm{C}$ & -5.97715900 & -0.79737700 & 1.27911500 \\
\hline $\mathrm{C}$ & -6.01605200 & -2.20739500 & 1.20966200 \\
\hline $\mathrm{C}$ & -4.85405900 & -2.98622800 & 0.98698600 \\
\hline $\mathrm{C}$ & -3.66899100 & -2.30429600 & 0.67826200 \\
\hline $\mathrm{H}$ & -2.75382600 & -2.85401500 & 0.50190400 \\
\hline $\mathrm{C}$ & -4.76821000 & 4.29170900 & -1.48674800 \\
\hline $\mathrm{C}$ & -6.16257700 & 3.94325900 & -2.05734100 \\
\hline $\mathrm{H}$ & -6.12177000 & 3.85198400 & -3.14595500 \\
\hline $\mathrm{H}$ & -6.88512900 & 4.72863900 & -1.80209200 \\
\hline $\mathrm{H}$ & -6.52772700 & 2.99704000 & -1.64412300 \\
\hline $\mathrm{C}$ & -4.89538100 & 4.44969600 & 0.04207800 \\
\hline $\mathrm{H}$ & -5.31286700 & 3.55668300 & 0.51858100 \\
\hline $\mathrm{H}$ & -5.57389200 & 5.28046800 & 0.26312600 \\
\hline $\mathrm{H}$ & -3.92674100 & 4.66560800 & 0.50675500 \\
\hline $\mathrm{C}$ & -4.34306600 & 5.66560400 & -2.04947400 \\
\hline $\mathrm{H}$ & -3.28813500 & 5.86723000 & -1.85047600 \\
\hline $\mathrm{H}$ & -4.93508900 & 6.45607800 & -1.57341000 \\
\hline $\mathrm{H}$ & -4.50577700 & 5.72805300 & -3.12430400 \\
\hline $\mathrm{C}$ & -2.64383400 & 1.06594800 & -4.98452800 \\
\hline $\mathrm{C}$ & -3.06941500 & -0.42533600 & -5.02754700 \\
\hline $\mathrm{H}$ & -2.51970600 & -1.05791100 & -4.32989100 \\
\hline $\mathrm{H}$ & -2.90219100 & -0.82450000 & -6.03473600 \\
\hline $\mathrm{H}$ & -4.13590400 & -0.52158600 & -4.79617600 \\
\hline $\mathrm{C}$ & -1.14230800 & 1.18081000 & -5.32943600 \\
\hline $\mathrm{H}$ & -0.83293300 & 2.22865700 & -5.40577500 \\
\hline $\mathrm{H}$ & -0.92503700 & 0.69279100 & -6.28724100 \\
\hline $\mathrm{H}$ & -0.52364200 & 0.71698700 & -4.55581700 \\
\hline $\mathrm{C}$ & -3.47405200 & 1.75030500 & -6.09121400 \\
\hline $\mathrm{H}$ & -4.53448100 & 1.78966500 & -5.82852300 \\
\hline $\mathrm{H}$ & -3.37268800 & 1.16083100 & -7.01036600 \\
\hline $\mathrm{H}$ & -3.14681100 & 2.76350400 & -6.31990100 \\
\hline C & -2.73872600 & 4.55199700 & -4.57257100 \\
\hline
\end{tabular}




\begin{tabular}{|c|c|c|c|}
\hline $\mathrm{H}$ & -3.15054200 & 5.31836500 & -5.23409500 \\
\hline $\mathrm{H}$ & -1.98237000 & 3.97699300 & -5.11725500 \\
\hline $\mathrm{H}$ & -2.26133700 & 5.02342000 & -3.70891700 \\
\hline $\mathrm{C}$ & -7.09866700 & 0.14044600 & 1.79108700 \\
\hline $\mathrm{C}$ & -7.64138200 & 0.98968900 & 0.62181000 \\
\hline $\mathrm{H}$ & -6.84011300 & 1.55702300 & 0.13774800 \\
\hline $\mathrm{H}$ & -8.39191900 & 1.70327400 & 0.98266900 \\
\hline $\mathrm{H}$ & -8.11152800 & 0.36361300 & -0.14432200 \\
\hline $\mathrm{C}$ & -6.47747900 & 1.09285100 & 2.84765700 \\
\hline $\mathrm{H}$ & -6.06977300 & 0.52010500 & 3.68824400 \\
\hline $\mathrm{H}$ & -7.24973700 & 1.76611800 & 3.23672800 \\
\hline $\mathrm{H}$ & -5.67233300 & 1.71324400 & 2.44638700 \\
\hline $\mathrm{C}$ & -8.27667000 & -0.55320300 & 2.50626000 \\
\hline $\mathrm{H}$ & -8.92794500 & -1.10739600 & 1.83084600 \\
\hline $\mathrm{H}$ & -8.89014600 & 0.21875800 & 2.98593400 \\
\hline $\mathrm{H}$ & -7.92881900 & -1.24153400 & 3.28064700 \\
\hline $\mathrm{C}$ & -4.85150900 & -4.51854800 & 1.18253900 \\
\hline $\mathrm{C}$ & -5.83143100 & -5.25082900 & 0.23933200 \\
\hline $\mathrm{H}$ & -6.87006800 & -5.07597800 & 0.51605000 \\
\hline $\mathrm{H}$ & -5.65173300 & -6.33126600 & 0.29364500 \\
\hline $\mathrm{H}$ & -5.68231500 & -4.94078500 & -0.80155100 \\
\hline $\mathrm{C}$ & -3.45705700 & -5.11705300 & 0.92268900 \\
\hline $\mathrm{H}$ & -3.14443900 & -4.95887700 & -0.11532400 \\
\hline $\mathrm{H}$ & -3.49578300 & -6.19695100 & 1.10576900 \\
\hline $\mathrm{H}$ & -2.68504100 & -4.69456800 & 1.56880400 \\
\hline $\mathrm{C}$ & -5.22826400 & -4.82224000 & 2.65070800 \\
\hline $\mathrm{H}$ & -5.22157600 & -5.90528000 & 2.82609400 \\
\hline $\mathrm{H}$ & -6.22384300 & -4.44048800 & 2.88799600 \\
\hline $\mathrm{H}$ & -4.50520500 & -4.36320800 & 3.33449500 \\
\hline $\mathrm{C}$ & -8.04403900 & -2.83624200 & 0.21415500 \\
\hline $\mathrm{H}$ & -8.31328600 & -1.81077500 & -0.05952600 \\
\hline $\mathrm{H}$ & -8.95012200 & -3.39640100 & 0.45775500 \\
\hline $\mathrm{H}$ & -7.54082400 & -3.30442100 & -0.63896200 \\
\hline $\mathrm{C}$ & -0.11737000 & 2.42027600 & 0.11832900 \\
\hline $\mathrm{C}$ & 0.66316700 & 1.57318900 & -0.71382500 \\
\hline $\mathrm{C}$ & 0.96562300 & 1.97560400 & -2.02570500 \\
\hline $\mathrm{H}$ & 1.54308700 & 1.30218900 & -2.64764400 \\
\hline $\mathrm{C}$ & 0.61666900 & 3.23008700 & -2.54540200 \\
\hline $\mathrm{H}$ & 0.86594400 & 3.51817400 & -3.56012500 \\
\hline $\mathrm{C}$ & -0.04428500 & 4.08238100 & -1.68379200 \\
\hline $\mathrm{C}$ & -0.40894800 & 3.67391000 & -0.40579700 \\
\hline $\mathrm{C}$ & -0.86364100 & 5.82905900 & -0.60091800 \\
\hline $\mathrm{H}$ & -0.00613000 & 6.36724000 & -0.17011800 \\
\hline $\mathrm{H}$ & -1.75021100 & 6.45706800 & -0.66323200 \\
\hline
\end{tabular}




\begin{tabular}{|c|c|c|c|}
\hline $\mathrm{C}$ & -1.74540500 & 1.04759700 & 1.54237400 \\
\hline $\mathrm{C}$ & -2.41823000 & 0.89238000 & 2.75983600 \\
\hline $\mathrm{H}$ & -3.15642400 & 0.10699600 & 2.85594000 \\
\hline $\mathrm{C}$ & -2.20574400 & 1.74184700 & 3.86071200 \\
\hline $\mathrm{H}$ & -2.76195800 & 1.62319400 & 4.78363800 \\
\hline $\mathrm{C}$ & -1.26768100 & 2.73793100 & 3.70134200 \\
\hline $\mathrm{C}$ & -0.55844800 & 2.87956400 & 2.51409500 \\
\hline $\mathrm{C}$ & -0.76065600 & 2.07313600 & 1.41029700 \\
\hline $\mathrm{C}$ & -0.07293300 & 4.60957400 & 3.81725400 \\
\hline $\mathrm{H}$ & 0.80299300 & 4.92435600 & 4.3846270 \\
\hline $\mathrm{H}$ & -0.70555700 & 5.46226300 & 3.53022900 \\
\hline $\mathrm{C}$ & 0.01546200 & -2.37911800 & -4.60459800 \\
\hline $\mathrm{C}$ & -0.68486300 & -3.41506000 & -5.10252300 \\
\hline $\mathrm{H}$ & -0.38162900 & -1.38146300 & -4.76101300 \\
\hline $\mathrm{C}$ & 1.28021600 & -2.42961400 & -3.88269300 \\
\hline $\mathrm{C}$ & 2.05535100 & -1.28426000 & -3.65905700 \\
\hline $\mathrm{H}$ & 1.75164700 & -3.40138700 & -3.76844900 \\
\hline $\mathrm{H}$ & 1.66861400 & -0.34526200 & -4.05239100 \\
\hline $\mathrm{C}$ & 3.49625700 & -1.28298700 & -3.36446300 \\
\hline $\mathrm{C}$ & 4.18516200 & -2.42591400 & -2.91506100 \\
\hline $\mathrm{C}$ & 4.23342500 & -0.10547600 & -3.57103600 \\
\hline $\mathrm{C}$ & 5.56489200 & -2.40089500 & -2.74503400 \\
\hline $\mathrm{H}$ & 3.63784500 & -3.33675700 & -2.69611900 \\
\hline $\mathrm{C}$ & 5.61411000 & -0.07916600 & -3.39421500 \\
\hline $\mathrm{H}$ & 3.71409200 & 0.79422300 & -3.89152300 \\
\hline $\mathrm{C}$ & 6.29066700 & -1.23359600 & -3.00223000 \\
\hline $\mathrm{H}$ & 6.07771400 & -3.29933300 & -2.41168400 \\
\hline $\mathrm{H}$ & 6.16086500 & 0.84197600 & -3.57341600 \\
\hline $\mathrm{H}$ & 7.37093000 & -1.22644500 & -2.89574700 \\
\hline $\mathrm{S}$ & -0.00781900 & -3.61107100 & -0.79507700 \\
\hline $\mathrm{C}$ & 1.51676700 & -4.59090200 & -0.77086400 \\
\hline $\mathrm{C}$ & 1.82978000 & -5.47684700 & -1.80264900 \\
\hline $\mathrm{C}$ & 2.42099400 & -4.33009800 & 0.26205200 \\
\hline $\mathrm{C}$ & 3.06964900 & -6.11837500 & -1.79317300 \\
\hline $\mathrm{H}$ & 1.11310000 & -5.65780000 & -2.59429000 \\
\hline $\mathrm{C}$ & 3.65015900 & -4.98736700 & 0.26712800 \\
\hline $\mathrm{H}$ & 2.16602600 & -3.62759700 & 1.04719100 \\
\hline $\mathrm{C}$ & 3.97852500 & -5.87430300 & -0.76141200 \\
\hline $\mathrm{H}$ & 3.32235800 & -6.81058900 & -2.59115000 \\
\hline $\mathrm{H}$ & 4.35129700 & -4.80514400 & 1.07424500 \\
\hline $\mathrm{H}$ & 4.94107000 & -6.37727500 & -0.75602900 \\
\hline $\mathrm{O}$ & -0.94820100 & -4.63422100 & -1.76149700 \\
\hline $\mathrm{O}$ & -0.57875900 & -3.73198100 & 0.58732800 \\
\hline $\mathrm{H}$ & -1.62185800 & -3.18629000 & -5.60779700 \\
\hline
\end{tabular}




$\begin{array}{lrrr}\mathrm{C} & -0.32775300 & -4.86922300 & -5.01953500 \\ \mathrm{H} & -0.49728200 & -5.37059400 & -5.98076500 \\ \mathrm{H} & 0.72027000 & -5.02385100 & -4.74555300 \\ \mathrm{H} & -0.94656800 & -5.38050700 & -4.26994700 \\ \mathrm{H} & -1.20626200 & -5.38171700 & -1.18292000\end{array}$<smiles>C/C=C/C1=C(P)[P+]1P(CP)c1ccccc1</smiles>

Int-7c

Total SCF energy (M06/SDD-6-311++G(d,p)/SMD(1,4-Dioxane)): -5497.847615a.u.

Thermal correction to Gibbs Free Energy at $298.15 \mathrm{~K}$ : 1.763288a.u

Gibbs free energy at 298.15 K (M06/SDD-6-311++G(d,p)/SMD(1,4-Dioxane)): -5496.084327a.u.

$\mathrm{Pd}$

$P$

$P$

$\mathrm{O}$

$\mathrm{O}$

$\mathrm{O}$

$\mathrm{O}$

$\mathrm{O}$

$\mathrm{O}$

$\mathrm{O}$

$\mathrm{O}$

C

C

$\mathrm{H}$

C

C

C

C

$\mathrm{H}$

C

C

$\mathrm{H}$

$\mathrm{H}$

$\mathrm{H}$

C

$\mathrm{H}$

$\mathrm{H}$

$\mathrm{H}$

$$
1.22863600
$$

$$
1.68395200
$$$$
-1.88182300
$$$$
7.47443600
$$

2.12577600

$-3.33631400$

$-7.41658200$

0.46300500

0.09580700

1.62901700

0.73206100

3.45091700

4.41778500

4.07997900

5.77772200

6.15216800

5.22742500

3.87370600

3.13728600

6.81845600

6.14367400

5.54828600

6.91799200

5.49448800

7.64314500

6.98853400

8.24691900

8.32431100
$-1.11332800 \quad-2.53491300$

$-0.31150600 \quad-0.36274800$

$-0.18873800 \quad 0.48285000$

$1.30691200 \quad-0.45009400$

$-4.15289000 \quad 4.23569200$

$5.56348400 \quad-0.46937400$

$-2.44977400$

0.18851600

5.16067400

1.49283200

3.48573400

3.02351200

1.12097600

4.23714700

$-0.24060600$

5.87023000

0.23144000

$-0.30502400$

$-0.68041800$

$-0.74100500$

$-1.64811500$

$-1.08969400$

$-0.37424000$

$-0.73674700$

0.92244400

$-0.28802600$

1.79670100

0.31556600

1.43286000

0.24622100

2.10990400

0.65159900

$-1.44849200$

$-1.11403600$

$-2.80067300$

$-1.41935100$

$-3.16302800$

$-0.57467100$

$-3.54723200$

$-1.62481300$

$-2.74869400$

$-2.30138700$

$-1.06022800$

$-2.36005500$

$-0.77842400$

$-3.19331300$

$-1.91595600$

$-2.68460000$

$-0.23553900$

$-2.15222100$ 


\begin{tabular}{|c|c|c|c|}
\hline $\mathrm{C}$ & 7.76447900 & -1.66310300 & 0.08979800 \\
\hline $\mathrm{H}$ & 8.29420400 & -0.74297700 & 0.34394100 \\
\hline $\mathrm{H}$ & 8.50511900 & -2.43670300 & -0.14699500 \\
\hline $\mathrm{H}$ & 7.19883900 & -1.99401700 & 0.96871000 \\
\hline $\mathrm{C}$ & 5.54478500 & 3.09269200 & 1.1007290 \\
\hline $\mathrm{C}$ & 5.00475600 & 4.31527100 & 0.3276470 \\
\hline $\mathrm{H}$ & 5.52244000 & 4.43847300 & -0.63023900 \\
\hline $\mathrm{H}$ & 5.15789600 & 5.23176300 & 0.9104270 \\
\hline $\mathrm{H}$ & 3.93445400 & 4.21816900 & 0.1185120 \\
\hline $\mathrm{C}$ & 4.81937900 & 3.01351500 & 2.4712380 \\
\hline $\mathrm{H}$ & 3.73465100 & 2.91414600 & 2.3826970 \\
\hline $\mathrm{H}$ & 5.02392800 & 3.92493700 & 3.0453010 \\
\hline $\mathrm{H}$ & 5.18400500 & 2.15801400 & 3.0489310 \\
\hline $\mathrm{C}$ & 7.03332000 & 3.31909100 & 1.4338470 \\
\hline $\mathrm{H}$ & 7.47873300 & 2.43763000 & 1.9020050 \\
\hline $\mathrm{H}$ & 7.10587300 & 4.15446500 & 2.1404750 \\
\hline $\mathrm{H}$ & 7.63614500 & 3.57946200 & 0.5638020 \\
\hline $\mathrm{C}$ & 7.66262600 & 2.17820500 & -1.56360300 \\
\hline $\mathrm{H}$ & 7.11219300 & 3.11703200 & -1.43787800 \\
\hline $\mathrm{H}$ & 7.33572700 & 1.70448300 & -2.49645300 \\
\hline $\mathrm{H}$ & 8.73336800 & 2.38983300 & -1.61568000 \\
\hline $\mathrm{C}$ & 1.73444600 & -1.46173900 & 1.07549200 \\
\hline $\mathrm{C}$ & 0.96485500 & -2.62258800 & 1.08968800 \\
\hline $\mathrm{H}$ & 0.26339000 & -2.78552500 & 0.28084600 \\
\hline $\mathrm{C}$ & 1.08762400 & -3.57936900 & 2.11524200 \\
\hline $\mathrm{C}$ & 2.00583600 & -3.30000300 & 3.15090100 \\
\hline $\mathrm{C}$ & 2.88112500 & -2.18625900 & 3.10758900 \\
\hline $\mathrm{C}$ & 2.69404900 & -1.27202200 & 2.07583800 \\
\hline $\mathrm{H}$ & 3.32186400 & -0.39817900 & 2.02614000 \\
\hline $\mathrm{C}$ & 4.11923800 & -2.05618300 & 4.02305300 \\
\hline $\mathrm{C}$ & 3.80798700 & -2.13390600 & 5.53368400 \\
\hline $\mathrm{H}$ & 2.97450900 & -1.48144000 & 5.80655800 \\
\hline $\mathrm{H}$ & 4.68929800 & -1.81952200 & 6.10537500 \\
\hline $\mathrm{H}$ & 3.55980800 & -3.14928100 & 5.84010800 \\
\hline $\mathrm{C}$ & 5.08295800 & -3.20815900 & 3.65456500 \\
\hline $\mathrm{H}$ & 4.61104100 & -4.17895200 & 3.82801600 \\
\hline $\mathrm{H}$ & 5.99442200 & -3.15178300 & 4.26296500 \\
\hline $\mathrm{H}$ & 5.37241100 & -3.14559800 & 2.59908400 \\
\hline $\mathrm{C}$ & 4.85653200 & -0.72307900 & 3.77730500 \\
\hline $\mathrm{H}$ & 5.22976400 & -0.63516200 & 2.75163400 \\
\hline $\mathrm{H}$ & 5.72174900 & -0.66239700 & 4.44623100 \\
\hline $\mathrm{H}$ & 4.20818100 & 0.13733200 & 3.9767970 \\
\hline $\mathrm{C}$ & 0.22802100 & -4.86258300 & 2.00977000 \\
\hline $\mathrm{C}$ & -1.21177200 & -4.53719200 & 2.46012500 \\
\hline
\end{tabular}




\begin{tabular}{|c|c|c|c|}
\hline $\mathrm{H}$ & -1.24589700 & -4.19242500 & 3.49796400 \\
\hline $\mathrm{H}$ & -1.84585800 & -5.42801300 & 2.37824200 \\
\hline $\mathrm{H}$ & -1.64442200 & -3.74719600 & 1.84059000 \\
\hline $\mathrm{C}$ & 0.18292000 & -5.33282800 & 0.53388100 \\
\hline $\mathrm{H}$ & -0.30558600 & -4.62263700 & -0.13275900 \\
\hline $\mathrm{H}$ & -0.37799400 & -6.27268600 & 0.47365100 \\
\hline $\mathrm{H}$ & 1.19567200 & -5.51941000 & 0.15765600 \\
\hline $\mathrm{C}$ & 0.75595300 & -6.07235400 & 2.81264500 \\
\hline $\mathrm{H}$ & 1.81610100 & -6.25260200 & 2.61254300 \\
\hline $\mathrm{H}$ & 0.19593400 & -6.96148200 & 2.49970000 \\
\hline $\mathrm{H}$ & 0.63151700 & -5.97281000 & 3.88969600 \\
\hline $\mathrm{C}$ & 1.17311200 & -3.90979700 & 5.27033200 \\
\hline $\mathrm{H}$ & 1.18214800 & -2.86170800 & 5.58260400 \\
\hline $\mathrm{H}$ & 1.46333200 & -4.54513900 & 6.11089500 \\
\hline $\mathrm{H}$ & 0.16025000 & -4.17577800 & 4.95224800 \\
\hline $\mathrm{C}$ & -2.36399300 & 1.57861100 & 0.26661100 \\
\hline $\mathrm{C}$ & -2.67603900 & 2.44316100 & 1.32279400 \\
\hline $\mathrm{H}$ & -2.59612000 & 2.06331200 & 2.33228900 \\
\hline $\mathrm{C}$ & -3.07801200 & 3.76233900 & 1.10450300 \\
\hline $\mathrm{C}$ & -3.11240200 & 4.21484700 & -0.24024000 \\
\hline $\mathrm{C}$ & -2.93399600 & 3.34380300 & -1.33113500 \\
\hline $\mathrm{C}$ & -2.52464200 & 2.03268800 & -1.03690100 \\
\hline $\mathrm{H}$ & -2.33068200 & 1.33887500 & -1.84928500 \\
\hline $\mathrm{C}$ & -3.56290100 & -0.93699100 & 0.69330800 \\
\hline $\mathrm{C}$ & -4.67336800 & -0.17904800 & 1.05383900 \\
\hline $\mathrm{H}$ & -4.52229800 & 0.84772000 & 1.35493500 \\
\hline $\mathrm{C}$ & -5.98186600 & -0.67637700 & 1.00729600 \\
\hline $\mathrm{C}$ & -6.14650700 & -1.97571200 & 0.48463800 \\
\hline $\mathrm{C}$ & -5.04412500 & -2.83435500 & 0.24109300 \\
\hline $\mathrm{C}$ & -3.76215200 & -2.28248700 & 0.35380900 \\
\hline $\mathrm{H}$ & -2.89681300 & -2.87970900 & 0.10437800 \\
\hline $\mathrm{C}$ & -3.62632400 & 4.61299900 & 2.27190400 \\
\hline $\mathrm{C}$ & -5.14566200 & 4.78751700 & 2.03952800 \\
\hline $\mathrm{H}$ & -5.33624900 & 5.30389100 & 1.09463300 \\
\hline $\mathrm{H}$ & -5.58865800 & 5.37827000 & 2.85116100 \\
\hline $\mathrm{H}$ & -5.65080800 & 3.81611000 & 2.01199600 \\
\hline $\mathrm{C}$ & -3.42578000 & 3.90169600 & 3.62505400 \\
\hline $\mathrm{H}$ & -3.96034000 & 2.94727400 & 3.67313200 \\
\hline $\mathrm{H}$ & -3.82145300 & 4.53482800 & 4.42641600 \\
\hline $\mathrm{H}$ & -2.36697000 & 3.70945400 & 3.82864400 \\
\hline $\mathrm{C}$ & -2.99058600 & 6.01562900 & 2.38997200 \\
\hline $\mathrm{H}$ & -1.90088500 & 5.96424400 & 2.34417500 \\
\hline $\mathrm{H}$ & -3.27159600 & 6.46518400 & 3.34969100 \\
\hline $\mathrm{H}$ & -3.33311200 & 6.67816300 & 1.59615100 \\
\hline
\end{tabular}




\begin{tabular}{|c|c|c|c|}
\hline $\mathrm{C}$ & -3.23968800 & 3.64773700 & -2.81682800 \\
\hline $\mathrm{C}$ & -4.36240500 & 2.66885800 & -3.23901900 \\
\hline $\mathrm{H}$ & -4.09458100 & 1.62823600 & -3.05801300 \\
\hline $\mathrm{H}$ & -4.57909400 & 2.78070900 & -4.30846500 \\
\hline $\mathrm{H}$ & -5.28050100 & 2.88253400 & -2.67946300 \\
\hline $\mathrm{C}$ & -1.97461200 & 3.39152900 & -3.66456000 \\
\hline $\mathrm{H}$ & -1.15954300 & 4.05340400 & -3.35293200 \\
\hline $\mathrm{H}$ & -2.18413400 & 3.58341200 & -4.72443700 \\
\hline $\mathrm{H}$ & -1.61274900 & 2.36330500 & -3.57620600 \\
\hline $\mathrm{C}$ & -3.76502700 & 5.05694700 & -3.15493300 \\
\hline $\mathrm{H}$ & -4.62553700 & 5.33237500 & -2.54307700 \\
\hline $\mathrm{H}$ & -4.07670000 & 5.05645000 & -4.20665100 \\
\hline $\mathrm{H}$ & -3.00647200 & 5.83395000 & -3.04775400 \\
\hline $\mathrm{C}$ & -2.12097400 & 6.27995500 & -0.70106500 \\
\hline $\mathrm{H}$ & -2.40571400 & 7.31240700 & -0.91781300 \\
\hline $\mathrm{H}$ & -1.57432900 & 5.86476700 & -1.55552900 \\
\hline $\mathrm{H}$ & -1.46677400 & 6.24755000 & 0.17490400 \\
\hline $\mathrm{C}$ & -7.09037600 & 0.25982900 & 1.54698100 \\
\hline $\mathrm{C}$ & -7.25985000 & 1.46431500 & 0.59392900 \\
\hline $\mathrm{H}$ & -6.31593800 & 2.00243500 & 0.45783300 \\
\hline $\mathrm{H}$ & -7.99755900 & 2.16857700 & 0.99720300 \\
\hline $\mathrm{H}$ & -7.60438400 & 1.14766200 & -0.39666100 \\
\hline $\mathrm{C}$ & -6.63796300 & 0.79118500 & 2.93270300 \\
\hline $\mathrm{H}$ & -6.48431200 & -0.03696000 & 3.63313700 \\
\hline $\mathrm{H}$ & -7.41102600 & 1.44935600 & 3.34512400 \\
\hline $\mathrm{H}$ & -5.71034600 & 1.36645300 & 2.88471800 \\
\hline $\mathrm{C}$ & -8.46201900 & -0.40405100 & 1.78717000 \\
\hline $\mathrm{H}$ & -8.99361800 & -0.64810200 & 0.86777700 \\
\hline $\mathrm{H}$ & -9.09155200 & 0.29656300 & 2.34837600 \\
\hline $\mathrm{H}$ & -8.36634600 & -1.32094000 & 2.37474300 \\
\hline $\mathrm{C}$ & -5.22887200 & -4.33055200 & -0.09840000 \\
\hline $\mathrm{C}$ & -5.96117300 & -4.54773400 & -1.44154900 \\
\hline $\mathrm{H}$ & -7.01550600 & -4.28095400 & -1.37910200 \\
\hline $\mathrm{H}$ & -5.90474800 & -5.60632800 & -1.72149200 \\
\hline $\mathrm{H}$ & -5.49458800 & -3.96790000 & -2.24703500 \\
\hline $\mathrm{C}$ & -3.87476900 & -5.05717000 & -0.20618200 \\
\hline $\mathrm{H}$ & -3.25109200 & -4.67122200 & -1.01784300 \\
\hline $\mathrm{H}$ & -4.05810700 & -6.12040800 & -0.39711100 \\
\hline $\mathrm{H}$ & -3.30010200 & -4.98323900 & 0.71939600 \\
\hline $\mathrm{C}$ & -6.03114300 & -5.00458000 & 1.03769500 \\
\hline $\mathrm{H}$ & -6.16296300 & -6.07207000 & 0.82200400 \\
\hline $\mathrm{H}$ & -7.01722900 & -4.54989800 & 1.15110400 \\
\hline $\mathrm{H}$ & -5.49418500 & -4.91661500 & 1.98907200 \\
\hline $\mathrm{C}$ & -7.93414300 & -1.93404800 & -1.03474000 \\
\hline
\end{tabular}




\begin{tabular}{|c|c|c|c|}
\hline $\mathrm{H}$ & -8.04856200 & -0.84591800 & -1.00067700 \\
\hline $\mathrm{H}$ & -8.91370900 & -2.39690100 & -1.17772400 \\
\hline $\mathrm{H}$ & -7.28346900 & -2.19100300 & -1.87914400 \\
\hline $\mathrm{C}$ & 0.50419000 & 1.57457000 & 1.49516200 \\
\hline $\mathrm{C}$ & 0.90802300 & 1.27497500 & 0.16466500 \\
\hline $\mathrm{C}$ & 1.02140600 & 2.30071100 & -0.78391300 \\
\hline $\mathrm{H}$ & 1.30137200 & 2.04802300 & -1.79920300 \\
\hline $\mathrm{C}$ & 0.86948800 & 3.65726600 & -0.45330100 \\
\hline $\mathrm{H}$ & 0.97373400 & 4.43282100 & -1.20222600 \\
\hline $\mathrm{C}$ & 0.63705400 & 3.93851100 & 0.87757500 \\
\hline $\mathrm{C}$ & 0.44011600 & 2.92196100 & 1.81127600 \\
\hline $\mathrm{C}$ & 0.49492200 & 4.84884000 & 2.89234800 \\
\hline $\mathrm{H}$ & 1.52465900 & 4.96545000 & 3.26174000 \\
\hline $\mathrm{H}$ & -0.20610500 & 5.48728500 & 3.42674800 \\
\hline $\mathrm{C}$ & -1.18856900 & -0.18587500 & 2.20093700 \\
\hline $\mathrm{C}$ & -1.72004900 & -1.00757400 & 3.19894200 \\
\hline $\mathrm{H}$ & -2.61306800 & -1.58330700 & 2.98444700 \\
\hline $\mathrm{C}$ & -1.14994600 & -1.10360500 & 4.48296800 \\
\hline $\mathrm{H}$ & -1.57669200 & -1.74352800 & 5.24709500 \\
\hline $\mathrm{C}$ & -0.03416900 & -0.33194000 & 4.72516200 \\
\hline $\mathrm{C}$ & 0.50590600 & 0.49336500 & 3.74362400 \\
\hline $\mathrm{C}$ & -0.02005800 & 0.59138600 & 2.47222400 \\
\hline $\mathrm{C}$ & 1.57932100 & 0.89479800 & 5.64672400 \\
\hline $\mathrm{H}$ & 2.58216900 & 0.67716100 & 6.01401400 \\
\hline $\mathrm{H}$ & 1.14341300 & 1.77400500 & 6.14267900 \\
\hline $\mathrm{C}$ & 2.67411600 & -2.55227700 & -4.75777100 \\
\hline $\mathrm{C}$ & 2.20265400 & -3.41824200 & -5.66809500 \\
\hline $\mathrm{H}$ & 3.45892700 & -2.87787600 & -4.07288600 \\
\hline $\mathrm{C}$ & 2.20478400 & -1.18102000 & -4.58629800 \\
\hline $\mathrm{C}$ & 2.88349000 & -0.26061000 & -3.77760900 \\
\hline $\mathrm{H}$ & 1.49459500 & -0.82255800 & -5.32946300 \\
\hline $\mathrm{H}$ & 3.78859000 & -0.60402100 & -3.28245000 \\
\hline $\mathrm{C}$ & 2.73514200 & 1.20211000 & -3.81632700 \\
\hline $\mathrm{C}$ & 1.65760400 & 1.85260800 & -4.44821400 \\
\hline $\mathrm{C}$ & 3.69886800 & 1.99976700 & -3.17182400 \\
\hline $\mathrm{C}$ & 1.55117400 & 3.24057600 & -4.43416200 \\
\hline $\mathrm{H}$ & 0.88601500 & 1.26529100 & -4.93601600 \\
\hline $\mathrm{C}$ & 3.58643100 & 3.38777600 & -3.15083600 \\
\hline $\mathrm{H}$ & 4.52952100 & 1.51709400 & -2.66722400 \\
\hline $\mathrm{C}$ & 2.51280300 & 4.01908800 & -3.78170800 \\
\hline $\mathrm{H}$ & 0.71086200 & 3.71692000 & -4.93036800 \\
\hline $\mathrm{H}$ & 4.33799800 & 3.97676700 & -2.63405400 \\
\hline $\mathrm{H}$ & 2.42663800 & 5.10206200 & -3.77024000 \\
\hline S & -0.71069900 & -2.44733100 & -2.65769400 \\
\hline
\end{tabular}




$\begin{array}{lrrr}\mathrm{C} & -2.06031100 & -1.55847800 & -3.47437000 \\ \mathrm{C} & -1.76823500 & -0.77101400 & -4.58927600 \\ \mathrm{C} & -3.36432000 & -1.71905400 & -3.01290900 \\ \mathrm{C} & -2.81537900 & -0.15823600 & -5.27532400 \\ \mathrm{H} & -0.73844000 & -0.65677800 & -4.91201500 \\ \mathrm{C} & -4.40506300 & -1.09936000 & -3.70600100 \\ \mathrm{H} & -3.55189000 & -2.30586500 & -2.12341000 \\ \mathrm{C} & -4.13293200 & -0.33338000 & -4.84105200 \\ \mathrm{H} & -2.60555800 & 0.45595200 & -6.14588200 \\ \mathrm{H} & -5.42517500 & -1.20854300 & -3.34915300 \\ \mathrm{H} & -4.94522600 & 0.14963100 & -5.37565800 \\ \mathrm{O} & -1.35602700 & -3.16325200 & -1.50986400 \\ \mathrm{O} & -0.48811800 & -3.62397700 & -3.84053300 \\ \mathrm{H} & -1.28588600 & -4.19111200 & -3.81103900 \\ \mathrm{H} & 1.40295000 & -3.09206800 & -6.33426000 \\ \mathrm{C} & 2.65486300 & -4.83859500 & -5.81064500 \\ \mathrm{H} & 3.00725900 & -5.05562600 & -6.82872000 \\ \mathrm{H} & 3.46739100 & -5.07060600 & -5.11329800 \\ \mathrm{H} & 1.82876100 & -5.53486300 & -5.60729900\end{array}$

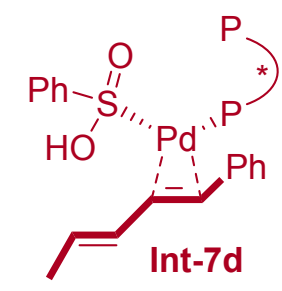

Total SCF energy (M06/SDD-6-311++G(d,p)/SMD(1,4-Dioxane)): -5497.846587a.u.

Thermal correction to Gibbs Free Energy at $298.15 \mathrm{~K}: 1.766164 \mathrm{a} . \mathrm{u}$

Gibbs free energy at 298.15 K (M06/SDD-6-311++G(d,p)/SMD(1,4-Dioxane)): -5496.080423a.u.

$\begin{array}{cccc}\text { Pd } & 0.94115700 & -1.78476300 & -1.68118600 \\ \text { P } & 1.50254200 & -0.02909400 & -0.22234900 \\ \text { P } & -2.03277000 & -0.03562200 & 0.20273100 \\ \text { O } & 7.30338200 & 1.53121000 & 0.12413200 \\ \text { O } & 1.13455500 & -0.29783200 & 5.77410200 \\ \text { O } & -3.57329300 & 3.51632500 & -4.40320600 \\ \text { O } & -7.39844200 & -2.39219400 & 1.53570100 \\ \text { O } & -0.37707800 & 5.31417000 & -2.07845200 \\ \text { O } & -0.99323800 & 4.72988800 & 0.05880500 \\ \text { O } & 0.55973800 & 3.98538100 & 2.51318300 \\ \text { O } & -0.57110100 & 3.85875600 & 4.52148000 \\ \text { C } & 3.28141000 & 0.48033900 & -0.28633600 \\ \text { C } & 4.23198200 & -0.46477200 & 0.11381100 \\ \text { H } & 3.89493300 & -1.47486100 & 0.30420400 \\ \text { C } & 5.56955200 & -0.12580200 & 0.31791000\end{array}$




\begin{tabular}{|c|c|c|c|}
\hline $\mathrm{C}$ & 5.95721600 & 1.21086200 & 0.02826900 \\
\hline $\mathrm{C}$ & 5.01844400 & 2.20709300 & -0.30289600 \\
\hline $\mathrm{C}$ & 3.68912800 & 1.79317500 & -0.47689900 \\
\hline $\mathrm{H}$ & 2.94877400 & 2.53411200 & -0.73519800 \\
\hline $\mathrm{C}$ & 6.52271800 & -1.12529900 & 1.01266200 \\
\hline $\mathrm{C}$ & 5.82928400 & -2.48115400 & 1.24889200 \\
\hline $\mathrm{H}$ & 4.94354400 & -2.38587300 & 1.88613400 \\
\hline $\mathrm{H}$ & 6.52682300 & -3.15809100 & 1.75408700 \\
\hline $\mathrm{H}$ & 5.52935400 & -2.94693500 & 0.30415700 \\
\hline $\mathrm{C}$ & 7.82330700 & -1.41921000 & 0.23646200 \\
\hline $\mathrm{H}$ & 7.60920400 & -1.74231900 & -0.78517100 \\
\hline $\mathrm{H}$ & 8.36786300 & -2.22832500 & 0.73759100 \\
\hline $\mathrm{H}$ & 8.47989500 & -0.55102800 & 0.20742500 \\
\hline $\mathrm{C}$ & 6.89151700 & -0.53142600 & 2.39150800 \\
\hline $\mathrm{H}$ & 7.41766100 & 0.41946400 & 2.27887700 \\
\hline $\mathrm{H}$ & 7.54019100 & -1.22420700 & 2.94187600 \\
\hline $\mathrm{H}$ & 5.99088000 & -0.36052500 & 2.98974200 \\
\hline $\mathrm{C}$ & 5.27818300 & 3.72738400 & -0.45144700 \\
\hline $\mathrm{C}$ & 5.14254800 & 4.11314900 & -1.94122800 \\
\hline $\mathrm{H}$ & 5.88427900 & 3.59293700 & -2.55769400 \\
\hline $\mathrm{H}$ & 5.29004500 & 5.19203900 & -2.07313000 \\
\hline $\mathrm{H}$ & 4.14963300 & 3.85566900 & -2.32602000 \\
\hline $\mathrm{C}$ & 4.20399100 & 4.50401900 & 0.35646400 \\
\hline $\mathrm{H}$ & 3.18172000 & 4.31257800 & 0.02141800 \\
\hline $\mathrm{H}$ & 4.38383300 & 5.58064000 & 0.25672400 \\
\hline $\mathrm{H}$ & 4.26058600 & 4.24644000 & 1.41851500 \\
\hline $\mathrm{C}$ & 6.63344100 & 4.23099400 & 0.08741800 \\
\hline $\mathrm{H}$ & 6.81483700 & 3.88159900 & 1.10682100 \\
\hline $\mathrm{H}$ & 6.61339400 & 5.32724200 & 0.10017500 \\
\hline $\mathrm{H}$ & 7.48208100 & 3.93399300 & -0.52785500 \\
\hline $\mathrm{C}$ & 7.97316600 & 1.56544900 & -1.13311500 \\
\hline $\mathrm{H}$ & 7.49376000 & 2.26524800 & -1.82596600 \\
\hline $\mathrm{H}$ & 7.99314100 & 0.57542000 & -1.59746200 \\
\hline $\mathrm{H}$ & 8.99464500 & 1.89750000 & -0.93160700 \\
\hline $\mathrm{C}$ & 1.36770000 & -0.13902100 & 1.61625400 \\
\hline $\mathrm{C}$ & 0.67970500 & -1.18118900 & 2.23173900 \\
\hline $\mathrm{H}$ & 0.15813500 & -1.90192100 & 1.61402300 \\
\hline $\mathrm{C}$ & 0.62629800 & -1.31178500 & 3.63503400 \\
\hline $\mathrm{C}$ & 1.26089900 & -0.31003000 & 4.39505400 \\
\hline $\mathrm{C}$ & 2.07888900 & 0.68729400 & 3.80380900 \\
\hline $\mathrm{C}$ & 2.08914600 & 0.75940700 & 2.41410100 \\
\hline $\mathrm{H}$ & 2.67848500 & 1.52024900 & 1.92674500 \\
\hline $\mathrm{C}$ & 3.05324200 & 1.55331700 & 4.63238300 \\
\hline $\mathrm{C}$ & 2.36851700 & 2.40033100 & 5.72685300 \\
\hline
\end{tabular}


$\mathrm{H}$

$\mathrm{H}$

$\mathrm{H}$

C

$\mathrm{H}$

$\mathrm{H}$

$\mathrm{H}$

C

$\mathrm{H}$

$\mathrm{H}$

$\mathrm{H}$

C

C

$\mathrm{H}$

$\mathrm{H}$

$\mathrm{H}$

C

$\mathrm{H}$

$\mathrm{H}$

$\mathrm{H}$

C

$\mathrm{H}$

$\mathrm{H}$

$\mathrm{H}$

C

$\mathrm{H}$

$\mathrm{H}$

$\mathrm{H}$

C

C

$\mathrm{H}$

C

C

C

C

$\mathrm{H}$

C

C

$\mathrm{H}$

C

C

C

C

$\mathrm{H}$
1.50241300

2.94037900

5.33724600

3.08217000

3.13510000

2.04023700

1.78275200

4.06627300

3.55795500

4.80435200

4.60311600

3.83705000

4.42787500

4.53204700

3.16482800

$-0.07284300$

$-1.57314000$

$-2.06477900$

$-2.07609100$

$-1.72462200$

0.57316400

0.40475700

0.13294500

1.65166000

0.06040400

1.10073500

$-0.33604000$

$-0.51216500$

$-0.07505700$

$-0.08004000$

$-0.12016600$

$-0.95003200$

$-2.55434100$

$-3.21792200$

$-3.39178600$

$-3.62641100$

$-3.30115400$

$-2.72697800$

$-2.32881400$

$-1.81024900$

$-3.66895600$

$-4.76166900$

$-4.63560400$

$-6.00483200$

$-6.14588800$

$-5.04261900$

$-3.81122100$

$-2.94195000$
0.60199800

$-0.08290100$

1.17855300

0.01029400

2.52465200

2.00310300

3.10698500

3.22405600

$-2.57614700$

$-2.52661700$

$-1.67015500$

$-3.43923100$

$-2.44726600$

$-3.81165500$

$-3.83267400$

$-4.72766000$

$-3.83681100$

$-2.82328200$

$-2.79297300$

$-3.82355900$

$-2.12174500$

0.31199400

1.38538000

0.14923400

$-0.14063300$

1.09806900

2.31468800

2.64826800

3.11343400

2.66579100

1.40306400

0.66158700

$-0.28286300$

$-0.72049800$

0.09341100

1.16584300

$-0.41852100$

$-1.82377500$

$-2.69133900$

$-2.10700200$

$-2.72974600$
6.11892800

6.56233200

5.30968200

5.99272000

5.88106600

4.56123700

3.72727200

2.96709300

4.34184500

3.21754900

4.19655500

3.83488500

4.31036100

4.17769500

2.75688700

3.52011600

2.44566100

3.93097400

3.71703100

5.71390000

6.04552700

5.92458800

6.32065700

6.22325500

6.01173400

7.30289900

5.74608700

$-1.15905400$

$-0.96617900$

0.04685800

$-2.03523700$

$-3.34253100$

$-3.58300800$

$-2.45869900$

$-2.59220500$

0.71768100

1.00983500

0.95864300

1.39413300

1.36138500

1.17184600

0.84357700

0.67696500 


\begin{tabular}{|c|c|c|c|}
\hline $\mathrm{C}$ & -4.53355800 & 4.33884900 & -1.78394000 \\
\hline $\mathrm{C}$ & -5.93493400 & 3.99667600 & -2.34145200 \\
\hline $\mathrm{H}$ & -5.88677400 & 3.81132200 & -3.41790400 \\
\hline $\mathrm{H}$ & -6.62831300 & 4.82838700 & -2.16409900 \\
\hline $\mathrm{H}$ & -6.34121500 & 3.10392500 & -1.85421300 \\
\hline $\mathrm{C}$ & -4.67063200 & 4.63368500 & -0.27628800 \\
\hline $\mathrm{H}$ & -5.13404400 & 3.80501800 & 0.26874400 \\
\hline $\mathrm{H}$ & -5.31319200 & 5.50965100 & -0.13788000 \\
\hline $\mathrm{H}$ & -3.69888200 & 4.84462500 & 0.18390000 \\
\hline $\mathrm{C}$ & -4.04723500 & 5.64073800 & -2.45818800 \\
\hline $\mathrm{H}$ & -2.98639400 & 5.81728600 & -2.26715200 \\
\hline $\mathrm{H}$ & -4.61162700 & 6.49203700 & -2.05967900 \\
\hline $\mathrm{H}$ & -4.19938700 & 5.61539500 & -3.53626900 \\
\hline $\mathrm{C}$ & -2.51124400 & 0.73925100 & -4.96297000 \\
\hline $\mathrm{C}$ & -3.06108800 & -0.70878600 & -4.89081100 \\
\hline $\mathrm{H}$ & -2.56137400 & -1.32198200 & -4.13828200 \\
\hline $\mathrm{H}$ & -2.92869700 & -1.19962600 & -5.86184400 \\
\hline $\mathrm{H}$ & -4.13104200 & -0.70125900 & -4.65690800 \\
\hline $\mathrm{C}$ & -0.99727300 & 0.69049800 & -5.26359700 \\
\hline $\mathrm{H}$ & -0.58615800 & 1.69798100 & -5.38918100 \\
\hline $\mathrm{H}$ & -0.80167200 & 0.12805900 & -6.18497100 \\
\hline $\mathrm{H}$ & -0.45041100 & 0.21592300 & -4.44362200 \\
\hline $\mathrm{C}$ & -3.24550900 & 1.39154200 & -6.15292400 \\
\hline $\mathrm{H}$ & -4.30688300 & 1.53847300 & -5.93802500 \\
\hline $\mathrm{H}$ & -3.16156500 & 0.72144500 & -7.01706800 \\
\hline $\mathrm{H}$ & -2.82578400 & 2.35203000 & -6.44890700 \\
\hline $\mathrm{C}$ & -2.42545800 & 4.24978300 & -4.83378300 \\
\hline $\mathrm{H}$ & -2.76922300 & 4.93222100 & -5.61510300 \\
\hline $\mathrm{H}$ & -1.65943000 & 3.58479900 & -5.24767200 \\
\hline $\mathrm{H}$ & -1.98378800 & 4.81936800 & -4.01122100 \\
\hline $\mathrm{C}$ & -7.06214500 & 0.61047600 & 1.86562100 \\
\hline $\mathrm{C}$ & -7.53969100 & 1.45351700 & 0.66374500 \\
\hline $\mathrm{H}$ & -6.69891100 & 1.94793300 & 0.16683900 \\
\hline $\mathrm{H}$ & -8.24227200 & 2.22836500 & 0.99368700 \\
\hline $\mathrm{H}$ & -8.04670600 & 0.83439700 & -0.08426700 \\
\hline $\mathrm{C}$ & -6.38519200 & 1.55532800 & 2.89406200 \\
\hline $\mathrm{H}$ & -6.02079500 & 0.98611800 & 3.75657900 \\
\hline $\mathrm{H}$ & -7.11348500 & 2.29090700 & 3.25412400 \\
\hline $\mathrm{H}$ & -5.53870100 & 2.10752600 & 2.47835600 \\
\hline $\mathrm{C}$ & -8.28860200 & 0.02209500 & 2.59368700 \\
\hline $\mathrm{H}$ & -8.97134300 & -0.50969100 & 1.93156900 \\
\hline $\mathrm{H}$ & -8.85138800 & 0.84900500 & 3.04319400 \\
\hline $\mathrm{H}$ & -7.99212100 & -0.66219500 & 3.39265900 \\
\hline $\mathrm{C}$ & -5.14831800 & -4.21293900 & 1.41772300 \\
\hline
\end{tabular}




\begin{tabular}{|c|c|c|c|}
\hline $\mathrm{C}$ & -6.14734000 & -4.90628000 & 0.46526800 \\
\hline $\mathrm{H}$ & -7.18023300 & -4.65093700 & 0.69977400 \\
\hline $\mathrm{H}$ & -6.04527200 & -5.99393300 & 0.55917300 \\
\hline $\mathrm{H}$ & -5.94266100 & -4.64306300 & -0.57907700 \\
\hline $\mathrm{C}$ & -3.79008300 & -4.91276200 & 1.22821600 \\
\hline $\mathrm{H}$ & -3.41886000 & -4.83336200 & 0.20213800 \\
\hline $\mathrm{H}$ & -3.90787800 & -5.97755700 & 1.45908600 \\
\hline $\mathrm{H}$ & -3.01651400 & -4.51382300 & 1.88794600 \\
\hline $\mathrm{C}$ & -5.59224400 & -4.44006000 & 2.88138700 \\
\hline $\mathrm{H}$ & -5.66221700 & -5.51495400 & 3.08942100 \\
\hline $\mathrm{H}$ & -6.56642500 & -3.98624200 & 3.07635100 \\
\hline $\mathrm{H}$ & -4.85991900 & -4.00915300 & 3.57382600 \\
\hline $\mathrm{C}$ & -8.20164400 & -2.33974000 & 0.35859800 \\
\hline $\mathrm{H}$ & -8.40779000 & -1.30755300 & 0.05570400 \\
\hline $\mathrm{H}$ & -9.14201600 & -2.84014800 & 0.60255600 \\
\hline $\mathrm{H}$ & -7.71564700 & -2.85814500 & -0.47527700 \\
\hline $\mathrm{C}$ & -0.03330800 & 2.44264200 & 0.05214400 \\
\hline $\mathrm{C}$ & 0.70081800 & 1.54193800 & -0.76410400 \\
\hline $\mathrm{C}$ & 0.99145800 & 1.88406600 & -2.09525500 \\
\hline $\mathrm{H}$ & 1.52880700 & 1.16524100 & -2.70324000 \\
\hline $\mathrm{C}$ & 0.67463400 & 3.13044100 & -2.65294300 \\
\hline $\mathrm{H}$ & 0.91138800 & 3.37418700 & -3.68222600 \\
\hline $\mathrm{C}$ & 0.05878500 & 4.03448200 & -1.81042400 \\
\hline $\mathrm{C}$ & -0.29353300 & 3.68552400 & -0.51155600 \\
\hline $\mathrm{C}$ & -0.70985100 & 5.84269900 & -0.78631800 \\
\hline $\mathrm{H}$ & 0.15686400 & 6.39238900 & -0.39055000 \\
\hline $\mathrm{H}$ & -1.59277900 & 6.47457100 & -0.86592300 \\
\hline $\mathrm{C}$ & -1.67112000 & 1.17198200 & 1.56146900 \\
\hline $\mathrm{C}$ & -2.30696100 & 1.07441700 & 2.80524800 \\
\hline $\mathrm{H}$ & -3.06681000 & 0.31692800 & 2.94821800 \\
\hline $\mathrm{C}$ & -2.02721500 & 1.94459000 & 3.87408900 \\
\hline $\mathrm{H}$ & -2.55340400 & 1.86895700 & 4.81881700 \\
\hline $\mathrm{C}$ & -1.05998300 & 2.90108000 & 3.65655400 \\
\hline $\mathrm{C}$ & -0.38956300 & 2.98703100 & 2.44242700 \\
\hline $\mathrm{C}$ & -0.65704900 & 2.15908300 & 1.36871700 \\
\hline $\mathrm{C}$ & 0.23436200 & 4.71317200 & 3.69843700 \\
\hline $\mathrm{H}$ & 1.15179300 & 4.96748100 & 4.2303740 \\
\hline $\mathrm{H}$ & -0.34560600 & 5.60819100 & 3.43063500 \\
\hline $\mathrm{C}$ & 0.07855900 & -2.94951100 & -4.39482900 \\
\hline $\mathrm{C}$ & -0.45721100 & -4.14932600 & -4.70983000 \\
\hline $\mathrm{H}$ & -0.44372200 & -2.04475600 & -4.69796500 \\
\hline $\mathrm{H}$ & 0.09239900 & -5.04949200 & -4.42756500 \\
\hline $\mathrm{C}$ & 1.33003100 & -2.77289500 & -3.66938800 \\
\hline $\mathrm{C}$ & 2.03233300 & -1.55435200 & -3.61331500 \\
\hline
\end{tabular}




\begin{tabular}{lccc}
$\mathrm{H}$ & 1.86692800 & -3.69226700 & -3.44287000 \\
$\mathrm{H}$ & 1.58569800 & -0.69852500 & -4.11669100 \\
$\mathrm{C}$ & 3.47775300 & -1.43619500 & -3.36398900 \\
$\mathrm{C}$ & 4.26135300 & -2.50688700 & -2.89291400 \\
$\mathrm{C}$ & 4.12086300 & -0.21692800 & -3.63521200 \\
$\mathrm{C}$ & 5.63932400 & -2.37424400 & -2.76421900 \\
$\mathrm{H}$ & 3.79107400 & -3.44813000 & -2.62647200 \\
$\mathrm{C}$ & 5.49872200 & -0.07974700 & -3.49096600 \\
$\mathrm{H}$ & 3.52842200 & 0.62817000 & -3.97692100 \\
$\mathrm{C}$ & 6.26869200 & -1.16615600 & -3.07756200 \\
$\mathrm{H}$ & 6.22782200 & -3.22002300 & -2.41880500 \\
$\mathrm{H}$ & 5.97051200 & 0.87322100 & -3.71279600 \\
$\mathrm{H}$ & 7.34737500 & -1.07619100 & -2.99915600 \\
$\mathrm{C}$ & -1.73562000 & -4.35999500 & -5.46680900 \\
$\mathrm{H}$ & -2.45063400 & -4.96277900 & -4.88984400 \\
$\mathrm{H}$ & -2.21758600 & -3.40930900 & -5.71215700 \\
$\mathrm{H}$ & -1.55568600 & -4.90222200 & -6.40506700 \\
$\mathrm{~S}$ & -0.19289300 & -3.63080900 & -0.68182600 \\
$\mathrm{C}$ & 1.22564200 & -4.75102700 & -0.59528900 \\
$\mathrm{C}$ & 1.34049700 & -5.87379500 & -1.41215000 \\
$\mathrm{C}$ & 2.26179100 & -4.36670300 & 0.26243400 \\
$\mathrm{C}$ & 2.51692700 & -6.62556500 & -1.36925600 \\
$\mathrm{H}$ & 0.51882900 & -6.15028600 & -2.06207800 \\
$\mathrm{C}$ & 3.42675500 & -5.12995900 & 0.30373000 \\
$\mathrm{H}$ & 2.15946800 & -3.48251400 & 0.88335000 \\
$\mathrm{C}$ & 3.55771800 & -6.25458700 & -0.51612900 \\
$\mathrm{H}$ & 2.61681300 & -7.50328900 & -2.00139100 \\
$\mathrm{H}$ & 4.23151500 & -4.84502900 & 0.97309700 \\
$\mathrm{H}$ & 4.47067800 & -6.84223200 & -0.48712000 \\
$\mathrm{O}$ & -1.13646300 & -4.48215400 & -1.76193800 \\
$\mathrm{O}$ & -0.90491200 & -3.72698500 & 0.62664800 \\
$\mathrm{H}$ & -1.02357200 & -4.05411800 & -2.64664100 \\
& & & \\
\hline & & & \\
$\mathrm{H}$ & &
\end{tabular}

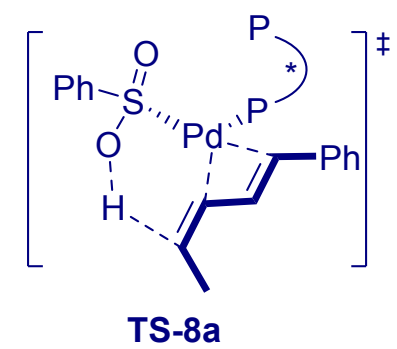

Total SCF energy (M06/SDD-6-311++G(d,p)/SMD(1,4-Dioxane)): -5497.833163a.u.

Thermal correction to Gibbs Free Energy at $298.15 \mathrm{~K}: 1.765712 \mathrm{a} . \mathrm{u}$

Gibbs free energy at 298.15 K (M06/SDD-6-311++G(d,p)/SMD(1,4-Dioxane)): -5496.067451a.u. Pd

$\begin{array}{lll}-1.08277400 & -0.85960100 \quad 2.61602200\end{array}$ 
P

$P$

O

O

O

O

O

O

O

O

C

C

$\mathrm{H}$

C

C

C

C

$\mathrm{H}$

C

C

$\mathrm{H}$

$\mathrm{H}$

$\mathrm{H}$

C

$\mathrm{H}$

$\mathrm{H}$

$\mathrm{H}$

C

$\mathrm{H}$

$\mathrm{H}$

$\mathrm{H}$

C

C

$\mathrm{H}$

$\mathrm{H}$

$\mathrm{H}$

C

$\mathrm{H}$

$\mathrm{H}$

$\mathrm{H}$

C

$\mathrm{H}$

$\mathrm{H}$

$\mathrm{H}$

\begin{tabular}{ccc}
-1.70832500 & -0.23283100 & 0.45204100 \\
1.89606700 & -0.20369900 & -0.38765000 \\
-7.50664000 & 1.34417000 & 0.65343100 \\
-2.57837900 & -4.66351500 & -3.48658100 \\
3.24298400 & 5.65446700 & -0.43136500 \\
7.45248300 & -2.46039100 & -0.07738800 \\
-0.57892200 & 4.89899400 & -2.21432000 \\
-0.17156900 & 3.01313100 & -3.46147100 \\
-1.66178200 & 0.49777100 & -4.28318300 \\
--0.84607300 & -1.18098800 & -5.63665200 \\
-3.47677500 & 0.30557600 & 0.45108800 \\
-4.41076400 & -0.56682900 & 1.01973500 \\
-4.04592900 & -1.49463100 & 1.44190800 \\
-5.77361400 & -0.27560300 & 1.04454800 \\
-6.18584000 & 0.96311700 & 0.47916200 \\
-5.30051200 & 1.77884200 & -0.25238300 \\
-3.93956100 & 1.43883200 & -0.20238800 \\
-3.23093800 & 2.07715800 & -0.70698000 \\
-6.78173900 & -1.31324500 & 1.57996700 \\
-6.07548500 & -2.61951900 & 1.99437800 \\
-5.51484800 & -3.06520600 & 1.16606400 \\
-6.82906700 & -3.34640800 & 2.31462700 \\
-5.38745300 & -2.46814800 & 2.83488900 \\
-7.55361800 & -0.80094200 & 2.81503400 \\
-6.86570100 & -0.42696900 & 3.58284800 \\
-8.13147200 & -1.62168700 & 3.25592300 \\
-8.25360800 & -0.00802200 & 2.55341400 \\
-7.77926900 & -1.66331100 & 0.45186000 \\
-8.33412300 & -0.78092300 & 0.12769700 \\
-8.49608300 & -2.41479200 & 0.80431100 \\
-7.25049300 & -2.07990500 & -0.41315400 \\
-5.67213200 & 2.98178200 & -1.15431300 \\
-5.15082700 & 4.29082300 & -0.52309500 \\
-5.65825400 & 4.50688900 & 0.42348600 \\
-5.33193300 & 5.13579300 & -1.19830000 \\
-4.07567200 & 4.23821800 & -0.32224200 \\
-4.97488800 & 2.79028800 & -2.52824000 \\
-3.88477000 & 2.75416000 & -2.45756000 \\
-5.23498300 & 3.62664300 & -3.18722700 \\
-5.30889900 & 1.86357700 & -3.00510200 \\
-7.17448300 & 3.12891700 & -1.47020900 \\
-7.60070300 & 2.19131700 & -1.83653700 \\
-7.29035800 & 3.88563000 & -2.25505300 \\
-7.76651300 & 3.45660400 & -0.61590400 \\
\hline & &
\end{tabular}




\begin{tabular}{|c|c|c|c|}
\hline $\mathrm{C}$ & -7.67104900 & 2.33283800 & 1.66798700 \\
\hline $\mathrm{H}$ & -7.15453100 & 3.26389400 & 1.41071600 \\
\hline $\mathrm{H}$ & -7.29138800 & 1.97677400 & 2.63278700 \\
\hline $\mathrm{H}$ & -8.74406300 & 2.52368700 & 1.74507200 \\
\hline $\mathrm{C}$ & -1.87289700 & -1.55531600 & -0.81181900 \\
\hline $\mathrm{C}$ & -1.23411100 & -2.77694000 & -0.63096700 \\
\hline $\mathrm{H}$ & -0.51888400 & -2.88101600 & 0.17500200 \\
\hline $\mathrm{C}$ & -1.50095600 & -3.87906700 & -1.46532700 \\
\hline $\mathrm{C}$ & -2.38380900 & -3.67072900 & -2.54186600 \\
\hline $\mathrm{C}$ & -3.13156800 & -2.47386000 & -2.69049500 \\
\hline $\mathrm{C}$ & -2.83589800 & -1.42859300 & -1.82251100 \\
\hline $\mathrm{H}$ & -3.37443800 & -0.49862300 & -1.90928500 \\
\hline $\mathrm{C}$ & -4.35827200 & -2.37790500 & -3.62357500 \\
\hline $\mathrm{C}$ & -4.06131600 & -2.72594400 & -5.09884600 \\
\hline $\mathrm{H}$ & -3.16994900 & -2.20872600 & -5.46192900 \\
\hline $\mathrm{H}$ & -4.91066100 & -2.42701400 & -5.72478200 \\
\hline $\mathrm{H}$ & -3.90908700 & -3.79629000 & -5.23245600 \\
\hline $\mathrm{C}$ & -5.42042400 & -3.36533600 & -3.08584300 \\
\hline $\mathrm{H}$ & -5.04252200 & -4.39093800 & -3.10538600 \\
\hline $\mathrm{H}$ & -6.32902200 & -3.31909600 & -3.69925600 \\
\hline $\mathrm{H}$ & -5.69181500 & -3.11431000 & -2.05380800 \\
\hline $\mathrm{C}$ & -4.97041600 & -0.96273400 & -3.59377600 \\
\hline $\mathrm{H}$ & -5.32596900 & -0.68813000 & -2.59496400 \\
\hline $\mathrm{H}$ & -5.83214000 & -0.92826700 & -4.26910600 \\
\hline $\mathrm{H}$ & -4.24966500 & -0.20397400 & -3.91770200 \\
\hline $\mathrm{C}$ & -0.84330100 & -5.22371600 & -1.07794700 \\
\hline $\mathrm{C}$ & 0.67137800 & -5.13299400 & -1.34633400 \\
\hline $\mathrm{H}$ & 0.88257500 & -4.97275500 & -2.41015600 \\
\hline $\mathrm{H}$ & 1.16819500 & -6.05955400 & -1.03625100 \\
\hline $\mathrm{H}$ & 1.11074100 & -4.30959000 & -0.78104300 \\
\hline $\mathrm{C}$ & -1.05988900 & -5.44978800 & 0.44228600 \\
\hline $\mathrm{H}$ & -0.54929400 & -4.70499200 & 1.05287900 \\
\hline $\mathrm{H}$ & -0.65574800 & -6.42857600 & 0.72463400 \\
\hline $\mathrm{H}$ & -2.12910700 & -5.44101400 & 0.68503700 \\
\hline $\mathrm{C}$ & -1.41395800 & -6.47683700 & -1.77375500 \\
\hline $\mathrm{H}$ & -2.50256500 & -6.53010000 & -1.68699000 \\
\hline $\mathrm{H}$ & -0.98993700 & -7.35959200 & -1.28082200 \\
\hline $\mathrm{H}$ & -1.15618700 & -6.54400000 & -2.83054000 \\
\hline $\mathrm{C}$ & -1.55823000 & -4.67917700 & -4.48675700 \\
\hline $\mathrm{H}$ & -1.51699400 & -3.72694100 & -5.02502300 \\
\hline $\mathrm{H}$ & -1.81755300 & -5.48383600 & -5.17907900 \\
\hline $\mathrm{H}$ & -0.57481600 & -4.87872100 & -4.04812000 \\
\hline $\mathrm{C}$ & 2.32251600 & 1.59011700 & -0.48748900 \\
\hline $\mathrm{C}$ & 2.61721900 & 2.27072600 & -1.6760990 \\
\hline
\end{tabular}




\begin{tabular}{|c|c|c|c|}
\hline $\mathrm{H}$ & 2.54682800 & 1.72377000 & -2.60658000 \\
\hline $\mathrm{C}$ & 3.00212200 & 3.61335200 & -1.68222100 \\
\hline $\mathrm{C}$ & 3.04058100 & 4.28291600 & -0.43128700 \\
\hline $\mathrm{C}$ & 2.89485200 & 3.60092800 & 0.79013700 \\
\hline $\mathrm{C}$ & 2.50014600 & 2.25370200 & 0.72126000 \\
\hline $\mathrm{H}$ & 2.34447000 & 1.69456200 & 1.63858500 \\
\hline $\mathrm{C}$ & 3.59477800 & -0.92647700 & -0.55050900 \\
\hline $\mathrm{C}$ & 4.71237700 & -0.12744400 & -0.77501200 \\
\hline $\mathrm{H}$ & 4.56749800 & 0.92059200 & -0.99097800 \\
\hline $\mathrm{C}$ & 6.02367700 & -0.61859300 & -0.72561300 \\
\hline $\mathrm{C}$ & 6.18405400 & -1.96618000 & -0.35080900 \\
\hline $\mathrm{C}$ & 5.08126300 & -2.85146600 & -0.25127300 \\
\hline $\mathrm{C}$ & 3.79826300 & -2.29840100 & -0.33988900 \\
\hline $\mathrm{H}$ & 2.93287100 & -2.92502200 & -0.17994300 \\
\hline $\mathrm{C}$ & 3.53339400 & 4.27442300 & -2.97365200 \\
\hline $\mathrm{C}$ & 5.04160000 & 4.55122700 & -2.76840200 \\
\hline $\mathrm{H}$ & 5.19811900 & 5.23835600 & -1.93267400 \\
\hline $\mathrm{H}$ & 5.46917000 & 5.00377200 & -3.67190400 \\
\hline $\mathrm{H}$ & 5.58651800 & 3.62486900 & -2.56274900 \\
\hline $\mathrm{C}$ & 3.37414000 & 3.33546400 & -4.18545600 \\
\hline $\mathrm{H}$ & 3.93285200 & 2.40225500 & -4.06028700 \\
\hline $\mathrm{H}$ & 3.76618400 & 3.83194000 & -5.07957100 \\
\hline $\mathrm{H}$ & 2.32333200 & 3.08370700 & -4.36523400 \\
\hline $\mathrm{C}$ & 2.84533000 & 5.61077900 & -3.32919700 \\
\hline $\mathrm{H}$ & 1.75896400 & 5.52937200 & -3.26441600 \\
\hline $\mathrm{H}$ & 3.10662000 & 5.89639300 & -4.35499200 \\
\hline $\mathrm{H}$ & 3.16524300 & 6.41386400 & -2.66630200 \\
\hline $\mathrm{C}$ & 3.24352900 & 4.14366500 & 2.19658400 \\
\hline $\mathrm{C}$ & 4.43891100 & 3.29654300 & 2.70023800 \\
\hline $\mathrm{H}$ & 4.23536800 & 2.22682500 & 2.65840400 \\
\hline $\mathrm{H}$ & 4.68102200 & 3.56012500 & 3.73773200 \\
\hline $\mathrm{H}$ & 5.32410300 & 3.49241100 & 2.08434200 \\
\hline $\mathrm{C}$ & 2.02986100 & 3.96228700 & 3.13437300 \\
\hline $\mathrm{H}$ & 1.17375000 & 4.54018600 & 2.76936700 \\
\hline $\mathrm{H}$ & 2.27509600 & 4.31585000 & 4.14370800 \\
\hline $\mathrm{H}$ & 1.71084300 & 2.91941900 & 3.21077700 \\
\hline $\mathrm{C}$ & 3.69868500 & 5.61275500 & 2.29843000 \\
\hline $\mathrm{H}$ & 4.52298700 & 5.83605200 & 1.61991900 \\
\hline $\mathrm{H}$ & 4.04096700 & 5.78832400 & 3.32566800 \\
\hline $\mathrm{H}$ & 2.89300800 & 6.32346700 & 2.10481300 \\
\hline $\mathrm{C}$ & 2.01863100 & 6.38054100 & -0.30303500 \\
\hline $\mathrm{H}$ & 2.28873900 & 7.43795000 & -0.24909300 \\
\hline $\mathrm{H}$ & 1.48312100 & 6.09337900 & 0.60926000 \\
\hline $\mathrm{H}$ & 1.36023600 & 6.20483600 & -1.15860100 \\
\hline
\end{tabular}




\begin{tabular}{|c|c|c|c|}
\hline $\mathrm{C}$ & 7.13137400 & 0.37452200 & -1.15496200 \\
\hline $\mathrm{C}$ & 7.17173500 & 1.56411100 & -0.16952800 \\
\hline $\mathrm{H}$ & 6.20784600 & 2.08038700 & -0.11434700 \\
\hline $\mathrm{H}$ & 7.92783900 & 2.29328700 & -0.48482800 \\
\hline $\mathrm{H}$ & 7.42619600 & 1.22891800 & 0.8426790 \\
\hline $\mathrm{C}$ & 6.75654200 & 0.89955600 & -2.56548200 \\
\hline $\mathrm{H}$ & 6.75062700 & 0.07815200 & -3.29051300 \\
\hline $\mathrm{H}$ & 7.49208700 & 1.64237300 & -2.89583100 \\
\hline $\mathrm{H}$ & 5.77184900 & 1.37124400 & -2.58874700 \\
\hline $\mathrm{C}$ & 8.55376100 & -0.20442900 & -1.29416800 \\
\hline $\mathrm{H}$ & 9.01935400 & -0.43679400 & -0.33572400 \\
\hline $\mathrm{H}$ & 9.18359100 & 0.54937800 & -1.78170400 \\
\hline $\mathrm{H}$ & 8.56654500 & -1.10837300 & -1.90700200 \\
\hline $\mathrm{C}$ & 5.26994000 & -4.37778900 & -0.10932600 \\
\hline $\mathrm{C}$ & 5.94683600 & -4.77573500 & 1.22056200 \\
\hline $\mathrm{H}$ & 6.99896000 & -4.49275000 & 1.23933000 \\
\hline $\mathrm{H}$ & 5.89491000 & -5.86349900 & 1.34670900 \\
\hline $\mathrm{H}$ & 5.44067600 & -4.32135300 & 2.08008400 \\
\hline $\mathrm{C}$ & 3.91967600 & -5.11305300 & -0.17284200 \\
\hline $\mathrm{H}$ & 3.24684400 & -4.84024700 & 0.64632500 \\
\hline $\mathrm{H}$ & 4.09749000 & -6.19268000 & -0.11608000 \\
\hline $\mathrm{H}$ & 3.40258900 & -4.91343100 & -1.11543700 \\
\hline $\mathrm{C}$ & 6.12956900 & -4.88596400 & -1.28971100 \\
\hline $\mathrm{H}$ & 6.25554200 & -5.97350300 & -1.21998100 \\
\hline $\mathrm{H}$ & 7.11768400 & -4.42209500 & -1.29111100 \\
\hline $\mathrm{H}$ & 5.63853700 & -4.66387200 & -2.24418200 \\
\hline $\mathrm{C}$ & 7.93645800 & -2.08702400 & 1.20879600 \\
\hline $\mathrm{H}$ & 7.87406400 & -1.00468200 & 1.36573500 \\
\hline $\mathrm{H}$ & 8.98194200 & -2.40191800 & 1.25393000 \\
\hline $\mathrm{H}$ & 7.37317300 & -2.58869600 & 2.00441700 \\
\hline $\mathrm{C}$ & -0.53976100 & 1.35917700 & -1.65011700 \\
\hline $\mathrm{C}$ & -0.94911600 & 1.26777200 & -0.29114000 \\
\hline $\mathrm{C}$ & -1.07681800 & 2.42586400 & 0.48813100 \\
\hline $\mathrm{H}$ & -1.36073100 & 2.33251200 & 1.52891700 \\
\hline $\mathrm{C}$ & -0.94279000 & 3.71564800 & -0.05141100 \\
\hline $\mathrm{H}$ & -1.05833200 & 4.59883200 & 0.56550800 \\
\hline $\mathrm{C}$ & -0.71807700 & 3.78742200 & -1.41173700 \\
\hline $\mathrm{C}$ & -0.50007700 & 2.64016900 & -2.17477400 \\
\hline $\mathrm{C}$ & -0.61574700 & 4.36524500 & -3.54633200 \\
\hline $\mathrm{H}$ & -1.65347500 & 4.38761700 & -3.91097100 \\
\hline $\mathrm{H}$ & 0.05623700 & 4.93155000 & -4.18776600 \\
\hline $\mathrm{C}$ & 1.12932800 & -0.52233600 & -2.05041300 \\
\hline $\mathrm{C}$ & 1.60074100 & -1.54884300 & -2.87543400 \\
\hline $\mathrm{H}$ & 2.47495700 & -2.11102900 & -2.56865400 \\
\hline
\end{tabular}




\begin{tabular}{|c|c|c|c|}
\hline $\mathrm{C}$ & 1.00197900 & -1.86771300 & -4.10874500 \\
\hline $\mathrm{H}$ & 1.38500300 & -2.66585800 & -4.73473800 \\
\hline $\mathrm{C}$ & -0.07429000 & -1.09799800 & -4.49395900 \\
\hline $\mathrm{C}$ & -0.56286000 & -0.07924200 & -3.68289500 \\
\hline $\mathrm{C}$ & -0.02014300 & 0.22728300 & -2.45108200 \\
\hline $\mathrm{C}$ & -1.61675000 & 0.02752100 & -5.63140500 \\
\hline $\mathrm{H}$ & -2.62867300 & -0.18432300 & -5.97507300 \\
\hline $\mathrm{H}$ & -1.11762000 & 0.77734200 & -6.26238100 \\
\hline $\mathrm{C}$ & -1.93857400 & -1.97183100 & 4.96113500 \\
\hline $\mathrm{C}$ & -1.05908200 & -2.81866200 & 5.65471700 \\
\hline $\mathrm{H}$ & -2.77225900 & -2.44239000 & 4.43743800 \\
\hline $\mathrm{C}$ & -1.79589000 & -0.57343900 & 4.77409300 \\
\hline $\mathrm{C}$ & -2.60932200 & 0.15060300 & 3.85821900 \\
\hline $\mathrm{H}$ & -1.06302600 & -0.04742000 & 5.37817700 \\
\hline $\mathrm{H}$ & -3.52700700 & -0.32651400 & 3.52060900 \\
\hline $\mathrm{C}$ & -2.60174800 & 1.61531700 & 3.73007300 \\
\hline $\mathrm{C}$ & -1.52173900 & 2.41089700 & 4.15956100 \\
\hline $\mathrm{C}$ & -3.69170200 & 2.25548900 & 3.11336100 \\
\hline $\mathrm{C}$ & -1.53363800 & 3.79082300 & 3.97681400 \\
\hline $\mathrm{H}$ & -0.65571000 & 1.94465400 & 4.61880400 \\
\hline $\mathrm{C}$ & -3.69769300 & 3.63478900 & 2.92257800 \\
\hline $\mathrm{H}$ & -4.52736400 & 1.65774800 & 2.76533800 \\
\hline $\mathrm{C}$ & -2.61970100 & 4.41137400 & 3.35128100 \\
\hline $\mathrm{H}$ & -0.68940900 & 4.38259400 & 4.31682500 \\
\hline $\mathrm{H}$ & -4.54575700 & 4.10240700 & 2.43296900 \\
\hline $\mathrm{H}$ & -2.62567800 & 5.48776000 & 3.20496100 \\
\hline $\mathrm{S}$ & 0.86657500 & -2.38659900 & 2.62813700 \\
\hline $\mathrm{C}$ & 2.29987800 & -1.41557400 & 3.14482800 \\
\hline $\mathrm{C}$ & 2.09488200 & -0.24671100 & 3.87771100 \\
\hline $\mathrm{C}$ & 3.58130700 & -1.88098600 & 2.86784400 \\
\hline $\mathrm{C}$ & 3.19764300 & 0.45514100 & 4.3632580 \\
\hline $\mathrm{H}$ & 1.08259400 & 0.10748000 & 4.0546210 \\
\hline $\mathrm{C}$ & 4.67992700 & -1.16256200 & 3.33751300 \\
\hline $\mathrm{H}$ & 3.70457500 & -2.78100700 & 2.28076700 \\
\hline $\mathrm{C}$ & 4.48977600 & -0.00414900 & 4.09321500 \\
\hline $\mathrm{H}$ & 3.05163700 & 1.36754200 & 4.9334990 \\
\hline $\mathrm{H}$ & 5.68358100 & -1.50730500 & 3.11100700 \\
\hline $\mathrm{H}$ & 5.34749100 & 0.55266600 & 4.4591320 \\
\hline $\mathrm{O}$ & 1.30004900 & -3.16871700 & 1.42082400 \\
\hline $\mathrm{O}$ & 0.75813800 & -3.37248600 & 3.85766100 \\
\hline $\mathrm{H}$ & -0.22863300 & -3.14552600 & 4.60335100 \\
\hline $\mathrm{H}$ & -1.46624500 & -3.79965600 & 5.90589200 \\
\hline $\mathrm{C}$ & -0.03189700 & -2.25883500 & 6.62530300 \\
\hline $\mathrm{H}$ & 0.64042300 & -3.05171000 & 6.96508700 \\
\hline
\end{tabular}




$$
\begin{array}{lrrr}
\mathrm{H} & 0.59327700 & -1.49707100 & 6.14533500 \\
\mathrm{H} & -0.49943100 & -1.80592100 & 7.50991300
\end{array}
$$

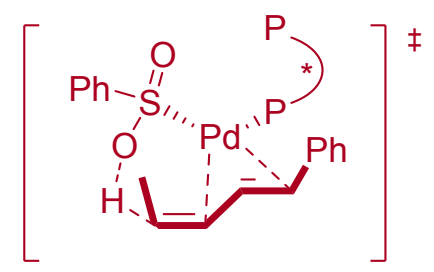

TS-8b

Total SCF energy (M06/SDD-6-311++G(d,p)/SMD(1,4-Dioxane)): -5497.824806a.u.

Thermal correction to Gibbs Free Energy at $298.15 \mathrm{~K}: 1.763972 \mathrm{a} . \mathrm{u}$

Gibbs free energy at 298.15 K (M06/SDD-6-311++G(d,p)/SMD(1,4-Dioxane)): -5496.060834a.u.

$\mathrm{Pd}$

$\mathrm{P}$

$P$

$\mathrm{O}$

$\mathrm{O}$

$\mathrm{O}$

$\mathrm{O}$

$\mathrm{O}$

$\mathrm{O}$

$\mathrm{O}$

$\mathrm{O}$

$\mathrm{C}$

$\mathrm{C}$

$\mathrm{H}$

$\mathrm{C}$

C

C

C

$\mathrm{H}$

C

C

$\mathrm{H}$

$\mathrm{H}$

$\mathrm{H}$

$\mathrm{C}$

$\mathrm{H}$

$\mathrm{H}$

$\mathrm{H}$

C

$\mathrm{H}$

$\mathrm{H}$

$\mathrm{H}$

$$
-0.99401300
$$$$
-1.49348500
$$

2.06726300

$-7.27770900$

$-1.18468500$

3.60704300

7.45357100

0.40984300

1.01564800

$-0.56359400$

0.55945300

$-3.26995300$

$-4.21520000$

$-3.87658200$

$-5.55042300$

$-5.94206700$

$-5.00740200$

$-3.68058300$

$-2.93641700$

$-6.49803500$

$-5.81195500$

$-4.92549700$

$-6.51172900$

$-5.51472900$

$-7.80691000$

$-7.60748900$

$-8.32784600$

$-8.48064200$

$-6.84379000$

$-7.34310700$

$-7.50807900$

$-5.93477300$
$1.49344700-1.99388600$

$-0.00605000 \quad-0.26133100$

$\begin{array}{ll}0.05393900 & 0.14040200\end{array}$

$\begin{array}{ll}-1.39073000 & 0.52310500\end{array}$

$1.23164200 \quad 5.61070300$

$-4.19841600 \quad-3.83039300$

$2.56108000 \quad 1.05110200$

$-5.58255200-1.17533500$

$-4.63369100 \quad 0.83140400$

$-3.47034300 \quad 3.11801000$

$-2.99544400 \quad 5.07754400$

$-0.50727700 \quad-0.25959500$

$0.50649700 \quad-0.09623000$

$1.53173100-0.15975800$

$0.22865500 \quad 0.19842200$

$-1.13591900$

0.25176500

0.12289200

$-0.14911400$

$-0.22532100$

0.60718700

0.44839900

1.08514400

0.74945000

$-0.58852800$

$-0.20940500$

$\begin{array}{ll}1.38495700 & -1.28261800 \\ 2.37362800 & -0.00273400\end{array}$

$0.62013500 \quad 0.05884000$

$1.19853300 \quad 2.10301000$

$0.24293600 \quad 2.27739600$

$2.00497700 \quad 2.43777100$

$1.23252200 \quad 2.71261300$ 


\begin{tabular}{|c|c|c|c|}
\hline $\mathrm{C}$ & -5.31235800 & -3.69607300 & 0.29509000 \\
\hline $\mathrm{C}$ & -5.77114400 & -4.29048100 & -1.05637100 \\
\hline $\mathrm{H}$ & -6.68793200 & -3.83133900 & -1.42972700 \\
\hline $\mathrm{H}$ & -5.95004100 & -5.36821300 & -0.95729500 \\
\hline $\mathrm{H}$ & -4.99320700 & -4.14383200 & -1.81454100 \\
\hline $\mathrm{C}$ & -4.03725300 & -4.47554600 & 0.70879100 \\
\hline $\mathrm{H}$ & -3.29364300 & -4.52971300 & -0.09253100 \\
\hline $\mathrm{H}$ & -4.31743300 & -5.50566700 & 0.95425900 \\
\hline $\mathrm{H}$ & -3.55773900 & -4.03480100 & 1.58894400 \\
\hline $\mathrm{C}$ & -6.34631400 & -3.97136600 & 1.40817800 \\
\hline $\mathrm{H}$ & -5.98868700 & -3.56882500 & 2.36242000 \\
\hline $\mathrm{H}$ & -6.47309900 & -5.05393600 & 1.52481400 \\
\hline $\mathrm{H}$ & -7.32109200 & -3.52991500 & 1.21579800 \\
\hline $\mathrm{C}$ & -8.09827900 & -1.79973100 & -0.56810000 \\
\hline $\mathrm{H}$ & -8.25805500 & -2.88295500 & -0.54912600 \\
\hline $\mathrm{H}$ & -7.65332000 & -1.51222900 & -1.52448900 \\
\hline $\mathrm{H}$ & -9.06799500 & -1.30558800 & -0.46007100 \\
\hline $\mathrm{C}$ & -1.36801600 & 0.40579100 & 1.53153500 \\
\hline $\mathrm{C}$ & -0.66724900 & 1.52174500 & 1.97875300 \\
\hline $\mathrm{H}$ & -0.12245100 & 2.12436200 & 1.26258200 \\
\hline $\mathrm{C}$ & -0.63251700 & 1.87698300 & 3.34308800 \\
\hline $\mathrm{C}$ & -1.29048500 & 1.01961700 & 4.24669500 \\
\hline $\mathrm{C}$ & -2.11167100 & -0.05403600 & 3.81330800 \\
\hline $\mathrm{C}$ & -2.11085200 & -0.34519900 & 2.45329500 \\
\hline $\mathrm{H}$ & -2.70682400 & -1.16630500 & 2.08906600 \\
\hline $\mathrm{C}$ & -3.10084500 & -0.77575800 & 4.75538400 \\
\hline $\mathrm{C}$ & -2.43798300 & -1.41526400 & 5.99478900 \\
\hline $\mathrm{H}$ & -1.55683800 & -2.00192700 & 5.72476300 \\
\hline $\mathrm{H}$ & -3.15530300 & -2.08176800 & 6.48883000 \\
\hline $\mathrm{H}$ & -2.13668900 & -0.65986100 & 6.71978400 \\
\hline $\mathrm{C}$ & -4.13518700 & 0.26763800 & 5.23590400 \\
\hline $\mathrm{H}$ & -3.64495500 & 1.07280300 & 5.78891200 \\
\hline $\mathrm{H}$ & -4.87421800 & -0.20583800 & 5.89428800 \\
\hline $\mathrm{H}$ & -4.66901500 & 0.70383300 & 4.38535200 \\
\hline $\mathrm{C}$ & -3.85940700 & -1.89543000 & 4.01330300 \\
\hline $\mathrm{H}$ & -4.44252500 & -1.51523400 & 3.16846200 \\
\hline $\mathrm{H}$ & -4.55946600 & -2.37497000 & 4.70606400 \\
\hline $\mathrm{H}$ & -3.17234600 & -2.66286300 & 3.63832000 \\
\hline $\mathrm{C}$ & 0.07754400 & 3.20837400 & 3.6987190 \\
\hline $\mathrm{C}$ & 1.58566400 & 3.07596100 & 3.3923150 \\
\hline $\mathrm{H}$ & 2.05033300 & 2.30844700 & 4.0220240 \\
\hline $\mathrm{H}$ & 2.09539600 & 4.02765600 & 3.5871230 \\
\hline $\mathrm{H}$ & 1.76123600 & 2.81123600 & 2.3479410 \\
\hline $\mathrm{C}$ & -0.52593100 & 4.31860000 & 2.80113900 \\
\hline
\end{tabular}




\begin{tabular}{|c|c|c|c|}
\hline $\mathrm{H}$ & -0.32858600 & 4.15041500 & 1.74480600 \\
\hline $\mathrm{H}$ & -0.07837100 & 5.28451400 & 3.06281300 \\
\hline $\mathrm{H}$ & -1.60865700 & 4.39414600 & 2.95657700 \\
\hline $\mathrm{C}$ & -0.09215300 & 3.71267600 & 5.14716900 \\
\hline $\mathrm{H}$ & -1.14170000 & 3.75006300 & 5.44838000 \\
\hline $\mathrm{H}$ & 0.31262300 & 4.73043500 & 5.19550400 \\
\hline $\mathrm{H}$ & 0.45288200 & 3.12004700 & 5.88200900 \\
\hline $\mathrm{C}$ & 0.01550700 & 0.70063700 & 6.17236100 \\
\hline $\mathrm{H}$ & 0.02049900 & -0.39280200 & 6.13473500 \\
\hline $\mathrm{H}$ & 0.04232100 & 1.03325600 & 7.21290200 \\
\hline $\mathrm{H}$ & 0.89980900 & 1.07163900 & 5.64497200 \\
\hline $\mathrm{C}$ & 2.57903700 & -1.29778500 & -1.01071800 \\
\hline $\mathrm{C}$ & 3.23675500 & -2.47107700 & -0.62636400 \\
\hline $\mathrm{H}$ & 3.40797100 & -2.64056600 & 0.42715300 \\
\hline $\mathrm{C}$ & 3.64572400 & -3.42859400 & -1.55605700 \\
\hline $\mathrm{C}$ & 3.32770700 & -3.19081600 & -2.91926600 \\
\hline $\mathrm{C}$ & 2.75803700 & -1.98006600 & -3.35813900 \\
\hline $\mathrm{C}$ & 2.35849400 & -1.07229700 & -2.36409100 \\
\hline $\mathrm{H}$ & 1.84620700 & -0.15749700 & -2.64384600 \\
\hline $\mathrm{C}$ & 3.71089500 & 0.80157100 & 0.52966700 \\
\hline $\mathrm{C}$ & 4.79948400 & 0.03829500 & 0.94793700 \\
\hline $\mathrm{H}$ & 4.66699400 & -1.02723400 & 1.07424000 \\
\hline $\mathrm{C}$ & 6.04794500 & 0.59840300 & 1.23715900 \\
\hline $\mathrm{C}$ & 6.19681900 & 1.97926000 & 0.97651200 \\
\hline $\mathrm{C}$ & 5.09749500 & 2.81039100 & 0.65222600 \\
\hline $\mathrm{C}$ & 3.86200600 & 2.18848300 & 0.42691600 \\
\hline $\mathrm{H}$ & 2.99465200 & 2.77829600 & 0.16392100 \\
\hline $\mathrm{C}$ & 4.54547400 & -4.60414400 & -1.11262500 \\
\hline $\mathrm{C}$ & 5.94762200 & -4.36941500 & -1.72141200 \\
\hline $\mathrm{H}$ & 5.89733500 & -4.35982800 & -2.81342400 \\
\hline $\mathrm{H}$ & 6.63412300 & -5.16800300 & -1.41328900 \\
\hline $\mathrm{H}$ & 6.36346000 & -3.41371200 & -1.38520300 \\
\hline $\mathrm{C}$ & 4.68803200 & -4.65131800 & 0.42239000 \\
\hline $\mathrm{H}$ & 5.15789700 & -3.74755500 & 0.82342900 \\
\hline $\mathrm{H}$ & 5.32706100 & -5.49676600 & 0.69840300 \\
\hline $\mathrm{H}$ & 3.71806900 & -4.77902800 & 0.91579400 \\
\hline $\mathrm{C}$ & 4.04431600 & -5.99362800 & -1.56563500 \\
\hline $\mathrm{H}$ & 2.97963800 & -6.12249900 & -1.35779400 \\
\hline $\mathrm{H}$ & 4.59445200 & -6.77483100 & -1.02789700 \\
\hline $\mathrm{H}$ & 4.20357500 & -6.14922800 & -2.63179000 \\
\hline $\mathrm{C}$ & 2.53893900 & -1.54595600 & -4.82704900 \\
\hline $\mathrm{C}$ & 3.04031900 & -0.08651600 & -4.98932700 \\
\hline $\mathrm{H}$ & 2.54629600 & 0.62107600 & -4.32096000 \\
\hline $\mathrm{H}$ & 2.86639100 & 0.25066900 & -6.01765400 \\
\hline
\end{tabular}


H

C

$\mathrm{H}$

$\mathrm{H}$

$\mathrm{H}$

C

$\mathrm{H}$

$\mathrm{H}$

$\mathrm{H}$

C

$\mathrm{H}$

$\mathrm{H}$

$\mathrm{H}$

C

C

$\mathrm{H}$

$\mathrm{H}$

$\mathrm{H}$

C

$\mathrm{H}$

$\mathrm{H}$

$\mathrm{H}$

C

$\mathrm{H}$

$\mathrm{H}$

$\mathrm{H}$

C

C

$\mathrm{H}$

$\mathrm{H}$

$\mathrm{H}$

C

$\mathrm{H}$

$\mathrm{H}$

$\mathrm{H}$

C

$\mathrm{H}$

$\mathrm{H}$

$\mathrm{H}$

C

$\mathrm{H}$

$\mathrm{H}$

$\mathrm{H}$

C

$\begin{array}{ccc}4.11547800 & -0.02895800 & -4.78871500 \\ 1.02774300 & -1.60029200 & -5.14178600 \\ 0.65530200 & -2.63003700 & -5.12559200 \\ 0.81982900 & -1.18004000 & -6.13340100 \\ 0.45425400 & -1.03931700 & -4.39841800 \\ 3.31015300 & -2.35580000 & -5.89031700 \\ 4.37075200 & -2.43665300 & -5.63877200 \\ 3.22626100 & -1.83232900 & -6.85032200 \\ 2.92183500 & -3.36280900 & -6.03631800 \\ 2.46551500 & -4.99494500 & -4.14914000 \\ 2.82481500 & -5.81493600 & -4.77599100 \\ 1.71884700 & -4.41830500 & -4.70591800 \\ 1.99475000 & -5.39752800 & -3.24746200 \\ 7.10404900 & -0.34758600 & 1.86084200 \\ 7.57018000 & -1.37340100 & 0.80553300 \\ 6.72359200 & -1.93514900 & 0.39815600 \\ 8.27138300 & -2.09030800 & 1.24980300 \\ 8.07481300 & -0.88331200 & -0.03414700 \\ 6.43139000 & -1.11325000 & 3.03111200 \\ 6.07353700 & -0.41193300 & 3.79317900 \\ 7.15997000 & -1.78422100 & 3.50038300 \\ 5.58103300 & -1.72207200 & 2.71398000 \\ 8.33799600 & 0.34329800 & 2.47772800 \\ 9.0173800 & 0.75962500 & 1.73452600 \\ 8.90126900 & -0.40405000 & 3.04944900 \\ 8.05003000 & 1.14769900 & 3.15930700 \\ 5.20642200 & 4.35143000 & 0.64587200 \\ 6.20221500 & 4.87776600 & -0.41100900 \\ 7.23578000 & 4.65969400 & -0.14337600 \\ 6.10400900 & 5.96673300 & -0.49378700 \\ 5.99066700 & 4.45002800 & -1.39793800 \\ 3.84730000 & 5.01376300 & 0.35051700 \\ 3.47033900 & 4.76970600 & -0.64728100 \\ 3.96869500 & 6.10167900 & 0.40629000 \\ 3.07528700 & 4.72734500 & 1.06832400 \\ 5.65806700 & 4.81265500 & 2.05089900 \\ 5.73049600 & 5.90696300 & 2.08144300 \\ 6.63269800 & 4.39435200 & 2.31228100 \\ 4.92874700 & 4.50129200 & 2.80764500 \\ 8.24718600 & 2.31850100 & -0.10827700 \\ 8.45281800 & 1.25076500 & -0.24117800 \\ 7.78377800 & 2.85219700 & 0.04315100 \\ 0.05008500 & -2.38776900 & 0.42567200\end{array}$




\begin{tabular}{|c|c|c|c|}
\hline $\mathrm{C}$ & -0.68753000 & -1.64416400 & -0.53425500 \\
\hline $\mathrm{C}$ & -0.98539700 & -2.21910000 & -1.77966600 \\
\hline $\mathrm{H}$ & -1.53599400 & -1.62658000 & -2.49799800 \\
\hline $\mathrm{C}$ & -0.66057700 & -3.54060800 & -2.11514600 \\
\hline $\mathrm{H}$ & -0.90001000 & -3.96044700 & -3.08532600 \\
\hline $\mathrm{C}$ & -0.03448400 & -4.27999800 & -1.13198100 \\
\hline $\mathrm{C}$ & 0.31400000 & -3.70834800 & 0.08648500 \\
\hline $\mathrm{C}$ & 0.75187600 & -5.87835700 & 0.18740500 \\
\hline $\mathrm{H}$ & -0.10485100 & -6.36844400 & 0.67249600 \\
\hline $\mathrm{H}$ & 1.64607600 & -6.49886800 & 0.21303800 \\
\hline $\mathrm{C}$ & 1.69932500 & -0.89218800 & 1.69043300 \\
\hline $\mathrm{C}$ & 2.33447400 & -0.58182900 & 2.89919600 \\
\hline $\mathrm{H}$ & 3.10423300 & 0.17908100 & 2.9069090 \\
\hline $\mathrm{C}$ & 2.03863800 & -1.24127700 & 4.10568300 \\
\hline $\mathrm{H}$ & 2.56267300 & -1.00332600 & 5.02434600 \\
\hline $\mathrm{C}$ & 1.06066800 & -2.21056600 & 4.05841000 \\
\hline $\mathrm{C}$ & 0.39314600 & -2.50578200 & 2.87601800 \\
\hline $\mathrm{C}$ & 0.67244600 & -1.88338800 & 1.67454200 \\
\hline $\mathrm{C}$ & -0.24189500 & -3.98098300 & 4.41311000 \\
\hline $\mathrm{H}$ & -1.16091300 & -4.14083800 & 4.97775500 \\
\hline $\mathrm{H}$ & 0.34011100 & -4.90786100 & 4.30615200 \\
\hline $\mathrm{C}$ & -0.11620100 & 2.05066200 & -4.54994900 \\
\hline $\mathrm{C}$ & 0.61184600 & 3.22051100 & -4.81441400 \\
\hline $\mathrm{H}$ & 0.43542800 & 1.11387100 & -4.56117600 \\
\hline $\mathrm{C}$ & -1.47875300 & 1.96303800 & -4.16888100 \\
\hline $\mathrm{C}$ & -2.08817700 & 0.73611000 & -3.77892900 \\
\hline $\mathrm{H}$ & -2.08620800 & 2.85991600 & -4.23683700 \\
\hline $\mathrm{H}$ & -1.55178700 & -0.17547600 & -4.03393000 \\
\hline $\mathrm{C}$ & -3.53726500 & 0.57044300 & -3.60666700 \\
\hline $\mathrm{C}$ & -4.40869100 & 1.66337000 & -3.44111200 \\
\hline $\mathrm{C}$ & -4.09232100 & -0.72034100 & -3.61643300 \\
\hline $\mathrm{C}$ & -5.78194000 & 1.47003100 & -3.34665000 \\
\hline $\mathrm{H}$ & -4.00796800 & 2.67023900 & -3.38208900 \\
\hline $\mathrm{C}$ & -5.46565800 & -0.91607300 & -3.49920200 \\
\hline $\mathrm{H}$ & -3.43606300 & -1.57898700 & -3.72824900 \\
\hline $\mathrm{C}$ & -6.31991600 & 0.18045500 & -3.38313800 \\
\hline $\mathrm{H}$ & -6.43739600 & 2.32954700 & -3.23894300 \\
\hline $\mathrm{H}$ & -5.86699200 & -1.92541600 & -3.50996600 \\
\hline $\mathrm{H}$ & -7.39405600 & 0.03570500 & -3.31762600 \\
\hline S & 0.27228600 & 3.45640900 & -1.25741400 \\
\hline $\mathrm{C}$ & -1.04996900 & 4.69124700 & -1.14530300 \\
\hline $\mathrm{C}$ & -0.94585900 & 5.92185900 & -1.78911300 \\
\hline $\mathrm{C}$ & -2.17957500 & 4.35746000 & -0.39410500 \\
\hline $\mathrm{C}$ & -1.99718200 & 6.83427100 & -1.68139100 \\
\hline
\end{tabular}




\begin{tabular}{|c|c|c|c|}
\hline $\mathrm{H}$ & -0.05441100 & 6.14525300 & -2.36417000 \\
\hline $\mathrm{C}$ & -3.22366800 & 5.27573900 & -0.29043700 \\
\hline $\mathrm{H}$ & -2.23461700 & 3.39556600 & 0.10761700 \\
\hline $\mathrm{C}$ & -3.13353100 & 6.51187700 & -0.93629500 \\
\hline $\mathrm{H}$ & -1.92798700 & 7.79742100 & -2.17927100 \\
\hline $\mathrm{H}$ & -4.10324000 & 5.02801200 & 0.29504100 \\
\hline $\mathrm{H}$ & -3.94957700 & 7.22448700 & -0.85663400 \\
\hline $\mathrm{O}$ & 1.17642100 & 4.07547000 & -2.39677900 \\
\hline $\mathrm{O}$ & 1.00813200 & 3.52262100 & 0.04833200 \\
\hline $\mathrm{H}$ & 0.98504000 & 3.52782600 & -3.50013800 \\
\hline $\mathrm{H}$ & 1.57958500 & 3.05639000 & -5.29169600 \\
\hline $\mathrm{C}$ & -0.08432200 & 4.52435700 & -5.17253500 \\
\hline $\mathrm{H}$ & -0.53272200 & 4.48809500 & -6.17425000 \\
\hline $\mathrm{H}$ & -0.87768000 & 4.76598900 & -4.45728300 \\
\hline $\mathrm{H}$ & 0.62856500 & 5.35451500 & -5.15283400 \\
\hline & $t$ & & \\
\hline
\end{tabular}

Total SCF energy (M06/SDD-6-311++G(d,p)/SMD(1,4-Dioxane)): -5497.828949a.u.

Thermal correction to Gibbs Free Energy at $298.15 \mathrm{~K}: 1.764762 \mathrm{a} . \mathrm{u}$

Gibbs free energy at 298.15 K (M06/SDD-6-311++G(d,p)/SMD(1,4-Dioxane)): -5496.064187a.u. $\mathrm{Pd}$ $\mathrm{P}$

$P$

$\mathrm{O}$

$\mathrm{O}$

$\mathrm{O}$

$\mathrm{O}$

$\mathrm{O}$

$\mathrm{O}$

$\mathrm{O}$

$\mathrm{O}$

C

C

$\mathrm{H}$

C

C

C

C

$\mathrm{H}$

\begin{tabular}{ccc}
1.33495700 & -0.73319300 & \multicolumn{1}{c}{-2.68181200} \\
1.63319900 & -0.29448000 & -0.38771700 \\
-1.92272000 & -0.11747100 & 0.34495000 \\
7.52654700 & 0.65917400 & 0.35307600 \\
1.30105900 & -4.75599100 & 3.61420700 \\
-2.93392100 & 5.77962400 & -0.29959000 \\
-7.55052400 & -2.20224500 & 0.24959600 \\
0.67019900 & 5.05084200 & 1.94773200 \\
0.03856200 & 3.26971600 & 3.25796700 \\
1.48254200 & 0.76453700 & 4.31173000 \\
0.46189100 & -0.66870000 & 5.80997300 \\
3.42957000 & 0.04397500 & -0.10123600 \\
4.34826300 & -0.91821300 & -0.53437800 \\
3.96184900 & -1.80277100 & -1.02463900 \\
5.72069500 & -0.77020400 & -0.34122200 \\
6.16307700 & 0.41527900 & 0.30956700 \\
5.26140400 & 1.31418000 & 0.91121100 \\
3.89800300 & 1.12168800 & 0.63723900 \\
3.18844200 & 1.82869700 & 1.03912500
\end{tabular}




\begin{tabular}{|c|c|c|c|}
\hline $\mathrm{C}$ & 6.68915500 & -1.90505600 & -0.73309100 \\
\hline $\mathrm{C}$ & 5.92830400 & -3.12764300 & -1.28370000 \\
\hline $\mathrm{H}$ & 5.19956800 & -3.51700900 & -0.56502800 \\
\hline $\mathrm{H}$ & 6.64483200 & -3.92766300 & -1.49664600 \\
\hline $\mathrm{H}$ & 5.40291700 & -2.90028200 & -2.21925900 \\
\hline $\mathrm{C}$ & 7.69756700 & -1.46893300 & -1.81770000 \\
\hline $\mathrm{H}$ & 7.18362800 & -1.01924300 & -2.67575600 \\
\hline $\mathrm{H}$ & 8.25123400 & -2.34289600 & -2.18046300 \\
\hline $\mathrm{H}$ & 8.42400400 & -0.75576900 & -1.42935600 \\
\hline $\mathrm{C}$ & 7.45114000 & -2.36475600 & 0.53121100 \\
\hline $\mathrm{H}$ & 8.03245200 & -1.54670900 & 0.96073200 \\
\hline $\mathrm{H}$ & 8.13631600 & -3.18345300 & 0.28000000 \\
\hline $\mathrm{H}$ & 6.75056500 & -2.73103000 & 1.29044600 \\
\hline $\mathrm{C}$ & 5.60264100 & 2.45609100 & 1.90021300 \\
\hline $\mathrm{C}$ & 5.28793000 & 3.82204600 & 1.25349800 \\
\hline $\mathrm{H}$ & 5.94356700 & 4.01548800 & 0.39723600 \\
\hline $\mathrm{H}$ & 5.44204200 & 4.62939100 & 1.97956300 \\
\hline $\mathrm{H}$ & 4.25279400 & 3.87327900 & 0.90039500 \\
\hline $\mathrm{C}$ & 4.70537800 & 2.28405700 & 3.15549900 \\
\hline $\mathrm{H}$ & 3.63597900 & 2.31327500 & 2.93481200 \\
\hline $\mathrm{H}$ & 4.92036600 & 3.08823500 & 3.86906600 \\
\hline $\mathrm{H}$ & 4.91454000 & 1.32845100 & 3.64655800 \\
\hline $\mathrm{C}$ & 7.05190500 & 2.46700600 & 2.42696400 \\
\hline $\mathrm{H}$ & 7.34626800 & 1.48839700 & 2.81431400 \\
\hline $\mathrm{H}$ & 7.11482500 & 3.19165400 & 3.24740600 \\
\hline $\mathrm{H}$ & 7.78303600 & 2.76684800 & 1.67600500 \\
\hline $\mathrm{C}$ & 7.94503800 & 1.63523100 & -0.59900600 \\
\hline $\mathrm{H}$ & 7.46945000 & 2.60512400 & -0.41685300 \\
\hline $\mathrm{H}$ & 7.70956500 & 1.31703000 & -1.62134700 \\
\hline $\mathrm{H}$ & 9.02721600 & 1.73519900 & -0.48683700 \\
\hline $\mathrm{C}$ & 1.44095700 & -1.64614700 & 0.84605600 \\
\hline $\mathrm{C}$ & 0.58937000 & -2.72535400 & 0.62004100 \\
\hline $\mathrm{H}$ & -0.04384000 & -2.71293400 & -0.25944400 \\
\hline $\mathrm{C}$ & 0.53758400 & -3.81942200 & 1.50753800 \\
\hline $\mathrm{C}$ & 1.36486100 & -3.76447700 & 2.64953500 \\
\hline $\mathrm{C}$ & 2.32600200 & -2.74063600 & 2.84266500 \\
\hline $\mathrm{C}$ & 2.30963000 & -1.68045200 & 1.94393200 \\
\hline $\mathrm{H}$ & 3.00194100 & -0.86691000 & 2.08000400 \\
\hline $\mathrm{C}$ & 3.47472900 & -2.85183400 & 3.87018900 \\
\hline $\mathrm{C}$ & 3.00691300 & -3.03982500 & 5.32944700 \\
\hline $\mathrm{H}$ & 2.24575800 & -2.30487200 & 5.60622700 \\
\hline $\mathrm{H}$ & 3.86215200 & -2.91671700 & 6.00499100 \\
\hline $\mathrm{H}$ & 2.59995900 & -4.03693600 & 5.49369400 \\
\hline $\mathrm{C}$ & 4.34236500 & -4.06507800 & 3.46176800 \\
\hline
\end{tabular}


$\mathrm{H}$

$\mathrm{H}$

$\mathrm{H}$

C

$\mathrm{H}$

$\mathrm{H}$

$\mathrm{H}$

C

C

$\mathrm{H}$

$\mathrm{H}$

$\mathrm{H}$

C

$\mathrm{H}$

$\mathrm{H}$

$\mathrm{H}$

C

$\mathrm{H}$

$\mathrm{H}$

$\mathrm{H}$

C

$\mathrm{H}$

$\mathrm{H}$

$\mathrm{H}$

C

C

$\mathrm{H}$

C

C

C

C

$\mathrm{H}$

C

C

$\mathrm{H}$

C

C

C

C

$\mathrm{H}$

C

C

$\mathrm{H}$

$\mathrm{H}$
3.75728500

5.19168300

4.73780900

4.37305700

4.83962900

5.17847800

3.81014900

$-0.40202500$

$-1.85734200$

$-2.00499500$

$-2.54970300$

$-2.13317200$

$-0.28083100$

$-0.61594700$

$-0.90057900$

0.75643500

$-0.09366400$

0.96293100

$-0.68432700$

$-0.35467300$

0.26389000

0.37458200

0.35499900

$-0.72542200$

$-2.30066800$

$-2.71044600$

$-2.78642200$

$-2.98958800$

$-2.78542500$

$-2.46350100$

$-2.19822600$

$-1.88849800$

$-3.62381900$

$-4.62198200$

$-4.37624300$

$-5.92796800$

$-6.23245800$

$-5.22855700$

$-3.92711300$

$-3.13105500$

$-3.63185900$

$-5.07725100$

$-5.07703700$

$-5.57926400$
$-4.98777100$

$-4.17297600$

$-3.93234600$

$-1.59799700$

$-1.44251200$

$-1.71485900$

$-0.69298200$

$-4.99463200$

$-4.58108500$

$-4.37703900$

$-5.38125300$

$-3.67805200$

$-5.28737900$

$-4.46309100$

$-6.15641400$

$-5.52868100$

$-6.33361600$

$-6.60157800$

$-7.11788100$

$-6.34380800$

$-4.55337100$

$-3.58994600$

$-5.36374800$

$-4.59383500$

1.68652800

2.49177100

2.04235200

3.85008900

4.40724100

3.61539600

2.25735700

1.61907900

$-0.82785000$

$-0.24892400$

0.61988200

$-0.74997800$

$-1.81144600$

$-2.50913500$

$-1.99363700$

$-2.47613300$

4.65321200

5.00573000

5.61918000

5.56596500
3.48770000

4.14801400

2.44787300

3.83862900

2.86018700

4.57169600

4.09163800

1.14104700

1.44764900

2.51338000

1.15946600

0.89882600

$-0.37617200$

$-1.00495900$

$-0.62518700$

$-0.63818500$

1.84673900

1.75765300

1.35905400

2.90415100

4.57266400

5.07931900

5.30014000

4.10657900

0.21720100

1.28476200

2.26504300

1.12182900

$-0.16761000$

$-1.28854500$

$-1.04744800$

$-1.86712600$

0.48216200

1.26210700

1.85692900

1.31133400

0.43014200

$-0.28487400$

$-0.23143300$

$-0.78176600$

2.27515100

1.85408800

0.94949000

2.65296800 


\begin{tabular}{|c|c|c|c|}
\hline $\mathrm{H}$ & -5.65884900 & 4.09878100 & 1.65819800 \\
\hline $\mathrm{C}$ & -3.69649100 & 3.81736200 & 3.56938100 \\
\hline $\mathrm{H}$ & -4.30446300 & 2.91449300 & 3.44989700 \\
\hline $\mathrm{H}$ & -4.15871900 & 4.41589100 & 4.36154600 \\
\hline $\mathrm{H}$ & -2.69783100 & 3.51738100 & 3.90650900 \\
\hline $\mathrm{C}$ & -2.89205400 & 5.96486000 & 2.61941000 \\
\hline $\mathrm{H}$ & -1.81527200 & 5.80320200 & 2.70320800 \\
\hline $\mathrm{H}$ & -3.25495800 & 6.35035400 & 3.57950500 \\
\hline $\mathrm{H}$ & -3.06361800 & 6.73248900 & 1.86627100 \\
\hline $\mathrm{C}$ & -2.38463300 & 4.10136200 & -2.75612700 \\
\hline $\mathrm{C}$ & -3.04893500 & 3.04165700 & -3.66601200 \\
\hline $\mathrm{H}$ & -2.51653500 & 2.09066700 & -3.67036300 \\
\hline $\mathrm{H}$ & -3.06541400 & 3.40201700 & -4.70056500 \\
\hline $\mathrm{H}$ & -4.08090600 & 2.84692700 & -3.35663200 \\
\hline $\mathrm{C}$ & -0.90536200 & 4.21703700 & -3.18091700 \\
\hline $\mathrm{H}$ & -0.36651200 & 4.96779200 & -2.59464200 \\
\hline $\mathrm{H}$ & -0.83633800 & 4.50080600 & -4.23893700 \\
\hline $\mathrm{H}$ & -0.38412800 & 3.26422400 & -3.04871200 \\
\hline $\mathrm{C}$ & -3.12120700 & 5.42542800 & -3.05069300 \\
\hline $\mathrm{H}$ & -4.14556900 & 5.40370700 & -2.66800600 \\
\hline $\mathrm{H}$ & -3.16766000 & 5.55991000 & -4.13799400 \\
\hline $\mathrm{H}$ & -2.62903700 & 6.30351400 & -2.63591900 \\
\hline $\mathrm{C}$ & -1.69466000 & 6.48944200 & -0.29024000 \\
\hline $\mathrm{H}$ & -1.94725200 & 7.55172800 & -0.24034000 \\
\hline $\mathrm{H}$ & -1.11924500 & 6.30188800 & -1.20279200 \\
\hline $\mathrm{H}$ & -1.08265900 & 6.21389200 & 0.57308700 \\
\hline $\mathrm{C}$ & -6.87493100 & -0.11348800 & 2.35783400 \\
\hline $\mathrm{C}$ & -7.20483900 & 1.33813800 & 1.94903700 \\
\hline $\mathrm{H}$ & -6.29297700 & 1.92963100 & 1.81881800 \\
\hline $\mathrm{H}$ & -7.81776000 & 1.82298400 & 2.71846700 \\
\hline $\mathrm{H}$ & -7.75853100 & 1.37391500 & 1.00463800 \\
\hline $\mathrm{C}$ & -6.12935700 & -0.08480300 & 3.71851600 \\
\hline $\mathrm{H}$ & -5.86059500 & -1.09965500 & 4.03188200 \\
\hline $\mathrm{H}$ & -6.77960500 & 0.34918500 & 4.48656000 \\
\hline $\mathrm{H}$ & -5.21227100 & 0.50902500 & 3.69065700 \\
\hline $\mathrm{C}$ & -8.18652000 & -0.88136500 & 2.62253300 \\
\hline $\mathrm{H}$ & -8.90109600 & -0.81454400 & 1.80262400 \\
\hline $\mathrm{H}$ & -8.66703400 & -0.44797000 & 3.50777500 \\
\hline $\mathrm{H}$ & -7.99925200 & -1.93987400 & 2.82089600 \\
\hline $\mathrm{C}$ & -5.52032700 & -3.81750400 & -1.05253300 \\
\hline $\mathrm{C}$ & -6.47953400 & -3.59177300 & -2.24218200 \\
\hline $\mathrm{H}$ & -7.49453300 & -3.36994100 & -1.91329300 \\
\hline $\mathrm{H}$ & -6.52122800 & -4.49927200 & -2.85595500 \\
\hline $\mathrm{H}$ & -6.12446000 & -2.77574500 & -2.88301600 \\
\hline
\end{tabular}




\begin{tabular}{|c|c|c|c|}
\hline $\mathrm{C}$ & -4.23321800 & -4.43894900 & -1.62863100 \\
\hline $\mathrm{H}$ & -3.74738600 & -3.80287900 & -2.37264000 \\
\hline $\mathrm{H}$ & -4.48898300 & -5.38677900 & -2.11508800 \\
\hline $\mathrm{H}$ & -3.49669800 & -4.65536100 & -0.85150800 \\
\hline $\mathrm{C}$ & -6.13652100 & -4.84529200 & -0.07624500 \\
\hline $\mathrm{H}$ & -6.33901100 & -5.78612800 & -0.60263000 \\
\hline $\mathrm{H}$ & -7.07192200 & -4.47930500 & 0.35146700 \\
\hline $\mathrm{H}$ & -5.43864900 & -5.06048100 & 0.74129900 \\
\hline $\mathrm{C}$ & -8.27920900 & -1.32561400 & -0.60653200 \\
\hline $\mathrm{H}$ & -8.37198200 & -0.32298600 & -0.17588000 \\
\hline $\mathrm{H}$ & -9.27388700 & -1.76275800 & -0.72341800 \\
\hline $\mathrm{H}$ & -7.79828000 & -1.24132600 & -1.58768800 \\
\hline $\mathrm{C}$ & 0.47601300 & 1.48644700 & 1.5919830 \\
\hline $\mathrm{C}$ & 0.95986400 & 1.28726300 & 0.2697530 \\
\hline $\mathrm{C}$ & 1.24560400 & 2.38875900 & -0.54961700 \\
\hline $\mathrm{H}$ & 1.60068000 & 2.21448200 & -1.55788400 \\
\hline $\mathrm{C}$ & 1.17150600 & 3.71390800 & -0.09327800 \\
\hline $\mathrm{H}$ & 1.41315200 & 4.54620900 & -0.74258100 \\
\hline $\mathrm{C}$ & 0.81741800 & 3.88396800 & 1.2294430 \\
\hline $\mathrm{C}$ & 0.45917600 & 2.80136300 & 2.0301340 \\
\hline $\mathrm{C}$ & 0.53443900 & 4.60585500 & 3.3056490 \\
\hline $\mathrm{H}$ & 1.52512000 & 4.61234800 & 3.7839790 \\
\hline $\mathrm{H}$ & -0.17693600 & 5.24151900 & 3.82965800 \\
\hline $\mathrm{C}$ & -1.31339000 & -0.25614100 & 2.08868400 \\
\hline $\mathrm{C}$ & -1.90472900 & -1.13784900 & 2.99979300 \\
\hline $\mathrm{H}$ & -2.79911200 & -1.67413400 & 2.70754600 \\
\hline $\mathrm{C}$ & -1.39403800 & -1.34472100 & 4.29483700 \\
\hline $\mathrm{H}$ & -1.87230000 & -2.02457200 & 4.99080100 \\
\hline $\mathrm{C}$ & -0.26241500 & -0.63693400 & 4.63610000 \\
\hline $\mathrm{C}$ & 0.34584500 & 0.23386600 & 3.73903100 \\
\hline $\mathrm{C}$ & -0.13234100 & 0.45319800 & 2.46294000 \\
\hline $\mathrm{C}$ & 1.40383300 & 0.40518200 & 5.6935290 \\
\hline $\mathrm{H}$ & 2.38287600 & 0.06540200 & 6.0346450 \\
\hline $\mathrm{H}$ & 1.04606400 & 1.26656500 & 6.2751490 \\
\hline $\mathrm{C}$ & 2.61937600 & -1.97819500 & -4.75892800 \\
\hline $\mathrm{C}$ & 1.86911000 & -2.79356400 & -5.61955800 \\
\hline $\mathrm{H}$ & 3.27434800 & -2.48389200 & -4.04546100 \\
\hline $\mathrm{H}$ & 1.40283200 & -2.28064300 & -6.46762500 \\
\hline $\mathrm{C}$ & 2.52761000 & -0.57057500 & -4.62010900 \\
\hline $\mathrm{C}$ & 3.19123100 & 0.10405400 & -3.55725600 \\
\hline $\mathrm{H}$ & 1.98519300 & -0.01023900 & -5.37803600 \\
\hline $\mathrm{H}$ & 3.97142100 & -0.44465900 & -3.03542800 \\
\hline $\mathrm{C}$ & 3.28710900 & 1.56315800 & -3.42970400 \\
\hline $\mathrm{C}$ & 2.38651000 & 2.44267300 & -4.06103100 \\
\hline
\end{tabular}




$\begin{array}{lrrr}\mathrm{C} & 4.28525500 & 2.11196100 & -2.60444500 \\ \mathrm{C} & 2.48456200 & 3.81864100 & -3.87255100 \\ \mathrm{H} & 1.58692800 & 2.04480900 & -4.67847600 \\ \mathrm{C} & 4.37599100 & 3.48755500 & -2.41144500 \\ \mathrm{H} & 4.97876100 & 1.44682200 & -2.10099600 \\ \mathrm{C} & 3.47721500 & 4.34932300 & -3.04311600 \\ \mathrm{H} & 1.77655500 & 4.47812800 & -4.36483700 \\ \mathrm{H} & 5.14803500 & 3.88683500 & -1.76171000 \\ \mathrm{H} & 3.54892800 & 5.42287500 & -2.89346200 \\ \mathrm{C} & 2.28438900 & -4.23119300 & -5.88061300 \\ \mathrm{H} & 3.03185300 & -4.30540700 & -6.68144400 \\ \mathrm{H} & 2.71396800 & -4.68126400 & -4.97788200 \\ \mathrm{H} & 1.42182000 & -4.83912000 & -6.17279200 \\ \mathrm{~S} & -0.70260000 & -2.01864200 & -3.17402500 \\ \mathrm{C} & -1.94420400 & -1.00327000 & -4.02335900 \\ \mathrm{C} & -1.62362700 & -0.44635200 & -5.26280100 \\ \mathrm{C} & -3.21571700 & -0.86006500 & -3.47568100 \\ \mathrm{C} & -2.60529400 & 0.23873400 & -5.97635100 \\ \mathrm{H} & -0.62847000 & -0.57800900 & -5.67649400 \\ \mathrm{C} & -4.19561300 & -0.17863600 & -4.20081200 \\ \mathrm{H} & -3.43763400 & -1.29064900 & -2.50920700 \\ \mathrm{C} & -3.89574600 & 0.36114000 & -5.45160300 \\ \mathrm{H} & -2.36924100 & 0.66622500 & -6.94678300 \\ \mathrm{H} & -5.19186500 & -0.07092000 & -3.78126800 \\ \mathrm{H} & -4.66038400 & 0.88948200 & -6.01353600 \\ \mathrm{O} & -1.44423500 & -2.70269000 & -2.06464800 \\ \mathrm{O} & -0.39804300 & -3.03234100 & -4.34363500 \\ \mathrm{H} & 0.76115200 & -2.91112100 & -4.84423100\end{array}$

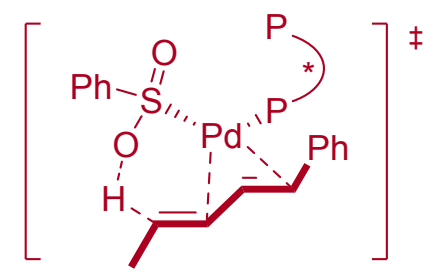

TS-8d

Total SCF energy (M06/SDD-6-311++G(d,p)/SMD(1,4-Dioxane)): -5497.823011a.u.

Thermal correction to Gibbs Free Energy at 298.15 K: 1.764592a.u

Gibbs free energy at 298.15 K (M06/SDD-6-311++G(d,p)/SMD(1,4-Dioxane)): -5496.058419a.u.

$\begin{array}{rrrc}\mathrm{Pd} & -0.92358000 & 1.44379200 & -2.05346700 \\ \mathrm{P} & -1.50727500 & 0.05229000 & -0.25683900 \\ \mathrm{P} & 2.05064500 & 0.06027400 & 0.14393000 \\ \mathrm{O} & -7.30583200 & -1.44319000 & 0.32323900 \\ \mathrm{O} & -1.19031300 & 1.52647500 & 5.56405800 \\ \mathrm{O} & 3.52420700 & -4.50945400 & -3.48604500\end{array}$




\begin{tabular}{|c|c|c|c|}
\hline $\mathrm{O}$ & 7.43918400 & 2.60625800 & 0.9070680 \\
\hline $\mathrm{O}$ & 0.27445100 & -5.60006900 & -0.84376400 \\
\hline $\mathrm{O}$ & 0.87985800 & -4.56046500 & 1.11622100 \\
\hline $\mathrm{O}$ & -0.64636400 & -3.22596100 & 3.32502500 \\
\hline $\mathrm{O}$ & 0.50118200 & -2.67717100 & 5.24926100 \\
\hline $\mathrm{C}$ & -3.28506200 & -0.46276400 & -0.24781300 \\
\hline $\mathrm{C}$ & -4.24931400 & 0.53708500 & -0.08276800 \\
\hline $\mathrm{H}$ & -3.92364900 & 1.56846500 & -0.11215300 \\
\hline $\mathrm{C}$ & -5.58906400 & 0.23483800 & 0.16528300 \\
\hline $\mathrm{C}$ & -5.96216400 & -1.13699100 & 0.17302200 \\
\hline $\mathrm{C}$ & -5.00966400 & -2.17116700 & 0.08718800 \\
\hline $\mathrm{C}$ & -3.68162900 & -1.79001700 & -0.15213500 \\
\hline $\mathrm{H}$ & -2.93285900 & -2.56189100 & -0.22711300 \\
\hline $\mathrm{C}$ & -6.56797900 & 1.35256600 & 0.59092000 \\
\hline $\mathrm{C}$ & -5.88687100 & 2.73362500 & 0.54546700 \\
\hline $\mathrm{H}$ & -5.02235200 & 2.78546800 & 1.21580000 \\
\hline $\mathrm{H}$ & -6.60149800 & 3.49764700 & 0.87011300 \\
\hline $\mathrm{H}$ & -5.55547200 & 2.99069200 & -0.46638900 \\
\hline $\mathrm{C}$ & -7.83870300 & 1.44893500 & -0.27935100 \\
\hline $\mathrm{H}$ & -7.58707000 & 1.51306000 & -1.34111400 \\
\hline $\mathrm{H}$ & -8.39455500 & 2.35482700 & -0.01016200 \\
\hline $\mathrm{H}$ & -8.50065400 & 0.59899700 & -0.11998900 \\
\hline $\mathrm{C}$ & -6.98547300 & 1.07879500 & 2.05428800 \\
\hline $\mathrm{H}$ & -7.50166000 & 0.12007100 & 2.14126700 \\
\hline $\mathrm{H}$ & -7.65834000 & 1.86901100 & 2.40967700 \\
\hline $\mathrm{H}$ & -6.10762700 & 1.06074800 & 2.70826500 \\
\hline $\mathrm{C}$ & -5.25341800 & -3.69045200 & 0.27224100 \\
\hline $\mathrm{C}$ & -5.10010100 & -4.39016500 & -1.09665800 \\
\hline $\mathrm{H}$ & -5.84136600 & -4.02701900 & -1.81728300 \\
\hline $\mathrm{H}$ & -5.23454400 & -5.47355800 & -0.99074700 \\
\hline $\mathrm{H}$ & -4.10617700 & -4.21037900 & -1.52116300 \\
\hline $\mathrm{C}$ & -4.17939800 & -4.26253100 & 1.23620700 \\
\hline $\mathrm{H}$ & -3.15602400 & -4.14252600 & 0.87258100 \\
\hline $\mathrm{H}$ & -4.35232400 & -5.33605100 & 1.37368900 \\
\hline $\mathrm{H}$ & -4.24429200 & -3.77912000 & 2.21559200 \\
\hline $\mathrm{C}$ & -6.60855700 & -4.07837700 & 0.90005100 \\
\hline $\mathrm{H}$ & -6.80262700 & -3.51373700 & 1.81533700 \\
\hline $\mathrm{H}$ & -6.57756900 & -5.14401900 & 1.15606800 \\
\hline $\mathrm{H}$ & -7.45460600 & -3.93605800 & 0.22874400 \\
\hline $\mathrm{C}$ & -7.96302200 & -1.78413700 & -0.89404300 \\
\hline $\mathrm{H}$ & -7.44850800 & -2.59740500 & -1.41616000 \\
\hline $\mathrm{H}$ & -8.02253200 & -0.92295300 & -1.56514000 \\
\hline $\mathrm{H}$ & -8.97072900 & -2.10758500 & -0.62200200 \\
\hline $\mathrm{C}$ & -1.38941100 & 0.55204800 & 1.51716800 \\
\hline
\end{tabular}




\begin{tabular}{|c|c|c|c|}
\hline $\mathrm{C}$ & -0.65142500 & 1.66108500 & 1.92577100 \\
\hline $\mathrm{H}$ & -0.08581700 & 2.22017600 & 1.18998200 \\
\hline $\mathrm{C}$ & -0.60692900 & 2.06648600 & 3.27670800 \\
\hline $\mathrm{C}$ & -1.29871800 & 1.26809200 & 4.20857000 \\
\hline $\mathrm{C}$ & -2.15923500 & 0.21224900 & 3.81191900 \\
\hline $\mathrm{C}$ & -2.16267800 & -0.13437500 & 2.46445600 \\
\hline $\mathrm{H}$ & -2.78369100 & -0.95012000 & 2.13072100 \\
\hline $\mathrm{C}$ & -3.18261300 & -0.42573000 & 4.77692300 \\
\hline $\mathrm{C}$ & -2.55428200 & -1.04143800 & 6.04601700 \\
\hline $\mathrm{H}$ & -1.69607300 & -1.67249800 & 5.80395000 \\
\hline $\mathrm{H}$ & -3.30009200 & -1.65993100 & 6.55971300 \\
\hline $\mathrm{H}$ & -2.22642200 & -0.27154800 & 6.74353100 \\
\hline $\mathrm{C}$ & -4.17171300 & 0.68227100 & 5.20588300 \\
\hline $\mathrm{H}$ & -3.65061600 & 1.48054400 & 5.74034600 \\
\hline $\mathrm{H}$ & -4.94343800 & 0.26800400 & 5.86656800 \\
\hline $\mathrm{H}$ & -4.66915400 & 1.11743500 & 4.33280000 \\
\hline $\mathrm{C}$ & -3.98213600 & -1.54315700 & 4.07781800 \\
\hline $\mathrm{H}$ & -4.54141200 & -1.17911700 & 3.20988000 \\
\hline $\mathrm{H}$ & -4.70746500 & -1.96248500 & 4.78337600 \\
\hline $\mathrm{H}$ & -3.32240600 & -2.35258900 & 3.74560500 \\
\hline $\mathrm{C}$ & 0.14436900 & 3.38686700 & 3.5910070 \\
\hline $\mathrm{C}$ & 1.64365200 & 3.20769800 & 3.2679040 \\
\hline $\mathrm{H}$ & 2.09745800 & 2.44782600 & 3.9143340 \\
\hline $\mathrm{H}$ & 2.17906700 & 4.15172900 & 3.4274740 \\
\hline $\mathrm{H}$ & 1.79928800 & 2.90824600 & 2.2299160 \\
\hline $\mathrm{C}$ & -0.44360900 & 4.49199700 & 2.67787400 \\
\hline $\mathrm{H}$ & -0.26051300 & 4.29882700 & 1.62335800 \\
\hline $\mathrm{H}$ & 0.02856100 & 5.45239000 & 2.9156630 \\
\hline $\mathrm{H}$ & -1.52235300 & 4.59599000 & 2.84508000 \\
\hline $\mathrm{C}$ & 0.01357400 & 3.93231300 & 5.0288250 \\
\hline $\mathrm{H}$ & -1.02886400 & 4.01492200 & 5.34448200 \\
\hline $\mathrm{H}$ & 0.45470600 & 4.93585100 & 5.0441460 \\
\hline $\mathrm{H}$ & 0.54991700 & 3.34049000 & 5.77062600 \\
\hline $\mathrm{C}$ & -0.01510400 & 0.96960500 & 6.15351300 \\
\hline $\mathrm{H}$ & -0.05720900 & -0.12346300 & 6.16537900 \\
\hline $\mathrm{H}$ & 0.02431800 & 1.34776900 & 7.17794600 \\
\hline $\mathrm{H}$ & 0.88487000 & 1.27800500 & 5.6128460 \\
\hline $\mathrm{C}$ & 2.55639300 & -1.37509200 & -0.90536300 \\
\hline $\mathrm{C}$ & 3.18760000 & -2.52882900 & -0.42738700 \\
\hline $\mathrm{H}$ & 3.34951400 & -2.61953700 & 0.63731300 \\
\hline $\mathrm{C}$ & 3.58030900 & -3.56465800 & -1.27692500 \\
\hline $\mathrm{C}$ & 3.27109800 & -3.42809600 & -2.65568300 \\
\hline $\mathrm{C}$ & 2.73173700 & -2.24291200 & -3.19055400 \\
\hline $\mathrm{C}$ & 2.34893600 & -1.25045000 & -2.27358300 \\
\hline
\end{tabular}




\begin{tabular}{|c|c|c|c|}
\hline $\mathrm{H}$ & 1.85883400 & -0.34901400 & -2.62807400 \\
\hline $\mathrm{C}$ & 3.69416000 & 0.82700900 & 0.48922100 \\
\hline $\mathrm{C}$ & 4.78630900 & 0.08257800 & 0.93081900 \\
\hline $\mathrm{H}$ & 4.65620400 & -0.97735200 & 1.09975700 \\
\hline $\mathrm{C}$ & 6.03590600 & 0.65488000 & 1.18861400 \\
\hline $\mathrm{C}$ & 6.18166300 & 2.02310900 & 0.86827200 \\
\hline $\mathrm{C}$ & 5.07934500 & 2.84043600 & 0.51832100 \\
\hline $\mathrm{C}$ & 3.84207500 & 2.20941100 & 0.32847300 \\
\hline $\mathrm{H}$ & 2.97213500 & 2.78929700 & 0.04929800 \\
\hline $\mathrm{C}$ & 4.45513300 & -4.71912800 & -0.73897200 \\
\hline $\mathrm{C}$ & 5.86306900 & -4.56035200 & -1.35884900 \\
\hline $\mathrm{H}$ & 5.81545600 & -4.63571900 & -2.44846700 \\
\hline $\mathrm{H}$ & 6.53292600 & -5.34556000 & -0.98648100 \\
\hline $\mathrm{H}$ & 6.29730400 & -3.58963900 & -1.09735000 \\
\hline $\mathrm{C}$ & 4.59158800 & -4.64898500 & 0.79555900 \\
\hline $\mathrm{H}$ & 5.07743900 & -3.72563100 & 1.12723000 \\
\hline $\mathrm{H}$ & 5.21298000 & -5.48237200 & 1.14003100 \\
\hline $\mathrm{H}$ & 3.61719100 & -4.72014600 & 1.29181100 \\
\hline $\mathrm{C}$ & 3.92874800 & -6.12948500 & -1.08485400 \\
\hline $\mathrm{H}$ & 2.86377400 & -6.22392000 & -0.86143800 \\
\hline $\mathrm{H}$ & 4.46902600 & -6.87756100 & -0.49284300 \\
\hline $\mathrm{H}$ & 4.07799400 & -6.36642300 & -2.13735500 \\
\hline $\mathrm{C}$ & 2.54192400 & -1.91248700 & -4.68996400 \\
\hline $\mathrm{C}$ & 3.14545000 & -0.50890100 & -4.95064000 \\
\hline $\mathrm{H}$ & 2.70153700 & 0.26824900 & -4.32621900 \\
\hline $\mathrm{H}$ & 2.99081300 & -0.22573600 & -5.99809200 \\
\hline $\mathrm{H}$ & 4.22271800 & -0.51312100 & -4.75277200 \\
\hline $\mathrm{C}$ & 1.03130900 & -1.87948100 & -5.00918600 \\
\hline $\mathrm{H}$ & 0.58119000 & -2.87183600 & -4.89693300 \\
\hline $\mathrm{H}$ & 0.85873900 & -1.54279800 & -6.03874200 \\
\hline $\mathrm{H}$ & 0.50192100 & -1.20475300 & -4.33007100 \\
\hline $\mathrm{C}$ & 3.25007200 & -2.84911600 & -5.69058800 \\
\hline $\mathrm{H}$ & 4.30377900 & -2.98566100 & -5.43597300 \\
\hline $\mathrm{H}$ & 3.19551000 & -2.39146900 & -6.68576800 \\
\hline $\mathrm{H}$ & 2.79027500 & -3.83404400 & -5.76384500 \\
\hline $\mathrm{C}$ & 2.35854800 & -5.29264600 & -3.74661200 \\
\hline $\mathrm{H}$ & 2.68448400 & -6.14035200 & -4.35446400 \\
\hline $\mathrm{H}$ & 1.60810100 & -4.71876600 & -4.30167500 \\
\hline $\mathrm{H}$ & 1.90426400 & -5.65322200 & -2.81939300 \\
\hline $\mathrm{C}$ & 7.09460500 & -0.26333500 & 1.84806600 \\
\hline $\mathrm{C}$ & 7.54514000 & -1.34337200 & 0.84121600 \\
\hline $\mathrm{H}$ & 6.69213400 & -1.92161900 & 0.47188800 \\
\hline $\mathrm{H}$ & 8.24832700 & -2.03977300 & 1.31399500 \\
\hline $\mathrm{H}$ & 8.04266000 & -0.89837800 & -0.02735600 \\
\hline
\end{tabular}




\begin{tabular}{|c|c|c|c|}
\hline $\mathrm{C}$ & 6.42892100 & -0.96532300 & 3.06154400 \\
\hline $\mathrm{H}$ & 6.08323800 & -0.22461300 & 3.79130400 \\
\hline $\mathrm{H}$ & 7.15764300 & -1.61741900 & 3.55651300 \\
\hline $\mathrm{H}$ & 5.57133000 & -1.58280000 & 2.78270000 \\
\hline $\mathrm{C}$ & 8.33808900 & 0.45053900 & 2.4175420 \\
\hline $\mathrm{H}$ & 9.01189500 & 0.82565400 & 1.6476820 \\
\hline $\mathrm{H}$ & 8.90350500 & -0.27088600 & 3.01963700 \\
\hline $\mathrm{H}$ & 8.06129500 & 1.28911800 & 3.0613780 \\
\hline $\mathrm{C}$ & 5.19142100 & 4.37971500 & 0.4474650 \\
\hline $\mathrm{C}$ & 6.17968400 & 4.85916300 & -0.63852500 \\
\hline $\mathrm{H}$ & 7.21518500 & 4.65180300 & -0.36995800 \\
\hline $\mathrm{H}$ & 6.08123700 & 5.94360800 & -0.76696600 \\
\hline $\mathrm{H}$ & 5.96005000 & 4.39007400 & -1.60470800 \\
\hline $\mathrm{C}$ & 3.83214400 & 5.03314400 & 0.1362570 \\
\hline $\mathrm{H}$ & 3.44656100 & 4.75164600 & -0.84813400 \\
\hline $\mathrm{H}$ & 3.95630100 & 6.12205700 & 0.1474650 \\
\hline $\mathrm{H}$ & 3.06468600 & 4.77827600 & 0.8703040 \\
\hline $\mathrm{C}$ & 5.65583900 & 4.89721500 & 1.8285950 \\
\hline $\mathrm{H}$ & 5.73056500 & 5.99163100 & 1.8129840 \\
\hline $\mathrm{H}$ & 6.63174700 & 4.48800800 & 2.0993780 \\
\hline $\mathrm{H}$ & 4.93198300 & 4.61941800 & 2.6034360 \\
\hline $\mathrm{C}$ & 8.22348900 & 2.31072200 & -0.24644200 \\
\hline $\mathrm{H}$ & 8.41783600 & 1.23658600 & -0.33853300 \\
\hline $\mathrm{H}$ & 9.17096400 & 2.84073700 & -0.12132900 \\
\hline $\mathrm{H}$ & 7.72800600 & 2.65568200 & -1.16061100 \\
\hline $\mathrm{C}$ & -0.00980300 & -2.31129700 & 0.57092500 \\
\hline $\mathrm{C}$ & -0.70630200 & -1.60068100 & -0.44271700 \\
\hline $\mathrm{C}$ & -0.98664900 & -2.22909500 & -1.66635600 \\
\hline $\mathrm{H}$ & -1.50370800 & -1.65953800 & -2.42794300 \\
\hline $\mathrm{C}$ & -0.69704600 & -3.57676300 & -1.92210800 \\
\hline $\mathrm{H}$ & -0.92563200 & -4.04067600 & -2.87460200 \\
\hline $\mathrm{C}$ & -0.12395200 & -4.28260000 & -0.88336200 \\
\hline $\mathrm{C}$ & 0.21951500 & -3.65608700 & 0.31001700 \\
\hline $\mathrm{C}$ & 0.57494900 & -5.82901300 & 0.54145000 \\
\hline $\mathrm{H}$ & -0.31281500 & -6.25171800 & 1.03436100 \\
\hline $\mathrm{H}$ & 1.43985500 & -6.48474300 & 0.62448700 \\
\hline $\mathrm{C}$ & 1.66908100 & -0.78407700 & 1.74960400 \\
\hline $\mathrm{C}$ & 2.32418900 & -0.43032900 & 2.93493900 \\
\hline $\mathrm{H}$ & 3.11504300 & 0.30757400 & 2.8969160 \\
\hline $\mathrm{C}$ & 2.02139100 & -1.01848400 & 4.17649000 \\
\hline $\mathrm{H}$ & 2.56124800 & -0.74925600 & 5.07720700 \\
\hline $\mathrm{C}$ & 1.01542200 & -1.95967600 & 4.18750500 \\
\hline $\mathrm{C}$ & 0.33076900 & -2.29911200 & 3.02645700 \\
\hline $\mathrm{C}$ & 0.61849100 & -1.75019200 & 1.79189700 \\
\hline
\end{tabular}




\begin{tabular}{|c|c|c|c|}
\hline $\mathrm{C}$ & -0.31802100 & -3.68262200 & 4.63798600 \\
\hline $\mathrm{H}$ & -1.23277000 & -3.80673500 & 5.21783000 \\
\hline $\mathrm{H}$ & 0.25326700 & -4.61965800 & 4.56657600 \\
\hline $\mathrm{C}$ & -0.01521400 & 1.95845000 & -4.65317700 \\
\hline $\mathrm{C}$ & 0.66500700 & 3.15132900 & -4.93919400 \\
\hline $\mathrm{H}$ & 0.55479200 & 1.02990900 & -4.66263600 \\
\hline $\mathrm{H}$ & 0.01860800 & 4.00488200 & -5.17072400 \\
\hline $\mathrm{C}$ & -1.36877000 & 1.87452000 & -4.24218400 \\
\hline $\mathrm{C}$ & -2.00328800 & 0.66791000 & -3.83108700 \\
\hline $\mathrm{H}$ & -1.95873600 & 2.78538000 & -4.31360100 \\
\hline $\mathrm{H}$ & -1.49368800 & -0.26251100 & -4.07362900 \\
\hline $\mathrm{C}$ & -3.45677500 & 0.55293400 & -3.65133400 \\
\hline $\mathrm{C}$ & -4.28355300 & 1.67766900 & -3.46746200 \\
\hline $\mathrm{C}$ & -4.06021700 & -0.71542900 & -3.67351200 \\
\hline $\mathrm{C}$ & -5.66345900 & 1.53756200 & -3.38103800 \\
\hline $\mathrm{H}$ & -3.84323600 & 2.66620800 & -3.38558300 \\
\hline $\mathrm{C}$ & -5.44099500 & -0.85721300 & -3.56454600 \\
\hline $\mathrm{H}$ & -3.43637600 & -1.59748000 & -3.79233000 \\
\hline $\mathrm{C}$ & -6.25139100 & 0.27126100 & -3.44249000 \\
\hline $\mathrm{H}$ & -6.28476900 & 2.42066400 & -3.26118200 \\
\hline $\mathrm{H}$ & -5.88322600 & -1.84894000 & -3.58898000 \\
\hline $\mathrm{H}$ & -7.33066400 & 0.16884500 & -3.39292500 \\
\hline $\mathrm{C}$ & 2.00845300 & 3.15371800 & -5.64558600 \\
\hline $\mathrm{H}$ & 2.56443200 & 4.06968900 & -5.41814400 \\
\hline $\mathrm{H}$ & 2.62484900 & 2.30752400 & -5.32692200 \\
\hline $\mathrm{H}$ & 1.89958400 & 3.09553900 & -6.73695100 \\
\hline S & 0.26622000 & 3.46821600 & -1.39352400 \\
\hline $\mathrm{C}$ & -1.16770200 & 4.57950600 & -1.37711100 \\
\hline $\mathrm{C}$ & -1.29443500 & 5.60122200 & -2.31512700 \\
\hline $\mathrm{C}$ & -2.18150500 & 4.31458000 & -0.45177200 \\
\hline $\mathrm{C}$ & -2.45542500 & 6.37772300 & -2.32248300 \\
\hline $\mathrm{H}$ & -0.48638500 & 5.78020900 & -3.01553900 \\
\hline $\mathrm{C}$ & -3.33203800 & 5.10126400 & -0.46074000 \\
\hline $\mathrm{H}$ & -2.06913700 & 3.50897400 & 0.26681400 \\
\hline $\mathrm{C}$ & -3.47188700 & 6.12868800 & -1.39844100 \\
\hline $\mathrm{H}$ & -2.56258900 & 7.18021800 & -3.04708100 \\
\hline $\mathrm{H}$ & -4.11760800 & 4.91387500 & 0.26375200 \\
\hline $\mathrm{H}$ & -4.37264900 & 6.73585600 & -1.40559000 \\
\hline $\mathrm{O}$ & 1.13711300 & 4.11151100 & -2.54554200 \\
\hline $\mathrm{O}$ & 0.96566300 & 3.64364200 & -0.07821500 \\
\hline $\mathrm{H}$ & 0.94969000 & 3.53698900 & -3.64170400 \\
\hline
\end{tabular}


<smiles>CCC1=CC(c2ccccc2)[P+]1P(O)(=P)S(=O)(=O)c1ccccc1</smiles>

Int-9a

Total SCF energy (M06/SDD-6-311++G(d,p)/SMD(1,4-Dioxane)): -5497.850832a.u.

Thermal correction to Gibbs Free Energy at 298.15 K: 1.769941a.u

Gibbs free energy at 298.15 K (M06/SDD-6-311++G(d,p)/SMD(1,4-Dioxane)): -5496.080891a.u.

$\mathrm{Pd}$

$P$

$P$

$\mathrm{O}$

$\mathrm{O}$

$\mathrm{O}$

$\mathrm{O}$

O

O

O

O

C

C

$\mathrm{H}$

C

C

C

C

$\mathrm{H}$

C

C

$\mathrm{H}$

$\mathrm{H}$

$\mathrm{H}$

C

$\mathrm{H}$

$\mathrm{H}$

$\mathrm{H}$

C

$\mathrm{H}$

$\mathrm{H}$

$\mathrm{H}$

C

C

H

$$
\begin{array}{ccc}
-2.12082900 & -1.68468300 & 2.31786100 \\
-1.74002900 & -0.39004700 & 0.35175700 \\
2.11636300 & -0.05064600 & 0.11471600
\end{array}
$$

$\begin{array}{lll}-7.25078700 & 1.60576800 & -0.98447100\end{array}$

$\begin{array}{lll}-1.00285900 & -3.68367600 & -4.60458400\end{array}$

$\begin{array}{lll}3.98338400 & 5.65617300 & 0.74294600\end{array}$

$\begin{array}{lll}7.31321000 & -3.06470600 & 0.00936300\end{array}$

$\begin{array}{lll}-0.13716600 & 5.24838300 & 0.07633700\end{array}$

$\begin{array}{lll}0.48169800 & 3.95779200 & -1.73073200\end{array}$

$\begin{array}{lll}-0.96056800 & 1.94968700 & -3.69053700\end{array}$

$\begin{array}{lll}0.11579700 & 0.90257400 & -5.44629800\end{array}$

$\begin{array}{lll}-3.40005600 & 0.28084500 & -0.10541600\end{array}$

$\begin{array}{lll}-4.46554300 & -0.62380900 & -0.18978800\end{array}$

$\begin{array}{lll}-4.26298600 & -1.66659200 & 0.01822800\end{array}$

$\begin{array}{lll}-5.75281100 & -0.22164900 & -0.53721300\end{array}$

$\begin{array}{lll}-5.95096500 & 1.16167300 & -0.80735100\end{array}$

$\begin{array}{lll}-4.87250500 & 2.05994600 & -0.91306900\end{array}$

$\begin{array}{lll}-3.61397300 & 1.59439200 & -0.49565600\end{array}$

$\begin{array}{lll}-2.78251100 & 2.28187200 & -0.50659300\end{array}$

$\begin{array}{lll}-6.87661500 & -1.26565200 & -0.70218600\end{array}$

$\begin{array}{lll}-6.34552700 & -2.69845700 & -0.49699100\end{array}$

$\begin{array}{lll}-5.53117600 & -2.93892500 & -1.18872000\end{array}$

$\begin{array}{lll}-7.15742100 & -3.40964900 & -0.68021300\end{array}$

$\begin{array}{lll}-5.98930300 & -2.86538700 & 0.52684000\end{array}$

$-8.02062500 \quad-1.05241000 \quad 0.31244600$

$\begin{array}{lll}-7.63230200 & -0.98016900 & 1.33556100\end{array}$

$\begin{array}{lll}-8.71031700 & -1.90376400 & 0.27627000\end{array}$

$\begin{array}{lll}-8.59443900 & -0.15322700 & 0.08972500\end{array}$

$\begin{array}{lll}-7.43612100 & -1.18209400 & -2.14084200\end{array}$

$\begin{array}{lll}-7.84193300 & -0.19079700 & -2.35035600\end{array}$

$\begin{array}{lll}-8.23614500 & -1.92021200 & -2.27426200\end{array}$

$-6.65097300 \quad-1.40027100 \quad-2.87323200$

$\begin{array}{lll}-4.90542400 & 3.48825200 & -1.50914100\end{array}$

$\begin{array}{lll}-4.60037100 & 4.52503700 & -0.40694000\end{array}$

$\begin{array}{lll}-5.38969200 & 4.53825600 & 0.35318200\end{array}$ 
$\mathrm{H}$

$\mathrm{H}$

C

$\mathrm{H}$

$\mathrm{H}$

$\mathrm{H}$

C

$\mathrm{H}$

$\mathrm{H}$

$\mathrm{H}$

C

$\mathrm{H}$

$\mathrm{H}$

$\mathrm{H}$

C

C

$\mathrm{H}$

C

C

C

C

$\mathrm{H}$

C

C

$\mathrm{H}$

$\mathrm{H}$

$\mathrm{H}$

C

$\mathrm{H}$

$\mathrm{H}$

$\mathrm{H}$

C

$\mathrm{H}$

$\mathrm{H}$

$\mathrm{H}$

C

C

$\mathrm{H}$

$\mathrm{H}$

$\mathrm{H}$

C

$\mathrm{H}$

$\mathrm{H}$

$\mathrm{H}$

\begin{tabular}{rrr}
-4.53734100 & 5.53081200 & -0.83954900 \\
-3.65323800 & 4.31134700 & 0.09894500 \\
-3.79537200 & 3.56977000 & -2.59115100 \\
-2.79328600 & 3.38222600 & -2.20133100 \\
-3.79801500 & 4.56934400 & -3.04208800 \\
-3.97874400 & 2.83684300 & -3.38413500 \\
-6.21346400 & 3.88016200 & -2.22530000 \\
-6.51298500 & 3.12627700 & -2.95756300 \\
-6.04480300 & 4.82403600 & -2.75700500 \\
-7.04909200 & 4.04005000 & -1.54378000 \\
-7.76440400 & 2.29062300 & 0.15670800 \\
-7.17442900 & 3.18356400 & 0.39009600 \\
-7.77467800 & 1.63797400 & 1.03754700 \\
-8.78647800 & 2.58551900 & -0.09199600 \\
-1.42236300 & -1.37447100 & -1.16365900 \\
-0.64872000 & -2.53083900 & -1.12419500 \\
-0.13392800 & -2.78358600 & -0.20558300 \\
-0.51730800 & -3.36725200 & -2.24896600 \\
-1.17149300 & -2.96619200 & -3.43257800 \\
-2.05203600 & -1.85423500 & -3.47330700 \\
-2.12585300 & -1.06137700 & -2.33398500 \\
-2.75538200 & -0.18822500 & -2.34292900 \\
-3.03995100 & -1.60465300 & -4.63524000 \\
-2.36852800 & -1.43979600 & -6.01520600 \\
-1.56419800 & -0.69915200 & -5.98461500 \\
-3.11500800 & -1.10211600 & -6.74449100 \\
-1.96164800 & -2.38368300 & -6.37624900 \\
-4.00431900 & -2.81255100 & -4.68775500 \\
-3.45750900 & -3.73795900 & -4.88498000 \\
-4.74679000 & -2.67185000 & -5.48325900 \\
-4.53969000 & -2.92137200 & -3.73717400 \\
-3.88623800 & -0.33798600 & -4.38630400 \\
-4.48676000 & -0.40993400 & -3.47386000 \\
-4.57707900 & -0.19769500 & -5.22448200 \\
-3.26210500 & 0.55893300 & -4.30564100 \\
0.30733300 & -4.66422200 & -2.05744800 \\
1.80376700 & -4.29443700 & -1.96620100 \\
2.16600400 & -3.84211600 & -2.89634600 \\
2.40411200 & -5.19127900 & -1.77198300 \\
\hline & -3.58381100 & -1.15694400 \\
-19233300 & -5.53688200 & -0.71671200
\end{tabular}




\begin{tabular}{|c|c|c|c|}
\hline $\mathrm{C}$ & 0.11359500 & -5.74468100 & -3.14249200 \\
\hline $\mathrm{H}$ & -0.94456000 & -5.96075700 & -3.31366700 \\
\hline $\mathrm{H}$ & 0.59578300 & -6.66530400 & -2.79385500 \\
\hline $\mathrm{H}$ & 0.56516800 & -5.48949000 & -4.10075600 \\
\hline $\mathrm{C}$ & 0.18359800 & -3.33228200 & -5.31485500 \\
\hline $\mathrm{H}$ & 0.16030100 & -2.28389200 & -5.62839900 \\
\hline $\mathrm{H}$ & 0.21885200 & -3.97884100 & -6.19503000 \\
\hline $\mathrm{H}$ & 1.07773900 & -3.49618600 & -4.70521800 \\
\hline $\mathrm{C}$ & 2.74644400 & 1.68538300 & 0.3009840 \\
\hline $\mathrm{C}$ & 3.27344700 & 2.41212500 & -0.76985300 \\
\hline $\mathrm{H}$ & 3.29561700 & 1.94478800 & -1.74317000 \\
\hline $\mathrm{C}$ & 3.74241100 & 3.71729000 & -0.63065600 \\
\hline $\mathrm{C}$ & 3.60293400 & 4.32601700 & 0.6426550 \\
\hline $\mathrm{C}$ & 3.16192700 & 3.60183100 & 1.7764860 \\
\hline $\mathrm{C}$ & 2.71548500 & 2.28628300 & 1.5578670 \\
\hline $\mathrm{H}$ & 2.32539100 & 1.70782900 & 2.3860540 \\
\hline $\mathrm{C}$ & 3.69187100 & -0.99947000 & -0.07640600 \\
\hline $\mathrm{C}$ & 4.80401700 & -0.51076200 & -0.75830500 \\
\hline $\mathrm{H}$ & 4.73629700 & 0.45447000 & -1.23981700 \\
\hline $\mathrm{C}$ & 6.00508400 & -1.22268800 & -0.85545700 \\
\hline $\mathrm{C}$ & 6.08934500 & -2.42612600 & -0.12224600 \\
\hline $\mathrm{C}$ & 4.95642400 & -3.01859300 & 0.48728600 \\
\hline $\mathrm{C}$ & 3.76776000 & -2.27746200 & 0.49445100 \\
\hline $\mathrm{H}$ & 2.87891800 & -2.68519300 & 0.95820900 \\
\hline $\mathrm{C}$ & 4.44496100 & 4.40874000 & -1.81971000 \\
\hline $\mathrm{C}$ & 5.86065900 & 4.85747500 & -1.39138100 \\
\hline $\mathrm{H}$ & 5.82129400 & 5.57662300 & -0.57271600 \\
\hline $\mathrm{H}$ & 6.37967800 & 5.31848800 & -2.24072100 \\
\hline $\mathrm{H}$ & 6.45120800 & 3.99368200 & -1.06483600 \\
\hline $\mathrm{C}$ & 4.61663800 & 3.44839700 & -3.01473800 \\
\hline $\mathrm{H}$ & 5.20408700 & 2.56407000 & -2.74875400 \\
\hline $\mathrm{H}$ & 5.15230500 & 3.96959600 & -3.81560500 \\
\hline $\mathrm{H}$ & 3.65590700 & 3.11535900 & -3.42234600 \\
\hline $\mathrm{C}$ & 3.64075900 & 5.61471400 & -2.34417900 \\
\hline $\mathrm{H}$ & 2.65868500 & 5.28457500 & -2.69785800 \\
\hline $\mathrm{H}$ & 4.16480300 & 6.07339400 & -3.19143400 \\
\hline $\mathrm{H}$ & 3.50779600 & 6.38284900 & -1.58329900 \\
\hline $\mathrm{C}$ & 3.16716200 & 4.15188600 & 3.2246840 \\
\hline $\mathrm{C}$ & 3.28681900 & 2.99323900 & 4.2411300 \\
\hline $\mathrm{H}$ & 2.39556000 & 2.36298000 & 4.2694370 \\
\hline $\mathrm{H}$ & 3.41824100 & 3.40964400 & 5.2460290 \\
\hline $\mathrm{H}$ & 4.15193600 & 2.35829700 & 4.0212240 \\
\hline $\mathrm{C}$ & 1.83514800 & 4.86963700 & 3.5392640 \\
\hline $\mathrm{H}$ & 1.64066200 & 5.71621400 & 2.8799750 \\
\hline
\end{tabular}


$\mathrm{H}$

$\mathrm{H}$

C

$\mathrm{H}$

$\mathrm{H}$

$\mathrm{H}$

C

$\mathrm{H}$

$\mathrm{H}$

$\mathrm{H}$

C

C

$\mathrm{H}$

$\mathrm{H}$

$\mathrm{H}$

C

$\mathrm{H}$

$\mathrm{H}$

$\mathrm{H}$

C

$\mathrm{H}$

$\mathrm{H}$

$\mathrm{H}$

C

C

$\mathrm{H}$

$\mathrm{H}$

$\mathrm{H}$

C

$\mathrm{H}$

$\mathrm{H}$

$\mathrm{H}$

C

$\mathrm{H}$

$\mathrm{H}$

$\mathrm{H}$

C

$\mathrm{H}$

$\mathrm{H}$

$\mathrm{H}$

C

C

C

$\mathrm{H}$
1.84180300

1.00105400

4.37550700

5.31159100

4.37730000

4.37937900

2.95091100

3.14795700

2.96057700

1.96622600

7.07501000

7.58562600

6.76577100

8.29698100

8.09561200

6.39213900

6.03457900

7.11249200

5.53849600

8.28454000

8.96692200

8.85429700

7.97189300

4.98422600

5.96516500

7.00488200

5.81431500

5.78715200

3.59669000

3.24561600

3.65791600

2.83082400

5.39657300

5.40610500

6.39104600

4.68078300

8.15077000

8.37410700

9.07901600

7.68407000

$-0.18111000$

$-0.79835100$

$-1.08766800$

$-1.54736900$
5.23942500

4.57225900

4.16876300

3.43404200

5.07226200

3.50822800

4.54421600

3.29456800

5.34435200

4.57011800

5.98713900

2.92150800

6.63086100

0.86957300

7.43235000

0.14949400

7.06530400

1.87531900

6.19734400

0.67764900

$-0.63556800$

$-1.80860700$

0.71230800

$-1.25389600$

1.42058400

$-1.09505000$

1.16816200

$-1.95319800$

0.57766300

$-0.29352100$

$-0.38946100$

$-3.18023200$

$-1.33511900$

$-3.60308900$

0.04719100

$-3.88168700$

0.28929800

$-3.11606400$

$-1.54414200$

$-2.10965600$

$-1.64805400$

$-1.26595600$

$-1.09522300$

$-2.93223700$

$-2.54473300$

$-2.41730200$

$-4.45501100$

1.05463300

$-4.60456600$

2.23847400

$-4.55252000$

1.91601700

$-5.57981600$

2.71607300

$-3.83320700$

2.99687500

$-4.88968900$

1.56376000

$-4.27826100$

2.39987500

$-5.92645300$

1.91252800

$-4.84554900$

0.78682200

$-5.42493800$

$-0.07648400$

$-6.45547400$

0.29931600

$-5.18764900$

$-0.46074200$

$-5.37445900$

$-0.90458600$

$-2.45629100$

0.99019200

$-1.41340000$

0.73961200

$-3.03270100$

1.00615200

$-2.48457200$

1.98108700

1.76594500

$-0.77937300$

1.18922000

0.37055500

1.97561200

1.49175900

1.51176300

2.35507800 


\begin{tabular}{|c|c|c|c|}
\hline $\mathrm{C}$ & -0.88890300 & 3.36567100 & 1.50870800 \\
\hline $\mathrm{H}$ & -1.14507900 & 3.96044400 & 2.37585000 \\
\hline $\mathrm{C}$ & -0.40851500 & 3.92694200 & 0.34526300 \\
\hline $\mathrm{C}$ & -0.04870600 & 3.14532400 & -0.75367300 \\
\hline $\mathrm{C}$ & 0.12057600 & 5.28163100 & -1.33413800 \\
\hline $\mathrm{H}$ & -0.79717800 & 5.58194500 & -1.86012700 \\
\hline $\mathrm{H}$ & 0.94673300 & 5.96141900 & -1.53420400 \\
\hline $\mathrm{C}$ & 1.52934800 & 0.10567000 & -1.64027700 \\
\hline $\mathrm{C}$ & 2.13700800 & -0.55705900 & -2.70832600 \\
\hline $\mathrm{H}$ & 2.96501900 & -1.22656300 & -2.51016800 \\
\hline $\mathrm{C}$ & 1.73998500 & -0.35433800 & -4.04442300 \\
\hline $\mathrm{H}$ & 2.23232700 & -0.86232200 & -4.86573300 \\
\hline $\mathrm{C}$ & 0.71018500 & 0.53486900 & -4.25763900 \\
\hline $\mathrm{C}$ & 0.07221500 & 1.17881200 & -3.20105500 \\
\hline $\mathrm{C}$ & 0.43527500 & 0.99108000 & -1.88386400 \\
\hline $\mathrm{C}$ & -0.76116900 & 1.98306200 & -5.10664200 \\
\hline $\mathrm{H}$ & -1.71884400 & 1.84269800 & -5.61017200 \\
\hline $\mathrm{H}$ & -0.29006200 & 2.93598900 & -5.38610800 \\
\hline $\mathrm{C}$ & -3.66018600 & -3.05971200 & 3.41839300 \\
\hline $\mathrm{C}$ & -3.29841900 & -4.31082900 & 4.16407200 \\
\hline $\mathrm{H}$ & -4.19415700 & -3.21528100 & 2.47780300 \\
\hline $\mathrm{H}$ & -4.08531700 & -4.50150600 & 4.91122200 \\
\hline $\mathrm{C}$ & -3.65974900 & -1.76496600 & 3.93052400 \\
\hline $\mathrm{C}$ & -3.95789700 & -0.66420100 & 3.06995100 \\
\hline $\mathrm{H}$ & -3.26796600 & -1.58849800 & 4.92990400 \\
\hline $\mathrm{H}$ & -4.66951100 & -0.83786500 & 2.26863300 \\
\hline $\mathrm{C}$ & -3.78351700 & 0.73560800 & 3.46368400 \\
\hline $\mathrm{C}$ & -2.87373200 & 1.12629300 & 4.46685900 \\
\hline $\mathrm{C}$ & -4.49999400 & 1.73743200 & 2.78507800 \\
\hline $\mathrm{C}$ & -2.69968800 & 2.47040600 & 4.78394100 \\
\hline $\mathrm{H}$ & -2.26376700 & 0.38004500 & 4.96648000 \\
\hline $\mathrm{C}$ & -4.32109300 & 3.08114100 & 3.10271500 \\
\hline $\mathrm{H}$ & -5.18803700 & 1.45205900 & 1.99585700 \\
\hline $\mathrm{C}$ & -3.42402400 & 3.45578600 & 4.10536000 \\
\hline $\mathrm{H}$ & -1.98572000 & 2.75073700 & 5.55335100 \\
\hline $\mathrm{H}$ & -4.87912500 & 3.83894800 & 2.56179300 \\
\hline $\mathrm{H}$ & -3.28547200 & 4.50451700 & 4.35244300 \\
\hline $\mathrm{C}$ & -3.16408800 & -5.51436000 & 3.22748700 \\
\hline $\mathrm{H}$ & -4.09669900 & -5.69225500 & 2.67673900 \\
\hline $\mathrm{H}$ & -2.35057800 & -5.34277000 & 2.51931200 \\
\hline $\mathrm{H}$ & -2.92572700 & -6.41941000 & 3.79420200 \\
\hline S & -0.12306100 & -2.97924800 & 2.64738300 \\
\hline $\mathrm{C}$ & 0.61681700 & -1.84954500 & 3.87086700 \\
\hline $\mathrm{C}$ & -0.04976000 & -1.64266000 & 5.08106800 \\
\hline
\end{tabular}




$\begin{array}{lrrr}\mathrm{C} & 1.83121200 & -1.22396900 & 3.60601900 \\ \mathrm{C} & 0.49515400 & -0.77471300 & 6.02695200 \\ \mathrm{H} & -0.97090900 & -2.17870200 & 5.28566800 \\ \mathrm{C} & 2.38704700 & -0.38028400 & 4.56909000 \\ \mathrm{H} & 2.32974700 & -1.40999700 & 2.66458100 \\ \mathrm{C} & 1.71552400 & -0.14206200 & 5.76999000 \\ \mathrm{H} & -0.01673100 & -0.60969400 & 6.97138600 \\ \mathrm{H} & 3.34593500 & 0.09054100 & 4.37861500 \\ \mathrm{H} & 2.14830300 & 0.52519900 & 6.51004700 \\ \mathrm{O} & 0.89895900 & -3.04581500 & 1.54418200 \\ \mathrm{O} & -0.33647200 & -4.25349900 & 3.40989100 \\ \mathrm{H} & -2.35800500 & -4.16847400 & 4.70369000\end{array}$

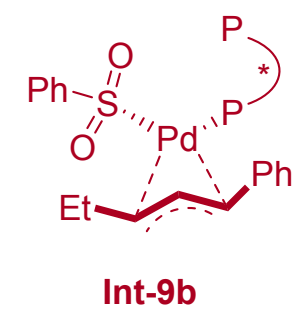

Total SCF energy (M06/SDD-6-311++G(d,p)/SMD(1,4-Dioxane)): -5497.848850a.u.

Thermal correction to Gibbs Free Energy at 298.15 K: 1.770834a.u

Gibbs free energy at 298.15 K (M06/SDD-6-311++G(d,p)/SMD(1,4-Dioxane)): -5496.078016a.u.

$\mathrm{Pd}$

$P$

$P$

$\mathrm{O}$

$\mathrm{O}$

$\mathrm{O}$

$\mathrm{O}$

$\mathrm{O}$

$\mathrm{O}$

O

O

C

C

$\mathrm{H}$

C

C

C

C

$\mathrm{H}$

C

C

$\mathrm{H}$

$\begin{array}{rcc}-0.85949300 & 1.43949200 & -2.01005700 \\ -1.48908400 & 0.00319100 & -0.24840400 \\ 2.08767800 & 0.09156900 & 0.09723600 \\ -7.30515900 & -1.15201400 & 0.56075700 \\ -1.35271000 & 1.12919100 & 5.64672900 \\ 3.57162500 & -4.28399900 & -3.76088700 \\ 7.43892300 & 2.65831600 & 1.05647000 \\ 0.38721300 & -5.60242600 & -1.04086300 \\ 1.05944800 & -4.58030400 & 0.90677900 \\ -0.46129700 & -3.41768500 & 3.17032600 \\ 0.66754300 & -2.88009500 & 5.10672300 \\ -3.27968200 & -0.43340000 & -0.28986000 \\ -4.18062000 & 0.61778000 & -0.11452900 \\ -3.80087600 & 1.62867300 & -0.17220300 \\ -5.52302800 & 0.39494900 & 0.19093800 \\ -5.96991600 & -0.95262300 & 0.24943700 \\ -5.08275500 & -2.04345500 & 0.09404600 \\ -3.74421700 & -1.73855600 & -0.18915500 \\ -3.03417300 & -2.54364400 & -0.27830300 \\ -6.41414800 & 1.58729600 & 0.60586800 \\ -5.69136600 & 2.93160100 & 0.38182900 \\ -4.78370200 & 3.01810100 & 0.98812500\end{array}$




\begin{tabular}{|c|c|c|c|}
\hline $\mathrm{H}$ & -6.35770000 & 3.74890400 & 0.67723800 \\
\hline $\mathrm{H}$ & -5.42150000 & 3.07870000 & -0.66966700 \\
\hline $\mathrm{C}$ & -7.75487400 & 1.66280900 & -0.15663800 \\
\hline $\mathrm{H}$ & -7.60890200 & 1.54608800 & -1.23335100 \\
\hline $\mathrm{H}$ & -8.21843700 & 2.64076500 & 0.01709500 \\
\hline $\mathrm{H}$ & -8.45503300 & 0.90435700 & 0.18763700 \\
\hline $\mathrm{C}$ & -6.70135800 & 1.45554000 & 2.11878500 \\
\hline $\mathrm{H}$ & -7.21055200 & 0.51448300 & 2.33989800 \\
\hline $\mathrm{H}$ & -7.33722400 & 2.28302600 & 2.45724600 \\
\hline $\mathrm{H}$ & -5.76742300 & 1.48988900 & 2.68931200 \\
\hline $\mathrm{C}$ & -5.45491000 & -3.53872100 & 0.23991000 \\
\hline $\mathrm{C}$ & -5.97781900 & -4.07526700 & -1.11273000 \\
\hline $\mathrm{H}$ & -6.87897100 & -3.56189000 & -1.45105600 \\
\hline $\mathrm{H}$ & -6.20859500 & -5.14467800 & -1.03380500 \\
\hline $\mathrm{H}$ & -5.21284200 & -3.94971700 & -1.88772300 \\
\hline $\mathrm{C}$ & -4.21034700 & -4.39039200 & 0.59771000 \\
\hline $\mathrm{H}$ & -3.48668000 & -4.45199000 & -0.22119500 \\
\hline $\mathrm{H}$ & -4.53409500 & -5.41389600 & 0.81499100 \\
\hline $\mathrm{H}$ & -3.69506800 & -4.00322400 & 1.48259000 \\
\hline $\mathrm{C}$ & -6.47090400 & -3.79499900 & 1.37426500 \\
\hline $\mathrm{H}$ & -6.06478200 & -3.44653600 & 2.32988800 \\
\hline $\mathrm{H}$ & -6.65372300 & -4.87248900 & 1.45878600 \\
\hline $\mathrm{H}$ & -7.42476500 & -3.29500800 & 1.22692500 \\
\hline $\mathrm{C}$ & -8.18399500 & -1.52009400 & -0.49976400 \\
\hline $\mathrm{H}$ & -8.38888900 & -2.59550100 & -0.47849400 \\
\hline $\mathrm{H}$ & -7.76499200 & -1.24581500 & -1.47103900 \\
\hline $\mathrm{H}$ & -9.12641500 & -0.98580500 & -0.35148300 \\
\hline $\mathrm{C}$ & -1.40688700 & 0.38798300 & 1.55040000 \\
\hline $\mathrm{C}$ & -0.75937600 & 1.51627700 & 2.03647100 \\
\hline $\mathrm{H}$ & -0.21468300 & 2.14988100 & 1.34888300 \\
\hline $\mathrm{C}$ & -0.77597900 & 1.84542500 & 3.40745400 \\
\hline $\mathrm{C}$ & -1.41666000 & 0.94316200 & 4.27706200 \\
\hline $\mathrm{C}$ & -2.18138000 & -0.15564500 & 3.80153800 \\
\hline $\mathrm{C}$ & -2.14282100 & -0.41485000 & 2.43594700 \\
\hline $\mathrm{H}$ & -2.69999400 & -1.24895400 & 2.04136300 \\
\hline $\mathrm{C}$ & -3.15264100 & -0.94186100 & 4.71012200 \\
\hline $\mathrm{C}$ & -2.48555500 & -1.55882800 & 5.95873900 \\
\hline $\mathrm{H}$ & -1.55922500 & -2.07817500 & 5.70524000 \\
\hline $\mathrm{H}$ & -3.17010300 & -2.28161300 & 6.41896700 \\
\hline $\mathrm{H}$ & -2.25652900 & -0.79865600 & 6.70471600 \\
\hline $\mathrm{C}$ & -4.25562400 & 0.03521600 & 5.17669700 \\
\hline $\mathrm{H}$ & -3.82400000 & 0.86788300 & 5.73790200 \\
\hline $\mathrm{H}$ & -4.97254500 & -0.48435800 & 5.82483200 \\
\hline $\mathrm{H}$ & -4.80428100 & 0.43757200 & 4.31933400 \\
\hline
\end{tabular}




\begin{tabular}{|c|c|c|c|}
\hline $\mathrm{C}$ & -3.82979700 & -2.09095700 & 3.93622500 \\
\hline $\mathrm{H}$ & -4.41473400 & -1.72800200 & 3.08521000 \\
\hline $\mathrm{H}$ & -4.51761400 & -2.61930300 & 4.60540200 \\
\hline $\mathrm{H}$ & -3.09249400 & -2.81253900 & 3.56582100 \\
\hline $\mathrm{C}$ & -0.12988900 & 3.19741000 & 3.80227500 \\
\hline $\mathrm{C}$ & 1.39570100 & 3.11869600 & 3.5685180 \\
\hline $\mathrm{H}$ & 1.85541300 & 2.35946600 & 4.2120240 \\
\hline $\mathrm{H}$ & 1.86151300 & 4.08466400 & 3.7997500 \\
\hline $\mathrm{H}$ & 1.62746000 & 2.87452600 & 2.5298720 \\
\hline $\mathrm{C}$ & -0.72607100 & 4.29365200 & 2.88069000 \\
\hline $\mathrm{H}$ & -0.46666500 & 4.14244800 & 1.83426900 \\
\hline $\mathrm{H}$ & -0.32885900 & 5.27204900 & 3.17513100 \\
\hline $\mathrm{H}$ & -1.81767000 & 4.32844400 & 2.98124400 \\
\hline $\mathrm{C}$ & -0.39259300 & 3.68183300 & 5.24366400 \\
\hline $\mathrm{H}$ & -1.45707100 & 3.67160000 & 5.49115800 \\
\hline $\mathrm{H}$ & -0.03560300 & 4.71557800 & 5.31954500 \\
\hline $\mathrm{H}$ & 0.13864000 & 3.10645000 & 6.0018730 \\
\hline $\mathrm{C}$ & -0.13668400 & 0.65416900 & 6.22593000 \\
\hline $\mathrm{H}$ & -0.06638600 & -0.43573800 & 6.16131000 \\
\hline $\mathrm{H}$ & -0.15557800 & 0.95994800 & 7.27478700 \\
\hline $\mathrm{H}$ & 0.73645000 & 1.09125800 & 5.7321020 \\
\hline $\mathrm{C}$ & 2.59160600 & -1.29512300 & -1.01593600 \\
\hline $\mathrm{C}$ & 3.24604800 & -2.46175600 & -0.60530400 \\
\hline $\mathrm{H}$ & 3.42647800 & -2.60330400 & 0.45083200 \\
\hline $\mathrm{C}$ & 3.64332400 & -3.44604200 & -1.51224300 \\
\hline $\mathrm{C}$ & 3.30786000 & -3.24788200 & -2.87740900 \\
\hline $\mathrm{C}$ & 2.73905300 & -2.04758900 & -3.34547700 \\
\hline $\mathrm{C}$ & 2.35991000 & -1.10737000 & -2.37376000 \\
\hline $\mathrm{H}$ & 1.85943800 & -0.19261800 & -2.67382100 \\
\hline $\mathrm{C}$ & 3.72746500 & 0.84925200 & 0.4823530 \\
\hline $\mathrm{C}$ & 4.83587300 & 0.08746100 & 0.8487970 \\
\hline $\mathrm{H}$ & 4.72878900 & -0.98583100 & 0.91941700 \\
\hline $\mathrm{C}$ & 6.07530700 & 0.65911600 & 1.15488100 \\
\hline $\mathrm{C}$ & 6.19302500 & 2.05502100 & 0.9668650 \\
\hline $\mathrm{C}$ & 5.07255100 & 2.87742700 & 0.7004330 \\
\hline $\mathrm{C}$ & 3.84862800 & 2.24189800 & 0.4568580 \\
\hline $\mathrm{H}$ & 2.96703800 & 2.82650900 & 0.2425180 \\
\hline $\mathrm{C}$ & 4.55083000 & -4.60797300 & -1.04884800 \\
\hline $\mathrm{C}$ & 5.94149100 & -4.39427900 & -1.69098500 \\
\hline $\mathrm{H}$ & 5.87096000 & -4.41883000 & -2.78156300 \\
\hline $\mathrm{H}$ & 6.63261000 & -5.18400900 & -1.37067100 \\
\hline $\mathrm{H}$ & 6.36483600 & -3.42958400 & -1.39253200 \\
\hline $\mathrm{C}$ & 4.72319400 & -4.60584500 & 0.48371000 \\
\hline $\mathrm{H}$ & 5.19900300 & -3.68880400 & 0.84572600 \\
\hline
\end{tabular}


H

$\mathrm{H}$

C

$\mathrm{H}$

$\mathrm{H}$

$\mathrm{H}$

C

C

$\mathrm{H}$

$\mathrm{H}$

$\mathrm{H}$

C

$\mathrm{H}$

$\mathrm{H}$

$\mathrm{H}$

C

$\mathrm{H}$

$\mathrm{H}$

$\mathrm{H}$

C

$\mathrm{H}$

$\mathrm{H}$

$\mathrm{H}$

C

C

$\mathrm{H}$

$\mathrm{H}$

$\mathrm{H}$

C

$\mathrm{H}$

$\mathrm{H}$

$\mathrm{H}$

C

$\mathrm{H}$

$\mathrm{H}$

$\mathrm{H}$

C

C

$\mathrm{H}$

$\mathrm{H}$

$\mathrm{H}$

C

$\mathrm{H}$

$\mathrm{H}$
$5.36937900 \quad-5.44097900$

$3.76349600-4.71956600$

$4.04222300 \quad-6.01076300$

2.98076300

4.59962100

4.18541300

2.50167000

3.00099300

2.52178200

2.81122300

4.07935300

0.98683200

0.61777100

0.76698700

0.41834400

3.26028000

4.32355800

3.16615400

2.86996100

2.42475600

2.77399100

1.67169700

1.96535300

7.15670900

7.62844000

6.78685900

8.34719900

8.11451300

6.51323200

6.15292800

7.25910100

5.66898900

8.38588900

9.04678700

8.97097300

8.09244700

5.14190200

6.10344600

7.14705600

5.98097700

5.87850500

3.75944000

3.36848100

3.85404100
$-6.13248700$

$-6.77507000$

$-6.20060200$

$-1.65977100$

$-0.20679600$

0.52471200

0.09711400

$-0.14484800$

$-1.72697100$

$-2.75680300$

$-1.33634700$

$-1.14636400$

$-2.50394600$

$-2.57751900$

$-2.01091500$

$-3.51480500$

$-5.08764900$

$-5.92859700$

$-4.52792200$

$-5.45971600$

$-0.29513300$

$-1.26066400$

$-1.81701200$

$-1.98546200$

$-0.72128800$

$-1.12759000$

$-0.47006400$

$-1.80751900$

$-1.73496500$

0.38848300

0.85367800

$-0.37494100$

1.15305300

4.41898100

5.01772600

4.80950800

6.10709100

4.63381800

5.05650600

4.84215300

6.14393300
0.77410300

1.00007000

$-1.44950600$

$-1.22247200$

$-0.89526400$

$-2.51242700$

$-4.82460400$

$-5.04090900$

$-4.38810300$

$-6.07683000$

$-4.86019200$

$-5.12059900$

$-5.07021200$

$-6.12169800$

$-4.38860000$

$-5.87037900$

$-5.62819600$

$-6.84543100$

$-5.98002700$

$-4.03827400$

$-4.64265100$

$-4.60373000$

$-3.11777500$

1.71995200

0.61174400

0.18682200

1.01327600

$-0.20844300$

2.86064700

3.65960600

3.28820100

2.52488900

2.35407000

1.62323000

2.88105200

3.07778800

0.76649600

$-0.28324100$

$-0.04766900$

$-0.31019000$

$-1.28493200$

0.52497100

$-0.47427500$

0.62691500 


\begin{tabular}{|c|c|c|c|}
\hline $\mathrm{H}$ & 3.01097200 & 4.71814100 & 1.24614100 \\
\hline $\mathrm{C}$ & 5.60929600 & 4.82668800 & 2.18259000 \\
\hline $\mathrm{H}$ & 5.65430100 & 5.92000700 & 2.26255700 \\
\hline $\mathrm{H}$ & 6.59921300 & 4.42265500 & 2.40688800 \\
\hline $\mathrm{H}$ & 4.90272500 & 4.46225300 & 2.93732000 \\
\hline $\mathrm{C}$ & 8.22015600 & 2.49363000 & -0.12460500 \\
\hline $\mathrm{H}$ & 8.44707900 & 1.43882500 & -0.31524200 \\
\hline $\mathrm{H}$ & 9.15213200 & 3.03991900 & 0.04115100 \\
\hline $\mathrm{H}$ & 7.70495900 & 2.90381500 & -1.00007300 \\
\hline $\mathrm{C}$ & 0.07093500 & -2.35378500 & 0.45836700 \\
\hline $\mathrm{C}$ & -0.70416200 & -1.64729300 & -0.50322900 \\
\hline $\mathrm{C}$ & -1.04380000 & -2.26967400 & -1.71455500 \\
\hline $\mathrm{H}$ & -1.63378000 & -1.71418400 & -2.42968200 \\
\hline $\mathrm{C}$ & -0.72172600 & -3.60008900 & -2.01614800 \\
\hline $\mathrm{H}$ & -0.99780900 & -4.05599500 & -2.95988000 \\
\hline $\mathrm{C}$ & -0.05444700 & -4.29983100 & -1.03158400 \\
\hline $\mathrm{C}$ & 0.33217600 & -3.68304800 & 0.15317500 \\
\hline $\mathrm{C}$ & 0.77271400 & -5.84778200 & 0.32124800 \\
\hline $\mathrm{H}$ & -0.06941300 & -6.31582600 & 0.85124500 \\
\hline $\mathrm{H}$ & 1.66576800 & -6.46966600 & 0.34186900 \\
\hline $\mathrm{C}$ & 1.71964100 & -0.80907400 & 1.67234600 \\
\hline $\mathrm{C}$ & 2.35689500 & -0.45818000 & 2.86903200 \\
\hline $\mathrm{H}$ & 3.11220100 & 0.31706200 & 2.85186900 \\
\hline $\mathrm{C}$ & 2.08288100 & -1.09540600 & 4.09221400 \\
\hline $\mathrm{H}$ & 2.60717200 & -0.82509700 & 5.00180500 \\
\hline $\mathrm{C}$ & 1.13301500 & -2.09321900 & 4.07097500 \\
\hline $\mathrm{C}$ & 0.46099200 & -2.42643300 & 2.90101600 \\
\hline $\mathrm{C}$ & 0.71020000 & -1.81687000 & 1.68564500 \\
\hline $\mathrm{C}$ & -0.08480300 & -3.91191500 & 4.45663600 \\
\hline $\mathrm{H}$ & -0.98075500 & -4.12924500 & 5.03793700 \\
\hline $\mathrm{H}$ & 0.55179400 & -4.80043400 & 4.33367800 \\
\hline $\mathrm{C}$ & -0.18669200 & 1.87308700 & -4.44992400 \\
\hline $\mathrm{C}$ & 0.53760700 & 3.07821900 & -4.95474200 \\
\hline $\mathrm{H}$ & 0.41142100 & 0.96976200 & -4.35895100 \\
\hline $\mathrm{C}$ & -1.54749500 & 1.76375300 & -4.24182000 \\
\hline $\mathrm{C}$ & -2.15089300 & 0.58788200 & -3.67702200 \\
\hline $\mathrm{H}$ & -2.16664300 & 2.64076400 & -4.40368700 \\
\hline $\mathrm{H}$ & -1.64256000 & -0.35107700 & -3.88210100 \\
\hline $\mathrm{C}$ & -3.60265000 & 0.49348900 & -3.50454200 \\
\hline $\mathrm{C}$ & -4.41173000 & 1.63778400 & -3.36093400 \\
\hline $\mathrm{C}$ & -4.23184300 & -0.76288000 & -3.52329600 \\
\hline $\mathrm{C}$ & -5.79622400 & 1.52833800 & -3.31424700 \\
\hline $\mathrm{H}$ & -3.95255500 & 2.61802400 & -3.27843000 \\
\hline $\mathrm{C}$ & -5.61768700 & -0.87377200 & -3.45464100 \\
\hline
\end{tabular}




\begin{tabular}{|c|c|c|c|}
\hline $\mathrm{H}$ & -3.62481700 & -1.65947800 & -3.61380800 \\
\hline $\mathrm{C}$ & -6.40814100 & 0.27323500 & -3.37237000 \\
\hline $\mathrm{H}$ & -6.40209500 & 2.42528000 & -3.22452600 \\
\hline $\mathrm{H}$ & -6.07916300 & -1.85651700 & -3.47941300 \\
\hline $\mathrm{H}$ & -7.49081600 & 0.19316300 & -3.34763100 \\
\hline S & 0.50502000 & 3.28049700 & -1.16275200 \\
\hline $\mathrm{C}$ & -0.79441700 & 4.55321200 & -1.28452600 \\
\hline $\mathrm{C}$ & -0.57364500 & 5.69814200 & -2.04401300 \\
\hline $\mathrm{C}$ & -2.00853600 & 4.34282000 & -0.62703400 \\
\hline $\mathrm{C}$ & -1.59068100 & 6.64959700 & -2.15314300 \\
\hline $\mathrm{H}$ & 0.38627700 & 5.82148700 & -2.53482000 \\
\hline $\mathrm{C}$ & -3.02162000 & 5.29404200 & -0.74353900 \\
\hline $\mathrm{H}$ & -2.14932700 & 3.45160500 & -0.02185900 \\
\hline $\mathrm{C}$ & -2.81351900 & 6.44520200 & -1.51005600 \\
\hline $\mathrm{H}$ & -1.42936900 & 7.54906400 & -2.74119400 \\
\hline $\mathrm{H}$ & -3.96664500 & 5.14333900 & -0.23062100 \\
\hline $\mathrm{H}$ & -3.60386000 & 7.18526000 & -1.60003400 \\
\hline $\mathrm{O}$ & 1.58208100 & 3.73084100 & -2.11450000 \\
\hline $\mathrm{O}$ & 0.92007100 & 3.34054600 & 0.28444800 \\
\hline $\mathrm{H}$ & 1.13712000 & 2.76374200 & -5.82232000 \\
\hline $\mathrm{C}$ & -0.34200400 & 4.27372500 & -5.31976300 \\
\hline $\mathrm{H}$ & -1.05336600 & 4.02771600 & -6.11750700 \\
\hline $\mathrm{H}$ & -0.90620900 & 4.62890800 & -4.45223200 \\
\hline $\mathrm{H}$ & 0.27900800 & 5.10464300 & -5.66894000 \\
\hline $\mathrm{H}$ & 1.25616900 & 3.36629100 & -4.17403800 \\
\hline
\end{tabular}

Total SCF energy (M06/SDD-6-311++G(d,p)/SMD(1,4-Dioxane)): -5497.845306a.u. Thermal correction to Gibbs Free Energy at $298.15 \mathrm{~K}: 1.766506 \mathrm{a}$.u

Gibbs free energy at 298.15 K (M06/SDD-6-311++G(d,p)/SMD(1,4-Dioxane)): -5496.078800a.u.

$\begin{array}{cccc}\mathrm{Pd} & 1.55005500 & -2.26568800 & 1.30484700 \\ \mathrm{P} & 1.75190700 & -0.19260200 & 0.28140800 \\ \mathrm{P} & -1.99147400 & 0.54449000 & 0.05951500 \\ \mathrm{O} & 7.53996700 & 1.18037400 & -0.43927600 \\ \mathrm{O} & 0.36654300 & 4.30256900 & 3.98278100 \\ \mathrm{O} & -2.59843000 & -2.70513100 & -4.95803400 \\ \mathrm{O} & -7.80523600 & 1.47222500 & 1.22424800 \\ \mathrm{O} & 0.53494800 & 0.24762700 & -5.49258400 \\ \mathrm{O} & -0.21427500 & 1.99354100 & -4.18721300 \\ \mathrm{O} & 1.54527100 & 3.77398600 & -2.26387100\end{array}$




\begin{tabular}{|c|c|c|c|}
\hline $\mathrm{O}$ & 0.71708900 & 5.71148600 & -1.32275100 \\
\hline $\mathrm{C}$ & 3.50035200 & 0.29190100 & 0.0004450 \\
\hline $\mathrm{C}$ & 4.34519100 & 0.33695400 & 1.1165790 \\
\hline $\mathrm{H}$ & 3.92392500 & 0.09604200 & 2.0837500 \\
\hline $\mathrm{C}$ & 5.68589200 & 0.69677000 & 1.0105790 \\
\hline $\mathrm{C}$ & 6.17499000 & 1.00194600 & -0.29062900 \\
\hline $\mathrm{C}$ & 5.31663300 & 1.14004400 & -1.39799100 \\
\hline $\mathrm{C}$ & 3.98559500 & 0.72212900 & -1.22569100 \\
\hline $\mathrm{H}$ & 3.31334600 & 0.75477800 & -2.07125400 \\
\hline $\mathrm{C}$ & 6.55693400 & 0.84342500 & 2.2750290 \\
\hline $\mathrm{C}$ & 5.73097500 & 0.60453000 & 3.5546990 \\
\hline $\mathrm{H}$ & 4.87741300 & 1.28705100 & 3.6266560 \\
\hline $\mathrm{H}$ & 6.36832900 & 0.77716900 & 4.4279590 \\
\hline $\mathrm{H}$ & 5.35710000 & -0.42439500 & 3.61984400 \\
\hline $\mathrm{C}$ & 7.73200400 & -0.15755300 & 2.29410800 \\
\hline $\mathrm{H}$ & 7.38000800 & -1.18220200 & 2.12426700 \\
\hline $\mathrm{H}$ & 8.22368100 & -0.13141300 & 3.27357100 \\
\hline $\mathrm{H}$ & 8.48151900 & 0.08635500 & 1.5417410 \\
\hline $\mathrm{C}$ & 7.10290000 & 2.28841700 & 2.34062000 \\
\hline $\mathrm{H}$ & 7.71544900 & 2.52008700 & 1.4674120 \\
\hline $\mathrm{H}$ & 7.71788300 & 2.41545200 & 3.2396390 \\
\hline $\mathrm{H}$ & 6.27920400 & 3.00944400 & 2.3910110 \\
\hline $\mathrm{C}$ & 5.66483200 & 1.77753200 & -2.76531000 \\
\hline $\mathrm{C}$ & 5.58586200 & 0.71398700 & -3.88129400 \\
\hline $\mathrm{H}$ & 6.36676300 & -0.04512900 & -3.76256400 \\
\hline $\mathrm{H}$ & 5.72578800 & 1.18446600 & -4.86183600 \\
\hline $\mathrm{H}$ & 4.61894200 & 0.20023600 & -3.88422900 \\
\hline $\mathrm{C}$ & 4.60426200 & 2.87353500 & -3.04857700 \\
\hline $\mathrm{H}$ & 3.58733900 & 2.48480600 & -3.11470000 \\
\hline $\mathrm{H}$ & 4.83603200 & 3.36931600 & -3.99861000 \\
\hline $\mathrm{H}$ & 4.61464800 & 3.62941800 & -2.25527100 \\
\hline $\mathrm{C}$ & 7.03090500 & 2.48884500 & -2.84205100 \\
\hline $\mathrm{H}$ & 7.16304300 & 3.19842900 & -2.02150400 \\
\hline $\mathrm{H}$ & 7.07407500 & 3.04639000 & -3.78502700 \\
\hline $\mathrm{H}$ & 7.87818700 & 1.80298000 & -2.83550900 \\
\hline $\mathrm{C}$ & 8.18656900 & 0.05068800 & -1.02321000 \\
\hline $\mathrm{H}$ & 7.80410800 & -0.15640400 & -2.02859900 \\
\hline $\mathrm{H}$ & 8.05161600 & -0.84482300 & -0.40531900 \\
\hline $\mathrm{H}$ & 9.24903400 & 0.29749300 & -1.08292400 \\
\hline $\mathrm{C}$ & 1.22960600 & 1.17556100 & 1.3736660 \\
\hline $\mathrm{C}$ & 0.32263700 & 0.96472700 & 2.4106370 \\
\hline $\mathrm{H}$ & -0.14509900 & -0.00566400 & 2.48976000 \\
\hline $\mathrm{C}$ & 0.00634100 & 1.98067000 & 3.3283180 \\
\hline $\mathrm{C}$ & 0.64092300 & 3.23453300 & 3.1469950 \\
\hline
\end{tabular}




\begin{tabular}{|c|c|c|c|}
\hline $\mathrm{C}$ & 1.64467000 & 3.44312900 & 2.16672300 \\
\hline $\mathrm{C}$ & 1.88798300 & 2.40398200 & 1.2746080 \\
\hline $\mathrm{H}$ & 2.61946300 & 2.53645500 & 0.4941270 \\
\hline $\mathrm{C}$ & 2.56688200 & 4.68226000 & 2.1516050 \\
\hline $\mathrm{C}$ & 1.81109800 & 6.01981000 & 2.0147780 \\
\hline $\mathrm{H}$ & 1.14484700 & 6.01448700 & 1.1486240 \\
\hline $\mathrm{H}$ & 2.53369700 & 6.83230700 & 1.8721080 \\
\hline $\mathrm{H}$ & 1.23553400 & 6.24698000 & 2.9095930 \\
\hline $\mathrm{C}$ & 3.37271500 & 4.68481000 & 3.4713880 \\
\hline $\mathrm{H}$ & 2.70735300 & 4.75252200 & 4.3350330 \\
\hline $\mathrm{H}$ & 4.06050900 & 5.53930200 & 3.4937970 \\
\hline $\mathrm{H}$ & 3.96737500 & 3.76795600 & 3.5621830 \\
\hline $\mathrm{C}$ & 3.57810500 & 4.61510300 & 0.9871810 \\
\hline $\mathrm{H}$ & 4.22930300 & 3.73667300 & 1.0493100 \\
\hline $\mathrm{H}$ & 4.21839400 & 5.50294200 & 1.0200160 \\
\hline $\mathrm{H}$ & 3.07588400 & 4.59887900 & 0.0141640 \\
\hline $\mathrm{C}$ & -0.97053700 & 1.64691000 & 4.48197400 \\
\hline $\mathrm{C}$ & -2.41156100 & 2.05936200 & 4.10338200 \\
\hline $\mathrm{H}$ & -2.52882300 & 3.13586100 & 3.97487400 \\
\hline $\mathrm{H}$ & -3.10768700 & 1.74105600 & 4.88893100 \\
\hline $\mathrm{H}$ & -2.71730800 & 1.57421200 & 3.17295800 \\
\hline $\mathrm{C}$ & -1.00972700 & 0.12225700 & 4.73776700 \\
\hline $\mathrm{H}$ & -1.51448600 & -0.43098300 & 3.94407500 \\
\hline $\mathrm{H}$ & -1.57698500 & -0.06849000 & 5.65503200 \\
\hline $\mathrm{H}$ & -0.00292300 & -0.29026200 & 4.87376200 \\
\hline $\mathrm{C}$ & -0.54430200 & 2.29277000 & 5.81867800 \\
\hline $\mathrm{H}$ & 0.46720800 & 1.96929500 & 6.0914510 \\
\hline $\mathrm{H}$ & -1.22697100 & 1.96141500 & 6.60936600 \\
\hline $\mathrm{H}$ & -0.54374500 & 3.38047300 & 5.79838700 \\
\hline $\mathrm{C}$ & -0.84409900 & 5.02146800 & 3.74686900 \\
\hline $\mathrm{H}$ & -1.30873800 & 4.71148900 & 2.80768200 \\
\hline $\mathrm{H}$ & -0.60702300 & 6.08835500 & 3.69291800 \\
\hline $\mathrm{H}$ & -1.54253200 & 4.85850100 & 4.57353600 \\
\hline $\mathrm{C}$ & -2.22082000 & -0.36148300 & -1.53042600 \\
\hline $\mathrm{C}$ & -2.72882700 & 0.18868500 & -2.71228500 \\
\hline $\mathrm{H}$ & -2.97276900 & 1.24159500 & -2.72635500 \\
\hline $\mathrm{C}$ & -2.90853900 & -0.58238500 & -3.86247600 \\
\hline $\mathrm{C}$ & -2.54246400 & -1.95441100 & -3.79086900 \\
\hline $\mathrm{C}$ & -2.14783800 & -2.56588300 & -2.58776300 \\
\hline $\mathrm{C}$ & -1.95510800 & -1.72466700 & -1.47952200 \\
\hline $\mathrm{H}$ & -1.58740500 & -2.13336700 & -0.54366800 \\
\hline $\mathrm{C}$ & -3.74233200 & 0.97813600 & 0.44752300 \\
\hline $\mathrm{C}$ & -4.48255400 & 1.93120900 & -0.24961700 \\
\hline $\mathrm{H}$ & -3.99400000 & 2.49490900 & -1.03453500 \\
\hline
\end{tabular}




\begin{tabular}{|c|c|c|c|}
\hline $\mathrm{C}$ & -5.82129300 & 2.21166600 & 0.05010400 \\
\hline $\mathrm{C}$ & -6.43377700 & 1.39720800 & 1.02715800 \\
\hline $\mathrm{C}$ & -5.69612000 & 0.49928400 & 1.83681500 \\
\hline $\mathrm{C}$ & -4.34856800 & 0.29692400 & 1.51139000 \\
\hline $\mathrm{H}$ & -3.75461900 & -0.41824900 & 2.07021000 \\
\hline $\mathrm{C}$ & -3.64128300 & 0.01225100 & -5.08639100 \\
\hline $\mathrm{C}$ & -5.04068900 & -0.64307400 & -5.14656000 \\
\hline $\mathrm{H}$ & -4.95394000 & -1.72547800 & -5.27587300 \\
\hline $\mathrm{H}$ & -5.61676100 & -0.23887600 & -5.98884800 \\
\hline $\mathrm{H}$ & -5.59827200 & -0.44766500 & -4.22394200 \\
\hline $\mathrm{C}$ & -3.83028200 & 1.53506600 & -4.93861600 \\
\hline $\mathrm{H}$ & -4.45582600 & 1.78818900 & -4.07782300 \\
\hline $\mathrm{H}$ & -4.33172200 & 1.92686900 & -5.83010000 \\
\hline $\mathrm{H}$ & -2.87157500 & 2.05392300 & -4.82629100 \\
\hline $\mathrm{C}$ & -2.93264900 & -0.22668700 & -6.43807900 \\
\hline $\mathrm{H}$ & -1.87421600 & 0.04139400 & -6.38999600 \\
\hline $\mathrm{H}$ & -3.40528800 & 0.39004500 & -7.21193900 \\
\hline $\mathrm{H}$ & -3.00585200 & -1.26748900 & -6.74979500 \\
\hline $\mathrm{C}$ & -1.96181400 & -4.07824700 & -2.32012700 \\
\hline $\mathrm{C}$ & -2.87663100 & -4.44391900 & -1.12283500 \\
\hline $\mathrm{H}$ & -2.66397400 & -3.85259600 & -0.23279600 \\
\hline $\mathrm{H}$ & -2.74558900 & -5.49776400 & -0.85701300 \\
\hline $\mathrm{H}$ & -3.92706500 & -4.28403000 & -1.39142100 \\
\hline $\mathrm{C}$ & -0.48714100 & -4.34396000 & -1.94229300 \\
\hline $\mathrm{H}$ & 0.18253400 & -4.09351500 & -2.77364200 \\
\hline $\mathrm{H}$ & -0.34523800 & -5.40262200 & -1.69328500 \\
\hline $\mathrm{H}$ & -0.18333300 & -3.74954200 & -1.07635500 \\
\hline $\mathrm{C}$ & -2.36206600 & -5.03946200 & -3.45686000 \\
\hline $\mathrm{H}$ & -3.36808100 & -4.83289000 & -3.82833100 \\
\hline $\mathrm{H}$ & -2.34833400 & -6.06061900 & -3.05665200 \\
\hline $\mathrm{H}$ & -1.67576800 & -5.01898000 & -4.30481600 \\
\hline $\mathrm{C}$ & -1.32032500 & -2.86780600 & -5.56946100 \\
\hline $\mathrm{H}$ & -1.46982100 & -3.51767600 & -6.43560600 \\
\hline $\mathrm{H}$ & -0.60745900 & -3.33693600 & -4.88234200 \\
\hline $\mathrm{H}$ & -0.90833900 & -1.90754600 & -5.89516600 \\
\hline $\mathrm{C}$ & -6.46162200 & 3.42250200 & -0.67269000 \\
\hline $\mathrm{C}$ & -6.63861900 & 3.10537700 & -2.17260500 \\
\hline $\mathrm{H}$ & -5.68085500 & 2.86050200 & -2.64211700 \\
\hline $\mathrm{H}$ & -7.06173200 & 3.96913800 & -2.69992800 \\
\hline $\mathrm{H}$ & -7.30977400 & 2.25336400 & -2.32560700 \\
\hline $\mathrm{C}$ & -5.49440100 & 4.62775600 & -0.53175500 \\
\hline $\mathrm{H}$ & -5.34464000 & 4.87597800 & 0.52512700 \\
\hline $\mathrm{H}$ & -5.92207100 & 5.50543000 & -1.03018600 \\
\hline $\mathrm{H}$ & -4.51236000 & 4.44337500 & -0.97433400 \\
\hline
\end{tabular}




\begin{tabular}{|c|c|c|c|}
\hline $\mathrm{C}$ & -7.80922700 & 3.90731900 & -0.09816000 \\
\hline $\mathrm{H}$ & -8.63711300 & 3.23034500 & -0.30757300 \\
\hline $\mathrm{H}$ & -8.05290100 & 4.87130200 & -0.56105200 \\
\hline $\mathrm{H}$ & -7.75763400 & 4.05194900 & 0.98384600 \\
\hline $\mathrm{C}$ & -6.30928800 & -0.15672200 & 3.09370500 \\
\hline $\mathrm{C}$ & -7.44908600 & -1.14036400 & 2.75013900 \\
\hline $\mathrm{H}$ & -8.34017900 & -0.62449800 & 2.39216100 \\
\hline $\mathrm{H}$ & -7.73056000 & -1.70282900 & 3.64852900 \\
\hline $\mathrm{H}$ & -7.12696000 & -1.86349500 & 1.99205800 \\
\hline $\mathrm{C}$ & -5.25297900 & -0.94641400 & 3.88753200 \\
\hline $\mathrm{H}$ & -4.83823400 & -1.78680000 & 3.32736000 \\
\hline $\mathrm{H}$ & -5.71944600 & -1.34139900 & 4.79752800 \\
\hline $\mathrm{H}$ & -4.41262400 & -0.31638300 & 4.19354000 \\
\hline $\mathrm{C}$ & -6.84921200 & 0.95656800 & 4.02134200 \\
\hline $\mathrm{H}$ & -7.26976600 & 0.51124500 & 4.93154600 \\
\hline $\mathrm{H}$ & -7.62793500 & 1.54465100 & 3.53200300 \\
\hline $\mathrm{H}$ & -6.03748300 & 1.63003400 & 4.32047900 \\
\hline $\mathrm{C}$ & -8.54319600 & 0.74908000 & 0.24198300 \\
\hline $\mathrm{H}$ & -8.40397800 & 1.17075400 & -0.75975700 \\
\hline $\mathrm{H}$ & -9.59600900 & 0.82811800 & 0.52460400 \\
\hline $\mathrm{H}$ & -8.24636800 & -0.30539200 & 0.22108200 \\
\hline $\mathrm{C}$ & 0.45227300 & 1.05500200 & -1.99948500 \\
\hline $\mathrm{C}$ & 1.07669400 & -0.08184400 & -1.41610800 \\
\hline $\mathrm{C}$ & 1.47058700 & -1.16511900 & -2.21616700 \\
\hline $\mathrm{H}$ & 1.93599100 & -2.02332500 & -1.74789900 \\
\hline $\mathrm{C}$ & 1.33679900 & -1.16488800 & -3.61149300 \\
\hline $\mathrm{H}$ & 1.65994200 & -2.00825500 & -4.20954600 \\
\hline $\mathrm{C}$ & 0.79174500 & -0.02737000 & -4.17111600 \\
\hline $\mathrm{C}$ & 0.35470200 & 1.03256700 & -3.38126600 \\
\hline $\mathrm{C}$ & 0.18218000 & 1.63997500 & -5.51107700 \\
\hline $\mathrm{H}$ & 1.06501900 & 2.22821200 & -5.80031000 \\
\hline $\mathrm{H}$ & -0.65175900 & 1.79417300 & -6.19475500 \\
\hline $\mathrm{C}$ & -1.26881300 & 2.17146300 & -0.44184500 \\
\hline $\mathrm{C}$ & -1.71988700 & 3.35468700 & 0.16515200 \\
\hline $\mathrm{H}$ & -2.57310600 & 3.30677300 & 0.83086500 \\
\hline $\mathrm{C}$ & -1.13783500 & 4.61100300 & -0.07531300 \\
\hline $\mathrm{H}$ & -1.51904500 & 5.51265900 & 0.39075400 \\
\hline $\mathrm{C}$ & -0.06454200 & 4.64062900 & -0.93944600 \\
\hline $\mathrm{C}$ & 0.42559000 & 3.47592700 & -1.51312600 \\
\hline $\mathrm{C}$ & -0.12123200 & 2.22562800 & -1.28677100 \\
\hline $\mathrm{C}$ & 1.55311700 & 5.20075200 & -2.36545400 \\
\hline $\mathrm{H}$ & 2.57179300 & 5.56669600 & -2.22776800 \\
\hline $\mathrm{H}$ & 1.14388900 & 5.49835900 & -3.34188600 \\
\hline $\mathrm{C}$ & 2.50985900 & -3.80941500 & 3.18490200 \\
\hline
\end{tabular}




\begin{tabular}{lrrr}
$\mathrm{C}$ & 1.75378900 & -4.80049600 & 4.01234800 \\
$\mathrm{H}$ & 2.75490700 & -2.86393600 & 3.67302800 \\
$\mathrm{H}$ & 1.53982800 & -5.69856500 & 3.42314100 \\
$\mathrm{C}$ & 2.99295200 & -4.03311400 & 1.92036000 \\
$\mathrm{C}$ & 3.54939400 & -2.96331000 & 1.10097300 \\
$\mathrm{H}$ & 2.79600600 & -4.99942100 & 1.45898600 \\
$\mathrm{H}$ & 4.19675800 & -2.24787500 & 1.60492200 \\
$\mathrm{C}$ & 3.89781200 & -3.22068200 & -0.31137800 \\
$\mathrm{C}$ & 3.16777600 & -4.13612200 & -1.09640900 \\
$\mathrm{C}$ & 4.92775800 & -2.49248200 & -0.92830000 \\
$\mathrm{C}$ & 3.45267700 & -4.30598100 & -2.44835000 \\
$\mathrm{H}$ & 2.33990200 & -4.68562300 & -0.65939500 \\
$\mathrm{C}$ & 5.21121100 & -2.66330300 & -2.28147600 \\
$\mathrm{H}$ & 5.49445900 & -1.77680800 & -0.34272700 \\
$\mathrm{C}$ & 4.47442800 & -3.56533800 & -3.05036100 \\
$\mathrm{H}$ & 2.86635300 & -5.00803600 & -3.03434800 \\
$\mathrm{H}$ & 6.00680600 & -2.08297600 & -2.73724600 \\
$\mathrm{H}$ & 4.69382600 & -3.69212300 & -4.10655000 \\
$\mathrm{C}$ & 2.50159100 & -5.16711500 & 5.30540300 \\
$\mathrm{H}$ & 3.46246500 & -5.64506900 & 5.08519200 \\
$\mathrm{H}$ & 2.69850700 & -4.27617900 & 5.91281600 \\
$\mathrm{H}$ & 1.90456200 & -5.85935100 & 5.90775900 \\
$\mathrm{~S}$ & -1.23517300 & -2.93171700 & 2.76696100 \\
$\mathrm{C}$ & -1.55556400 & -4.63258100 & 2.16679000 \\
$\mathrm{C}$ & -2.76597900 & -5.24048100 & 2.49336600 \\
$\mathrm{C}$ & -0.57648600 & -5.33490100 & 1.46349100 \\
$\mathrm{C}$ & -2.99892700 & -6.56253600 & 2.10862200 \\
$\mathrm{H}$ & -3.51498800 & -4.66111900 & 3.02374700 \\
$\mathrm{C}$ & -0.80747600 & -6.65777200 & 1.08815700 \\
$\mathrm{H}$ & 0.34591300 & -4.83753400 & 1.18413800 \\
$\mathrm{H}$ & -2.01912100 & -7.27431000 & 1.41311600 \\
$\mathrm{H}$ & -3.94641400 & -7.03715500 & 2.34926100 \\
$\mathrm{H}$ & -0.05129800 & -7.20341000 & 0.52920400 \\
$\mathrm{H}$ & -2.2080400 & -8.30407200 & 1.11813600 \\
$\mathrm{H}$ & -2.6574900 & -2.28990400 & 1.46101800 \\
$\mathrm{H}$ & -2.37572900 & 2.99706100 \\
& -4.35450700 & 4.27064200 \\
\hline
\end{tabular}<smiles></smiles>

Int-10b 
Total SCF energy (M06/SDD-6-311++G(d,p)/SMD(1,4-Dioxane)): -5497.848828a.u.

Thermal correction to Gibbs Free Energy at 298.15 K: 1.766551a.u

Gibbs free energy at $298.15 \mathrm{~K}$ (M06/SDD-6-311++G(d,p)/SMD(1,4-Dioxane)): -5496.082277a.u.

\begin{tabular}{|c|c|c|c|}
\hline $\mathrm{Pd}$ & -1.25391200 & -2.03928700 & 1.97515900 \\
\hline $\mathrm{P}$ & -1.67875600 & -0.43593500 & 0.28415200 \\
\hline $\mathrm{P}$ & 1.89962300 & 0.42538400 & -0.35606400 \\
\hline $\mathrm{O}$ & -7.49851800 & 0.13523900 & -1.09913900 \\
\hline $\mathrm{O}$ & -0.35421400 & -1.73084500 & -5.41956700 \\
\hline $\mathrm{O}$ & 2.26302500 & 3.75304300 & 4.62741300 \\
\hline $\mathrm{O}$ & 7.77495200 & 0.02175000 & -1.51219700 \\
\hline $\mathrm{O}$ & -1.07468000 & 5.23612800 & 1.92679200 \\
\hline $\mathrm{O}$ & -0.32427100 & 4.74358900 & -0.19753800 \\
\hline $\mathrm{O}$ & -1.84016700 & 3.50063900 & -2.56285100 \\
\hline $\mathrm{O}$ & -0.85412200 & 3.57189400 & -4.65087500 \\
\hline $\mathrm{C}$ & -3.49050400 & -0.22295200 & 0.01257200 \\
\hline $\mathrm{C}$ & -4.19366500 & -1.32030100 & -0.49905700 \\
\hline $\mathrm{H}$ & -3.66464500 & -2.25805800 & -0.60740200 \\
\hline $\mathrm{C}$ & -5.52125200 & -1.22002900 & -0.91031100 \\
\hline $\mathrm{C}$ & -6.15563100 & 0.04571700 & -0.76963000 \\
\hline $\mathrm{C}$ & -5.44413500 & 1.19701900 & -0.37562100 \\
\hline $\mathrm{C}$ & -4.12446900 & 1.00929100 & 0.06869700 \\
\hline $\mathrm{H}$ & -3.56533100 & 1.86673800 & 0.41279900 \\
\hline $\mathrm{C}$ & -6.21682500 & -2.42736200 & -1.57591500 \\
\hline $\mathrm{C}$ & -5.24168300 & -3.60813200 & -1.75667200 \\
\hline $\mathrm{H}$ & -4.36751900 & -3.32997800 & -2.35481600 \\
\hline $\mathrm{H}$ & -5.75803500 & -4.41948000 & -2.28015200 \\
\hline $\mathrm{H}$ & -4.89047900 & -4.00698600 & -0.79773600 \\
\hline $\mathrm{C}$ & -7.40379200 & -2.94146700 & -0.73459800 \\
\hline $\mathrm{H}$ & -7.09714800 & -3.14312900 & 0.29868800 \\
\hline $\mathrm{H}$ & -7.78140400 & -3.87927300 & -1.15881500 \\
\hline $\mathrm{H}$ & -8.22895800 & -2.23003900 & -0.72474100 \\
\hline $\mathrm{C}$ & -6.71642300 & -2.02981400 & -2.98392200 \\
\hline $\mathrm{H}$ & -7.43486900 & -1.21080200 & -2.93532800 \\
\hline $\mathrm{H}$ & -7.19910400 & -2.88996000 & -3.46357600 \\
\hline $\mathrm{H}$ & -5.87853300 & -1.71977500 & -3.61644700 \\
\hline $\mathrm{C}$ & -5.91891700 & 2.67022800 & -0.47489300 \\
\hline $\mathrm{C}$ & -6.08306600 & 3.26261400 & 0.94063000 \\
\hline $\mathrm{H}$ & -6.87217000 & 2.74978900 & 1.49977500 \\
\hline $\mathrm{H}$ & -6.34631700 & 4.32549100 & 0.87767300 \\
\hline $\mathrm{H}$ & -5.15893900 & 3.17251700 & 1.52098300 \\
\hline $\mathrm{C}$ & -4.82249600 & 3.47364000 & -1.22350400 \\
\hline $\mathrm{H}$ & -3.85836800 & 3.47783100 & -0.71314500 \\
\hline $\mathrm{H}$ & -5.14416200 & 4.51634900 & -1.33048200 \\
\hline $\mathrm{H}$ & -4.65900600 & 3.06012200 & -2.22374100 \\
\hline
\end{tabular}




\begin{tabular}{|c|c|c|c|}
\hline $\mathrm{C}$ & -7.21380400 & 2.90607500 & -1.28000100 \\
\hline $\mathrm{H}$ & -7.17999800 & 2.40970900 & -2.25282300 \\
\hline $\mathrm{H}$ & -7.31932800 & 3.98387700 & -1.45032600 \\
\hline $\mathrm{H}$ & -8.11418100 & 2.57854600 & -0.76118100 \\
\hline $\mathrm{C}$ & -8.39005700 & 0.26657700 & 0.00265000 \\
\hline $\mathrm{H}$ & -8.05390700 & 1.03202800 & 0.70703000 \\
\hline $\mathrm{H}$ & -8.49694600 & -0.68056200 & 0.54278100 \\
\hline $\mathrm{H}$ & -9.35837600 & 0.55221000 & -0.41481200 \\
\hline $\mathrm{C}$ & -1.19685900 & -0.81427400 & -1.44187400 \\
\hline $\mathrm{C}$ & -0.19299800 & -1.73700900 & -1.72887200 \\
\hline $\mathrm{H}$ & 0.31769900 & -2.20427300 & -0.89961700 \\
\hline $\mathrm{C}$ & 0.14167100 & -2.06915400 & -3.05347900 \\
\hline $\mathrm{C}$ & -0.60118700 & -1.44698100 & -4.08641100 \\
\hline $\mathrm{C}$ & -1.69816000 & -0.58778700 & -3.81845000 \\
\hline $\mathrm{C}$ & -1.94029200 & -0.25931600 & -2.48760300 \\
\hline $\mathrm{H}$ & -2.74127200 & 0.42155400 & -2.24878300 \\
\hline $\mathrm{C}$ & -2.70939200 & -0.14696900 & -4.90078200 \\
\hline $\mathrm{C}$ & -2.07748600 & 0.64930500 & -6.06047800 \\
\hline $\mathrm{H}$ & -1.50751900 & 1.50769100 & -5.69647000 \\
\hline $\mathrm{H}$ & -2.87255000 & 1.03089500 & -6.71293600 \\
\hline $\mathrm{H}$ & -1.42941300 & 0.02471700 & -6.67125100 \\
\hline $\mathrm{C}$ & -3.38100500 & -1.42006300 & -5.46556900 \\
\hline $\mathrm{H}$ & -2.64495300 & -2.06885700 & -5.94491300 \\
\hline $\mathrm{H}$ & -4.14415100 & -1.14987600 & -6.20615500 \\
\hline $\mathrm{H}$ & -3.86966100 & -1.98603900 & -4.66461400 \\
\hline $\mathrm{C}$ & -3.81887300 & 0.74286900 & -4.30037900 \\
\hline $\mathrm{H}$ & -4.38971700 & 0.23193800 & -3.51855300 \\
\hline $\mathrm{H}$ & -4.52151000 & 1.02089400 & -5.09321000 \\
\hline $\mathrm{H}$ & -3.41056700 & 1.66491900 & -3.87203300 \\
\hline $\mathrm{C}$ & 1.27481700 & -3.09798500 & -3.28484600 \\
\hline $\mathrm{C}$ & 2.60886000 & -2.36592900 & -3.55657200 \\
\hline $\mathrm{H}$ & 2.58240300 & -1.74222700 & -4.45007000 \\
\hline $\mathrm{H}$ & 3.41686800 & -3.09722200 & -3.68380300 \\
\hline $\mathrm{H}$ & 2.87165300 & -1.72536000 & -2.71148900 \\
\hline $\mathrm{C}$ & 1.49829100 & -3.95547500 & -2.02064600 \\
\hline $\mathrm{H}$ & 1.87890100 & -3.38141100 & -1.17443100 \\
\hline $\mathrm{H}$ & 2.23351700 & -4.73699400 & -2.23997100 \\
\hline $\mathrm{H}$ & 0.57321600 & -4.44315200 & -1.69950400 \\
\hline $\mathrm{C}$ & 0.94822800 & -4.09344700 & -4.41965500 \\
\hline $\mathrm{H}$ & 0.01022300 & -4.61849200 & -4.20447800 \\
\hline $\mathrm{H}$ & 1.74597900 & -4.84284300 & -4.48015000 \\
\hline $\mathrm{H}$ & 0.84178600 & -3.62422600 & -5.39537300 \\
\hline $\mathrm{C}$ & 0.74265900 & -1.07019000 & -6.04775900 \\
\hline $\mathrm{H}$ & 1.08012900 & -0.21633500 & -5.45474300 \\
\hline
\end{tabular}




\begin{tabular}{|c|c|c|c|}
\hline $\mathrm{H}$ & 0.40831000 & -0.71620600 & -7.02745500 \\
\hline $\mathrm{H}$ & 1.57288100 & -1.76788400 & -6.19583400 \\
\hline $\mathrm{C}$ & 2.05353100 & 1.54219100 & 1.10054900 \\
\hline $\mathrm{C}$ & 2.34664600 & 2.90843800 & 1.06285000 \\
\hline $\mathrm{H}$ & 2.45376300 & 3.38490500 & 0.09813600 \\
\hline $\mathrm{C}$ & 2.49041500 & 3.65955800 & 2.23146300 \\
\hline $\mathrm{C}$ & 2.28155300 & 2.98856300 & 3.46732700 \\
\hline $\mathrm{C}$ & 2.10613800 & 1.59443700 & 3.54606400 \\
\hline $\mathrm{C}$ & 1.96939000 & 0.90542000 & 2.33209500 \\
\hline $\mathrm{H}$ & 1.81781600 & -0.16723300 & 2.33298000 \\
\hline $\mathrm{C}$ & 3.67114800 & 0.34449300 & -0.86220400 \\
\hline $\mathrm{C}$ & 4.39341700 & 1.43326700 & -1.34616400 \\
\hline $\mathrm{H}$ & 3.87838700 & 2.37499400 & -1.48838900 \\
\hline $\mathrm{C}$ & 5.75079400 & 1.34645100 & -1.67941400 \\
\hline $\mathrm{C}$ & 6.39523900 & 0.12009100 & -1.39956700 \\
\hline $\mathrm{C}$ & 5.68066800 & -1.03674800 & -1.00656800 \\
\hline $\mathrm{C}$ & 4.31762200 & -0.88711600 & -0.72869400 \\
\hline $\mathrm{H}$ & 3.74154500 & -1.72132000 & -0.35301500 \\
\hline $\mathrm{C}$ & 3.02606400 & 5.10772200 & 2.16001100 \\
\hline $\mathrm{C}$ & 4.44767800 & 5.10213600 & 2.76918500 \\
\hline $\mathrm{H}$ & 4.41845700 & 4.78878400 & 3.81614300 \\
\hline $\mathrm{H}$ & 4.88687700 & 6.10669500 & 2.72056900 \\
\hline $\mathrm{H}$ & 5.10072900 & 4.41557400 & 2.21910400 \\
\hline $\mathrm{C}$ & 3.13829700 & 5.59184600 & 0.70037100 \\
\hline $\mathrm{H}$ & 3.83767700 & 4.98335000 & 0.11900400 \\
\hline $\mathrm{H}$ & 3.51512900 & 6.62018400 & 0.68912300 \\
\hline $\mathrm{H}$ & 2.17062900 & 5.57491500 & 0.18716800 \\
\hline $\mathrm{C}$ & 2.17013000 & 6.14589600 & 2.91855600 \\
\hline $\mathrm{H}$ & 1.11188800 & 6.06265700 & 2.65717600 \\
\hline $\mathrm{H}$ & 2.50832000 & 7.15658800 & 2.65962000 \\
\hline $\mathrm{H}$ & 2.26736000 & 6.03131800 & 3.99746500 \\
\hline $\mathrm{C}$ & 2.08477500 & 0.71543700 & 4.81936800 \\
\hline $\mathrm{C}$ & 3.15276700 & -0.39697300 & 4.64618800 \\
\hline $\mathrm{H}$ & 3.01262700 & -1.00053100 & 3.74650200 \\
\hline $\mathrm{H}$ & 3.12703700 & -1.06705200 & 5.51459500 \\
\hline $\mathrm{H}$ & 4.15258500 & 0.04881200 & 4.59444000 \\
\hline $\mathrm{C}$ & 0.69151300 & 0.05758600 & 4.94259100 \\
\hline $\mathrm{H}$ & -0.09266400 & 0.81434800 & 5.07109400 \\
\hline $\mathrm{H}$ & 0.65975600 & -0.61587700 & 5.80853800 \\
\hline $\mathrm{H}$ & 0.44972800 & -0.52294800 & 4.04653000 \\
\hline $\mathrm{C}$ & 2.41922800 & 1.41998600 & 6.14865200 \\
\hline $\mathrm{H}$ & 3.35464200 & 1.97991600 & 6.08451200 \\
\hline $\mathrm{H}$ & 2.53248800 & 0.65224100 & 6.92365600 \\
\hline $\mathrm{H}$ & 1.63774500 & 2.10277900 & 6.48527200 \\
\hline
\end{tabular}




\begin{tabular}{|c|c|c|c|}
\hline $\mathrm{C}$ & 0.93901700 & 4.06910200 & 5.04984800 \\
\hline $\mathrm{H}$ & 1.03396700 & 4.62994700 & 5.9832270 \\
\hline $\mathrm{H}$ & 0.35089300 & 3.16156600 & 5.2295480 \\
\hline $\mathrm{H}$ & 0.41762500 & 4.67948100 & 4.3049810 \\
\hline $\mathrm{C}$ & 6.38118400 & 2.58412900 & -2.36454900 \\
\hline $\mathrm{C}$ & 6.50213400 & 3.73863300 & -1.34681500 \\
\hline $\mathrm{H}$ & 5.52805800 & 3.99022200 & -0.91507900 \\
\hline $\mathrm{H}$ & 6.90084800 & 4.63805200 & -1.83183000 \\
\hline $\mathrm{H}$ & 7.17047300 & 3.47584100 & -0.51990400 \\
\hline $\mathrm{C}$ & 5.43778000 & 3.03281100 & -3.51200600 \\
\hline $\mathrm{H}$ & 5.32036700 & 2.22896900 & -4.24750500 \\
\hline $\mathrm{H}$ & 5.86644200 & 3.90236400 & -4.02343500 \\
\hline $\mathrm{H}$ & 4.44115700 & 3.31496000 & -3.16385100 \\
\hline $\mathrm{C}$ & 7.75324800 & 2.34903700 & -3.03063100 \\
\hline $\mathrm{H}$ & 8.56525400 & 2.21900100 & -2.31594200 \\
\hline $\mathrm{H}$ & 7.99512000 & 3.22768700 & -3.64076800 \\
\hline $\mathrm{H}$ & 7.73743200 & 1.47366900 & -3.68501000 \\
\hline $\mathrm{C}$ & 6.32868900 & -2.43743400 & -0.96372100 \\
\hline $\mathrm{C}$ & 7.42743500 & -2.54775200 & 0.11540700 \\
\hline $\mathrm{H}$ & 8.31520200 & -1.97215000 & -0.14684000 \\
\hline $\mathrm{H}$ & 7.73304200 & -3.59566300 & 0.22254600 \\
\hline $\mathrm{H}$ & 7.05588600 & -2.20657100 & 1.08846700 \\
\hline $\mathrm{C}$ & 5.27982100 & -3.52155100 & -0.65261200 \\
\hline $\mathrm{H}$ & 4.81015600 & -3.37411400 & 0.32220600 \\
\hline $\mathrm{H}$ & 5.76576200 & -4.50355000 & -0.64939000 \\
\hline $\mathrm{H}$ & 4.48468100 & -3.54987400 & -1.40368500 \\
\hline $\mathrm{C}$ & 6.93238700 & -2.75341800 & -2.35089300 \\
\hline $\mathrm{H}$ & 7.37419800 & -3.75773700 & -2.34670900 \\
\hline $\mathrm{H}$ & 7.70812900 & -2.03426000 & -2.62129400 \\
\hline $\mathrm{H}$ & 6.15222700 & -2.73156100 & -3.12074900 \\
\hline $\mathrm{C}$ & 8.46268100 & 0.56655400 & -0.38830800 \\
\hline $\mathrm{H}$ & 8.30852800 & 1.64791700 & -0.30473300 \\
\hline $\mathrm{H}$ & 9.52502800 & 0.36450000 & -0.54706000 \\
\hline $\mathrm{H}$ & 8.13384500 & 0.09467200 & 0.5440290 \\
\hline $\mathrm{C}$ & -0.71257600 & 2.30106200 & -0.10975100 \\
\hline $\mathrm{C}$ & -1.18750500 & 1.27469200 & 0.75582100 \\
\hline $\mathrm{C}$ & -1.55562400 & 1.58886800 & 2.07123500 \\
\hline $\mathrm{H}$ & -1.88951600 & 0.78723200 & 2.71137400 \\
\hline $\mathrm{C}$ & -1.56651500 & 2.89444800 & 2.57893200 \\
\hline $\mathrm{H}$ & -1.86068600 & 3.10993600 & 3.59925200 \\
\hline $\mathrm{C}$ & -1.18660800 & 3.88742500 & 1.70155800 \\
\hline $\mathrm{C}$ & -0.75779400 & 3.58601000 & 0.41250700 \\
\hline $\mathrm{C}$ & -0.79575800 & 5.80142400 & 0.63585100 \\
\hline $\mathrm{H}$ & -1.72459100 & 6.21416300 & 0.21760600 \\
\hline
\end{tabular}




\begin{tabular}{|c|c|c|c|}
\hline $\mathrm{H}$ & -0.02025600 & 6.56101100 & 0.73457000 \\
\hline $\mathrm{C}$ & 1.15140700 & 1.48198800 & -1.67724500 \\
\hline $\mathrm{C}$ & 1.69584800 & 1.48194300 & -2.97067300 \\
\hline $\mathrm{H}$ & 2.62332700 & 0.95213400 & -3.14765200 \\
\hline $\mathrm{C}$ & 1.11198800 & 2.17051700 & -4.04821500 \\
\hline $\mathrm{H}$ & 1.57036500 & 2.18288200 & -5.03079800 \\
\hline $\mathrm{C}$ & -0.06216400 & 2.84310500 & -3.78899300 \\
\hline $\mathrm{C}$ & -0.64425800 & 2.81306900 & -2.52948500 \\
\hline $\mathrm{C}$ & -0.08944600 & 2.14947900 & -1.44982500 \\
\hline $\mathrm{C}$ & -1.84661700 & 4.18275600 & -3.81962300 \\
\hline $\mathrm{H}$ & -2.82794400 & 4.07254600 & -4.28470500 \\
\hline $\mathrm{H}$ & -1.59018300 & 5.24007400 & -3.66181100 \\
\hline $\mathrm{C}$ & -1.17131900 & -3.37356500 & 3.86005800 \\
\hline $\mathrm{C}$ & -0.85017300 & -4.84117200 & 3.74850800 \\
\hline $\mathrm{H}$ & -0.41049400 & -2.74159600 & 4.31655300 \\
\hline $\mathrm{C}$ & -2.44794300 & -2.85447800 & 3.67492400 \\
\hline $\mathrm{C}$ & -2.65305700 & -1.44534400 & 3.57267200 \\
\hline $\mathrm{H}$ & -3.25683000 & -3.53111100 & 3.40621500 \\
\hline $\mathrm{H}$ & -1.96220200 & -0.82694000 & 4.14606000 \\
\hline $\mathrm{C}$ & -3.98116100 & -0.84779000 & 3.37504000 \\
\hline $\mathrm{C}$ & -5.00515800 & -1.50913100 & 2.67835500 \\
\hline $\mathrm{C}$ & -4.26055600 & 0.40912700 & 3.93577900 \\
\hline $\mathrm{C}$ & -6.27468000 & -0.95148900 & 2.58722700 \\
\hline $\mathrm{H}$ & -4.80092400 & -2.45749600 & 2.19295700 \\
\hline $\mathrm{C}$ & -5.52796300 & 0.97676200 & 3.83044300 \\
\hline $\mathrm{H}$ & -3.47914800 & 0.93161600 & 4.48181300 \\
\hline $\mathrm{C}$ & -6.54437400 & 0.29091400 & 3.16528700 \\
\hline $\mathrm{H}$ & -7.05359800 & -1.48750500 & 2.05666000 \\
\hline $\mathrm{H}$ & -5.72435700 & 1.94754100 & 4.27606400 \\
\hline $\mathrm{H}$ & -7.53883500 & 0.72136400 & 3.09647400 \\
\hline S & 1.61559900 & -3.21857400 & 2.02274400 \\
\hline $\mathrm{C}$ & 2.18170300 & -4.86903200 & 1.48756200 \\
\hline $\mathrm{C}$ & 3.47739500 & -5.25566700 & 1.83454000 \\
\hline $\mathrm{C}$ & 1.32928100 & -5.75104200 & 0.82416700 \\
\hline $\mathrm{C}$ & 3.92839900 & -6.53188400 & 1.49901600 \\
\hline $\mathrm{H}$ & 4.12830000 & -4.55096500 & 2.34240500 \\
\hline $\mathrm{C}$ & 1.78635100 & -7.02667400 & 0.48757100 \\
\hline $\mathrm{H}$ & 0.33528700 & -5.41625700 & 0.54993600 \\
\hline $\mathrm{C}$ & 3.08356700 & -7.41774900 & 0.82487200 \\
\hline $\mathrm{H}$ & 4.93977600 & -6.83347700 & 1.75743100 \\
\hline $\mathrm{H}$ & 1.13229300 & -7.71140400 & -0.04568800 \\
\hline $\mathrm{H}$ & 3.43732600 & -8.41075300 & 0.56168600 \\
\hline $\mathrm{O}$ & 2.77019600 & -2.30617400 & 1.65847400 \\
\hline & 0.38327700 & -3.05693400 & 1.03096400 \\
\hline
\end{tabular}




$\begin{array}{lrrr}\mathrm{H} & -0.41802700 & -5.03913600 & 2.76167900 \\ \mathrm{C} & 0.13785300 & -5.31373600 & 4.81998800 \\ \mathrm{H} & 1.08097500 & -4.76363200 & 4.73171500 \\ \mathrm{H} & -0.25865400 & -5.15610300 & 5.82967100 \\ \mathrm{H} & 0.35897500 & -6.37909600 & 4.70008800 \\ \mathrm{H} & -1.78110900 & -5.42182800 & 3.79507400\end{array}$<smiles>CC/C=C\C(c1ccccc1)[P+]1(OOS(=O)c2ccccc2)PCCCP1</smiles>

Int-11a

Total SCF energy (M06/SDD-6-311++G(d,p)/SMD(1,4-Dioxane)): -5487.868401a.u.

Thermal correction to Gibbs Free Energy at $298.15 \mathrm{~K}: 1.769805 \mathrm{a}$.u

Gibbs free energy at 298.15 K (M06/SDD-6-311++G(d,p)/SMD(1,4-Dioxane)): -5496.098596a.u.

$\mathrm{Pd}$

$\mathrm{P}$

$\begin{array}{lll}0.21142100 & 0.58835600 \quad 1.43410600\end{array}$

$P$

1.93744800

$-0.12961300$

0.17072300

$\mathrm{O}$

$-1.32002600$

$-0.07310900$

$-0.39160200$

$\mathrm{O}$

7.48218900

0.18552700

2.35888300

$\mathrm{O}$

2.99013400

2.63341500

$-5.01790100$

$\mathrm{O}$

$-3.94596700$

$-5.35877100$

0.67137400

$\mathrm{O}$

$-5.70971000$

3.94203300

$-0.81099200$

$\mathrm{O}$

0.19538700

$-5.78426700$

$-0.45463300$

$\mathrm{O}$

$-0.29420700$

$-4.34911600$

$-2.19687000$

$\mathrm{O}$

1.59291800

$-2.55875400$

$-4.05590700$

0.86423600

$-1.26725700$

$-5.82495600$

C

3.67029000

$-0.12727600$

0.74349700

C

4.20928300

1.12758000

1.05088200

$\mathrm{H}$

3.58765200

2.00189000

0.90973100

C

5.50743500

1.27026700

1.53263100

C

6.26756800

0.08159900

1.70583600

C

5.82719300

$-1.16573200$

1.21744100

C

4.49468800

$-1.24227800$

0.78219200

$\mathrm{H}$

4.09805800

$-2.19819800$

0.47002000

6.09136900

2.67526300

1.78510100

C

5.09356800

3.77811700

1.37657900

$\mathrm{H}$

4.79701800

3.69791100

0.32496100

$\mathrm{H}$

5.56816400

4.75523200

1.51300200

$\mathrm{H}$

4.18762000

3.76487300

1.99359800

C

6.43776100

2.90418700

3.27229900

$\mathrm{H}$

5.58561000

2.66028100

3.91708200

$\mathrm{H}$

6.68724500

3.95941100

3.43327700 


\begin{tabular}{|c|c|c|c|}
\hline $\mathrm{H}$ & 7.29773200 & 2.31116100 & 3.58253600 \\
\hline $\mathrm{C}$ & 7.36072700 & 2.84978900 & 0.92067900 \\
\hline $\mathrm{H}$ & 8.11970900 & 2.10884000 & 1.17887400 \\
\hline $\mathrm{H}$ & 7.78440400 & 3.84926600 & 1.07575500 \\
\hline $\mathrm{H}$ & 7.11885000 & 2.74632600 & -0.14344700 \\
\hline $\mathrm{C}$ & 6.67528400 & -2.45196300 & 1.07147500 \\
\hline $\mathrm{C}$ & 6.17811100 & -3.52189400 & 2.06756400 \\
\hline $\mathrm{H}$ & 6.33985100 & -3.20722700 & 3.10414200 \\
\hline $\mathrm{H}$ & 6.71893500 & -4.46344000 & 1.91487400 \\
\hline $\mathrm{H}$ & 5.10800600 & -3.71901800 & 1.94511700 \\
\hline $\mathrm{C}$ & 6.48075700 & -2.99054400 & -0.37073800 \\
\hline $\mathrm{H}$ & 5.44529200 & -3.26405300 & -0.58872100 \\
\hline $\mathrm{H}$ & 7.09303400 & -3.88788400 & -0.51345900 \\
\hline $\mathrm{H}$ & 6.79467900 & -2.24346000 & -1.10795400 \\
\hline $\mathrm{C}$ & 8.19664000 & -2.26427300 & 1.24309800 \\
\hline $\mathrm{H}$ & 8.57467400 & -1.45056100 & 0.61870500 \\
\hline $\mathrm{H}$ & 8.69491100 & -3.19062700 & 0.93426700 \\
\hline $\mathrm{H}$ & 8.49437400 & -2.06486300 & 2.27207900 \\
\hline $\mathrm{C}$ & 7.42385800 & -0.25445300 & 3.71652000 \\
\hline $\mathrm{H}$ & 7.19148600 & -1.32257600 & 3.78317700 \\
\hline $\mathrm{H}$ & 6.66892200 & 0.30495400 & 4.28005800 \\
\hline $\mathrm{H}$ & 8.41237700 & -0.07070000 & 4.14341000 \\
\hline $\mathrm{C}$ & 2.20165000 & 0.68251200 & -1.43953500 \\
\hline $\mathrm{C}$ & 1.63899300 & 1.92602300 & -1.68176500 \\
\hline $\mathrm{H}$ & 0.94984500 & 2.32676400 & -0.94667100 \\
\hline $\mathrm{C}$ & 1.94445400 & 2.66360700 & -2.83779100 \\
\hline $\mathrm{C}$ & 2.77684800 & 2.03902600 & -3.78755400 \\
\hline $\mathrm{C}$ & 3.44803400 & 0.81249700 & -3.52567100 \\
\hline $\mathrm{C}$ & 3.13427500 & 0.15660000 & -2.33910800 \\
\hline $\mathrm{H}$ & 3.61572700 & -0.77910800 & -2.09174100 \\
\hline $\mathrm{C}$ & 4.59333200 & 0.28723800 & -4.41772300 \\
\hline $\mathrm{C}$ & 4.19628900 & 0.10347800 & -5.89914300 \\
\hline $\mathrm{H}$ & 3.25150000 & -0.43810600 & -5.99575500 \\
\hline $\mathrm{H}$ & 4.97411100 & -0.46988300 & -6.41709600 \\
\hline $\mathrm{H}$ & 4.09588500 & 1.06073200 & -6.40902400 \\
\hline $\mathrm{C}$ & 5.75522500 & 1.30384200 & -4.33308200 \\
\hline $\mathrm{H}$ & 5.44089600 & 2.28384700 & -4.70120700 \\
\hline $\mathrm{H}$ & 6.60288800 & 0.96221300 & -4.93989500 \\
\hline $\mathrm{H}$ & 6.09861600 & 1.41399300 & -3.29820200 \\
\hline $\mathrm{C}$ & 5.10999500 & -1.07547800 & -3.91340100 \\
\hline $\mathrm{H}$ & 5.51756000 & -1.01380800 & -2.89899500 \\
\hline $\mathrm{H}$ & 5.91751900 & -1.41851800 & -4.56857500 \\
\hline $\mathrm{H}$ & 4.31981500 & -1.83537100 & -3.91953600 \\
\hline $\mathrm{C}$ & 1.36691600 & 4.09642900 & -2.91313200 \\
\hline
\end{tabular}




\begin{tabular}{|c|c|c|c|}
\hline $\mathrm{C}$ & -0.16331000 & 4.02016000 & -3.10696700 \\
\hline $\mathrm{H}$ & -0.41734000 & 3.55046800 & -4.06372100 \\
\hline $\mathrm{H}$ & -0.59876000 & 5.02587400 & -3.09828300 \\
\hline $\mathrm{H}$ & -0.64696200 & 3.43932500 & -2.31639600 \\
\hline $\mathrm{C}$ & 1.68006800 & 4.80536800 & -1.56868400 \\
\hline $\mathrm{H}$ & 1.24818800 & 4.30038500 & -0.70272600 \\
\hline $\mathrm{H}$ & 1.27550300 & 5.82299500 & -1.58491400 \\
\hline $\mathrm{H}$ & 2.76351100 & 4.87055400 & -1.41553100 \\
\hline $\mathrm{C}$ & 1.96824400 & 5.00644900 & -4.00421200 \\
\hline $\mathrm{H}$ & 3.06069000 & 5.00853200 & -3.97251800 \\
\hline $\mathrm{H}$ & 1.61970600 & 6.02998500 & -3.82379700 \\
\hline $\mathrm{H}$ & 1.65951800 & 4.73535600 & -5.01356200 \\
\hline $\mathrm{C}$ & 1.92640100 & 2.40193700 & -5.94373000 \\
\hline $\mathrm{H}$ & 1.79955700 & 1.33324900 & -6.14254600 \\
\hline $\mathrm{H}$ & 2.20353700 & 2.91835900 & -6.86562300 \\
\hline $\mathrm{H}$ & 0.97945700 & 2.80522200 & -5.57086100 \\
\hline $\mathrm{C}$ & -2.15056500 & -1.67581500 & -0.06623800 \\
\hline $\mathrm{C}$ & -2.78556300 & -2.36957300 & -1.09940800 \\
\hline $\mathrm{H}$ & -2.73847400 & -1.96257900 & -2.09883900 \\
\hline $\mathrm{C}$ & -3.46940700 & -3.56812500 & -0.87636000 \\
\hline $\mathrm{C}$ & -3.41949700 & -4.09557300 & 0.43604300 \\
\hline $\mathrm{C}$ & -2.90934000 & -3.35665200 & 1.53057000 \\
\hline $\mathrm{C}$ & -2.23843100 & -2.16193200 & 1.23880500 \\
\hline $\mathrm{H}$ & -1.81631700 & -1.56207500 & 2.03224400 \\
\hline $\mathrm{C}$ & -2.64639400 & 1.15106700 & -0.63930500 \\
\hline $\mathrm{C}$ & -3.84842500 & 0.82772200 & -1.26370200 \\
\hline $\mathrm{H}$ & -4.00120900 & -0.18443100 & -1.61391600 \\
\hline $\mathrm{C}$ & -4.86533800 & 1.77030600 & -1.44637900 \\
\hline $\mathrm{C}$ & -4.65896800 & 3.04643700 & -0.87593600 \\
\hline $\mathrm{C}$ & -3.40572200 & 3.45209500 & -0.35599500 \\
\hline $\mathrm{C}$ & -2.41987100 & 2.46850900 & -0.23150100 \\
\hline $\mathrm{H}$ & -1.47991700 & 2.70964300 & 0.24389000 \\
\hline $\mathrm{C}$ & -4.39260300 & -4.13839200 & -1.97818800 \\
\hline $\mathrm{C}$ & -5.84433500 & -4.04866400 & -1.45147100 \\
\hline $\mathrm{H}$ & -5.96904500 & -4.65339200 & -0.55000400 \\
\hline $\mathrm{H}$ & -6.54709200 & -4.40957800 & -2.21255300 \\
\hline $\mathrm{H}$ & -6.10597900 & -3.01203000 & -1.21254200 \\
\hline $\mathrm{C}$ & -4.30375300 & -3.30585500 & -3.27247200 \\
\hline $\mathrm{H}$ & -4.59827000 & -2.26396700 & -3.11531400 \\
\hline $\mathrm{H}$ & -4.98734100 & -3.72699900 & -4.01706700 \\
\hline $\mathrm{H}$ & -3.29368500 & -3.31970600 & -3.69778900 \\
\hline $\mathrm{C}$ & -4.10095700 & -5.59910300 & -2.37169400 \\
\hline $\mathrm{H}$ & -4.34653900 & -6.29070500 & -1.56745800 \\
\hline $\mathrm{H}$ & -3.05123900 & -5.72750000 & -2.65607600 \\
\hline
\end{tabular}




\begin{tabular}{|c|c|c|c|}
\hline $\mathrm{H}$ & -4.71173100 & -5.87248800 & -3.23990300 \\
\hline $\mathrm{C}$ & -3.21201000 & -3.72356400 & 3.00303100 \\
\hline $\mathrm{C}$ & -2.74759500 & -2.60464000 & 3.95537000 \\
\hline $\mathrm{H}$ & -1.66233100 & -2.48995900 & 3.93945000 \\
\hline $\mathrm{H}$ & -3.04232700 & -2.86549000 & 4.97761500 \\
\hline $\mathrm{H}$ & -3.19206000 & -1.63609700 & 3.71495800 \\
\hline $\mathrm{C}$ & -2.52007900 & -5.02167400 & 3.47052300 \\
\hline $\mathrm{H}$ & -2.98052500 & -5.91325800 & 3.04524200 \\
\hline $\mathrm{H}$ & -2.60026600 & -5.10430300 & 4.56080400 \\
\hline $\mathrm{H}$ & -1.45405800 & -5.01513200 & 3.21373400 \\
\hline $\mathrm{C}$ & -4.74346900 & -3.85755400 & 3.17034900 \\
\hline $\mathrm{H}$ & -5.23761000 & -2.91171600 & 2.92160900 \\
\hline $\mathrm{H}$ & -4.98261800 & -4.09696200 & 4.21361800 \\
\hline $\mathrm{H}$ & -5.14846100 & -4.64548100 & 2.53263000 \\
\hline $\mathrm{C}$ & -2.97152400 & -6.40565600 & 0.61531800 \\
\hline $\mathrm{H}$ & -2.77255500 & -6.70424000 & -0.41836700 \\
\hline $\mathrm{H}$ & -3.39253800 & -7.25629700 & 1.15693800 \\
\hline $\mathrm{H}$ & -2.02882100 & -6.10150100 & 1.07358000 \\
\hline $\mathrm{C}$ & -6.07211600 & 1.32787900 & -2.30878800 \\
\hline $\mathrm{C}$ & -6.84777900 & 0.20488500 & -1.58795400 \\
\hline $\mathrm{H}$ & -6.20136000 & -0.65050300 & -1.36485500 \\
\hline $\mathrm{H}$ & -7.67463300 & -0.15044900 & -2.21472900 \\
\hline $\mathrm{H}$ & -7.26303900 & 0.55917700 & -0.63942200 \\
\hline $\mathrm{C}$ & -5.51101400 & 0.77602800 & -3.64543200 \\
\hline $\mathrm{H}$ & -4.97031000 & 1.56089100 & -4.18640400 \\
\hline $\mathrm{H}$ & -6.33386200 & 0.42842600 & -4.28073600 \\
\hline $\mathrm{H}$ & -4.82472700 & -0.06240100 & -3.50362100 \\
\hline $\mathrm{C}$ & -7.06317500 & 2.44104600 & -2.70523100 \\
\hline $\mathrm{H}$ & -7.66721000 & 2.79495400 & -1.86996300 \\
\hline $\mathrm{H}$ & -7.75175500 & 2.03425100 & -3.45580600 \\
\hline $\mathrm{H}$ & -6.55196400 & 3.30221700 & -3.14171300 \\
\hline $\mathrm{C}$ & -3.10317100 & 4.91991300 & 0.01416800 \\
\hline $\mathrm{C}$ & -3.91564200 & 5.38942300 & 1.24040200 \\
\hline $\mathrm{H}$ & -4.97463300 & 5.50254500 & 1.00578100 \\
\hline $\mathrm{H}$ & -3.54439400 & 6.36910800 & 1.56572200 \\
\hline $\mathrm{H}$ & -3.78651400 & 4.68521000 & 2.06616500 \\
\hline $\mathrm{C}$ & -1.61323200 & 5.10606900 & 0.35884500 \\
\hline $\mathrm{H}$ & -1.33371200 & 4.52809900 & 1.24150700 \\
\hline $\mathrm{H}$ & -1.42685800 & 6.16510900 & 0.56961300 \\
\hline $\mathrm{H}$ & -0.96980800 & 4.82130100 & -0.47788200 \\
\hline $\mathrm{C}$ & -3.41635400 & 5.82094600 & -1.20227300 \\
\hline $\mathrm{H}$ & -3.17409200 & 6.86324800 & -0.96123400 \\
\hline $\mathrm{H}$ & -4.47025200 & 5.76656800 & -1.48081300 \\
\hline & -2.81134500 & 5.52685900 & -2.06841200 \\
\hline
\end{tabular}




\begin{tabular}{|c|c|c|c|}
\hline $\mathrm{C}$ & -6.59610700 & 3.65893600 & 0.27577100 \\
\hline $\mathrm{H}$ & -7.08321800 & 2.68615300 & 0.15346600 \\
\hline $\mathrm{H}$ & -7.34891100 & 4.45099800 & 0.27160500 \\
\hline $\mathrm{H}$ & -6.06098900 & 3.65295100 & 1.22841100 \\
\hline $\mathrm{C}$ & 0.74012900 & -2.32488600 & -1.21394100 \\
\hline $\mathrm{C}$ & 1.47631000 & -1.88270300 & -0.07873700 \\
\hline $\mathrm{C}$ & 1.72003200 & -2.75283100 & 0.99612100 \\
\hline $\mathrm{H}$ & 2.21450000 & -2.38090800 & 1.88464200 \\
\hline $\mathrm{C}$ & 1.32645600 & -4.09814800 & 0.97227500 \\
\hline $\mathrm{H}$ & 1.51031700 & -4.75282900 & 1.81603200 \\
\hline $\mathrm{C}$ & 0.69211500 & -4.53450100 & -0.17299700 \\
\hline $\mathrm{C}$ & 0.40712900 & -3.67052400 & -1.22675400 \\
\hline $\mathrm{C}$ & -0.18203900 & -5.72800300 & -1.83538700 \\
\hline $\mathrm{H}$ & 0.59932800 & -6.20324300 & -2.44550600 \\
\hline $\mathrm{H}$ & -1.14601400 & -6.21813200 & -1.96540100 \\
\hline $\mathrm{C}$ & -0.68587900 & -0.38628900 & -2.09665300 \\
\hline $\mathrm{C}$ & -1.10509600 & 0.39922300 & -3.17330200 \\
\hline $\mathrm{H}$ & -1.81784400 & 1.19510900 & -2.99818600 \\
\hline $\mathrm{C}$ & -0.64721800 & 0.18350700 & -4.48607300 \\
\hline $\mathrm{H}$ & -0.98170700 & 0.80072300 & -5.31188500 \\
\hline $\mathrm{C}$ & 0.23743300 & -0.85633400 & -4.66940100 \\
\hline $\mathrm{C}$ & 0.66982500 & -1.64231100 & -3.60364600 \\
\hline $\mathrm{C}$ & 0.25161500 & -1.44273100 & -2.30369300 \\
\hline $\mathrm{C}$ & 1.48680900 & -2.51328800 & -5.48077400 \\
\hline $\mathrm{H}$ & 2.48378200 & -2.55332300 & -5.91991800 \\
\hline $\mathrm{H}$ & 0.85323500 & -3.34144500 & -5.82811600 \\
\hline $\mathrm{C}$ & 1.09017500 & 3.28926800 & 3.9620020 \\
\hline $\mathrm{C}$ & 0.42841200 & 4.30866000 & 4.8408000 \\
\hline $\mathrm{H}$ & 1.84548500 & 3.66905700 & 3.2684060 \\
\hline $\mathrm{H}$ & 1.18213100 & 4.74561300 & 5.5152090 \\
\hline $\mathrm{C}$ & 0.81707000 & 1.97387600 & 3.9585000 \\
\hline $\mathrm{C}$ & 1.51133500 & 0.99225700 & 3.1102880 \\
\hline $\mathrm{H}$ & 0.05530100 & 1.59382800 & 4.6358630 \\
\hline $\mathrm{H}$ & 2.42404900 & 1.41992900 & 2.7003330 \\
\hline $\mathrm{C}$ & 1.77553300 & -0.34843700 & 3.70785100 \\
\hline $\mathrm{C}$ & 0.72875400 & -1.16566200 & 4.17585100 \\
\hline $\mathrm{C}$ & 3.09081100 & -0.83561100 & 3.80402900 \\
\hline $\mathrm{C}$ & 0.99103900 & -2.43474200 & 4.68661700 \\
\hline $\mathrm{H}$ & -0.28967000 & -0.79789800 & 4.09710100 \\
\hline $\mathrm{C}$ & 3.35090400 & -2.10893300 & 4.31276200 \\
\hline $\mathrm{H}$ & 3.91345100 & -0.21067900 & 3.47424200 \\
\hline $\mathrm{C}$ & 2.30169500 & -2.92002700 & 4.74797200 \\
\hline $\mathrm{H}$ & 0.16928700 & -3.05354600 & 5.03598200 \\
\hline $\mathrm{H}$ & 4.37598300 & -2.46438100 & 4.36890900 \\
\hline
\end{tabular}




$\begin{array}{lrrr}\mathrm{H} & 2.50152200 & -3.91170300 & 5.14474100 \\ \mathrm{C} & -0.24869700 & 5.43589500 & 4.04491200 \\ \mathrm{H} & 0.45896700 & 5.91050000 & 3.35300500 \\ \mathrm{H} & -1.07921500 & 5.02409400 & 3.468866000 \\ \mathrm{H} & -0.63034900 & 6.21300700 & 4.71808800 \\ \mathrm{~S} & -2.38217600 & 1.72420900 & 3.42303900 \\ \mathrm{C} & -4.13615400 & 1.36366500 & 3.00656000 \\ \mathrm{C} & -5.10998900 & 2.17267600 & 3.59735500 \\ \mathrm{C} & -4.50777200 & 0.30227900 & 2.18646700 \\ \mathrm{C} & -6.46162600 & 1.90701000 & 3.37453100 \\ \mathrm{H} & -4.80931900 & 3.00634600 & 4.22707200 \\ \mathrm{C} & -5.86052000 & 0.04094100 & 1.95887800 \\ \mathrm{H} & -3.74159800 & -0.30870500 & 1.73251900 \\ \mathrm{C} & -6.83919900 & 0.83714500 & 2.55654700 \\ \mathrm{H} & -7.22062700 & 2.53203900 & 3.83803100 \\ \mathrm{H} & -6.14550500 & -0.78738200 & 1.31619100 \\ \mathrm{H} & -7.89205900 & 0.62519800 & 2.38981400 \\ \mathrm{O} & -1.67850900 & 0.56133200 & 2.62074800 \\ \mathrm{O} & -2.14390300 & 3.08025000 & 2.79223200 \\ \mathrm{H} & -0.31426100 & 3.81162000 & 5.47646100\end{array}$<smiles>C/C=C\C(=C/C(=N)OS(=O)(=O)c1ccccc1)[Ge]1PCCCP1</smiles>

Int-11b

Total SCF energy (M06/SDD-6-311++G(d,p)/SMD(1,4-Dioxane)): -5497.871269a.u.

Thermal correction to Gibbs Free Energy at 298.15 K: 1.768311a.u

Gibbs free energy at 298.15 K (M06/SDD-6-311++G(d,p)/SMD(1,4-Dioxane)):-5496.102958 a.u.

$\begin{array}{cccc}\mathrm{Pd} & 0.21142100 & 0.58835600 & 1.43410600 \\ \mathrm{P} & 1.93744800 & -0.12961300 & 0.17072300 \\ \mathrm{P} & -1.32002600 & -0.07310900 & -0.39160200 \\ \mathrm{O} & 7.48218900 & 0.18552700 & 2.35888300 \\ \mathrm{O} & 2.99013400 & 2.63341500 & -5.01790100 \\ \mathrm{O} & -3.94596700 & -5.35877100 & 0.67137400 \\ \mathrm{O} & -5.70971000 & 3.94203300 & -0.81099200 \\ \mathrm{O} & 0.19538700 & -5.78426700 & -0.45463300 \\ \mathrm{O} & -0.29420700 & -4.34911600 & -2.19687000 \\ \mathrm{O} & 1.59291800 & -2.55875400 & -4.05590700 \\ \mathrm{O} & 0.86423600 & -1.26725700 & -5.82495600 \\ \mathrm{C} & 3.67029000 & -0.12727600 & 0.74349700 \\ \mathrm{C} & 4.20928300 & 1.12758000 & 1.05088200 \\ \mathrm{H} & 3.58765200 & 2.00189000 & 0.90973100\end{array}$




\begin{tabular}{|c|c|c|c|}
\hline $\mathrm{C}$ & 5.50743500 & 1.27026700 & 1.53263100 \\
\hline $\mathrm{C}$ & 6.26756800 & 0.08159900 & 1.7058360 \\
\hline $\mathrm{C}$ & 5.82719300 & -1.16573200 & 1.21744100 \\
\hline $\mathrm{C}$ & 4.49468800 & -1.24227800 & 0.78219200 \\
\hline $\mathrm{H}$ & 4.09805800 & -2.19819800 & 0.47002000 \\
\hline $\mathrm{C}$ & 6.09136900 & 2.67526300 & 1.7851010 \\
\hline $\mathrm{C}$ & 5.09356800 & 3.77811700 & 1.3765790 \\
\hline $\mathrm{H}$ & 4.79701800 & 3.69791100 & 0.3249610 \\
\hline $\mathrm{H}$ & 5.56816400 & 4.75523200 & 1.5130020 \\
\hline $\mathrm{H}$ & 4.18762000 & 3.76487300 & 1.9935980 \\
\hline $\mathrm{C}$ & 6.43776100 & 2.90418700 & 3.2722990 \\
\hline $\mathrm{H}$ & 5.58561000 & 2.66028100 & 3.9170820 \\
\hline $\mathrm{H}$ & 6.68724500 & 3.95941100 & 3.4332770 \\
\hline $\mathrm{H}$ & 7.29773200 & 2.31116100 & 3.5825360 \\
\hline $\mathrm{C}$ & 7.36072700 & 2.84978900 & 0.9206790 \\
\hline $\mathrm{H}$ & 8.11970900 & 2.10884000 & 1.1788740 \\
\hline $\mathrm{H}$ & 7.78440400 & 3.84926600 & 1.0757550 \\
\hline $\mathrm{H}$ & 7.11885000 & 2.74632600 & -0.14344700 \\
\hline $\mathrm{C}$ & 6.67528400 & -2.45196300 & 1.07147500 \\
\hline $\mathrm{C}$ & 6.17811100 & -3.52189400 & 2.06756400 \\
\hline $\mathrm{H}$ & 6.33985100 & -3.20722700 & 3.10414200 \\
\hline $\mathrm{H}$ & 6.71893500 & -4.46344000 & 1.91487400 \\
\hline $\mathrm{H}$ & 5.10800600 & -3.71901800 & 1.94511700 \\
\hline $\mathrm{C}$ & 6.48075700 & -2.99054400 & -0.37073800 \\
\hline $\mathrm{H}$ & 5.44529200 & -3.26405300 & -0.58872100 \\
\hline $\mathrm{H}$ & 7.09303400 & -3.88788400 & -0.51345900 \\
\hline $\mathrm{H}$ & 6.79467900 & -2.24346000 & -1.10795400 \\
\hline $\mathrm{C}$ & 8.19664000 & -2.26427300 & 1.24309800 \\
\hline $\mathrm{H}$ & 8.57467400 & -1.45056100 & 0.61870500 \\
\hline $\mathrm{H}$ & 8.69491100 & -3.19062700 & 0.93426700 \\
\hline $\mathrm{H}$ & 8.49437400 & -2.06486300 & 2.27207900 \\
\hline $\mathrm{C}$ & 7.42385800 & -0.25445300 & 3.71652000 \\
\hline $\mathrm{H}$ & 7.19148600 & -1.32257600 & 3.78317700 \\
\hline $\mathrm{H}$ & 6.66892200 & 0.30495400 & 4.2800580 \\
\hline $\mathrm{H}$ & 8.41237700 & -0.07070000 & 4.14341000 \\
\hline $\mathrm{C}$ & 2.20165000 & 0.68251200 & -1.43953500 \\
\hline $\mathrm{C}$ & 1.63899300 & 1.92602300 & -1.68176500 \\
\hline $\mathrm{H}$ & 0.94984500 & 2.32676400 & -0.94667100 \\
\hline $\mathrm{C}$ & 1.94445400 & 2.66360700 & -2.83779100 \\
\hline $\mathrm{C}$ & 2.77684800 & 2.03902600 & -3.78755400 \\
\hline $\mathrm{C}$ & 3.44803400 & 0.81249700 & -3.52567100 \\
\hline $\mathrm{C}$ & 3.13427500 & 0.15660000 & -2.33910800 \\
\hline $\mathrm{H}$ & 3.61572700 & -0.77910800 & -2.09174100 \\
\hline $\mathrm{C}$ & 4.59333200 & 0.28723800 & -4.41772300 \\
\hline
\end{tabular}




\begin{tabular}{|c|c|c|c|}
\hline $\mathrm{C}$ & 4.19628900 & 0.10347800 & -5.89914300 \\
\hline $\mathrm{H}$ & 3.25150000 & -0.43810600 & -5.99575500 \\
\hline $\mathrm{H}$ & 4.97411100 & -0.46988300 & -6.41709600 \\
\hline $\mathrm{H}$ & 4.09588500 & 1.06073200 & -6.40902400 \\
\hline $\mathrm{C}$ & 5.75522500 & 1.30384200 & -4.33308200 \\
\hline $\mathrm{H}$ & 5.44089600 & 2.28384700 & -4.70120700 \\
\hline $\mathrm{H}$ & 6.60288800 & 0.96221300 & -4.93989500 \\
\hline $\mathrm{H}$ & 6.09861600 & 1.41399300 & -3.29820200 \\
\hline $\mathrm{C}$ & 5.10999500 & -1.07547800 & -3.91340100 \\
\hline $\mathrm{H}$ & 5.51756000 & -1.01380800 & -2.89899500 \\
\hline $\mathrm{H}$ & 5.91751900 & -1.41851800 & -4.56857500 \\
\hline $\mathrm{H}$ & 4.31981500 & -1.83537100 & -3.91953600 \\
\hline $\mathrm{C}$ & 1.36691600 & 4.09642900 & -2.91313200 \\
\hline $\mathrm{C}$ & -0.16331000 & 4.02016000 & -3.10696700 \\
\hline $\mathrm{H}$ & -0.41734000 & 3.55046800 & -4.06372100 \\
\hline $\mathrm{H}$ & -0.59876000 & 5.02587400 & -3.09828300 \\
\hline $\mathrm{H}$ & -0.64696200 & 3.43932500 & -2.31639600 \\
\hline $\mathrm{C}$ & 1.68006800 & 4.80536800 & -1.56868400 \\
\hline $\mathrm{H}$ & 1.24818800 & 4.30038500 & -0.70272600 \\
\hline $\mathrm{H}$ & 1.27550300 & 5.82299500 & -1.58491400 \\
\hline $\mathrm{H}$ & 2.76351100 & 4.87055400 & -1.41553100 \\
\hline $\mathrm{C}$ & 1.96824400 & 5.00644900 & -4.00421200 \\
\hline $\mathrm{H}$ & 3.06069000 & 5.00853200 & -3.97251800 \\
\hline $\mathrm{H}$ & 1.61970600 & 6.02998500 & -3.82379700 \\
\hline $\mathrm{H}$ & 1.65951800 & 4.73535600 & -5.01356200 \\
\hline $\mathrm{C}$ & 1.92640100 & 2.40193700 & -5.94373000 \\
\hline $\mathrm{H}$ & 1.79955700 & 1.33324900 & -6.14254600 \\
\hline $\mathrm{H}$ & 2.20353700 & 2.91835900 & -6.86562300 \\
\hline $\mathrm{H}$ & 0.97945700 & 2.80522200 & -5.57086100 \\
\hline $\mathrm{C}$ & -2.15056500 & -1.67581500 & -0.06623800 \\
\hline $\mathrm{C}$ & -2.78556300 & -2.36957300 & -1.09940800 \\
\hline $\mathrm{H}$ & -2.73847400 & -1.96257900 & -2.09883900 \\
\hline $\mathrm{C}$ & -3.46940700 & -3.56812500 & -0.87636000 \\
\hline $\mathrm{C}$ & -3.41949700 & -4.09557300 & 0.43604300 \\
\hline $\mathrm{C}$ & -2.90934000 & -3.35665200 & 1.53057000 \\
\hline $\mathrm{C}$ & -2.23843100 & -2.16193200 & 1.23880500 \\
\hline $\mathrm{H}$ & -1.81631700 & -1.56207500 & 2.03224400 \\
\hline $\mathrm{C}$ & -2.64639400 & 1.15106700 & -0.63930500 \\
\hline $\mathrm{C}$ & -3.84842500 & 0.82772200 & -1.26370200 \\
\hline $\mathrm{H}$ & -4.00120900 & -0.18443100 & -1.61391600 \\
\hline $\mathrm{C}$ & -4.86533800 & 1.77030600 & -1.44637900 \\
\hline $\mathrm{C}$ & -4.65896800 & 3.04643700 & -0.87593600 \\
\hline $\mathrm{C}$ & -3.40572200 & 3.45209500 & -0.35599500 \\
\hline $\mathrm{C}$ & -2.41987100 & 2.46850900 & -0.23150100 \\
\hline
\end{tabular}




\begin{tabular}{|c|c|c|c|}
\hline $\mathrm{H}$ & -1.47991700 & 2.70964300 & 0.24389000 \\
\hline $\mathrm{C}$ & -4.39260300 & -4.13839200 & -1.97818800 \\
\hline $\mathrm{C}$ & -5.84433500 & -4.04866400 & -1.45147100 \\
\hline $\mathrm{H}$ & -5.96904500 & -4.65339200 & -0.55000400 \\
\hline $\mathrm{H}$ & -6.54709200 & -4.40957800 & -2.21255300 \\
\hline $\mathrm{H}$ & -6.10597900 & -3.01203000 & -1.21254200 \\
\hline $\mathrm{C}$ & -4.30375300 & -3.30585500 & -3.27247200 \\
\hline $\mathrm{H}$ & -4.59827000 & -2.26396700 & -3.11531400 \\
\hline $\mathrm{H}$ & -4.98734100 & -3.72699900 & -4.01706700 \\
\hline $\mathrm{H}$ & -3.29368500 & -3.31970600 & -3.69778900 \\
\hline $\mathrm{C}$ & -4.10095700 & -5.59910300 & -2.37169400 \\
\hline $\mathrm{H}$ & -4.34653900 & -6.29070500 & -1.56745800 \\
\hline $\mathrm{H}$ & -3.05123900 & -5.72750000 & -2.65607600 \\
\hline $\mathrm{H}$ & -4.71173100 & -5.87248800 & -3.23990300 \\
\hline C & -3.21201000 & -3.72356400 & 3.00303100 \\
\hline $\mathrm{C}$ & -2.74759500 & -2.60464000 & 3.95537000 \\
\hline $\mathrm{H}$ & -1.66233100 & -2.48995900 & 3.93945000 \\
\hline $\mathrm{H}$ & -3.04232700 & -2.86549000 & 4.97761500 \\
\hline $\mathrm{H}$ & -3.19206000 & -1.63609700 & 3.71495800 \\
\hline $\mathrm{C}$ & -2.52007900 & -5.02167400 & 3.47052300 \\
\hline $\mathrm{H}$ & -2.98052500 & -5.91325800 & 3.04524200 \\
\hline $\mathrm{H}$ & -2.60026600 & -5.10430300 & 4.56080400 \\
\hline $\mathrm{H}$ & -1.45405800 & -5.01513200 & 3.21373400 \\
\hline $\mathrm{C}$ & -4.74346900 & -3.85755400 & 3.17034900 \\
\hline $\mathrm{H}$ & -5.23761000 & -2.91171600 & 2.92160900 \\
\hline $\mathrm{H}$ & -4.98261800 & -4.09696200 & 4.21361800 \\
\hline $\mathrm{H}$ & -5.14846100 & -4.64548100 & 2.53263000 \\
\hline $\mathrm{C}$ & -2.97152400 & -6.40565600 & 0.61531800 \\
\hline $\mathrm{H}$ & -2.77255500 & -6.70424000 & -0.41836700 \\
\hline $\mathrm{H}$ & -3.39253800 & -7.25629700 & 1.15693800 \\
\hline $\mathrm{H}$ & -2.02882100 & -6.10150100 & 1.07358000 \\
\hline $\mathrm{C}$ & -6.07211600 & 1.32787900 & -2.30878800 \\
\hline $\mathrm{C}$ & -6.84777900 & 0.20488500 & -1.58795400 \\
\hline $\mathrm{H}$ & -6.20136000 & -0.65050300 & -1.36485500 \\
\hline $\mathrm{H}$ & -7.67463300 & -0.15044900 & -2.21472900 \\
\hline $\mathrm{H}$ & -7.26303900 & 0.55917700 & -0.63942200 \\
\hline $\mathrm{C}$ & -5.51101400 & 0.77602800 & -3.64543200 \\
\hline $\mathrm{H}$ & -4.97031000 & 1.56089100 & -4.18640400 \\
\hline $\mathrm{H}$ & -6.33386200 & 0.42842600 & -4.28073600 \\
\hline $\mathrm{H}$ & -4.82472700 & -0.06240100 & -3.50362100 \\
\hline $\mathrm{C}$ & -7.06317500 & 2.44104600 & -2.70523100 \\
\hline $\mathrm{H}$ & -7.66721000 & 2.79495400 & -1.86996300 \\
\hline $\mathrm{H}$ & -7.75175500 & 2.03425100 & -3.45580600 \\
\hline $\mathrm{H}$ & -6.55196400 & 3.30221700 & -3.14171300 \\
\hline
\end{tabular}




\begin{tabular}{|c|c|c|c|}
\hline $\mathrm{C}$ & -3.10317100 & 4.91991300 & 0.01416800 \\
\hline $\mathrm{C}$ & -3.91564200 & 5.38942300 & 1.24040200 \\
\hline $\mathrm{H}$ & -4.97463300 & 5.50254500 & 1.00578100 \\
\hline $\mathrm{H}$ & -3.54439400 & 6.36910800 & 1.56572200 \\
\hline $\mathrm{H}$ & -3.78651400 & 4.68521000 & 2.06616500 \\
\hline $\mathrm{C}$ & -1.61323200 & 5.10606900 & 0.35884500 \\
\hline $\mathrm{H}$ & -1.33371200 & 4.52809900 & 1.24150700 \\
\hline $\mathrm{H}$ & -1.42685800 & 6.16510900 & 0.56961300 \\
\hline $\mathrm{H}$ & -0.96980800 & 4.82130100 & -0.47788200 \\
\hline $\mathrm{C}$ & -3.41635400 & 5.82094600 & -1.20227300 \\
\hline $\mathrm{H}$ & -3.17409200 & 6.86324800 & -0.96123400 \\
\hline $\mathrm{H}$ & -4.47025200 & 5.76656800 & -1.48081300 \\
\hline $\mathrm{H}$ & -2.81134500 & 5.52685900 & -2.06841200 \\
\hline $\mathrm{C}$ & -6.59610700 & 3.65893600 & 0.27577100 \\
\hline $\mathrm{H}$ & -7.08321800 & 2.68615300 & 0.15346600 \\
\hline $\mathrm{H}$ & -7.34891100 & 4.45099800 & 0.27160500 \\
\hline $\mathrm{H}$ & -6.06098900 & 3.65295100 & 1.22841100 \\
\hline $\mathrm{C}$ & 0.74012900 & -2.32488600 & -1.21394100 \\
\hline $\mathrm{C}$ & 1.47631000 & -1.88270300 & -0.07873700 \\
\hline $\mathrm{C}$ & 1.72003200 & -2.75283100 & 0.99612100 \\
\hline $\mathrm{H}$ & 2.21450000 & -2.38090800 & 1.88464200 \\
\hline $\mathrm{C}$ & 1.32645600 & -4.09814800 & 0.97227500 \\
\hline $\mathrm{H}$ & 1.51031700 & -4.75282900 & 1.81603200 \\
\hline $\mathrm{C}$ & 0.69211500 & -4.53450100 & -0.17299700 \\
\hline $\mathrm{C}$ & 0.40712900 & -3.67052400 & -1.22675400 \\
\hline $\mathrm{C}$ & -0.18203900 & -5.72800300 & -1.83538700 \\
\hline $\mathrm{H}$ & 0.59932800 & -6.20324300 & -2.44550600 \\
\hline $\mathrm{H}$ & -1.14601400 & -6.21813200 & -1.96540100 \\
\hline $\mathrm{C}$ & -0.68587900 & -0.38628900 & -2.09665300 \\
\hline $\mathrm{C}$ & -1.10509600 & 0.39922300 & -3.17330200 \\
\hline $\mathrm{H}$ & -1.81784400 & 1.19510900 & -2.99818600 \\
\hline $\mathrm{C}$ & -0.64721800 & 0.18350700 & -4.48607300 \\
\hline $\mathrm{H}$ & -0.98170700 & 0.80072300 & -5.31188500 \\
\hline $\mathrm{C}$ & 0.23743300 & -0.85633400 & -4.66940100 \\
\hline $\mathrm{C}$ & 0.66982500 & -1.64231100 & -3.60364600 \\
\hline $\mathrm{C}$ & 0.25161500 & -1.44273100 & -2.30369300 \\
\hline $\mathrm{C}$ & 1.48680900 & -2.51328800 & -5.48077400 \\
\hline $\mathrm{H}$ & 2.48378200 & -2.55332300 & -5.91991800 \\
\hline $\mathrm{H}$ & 0.85323500 & -3.34144500 & -5.82811600 \\
\hline $\mathrm{C}$ & 1.09017500 & 3.28926800 & 3.9620020 \\
\hline $\mathrm{C}$ & 0.42841200 & 4.30866000 & 4.8408000 \\
\hline $\mathrm{H}$ & 1.84548500 & 3.66905700 & 3.2684060 \\
\hline $\mathrm{H}$ & 1.18213100 & 4.74561300 & 5.5152090 \\
\hline $\mathrm{C}$ & 0.81707000 & 1.97387600 & 3.958500 \\
\hline
\end{tabular}




$\begin{array}{lrrr}\mathrm{C} & 1.51133500 & 0.99225700 & 3.11028800 \\ \mathrm{H} & 0.05530100 & 1.59382800 & 4.63586300 \\ \mathrm{H} & 2.42404900 & 1.41992900 & 2.70033300 \\ \mathrm{C} & 1.77553300 & -0.34843700 & 3.70785100 \\ \mathrm{C} & 0.72875400 & -1.16566200 & 4.17585100 \\ \mathrm{C} & 3.09081100 & -0.83561100 & 3.80402900 \\ \mathrm{C} & 0.99103900 & -2.43474200 & 4.68661700 \\ \mathrm{H} & -0.28967000 & -0.79789800 & 4.09710100 \\ \mathrm{C} & 3.35090400 & -2.10893300 & 4.31276200 \\ \mathrm{H} & 3.91345100 & -0.21067900 & 3.47424200 \\ \mathrm{C} & 2.30169500 & -2.92002700 & 4.74797200 \\ \mathrm{H} & 0.16928700 & -3.05354600 & 5.03598200 \\ \mathrm{H} & 4.37598300 & -2.46438100 & 4.36890900 \\ \mathrm{H} & 2.50152200 & -3.91170300 & 5.14474100 \\ \mathrm{C} & -0.24869700 & 5.43589500 & 4.04491200 \\ \mathrm{H} & 0.45896700 & 5.91050000 & 3.35300500 \\ \mathrm{H} & -1.07921500 & 5.02409400 & 3.46886600 \\ \mathrm{H} & -0.63034900 & 6.21300700 & 4.71808800 \\ \mathrm{~S} & -2.38217600 & 1.72420900 & 3.42303900 \\ \mathrm{C} & -4.13615400 & 1.36366500 & 3.00656000 \\ \mathrm{C} & -5.10998900 & 2.17267600 & 3.59735500 \\ \mathrm{C} & -4.50777200 & 0.30227900 & 2.18646700 \\ \mathrm{C} & -6.46162600 & 1.90701000 & 3.37453100 \\ \mathrm{H} & -4.80931900 & 3.00634600 & 4.22707200 \\ \mathrm{C} & -5.86052000 & 0.04094100 & 1.95887800 \\ \mathrm{H} & -3.74159800 & -0.30870500 & 1.73251900 \\ \mathrm{C} & -6.83919900 & 0.83714500 & 2.55654700 \\ \mathrm{H} & -7.22062700 & 2.53203900 & 3.83803100 \\ \mathrm{H} & -6.14550500 & -0.78738200 & 1.31619100 \\ \mathrm{H} & -7.89205900 & 0.62519800 & 2.38981400 \\ \mathrm{O} & -1.67850900 & 0.56133200 & 2.62074800 \\ & -2.14390300 & 3.08025000 & 2.79223200 \\ -0.31426100 & 3.81162000 & 5.47646100\end{array}$<smiles></smiles>

TS-12a

Total SCF energy (M06/SDD-6-311++G(d,p)/SMD(1,4-Dioxane)): -5497.840146a.u.

Thermal correction to Gibbs Free Energy at 298.15 K: 1.770720a.u

Gibbs free energy at 298.15 K (M06/SDD-6-311++G(d,p)/SMD(1,4-Dioxane)): -5496.069426a.u. $\mathrm{Pd}$

$0.01476100 \quad-0.65070300 \quad-1.46503100$ 
1.81432100

$-1.46220700$

7.43881900

3.45261000

$-3.23328200$

$-6.70563200$

0.62088800

0.13420200

1.98946400

1.33222200

3.52622500

4.01078400

3.33070200

5.33079000

6.16738800

5.75274500

4.40550600

4.05256200

5.85938500

4.76839900

4.37721500

5.20036200

3.92663900

6.34956500

5.59143600

6.54694800

7.27448800

7.02001200

7.83824700

7.40676500

6.67023900

6.64294400

6.27856300

6.49260900

6.85543200

5.21344600

6.35847700

5.32994200

7.01444200

6.55776400

8.16393100

8.44637600

8.67667500

8.54256100
0.14429300

0.05136300

$-0.31729600$

$-3.46866200$

5.69750800

$-2.81925500$

5.63837000

3.87626100

1.65544800

0.09835300

0.16674800

$-1.09533300$

$-1.93521700$

$-1.29667400$

$-0.14959100$

1.10999400

1.23691900

2.19469200

$-2.73070700$

$-3.78103900$

$-3.69345000$

$-4.78167600$

$-3.71190000$

$-2.97405100$

$-2.67169000$

$-4.04221300$

$-2.43688100$

$-2.98241300$

$-2.27839700$

$-4.00088700$

$-2.88087500$

2.36325300

3.41744900

3.05511500

4.33653300

3.66784100

2.96969200

3.31994300

3.83191100

2.23677700

2.10378700

1.28856700

3.01352700

1.86720400
$-0.29663900$

0.54183400

$-2.31464900$

4.18728600

$-0.48489800$

0.99075800

1.69984800

3.09969200

4.26591000

5.83639300

$-0.96231000$

$-1.32711200$

$-1.27244300$

$-1.71329400$

$-1.78809000$

$-1.31206400$

$-0.92964200$

$-0.57787600$

$-1.92890800$

$-1.63403700$

$-0.61473100$

$-1.73912900$

$-2.33302600$

$-3.37216100$

$-4.10459200$

$-3.51996200$

$-3.58049200$

$-0.93898700$

$-1.10124400$

$-1.06584700$

0.09460900

$-1.12273200$

$-2.19078300$

$-3.20162500$

$-2.03115700$

$-2.15271300$

0.27700300

0.39405600

0.44051400

1.06609300

$-1.15544500$

$-0.48403600$

$-0.82167000$

$-2.14900300$ 


\begin{tabular}{|c|c|c|c|}
\hline $\mathrm{C}$ & 7.55997900 & 0.11073100 & -3.66787400 \\
\hline $\mathrm{H}$ & 7.35150100 & 1.18057100 & -3.77369400 \\
\hline $\mathrm{H}$ & 6.88018700 & -0.44606400 & -4.32260100 \\
\hline $\mathrm{H}$ & 8.59290500 & -0.08746300 & -3.96416600 \\
\hline $\mathrm{C}$ & 2.20449400 & -0.88838500 & 1.16901200 \\
\hline $\mathrm{C}$ & 1.60703400 & -2.13451500 & 1.29283400 \\
\hline $\mathrm{H}$ & 0.81996500 & -2.38724700 & 0.59356200 \\
\hline $\mathrm{C}$ & 2.01322900 & -3.06254000 & 2.26511100 \\
\hline $\mathrm{C}$ & 3.02638500 & -2.64704500 & 3.15743500 \\
\hline $\mathrm{C}$ & 3.70793800 & -1.40797900 & 3.00974800 \\
\hline $\mathrm{C}$ & 3.26374700 & -0.54546700 & 2.01131700 \\
\hline $\mathrm{H}$ & 3.75598100 & 0.40374300 & 1.8557360 \\
\hline $\mathrm{C}$ & 4.99236800 & -1.06861000 & 3.79623900 \\
\hline $\mathrm{C}$ & 4.82687900 & -1.17670000 & 5.32957500 \\
\hline $\mathrm{H}$ & 3.87933600 & -0.74462200 & 5.66307700 \\
\hline $\mathrm{H}$ & 5.64260300 & -0.64056700 & 5.82858900 \\
\hline $\mathrm{H}$ & 4.86121000 & -2.21244300 & 5.66399100 \\
\hline $\mathrm{C}$ & 6.09807400 & -2.04748000 & 3.33886000 \\
\hline $\mathrm{H}$ & 5.80743000 & -3.08163900 & 3.53963100 \\
\hline $\mathrm{H}$ & 7.03461900 & -1.84020600 & 3.87174900 \\
\hline $\mathrm{H}$ & 6.28677700 & -1.93970900 & 2.26469000 \\
\hline $\mathrm{C}$ & 5.47068800 & 0.36512300 & 3.4875890 \\
\hline $\mathrm{H}$ & 5.72569300 & 0.49421400 & 2.4308720 \\
\hline $\mathrm{H}$ & 6.37435200 & 0.57592900 & 4.0691250 \\
\hline $\mathrm{H}$ & 4.71196400 & 1.11140200 & 3.7500680 \\
\hline $\mathrm{C}$ & 1.32764900 & -4.44842700 & 2.25819900 \\
\hline $\mathrm{C}$ & 0.04720500 & -4.38840700 & 3.11871400 \\
\hline $\mathrm{H}$ & 0.25853300 & -4.14438400 & 4.16236000 \\
\hline $\mathrm{H}$ & -0.47469900 & -5.35169200 & 3.09281900 \\
\hline $\mathrm{H}$ & -0.63133100 & -3.62113500 & 2.73551600 \\
\hline $\mathrm{C}$ & 0.90401800 & -4.82816900 & 0.81565600 \\
\hline $\mathrm{H}$ & 0.12054300 & -4.18958500 & 0.40167900 \\
\hline $\mathrm{H}$ & 0.50883400 & -5.84923000 & 0.81598500 \\
\hline $\mathrm{H}$ & 1.76176000 & -4.79694100 & 0.13379800 \\
\hline $\mathrm{C}$ & 2.24872200 & -5.59079900 & 2.73775200 \\
\hline $\mathrm{H}$ & 3.18603000 & -5.59475500 & 2.17076200 \\
\hline $\mathrm{H}$ & 1.74498300 & -6.54830600 & 2.56340400 \\
\hline $\mathrm{H}$ & 2.50482300 & -5.53252600 & 3.79346500 \\
\hline $\mathrm{C}$ & 2.64490400 & -3.45661600 & 5.36497900 \\
\hline $\mathrm{H}$ & 2.20057700 & -2.47088600 & 5.53306900 \\
\hline $\mathrm{H}$ & 3.30110600 & -3.70378600 & 6.20342500 \\
\hline $\mathrm{H}$ & 1.85266300 & -4.20918300 & 5.30806300 \\
\hline $\mathrm{C}$ & -1.97529200 & 1.79285700 & 0.27318200 \\
\hline $\mathrm{C}$ & -2.45244300 & 2.60979300 & 1.30202800 \\
\hline
\end{tabular}




\begin{tabular}{|c|c|c|c|}
\hline $\mathrm{H}$ & -2.45833600 & 2.21420400 & 2.30824800 \\
\hline $\mathrm{C}$ & -2.91123600 & 3.90540200 & 1.05785600 \\
\hline $\mathrm{C}$ & -2.83472500 & 4.38879600 & -0.27503300 \\
\hline $\mathrm{C}$ & -2.42609100 & 3.57027900 & -1.35257300 \\
\hline $\mathrm{C}$ & -1.97448200 & 2.28132500 & -1.02942500 \\
\hline $\mathrm{H}$ & -1.60080100 & 1.62631000 & -1.80722300 \\
\hline $\mathrm{C}$ & -3.05954900 & -0.80796200 & 0.85293000 \\
\hline $\mathrm{C}$ & -4.23939600 & -0.11254600 & 1.09554100 \\
\hline $\mathrm{H}$ & -4.20862900 & 0.96575300 & 1.15756300 \\
\hline $\mathrm{C}$ & -5.47405500 & -0.75775900 & 1.24995100 \\
\hline $\mathrm{C}$ & -5.49492500 & -2.15191300 & 1.04470200 \\
\hline $\mathrm{C}$ & -4.30511100 & -2.91255800 & 0.90687300 \\
\hline $\mathrm{C}$ & -3.10153000 & -2.20891900 & 0.81258000 \\
\hline $\mathrm{H}$ & -2.18439700 & -2.74750600 & 0.63779900 \\
\hline $\mathrm{C}$ & -3.59485600 & 4.70680600 & 2.18699500 \\
\hline $\mathrm{C}$ & -5.10715700 & 4.76961800 & 1.86993000 \\
\hline $\mathrm{H}$ & -5.27945400 & 5.26455300 & 0.91050300 \\
\hline $\mathrm{H}$ & -5.63423100 & 5.33340200 & 2.64976400 \\
\hline $\mathrm{H}$ & -5.54150500 & 3.76558500 & 1.82645000 \\
\hline $\mathrm{C}$ & -3.41488300 & 4.01437700 & 3.55370900 \\
\hline $\mathrm{H}$ & -3.87776300 & 3.02361800 & 3.58409900 \\
\hline $\mathrm{H}$ & -3.90024500 & 4.61848900 & 4.32732300 \\
\hline $\mathrm{H}$ & -2.35679900 & 3.90443500 & 3.81414800 \\
\hline $\mathrm{C}$ & -3.06105800 & 6.15268800 & 2.33044600 \\
\hline $\mathrm{H}$ & -1.98136100 & 6.20258100 & 2.17097400 \\
\hline $\mathrm{H}$ & -3.27735400 & 6.52991500 & 3.33661800 \\
\hline $\mathrm{H}$ & -3.53626100 & 6.82608800 & 1.61812400 \\
\hline $\mathrm{C}$ & -2.42895500 & 3.98650700 & -2.84251200 \\
\hline $\mathrm{C}$ & -2.61660500 & 2.74687400 & -3.75136800 \\
\hline $\mathrm{H}$ & -1.77134700 & 2.05698700 & -3.71576200 \\
\hline $\mathrm{H}$ & -2.71222100 & 3.07677300 & -4.79108100 \\
\hline $\mathrm{H}$ & -3.51785300 & 2.18484500 & -3.48875100 \\
\hline $\mathrm{C}$ & -1.06296600 & 4.61596400 & -3.20002700 \\
\hline $\mathrm{H}$ & -0.88794500 & 5.55628800 & -2.67402600 \\
\hline $\mathrm{H}$ & -1.00432700 & 4.81399500 & -4.27718200 \\
\hline $\mathrm{H}$ & -0.25058600 & 3.93584900 & -2.92928500 \\
\hline $\mathrm{C}$ & -3.58692700 & 4.93974200 & -3.20897700 \\
\hline $\mathrm{H}$ & -4.54933500 & 4.49493300 & -2.93251100 \\
\hline $\mathrm{H}$ & -3.58898500 & 5.09857700 & -4.29336500 \\
\hline $\mathrm{H}$ & -3.52567400 & 5.91034800 & -2.72336900 \\
\hline $\mathrm{C}$ & -2.19032800 & 6.66445200 & -0.62443700 \\
\hline $\mathrm{H}$ & -2.52589700 & 7.58160900 & -0.13391700 \\
\hline $\mathrm{H}$ & -2.00402400 & 6.88330800 & -1.68056500 \\
\hline $\mathrm{H}$ & -1.26509600 & 6.32349800 & -0.15042300 \\
\hline
\end{tabular}




\begin{tabular}{|c|c|c|c|}
\hline $\mathrm{C}$ & -6.66404300 & 0.13608900 & 1.67825600 \\
\hline $\mathrm{C}$ & -7.00872500 & 1.12281300 & 0.54118400 \\
\hline $\mathrm{H}$ & -6.14936100 & 1.74907700 & 0.27829600 \\
\hline $\mathrm{H}$ & -7.82751400 & 1.78549900 & 0.84674200 \\
\hline $\mathrm{H}$ & -7.31988400 & 0.59028400 & -0.36358100 \\
\hline $\mathrm{C}$ & -6.21983500 & 0.94564900 & 2.92387600 \\
\hline $\mathrm{H}$ & -5.97588000 & 0.27269800 & 3.75328200 \\
\hline $\mathrm{H}$ & -7.03077500 & 1.60838200 & 3.24744100 \\
\hline $\mathrm{H}$ & -5.34371100 & 1.56729600 & 2.72971700 \\
\hline $\mathrm{C}$ & -7.94795000 & -0.60365100 & 2.10721400 \\
\hline $\mathrm{H}$ & -8.48203600 & -1.06005100 & 1.27417400 \\
\hline $\mathrm{H}$ & -8.62629700 & 0.12661500 & 2.56479300 \\
\hline $\mathrm{H}$ & -7.73863500 & -1.38321700 & 2.84326700 \\
\hline $\mathrm{C}$ & -4.30802800 & -4.45787700 & 0.94123300 \\
\hline $\mathrm{C}$ & -5.10246800 & -5.09173000 & -0.22099800 \\
\hline $\mathrm{H}$ & -6.17325400 & -4.91402600 & -0.13095600 \\
\hline $\mathrm{H}$ & -4.94678600 & -6.17699200 & -0.20696600 \\
\hline $\mathrm{H}$ & -4.74141400 & -4.72502600 & -1.18590500 \\
\hline $\mathrm{C}$ & -2.87935700 & -5.02362600 & 0.84693900 \\
\hline $\mathrm{H}$ & -2.40925000 & -4.78608200 & -0.11117100 \\
\hline $\mathrm{H}$ & -2.92553400 & -6.11489300 & 0.93309800 \\
\hline $\mathrm{H}$ & -2.24398300 & -4.65946100 & 1.65700900 \\
\hline $\mathrm{C}$ & -4.91254900 & -4.91009300 & 2.29013100 \\
\hline $\mathrm{H}$ & -4.90874900 & -6.00522200 & 2.35261400 \\
\hline $\mathrm{H}$ & -5.94171300 & -4.56136800 & 2.40071000 \\
\hline $\mathrm{H}$ & -4.32049400 & -4.52222000 & 3.12791000 \\
\hline $\mathrm{C}$ & -7.35691400 & -2.68913700 & -0.27367500 \\
\hline $\mathrm{H}$ & -7.56420800 & -1.64135800 & -0.51354200 \\
\hline $\mathrm{H}$ & -8.29691400 & -3.24077500 & -0.19345100 \\
\hline $\mathrm{H}$ & -6.74516100 & -3.10650700 & -1.07686500 \\
\hline $\mathrm{C}$ & 0.86230500 & 2.06239600 & 1.5787690 \\
\hline $\mathrm{C}$ & 1.47223900 & 1.83539300 & 0.3137550 \\
\hline $\mathrm{C}$ & 1.72223000 & 2.91466200 & -0.54854400 \\
\hline $\mathrm{H}$ & 2.12523500 & 2.71910000 & -1.53370400 \\
\hline $\mathrm{C}$ & 1.46672600 & 4.24637000 & -0.18659000 \\
\hline $\mathrm{H}$ & 1.66190500 & 5.06659400 & -0.86802100 \\
\hline $\mathrm{C}$ & 0.96784000 & 4.45417300 & 1.08323700 \\
\hline $\mathrm{C}$ & 0.67830500 & 3.38993800 & 1.9302870 \\
\hline $\mathrm{C}$ & 0.43611100 & 5.27206900 & 3.0745940 \\
\hline $\mathrm{H}$ & 1.37131600 & 5.45301200 & 3.62401300 \\
\hline $\mathrm{H}$ & -0.39798800 & 5.83201500 & 3.49322300 \\
\hline $\mathrm{C}$ & -0.69112100 & 0.10780900 & 2.22232600 \\
\hline $\mathrm{C}$ & -1.08906300 & -0.81960900 & 3.19229100 \\
\hline $\mathrm{H}$ & -1.91029800 & -1.49014500 & 2.97190800 \\
\hline
\end{tabular}




\begin{tabular}{|c|c|c|c|}
\hline $\mathrm{C}$ & -0.47488600 & -0.91325500 & 4.45325300 \\
\hline $\mathrm{H}$ & -0.79281500 & -1.64803500 & 5.18391300 \\
\hline $\mathrm{C}$ & 0.54172400 & -0.02051700 & 4.71295200 \\
\hline $\mathrm{C}$ & 0.93773400 & 0.91919400 & 3.7671430 \\
\hline $\mathrm{C}$ & 0.37568600 & 1.01237300 & 2.5080110 \\
\hline $\mathrm{C}$ & 2.02323200 & 1.34248400 & 5.6600120 \\
\hline $\mathrm{H}$ & 3.05847900 & 1.23206900 & 5.9814060 \\
\hline $\mathrm{H}$ & 1.50106500 & 2.12867200 & 6.2239750 \\
\hline $\mathrm{C}$ & -0.97448100 & -2.01760000 & -4.52732000 \\
\hline $\mathrm{C}$ & -2.06818200 & -1.96131000 & -5.57269100 \\
\hline $\mathrm{H}$ & -0.34297000 & -2.90614400 & -4.54813900 \\
\hline $\mathrm{H}$ & -1.68639600 & -1.37355800 & -6.42070800 \\
\hline $\mathrm{C}$ & -0.40208000 & -0.84933600 & -4.02442500 \\
\hline $\mathrm{C}$ & 0.89811800 & -0.80185300 & -3.40792400 \\
\hline $\mathrm{H}$ & -0.89946800 & 0.09060700 & -4.25386600 \\
\hline $\mathrm{H}$ & 1.42560300 & -1.75846900 & -3.40436800 \\
\hline $\mathrm{C}$ & 1.78894900 & 0.33949900 & -3.74401700 \\
\hline $\mathrm{C}$ & 1.33360000 & 1.66795200 & -3.75317600 \\
\hline $\mathrm{C}$ & 3.11732100 & 0.10109100 & -4.12938100 \\
\hline $\mathrm{C}$ & 2.17156300 & 2.71559500 & -4.12739700 \\
\hline $\mathrm{H}$ & 0.31765800 & 1.87828600 & -3.44114000 \\
\hline $\mathrm{C}$ & 3.96227900 & 1.14744400 & -4.49378100 \\
\hline $\mathrm{H}$ & 3.49005500 & -0.91788500 & -4.13581500 \\
\hline $\mathrm{C}$ & 3.49598000 & 2.46210200 & -4.49411900 \\
\hline $\mathrm{H}$ & 1.78995400 & 3.73331200 & -4.12823900 \\
\hline $\mathrm{H}$ & 4.98682400 & 0.93356700 & -4.78004300 \\
\hline $\mathrm{H}$ & 4.15250600 & 3.27849100 & -4.78014700 \\
\hline $\mathrm{C}$ & -2.52326100 & -3.33291500 & -6.07898400 \\
\hline $\mathrm{H}$ & -1.69577000 & -3.85583100 & -6.57320600 \\
\hline $\mathrm{H}$ & -2.87152300 & -3.97178900 & -5.26283000 \\
\hline $\mathrm{H}$ & -3.33485200 & -3.22686500 & -6.80664500 \\
\hline S & -2.22736400 & -3.05294000 & -2.73154500 \\
\hline $\mathrm{C}$ & -3.78961100 & -2.13714800 & -2.70873800 \\
\hline $\mathrm{C}$ & -4.91354100 & -2.70429200 & -3.31584800 \\
\hline $\mathrm{C}$ & -3.80898000 & -0.80845900 & -2.27944600 \\
\hline $\mathrm{C}$ & -6.07048900 & -1.93951600 & -3.46862700 \\
\hline $\mathrm{H}$ & -4.87048100 & -3.73594000 & -3.64821200 \\
\hline $\mathrm{C}$ & -4.97380700 & -0.05524300 & -2.41900000 \\
\hline $\mathrm{H}$ & -2.92762200 & -0.38037700 & -1.82664300 \\
\hline $\mathrm{C}$ & -6.10365400 & -0.61669000 & -3.01772900 \\
\hline $\mathrm{H}$ & -6.94958400 & -2.37807800 & -3.93321400 \\
\hline $\mathrm{H}$ & -4.99742300 & 0.96463500 & -2.04721800 \\
\hline $\mathrm{H}$ & -7.00806000 & -0.02550500 & -3.13220900 \\
\hline $\mathrm{O}$ & -1.46256900 & -2.60944100 & -1.49700200 \\
\hline
\end{tabular}




$\begin{array}{llll}\mathrm{O} & -2.59855300 & -4.50995800 & -2.78214600 \\ \mathrm{H} & -2.92104400 & -1.39417500 & -5.17886400\end{array}$

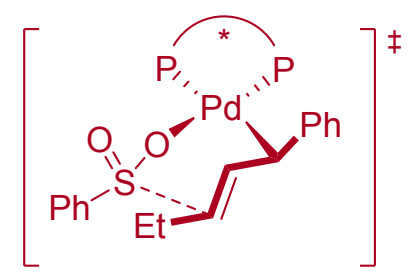

TS-12b

Total SCF energy (M06/SDD-6-311++G(d,p)/SMD(1,4-Dioxane)): -5497.840824a.u.

Thermal correction to Gibbs Free Energy at 298.15 K: 1.769026a.u

Gibbs free energy at 298.15 K (M06/SDD-6-311++G(d,p)/SMD(1,4-Dioxane)): -5496.071798a.u.

$\begin{array}{lccc}\mathrm{Pd} & -0.06770100 & -1.06216400 & -0.72149100 \\ \mathrm{P} & 1.20261200 & 0.99983200 & -0.35230500 \\ \mathrm{P} & -1.86622000 & -0.16137200 & 0.45303200 \\ \mathrm{O} & 5.29840600 & 0.04200600 & -4.43250900 \\ \mathrm{O} & 3.92608100 & 0.43396000 & 4.96285800 \\ \mathrm{O} & -4.48631000 & 4.38167900 & -2.41855400 \\ \mathrm{O} & -5.73682100 & -4.59880800 & 1.33530800 \\ \mathrm{O} & -1.61155700 & 6.20529100 & 0.37014800 \\ \mathrm{O} & -1.94202800 & 4.57154000 & 1.95627500 \\ \mathrm{O} & 0.37824900 & 3.51424300 & 3.61658300 \\ \mathrm{O} & -0.01725600 & 2.34969300 & 5.56810200 \\ \mathrm{C} & 2.45443500 & 1.20188500 & -1.66133900 \\ \mathrm{C} & 3.79371100 & 1.48867200 & -1.43182500 \\ \mathrm{H} & 4.09876500 & 1.85317500 & -0.46024200 \\ \mathrm{C} & 4.77953000 & 1.24808600 & -2.40064200 \\ \mathrm{C} & 4.36439900 & 0.60377000 & -3.58398800 \\ \mathrm{C} & 2.99428800 & 0.47578800 & -3.92830000 \\ \mathrm{C} & 2.06423200 & 0.77919200 & -2.93856800 \\ \mathrm{H} & 1.01393900 & 0.57754200 & -3.11062100 \\ \mathrm{C} & 6.21304400 & 1.72498600 & -2.07084300 \\ \mathrm{C} & 6.12599000 & 3.22288500 & -1.68061700 \\ \mathrm{H} & 5.74918900 & 3.81842700 & -2.51960300 \\ \mathrm{H} & 7.12130500 & 3.59761500 & -1.41459300 \\ \mathrm{H} & 5.46649300 & 3.39682900 & -0.82655200 \\ \mathrm{C} & 6.77294900 & 0.93103800 & -0.87141900 \\ \mathrm{H} & 6.14426100 & 1.04865500 & 0.01713700 \\ \mathrm{H} & 7.78115100 & 1.28305600 & -0.62032500 \\ \mathrm{H} & 6.82833100 & -0.13989600 & -1.08807100 \\ \mathrm{C} & 7.22054100 & 1.65545600 & -3.23616800 \\ \mathrm{H} & 7.51173700 & 0.63721700 & -3.49236800 \\ \mathrm{H} & 8.12957300 & 2.19152500 & -2.93713100 \\ & & & \\ & & & \\ & & \end{array}$




\begin{tabular}{|c|c|c|c|}
\hline $\mathrm{H}$ & 6.82613700 & 2.12998800 & -4.13834100 \\
\hline $\mathrm{C}$ & 2.53183100 & -0.01191200 & -5.31517500 \\
\hline $\mathrm{C}$ & 2.88221800 & -1.50041700 & -5.53922100 \\
\hline $\mathrm{H}$ & 3.94962500 & -1.63666000 & -5.71531900 \\
\hline $\mathrm{H}$ & 2.35033900 & -1.87367700 & -6.42341400 \\
\hline $\mathrm{H}$ & 2.59953700 & -2.11153900 & -4.67618200 \\
\hline $\mathrm{C}$ & 1.00512700 & 0.13024100 & -5.47316600 \\
\hline $\mathrm{H}$ & 0.45453800 & -0.52940300 & -4.79507200 \\
\hline $\mathrm{H}$ & 0.72414500 & -0.14395900 & -6.49587300 \\
\hline $\mathrm{H}$ & 0.66819700 & 1.15722300 & -5.29858200 \\
\hline $\mathrm{C}$ & 3.18948800 & 0.85531100 & -6.41156200 \\
\hline $\mathrm{H}$ & 2.89426200 & 1.90599300 & -6.30334900 \\
\hline $\mathrm{H}$ & 2.86589500 & 0.51390600 & -7.40264100 \\
\hline $\mathrm{H}$ & 4.27780500 & 0.79257800 & -6.36222400 \\
\hline $\mathrm{C}$ & 5.78000900 & -1.22191900 & -3.94475900 \\
\hline $\mathrm{H}$ & 6.37244400 & -1.65259100 & -4.75579500 \\
\hline $\mathrm{H}$ & 4.95531900 & -1.88992100 & -3.67955800 \\
\hline $\mathrm{H}$ & 6.41733200 & -1.08671500 & -3.06368100 \\
\hline $\mathrm{C}$ & 2.08164500 & 0.85181700 & 1.24585500 \\
\hline $\mathrm{C}$ & 1.93444500 & -0.33306700 & 1.96374200 \\
\hline $\mathrm{H}$ & 1.33053300 & -1.10876000 & 1.51263400 \\
\hline $\mathrm{C}$ & 2.55102500 & -0.55001700 & 3.20215200 \\
\hline $\mathrm{C}$ & 3.31654100 & 0.52285200 & 3.72339500 \\
\hline $\mathrm{C}$ & 3.56484100 & 1.70734000 & 2.97895700 \\
\hline $\mathrm{C}$ & 2.89608600 & 1.85928100 & 1.76256700 \\
\hline $\mathrm{H}$ & 3.00154200 & 2.77759100 & 1.20175500 \\
\hline $\mathrm{C}$ & 4.58807200 & 2.77267000 & 3.42800600 \\
\hline $\mathrm{C}$ & 4.13141900 & 3.50022500 & 4.70804400 \\
\hline $\mathrm{H}$ & 3.15753700 & 3.97155300 & 4.53904800 \\
\hline $\mathrm{H}$ & 4.84711400 & 4.29010300 & 4.96572800 \\
\hline $\mathrm{H}$ & 4.05492800 & 2.82931200 & 5.56179300 \\
\hline $\mathrm{C}$ & 5.96449900 & 2.10401200 & 3.64795500 \\
\hline $\mathrm{H}$ & 5.91699200 & 1.33246800 & 4.41695200 \\
\hline $\mathrm{H}$ & 6.70288600 & 2.85668400 & 3.95005100 \\
\hline $\mathrm{H}$ & 6.31650100 & 1.64703200 & 2.71506900 \\
\hline $\mathrm{C}$ & 4.78485700 & 3.86015700 & 2.35248700 \\
\hline $\mathrm{H}$ & 5.12776500 & 3.43899700 & 1.40202600 \\
\hline $\mathrm{H}$ & 5.55083000 & 4.56426200 & 2.69454100 \\
\hline $\mathrm{H}$ & 3.86952800 & 4.43316900 & 2.16832500 \\
\hline $\mathrm{C}$ & 2.39844800 & -1.94369000 & 3.86405800 \\
\hline $\mathrm{C}$ & 1.21697000 & -1.95793000 & 4.85855200 \\
\hline $\mathrm{H}$ & 1.30676000 & -1.20701600 & 5.64420300 \\
\hline $\mathrm{H}$ & 1.14452500 & -2.94279200 & 5.33628300 \\
\hline $\mathrm{H}$ & 0.28084100 & -1.76465300 & 4.32814200 \\
\hline
\end{tabular}




\begin{tabular}{|c|c|c|c|}
\hline $\mathrm{C}$ & 2.07009800 & -3.01833400 & 2.80037200 \\
\hline $\mathrm{H}$ & 1.08203000 & -2.87288500 & 2.35375300 \\
\hline $\mathrm{H}$ & 2.06204500 & -4.00312200 & 3.27997500 \\
\hline $\mathrm{H}$ & 2.81505100 & -3.03540000 & 2.00109000 \\
\hline $\mathrm{C}$ & 3.70292300 & -2.41211700 & 4.54533400 \\
\hline $\mathrm{H}$ & 4.52320200 & -2.42964300 & 3.82037100 \\
\hline $\mathrm{H}$ & 3.56428500 & -3.43196600 & 4.92244500 \\
\hline $\mathrm{H}$ & 4.00827400 & -1.77770300 & 5.37490800 \\
\hline $\mathrm{C}$ & 3.10578400 & 0.60032400 & 6.1189880 \\
\hline $\mathrm{H}$ & 2.17267000 & 1.11988300 & 5.8803160 \\
\hline $\mathrm{H}$ & 3.67589700 & 1.19207600 & 6.8417730 \\
\hline $\mathrm{H}$ & 2.87918500 & -0.36971400 & 6.57272300 \\
\hline $\mathrm{C}$ & -2.74057000 & 1.17215400 & -0.43078800 \\
\hline $\mathrm{C}$ & -3.67349000 & 1.98850600 & 0.21662600 \\
\hline $\mathrm{H}$ & -3.88305700 & 1.80010400 & 1.26064500 \\
\hline $\mathrm{C}$ & -4.31188000 & 3.03531300 & -0.44747100 \\
\hline $\mathrm{C}$ & -3.93446600 & 3.27698200 & -1.79649200 \\
\hline $\mathrm{C}$ & -3.06913900 & 2.41785000 & -2.50859400 \\
\hline $\mathrm{C}$ & -2.46927300 & 1.37969500 & -1.77801900 \\
\hline $\mathrm{H}$ & -1.75887800 & 0.71511100 & -2.25520700 \\
\hline $\mathrm{C}$ & -3.15373800 & -1.35855200 & 0.94458400 \\
\hline $\mathrm{C}$ & -4.50760900 & -1.20264900 & 0.67297000 \\
\hline $\mathrm{H}$ & -4.84788500 & -0.26541700 & 0.25424600 \\
\hline $\mathrm{C}$ & -5.43870000 & -2.22782100 & 0.90521300 \\
\hline $\mathrm{C}$ & -4.92600600 & -3.47764200 & 1.31725300 \\
\hline $\mathrm{C}$ & -3.57440700 & -3.64019200 & 1.72321300 \\
\hline $\mathrm{C}$ & -2.71804500 & -2.56144300 & 1.51721900 \\
\hline $\mathrm{H}$ & -1.66877400 & -2.65622100 & 1.76382400 \\
\hline $\mathrm{C}$ & -5.47403000 & 3.78610100 & 0.23953700 \\
\hline $\mathrm{C}$ & -6.77992900 & 3.37820300 & -0.48070100 \\
\hline $\mathrm{H}$ & -6.74347300 & 3.66145700 & -1.53601100 \\
\hline $\mathrm{H}$ & -7.64093500 & 3.87607200 & -0.01763200 \\
\hline $\mathrm{H}$ & -6.93916100 & 2.29584000 & -0.41743000 \\
\hline $\mathrm{C}$ & -5.59154000 & 3.39278900 & 1.72615900 \\
\hline $\mathrm{H}$ & -5.80110900 & 2.32746000 & 1.86312600 \\
\hline $\mathrm{H}$ & -6.42134500 & 3.94552400 & 2.17894800 \\
\hline $\mathrm{H}$ & -4.67649100 & 3.63873600 & 2.27701000 \\
\hline $\mathrm{C}$ & -5.34551300 & 5.32420100 & 0.19677100 \\
\hline $\mathrm{H}$ & -4.34759000 & 5.64358900 & 0.50552200 \\
\hline $\mathrm{H}$ & -6.07257200 & 5.77043700 & 0.88523400 \\
\hline $\mathrm{H}$ & -5.54227200 & 5.72001900 & -0.79801800 \\
\hline $\mathrm{C}$ & -2.73004400 & 2.50928900 & -4.01745200 \\
\hline $\mathrm{C}$ & -2.70278800 & 1.08045900 & -4.60910900 \\
\hline $\mathrm{H}$ & -1.91532900 & 0.45567800 & -4.18774700 \\
\hline
\end{tabular}


$\mathrm{H}$

$\mathrm{H}$

C

$\mathrm{H}$

$\mathrm{H}$

$\mathrm{H}$

C

$\mathrm{H}$

$\mathrm{H}$

$\mathrm{H}$

C

$\mathrm{H}$

$\mathrm{H}$

$\mathrm{H}$

C

C

$\mathrm{H}$

$\mathrm{H}$

$\mathrm{H}$

C

$\mathrm{H}$

$\mathrm{H}$

$\mathrm{H}$

C

$\mathrm{H}$

$\mathrm{H}$

$\mathrm{H}$

C

C

$\mathrm{H}$

$\mathrm{H}$

$\mathrm{H}$

C

$\mathrm{H}$

$\mathrm{H}$

$\mathrm{H}$

C

$\mathrm{H}$

$\mathrm{H}$

$\mathrm{H}$

C

$\mathrm{H}$

$\mathrm{H}$

$\mathrm{H}$

$\begin{array}{rrr}-2.52360600 & 1.13529700 & -5.68867600 \\ -3.65332100 & 0.56452300 & -4.44396400 \\ -1.32211000 & 3.11913500 & -4.19322700 \\ -1.26478300 & 4.14165800 & -3.80885900 \\ -1.04944100 & 3.13840500 & -5.25523200 \\ -0.57134200 & 2.52877100 & -3.65926700 \\ -3.75180500 & 3.29193800 & -4.87042400 \\ -4.77307100 & 2.94875500 & -4.68153700 \\ -3.52444000 & 3.11376300 & -5.92783900 \\ -3.72554900 & 4.36852800 & -4.71090400 \\ -3.61775800 & 5.51616700 & -2.47339200 \\ -4.20810200 & 6.33157800 & -2.89820600 \\ -2.75262800 & 5.32662200 & -3.11483800 \\ -3.26316800 & 5.79740700 & -1.47857100 \\ -6.93179900 & -1.86711300 & 0.71179000 \\ -7.24513300 & -1.69339200 & -0.79004800 \\ -6.58156200 & -0.95510000 & -1.25233700 \\ -8.27931800 & -1.35486500 & -0.92505200 \\ -7.12742400 & -2.63410100 & -1.33774300 \\ -7.19456100 & -0.51159100 & 1.41919800 \\ -6.93385400 & -0.56869400 & 2.48167700 \\ -8.25696800 & -0.25596000 & 1.34078100 \\ -6.62983000 & 0.31199500 & 0.97657600 \\ -7.92991500 & -2.86313200 & 1.33791000 \\ -7.98788100 & -3.81246400 & 0.80716000 \\ -8.92931200 & -2.41350000 & 1.31267200 \\ -7.68008700 & -3.07637000 & 2.38104300 \\ -3.04262500 & -4.93224900 & 2.37767300 \\ -3.06647100 & -6.12380500 & 1.39497500 \\ -4.08296100 & -6.45478500 & 1.18409800 \\ -2.52634500 & -6.97202900 & 1.83183900 \\ -2.57363700 & -5.86419900 & 0.45097400 \\ -1.58038700 & -4.76698600 & 2.84179600 \\ -0.89261000 & -4.62452200 & 2.00053600 \\ -1.26918300 & -5.67689300 & 3.36544400 \\ -1.45577900 & -3.92825500 & 3.53560300 \\ -3.89351300 & -5.26177600 & 3.62410500 \\ -3.52197700 & -6.17748700 & 4.09961200 \\ -4.94171500 & -5.40935500 & 3.35888300 \\ -3.83068400 & -4.45045100 & 4.35868500 \\ -5.95459600 & -5.15968100 & 0.03910800 \\ -6.46630100 & -6.11183600 & 0.19734500 \\ & -5.33095400 & -0.48187000\end{array}$




\begin{tabular}{|c|c|c|c|}
\hline $\mathrm{C}$ & -0.48513400 & 2.84599300 & 0.93063500 \\
\hline $\mathrm{C}$ & 0.37987100 & 2.62465500 & -0.17919300 \\
\hline $\mathrm{C}$ & 0.55724900 & 3.61848600 & -1.14909300 \\
\hline $\mathrm{H}$ & 1.22929700 & 3.42268200 & -1.97691000 \\
\hline $\mathrm{C}$ & -0.07585800 & 4.87043200 & -1.07027900 \\
\hline $\mathrm{H}$ & 0.08310500 & 5.63767600 & -1.81959100 \\
\hline $\mathrm{C}$ & -0.88222500 & 5.08259200 & 0.02776500 \\
\hline $\mathrm{C}$ & -1.07945700 & 4.09533700 & 0.98873400 \\
\hline $\mathrm{C}$ & -1.96187200 & 5.98285600 & 1.74139800 \\
\hline $\mathrm{H}$ & -1.21084300 & 6.46046700 & 2.38803300 \\
\hline $\mathrm{H}$ & -2.96376100 & 6.36639600 & 1.92896100 \\
\hline $\mathrm{C}$ & -1.38978700 & 0.65371400 & 2.04409400 \\
\hline $\mathrm{C}$ & -1.63623200 & -0.02272200 & 3.24752800 \\
\hline $\mathrm{H}$ & -2.18562700 & -0.95399800 & 3.22583500 \\
\hline $\mathrm{C}$ & -1.23277100 & 0.46394000 & 4.49988600 \\
\hline $\mathrm{H}$ & -1.44478700 & -0.07659400 & 5.41490600 \\
\hline $\mathrm{C}$ & -0.55418900 & 1.66116600 & 4.50241700 \\
\hline $\mathrm{C}$ & -0.31355800 & 2.35717100 & 3.32358800 \\
\hline $\mathrm{C}$ & -0.71408600 & 1.91447400 & 2.07073300 \\
\hline $\mathrm{C}$ & 0.28756200 & 3.64526700 & 5.0360620 \\
\hline $\mathrm{H}$ & 1.24409100 & 3.98148000 & 5.4344290 \\
\hline $\mathrm{H}$ & -0.52684500 & 4.34031500 & 5.28703000 \\
\hline $\mathrm{C}$ & 1.95005800 & -5.54887100 & -0.94200700 \\
\hline $\mathrm{C}$ & 1.30264700 & -4.20390000 & -0.68962300 \\
\hline $\mathrm{C}$ & 0.32632800 & -3.70688900 & -1.57682800 \\
\hline $\mathrm{C}$ & -0.87013600 & -3.03096600 & -1.20919100 \\
\hline $\mathrm{H}$ & -1.30777300 & -3.30464100 & -0.25235600 \\
\hline $\mathrm{C}$ & -1.86532800 & -2.76479500 & -2.27372100 \\
\hline $\mathrm{C}$ & -3.24244000 & -2.87759100 & -2.01697100 \\
\hline $\mathrm{C}$ & -1.46242700 & -2.43147900 & -3.58214200 \\
\hline $\mathrm{C}$ & -4.17981300 & -2.66392100 & -3.02545300 \\
\hline $\mathrm{H}$ & -3.57377900 & -3.15208000 & -1.02373400 \\
\hline $\mathrm{C}$ & -2.39934600 & -2.23508600 & -4.59239200 \\
\hline $\mathrm{H}$ & -0.40449800 & -2.33099800 & -3.80291100 \\
\hline $\mathrm{C}$ & -3.76548700 & -2.34606100 & -4.32039500 \\
\hline $\mathrm{H}$ & -5.23819000 & -2.75851200 & -2.79992900 \\
\hline $\mathrm{H}$ & -2.06144000 & -1.97973400 & -5.59302100 \\
\hline $\mathrm{H}$ & -4.49608400 & -2.18739900 & -5.10854600 \\
\hline $\mathrm{H}$ & 2.25553900 & -5.61060600 & -1.99161300 \\
\hline $\mathrm{C}$ & 0.98500500 & -6.69246300 & -0.59453400 \\
\hline $\mathrm{H}$ & 1.46373500 & -7.66721400 & -0.74354400 \\
\hline $\mathrm{H}$ & 0.66047800 & -6.63240700 & 0.45184700 \\
\hline $\mathrm{H}$ & 0.08955300 & -6.64763000 & -1.22295300 \\
\hline $\mathrm{H}$ & 2.86106800 & -5.64076200 & -0.33603500 \\
\hline
\end{tabular}




$\begin{array}{lccc}\mathrm{S} & 3.03276500 & -2.86989100 & -1.19845800 \\ \mathrm{O} & 2.46878700 & -1.48044300 & -1.14377300 \\ \mathrm{O} & 3.46241700 & -3.37557300 & -2.54488900 \\ \mathrm{C} & 4.53486200 & -2.76011100 & -0.20323800 \\ \mathrm{C} & 4.73261500 & -1.67565100 & 0.65199600 \\ \mathrm{C} & 5.45751400 & -3.80830400 & -0.26809200 \\ \mathrm{C} & 5.86423900 & -1.64998500 & 1.46798800 \\ \mathrm{H} & 4.01803000 & -0.86518200 & 0.65850500 \\ \mathrm{C} & 6.58774600 & -3.76986200 & 0.54716400 \\ \mathrm{H} & 5.29550800 & -4.62332000 & -0.96603000 \\ \mathrm{C} & 6.78848600 & -2.69605700 & 1.42063500 \\ \mathrm{H} & 6.02224000 & -0.80720000 & 2.13468000 \\ \mathrm{H} & 7.31472400 & -4.57582300 & 0.49959700 \\ \mathrm{H} & 7.66801900 & -2.67266800 & 2.05773100 \\ \mathrm{H} & 1.14706500 & -3.97224400 & 0.36553800 \\ \mathrm{H} & 0.50858600 & -3.89181300 & -2.63330800\end{array}$<smiles>CCC(C=C(c1ccccc1)[P+]1PCCCP1)C(C)S(=O)(=O)c1ccccc1</smiles>

\section{Int-13a}

Total SCF energy (M06/SDD-6-311++G(d,p)/SMD(1,4-Dioxane)): -5497.869559a.u.

Thermal correction to Gibbs Free Energy at 298.15 K: 1.773361a.u

Gibbs free energy at 298.15 K (M06/SDD-6-311++G(d,p)/SMD(1,4-Dioxane)): -5496.095198a.u.

$\begin{array}{crcc}\mathrm{Pd} & 0.11206900 & -1.36248700 & -0.54223400 \\ \mathrm{P} & 2.00681800 & -0.08014700 & -0.05075100 \\ \mathrm{P} & -1.38525600 & 0.37315700 & 0.30471500 \\ \mathrm{O} & 7.18159000 & -2.65551200 & -1.59550800 \\ \mathrm{O} & 3.37540400 & 1.87512800 & 5.44592500 \\ \mathrm{O} & -3.76993100 & 3.71378900 & -4.08117900 \\ \mathrm{O} & -6.16215000 & -1.90504500 & 3.05687200 \\ \mathrm{O} & 0.59578500 & 4.45535500 & -3.64774500 \\ \mathrm{O} & -0.08357200 & 4.60002500 & -1.44935000 \\ \mathrm{O} & 1.78606800 & 4.56304600 & 1.14202600 \\ \mathrm{O} & 1.07971000 & 4.93601100 & 3.30515900 \\ \mathrm{C} & 3.65677500 & -0.73685700 & -0.50019500 \\ \mathrm{C} & 3.92283100 & -2.04025300 & -0.06880100 \\ \mathrm{H} & 3.14517900 & -2.56123000 & 0.47550900 \\ \mathrm{C} & 5.12906000 & -2.67931800 & -0.35193700 \\ \mathrm{C} & 6.06992400 & -1.96413600 & -1.13937100 \\ \mathrm{C} & 5.90514500 & -0.59740800 & -1.44790200\end{array}$




\begin{tabular}{|c|c|c|c|}
\hline $\mathrm{C}$ & 4.66406200 & -0.02161900 & -1.13362700 \\
\hline $\mathrm{H}$ & 4.47674800 & 1.00781600 & -1.40982900 \\
\hline $\mathrm{C}$ & 5.42339300 & -4.07808000 & 0.22935200 \\
\hline $\mathrm{C}$ & 4.28762700 & -4.54672800 & 1.16119700 \\
\hline $\mathrm{H}$ & 4.10862400 & -3.84096200 & 1.97921500 \\
\hline $\mathrm{H}$ & 4.56507600 & -5.50803300 & 1.60603800 \\
\hline $\mathrm{H}$ & 3.34411100 & -4.69442400 & 0.62295700 \\
\hline $\mathrm{C}$ & 5.58173400 & -5.15319100 & -0.86778400 \\
\hline $\mathrm{H}$ & 4.73405500 & -5.13702400 & -1.56324800 \\
\hline $\mathrm{H}$ & 5.61481400 & -6.14740600 & -0.40687700 \\
\hline $\mathrm{H}$ & 6.50381600 & -5.02212100 & -1.43337300 \\
\hline $\mathrm{C}$ & 6.71698600 & -4.00577000 & 1.07230400 \\
\hline $\mathrm{H}$ & 7.56854000 & -3.69822200 & 0.46251700 \\
\hline $\mathrm{H}$ & 6.93679400 & -4.98877100 & 1.50630200 \\
\hline $\mathrm{H}$ & 6.60007900 & -3.29109300 & 1.89475500 \\
\hline $\mathrm{C}$ & 6.96743000 & 0.34175700 & -2.06933400 \\
\hline $\mathrm{C}$ & 6.55164800 & 0.71286100 & -3.50982700 \\
\hline $\mathrm{H}$ & 6.54012200 & -0.16613700 & -4.16279700 \\
\hline $\mathrm{H}$ & 7.25629300 & 1.43799700 & -3.93454700 \\
\hline $\mathrm{H}$ & 5.54976200 & 1.15430600 & -3.53470700 \\
\hline $\mathrm{C}$ & 7.02034400 & 1.64098800 & -1.22363500 \\
\hline $\mathrm{H}$ & 6.07885900 & 2.19544900 & -1.23265500 \\
\hline $\mathrm{H}$ & 7.79385000 & 2.30761600 & -1.62089700 \\
\hline $\mathrm{H}$ & 7.26910200 & 1.41345000 & -0.18141300 \\
\hline $\mathrm{C}$ & 8.41099200 & -0.20315400 & -2.07590900 \\
\hline $\mathrm{H}$ & 8.70727000 & -0.56576700 & -1.08809200 \\
\hline $\mathrm{H}$ & 9.08673500 & 0.61349700 & -2.35594500 \\
\hline $\mathrm{H}$ & 8.56752100 & -1.01026200 & -2.79062100 \\
\hline $\mathrm{C}$ & 7.07308800 & -3.05557800 & -2.95967000 \\
\hline $\mathrm{H}$ & 7.05753600 & -2.19207700 & -3.63343800 \\
\hline $\mathrm{H}$ & 6.16743400 & -3.64889700 & -3.12869800 \\
\hline $\mathrm{H}$ & 7.95432000 & -3.66436500 & -3.17596800 \\
\hline $\mathrm{C}$ & 2.40254000 & 0.58014200 & 1.61268700 \\
\hline $\mathrm{C}$ & 1.82881800 & -0.03740200 & 2.71542100 \\
\hline $\mathrm{H}$ & 1.09732600 & -0.81575500 & 2.53308100 \\
\hline $\mathrm{C}$ & 2.17410800 & 0.31191900 & 4.03098500 \\
\hline $\mathrm{C}$ & 3.07088900 & 1.39007500 & 4.1850380 \\
\hline $\mathrm{C}$ & 3.73312400 & 1.99513600 & 3.0818490 \\
\hline $\mathrm{C}$ & 3.37352200 & 1.56541700 & 1.8054990 \\
\hline $\mathrm{H}$ & 3.84544100 & 1.99742800 & 0.9342440 \\
\hline $\mathrm{C}$ & 4.91536100 & 2.97383200 & 3.2592550 \\
\hline $\mathrm{C}$ & 4.60314700 & 4.18163300 & 4.1711790 \\
\hline $\mathrm{H}$ & 3.64367500 & 4.63902400 & 3.9162440 \\
\hline $\mathrm{H}$ & 5.38496700 & 4.94099000 & 4.0523060 \\
\hline
\end{tabular}




\begin{tabular}{|c|c|c|c|}
\hline $\mathrm{H}$ & 4.57561200 & 3.89674100 & 5.22169700 \\
\hline $\mathrm{C}$ & 6.09153800 & 2.17999100 & 3.87256700 \\
\hline $\mathrm{H}$ & 5.81503100 & 1.77176900 & 4.84824800 \\
\hline $\mathrm{H}$ & 6.96359500 & 2.83281000 & 4.00462300 \\
\hline $\mathrm{H}$ & 6.38122000 & 1.35048000 & 3.21780000 \\
\hline $\mathrm{C}$ & 5.37425300 & 3.54248300 & 1.90121200 \\
\hline $\mathrm{H}$ & 5.74123100 & 2.76171100 & 1.22885800 \\
\hline $\mathrm{H}$ & 6.19928900 & 4.24394000 & 2.06426700 \\
\hline $\mathrm{H}$ & 4.56363000 & 4.07867700 & 1.39409300 \\
\hline $\mathrm{C}$ & 1.55625900 & -0.53200300 & 5.17195900 \\
\hline $\mathrm{C}$ & 0.10293300 & -0.07525500 & 5.42276600 \\
\hline $\mathrm{H}$ & 0.05447900 & 0.96394100 & 5.76101300 \\
\hline $\mathrm{H}$ & -0.36802500 & -0.70316800 & 6.18812900 \\
\hline $\mathrm{H}$ & -0.49141500 & -0.14830300 & 4.50746000 \\
\hline $\mathrm{C}$ & 1.52501100 & -2.02208600 & 4.74101900 \\
\hline $\mathrm{H}$ & 0.86794000 & -2.21308100 & 3.89021000 \\
\hline $\mathrm{H}$ & 1.15404500 & -2.63269500 & 5.57097700 \\
\hline $\mathrm{H}$ & 2.53004600 & -2.37274800 & 4.48088100 \\
\hline $\mathrm{C}$ & 2.35083700 & -0.50223000 & 6.49528100 \\
\hline $\mathrm{H}$ & 3.40933600 & -0.72159700 & 6.32606500 \\
\hline $\mathrm{H}$ & 1.94631400 & -1.27350900 & 7.16094500 \\
\hline $\mathrm{H}$ & 2.28866100 & 0.44897300 & 7.02078300 \\
\hline $\mathrm{C}$ & 2.40443000 & 2.77957400 & 5.97445700 \\
\hline $\mathrm{H}$ & 2.13439000 & 3.54702400 & 5.24338600 \\
\hline $\mathrm{H}$ & 2.86402000 & 3.25049700 & 6.84701700 \\
\hline $\mathrm{H}$ & 1.49814600 & 2.25383200 & 6.29032700 \\
\hline $\mathrm{C}$ & -2.14215700 & 1.37702300 & -1.03756500 \\
\hline $\mathrm{C}$ & -2.67710600 & 2.64081100 & -0.77085100 \\
\hline $\mathrm{H}$ & -2.60630000 & 3.02144800 & 0.23744300 \\
\hline $\mathrm{C}$ & -3.28316900 & 3.41290200 & -1.76008700 \\
\hline $\mathrm{C}$ & -3.28471000 & 2.89014100 & -3.07921100 \\
\hline $\mathrm{C}$ & -2.84781200 & 1.57696400 & -3.37144200 \\
\hline $\mathrm{C}$ & -2.25018200 & 0.85782100 & -2.32270100 \\
\hline $\mathrm{H}$ & -1.84045500 & -0.12815800 & -2.50000100 \\
\hline $\mathrm{C}$ & -2.84124300 & -0.10423100 & 1.32665800 \\
\hline $\mathrm{C}$ & -4.10810000 & 0.44549100 & 1.14791400 \\
\hline $\mathrm{H}$ & -4.22943600 & 1.27063800 & 0.45985600 \\
\hline $\mathrm{C}$ & -5.24103500 & -0.05422900 & 1.80458600 \\
\hline $\mathrm{C}$ & -5.05944100 & -1.20920600 & 2.59161300 \\
\hline $\mathrm{C}$ & -3.77265000 & -1.70131500 & 2.92654400 \\
\hline $\mathrm{C}$ & -2.68121000 & -1.13005000 & 2.26730100 \\
\hline $\mathrm{H}$ & -1.69223300 & -1.53232600 & 2.42824300 \\
\hline $\mathrm{C}$ & -4.03060600 & 4.70651200 & -1.36702500 \\
\hline $\mathrm{C}$ & -5.53926800 & 4.46116900 & -1.60026900 \\
\hline
\end{tabular}


$\mathrm{H}$

$\mathrm{H}$

$\mathrm{H}$

C

$\mathrm{H}$

$\mathrm{H}$

$\mathrm{H}$

C

$\mathrm{H}$

$\mathrm{H}$

$\mathrm{H}$

C

C

$\mathrm{H}$

$\mathrm{H}$

$\mathrm{H}$

C

$\mathrm{H}$

$\mathrm{H}$

$\mathrm{H}$

C

$\mathrm{H}$

$\mathrm{H}$

$\mathrm{H}$

C

$\mathrm{H}$

$\mathrm{H}$

$\mathrm{H}$

C

C

$\mathrm{H}$

$\mathrm{H}$

$\mathrm{H}$

C

$\mathrm{H}$

$\mathrm{H}$

$\mathrm{H}$

C

$\mathrm{H}$

$\mathrm{H}$

$\mathrm{H}$

C

C

$\mathrm{H}$

\begin{tabular}{rrr}
-5.73867600 & 4.24519300 & -2.65314800 \\
-6.11758400 & 5.34778500 & -1.31235300 \\
-5.89373700 & 3.61628800 & -0.99951700 \\
-3.81553600 & 5.05160500 & 0.12059200 \\
-4.18680000 & 4.27231900 & 0.79189300 \\
-4.36251900 & 5.97028300 & 0.35728400 \\
-2.75598400 & 5.22100900 & 0.34357400 \\
-3.59803400 & 5.95229200 & -2.16526000 \\
-2.51730100 & 6.10367000 & -2.09112200 \\
-4.08402800 & 6.84050200 & -1.74503200 \\
-3.88139100 & 5.88403000 & -3.21291200 \\
-2.96910700 & 0.88906400 & -4.75218900 \\
-3.11918800 & -0.64254600 & -4.58102200 \\
-2.23474800 & -1.11825300 & -4.15338200 \\
-3.27975600 & -1.09795300 & -5.56386600 \\
-3.97307900 & -0.89508700 & -3.94578400 \\
-1.67944400 & 1.14351700 & -5.56440900 \\
-1.55691600 & 2.19524200 & -5.82965900 \\
-1.69338900 & 0.56068800 & -6.49388000 \\
-0.80142600 & 0.84836200 & -4.98287600 \\
-4.21748900 & 1.32678400 & -5.54912300 \\
-5.12483500 & 1.17223600 & -4.95456300 \\
-4.29801200 & 0.71005800 & -6.45157300 \\
-4.20123800 & 2.37025100 & -5.85292600 \\
-2.78477100 & 4.32822300 & -4.91628500 \\
-3.00569200 & 5.39827500 & -4.97890300 \\
-2.83071400 & 3.90567900 & -5.92538100 \\
-1.77695900 & 4.18978200 & -4.51431900 \\
-6.55539700 & 0.74589400 & 1.63245300 \\
-7.03143200 & 0.67956700 & 0.16546000 \\
-6.27141500 & 1.06612600 & -0.52211500 \\
-7.93956700 & 1.28013400 & 0.03381400 \\
-7.25755900 & -0.34935800 & -0.13150100 \\
-6.24986300 & 2.22384500 & 1.98522500 \\
-5.90710000 & 2.31085800 & 3.02208300 \\
-7.15547600 & 2.83076000 & 1.87100700 \\
-5.48085500 & 2.65442400 & 1.34076600 \\
-7.72012800 & 0.32935800 & 2.55362500 \\
-8.15945200 & -0.63120300 & 2.28447800 \\
-7.51096000 & 1.08442400 & 2.46848100 \\
\hline & 0.27721900 & 3.59976800 \\
-2.5649900 & -4.11868000 & 3.60363200
\end{tabular}




\begin{tabular}{|c|c|c|c|}
\hline $\mathrm{H}$ & -3.87531400 & -4.90194600 & 4.35029100 \\
\hline $\mathrm{H}$ & -3.81244300 & -4.48012100 & 2.63824400 \\
\hline $\mathrm{C}$ & -2.07409100 & -3.02298900 & 4.29041500 \\
\hline $\mathrm{H}$ & -1.55837600 & -3.43178400 & 3.41833000 \\
\hline $\mathrm{H}$ & -1.97855300 & -3.74387900 & 5.11006700 \\
\hline $\mathrm{H}$ & -1.57174100 & -2.10450500 & 4.60316300 \\
\hline $\mathrm{C}$ & -4.21618600 & -2.29090900 & 5.32465500 \\
\hline $\mathrm{H}$ & -4.06579500 & -3.03624900 & 6.11521000 \\
\hline $\mathrm{H}$ & -5.28881700 & -2.12646900 & 5.20494800 \\
\hline $\mathrm{H}$ & -3.75293600 & -1.35293000 & 5.65358900 \\
\hline $\mathrm{C}$ & -6.73985300 & -2.73901300 & 2.05114200 \\
\hline $\mathrm{H}$ & -7.12903900 & -2.14932400 & 1.21449300 \\
\hline $\mathrm{H}$ & -7.55955500 & -3.27904500 & 2.53136300 \\
\hline $\mathrm{H}$ & -6.00629500 & -3.44908000 & 1.65788500 \\
\hline $\mathrm{C}$ & 0.89978200 & 2.45030200 & -0.68878400 \\
\hline $\mathrm{C}$ & 1.70899900 & 1.36531600 & -1.13708600 \\
\hline $\mathrm{C}$ & 2.09769700 & 1.28341400 & -2.48204200 \\
\hline $\mathrm{H}$ & 2.66035800 & 0.42455800 & -2.82222300 \\
\hline $\mathrm{C}$ & 1.76771000 & 2.27169800 & -3.42164500 \\
\hline $\mathrm{H}$ & 2.06724300 & 2.18314500 & -4.45911600 \\
\hline $\mathrm{C}$ & 1.04904400 & 3.35077400 & -2.95395400 \\
\hline $\mathrm{C}$ & 0.63462000 & 3.43495900 & -1.62744100 \\
\hline $\mathrm{C}$ & 0.18912800 & 5.36779500 & -2.62379900 \\
\hline $\mathrm{H}$ & 1.00853300 & 6.07185800 & -2.41585400 \\
\hline $\mathrm{H}$ & -0.71474400 & 5.88402000 & -2.94282300 \\
\hline $\mathrm{C}$ & -0.62584600 & 1.75084800 & 1.28643700 \\
\hline $\mathrm{C}$ & -1.04167500 & 1.98712400 & 2.59992600 \\
\hline $\mathrm{H}$ & -1.80572500 & 1.35144300 & 3.03002200 \\
\hline $\mathrm{C}$ & -0.52674400 & 3.03566000 & 3.38391700 \\
\hline $\mathrm{H}$ & -0.85798600 & 3.19717900 & 4.40323900 \\
\hline $\mathrm{C}$ & 0.40295400 & 3.85428000 & 2.78246100 \\
\hline $\mathrm{C}$ & 0.82514500 & 3.63264900 & 1.47453600 \\
\hline $\mathrm{C}$ & 0.36344600 & 2.59197900 & 0.69176600 \\
\hline $\mathrm{C}$ & 1.70150500 & 5.55195500 & 2.16847600 \\
\hline $\mathrm{H}$ & 2.70409300 & 5.88300400 & 2.43734000 \\
\hline $\mathrm{H}$ & 1.07369900 & 6.38609600 & 1.82305900 \\
\hline $\mathrm{C}$ & -0.96383000 & -4.43478000 & -1.35524700 \\
\hline $\mathrm{C}$ & -1.48862000 & -5.34722000 & -2.48554600 \\
\hline $\mathrm{H}$ & -0.10703100 & -4.90837800 & -0.85923600 \\
\hline $\mathrm{H}$ & -0.89338400 & -5.09838500 & -3.37195000 \\
\hline $\mathrm{C}$ & -0.54883000 & -3.08076600 & -1.88657500 \\
\hline $\mathrm{C}$ & 0.84269500 & -2.83591900 & -1.98856400 \\
\hline $\mathrm{H}$ & -1.20747400 & -2.69717700 & -2.66244400 \\
\hline $\mathrm{H}$ & 1.49591900 & -3.52188200 & -1.44958600 \\
\hline
\end{tabular}




$\begin{array}{lrrr}\mathrm{C} & 1.51760700 & -2.16031200 & -3.11601100 \\ \mathrm{C} & 0.83222300 & -1.40986300 & -4.08813700 \\ \mathrm{C} & 2.90974500 & -2.29872500 & -3.26522000 \\ \mathrm{C} & 1.50694700 & -0.82487100 & -5.15617300 \\ \mathrm{H} & -0.23943000 & -1.28159000 & -4.00370600 \\ \mathrm{C} & 3.58930400 & -1.69751100 & -4.32208300 \\ \mathrm{H} & 3.46384400 & -2.87299400 & -2.53188100 \\ \mathrm{C} & 2.89312400 & -0.95453200 & -5.27739700 \\ \mathrm{H} & 0.94756200 & -0.25991500 & -5.89585600 \\ \mathrm{H} & 4.66575900 & -1.81504000 & -4.39855600 \\ \mathrm{H} & 3.41935100 & -0.49204600 & -6.10763400 \\ \mathrm{C} & -1.39379300 & -6.85214000 & -2.22078200 \\ \mathrm{H} & -0.35596200 & -7.14220900 & -2.01607800 \\ \mathrm{H} & -2.00008000 & -7.14691900 & -1.36285800 \\ \mathrm{H} & -1.72859800 & -7.41219200 & -3.10127300 \\ \mathrm{~S} & -2.14027200 & -4.38063600 & 0.05738800 \\ \mathrm{C} & -3.63929100 & -3.64411300 & -0.60652500 \\ \mathrm{C} & -4.75852500 & -4.45873800 & -0.79154400 \\ \mathrm{C} & -3.66034400 & -2.29066300 & -0.93543100 \\ \mathrm{C} & -5.92201900 & -3.89513400 & -1.31618500 \\ \mathrm{H} & -4.70395300 & -5.50622300 & -0.51733900 \\ \mathrm{C} & -4.82936100 & -1.73610600 & -1.45198400 \\ \mathrm{H} & -2.77191000 & -1.69477000 & -0.78677700 \\ \mathrm{C} & -5.95672500 & -2.53703400 & -1.64545300 \\ \mathrm{H} & -6.80127400 & -4.51507100 & -1.46549300 \\ \mathrm{H} & -4.85319700 & -0.67861900 & -1.69135400 \\ \mathrm{H} & -6.86564600 & -2.10173000 & -2.05107600 \\ \mathrm{O} & -1.55166800 & -3.49324500 & 1.07646000 \\ \mathrm{O} & -2.49240600 & -5.76328200 & 0.44643500 \\ \mathrm{H} & -2.52095200 & -5.06113900 & -2.72707000 \\ & & & \\ & & & \\ & & \end{array}$<smiles>CCC(c1c[pH]p1[Pb]1PCCCP1)S(=O)(=O)c1ccccc1</smiles>

Int-13b

Total SCF energy (M06/SDD-6-311++G(d,p)/SMD(1,4-Dioxane)): -5497.872430a.u.

Thermal correction to Gibbs Free Energy at $298.15 \mathrm{~K}$ : 1.767732a.u

Gibbs free energy at 298.15 K (M06/SDD-6-311++G(d,p)/SMD(1,4-Dioxane)): -5496.104698a.u.

$\mathrm{Pd}$

$$
\begin{array}{rrrr}
\mathrm{Pd} & -0.02304400 & 1.01582500 & -0.46114300 \\
\mathrm{P} & -1.10906000 & -1.06195200 & -0.37758000 \\
\mathrm{P} & 1.95873600 & 0.22069400 & 0.50156200
\end{array}
$$




\begin{tabular}{|c|c|c|c|}
\hline $\mathrm{O}$ & -5.33041600 & -0.49903300 & -4.53201200 \\
\hline $\mathrm{O}$ & -3.49273400 & -2.17345300 & 5.00039600 \\
\hline $\mathrm{O}$ & 5.42671200 & -3.17084900 & -2.98961100 \\
\hline $\mathrm{O}$ & 4.79091500 & 5.25074600 & 1.9718850 \\
\hline $\mathrm{O}$ & 2.53018200 & -5.77521400 & -0.94512200 \\
\hline $\mathrm{O}$ & 2.69873600 & -4.51445900 & 0.97285500 \\
\hline $\mathrm{O}$ & 0.33890700 & -4.19224600 & 2.96080300 \\
\hline $\mathrm{O}$ & 0.72720300 & -3.39474600 & 5.08959900 \\
\hline $\mathrm{C}$ & -2.39317500 & -1.21158100 & -1.66210300 \\
\hline $\mathrm{C}$ & -3.71067600 & -1.55428700 & -1.39704900 \\
\hline $\mathrm{H}$ & -3.96286000 & -1.89561700 & -0.40538700 \\
\hline $\mathrm{C}$ & -4.73058700 & -1.38303500 & -2.34344400 \\
\hline $\mathrm{C}$ & -4.36857600 & -0.81866600 & -3.58682800 \\
\hline $\mathrm{C}$ & -3.01403700 & -0.56677200 & -3.93243600 \\
\hline $\mathrm{C}$ & -2.05711600 & -0.74844300 & -2.93743300 \\
\hline $\mathrm{H}$ & -1.02765900 & -0.46822000 & -3.12218100 \\
\hline $\mathrm{C}$ & -6.16647600 & -1.74681300 & -1.90089600 \\
\hline $\mathrm{C}$ & -6.13908800 & -2.90452600 & -0.87102000 \\
\hline $\mathrm{H}$ & -5.56878700 & -3.76082600 & -1.24808900 \\
\hline $\mathrm{H}$ & -7.16402400 & -3.23841900 & -0.67595700 \\
\hline $\mathrm{H}$ & -5.71416200 & -2.60523800 & 0.09115900 \\
\hline $\mathrm{C}$ & -6.79521400 & -0.52645100 & -1.18707000 \\
\hline $\mathrm{H}$ & -6.17708200 & -0.23273800 & -0.33244000 \\
\hline $\mathrm{H}$ & -7.80012700 & -0.77442100 & -0.82202800 \\
\hline $\mathrm{H}$ & -6.87640000 & 0.34302300 & -1.84166200 \\
\hline $\mathrm{C}$ & -7.05906900 & -2.24710800 & -3.05615500 \\
\hline $\mathrm{H}$ & -7.23039100 & -1.50438800 & -3.83067200 \\
\hline $\mathrm{H}$ & -8.03109600 & -2.55439100 & -2.65253900 \\
\hline $\mathrm{H}$ & -6.60357600 & -3.12001500 & -3.53764100 \\
\hline $\mathrm{C}$ & -2.57544100 & -0.06176800 & -5.32301100 \\
\hline $\mathrm{C}$ & -3.06302500 & 1.38413800 & -5.56491900 \\
\hline $\mathrm{H}$ & -4.13757300 & 1.42862800 & -5.73596700 \\
\hline $\mathrm{H}$ & -2.56996500 & 1.79940300 & -6.45261700 \\
\hline $\mathrm{H}$ & -2.82706400 & 2.02092300 & -4.70574300 \\
\hline $\mathrm{C}$ & -1.03792400 & -0.03973400 & -5.45546300 \\
\hline $\mathrm{H}$ & -0.56941900 & 0.68825300 & -4.78396300 \\
\hline $\mathrm{H}$ & -0.77288000 & 0.24484300 & -6.47956400 \\
\hline $\mathrm{H}$ & -0.59457700 & -1.02092200 & -5.25815200 \\
\hline $\mathrm{C}$ & -3.11067600 & -0.99950800 & -6.42719300 \\
\hline $\mathrm{H}$ & -2.70288100 & -2.00977100 & -6.30242900 \\
\hline $\mathrm{H}$ & -2.80149700 & -0.63126600 & -7.41339900 \\
\hline $\mathrm{H}$ & -4.19894600 & -1.06154000 & -6.40127500 \\
\hline $\mathrm{C}$ & -6.04974800 & 0.72033800 & -4.32595600 \\
\hline $\mathrm{H}$ & -6.19387000 & 1.18256800 & -5.30726400 \\
\hline
\end{tabular}




\begin{tabular}{|c|c|c|c|}
\hline $\mathrm{H}$ & -5.49623200 & 1.39629100 & -3.67050300 \\
\hline $\mathrm{H}$ & -7.03632900 & 0.51633700 & -3.89526100 \\
\hline $\mathrm{C}$ & -1.88353600 & -1.43163400 & 1.22932800 \\
\hline $\mathrm{C}$ & -1.88094600 & -0.42757900 & 2.18965400 \\
\hline $\mathrm{H}$ & -1.44043000 & 0.51806000 & 1.90763300 \\
\hline $\mathrm{C}$ & -2.43954900 & -0.60024700 & 3.45963500 \\
\hline $\mathrm{C}$ & -2.97006000 & -1.88183800 & 3.75042200 \\
\hline $\mathrm{C}$ & -3.06701100 & -2.90212500 & 2.76633200 \\
\hline $\mathrm{C}$ & -2.48351600 & -2.65545100 & 1.52174000 \\
\hline $\mathrm{H}$ & -2.49252300 & -3.42126700 & 0.75806400 \\
\hline $\mathrm{C}$ & -3.85147200 & -4.21213600 & 2.99758900 \\
\hline $\mathrm{C}$ & -3.18816000 & -5.09721200 & 4.07118900 \\
\hline $\mathrm{H}$ & -2.16859900 & -5.35055700 & 3.76361800 \\
\hline $\mathrm{H}$ & -3.74802600 & -6.03382300 & 4.18160600 \\
\hline $\mathrm{H}$ & -3.15434700 & -4.61335800 & 5.04584500 \\
\hline $\mathrm{C}$ & -5.30508600 & -3.87748400 & 3.40251800 \\
\hline $\mathrm{H}$ & -5.33925100 & -3.30279100 & 4.32867600 \\
\hline $\mathrm{H}$ & -5.87719500 & -4.80315400 & 3.54120100 \\
\hline $\mathrm{H}$ & -5.79459400 & -3.29417700 & 2.61407200 \\
\hline $\mathrm{C}$ & -3.92155000 & -5.06570000 & 1.71460200 \\
\hline $\mathrm{H}$ & -4.39540300 & -4.52909200 & 0.88730600 \\
\hline $\mathrm{H}$ & -4.52172800 & -5.95976600 & 1.91561100 \\
\hline $\mathrm{H}$ & -2.93053800 & -5.40012800 & 1.38830300 \\
\hline $\mathrm{C}$ & -2.48198300 & 0.62989100 & 4.39944100 \\
\hline $\mathrm{C}$ & -1.24959700 & 0.66186800 & 5.32945000 \\
\hline $\mathrm{H}$ & -1.17806900 & -0.21570100 & 5.97327000 \\
\hline $\mathrm{H}$ & -1.28658800 & 1.55103200 & 5.97144000 \\
\hline $\mathrm{H}$ & -0.33326600 & 0.70582700 & 4.73365900 \\
\hline $\mathrm{C}$ & -2.43191300 & 1.93908300 & 3.57176200 \\
\hline $\mathrm{H}$ & -1.45132200 & 2.10681100 & 3.11608400 \\
\hline $\mathrm{H}$ & -2.62807100 & 2.79120500 & 4.23163500 \\
\hline $\mathrm{H}$ & -3.17734900 & 1.93465600 & 2.77243200 \\
\hline $\mathrm{C}$ & -3.79337500 & 0.69361500 & 5.21137600 \\
\hline $\mathrm{H}$ & -4.65647500 & 0.70721600 & 4.53532400 \\
\hline $\mathrm{H}$ & -3.81104700 & 1.61674400 & 5.80245400 \\
\hline $\mathrm{H}$ & -3.92472300 & -0.15064500 & 5.88490400 \\
\hline $\mathrm{C}$ & -2.57090100 & -2.41775000 & 6.06130700 \\
\hline $\mathrm{H}$ & -1.57644900 & -2.67207200 & 5.68263800 \\
\hline $\mathrm{H}$ & -2.96015800 & -3.25509300 & 6.64915500 \\
\hline $\mathrm{H}$ & -2.49848000 & -1.54403500 & 6.71763500 \\
\hline $\mathrm{C}$ & 3.08186600 & -0.75975200 & -0.54448700 \\
\hline $\mathrm{C}$ & 4.17068300 & -1.46071700 & -0.01885100 \\
\hline $\mathrm{H}$ & 4.35144100 & -1.40464100 & 1.04608600 \\
\hline $\mathrm{C}$ & 5.00423000 & -2.22597900 & -0.83365200 \\
\hline
\end{tabular}




\begin{tabular}{|c|c|c|c|}
\hline $\mathrm{C}$ & 4.67001200 & -2.31073400 & -2.21293000 \\
\hline $\mathrm{C}$ & 3.64233500 & -1.53559600 & -2.79380600 \\
\hline $\mathrm{C}$ & 2.84518900 & -0.78781800 & -1.91369000 \\
\hline $\mathrm{H}$ & 2.00763400 & -0.21257500 & -2.28934600 \\
\hline $\mathrm{C}$ & 2.96880600 & 1.59789400 & 1.1527380 \\
\hline $\mathrm{C}$ & 4.31656700 & 1.77561300 & 0.8737520 \\
\hline $\mathrm{H}$ & 4.84396900 & 0.99131900 & 0.3478920 \\
\hline $\mathrm{C}$ & 5.00600200 & 2.94833900 & 1.2280760 \\
\hline $\mathrm{C}$ & 4.24410200 & 3.98996800 & 1.7971470 \\
\hline $\mathrm{C}$ & 2.89615900 & 3.80038200 & 2.2098270 \\
\hline $\mathrm{C}$ & 2.28874800 & 2.59821500 & 1.8595350 \\
\hline $\mathrm{H}$ & 1.24228300 & 2.43600900 & 2.0827460 \\
\hline $\mathrm{C}$ & 6.30072100 & -2.83431400 & -0.25604700 \\
\hline $\mathrm{C}$ & 7.49942000 & -2.13699700 & -0.93920600 \\
\hline $\mathrm{H}$ & 7.48080900 & -2.30113900 & -2.01918200 \\
\hline $\mathrm{H}$ & 8.44348600 & -2.53168700 & -0.54345200 \\
\hline $\mathrm{H}$ & 7.47812400 & -1.05741900 & -0.75100200 \\
\hline $\mathrm{C}$ & 6.40551600 & -2.59109400 & 1.26285800 \\
\hline $\mathrm{H}$ & 6.41389300 & -1.52614800 & 1.51545500 \\
\hline $\mathrm{H}$ & 7.34357600 & -3.02050800 & 1.63023900 \\
\hline $\mathrm{H}$ & 5.58094100 & -3.06810700 & 1.80499300 \\
\hline $\mathrm{C}$ & 6.41561900 & -4.35847600 & -0.46730500 \\
\hline $\mathrm{H}$ & 5.50675400 & -4.86318900 & -0.12824300 \\
\hline $\mathrm{H}$ & 7.25540900 & -4.74858000 & 0.11965400 \\
\hline $\mathrm{H}$ & 6.59107500 & -4.61426000 & -1.51036500 \\
\hline $\mathrm{C}$ & 3.32524500 & -1.42249000 & -4.30502900 \\
\hline $\mathrm{C}$ & 2.95623900 & 0.04507800 & -4.63336100 \\
\hline $\mathrm{H}$ & 2.03106000 & 0.37374300 & -4.15776700 \\
\hline $\mathrm{H}$ & 2.81337400 & 0.15078600 & -5.71461100 \\
\hline $\mathrm{H}$ & 3.74630300 & 0.73549400 & -4.32290000 \\
\hline $\mathrm{C}$ & 2.10072900 & -2.30000300 & -4.64395500 \\
\hline $\mathrm{H}$ & 2.29137600 & -3.36192200 & -4.46384400 \\
\hline $\mathrm{H}$ & 1.82664700 & -2.17608100 & -5.69857800 \\
\hline $\mathrm{H}$ & 1.24060500 & -2.01615700 & -4.03008800 \\
\hline $\mathrm{C}$ & 4.50528200 & -1.77464800 & -5.23575100 \\
\hline $\mathrm{H}$ & 5.40909400 & -1.22749400 & -4.95057300 \\
\hline $\mathrm{H}$ & 4.24033600 & -1.48111100 & -6.25812000 \\
\hline $\mathrm{H}$ & 4.75033100 & -2.83502200 & -5.25293400 \\
\hline $\mathrm{C}$ & 4.79435900 & -4.42523900 & -3.25550700 \\
\hline $\mathrm{H}$ & 5.57308800 & -5.08734800 & -3.64247300 \\
\hline $\mathrm{H}$ & 4.00717100 & -4.32432600 & -4.00867500 \\
\hline $\mathrm{H}$ & 4.35909100 & -4.85500100 & -2.34895800 \\
\hline $\mathrm{C}$ & 6.53420000 & 2.95643000 & 0.9797280 \\
\hline $\mathrm{C}$ & 6.82442000 & 3.07535000 & -0.53220400 \\
\hline
\end{tabular}


$\mathrm{H}$

$\mathrm{H}$

$\mathrm{H}$

C

$\mathrm{H}$

$\mathrm{H}$

$\mathrm{H}$

C

$\mathrm{H}$

$\mathrm{H}$

$\mathrm{H}$

C

C

$\mathrm{H}$

$\mathrm{H}$

$\mathrm{H}$

C

$\mathrm{H}$

$\mathrm{H}$

$\mathrm{H}$

C

$\mathrm{H}$

$\mathrm{H}$

$\mathrm{H}$

C

$\mathrm{H}$

$\mathrm{H}$

$\mathrm{H}$

C

C

C

$\mathrm{H}$

C

$\mathrm{H}$

C

C

C

$\mathrm{H}$

$\mathrm{H}$

C

C

$\mathrm{H}$

C

$\mathrm{H}$

$\begin{array}{rrr}6.31450100 & 2.28778900 & -1.09686300 \\ 7.90143500 & 2.98876200 & -0.71984800 \\ 6.49185700 & 4.03793900 & -0.93344100 \\ 7.11513400 & 1.60477000 & 1.47345400 \\ 6.87464600 & 1.43766800 & 2.52908500 \\ 8.20558000 & 1.61445000 & 1.36838700 \\ 6.74367900 & 0.75056600 & 0.90234900 \\ 7.31051900 & 4.04698000 & 1.74701700 \\ 7.13772500 & 5.05291500 & 1.36726100 \\ 8.38312900 & 3.84151100 & 1.65239300 \\ 7.05678200 & 4.04236400 & 2.81098300 \\ 2.11630900 & 4.84959500 & 3.02950000 \\ 1.87534100 & 6.14865900 & 2.22952000 \\ 2.79545500 & 6.71493600 & 2.08938400 \\ 1.17098900 & 6.79065400 & 2.77186300 \\ 1.44186500 & 5.93059400 & 1.24639300 \\ 0.72936800 & 4.31788600 & 3.44645300 \\ 0.07558600 & 4.14645200 & 2.58320300 \\ 0.23954600 & 5.06049400 & 4.08509900 \\ 0.79643000 & 3.38440500 & 4.01542000 \\ 2.89979700 & 5.17002100 & 4.32189200 \\ 2.35467300 & 5.91234100 & 4.91762600 \\ 3.89002800 & 5.56779600 & 4.09327400 \\ 3.01903700 & 4.26780200 & 4.93294000 \\ 4.84813500 & 6.01660000 & 0.76752400 \\ 5.60089800 & 5.62510700 & 0.07622500 \\ 5.12326400 & 7.03271300 & 1.06041200 \\ 3.87875000 & 6.02846800 & 0.25815200 \\ 0.93826500 & -2.85937000 & 0.41354100 \\ -0.01558800 & -2.52999700 & -0.59738000 \\ -0.09013900 & -3.29483800 & -1.76677900 \\ -0.82982000 & -3.03350800 & -2.51447100 \\ 0.73189300 & -4.41142400 & -1.99972100 \\ 0.64986500 & -4.99906300 & -2.90695800 \\ 1.62340500 & -4.73468100 & -1.00147800 \\ 1.72056600 & -3.97406900 & 0.16079100 \\ 2.91872500 & -5.81717400 & 0.43265900 \\ 2.28657000 & -6.54381800 & 0.96474900 \\ 3.97532200 & -6.07052000 & 0.50459800 \\ 1.67393500 & -0.88433700 & 1.94497500 \\ 1.91270000 & -0.42208000 & 3.24394200 \\ 2.35215800 & 0.55834800 & 3.38120000 \\ 1.63119000 & -1.19090800 & 4.38582000 \\ 1.83042000 & -0.82247500 & 5.38556900\end{array}$




\begin{tabular}{|c|c|c|c|}
\hline $\mathrm{C}$ & 1.10064200 & -2.44139200 & 4.16601200 \\
\hline $\mathrm{C}$ & 0.86076200 & -2.91837000 & 2.87985500 \\
\hline $\mathrm{C}$ & 1.12268200 & -2.18543800 & 1.73347300 \\
\hline $\mathrm{C}$ & 0.55670900 & -4.58837500 & 4.31600000 \\
\hline $\mathrm{H}$ & -0.31204700 & -5.13297200 & 4.68267600 \\
\hline $\mathrm{H}$ & 1.47386400 & -5.19261900 & 4.37554800 \\
\hline $\mathrm{C}$ & -2.60339900 & 4.72169900 & -0.55144900 \\
\hline $\mathrm{C}$ & -2.29852100 & 3.22504000 & -0.30305100 \\
\hline $\mathrm{H}$ & -2.07207300 & 3.06078000 & 0.75529800 \\
\hline $\mathrm{C}$ & -1.18657200 & 2.69454700 & -1.18579200 \\
\hline $\mathrm{C}$ & 0.14764300 & 3.10422100 & -0.91429500 \\
\hline $\mathrm{H}$ & -1.49409700 & 2.54289400 & -2.21639900 \\
\hline $\mathrm{H}$ & 0.34385700 & 3.63398400 & 0.0180450 \\
\hline $\mathrm{C}$ & 1.18590300 & 3.29066700 & -1.94676400 \\
\hline $\mathrm{C}$ & 2.47576400 & 3.70775100 & -1.56503800 \\
\hline $\mathrm{C}$ & 0.94860200 & 3.07383300 & -3.31759200 \\
\hline $\mathrm{C}$ & 3.48510800 & 3.88484900 & -2.50656600 \\
\hline $\mathrm{H}$ & 2.68309500 & 3.87529500 & -0.51416900 \\
\hline $\mathrm{C}$ & 1.95762300 & 3.26140500 & -4.25955600 \\
\hline $\mathrm{H}$ & -0.03758500 & 2.76758100 & -3.65145900 \\
\hline $\mathrm{C}$ & 3.23517300 & 3.66338300 & -3.86328600 \\
\hline $\mathrm{H}$ & 4.47201400 & 4.19850600 & -2.17798100 \\
\hline $\mathrm{H}$ & 1.74420200 & 3.08660600 & -5.31089300 \\
\hline $\mathrm{H}$ & 4.02085100 & 3.80503000 & -4.59995500 \\
\hline $\mathrm{H}$ & -1.66491400 & 5.16666500 & -0.89733100 \\
\hline $\mathrm{C}$ & -3.08668100 & 5.48107400 & 0.68749500 \\
\hline $\mathrm{H}$ & -3.23315500 & 6.54191100 & 0.45662200 \\
\hline $\mathrm{H}$ & -4.03060900 & 5.09029500 & 1.07548600 \\
\hline $\mathrm{H}$ & -2.34099100 & 5.41397300 & 1.48945800 \\
\hline $\mathrm{H}$ & -3.30885300 & 4.83169900 & -1.38314400 \\
\hline $\mathrm{S}$ & -3.77675600 & 2.15725100 & -0.61242200 \\
\hline $\mathrm{O}$ & -3.69009200 & 0.97021800 & 0.25887200 \\
\hline $\mathrm{O}$ & -3.92019700 & 2.02771400 & -2.07868100 \\
\hline $\mathrm{C}$ & -5.22994900 & 3.05980900 & -0.05660300 \\
\hline $\mathrm{C}$ & -5.67846700 & 2.88021300 & 1.25203900 \\
\hline $\mathrm{C}$ & -5.90008400 & 3.89683800 & -0.94898000 \\
\hline $\mathrm{C}$ & -6.80433500 & 3.58131400 & 1.68315800 \\
\hline $\mathrm{H}$ & -5.16214000 & 2.18773400 & 1.90643800 \\
\hline $\mathrm{C}$ & -7.02328900 & 4.59515800 & -0.50741200 \\
\hline $\mathrm{H}$ & -5.54894400 & 3.98025500 & -1.97135300 \\
\hline $\mathrm{C}$ & -7.46937500 & 4.44330700 & 0.80784800 \\
\hline $\mathrm{H}$ & -7.16536000 & 3.44944100 & 2.69875800 \\
\hline $\mathrm{H}$ & -7.55410800 & 5.25162200 & -1.19047500 \\
\hline $\mathrm{H}$ & -8.34471900 & 4.98912200 & 1.14782600 \\
\hline
\end{tabular}


<smiles>CCC(/C=C/c1ccccc1)S(=O)(=O)c1ccccc1</smiles>

Total SCF energy (M06/SDD-6-311++G(d,p)/SMD(1,4-Dioxane)): -1206.848299a.u.

Thermal correction to Gibbs Free Energy at $298.15 \mathrm{~K}$ : 0.262603a.u

Gibbs free energy at 298.15 K (M06/SDD-6-311++G(d,p)/SMD(1,4-Dioxane)): -1206.585696a.u.

$\begin{array}{lrrc}\mathrm{S} & 1.96770900 & -0.69458500 & -1.08227500 \\ \mathrm{O} & 1.18883700 & -0.74957300 & -2.33135000 \\ \mathrm{O} & 3.39122300 & -1.08061700 & -1.05994800 \\ \mathrm{C} & -3.79721100 & -1.07347000 & -0.68456500 \\ \mathrm{C} & -5.02228000 & -0.42665600 & -0.53885300 \\ \mathrm{C} & -5.10585600 & 0.75330700 & 0.20099400 \\ \mathrm{C} & -3.95305900 & 1.28377300 & 0.78662700 \\ \mathrm{C} & -2.72979500 & 0.63746000 & 0.64160000 \\ \mathrm{C} & -2.63321100 & -0.56239100 & -0.08714500 \\ \mathrm{C} & -1.36423300 & -1.27523300 & -0.26231000 \\ \mathrm{C} & -0.25282500 & -1.09360400 & 0.46648000 \\ \mathrm{C} & 1.05970000 & -1.73912900 & 0.16850600 \\ \mathrm{C} & 1.93084400 & -1.98731700 & 1.40143300 \\ \mathrm{C} & 1.28871500 & -3.00824100 & 2.34540900 \\ \mathrm{C} & 1.82995100 & 0.96669400 & -0.41755000 \\ \mathrm{C} & 0.70081900 & 1.72846600 & -0.72460500 \\ \mathrm{C} & 0.56568200 & 2.99147000 & -0.15015700 \\ \mathrm{C} & 1.55165700 & 3.47786900 & 0.71244100 \\ \mathrm{C} & 2.68202400 & 2.70966300 & 0.99897600 \\ \mathrm{C} & 2.82722800 & 1.44266400 & 0.43315200 \\ \mathrm{H} & -3.73425200 & -1.99129500 & -1.26364600 \\ \mathrm{H} & -5.91084900 & -0.84196400 & -1.00576400 \\ \mathrm{H} & -6.05871800 & 1.26265700 & 0.31229600 \\ \mathrm{H} & -4.00764300 & 2.21131600 & 1.34990900 \\ \mathrm{H} & -1.83638600 & 1.07805500 & 1.07330900 \\ \mathrm{H} & -1.33649000 & -1.99863600 & -1.07600800 \\ \mathrm{H} & -0.25046600 & -0.39001000 & 1.29632600 \\ \mathrm{H} & 0.92221200 & -2.66454800 & -0.40451500 \\ \mathrm{H} & 2.08159800 & -1.03516400 & 1.92720600 \\ \mathrm{H} & 2.91633800 & -2.33215200 & 1.07679800 \\ \mathrm{H} & 1.91904900 & -3.17074700 & 3.22582100 \\ \mathrm{H} & 1.15453900 & -3.97414200 & 1.84474100 \\ \mathrm{H} & 0.30417600 & -2.67267600 & 2.68856500 \\ \mathrm{H} & & 1.33354000 & -1.40342700 \\ \mathrm{H} & & & \\ \mathrm{H} & -0.5966000 & & \\ & & & \end{array}$


0.62715200

H

1.44186400

4.46286400

1.15701000 


\section{Copies of NMR Spectra}

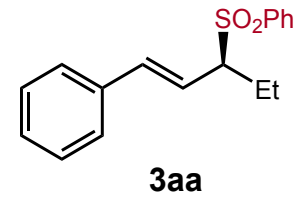

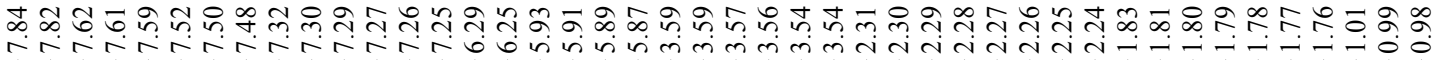
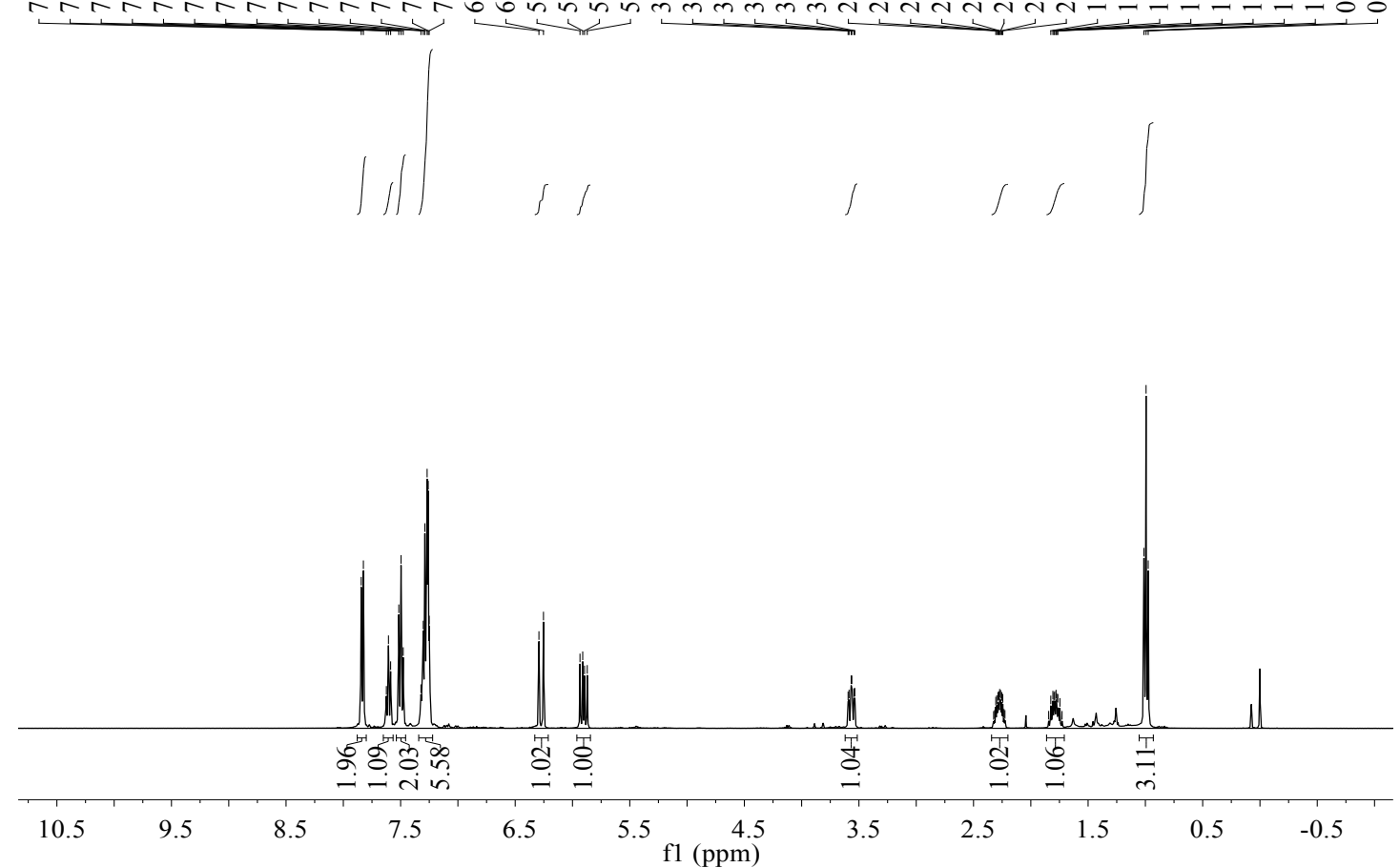

๓ंกิ

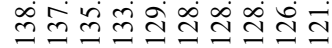
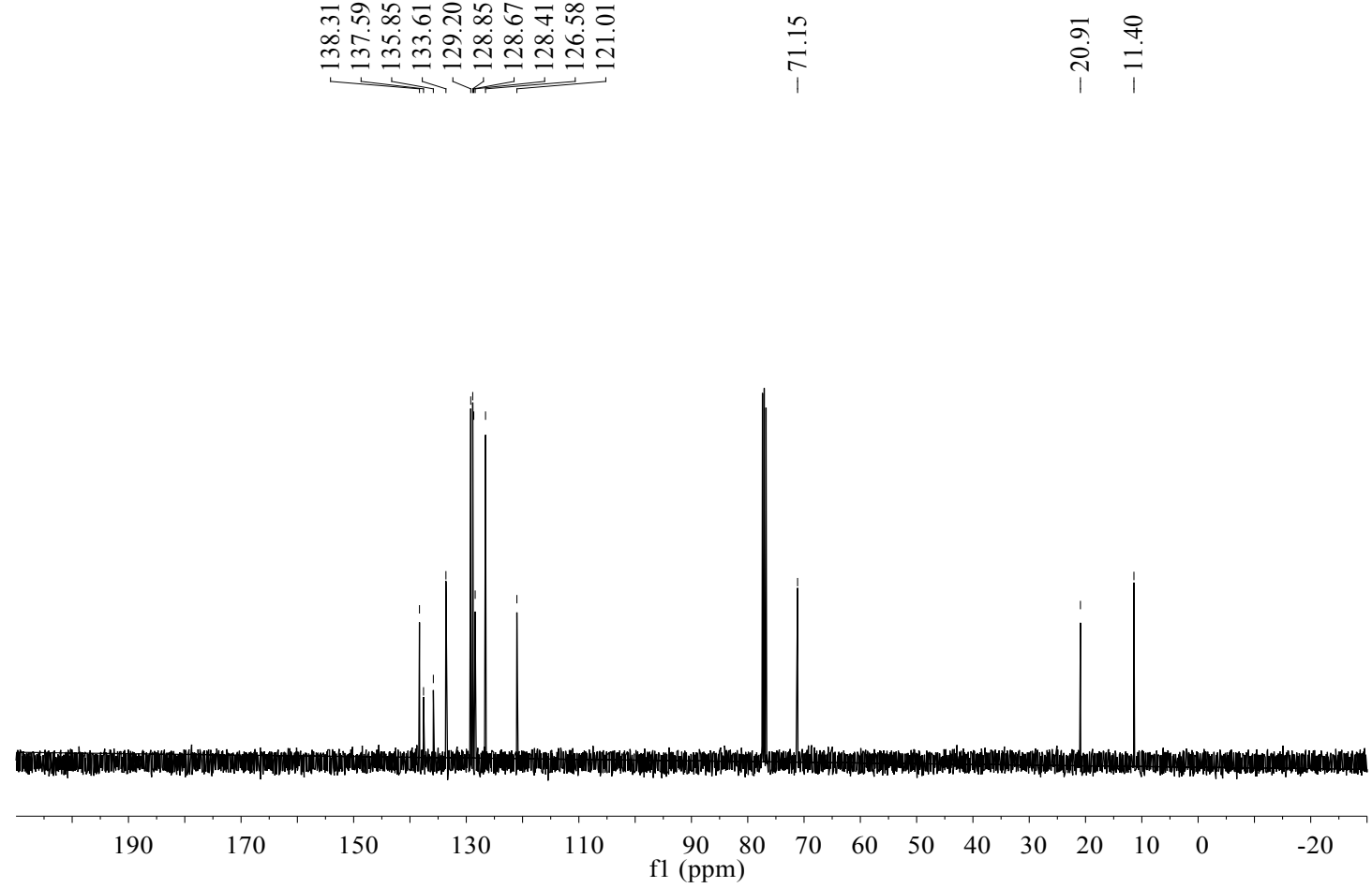


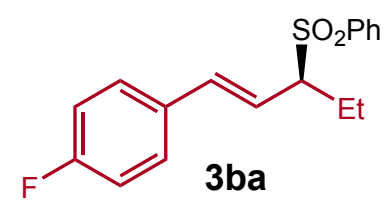

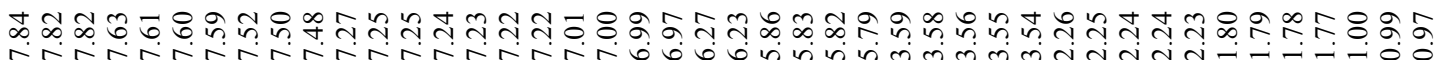
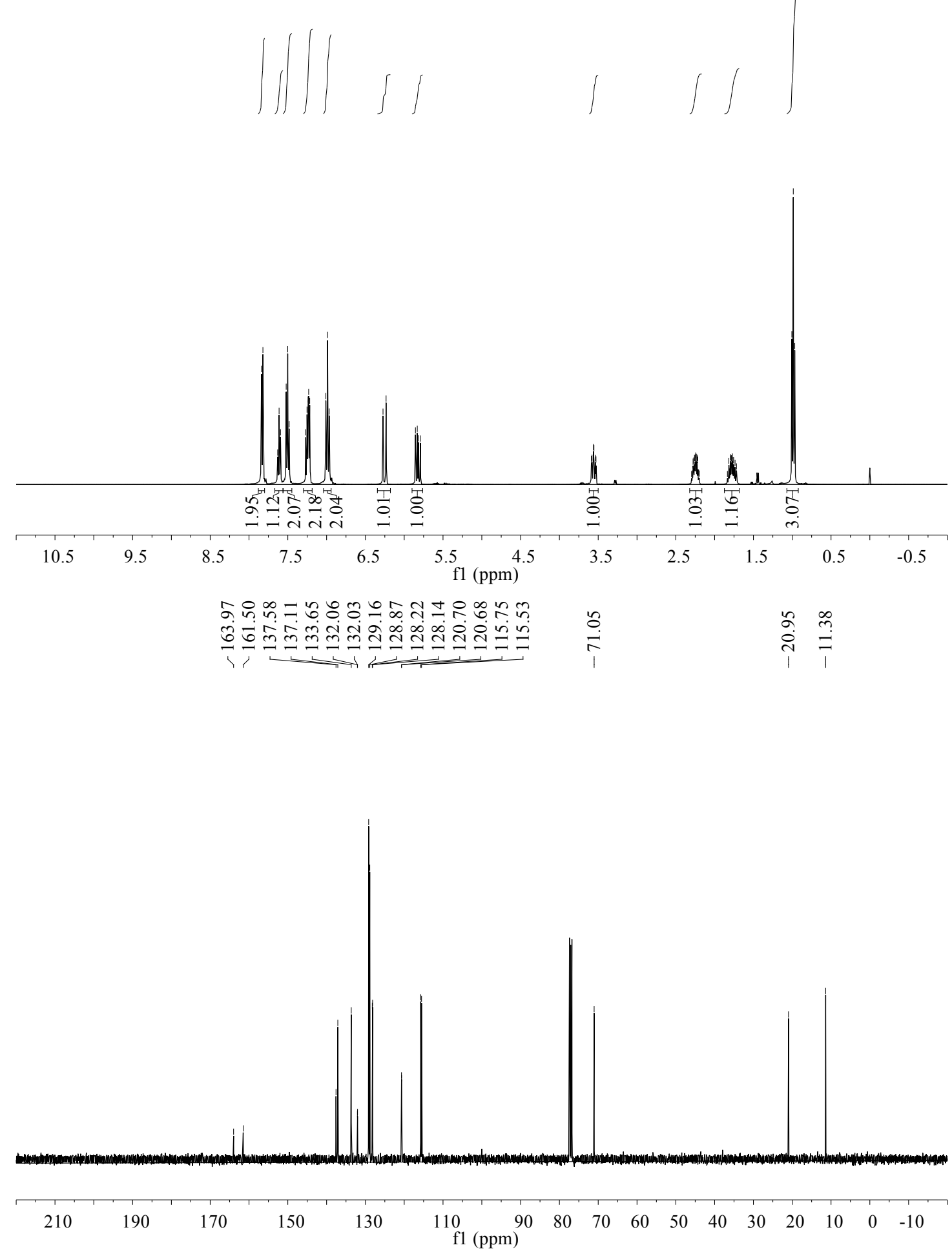


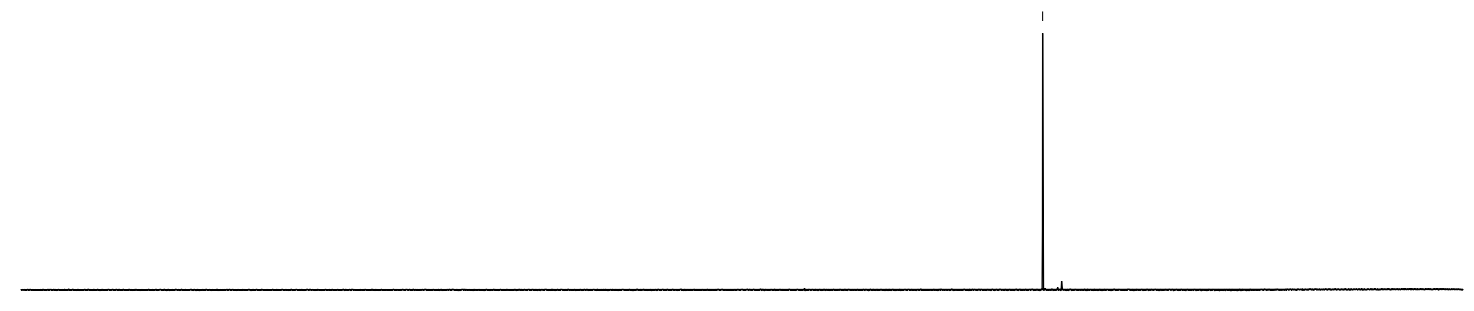

\begin{tabular}{|c|c|c|c|c|c|c|c|c|c|c|}
\hline 80 & 60 & 40 & 20 & 0 & -30 & f1 (ppm) & -90 & -120 & -150 & -180 \\
\hline
\end{tabular}<smiles>CCC(/C=C/c1cccc(F)c1)S(=O)(=O)c1ccccc1</smiles>

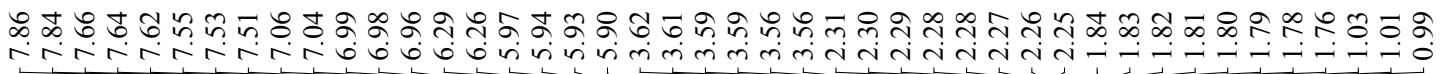
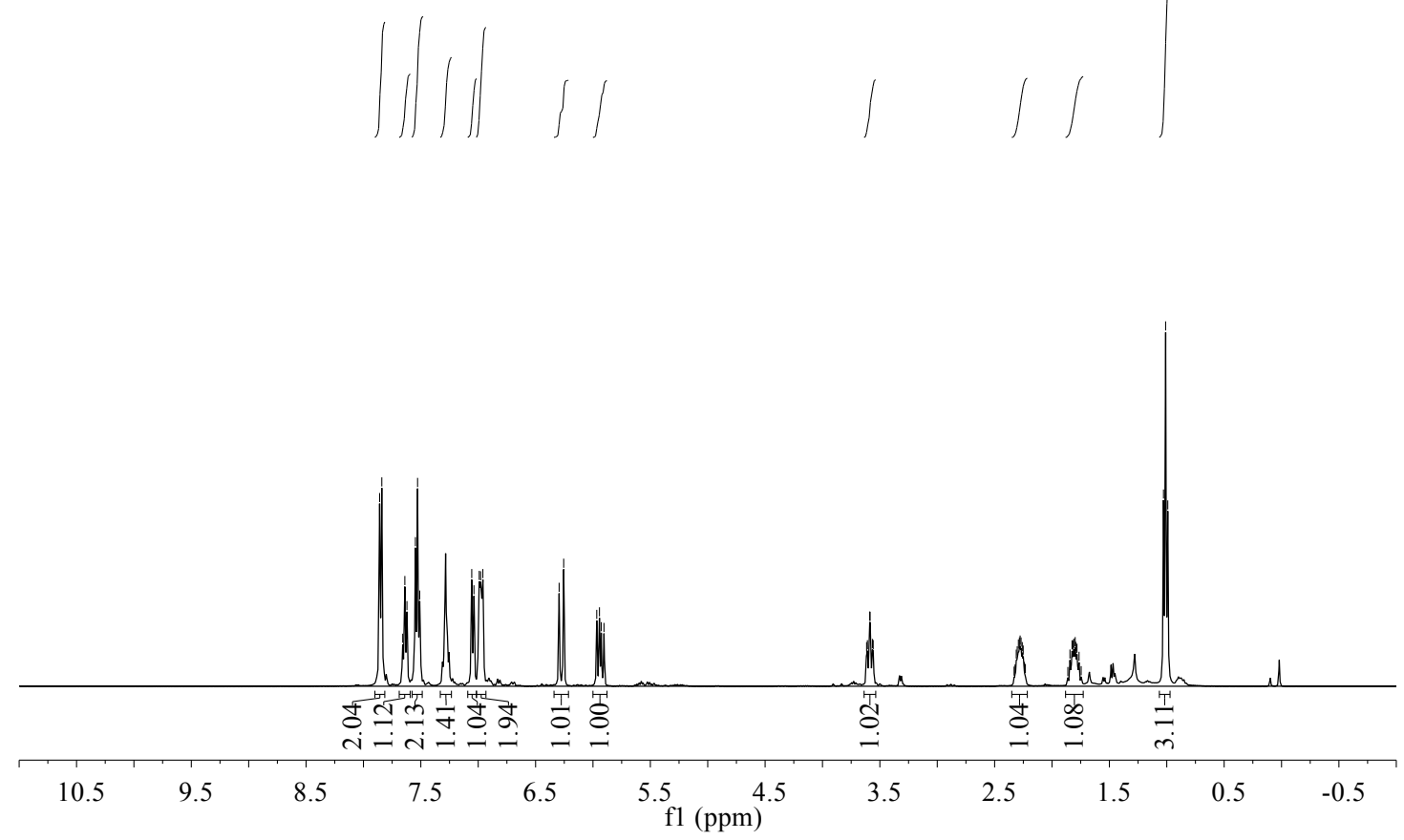

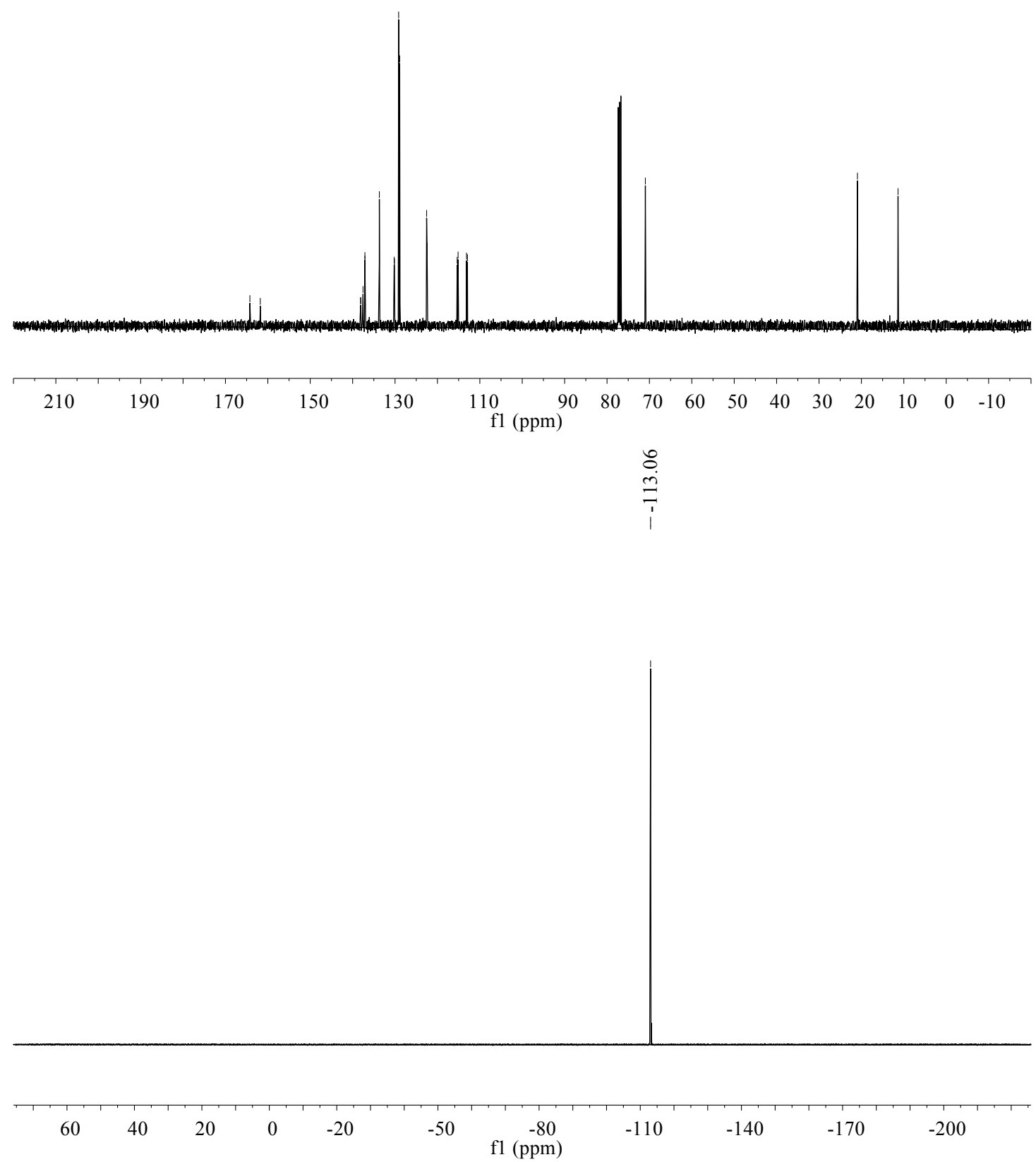
<smiles>CCC(/C=C/c1ccccc1F)[Sb](=O)(=O)c1ccccc1</smiles>

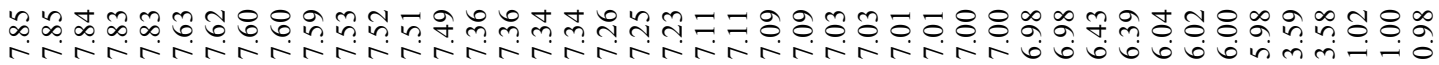
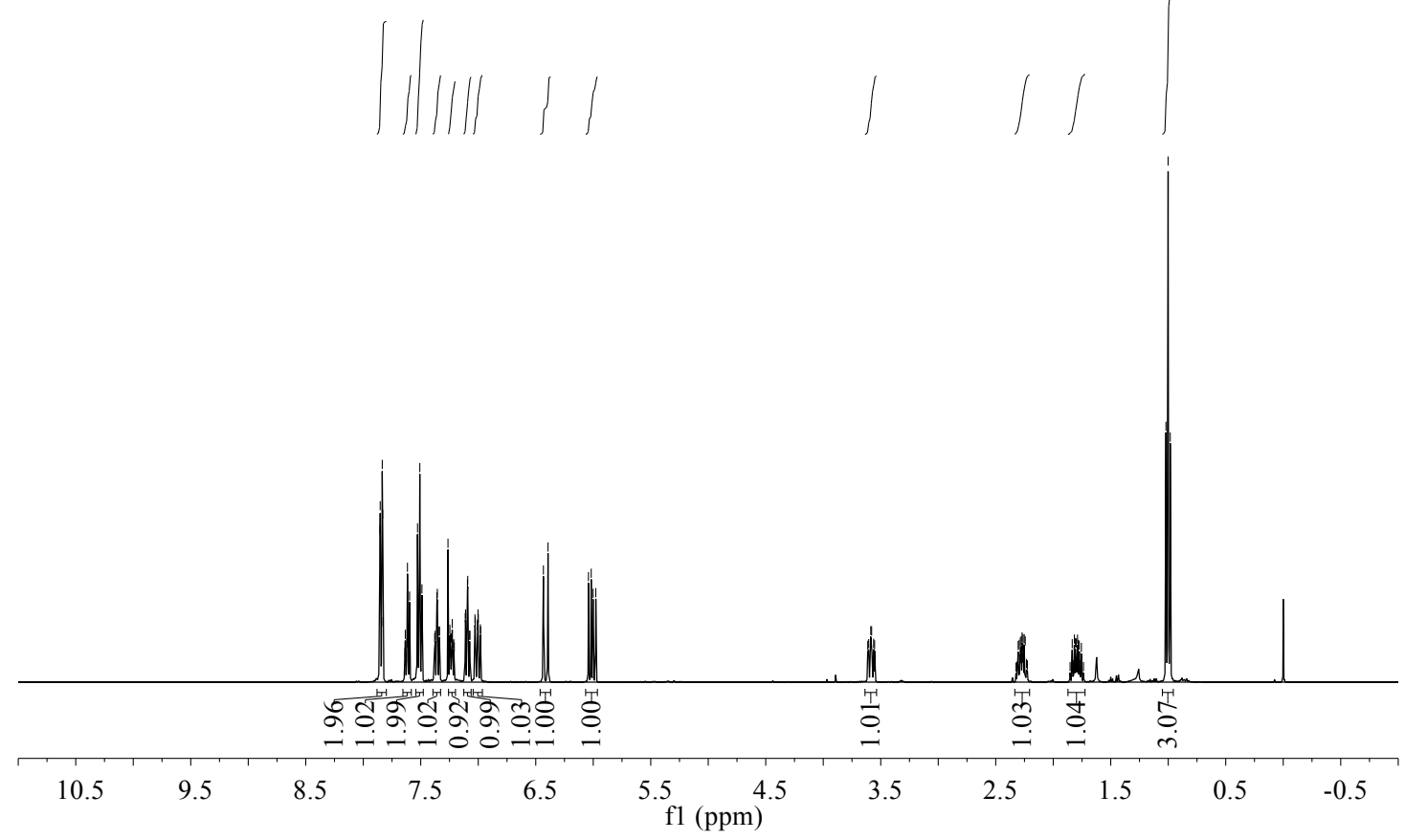

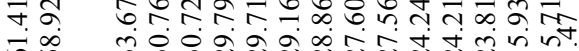

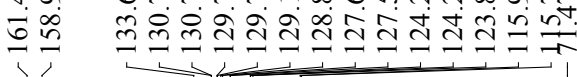

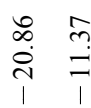

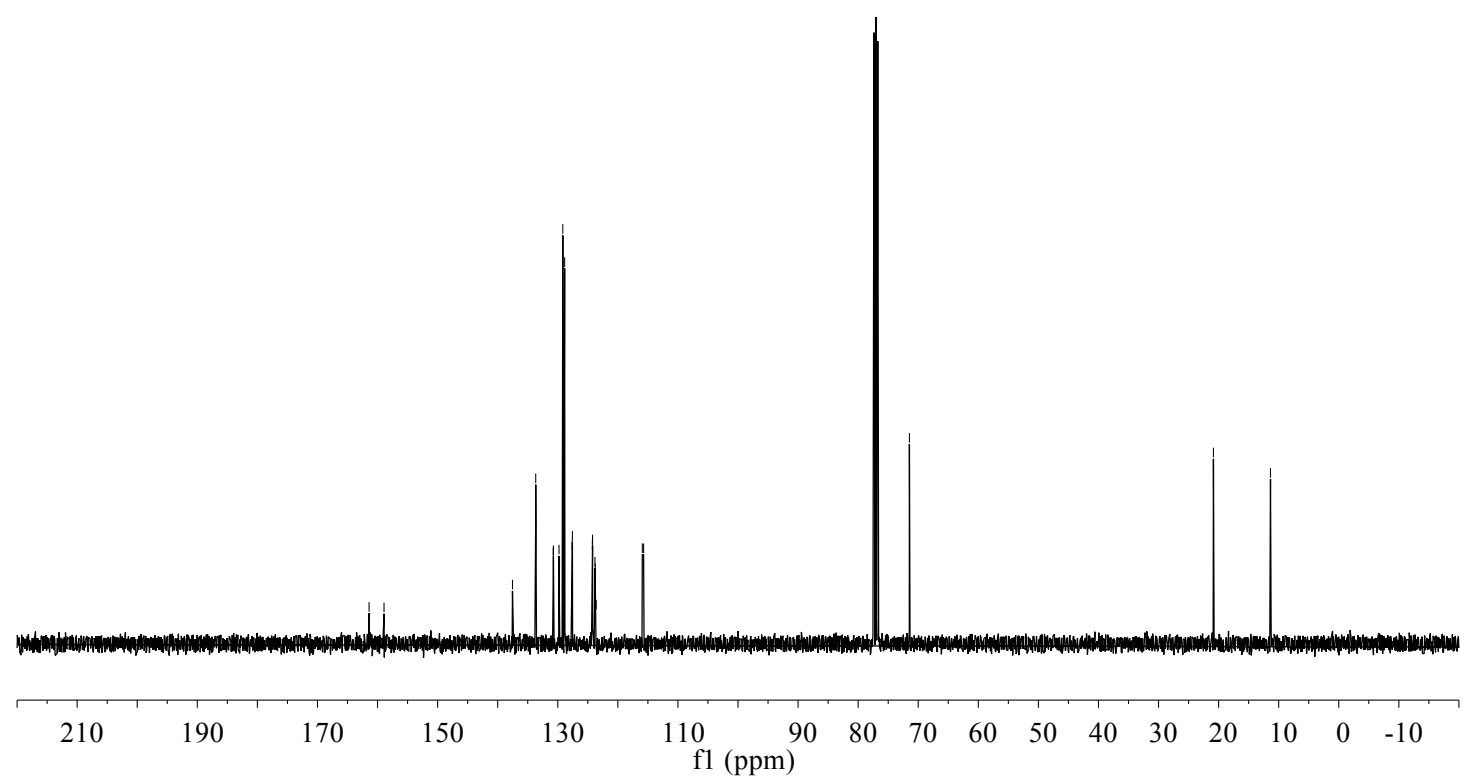




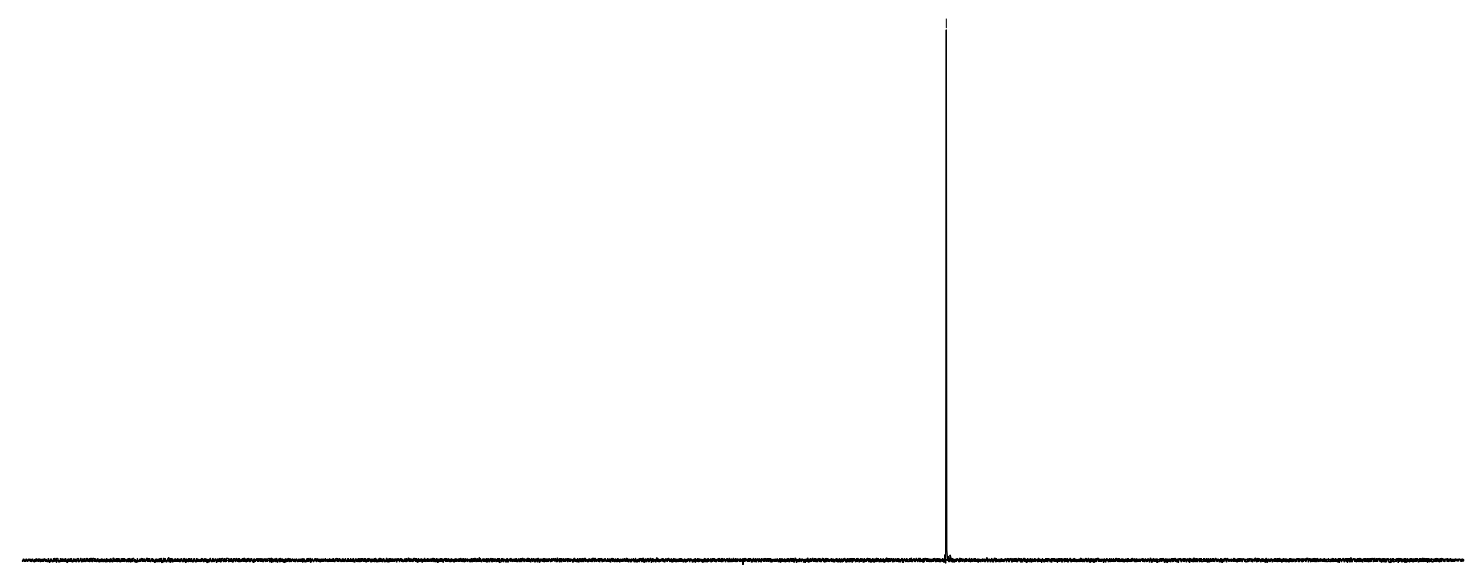

\begin{tabular}{|c|c|c|c|c|c|c|c|c|c|c|}
\hline 60 & 40 & 20 & 0 & -20 & -50 & f1 $\left(\begin{array}{c}-80 \\
\text { ppm })\end{array}\right.$ & -110 & -140 & -170 & -200 \\
\hline
\end{tabular}<smiles>CC[C@H](/C=C/c1ccc(Cl)cc1)[S@@](=O)(O)c1ccccc1</smiles>

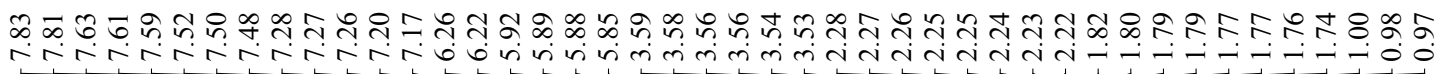
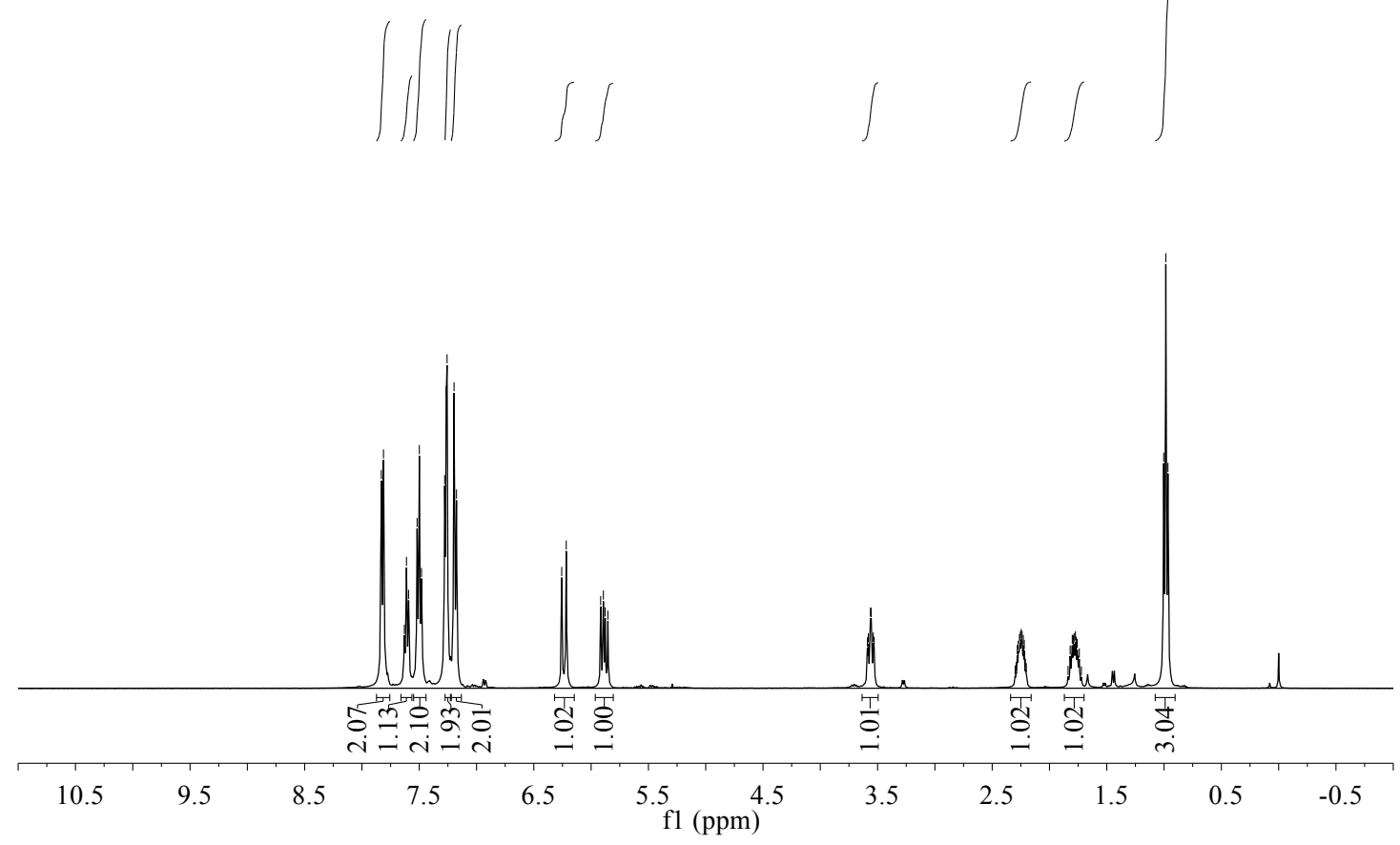


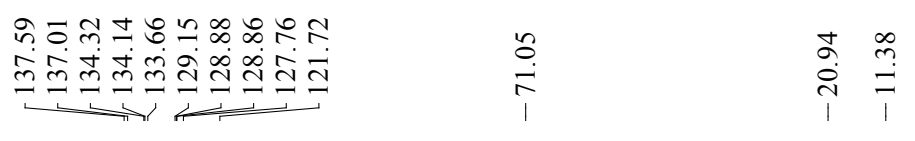

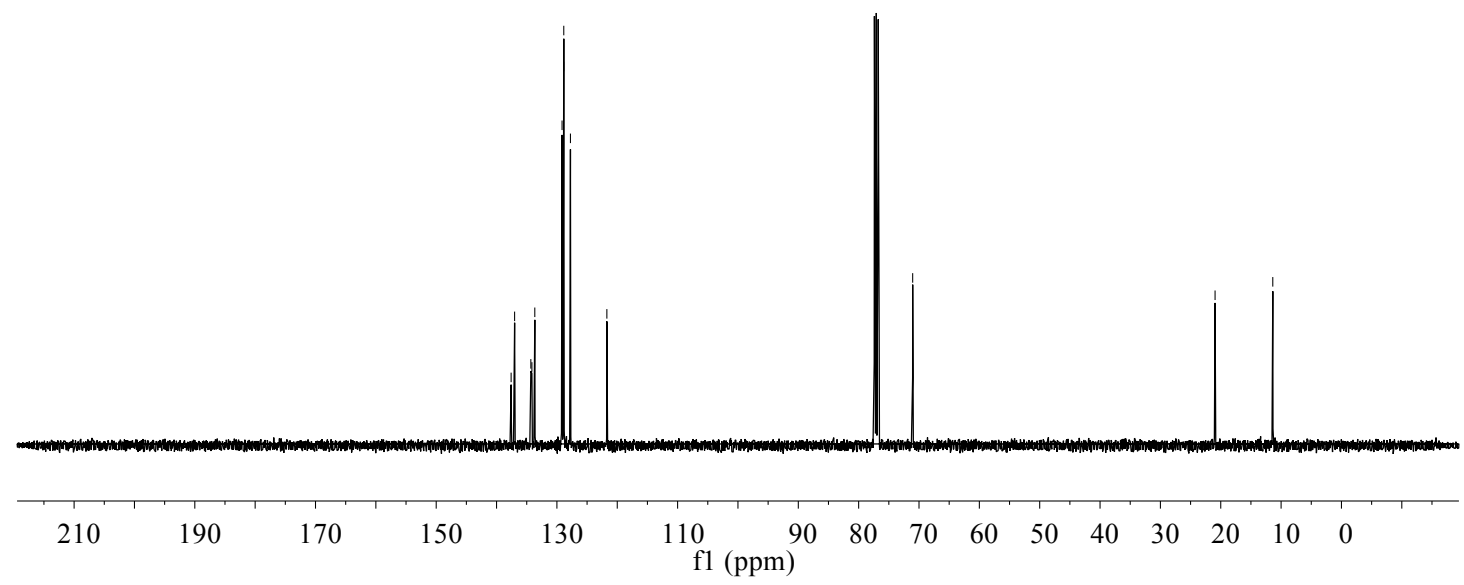<smiles>CC[C@H](/C=C/c1ccc(C(F)(F)F)cc1)C(=O)Oc1ccccc1</smiles>

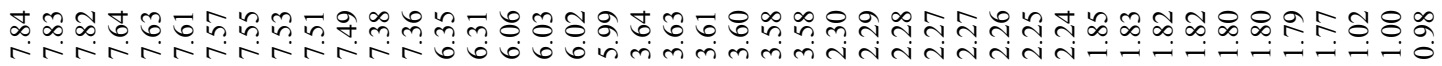
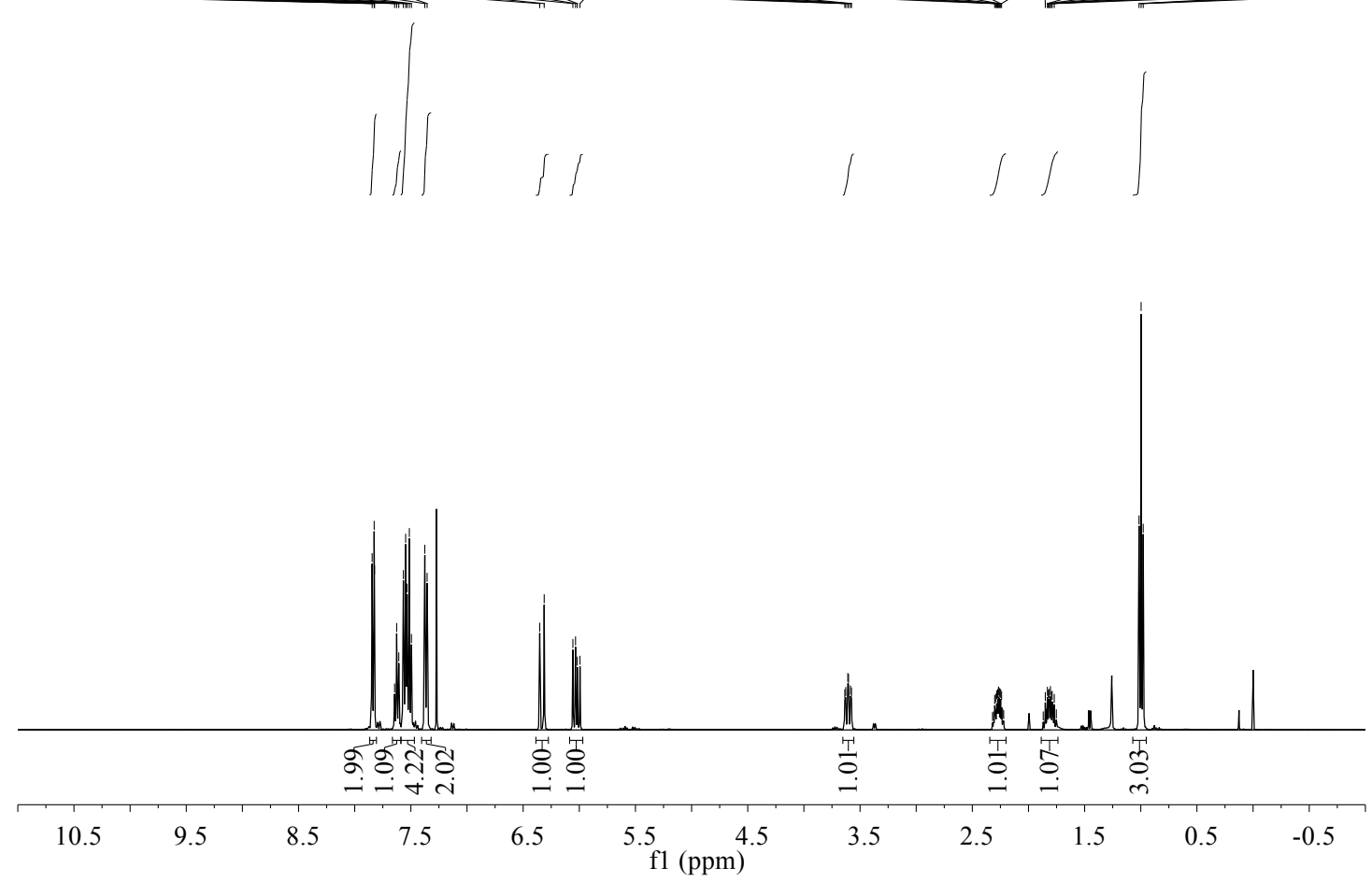


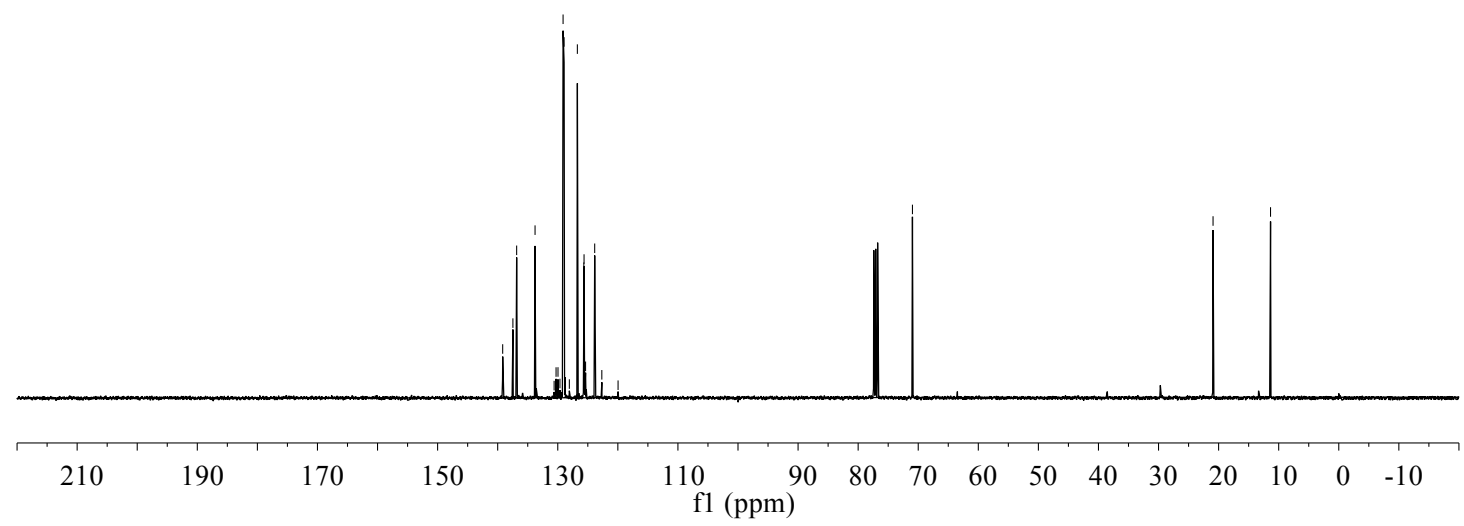

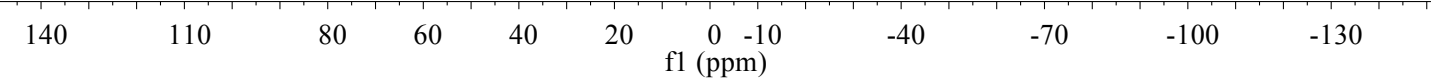




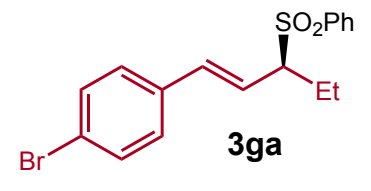

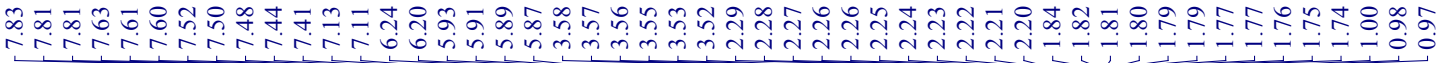
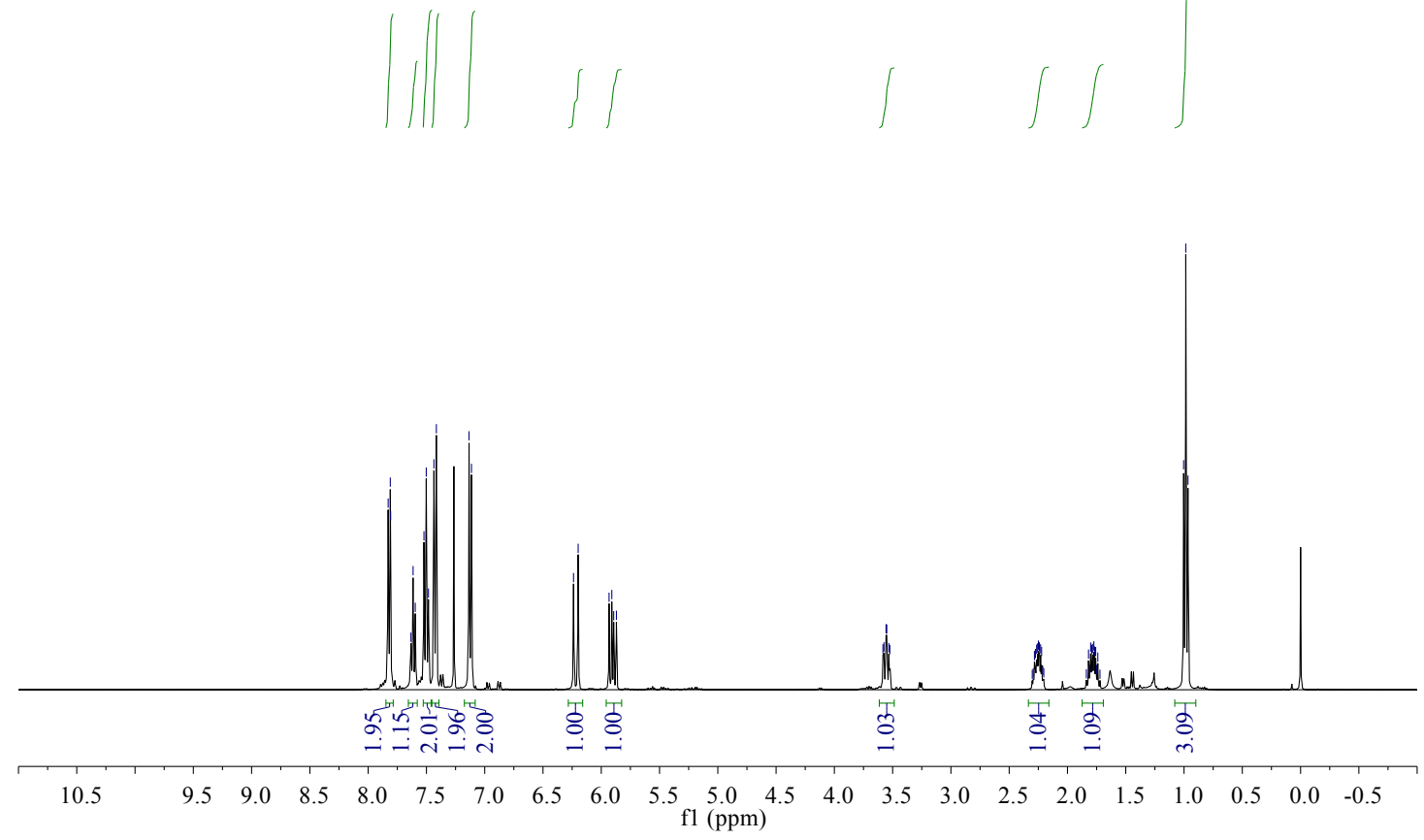

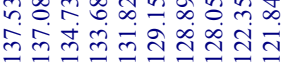
$\stackrel{8}{\stackrel{i}{i}}$

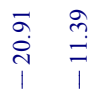

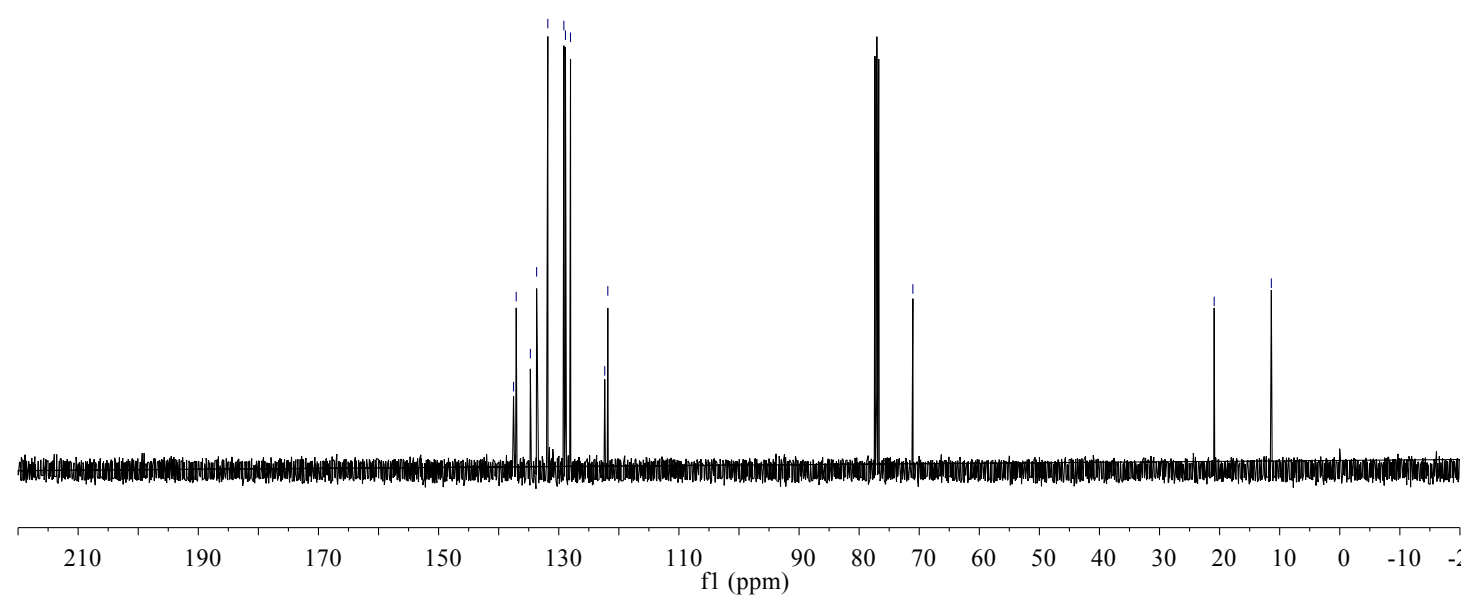


<smiles></smiles>

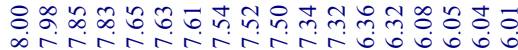

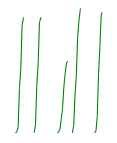

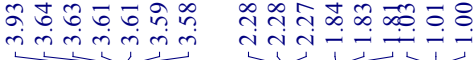

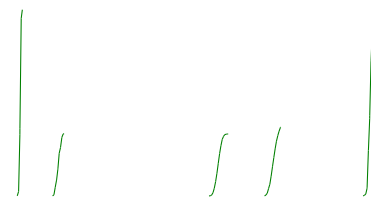

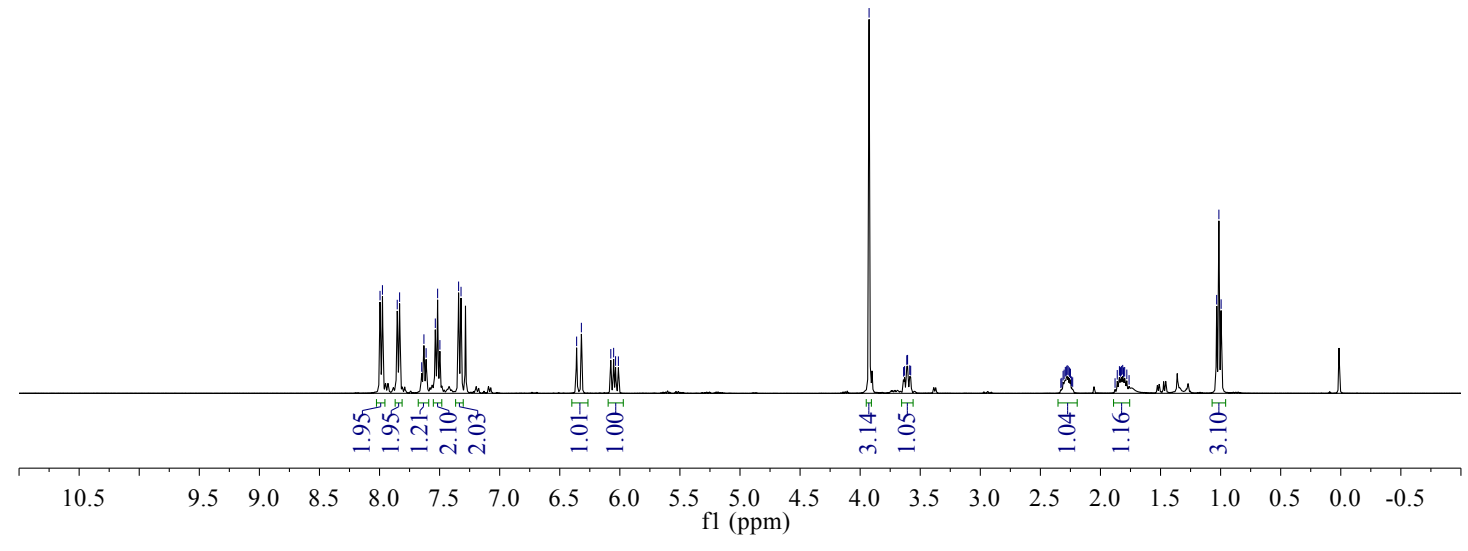

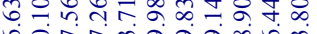

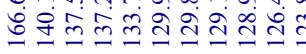

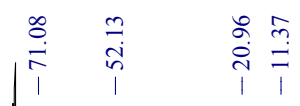

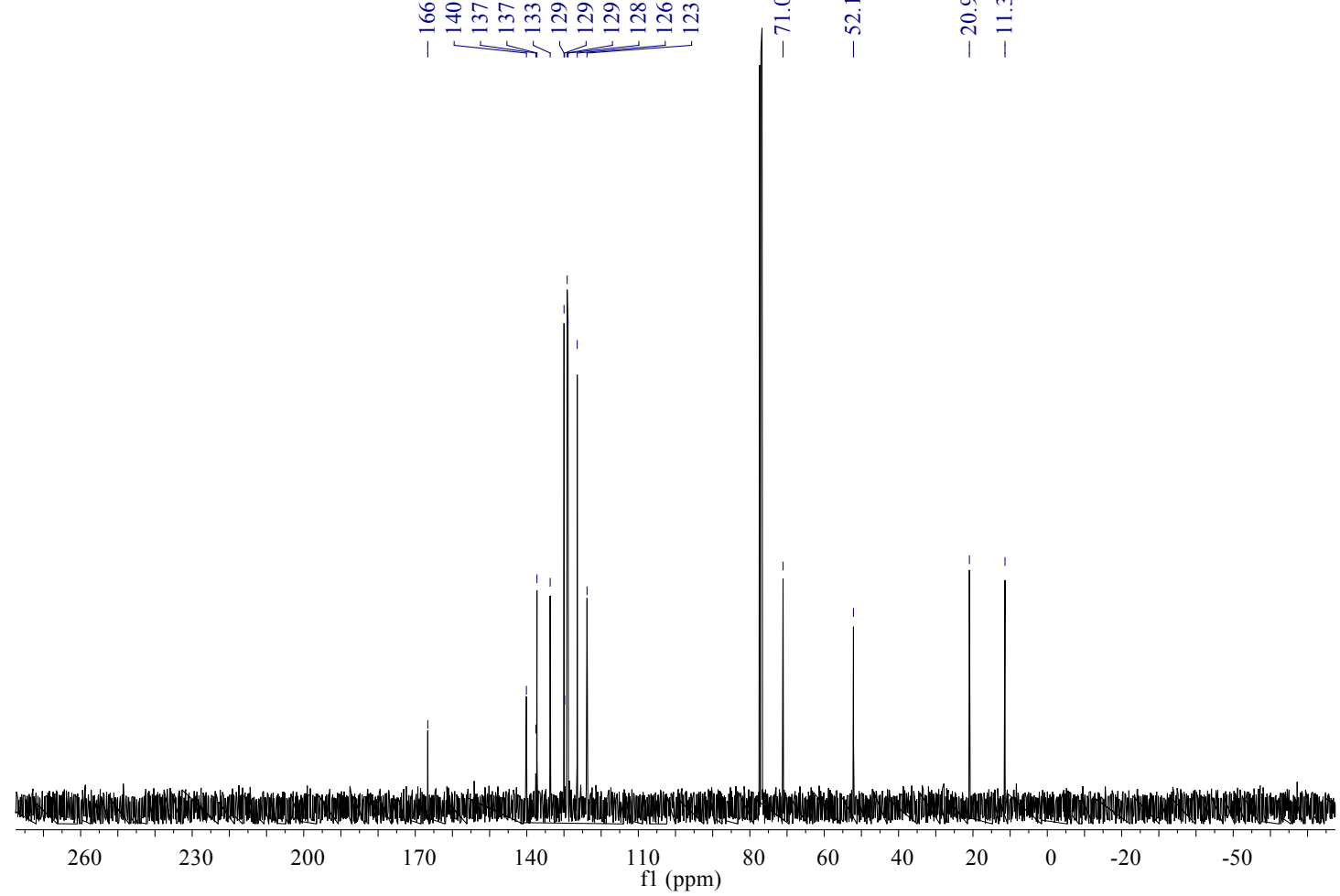


<smiles>CC[C@H](/C=C/c1ccc(OC)cc1)C(=O)O[R6](=O)(c1ccccc1)c1ccccc1</smiles>

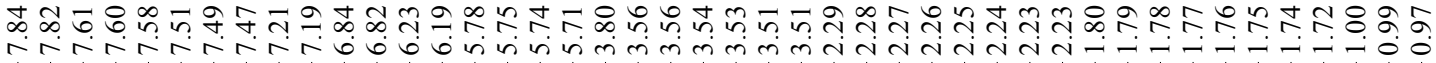

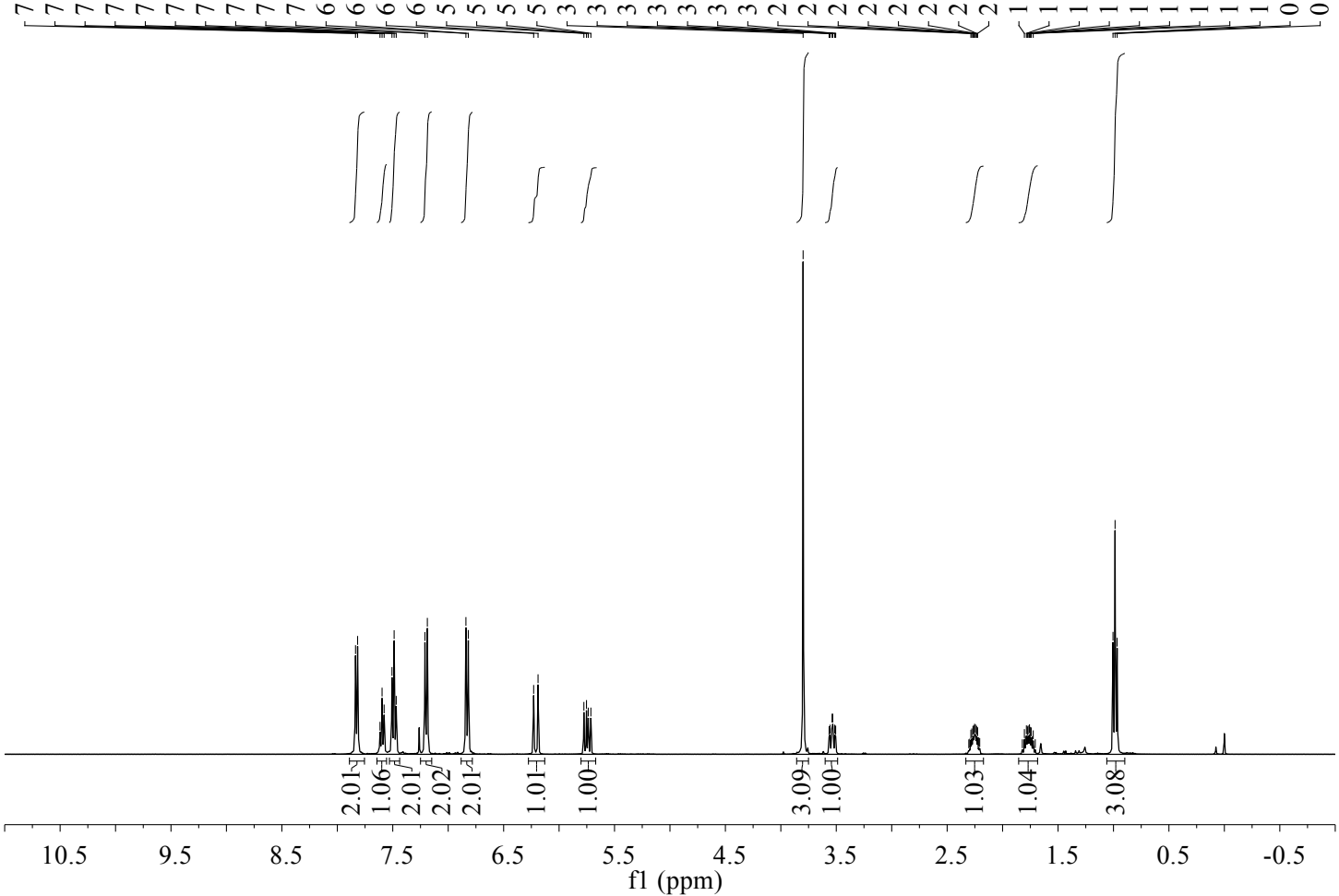

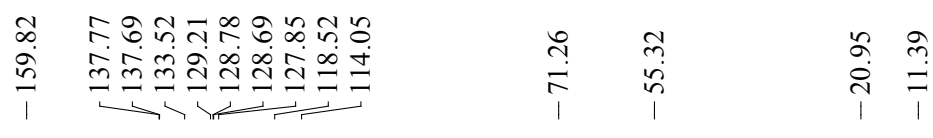

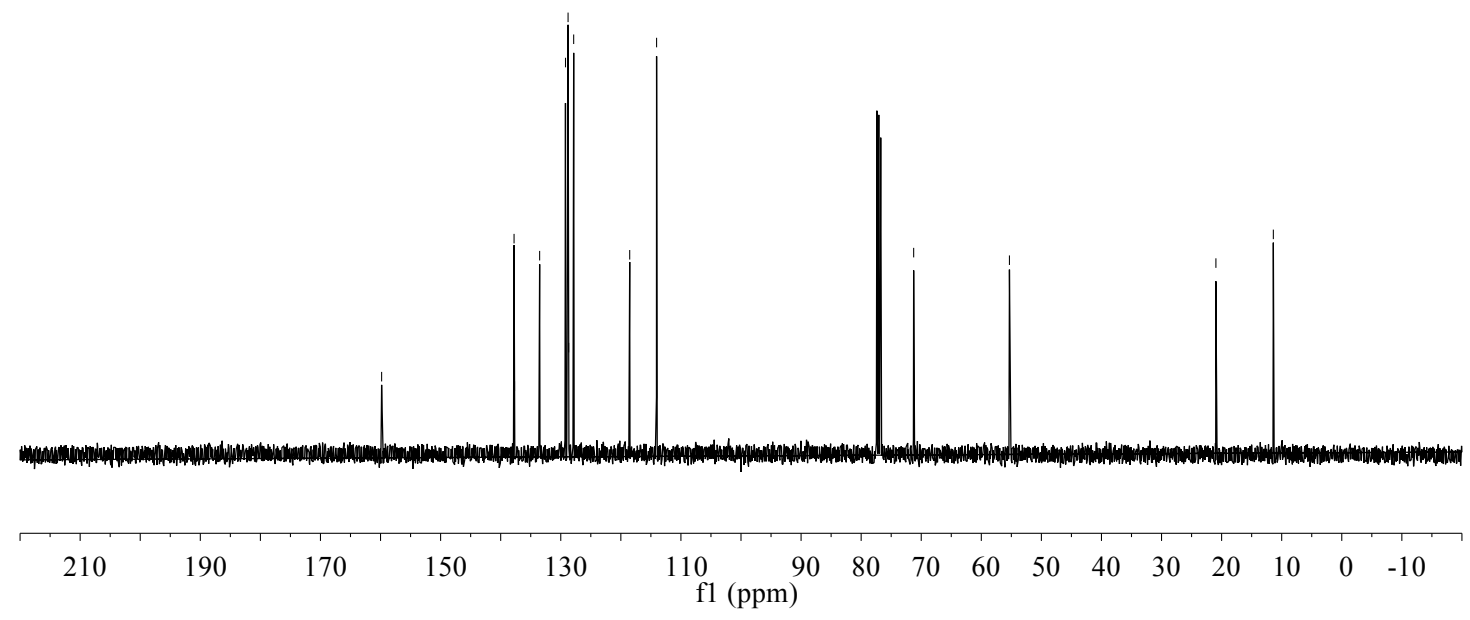


<smiles>CC[C@H](/C=C/c1ccc(C)cc1)S(=O)(=O)c1ccccc1</smiles>

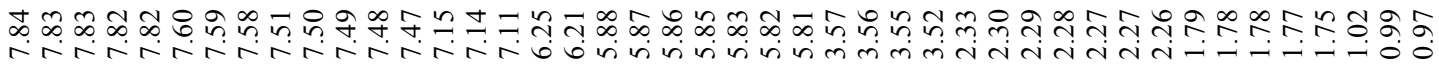
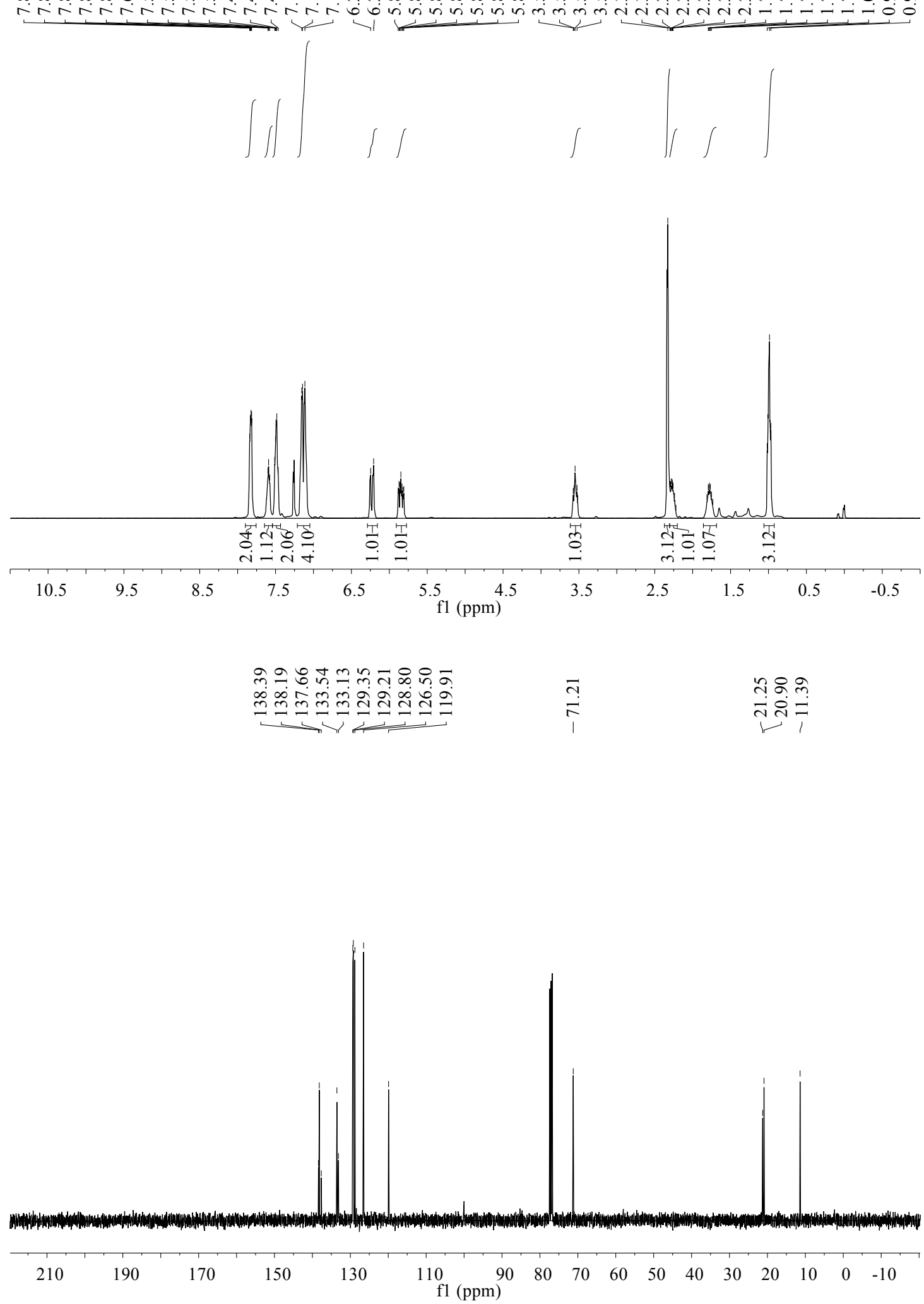


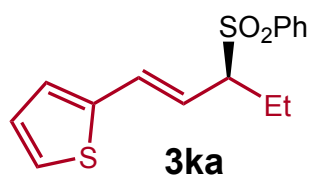

屯

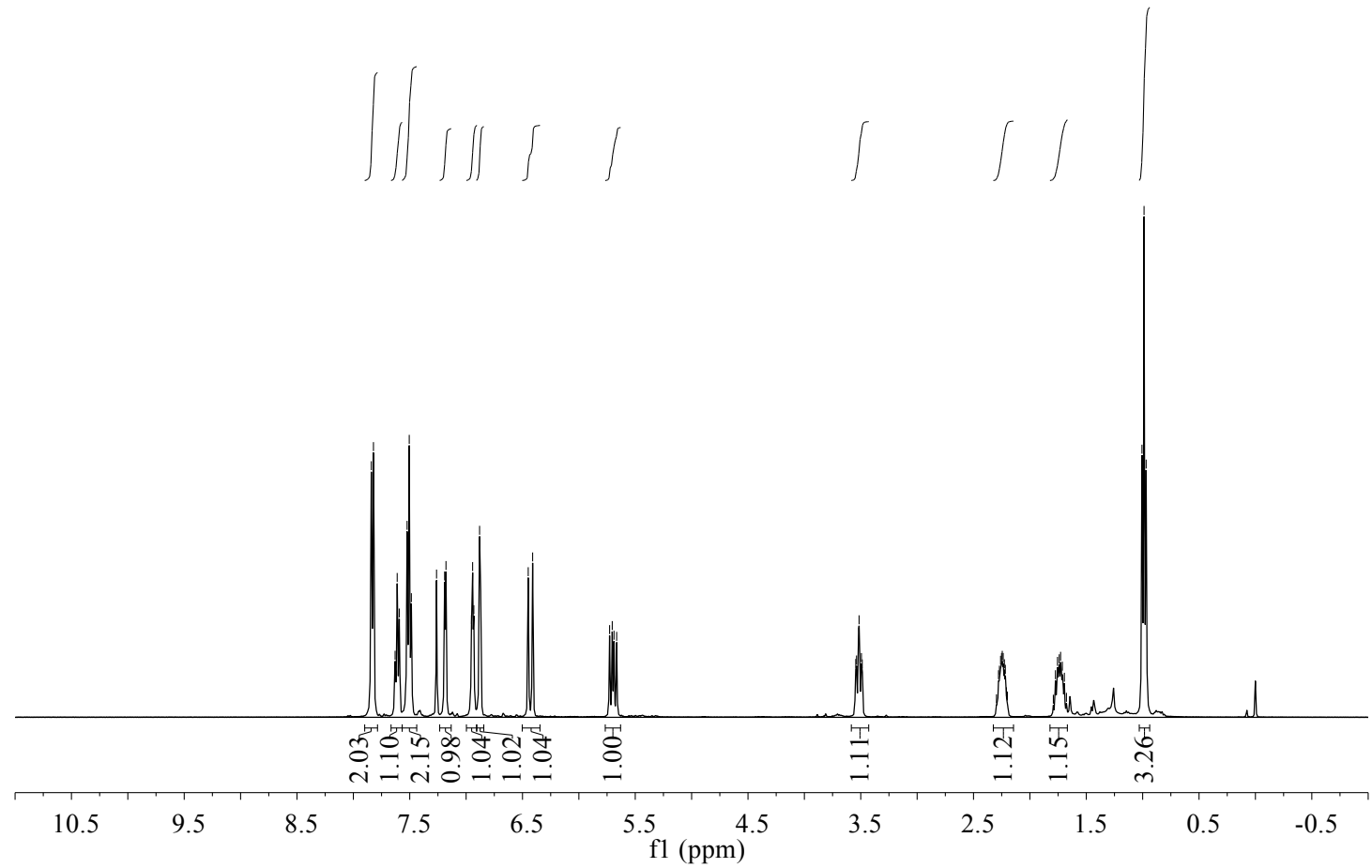

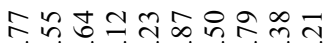

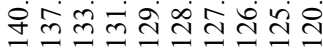
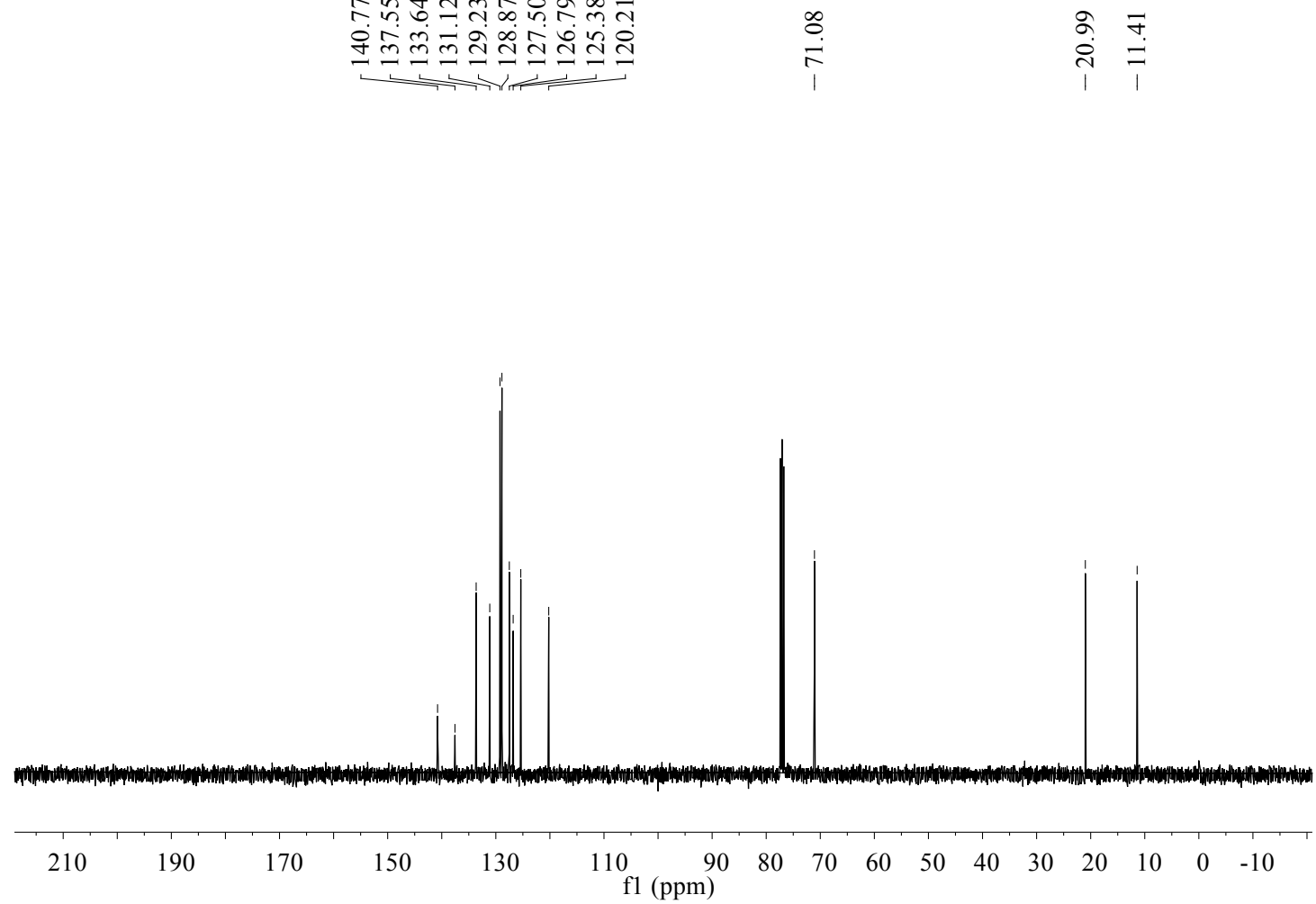
<smiles>CCC(/C=C/c1ccco1)S(=O)(=O)c1ccccc1</smiles>
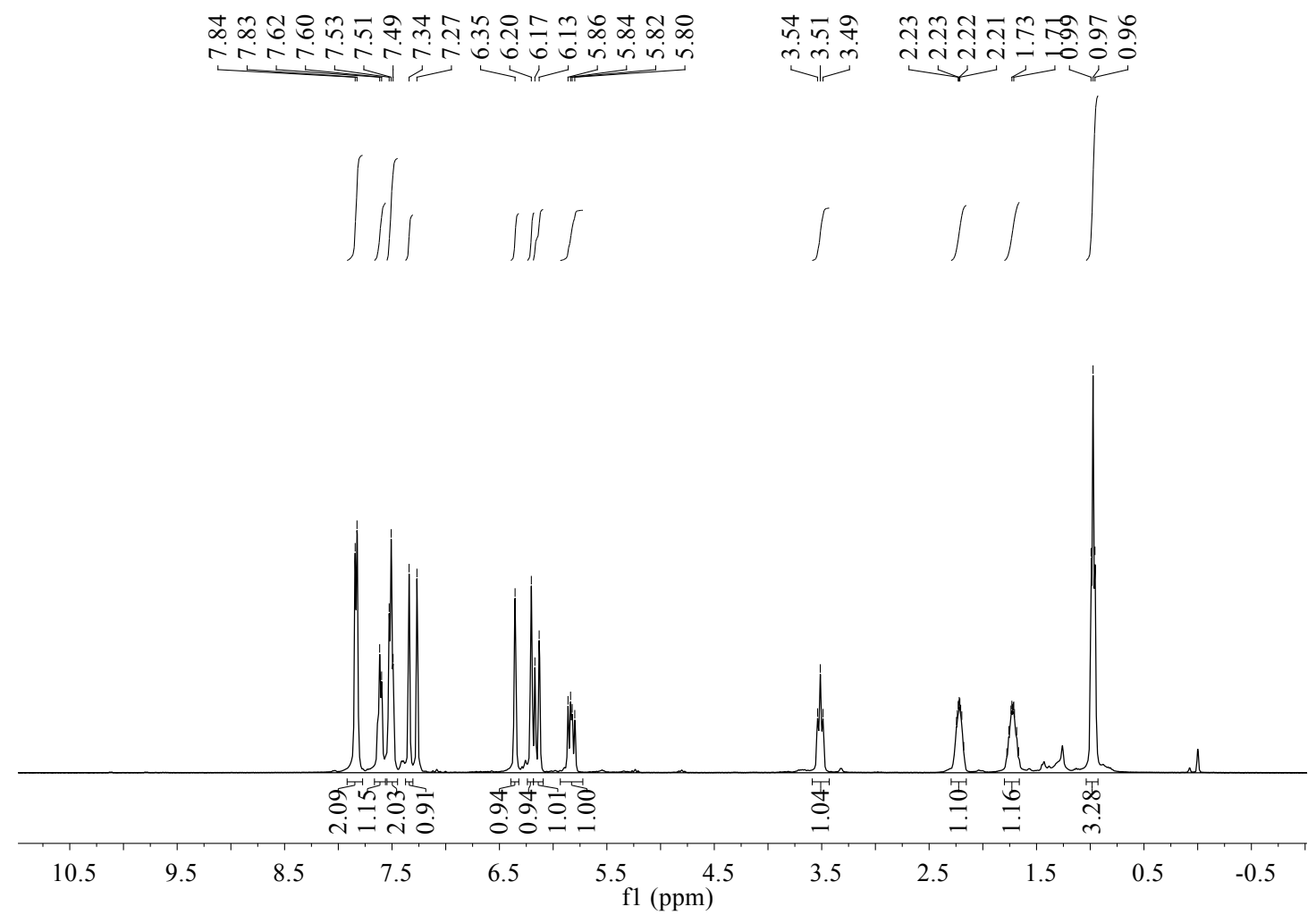

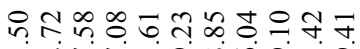

$$
\begin{aligned}
& \text { ธุษ }
\end{aligned}
$$

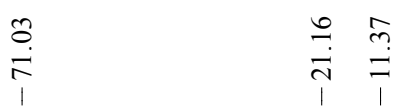

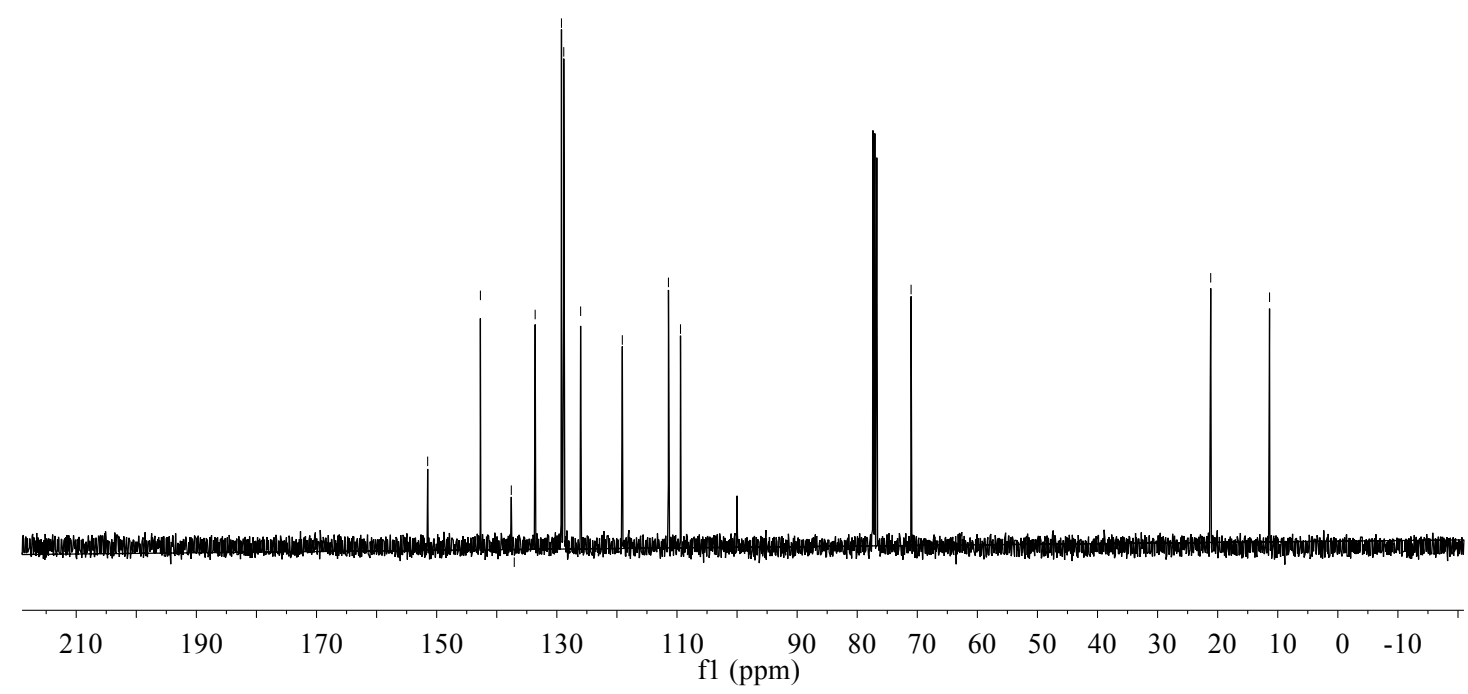




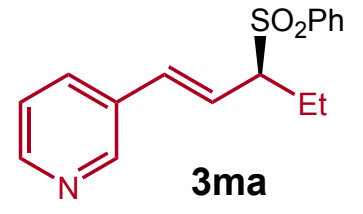

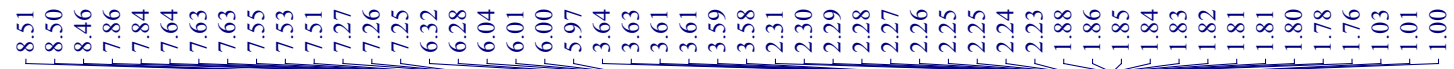
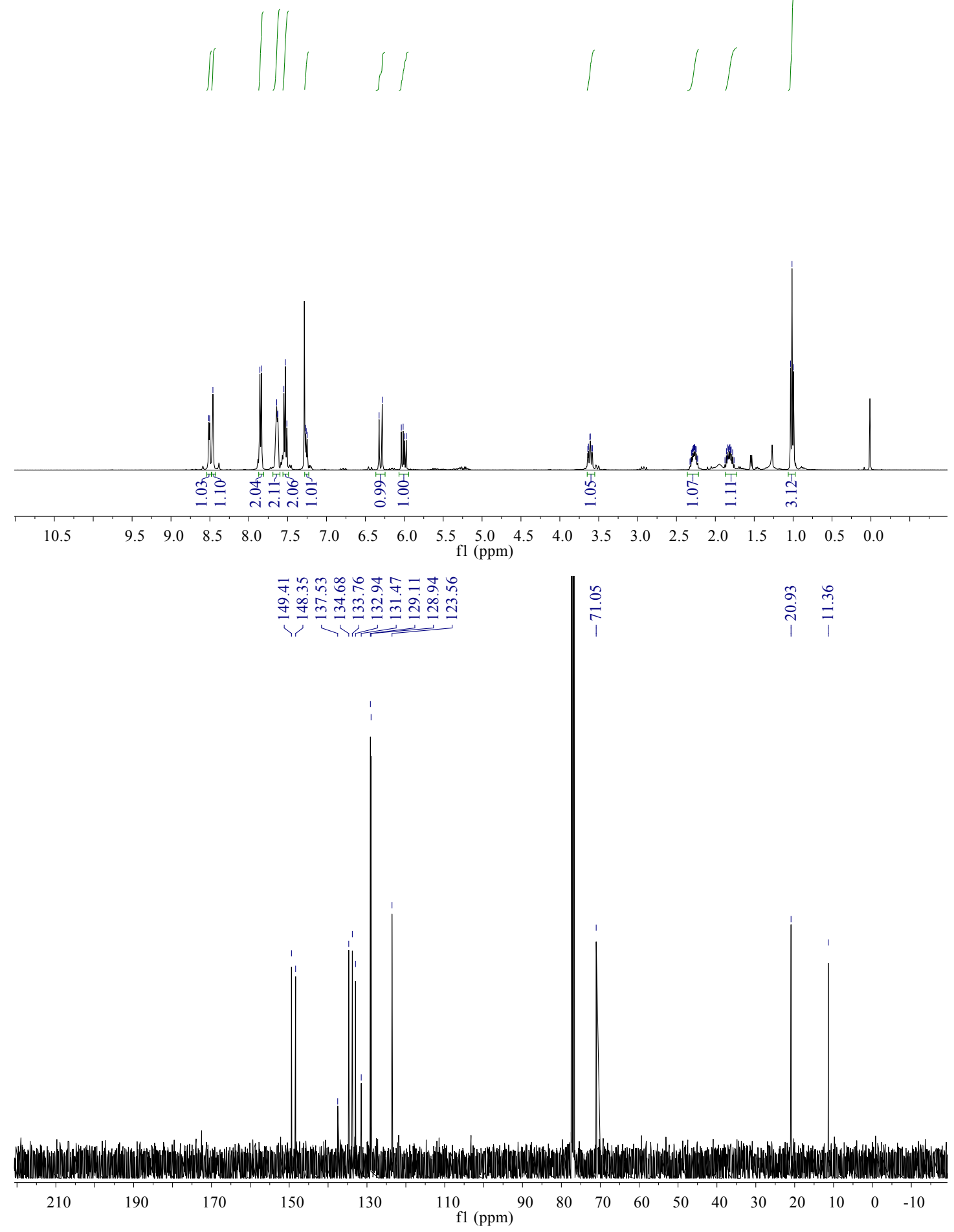
<smiles>CC[C@H](/C=C/C1CCCCC1)S(=O)(=O)O</smiles>

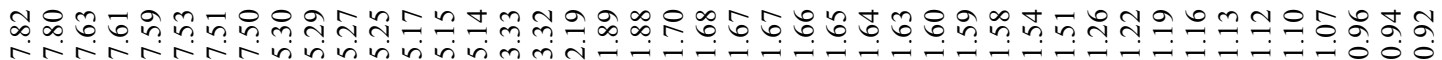
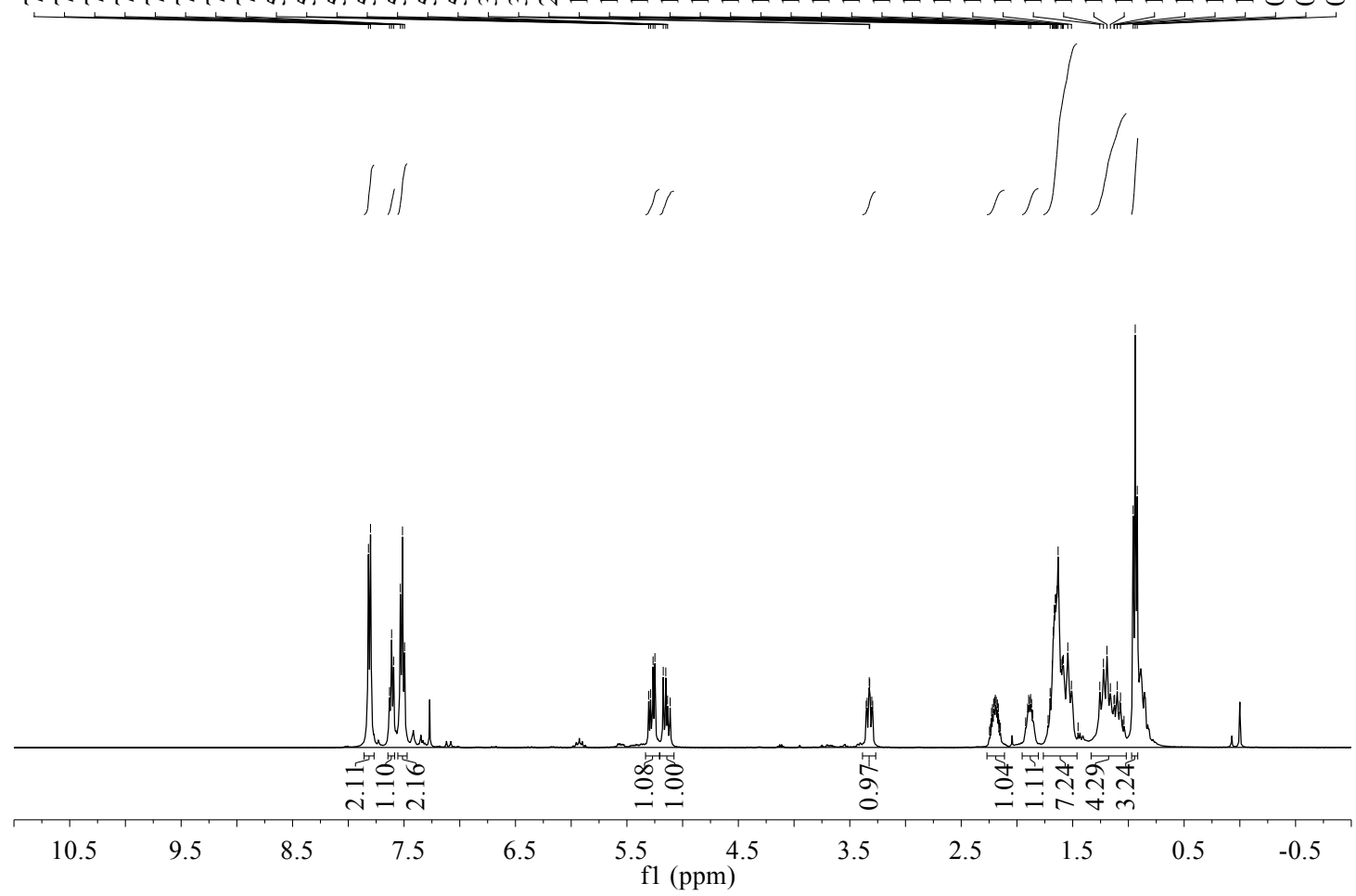

구ำำํํำ 7

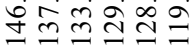

म.

श क्षंतेंते

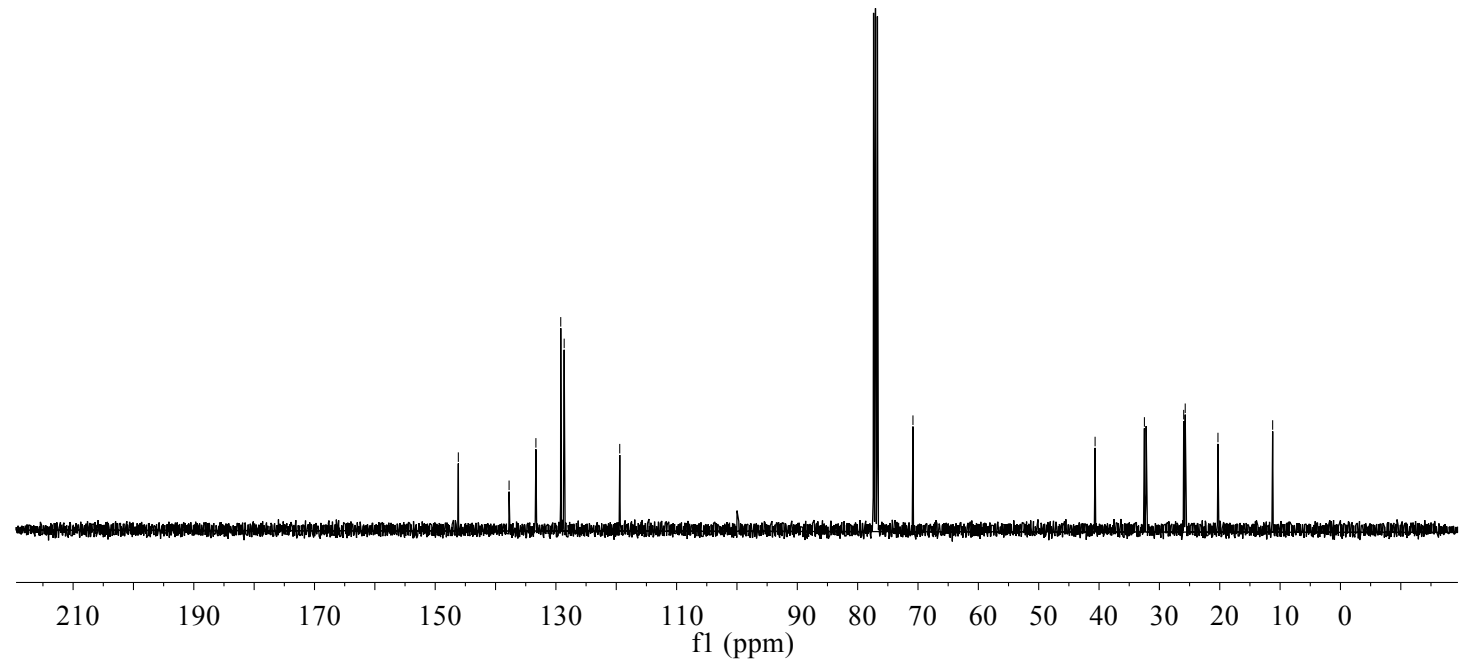


<smiles>CCC[C@H](/C=C/c1ccccc1)[Se](=O)(=O)c1ccccc1</smiles>
荧
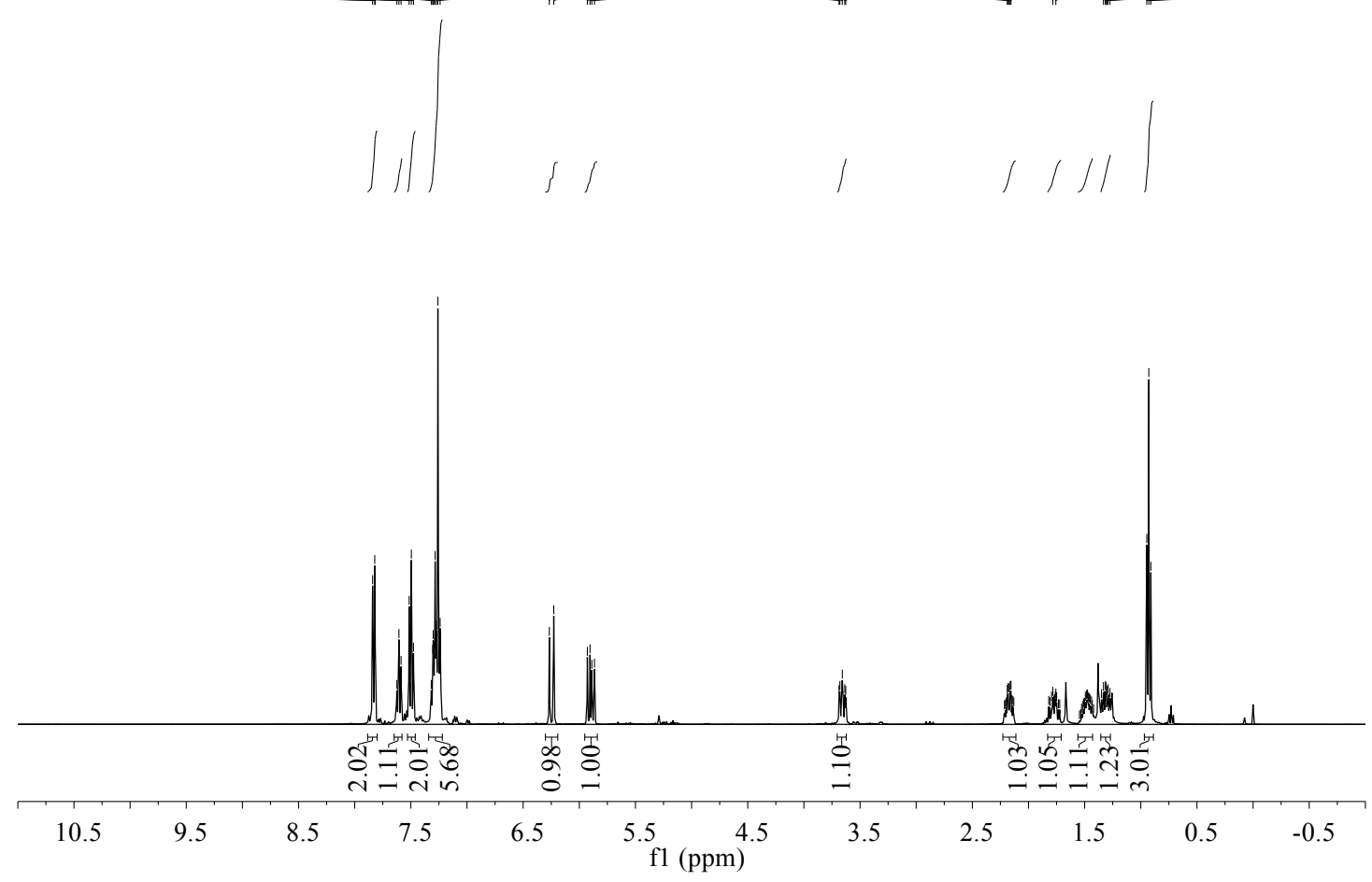

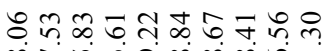

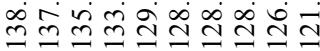
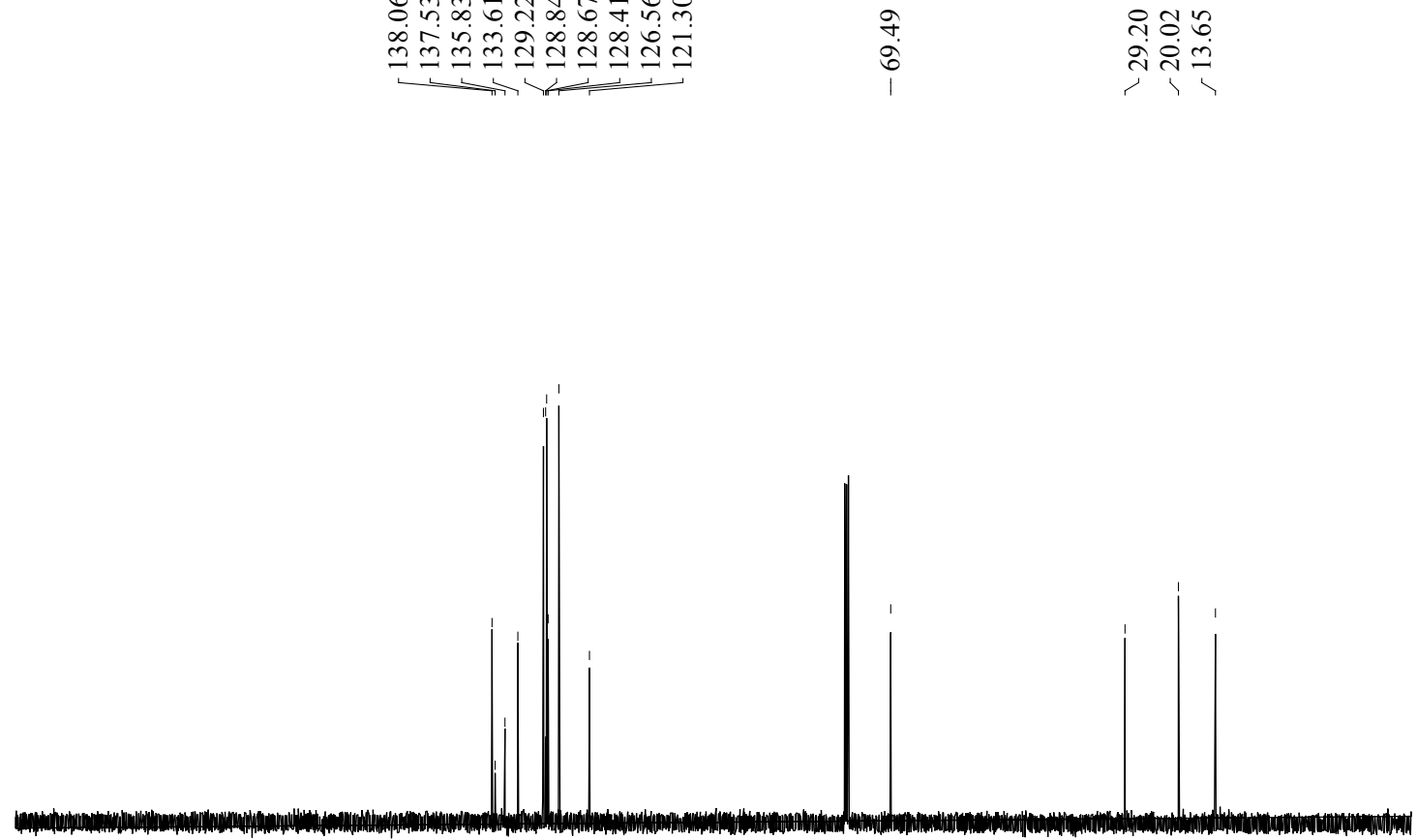
210
190
170
150
130
110 f1 (ppm)
$\begin{array}{lllllllll}80 & 70 & 60 & 50 & 40 & 30 & 20 & 10 & 0\end{array}$ 


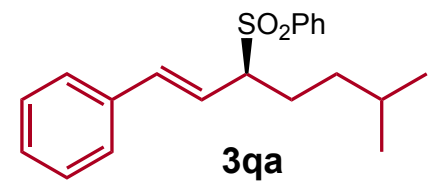

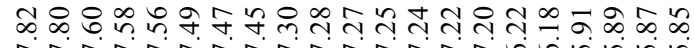
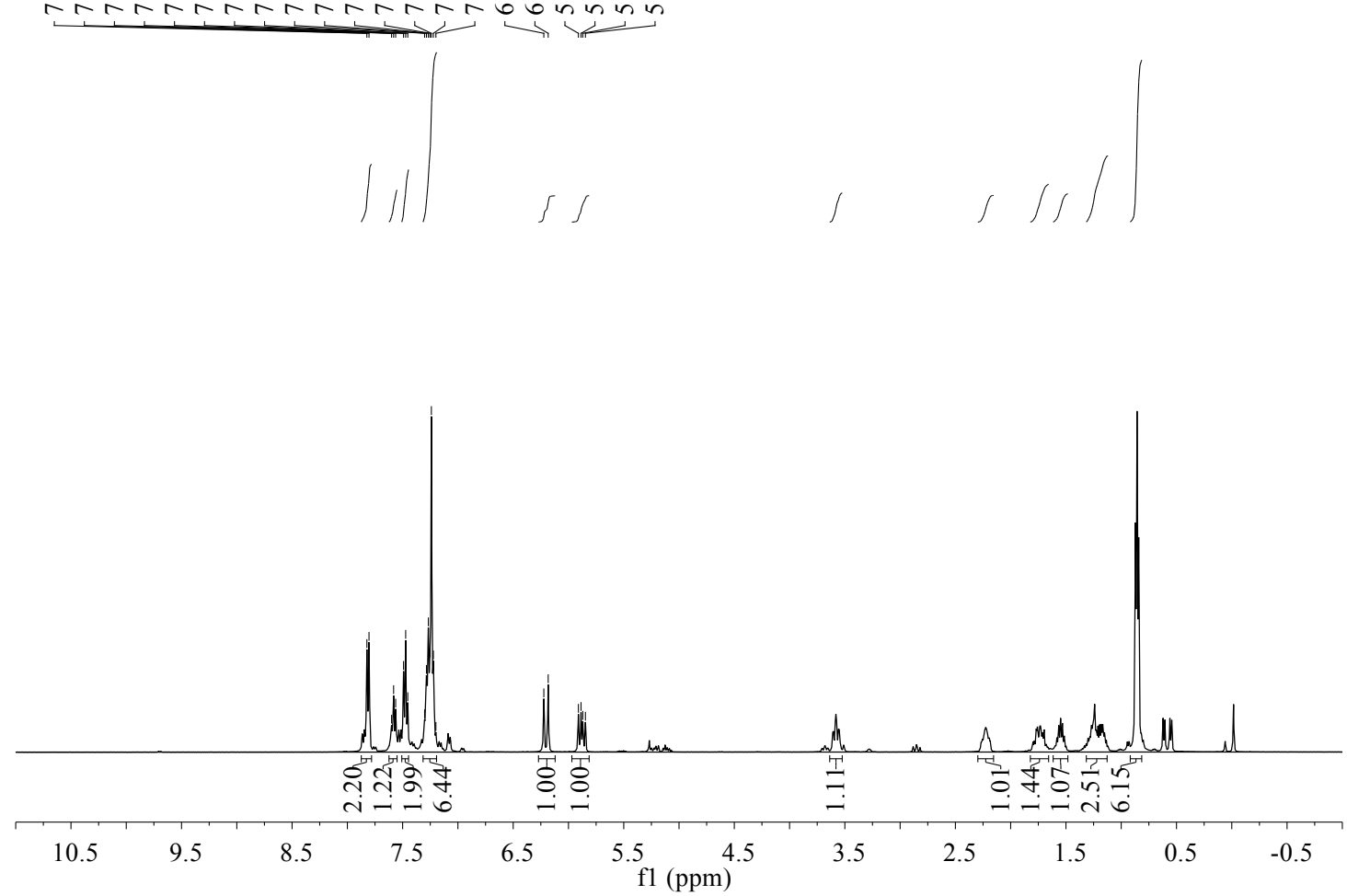

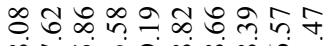

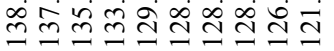

ลิ

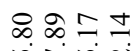
लेतูก

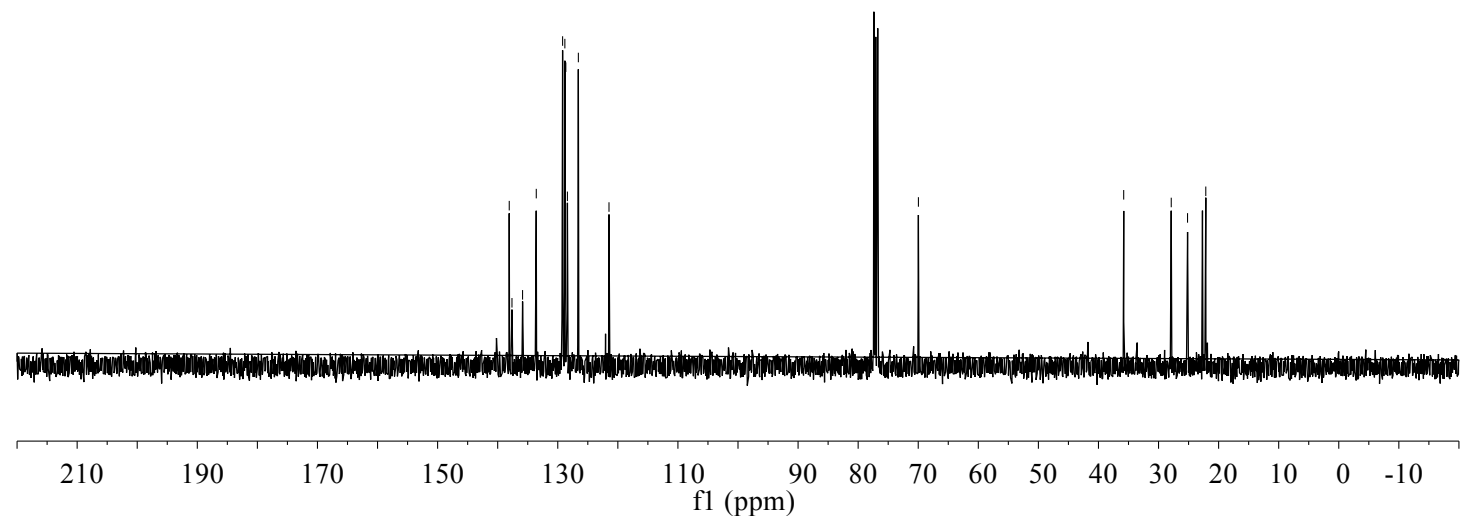


<smiles>COCCC[C@H](/C=C/c1ccccc1)S(=O)(=O)c1ccccc1</smiles>

我
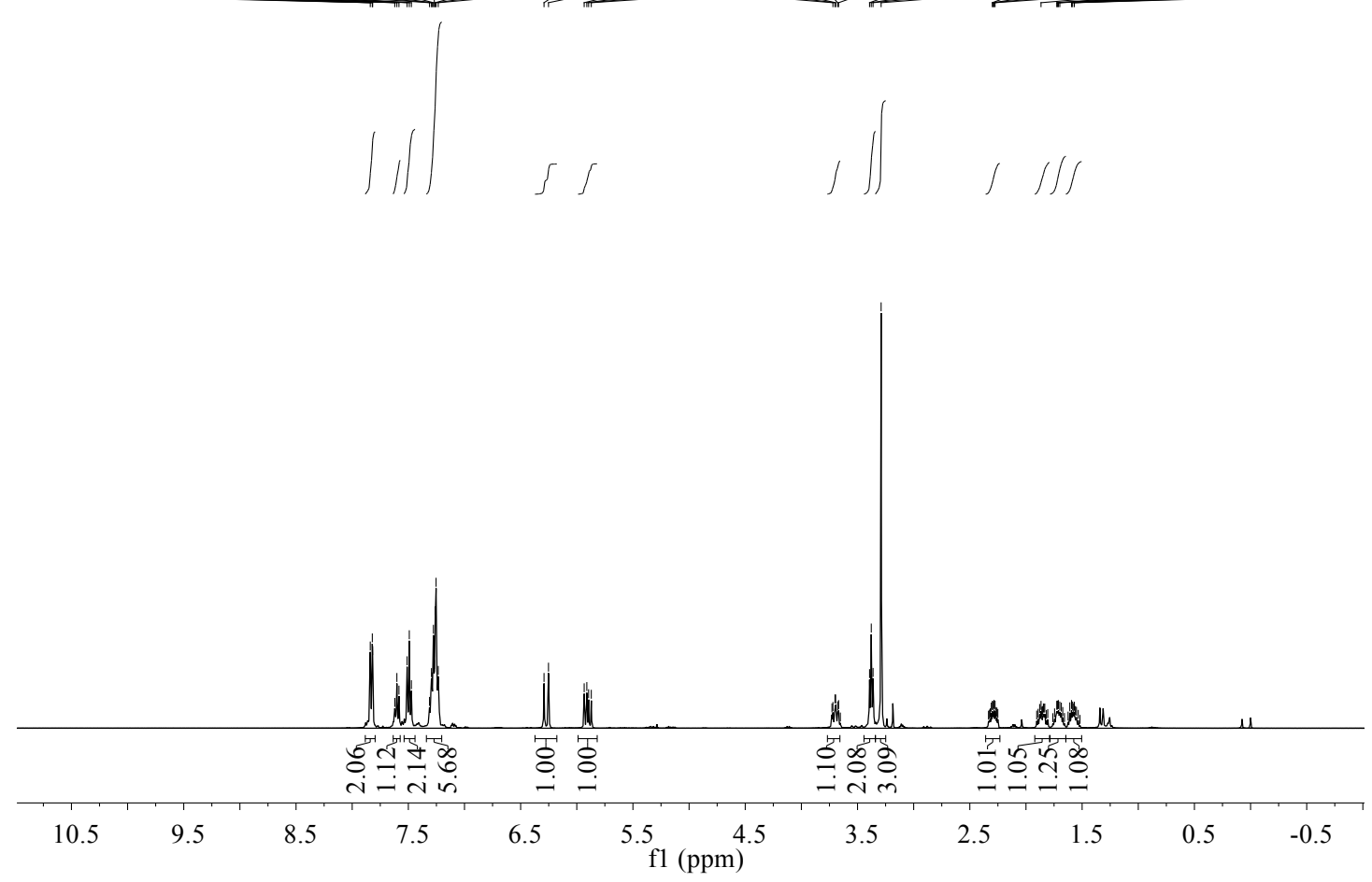

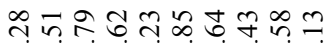

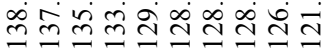

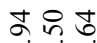

तें

क⿺⿻一𠃋十

กับ

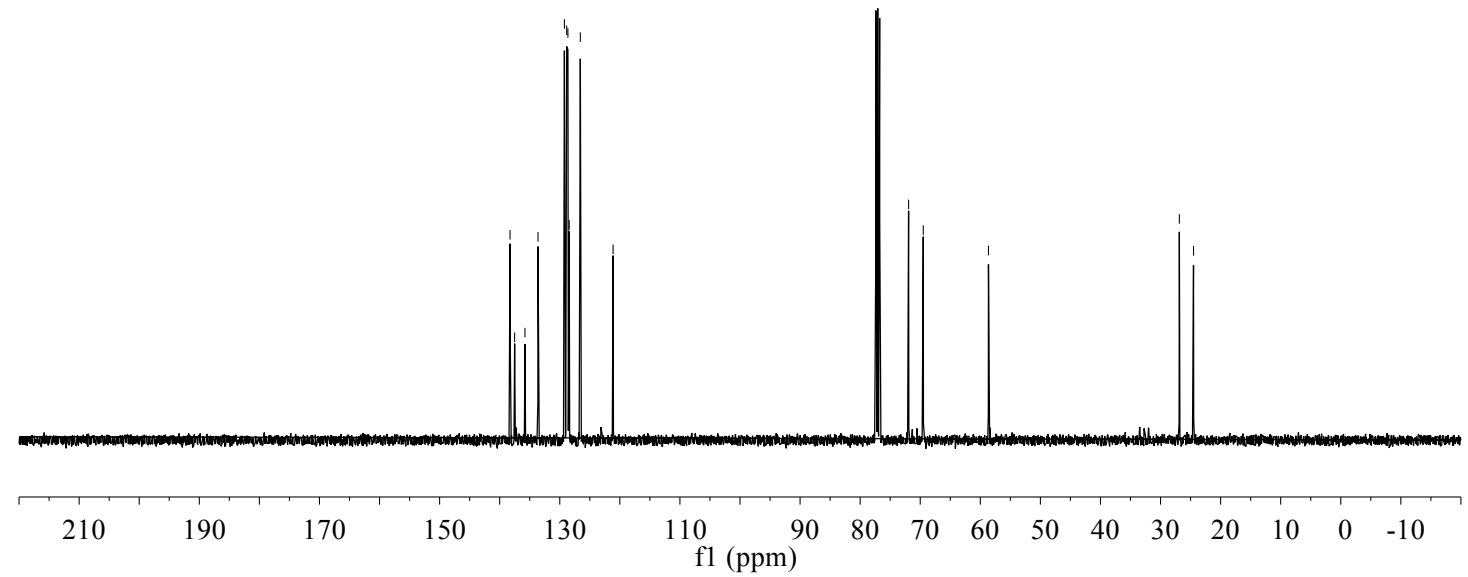


<smiles>CC(=O)C[C@H](/C=C/c1ccccc1)S(=O)(=O)c1ccccc1</smiles>

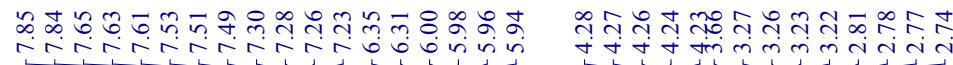
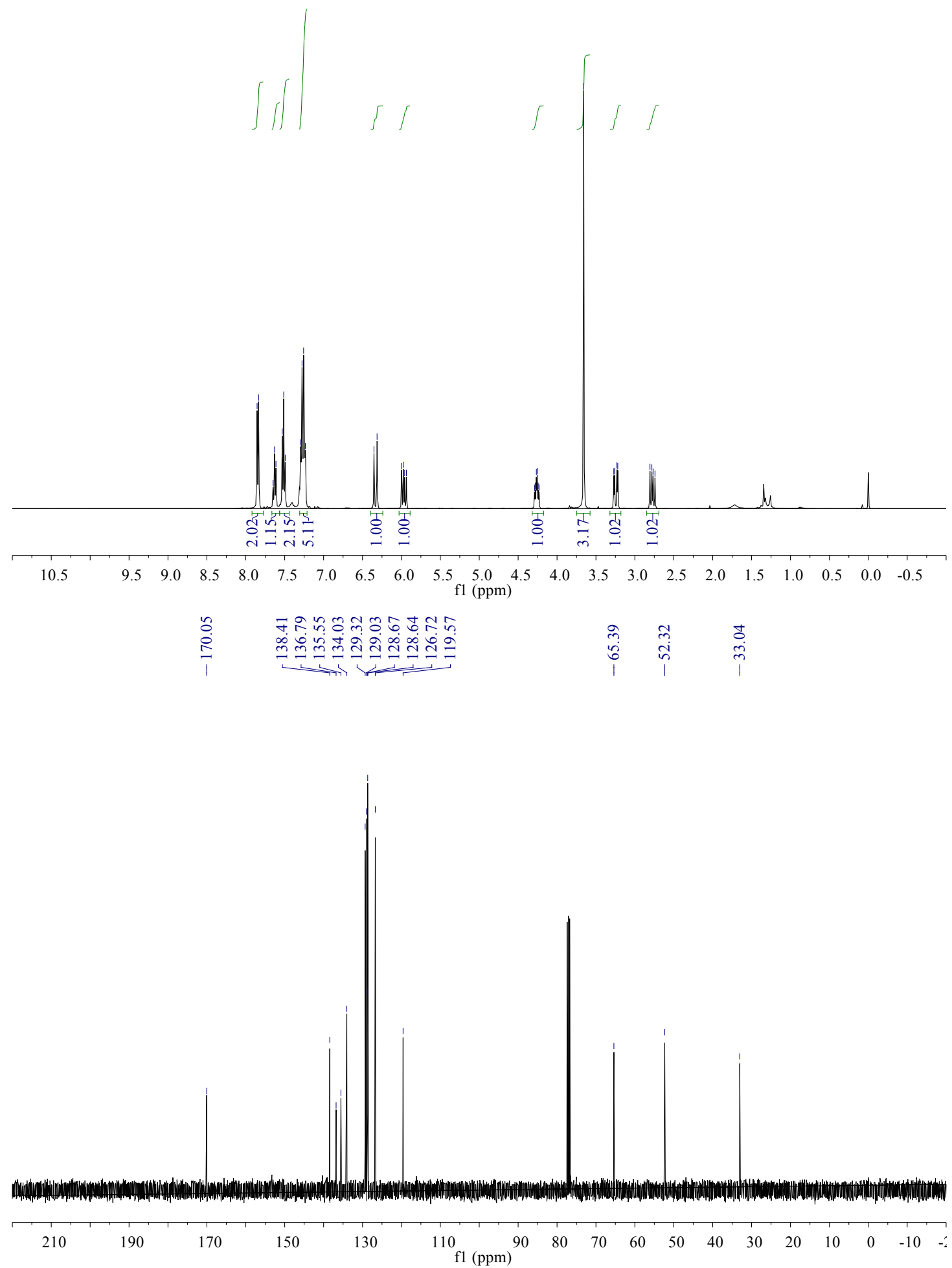
<smiles>CC(/C=C/c1ccccc1)[Sb](=O)(=O)c1ccccc1</smiles>
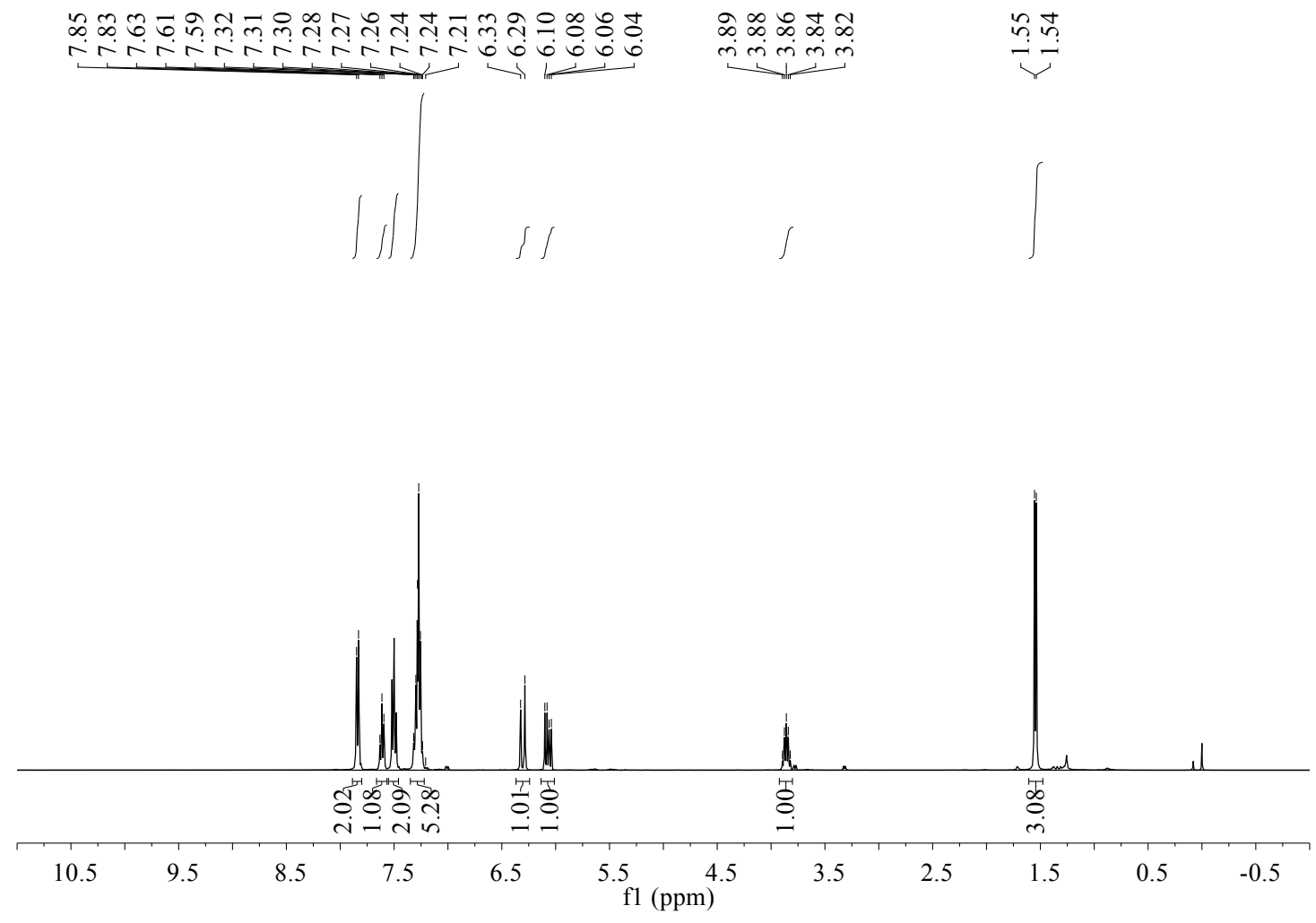

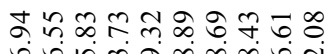

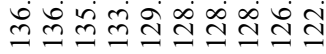

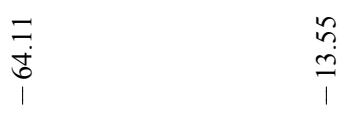

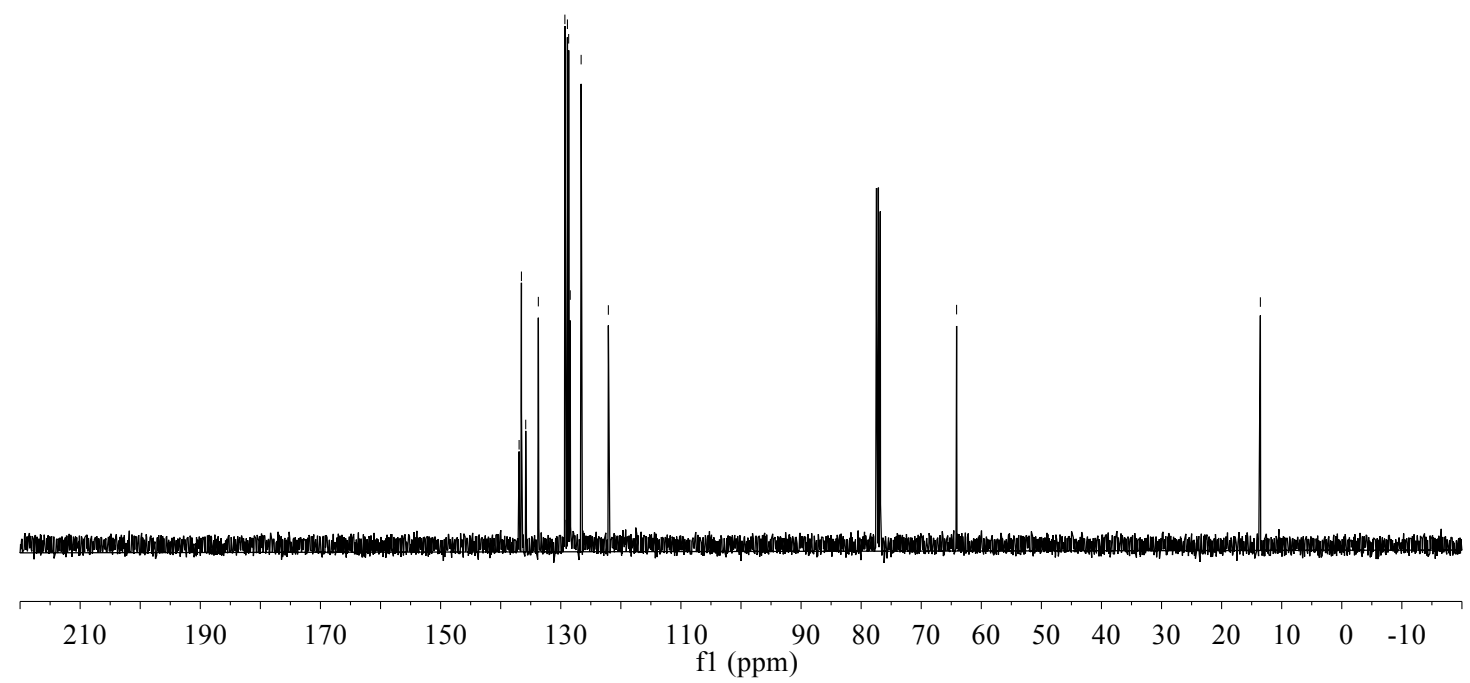




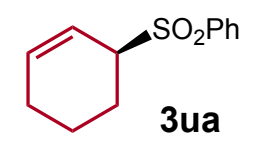

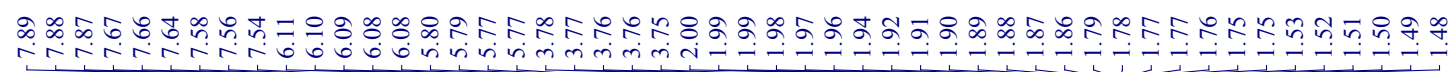
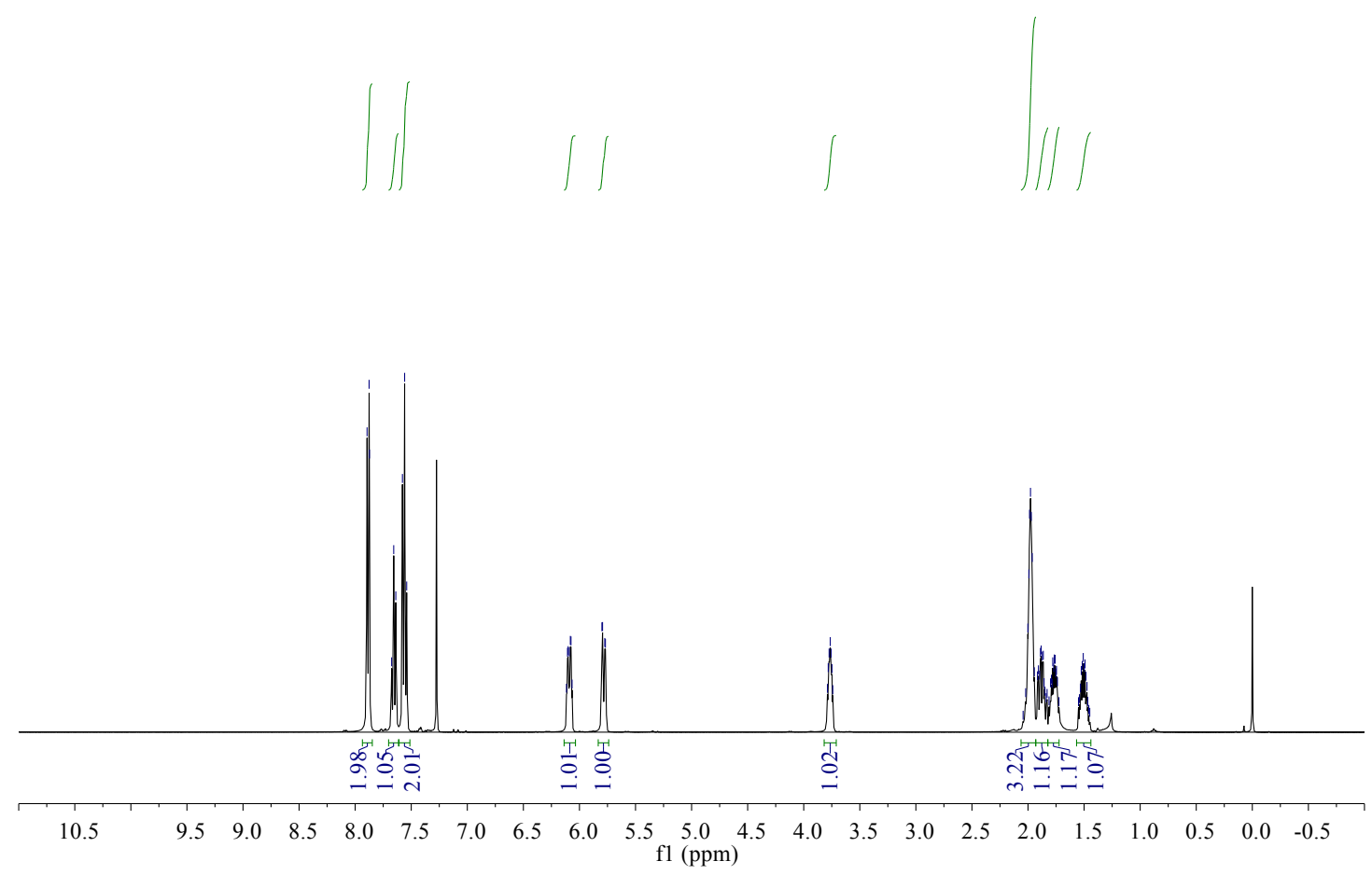

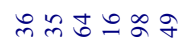

ind

ำ

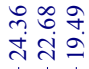

is

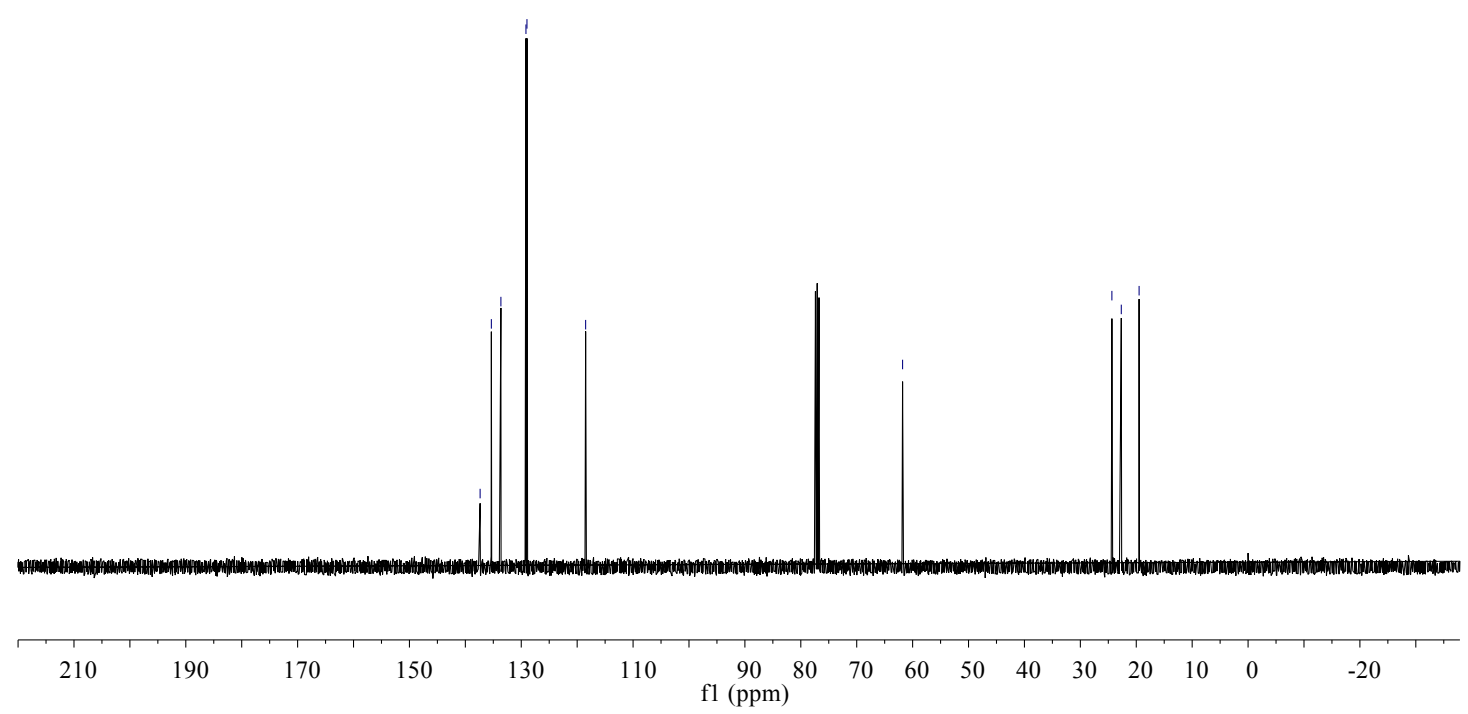


$\underbrace{\mathrm{SO}_{2} \mathrm{Ph}}_{3 \mathbf{v a}}$

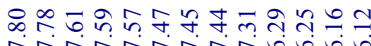

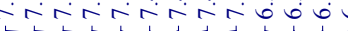

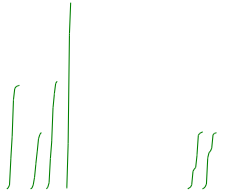

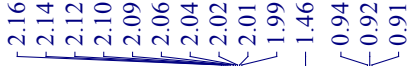

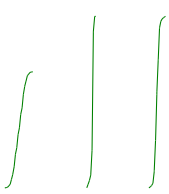

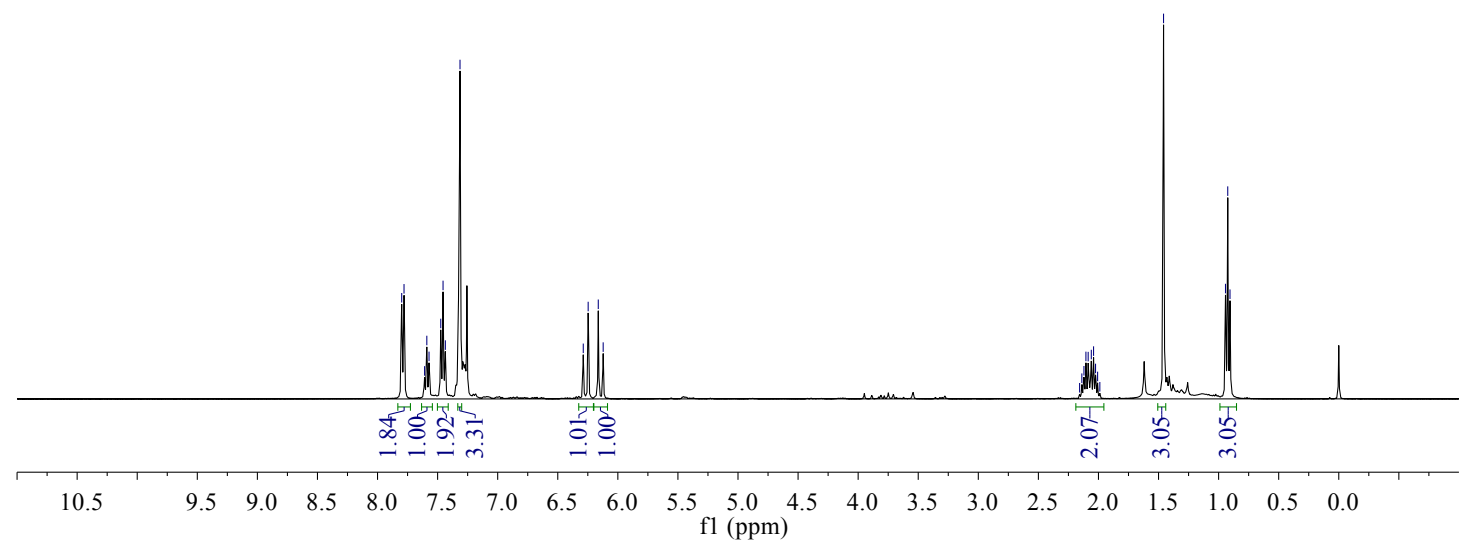

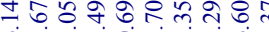

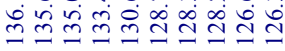

8
0
0
0
1

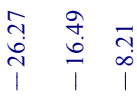

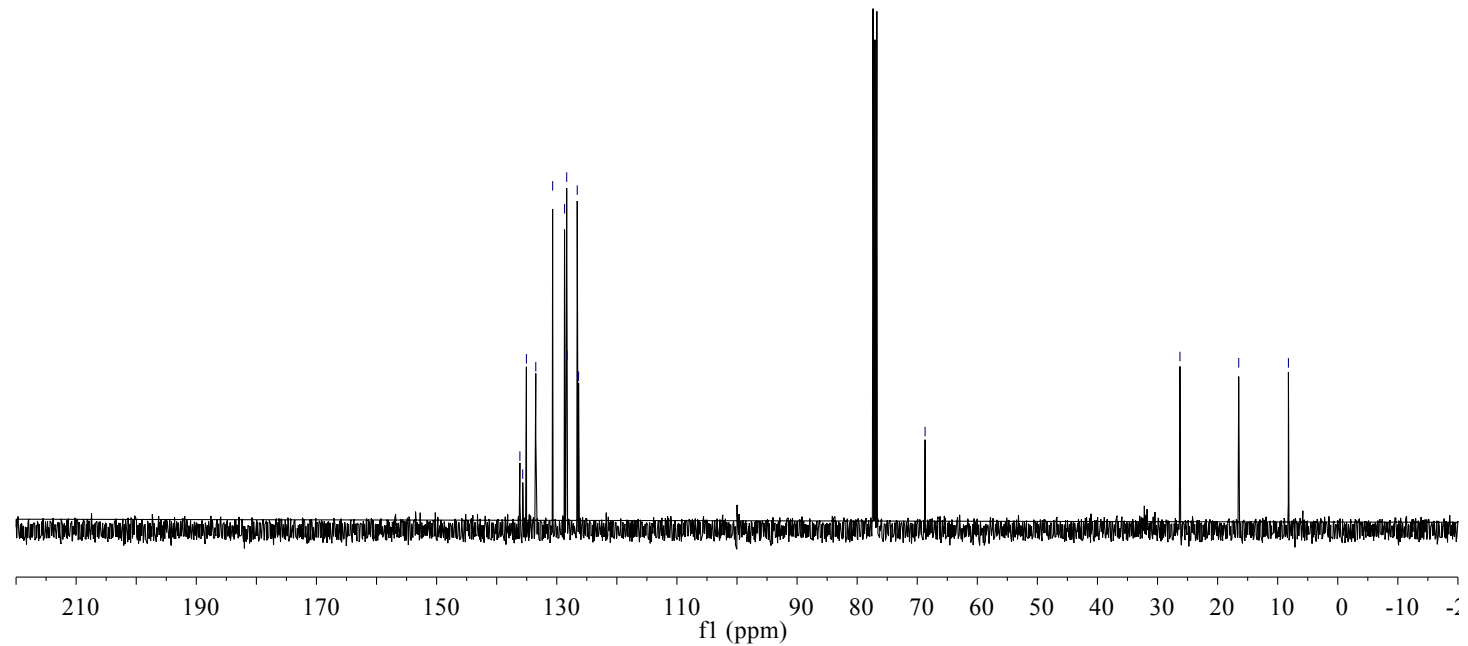


<smiles>COc1ccc(/C=C/[C@H](Cc2ccc(C(F)(F)F)cc2)[SnH+](/C=C/c2ccc(OC)cc2)C(/C=C/c2ccc(C(F)(F)F)cc2)c2ccccc2)cc1</smiles>

$3 w a+3 x a$

2.8:1 rr

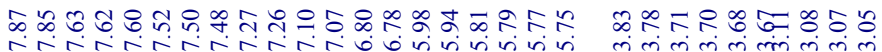

rrarra

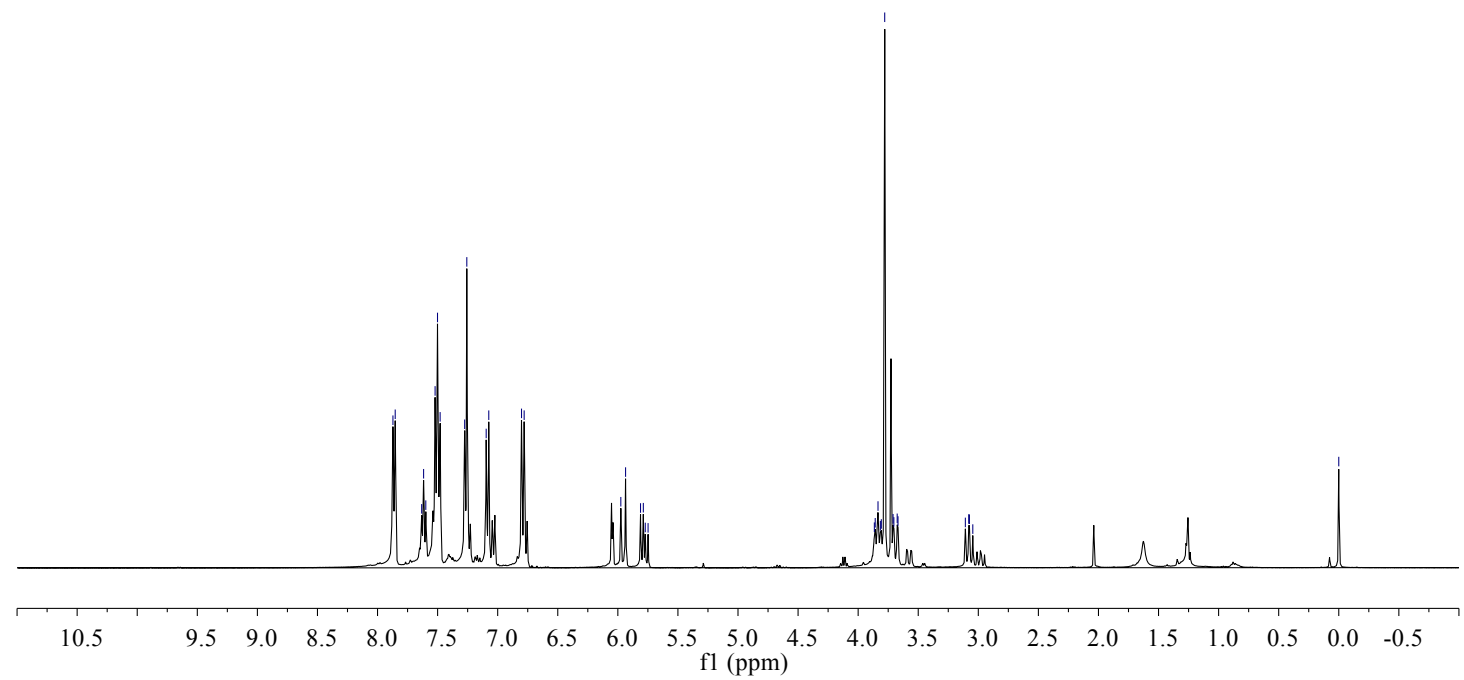

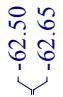

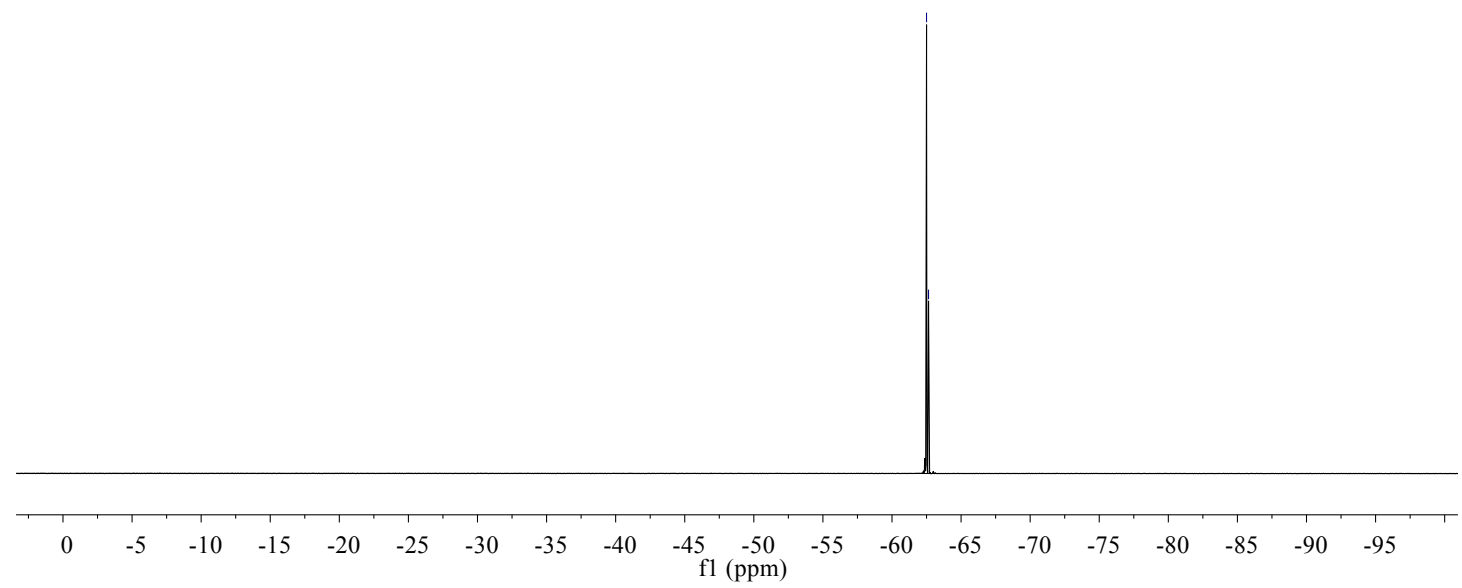


<smiles>CCC(/C=C/c1ccccc1)S(=O)(=O)c1ccc(Cl)cc1</smiles>

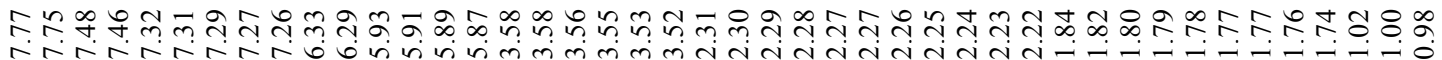
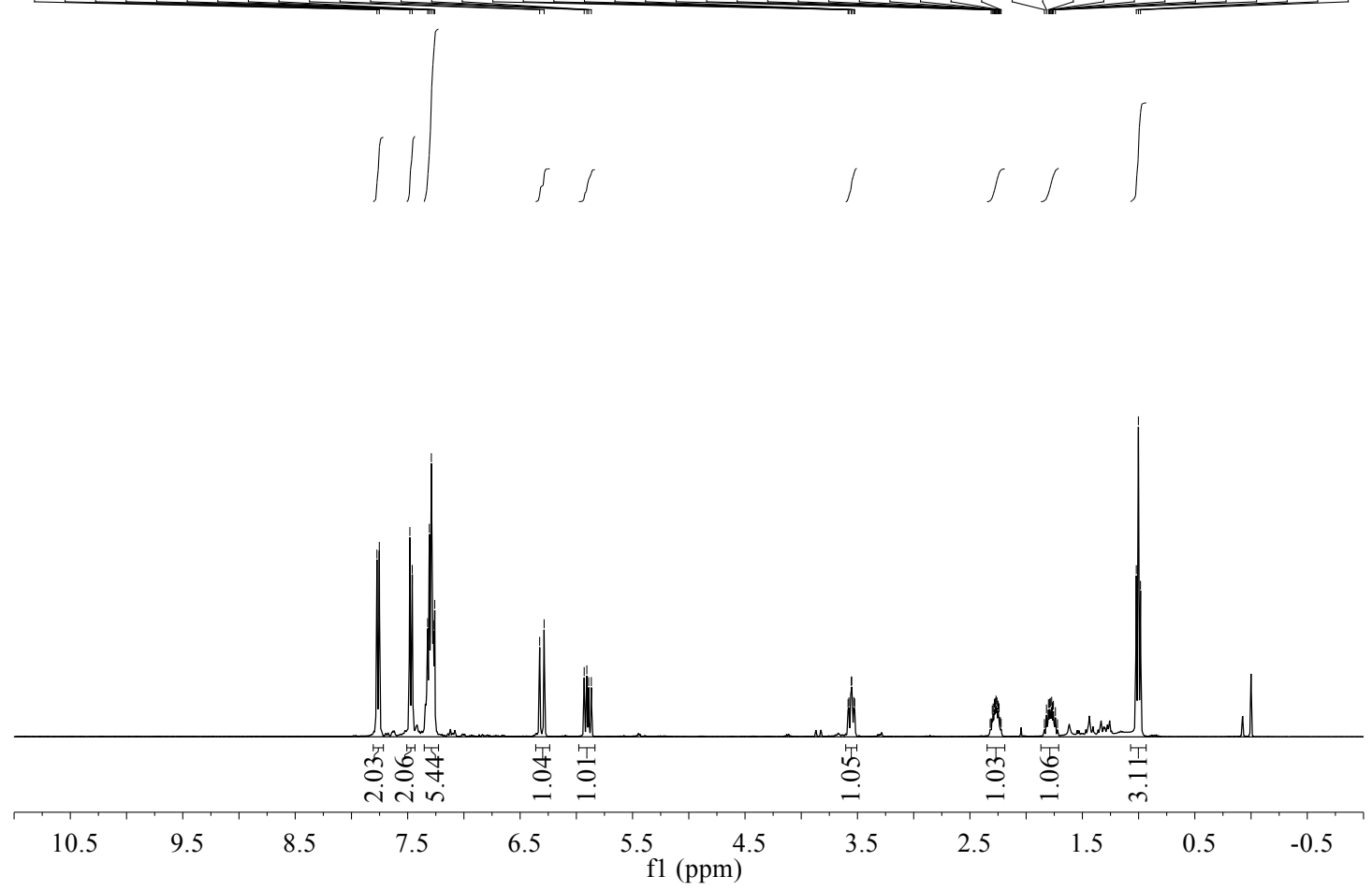

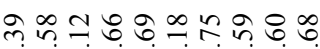

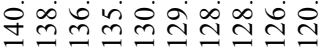

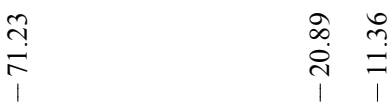

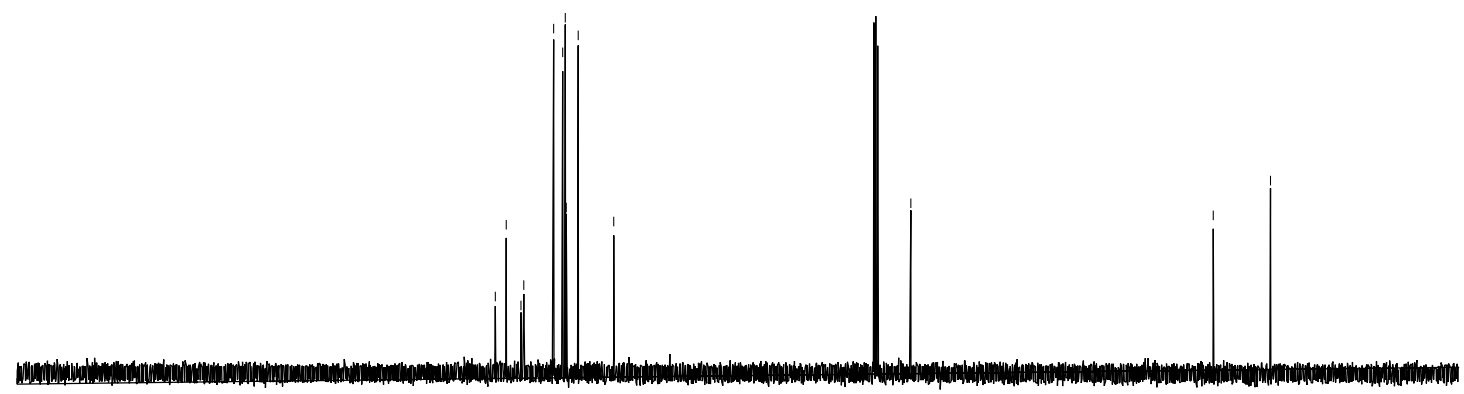

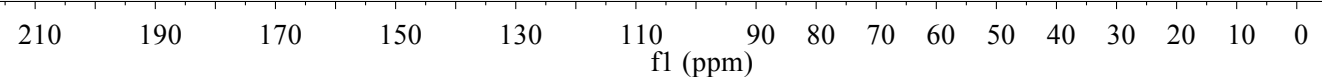


<smiles>CCC(/C=C/c1ccccc1)S(=O)(=O)c1ccc(C)cc1</smiles>

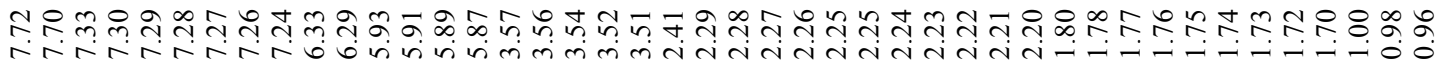
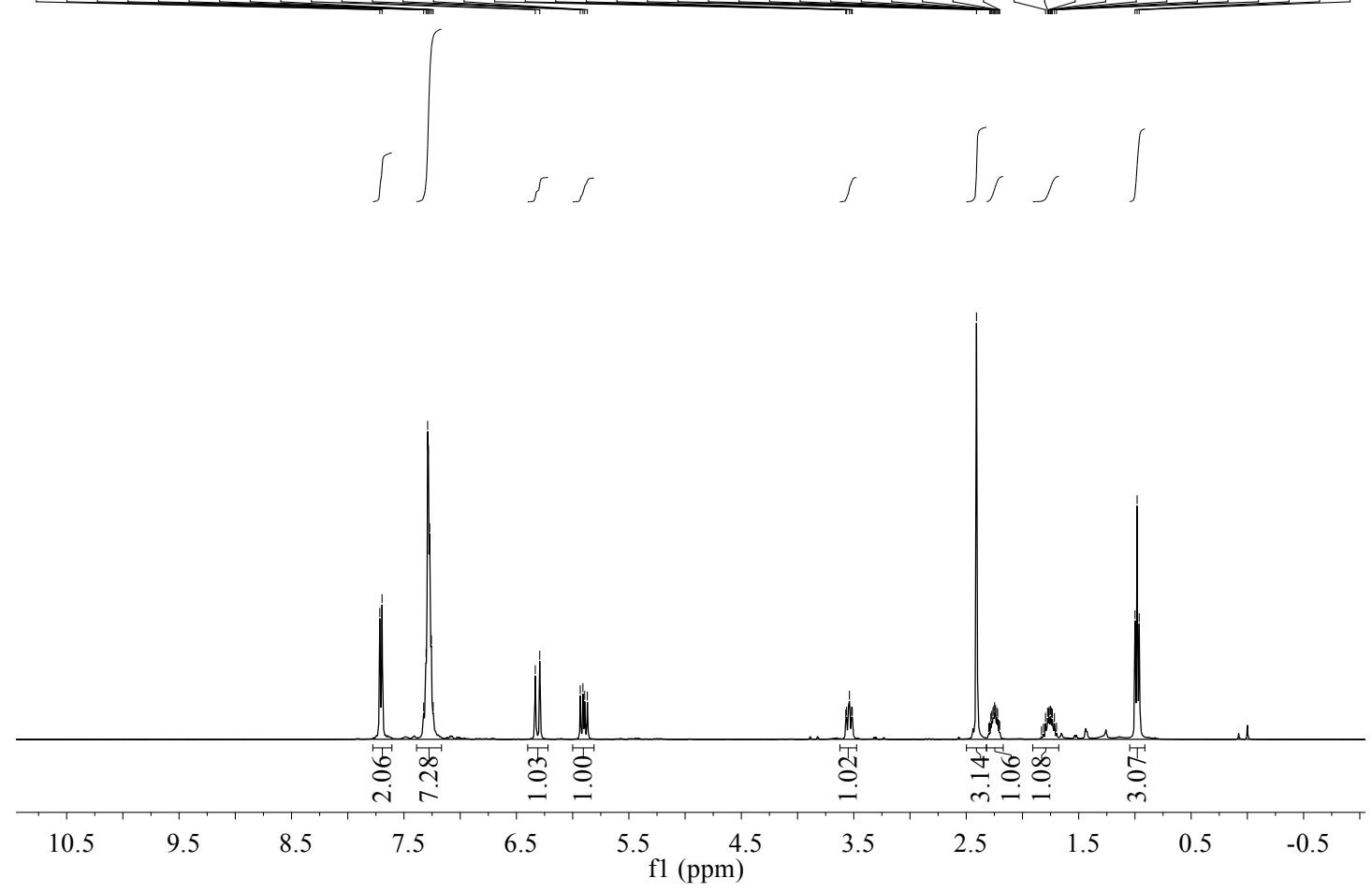

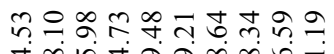

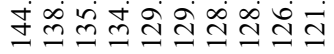

水

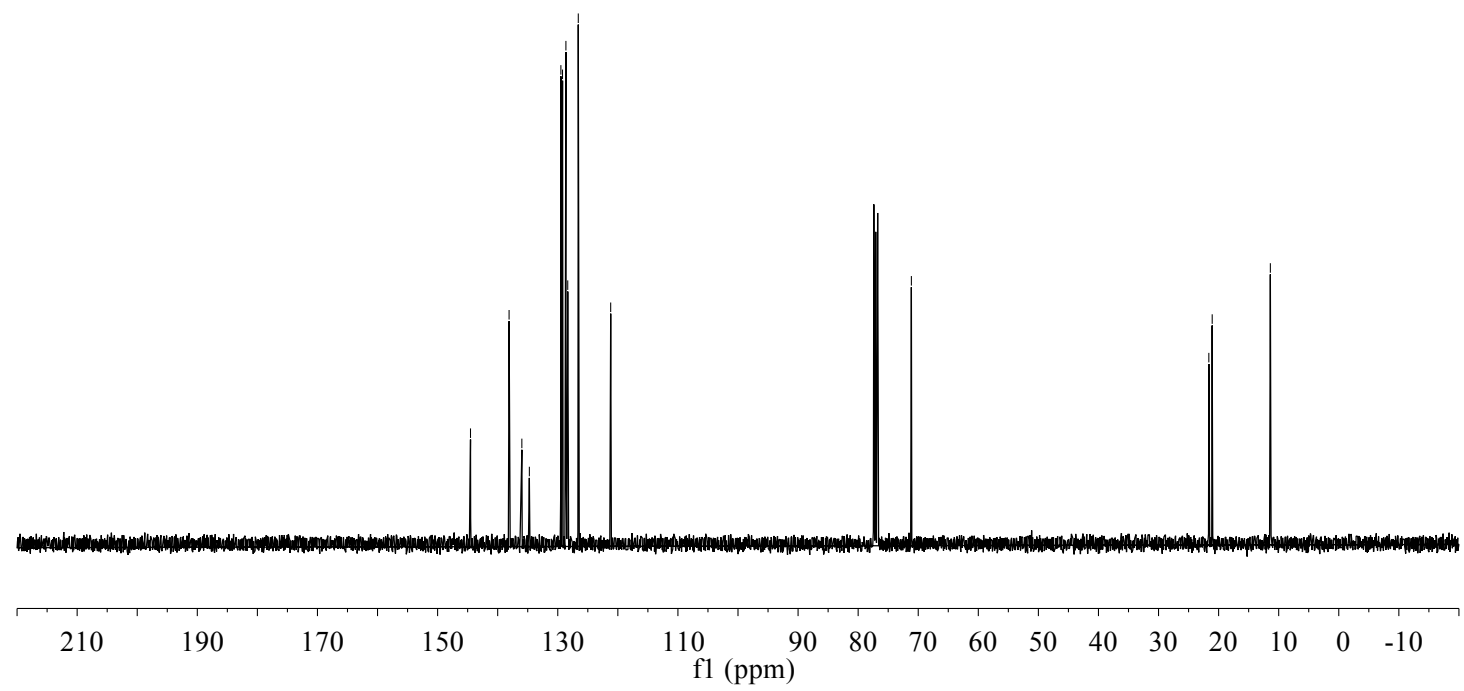




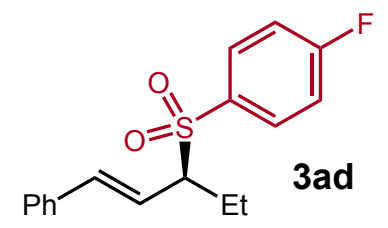

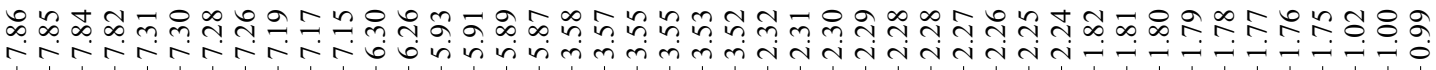
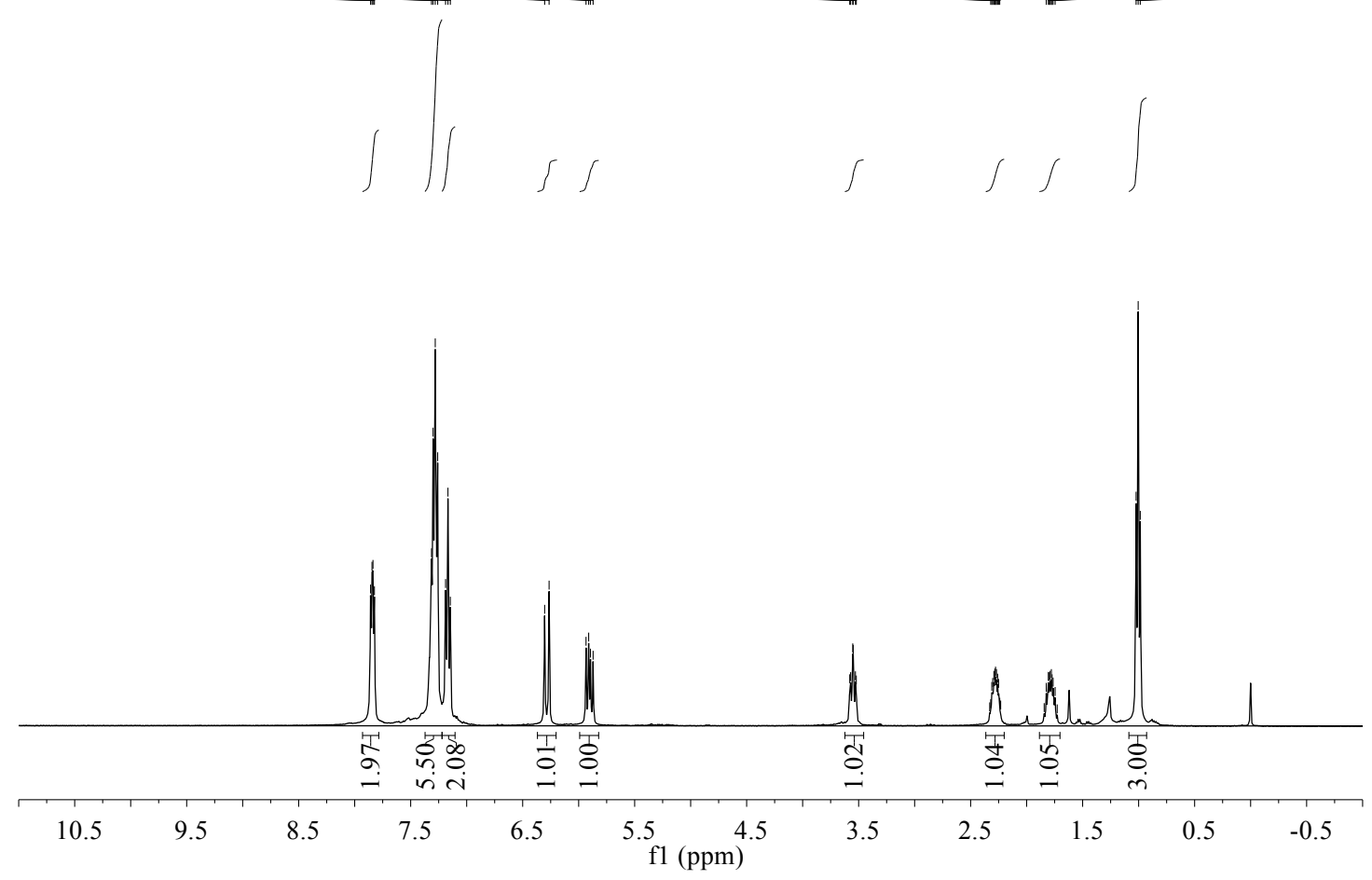

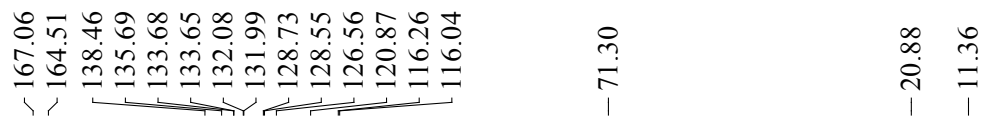

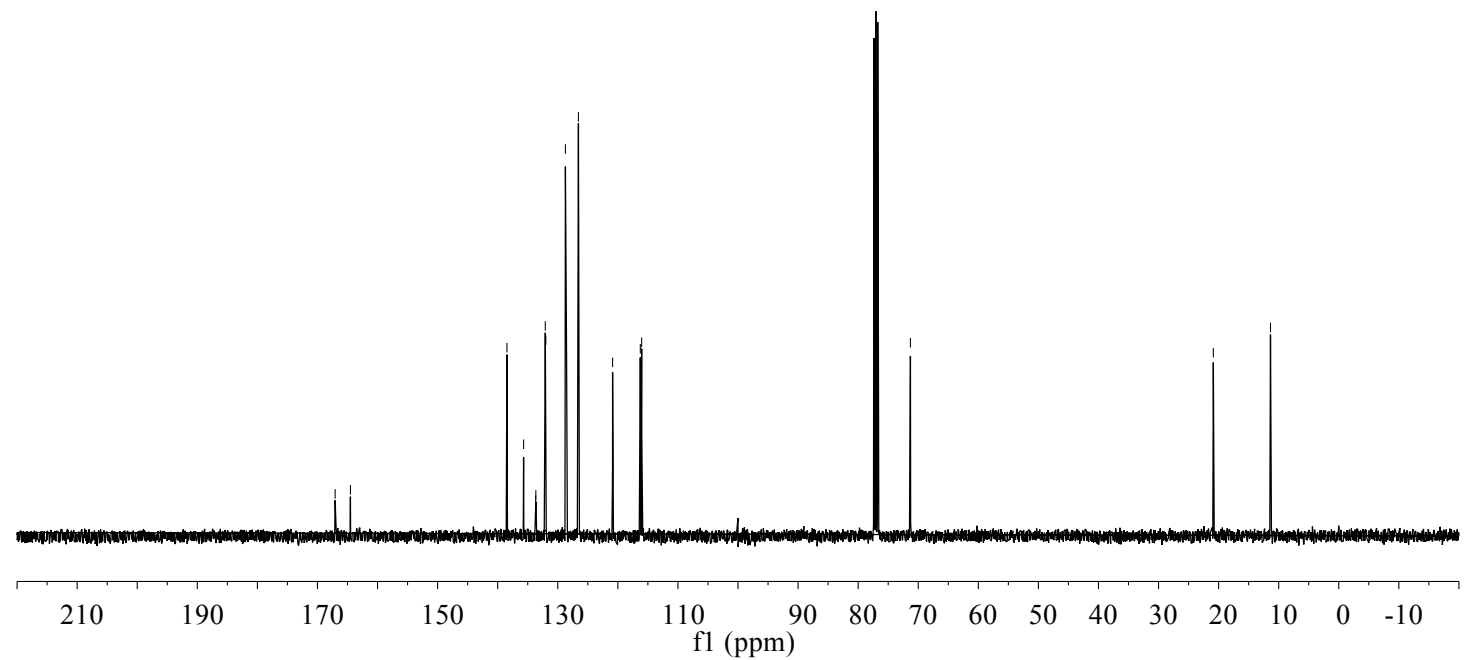



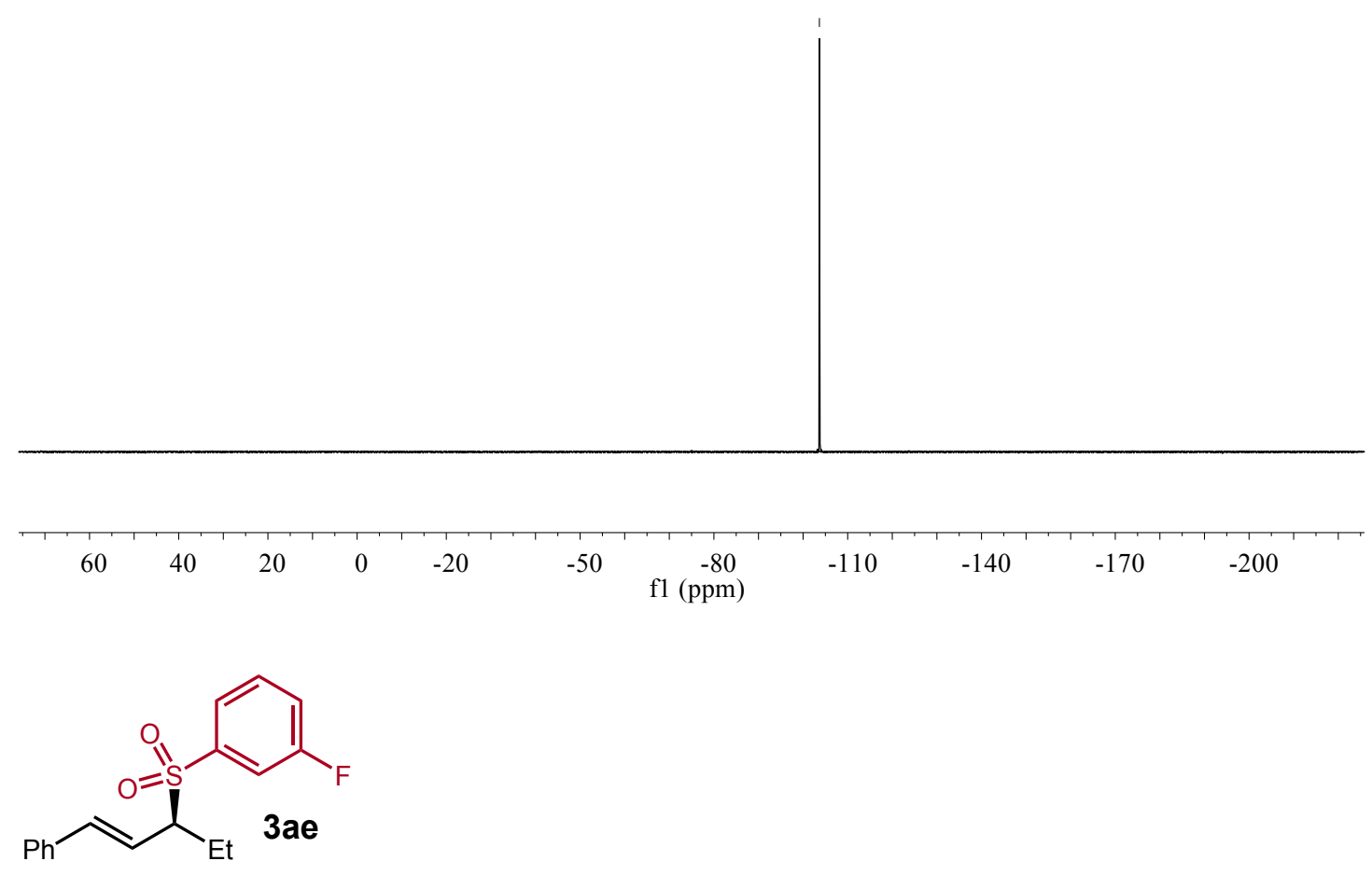

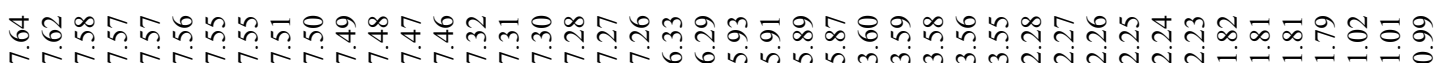

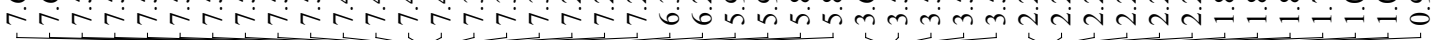
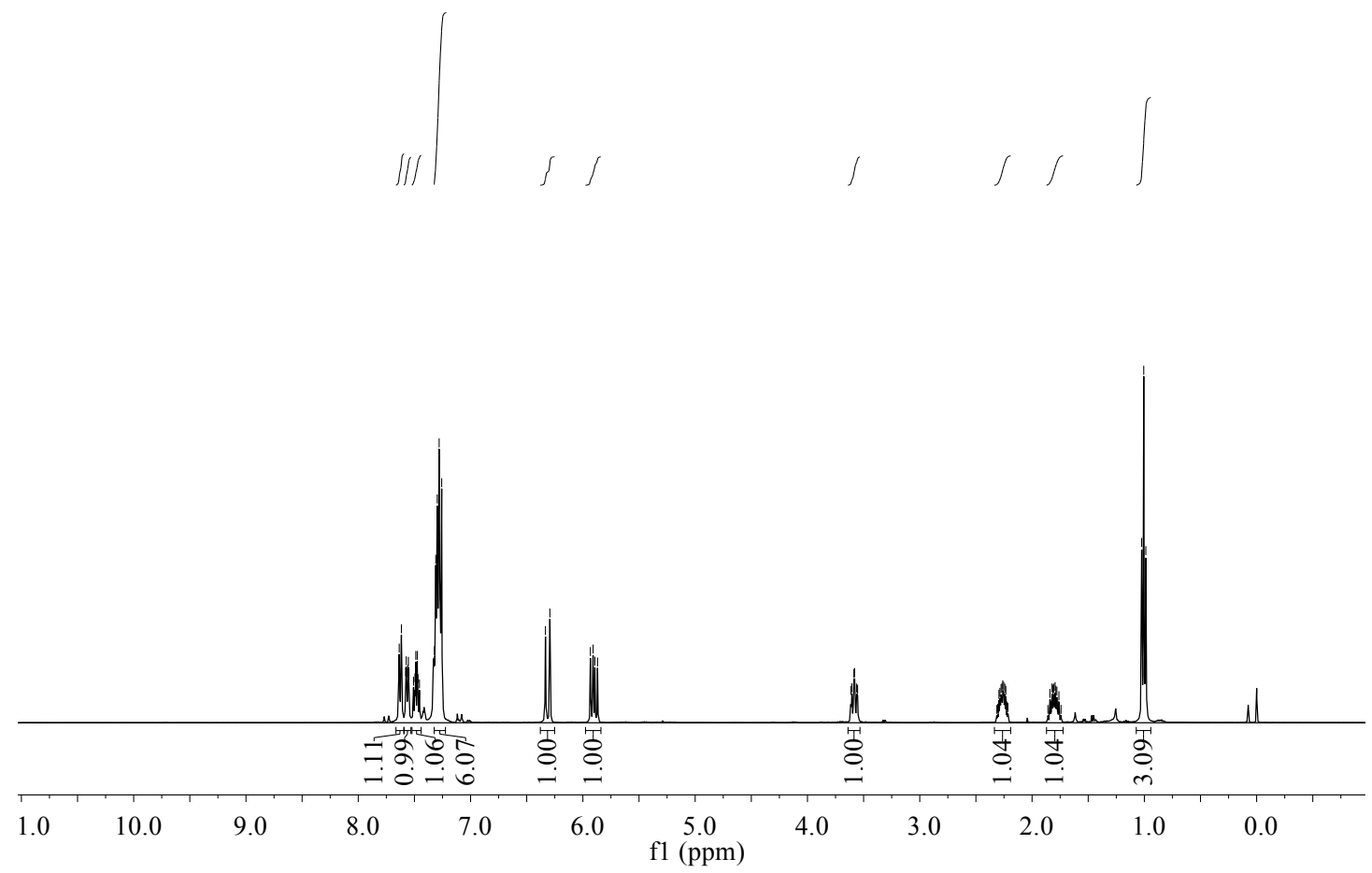

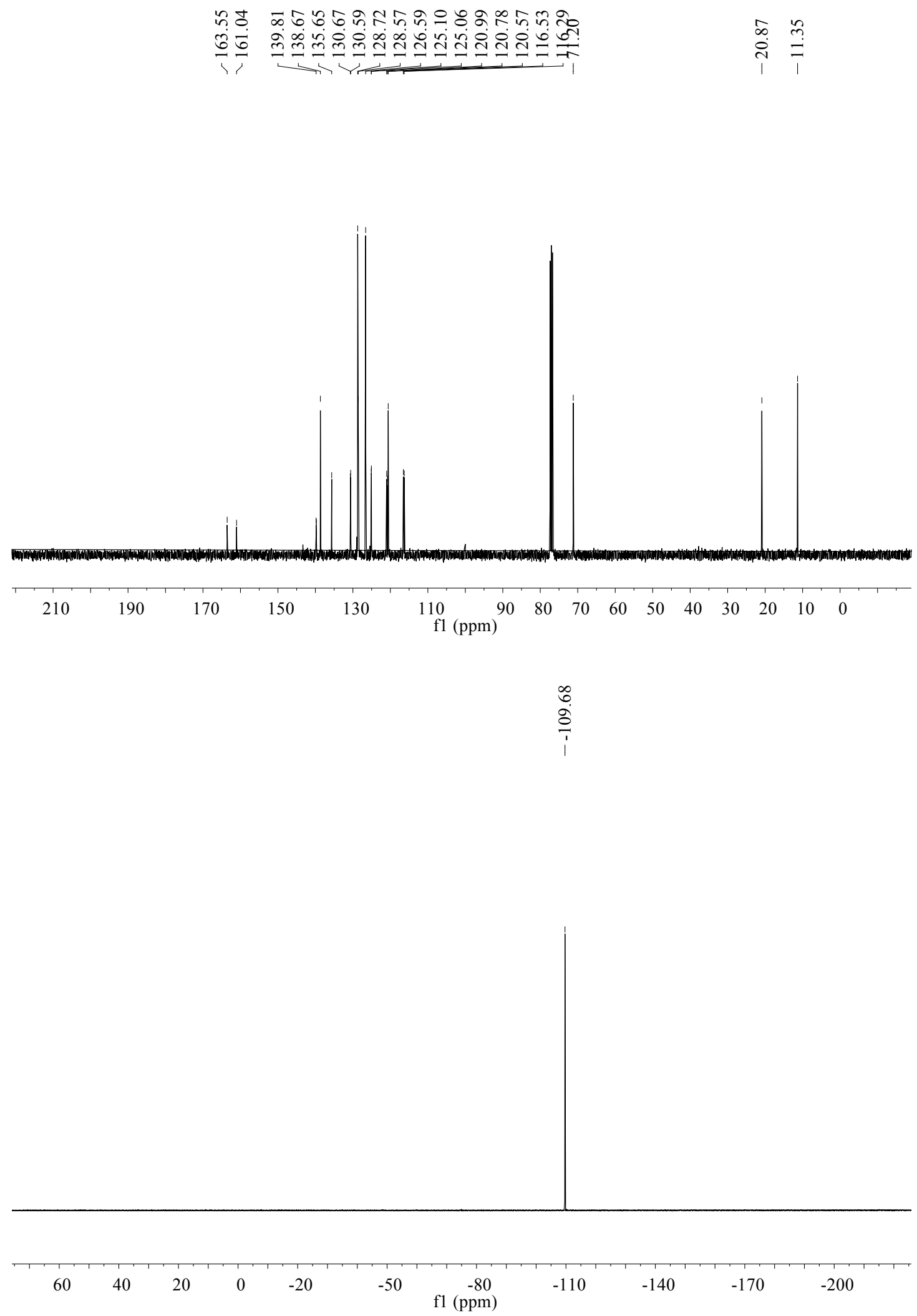
<smiles>O=[10B][C@H](/C=C/c1ccccc1)S(=O)(=O)c1ccccc1F</smiles>

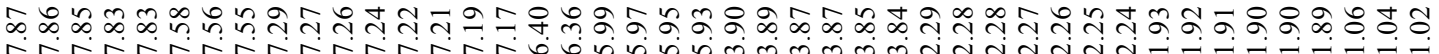

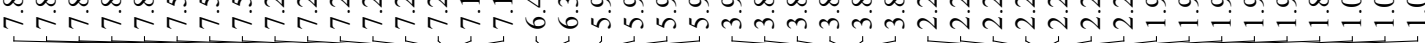
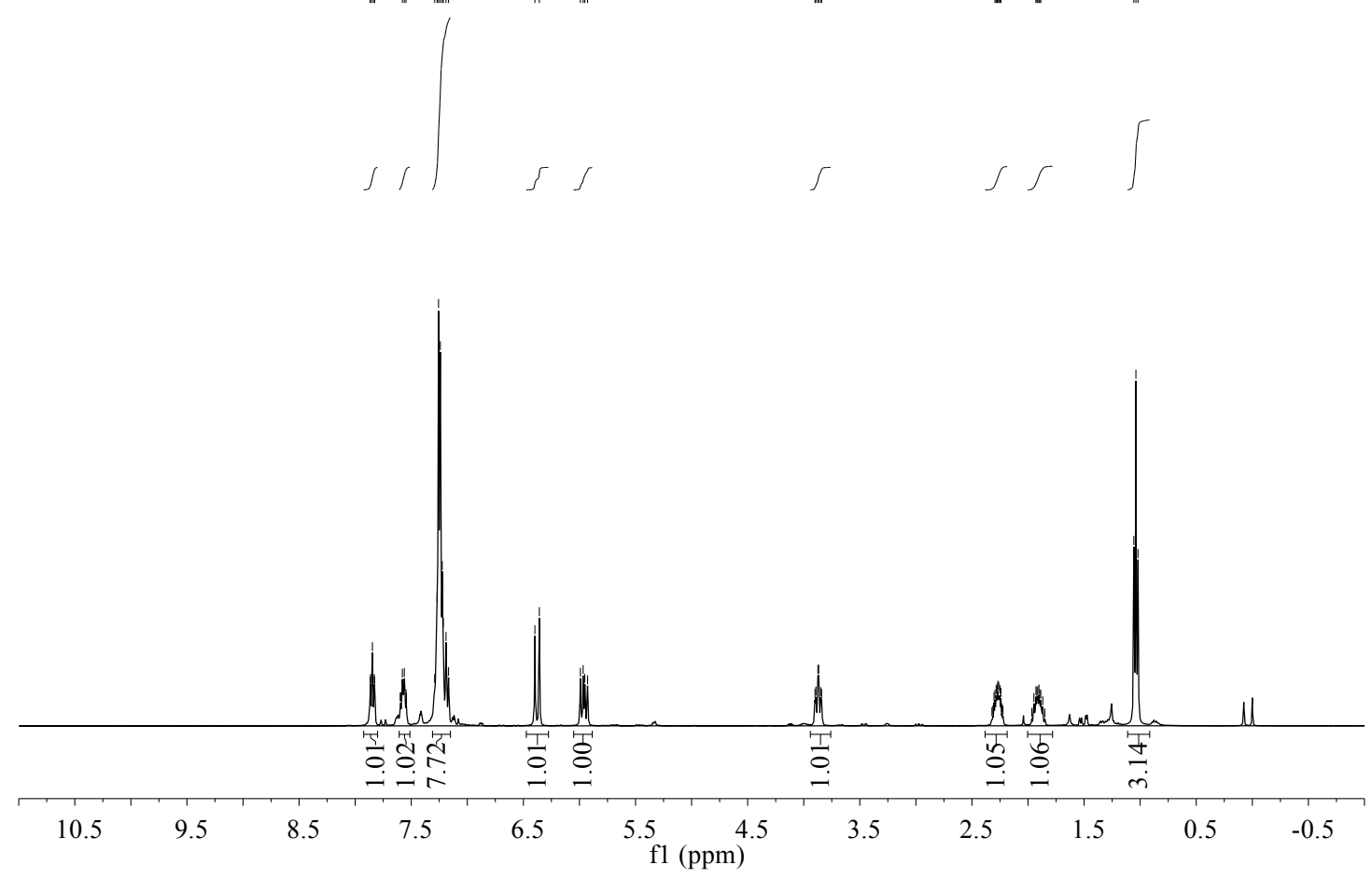

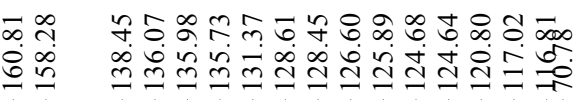

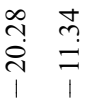

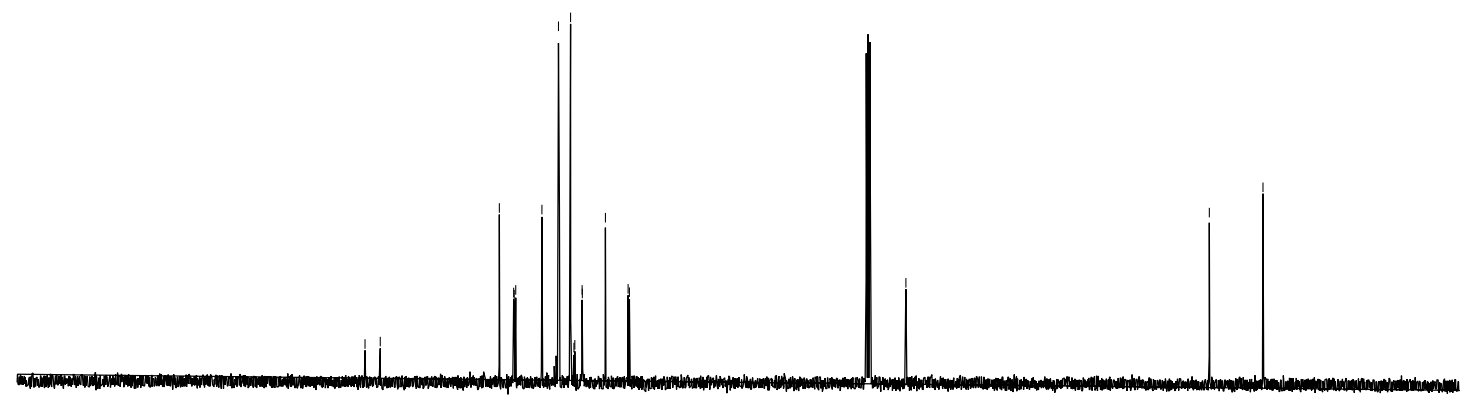

$\begin{array}{lllllllllllllllll}210 & 190 & 170 & 150 & 130 & \begin{array}{c}110 \\ \mathrm{f} 1(\mathrm{ppm})\end{array} & 80 & 70 & 60 & 50 & 40 & 30 & 20 & 10 & 0 & -10\end{array}$ 

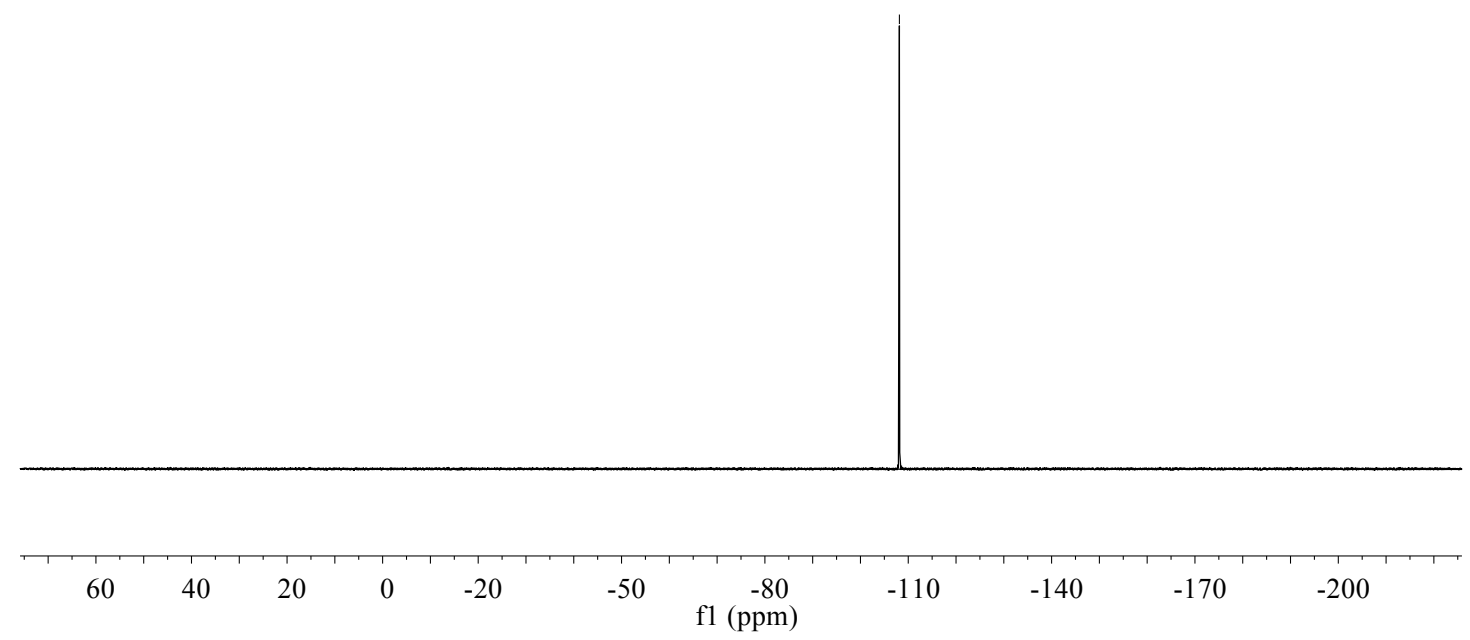<smiles>CC(/C=C/c1ccccc1)S(=O)(=O)c1ccc(C(F)(F)F)cc1</smiles>

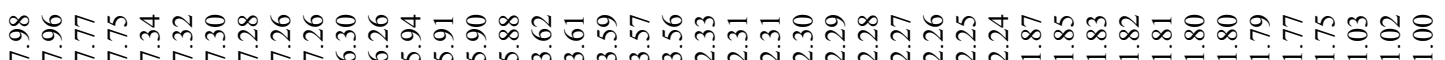

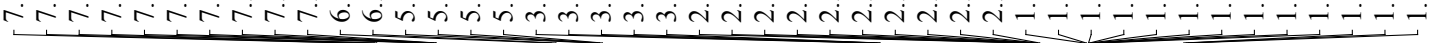
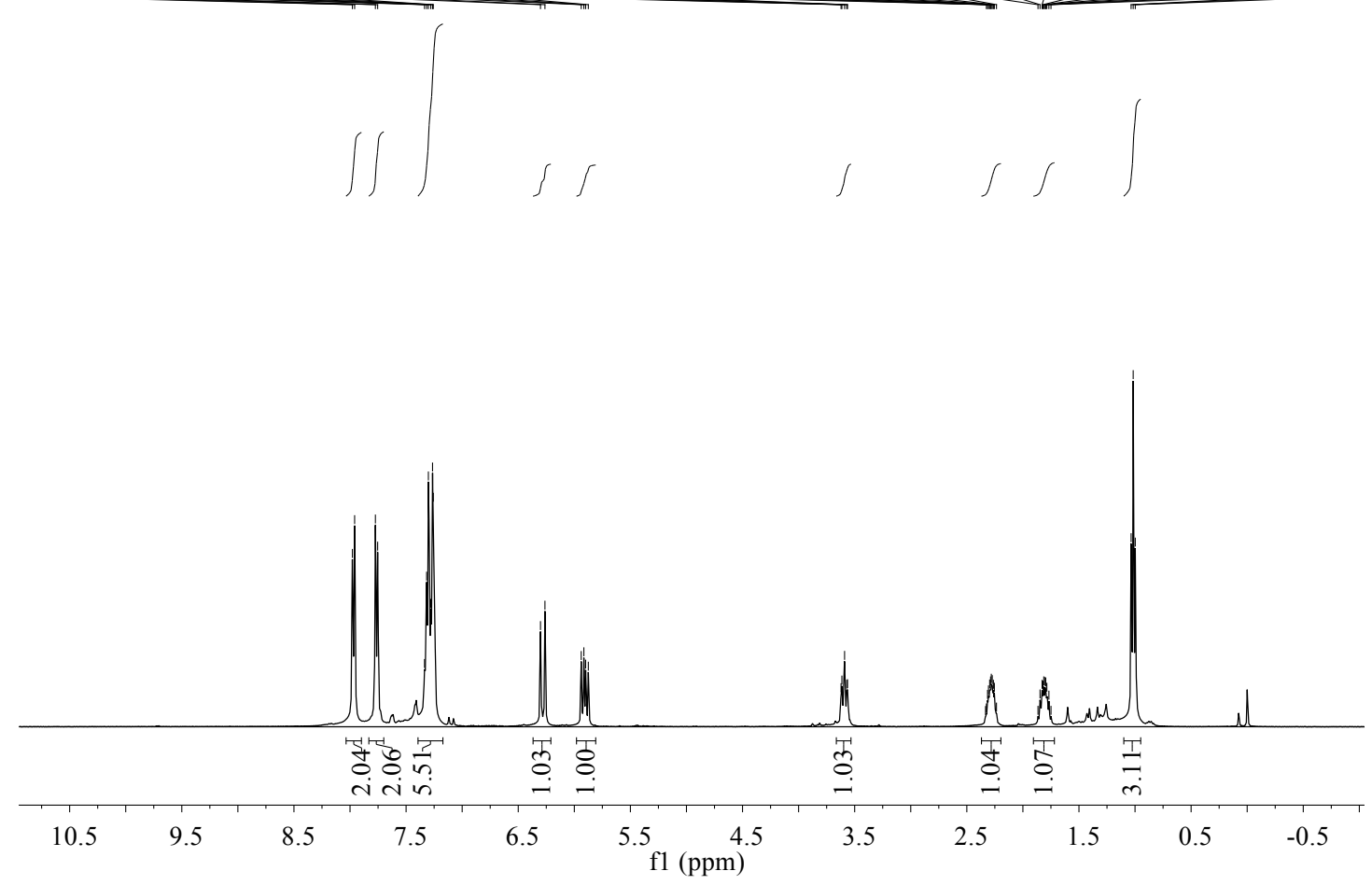


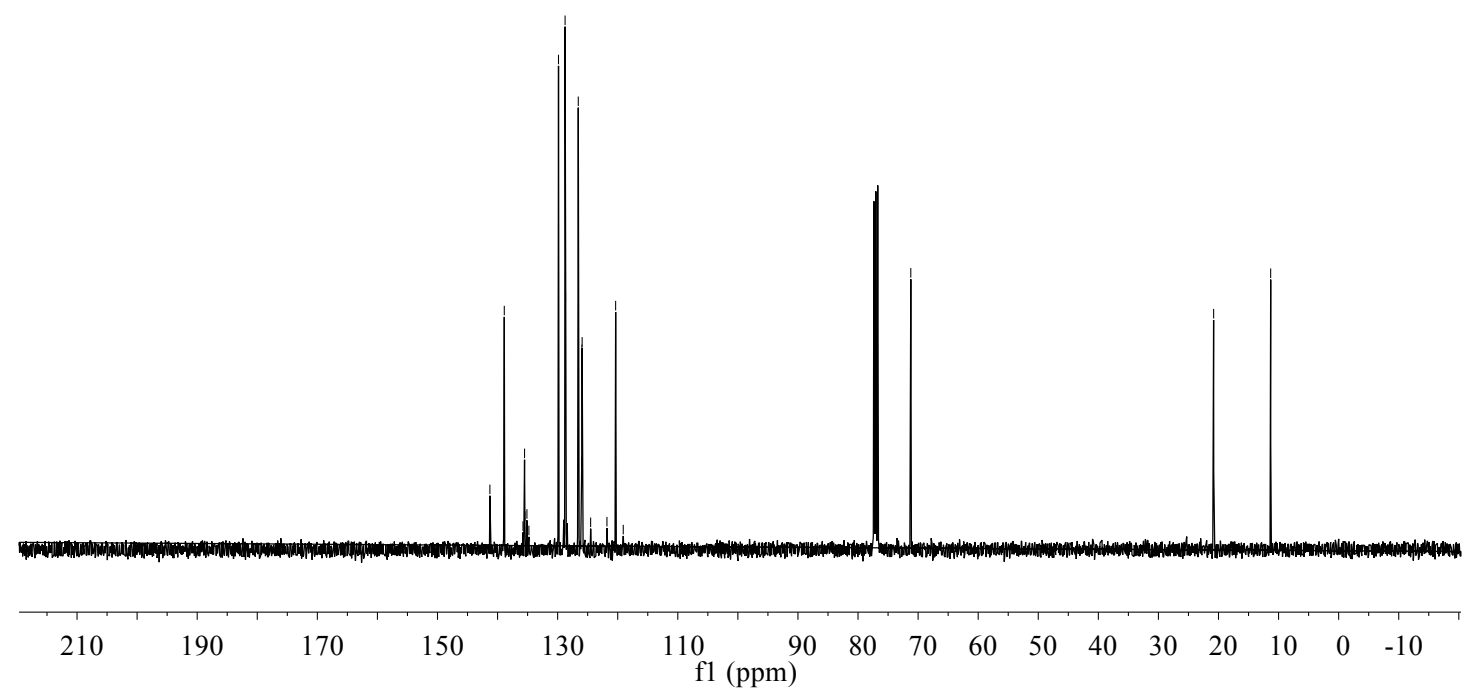

\pm
लூ
1

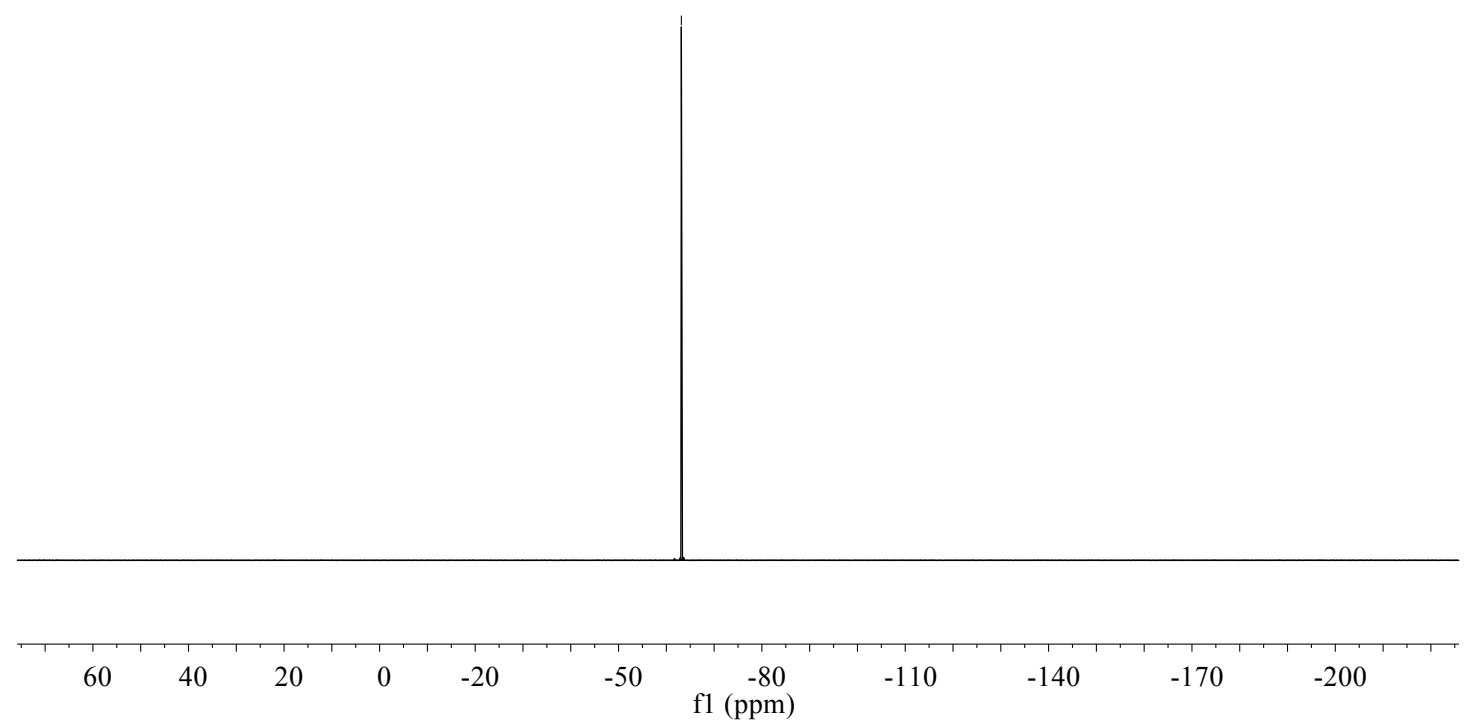


<smiles>CC(/C=C/c1ccccc1)S(=O)(=O)c1ccc(C#N)cc1</smiles>

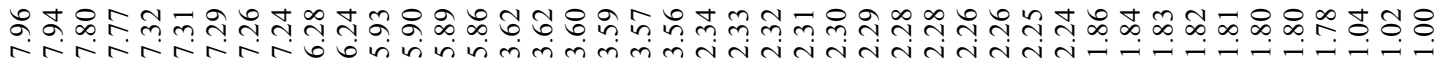

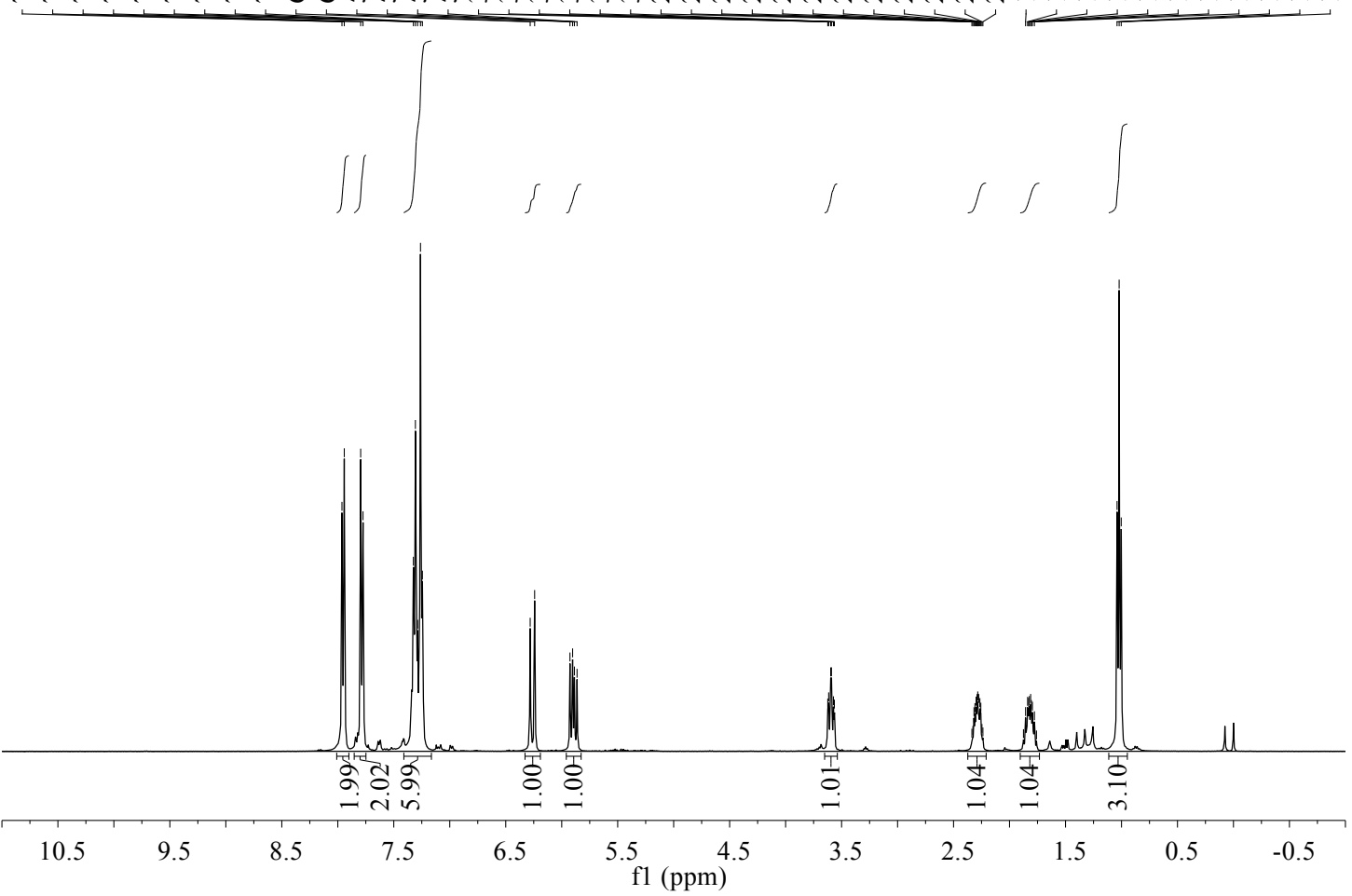

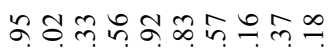

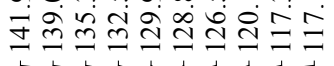

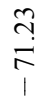

$\stackrel{\overrightarrow{0}}{\stackrel{m}{*}}$

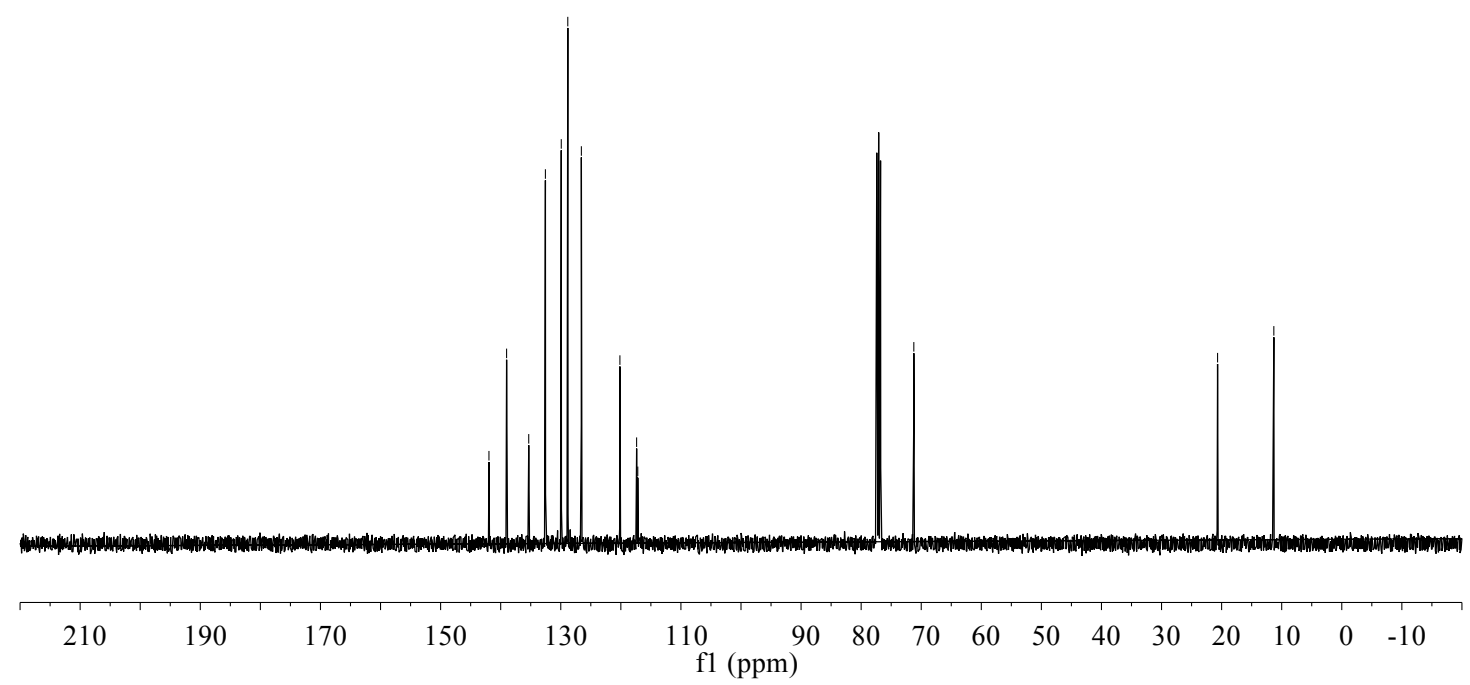


<smiles>CCC(/C=C/c1ccccc1)S(=O)(=O)c1cc(C(F)(F)F)cc(C(F)(F)F)c1</smiles>
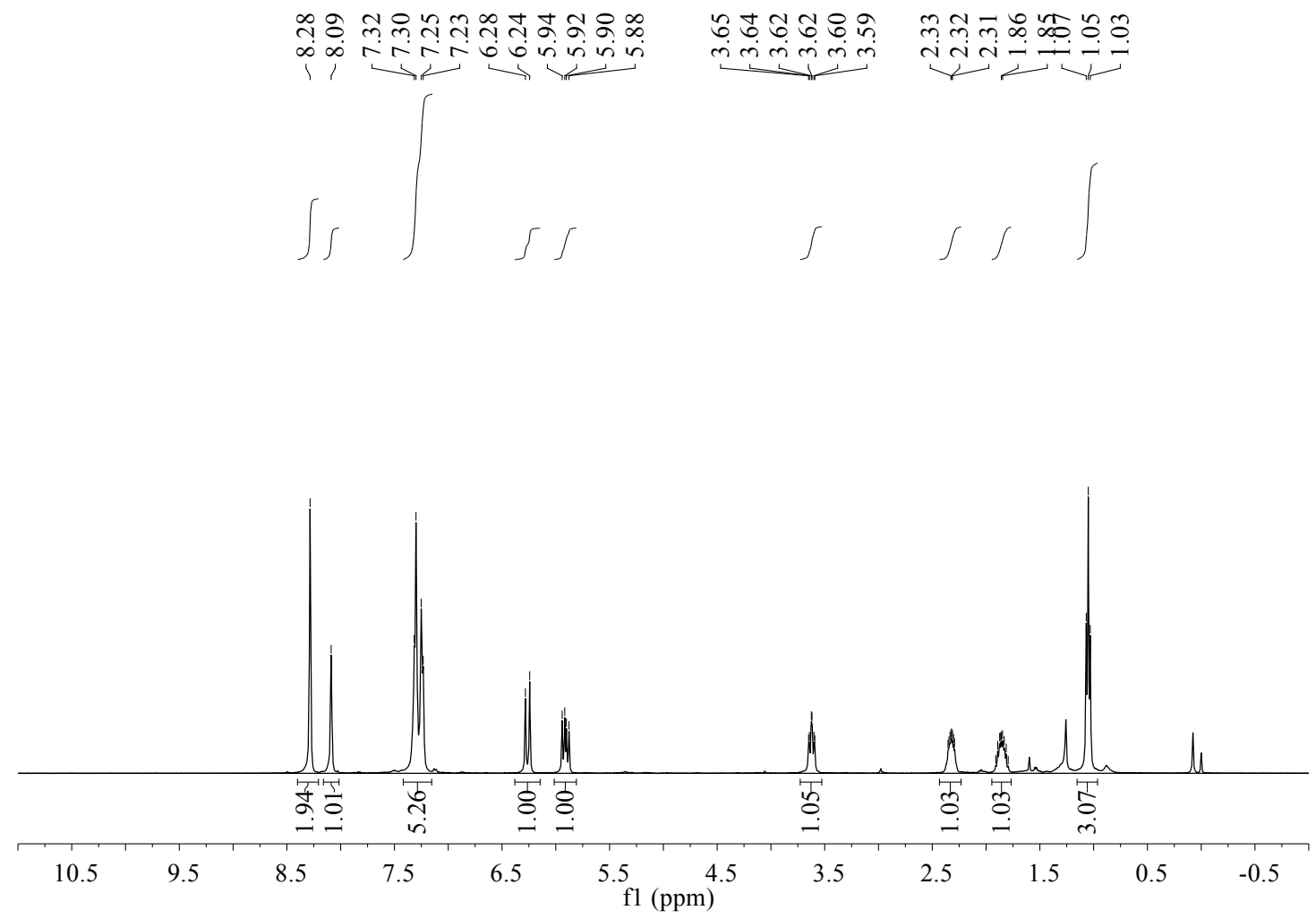

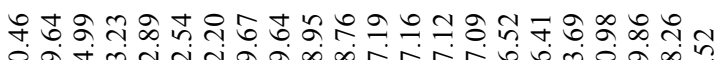

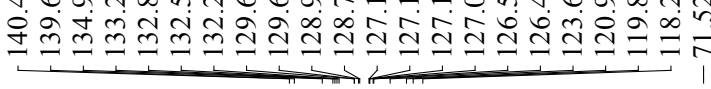

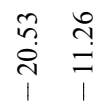

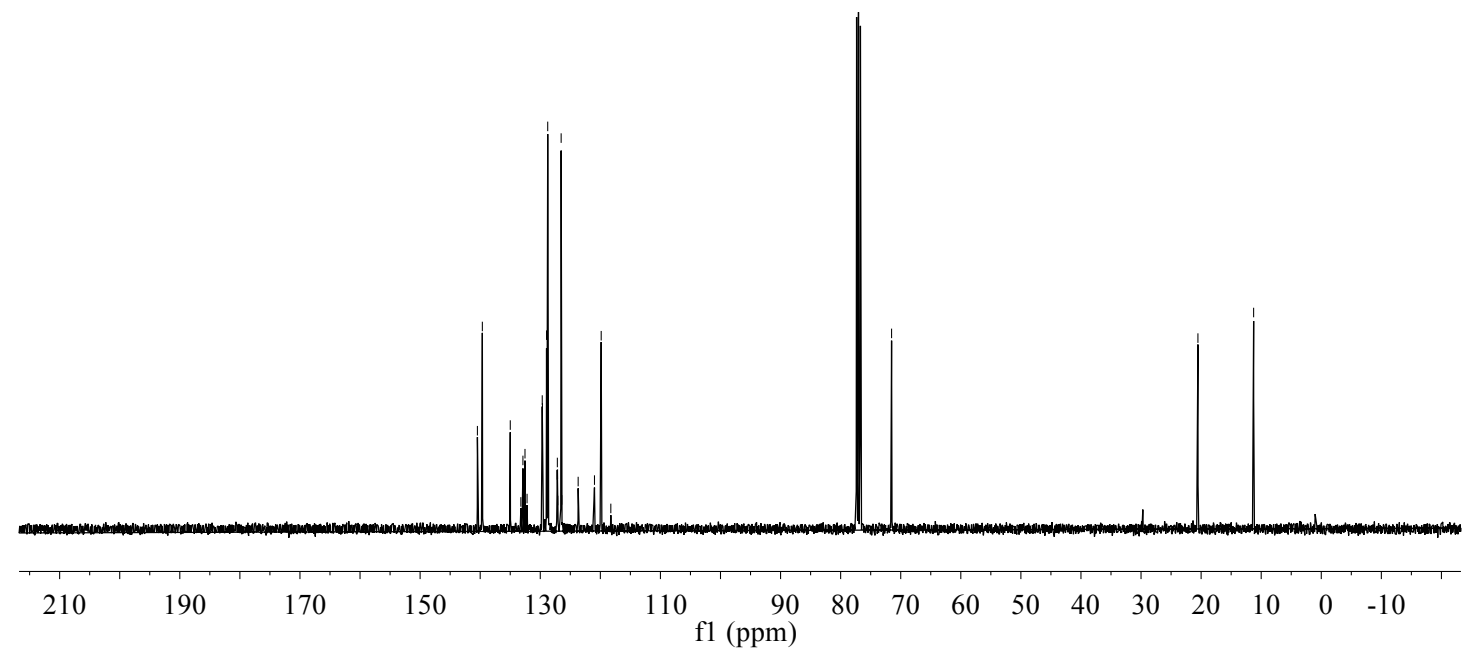



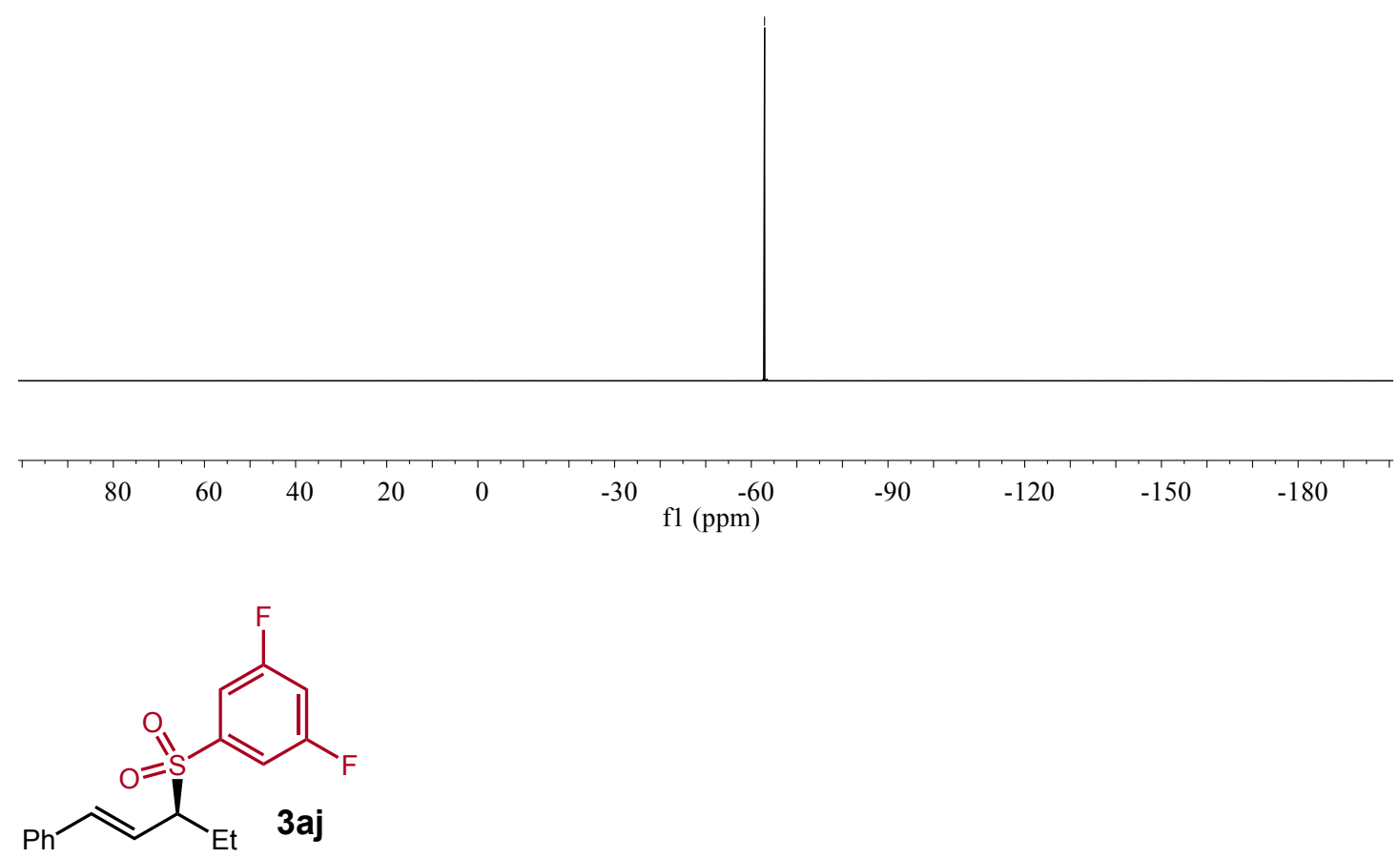

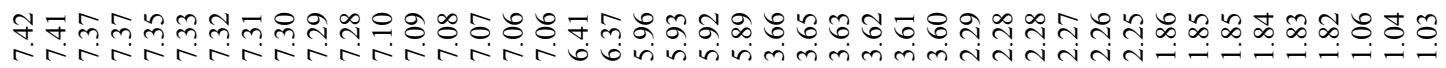
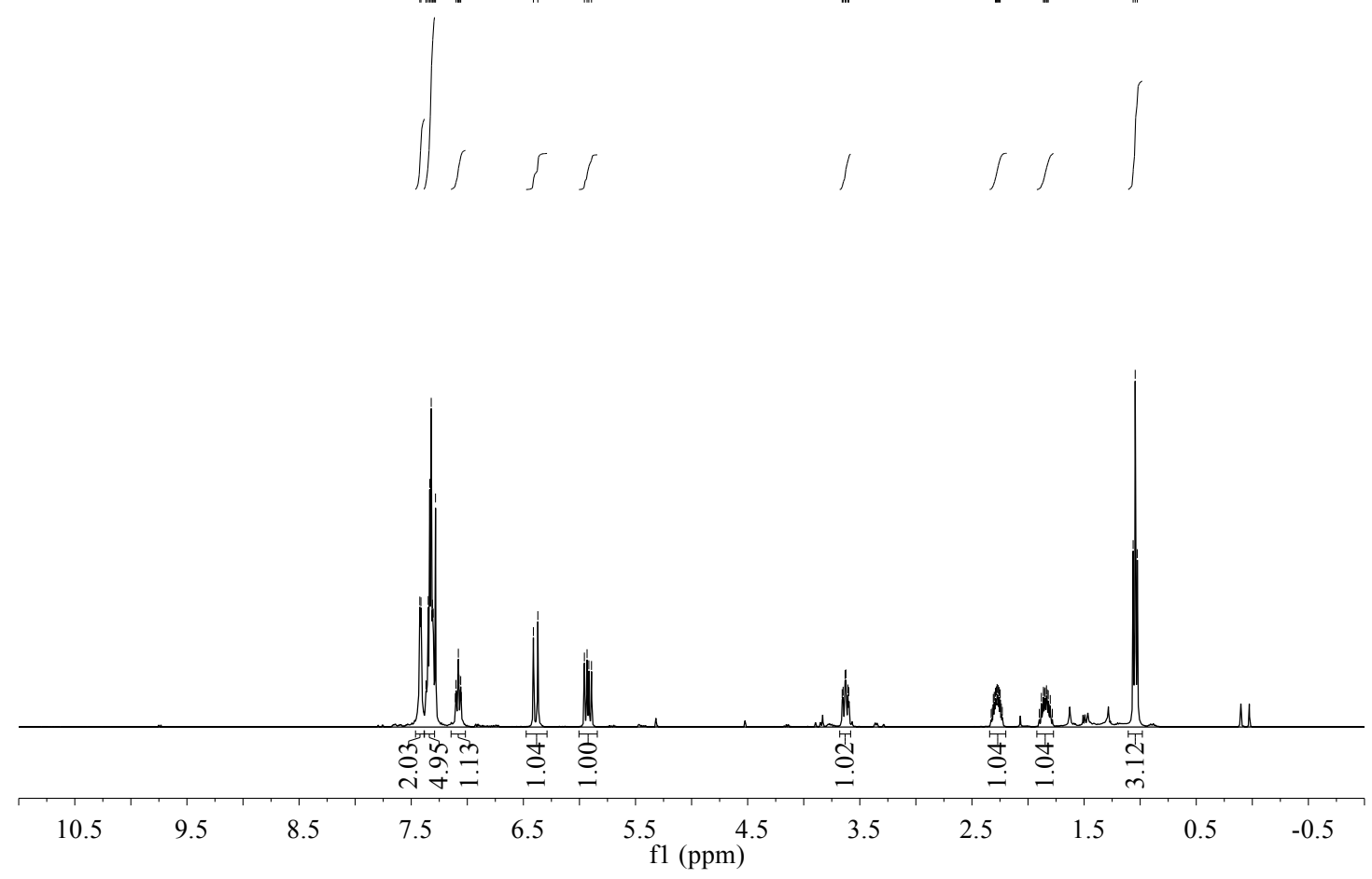


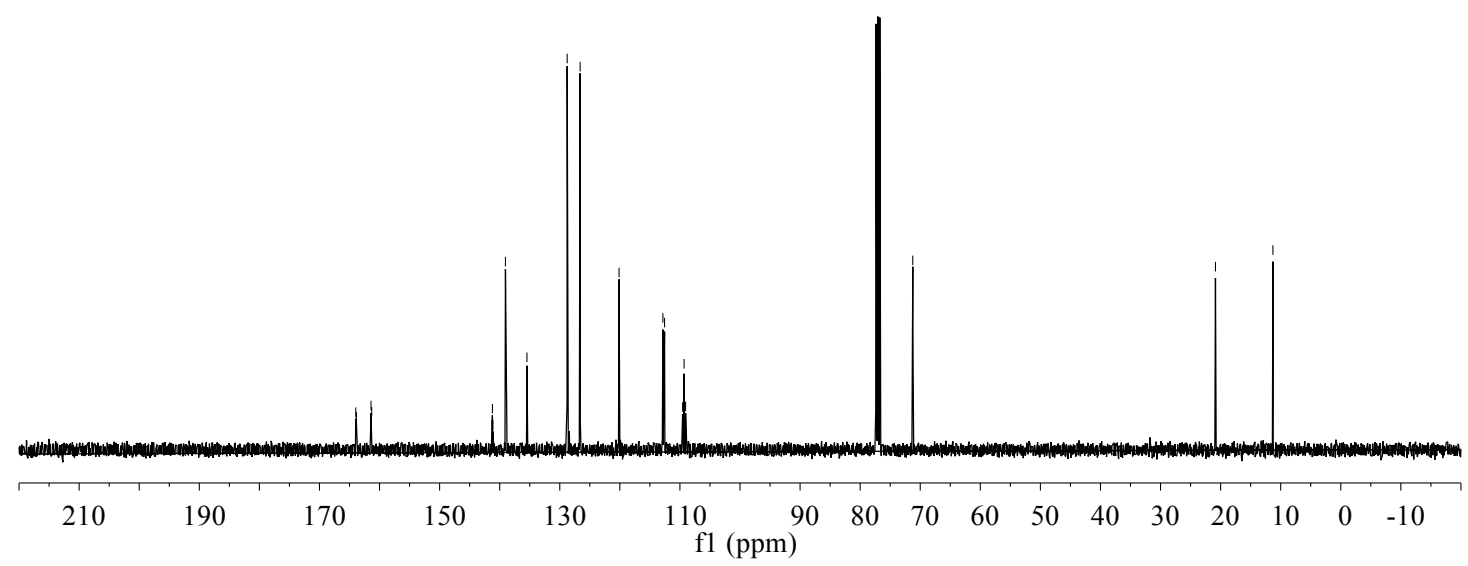

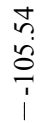

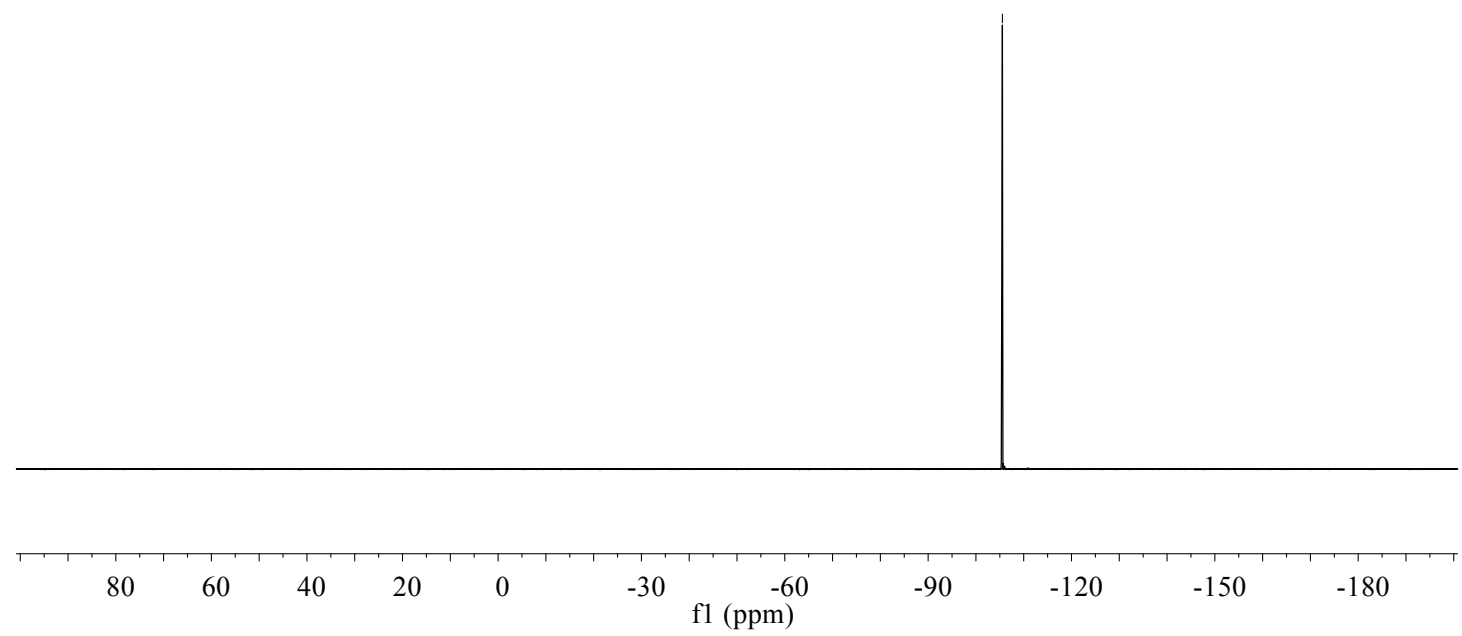




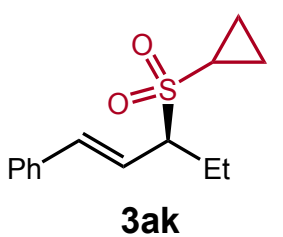

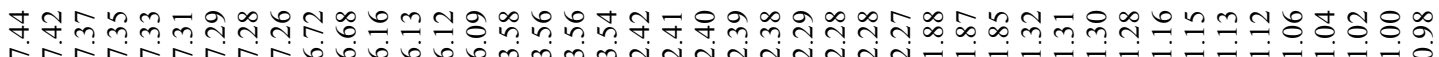

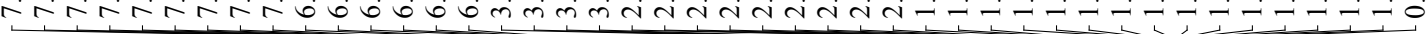
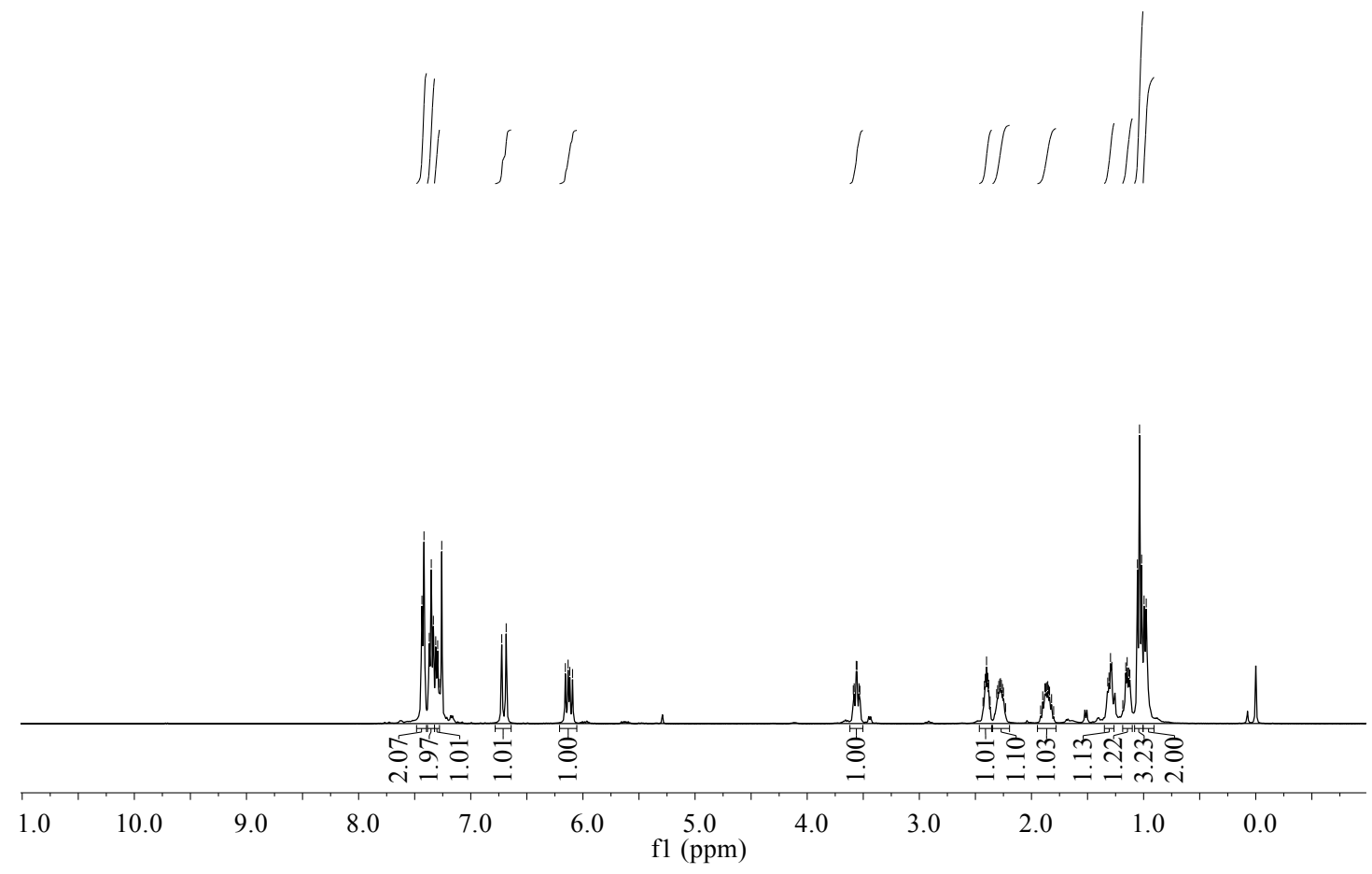

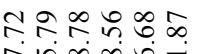

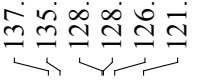
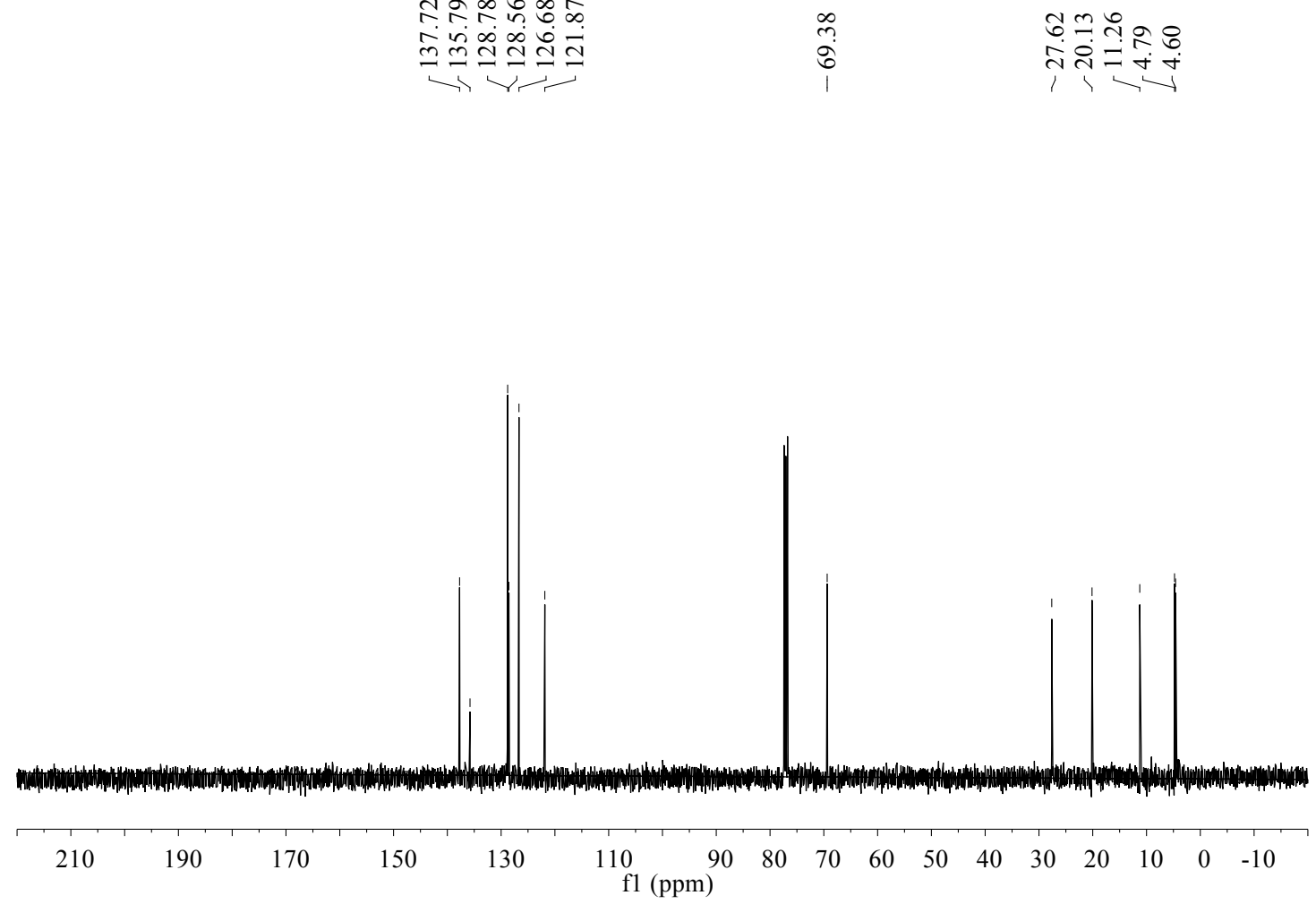
<smiles>CCC(/C=C/c1ccccc1)S(=O)(=O)C(C)C</smiles>

3al

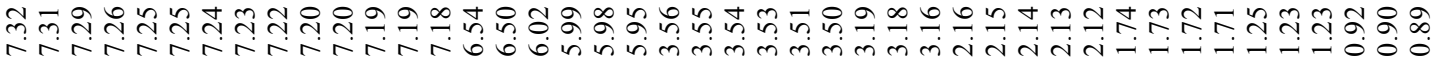

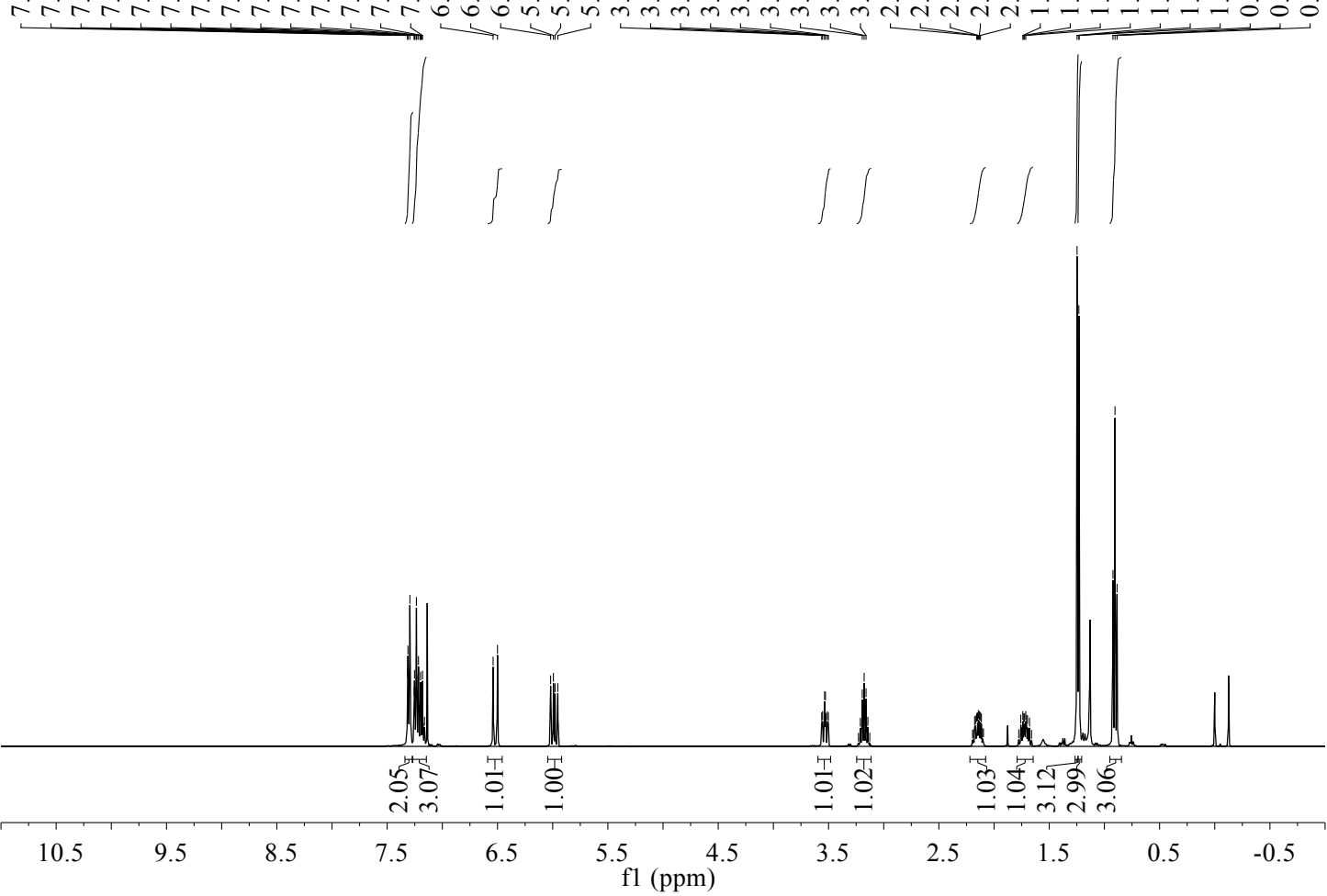

운웅요웅

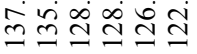

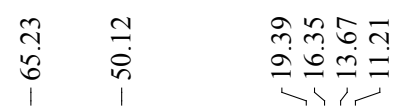

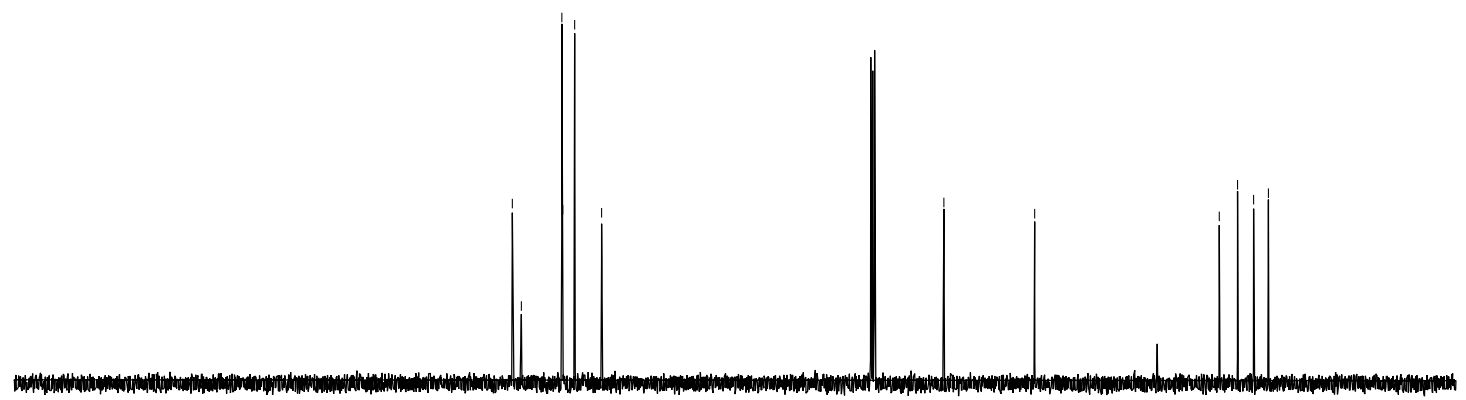

210

190

170

150

130

110 f1 (ppm)

$\begin{array}{llllllllll}80 & 70 & 60 & 50 & 40 & 30 & 20 & 10 & 0 & -10\end{array}$ 


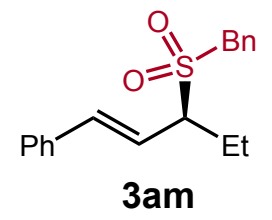

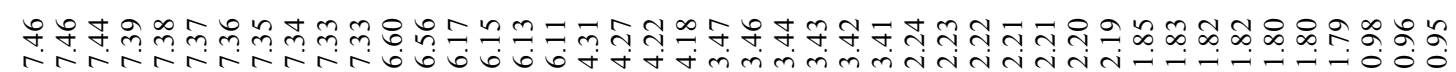

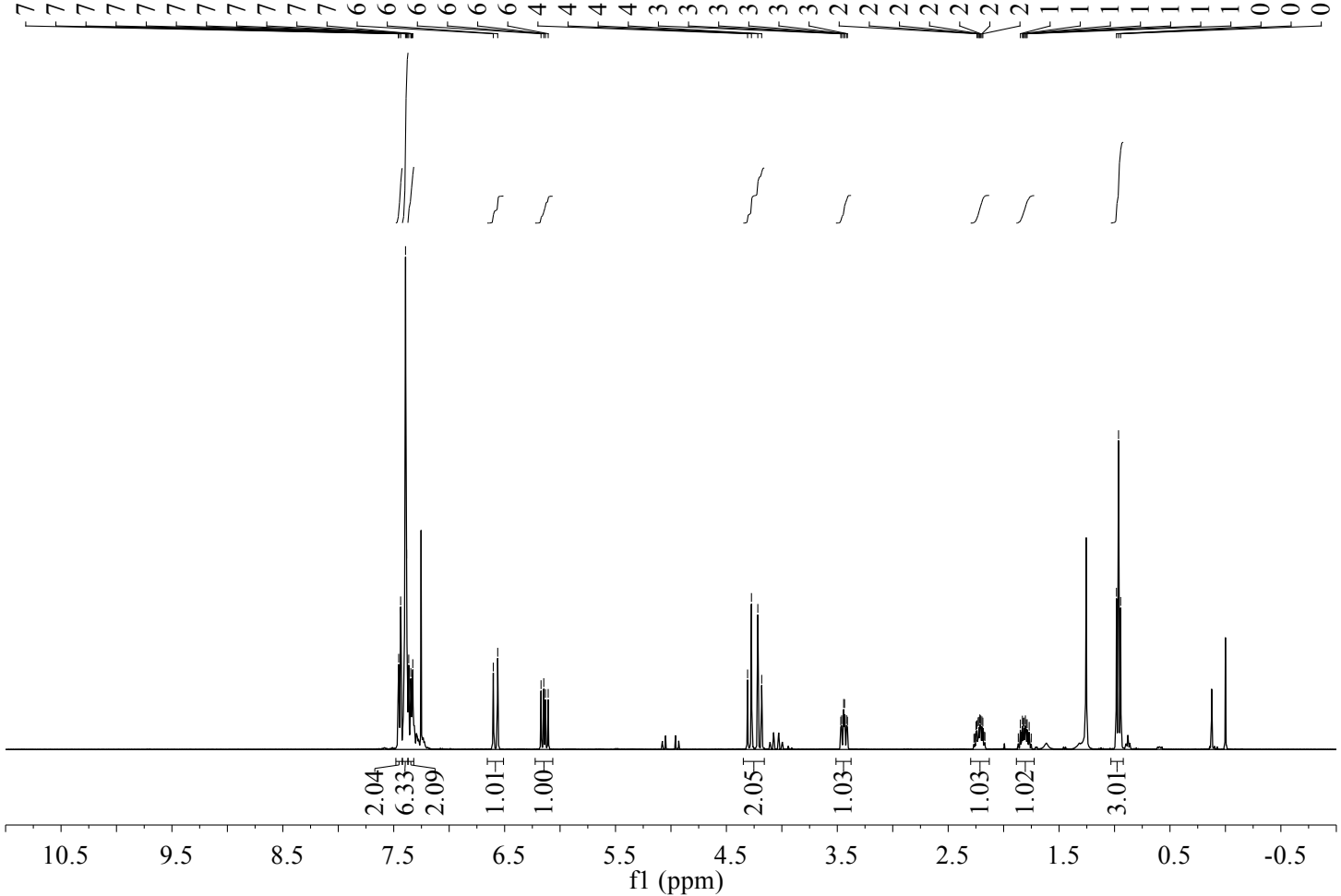

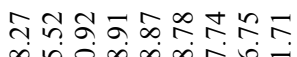

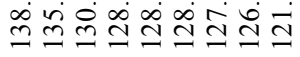

$\begin{array}{cc}\text { aे } & 0 \\ \text { ठ } & \text { in } \\ 1 & 1\end{array}$

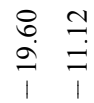

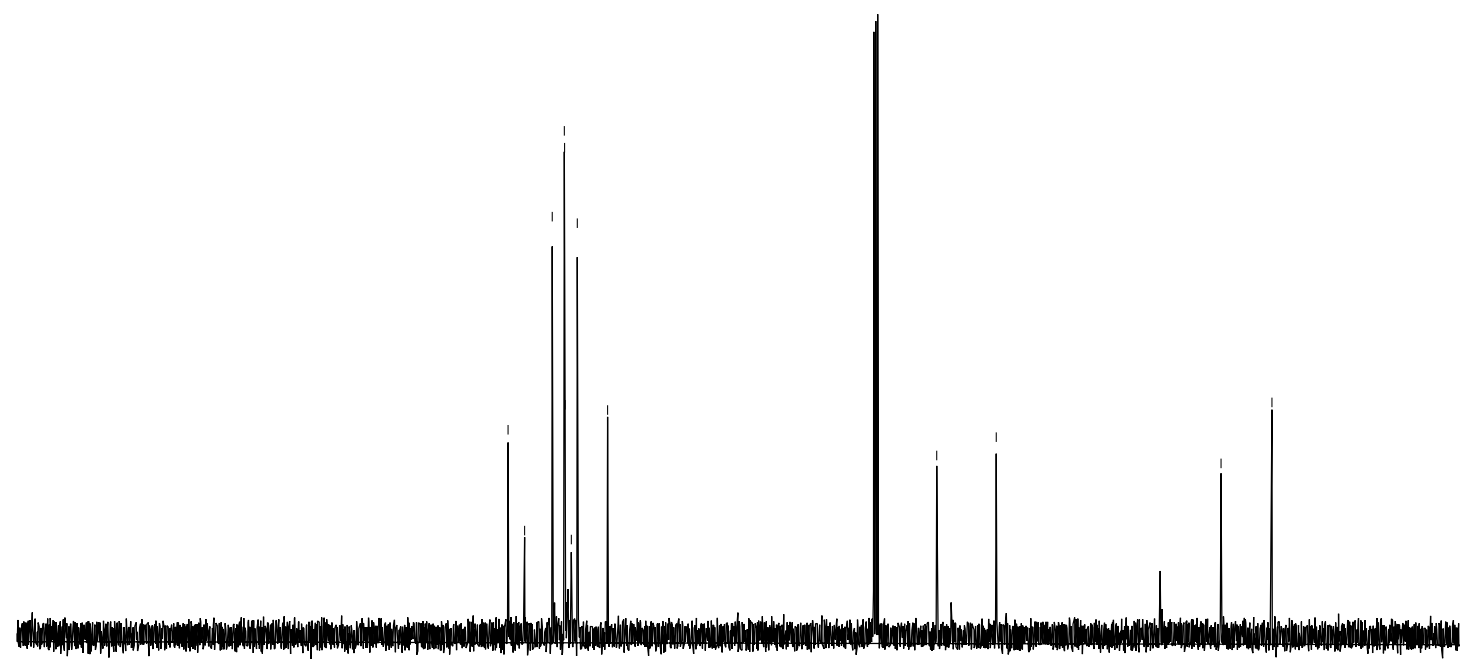

$\begin{array}{llllllllllllllll}210 & 190 & 170 & 150 & 130 & \begin{array}{c}110 \\ \mathrm{f} 1(\mathrm{ppm})\end{array} & 80 & 70 & 60 & 50 & 40 & 30 & 20 & 10 & 0 & -10\end{array}$


<smiles>CCC(/C=C/c1ccccc1)S(C)(=O)=O</smiles>

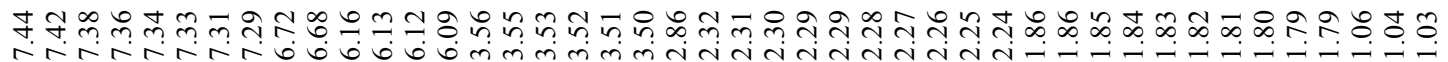

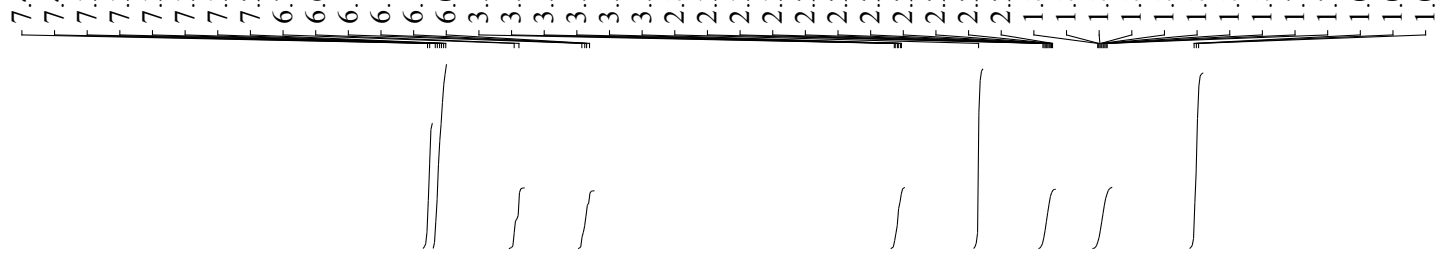

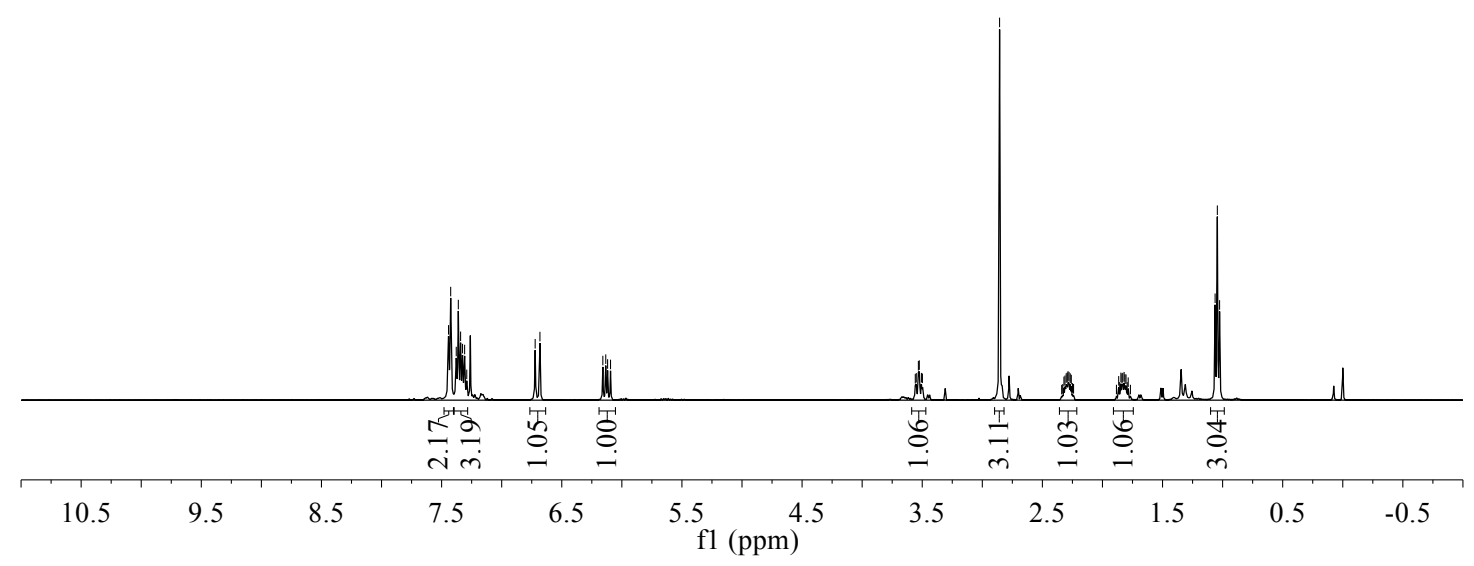

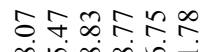

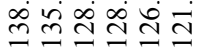

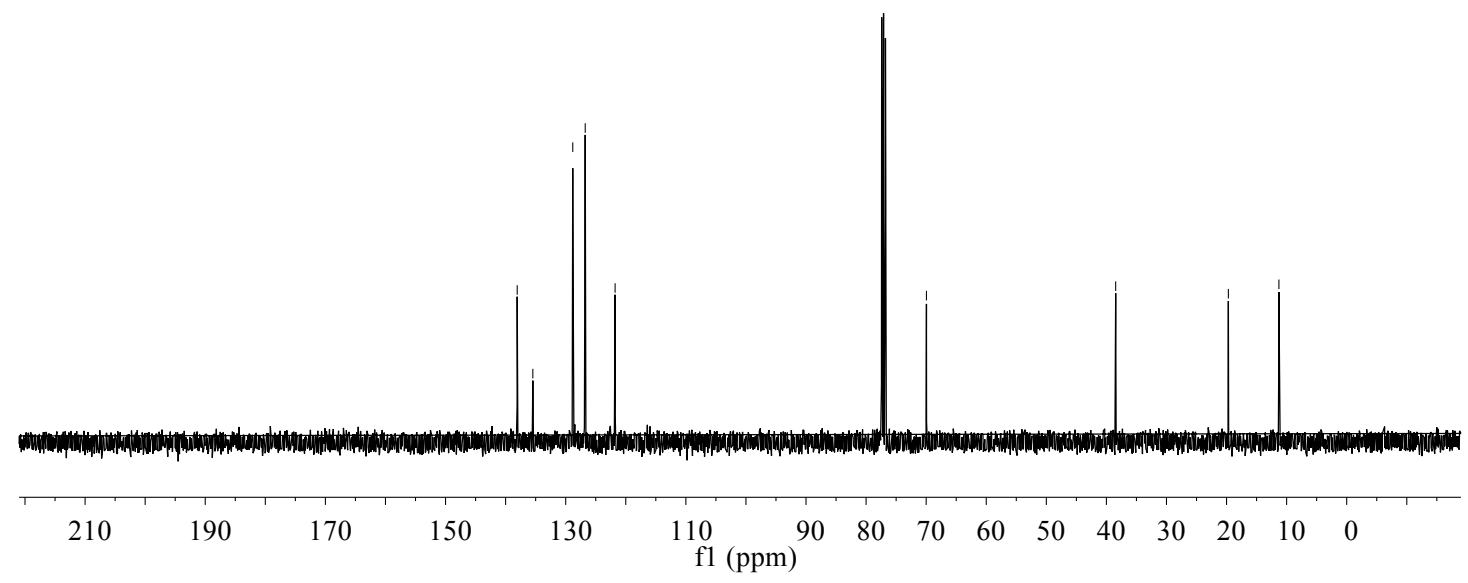




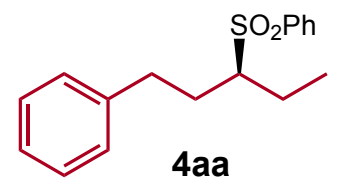

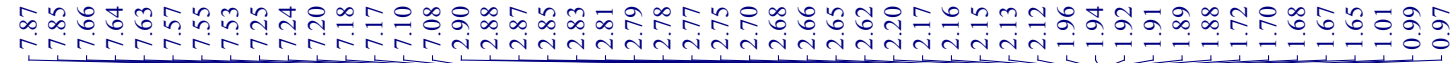
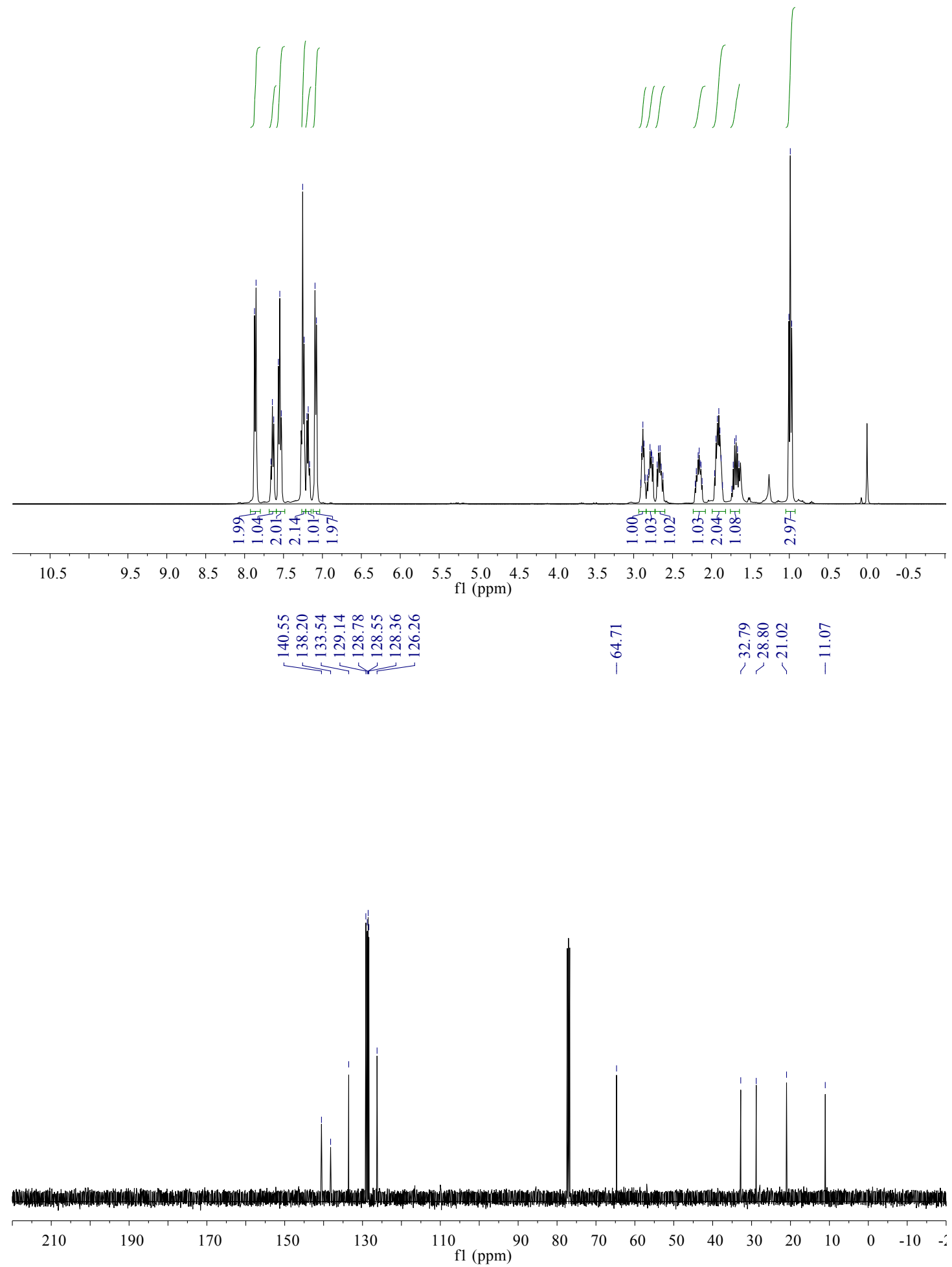
<smiles>CC[C@@H](CO)[Sb](=O)(=O)c1ccccc1</smiles>

$5 a a$

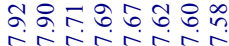

तN

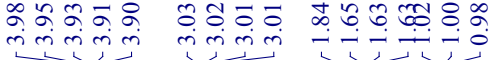
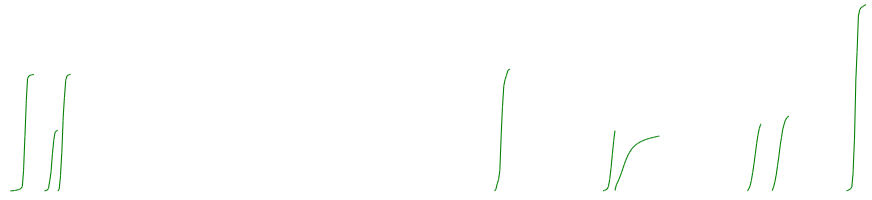
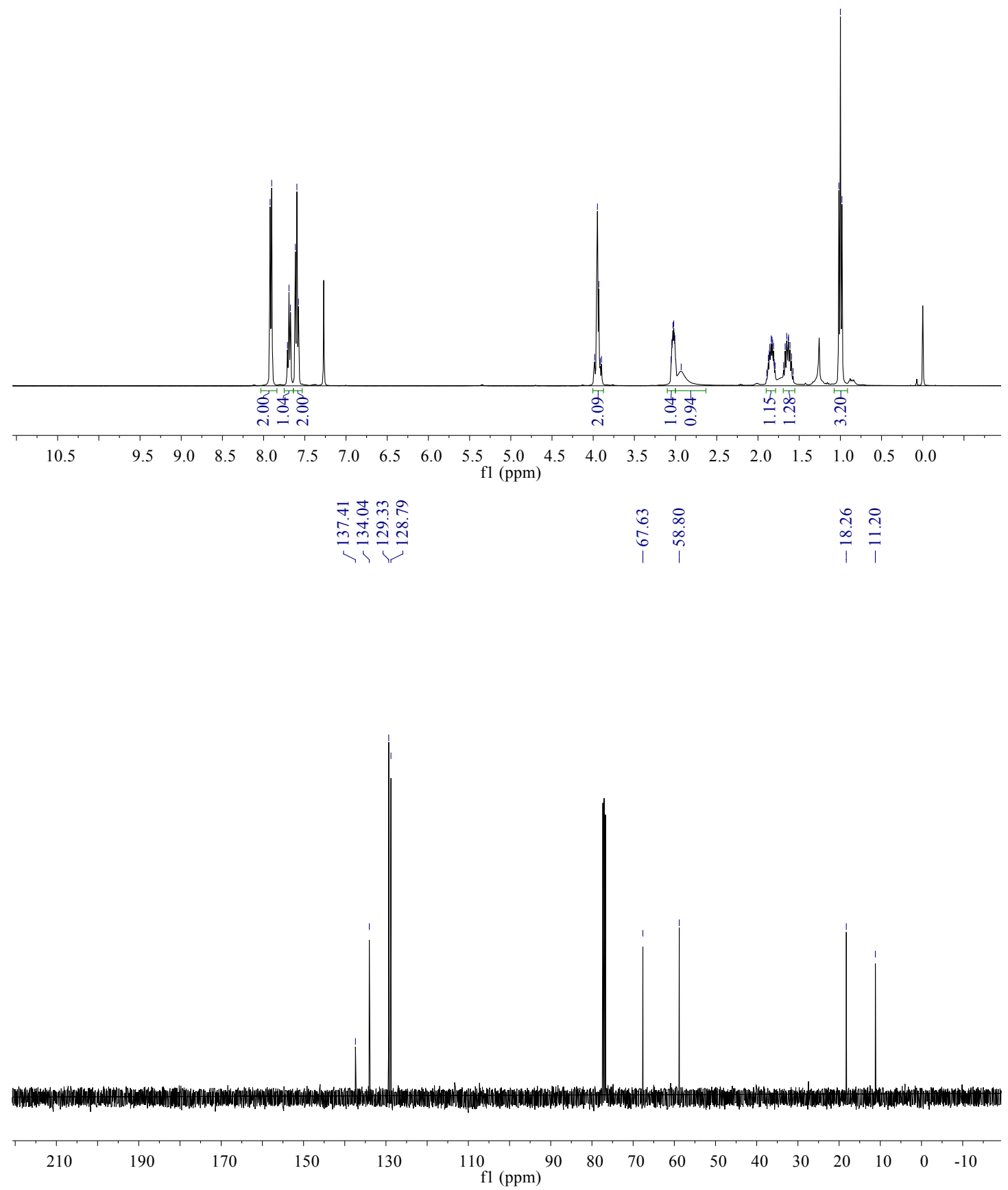
<smiles>CC[C@H](C1O[C@H]1c1ccccc1)[C@@]1(S(=O)(=O)c2ccccc2)O[C@@H]1c1ccccc1</smiles>

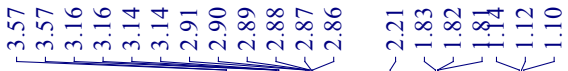
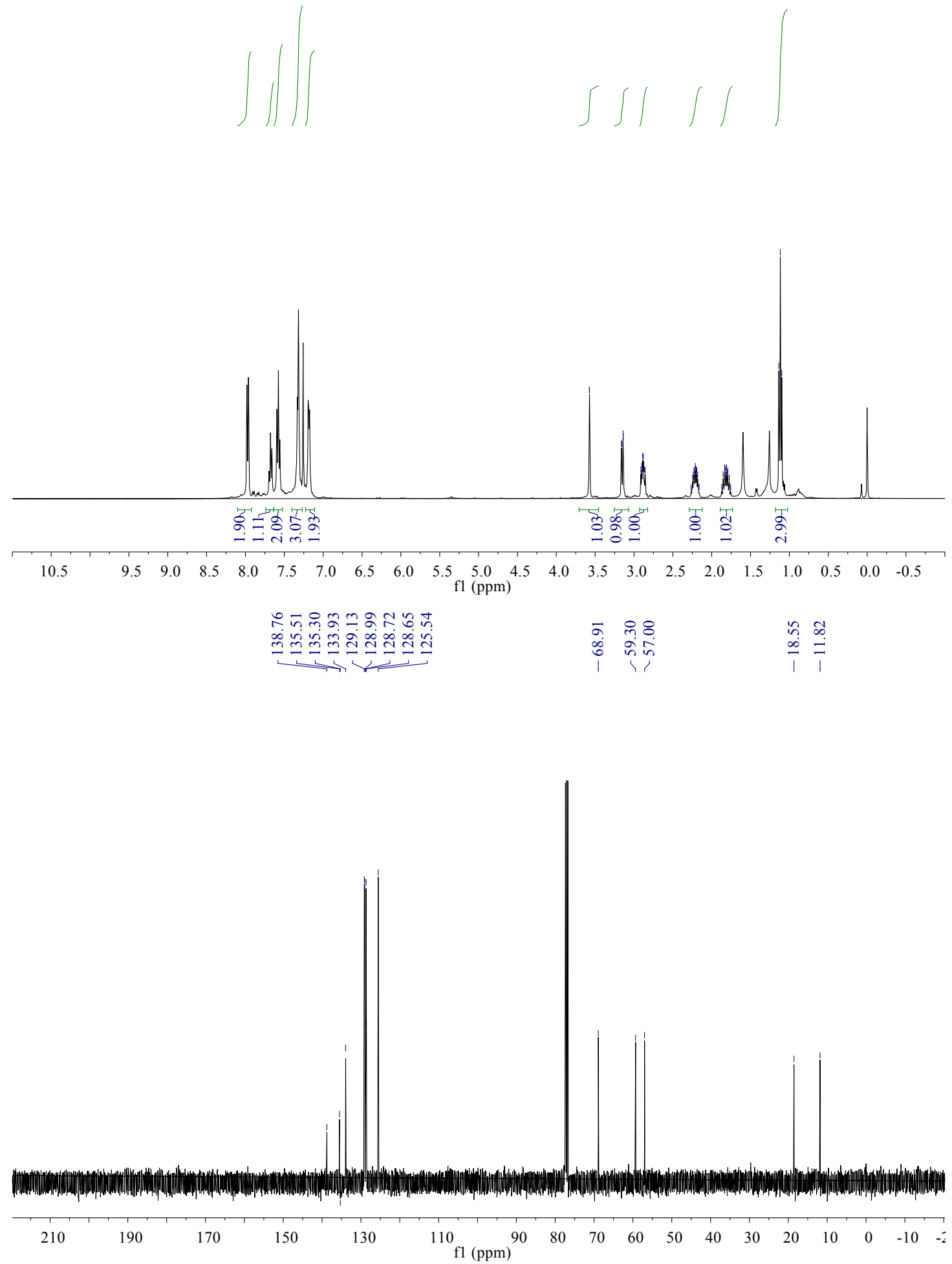